University of Louisville

ThinkIR: The University of Louisville's Institutional Repository

Electronic Theses and Dissertations

$5-2018$

\title{
The measurement of the adhesion of glaze ice.
}

Andrew Hardesty Work Jr.

University of Louisville

Follow this and additional works at: https://ir.library.louisville.edu/etd

Part of the Other Aerospace Engineering Commons

\section{Recommended Citation}

Work, Andrew Hardesty Jr., "The measurement of the adhesion of glaze ice." (2018). Electronic Theses and Dissertations. Paper 2987.

https://doi.org/10.18297/etd/2987

This Doctoral Dissertation is brought to you for free and open access by ThinkIR: The University of Louisville's Institutional Repository. It has been accepted for inclusion in Electronic Theses and Dissertations by an authorized administrator of ThinkIR: The University of Louisville's Institutional Repository. This title appears here courtesy of the author, who has retained all other copyrights. For more information, please contact thinkir@louisville.edu. 


\title{
THE MEASUREMENT OF THE ADHESION OF GLAZE ICE
}

\author{
By \\ Andrew Hardesty Work Jr. \\ B.S, University of Louisville 2011 \\ M.Eng., University of Louisville 2012
}

\begin{abstract}
A Dissertation
Submitted to the Faculty of the

J.B. Speed School of Engineering of the University of Louisville in Partial Fulfillment of the Requirements

for the Degree of

Doctor of Philosophy

in Mechanical Engineering

Department of Mechanical Engineering

University of Louisville

Louisville, Kentucky
\end{abstract}

May 2018 



\title{
THE MEASUREMENT OF THE ADHESION OF GLAZE ICE
}

\author{
By \\ Andrew Hardesty Work Jr. \\ B.S, University of Louisville 2011 \\ M.Eng., University of Louisville 2012 \\ A Dissertation Approved on
}

April 19, 2018

By the following Dissertation Committee:

Dr. Yongsheng Lian

Dr. Geoffrey Cobourn

Dr. Stuart Williams

Dr. Gerold Willing 


\section{ACKNOWLEDGEMENTS}

First and foremost, I would like to thank my advisor Dr. Yongsheng Lian for his support in advising me across two projects during my dissertation, and to Dr. Stuart Williams, Dr. Gerold Willing, and Dr. Geoffrey Cobourn for their guidance and support in serving on my technical committee.

I would like to thank the Icing Branch at NASA Glenn for their support of my work by providing countless hours of patient guidance; I would especially like to thank Mario Vargas for his reliable support, guidance, and encouragement. I would also like to thank Peter Struk and Paul Tsao for their support and mentorship. Thanks to Andy Broeren for his help in designing the XT model and for the other guidance he provided, to Chris Porter for his help in the aerodynamic design of the XT model, to Bill Wright for the numerous helpful discussions on LEWICE and past experiments performed in the IRT, and to Tom Ratvasky for his support. Thanks to Mary Wadel, Eric Kreeger, and Mark Potapczuk for their guidance and leadership, and to Justyna Ragiel-Smith for her extensive administrative support. I would also like to thank the RVLT and AATT projects for providing the opportunity to do the work, and to Tony Nerone for his help supporting my work. I would especially like to thank Susan Johnson for her flexibility in consistently going out of her way to support my work and planning ahead to account for the expenses due to my mistakes.

My thanks to Andy Gyekenyesi and Jon Salem for their extensive technical guidance and test support, and for catching many of my mistakes. Thanks to Dan Scheiman 
for his generous support in providing test data supporting the XT model, and to John Ramsey for his help in the mechanical analysis of the XT model.

I would like to thank Carl Blaser for his advice over the last two years and instrumental help designing and drafting components, as well as his general support in getting the parts produced. Thanks to Tim Heineke for his help in designing and machining components. Thanks to Quentin Schwinn and Jordan Salkin for their imaging support and discussions during piggyback testing. I would especially like to thank the exceptional IRT staff for their generous help during tests, especially Shaun McNea for his support during the first IRT test and making sure the test was successful.

I would also like to express my gratitude to my interns for supporting this work and their patience with my novice guidance: Tina Schirmer, Jaime McCarrell, Rebekah Doughlass, Zak Koster, Matthew Gazella, Jodi Turk, and Shawn McTaggart. I would also like to extend my thanks the Ohio Aerospace Institute for their support both in funding several of my students, and for their flexibility in helping collaboration on publications to occur. I would especially to Ann Heyward and Tony Smith.

I would like to thank my friends, Seth Tucker, Kyle Hord, Daniel Porter, Russ Prater, Guibo Li, and Yisen Guo, for their support and technical assistance throughout my time at the University of Louisville, for sharing their work with me, and for including me in their projects.

None of this would have been possible without the loving patience of my dear wife Mara, who has selflessly sacrificed years of effort to protect my time and keep me sane when I felt like I had lost my path. 


\section{ABSTRACT \\ THE MEASUREMENT OF THE ADHESION OF GLAZE ICE \\ Andrew Work \\ April $19^{\text {th }}, 2018$}

Icing is a lethal and costly aviation hazard affecting aircraft of all sizes ranging from small UAVs to large commercial craft such as the Boeing 787, resulting in hundreds of deaths over the last century and resulting in billions of dollars of economic impact. Three predominant types of icing, namely, airframe icing, engine icing, and rotorcraft icing, dominate aircraft icing research. Each type poses unique challenges. In the case of rotor icing, attention needs to give to the rotating environment with large oscillatory loads and icing conditions. In the case of airframe icing, special attention needs to be paid to a variety of loading cases from the available options for de-icing and anti-icing equipment. In the case of engine icing, the formation mechanism is poorly understood and the structure of the ice needs to be studied in greater detail. Airframe icing requires However, all three share the same fundamental problem that ice sticks to surfaces. How strongly ice adheres to a surface dictates how hard it is to remove. The adhesion strength then regulates the primary threat from icing - the maximum aerodynamic penalty that accretion will have. It also dictates the threat of impingement from shed ice elsewhere on the aircraft, such that a piece of ice from the main rotor could strike the tail rotor on a helicopter, destroying it. 
There exist many methods to evaluate the adhesion of ice to a given substrate, the most common being pusher tests and centrifuge tests. These and other methods are problematic in evaluating the adhesive properties of ice to a substrate in aircraft icing conditions; no data in the literature exists that accounts for stress concentrations at the interface and the strain rate at the interface, and no data was found in the literature on the grain structure of impact ice at speeds relevant to aircraft icing. A new method to measure the adhesion of impact ice has been developed based on a lap joint shear test. Lap joint tests are common in adhesion measurements since they produce nearly uniform stress at the interface of interest. In support of this end, a new shear rig and a new wind tunnel model for the Icing Research Tunnel were designed and fabricated. Six nights of testing in the Icing Research Tunnel were conducted to obtain samples, which were later tested in a laboratory environment. The tests were displacement controlled and samples were tested at four crosshead speed rates. The grain structure of the ice was documented using a crosspolarized optical microscope for the first two nights of testing, showing significant differences in the grain structure dependent on velocity and whether the cloud was in the SLD range or not. Correlations with temperature and test section velocity are demonstrated. It was also demonstrated that residual stresses, which are unaccounted for in the literature, play a significant role in the adhesion of impact ice. Options to improve the test methodology further are discussed. Finally, a shedding model predicting the trajectory of ice at a shed event has been developed and validated against test data. This model successfully predicted the front of a multi-break shed event in the Icing Research Tunnel. 


\section{TABLE OF CONTENTS}

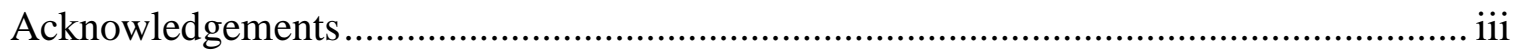

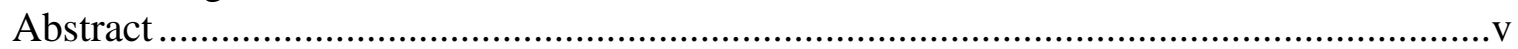

List of Tables

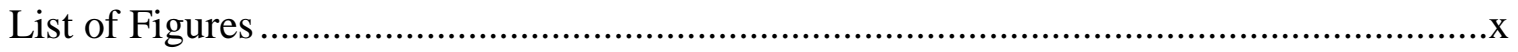

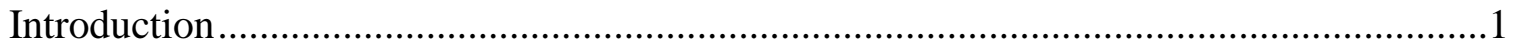

The High Cost of Aircraft Icing ............................................................................. 3

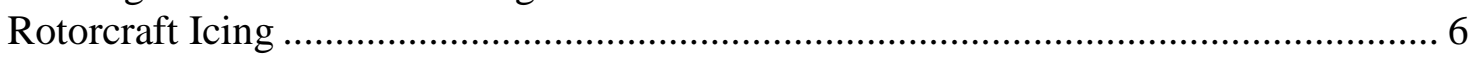

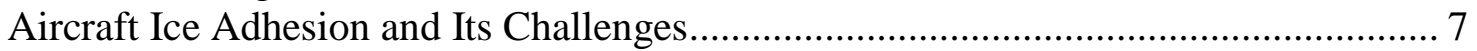

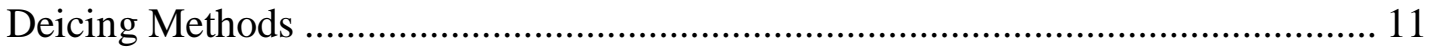

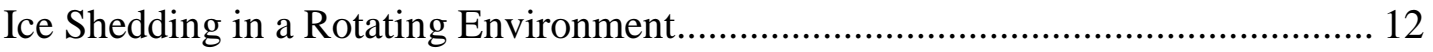

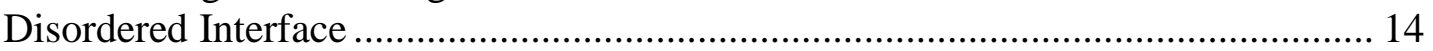

Roughness and Clamping ................................................................................. 15

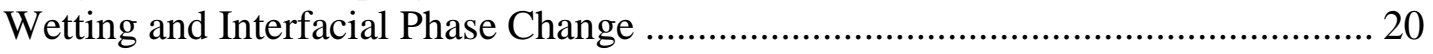

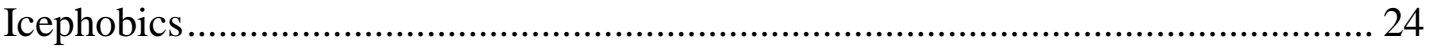

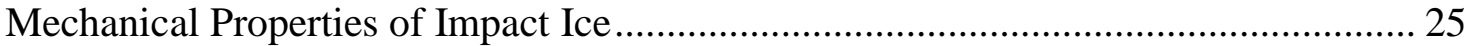

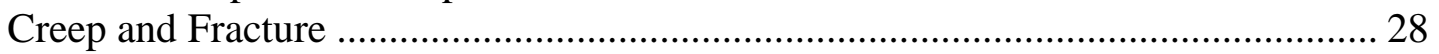

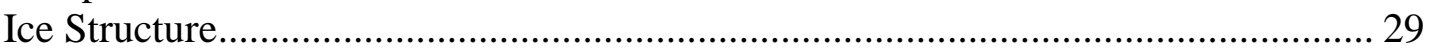

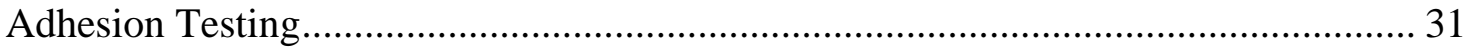

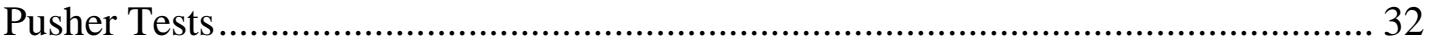

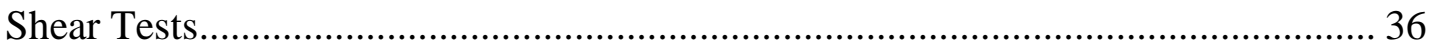

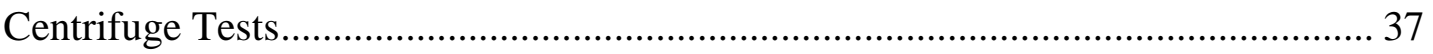

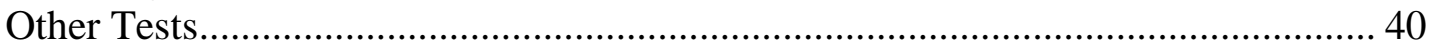

Comparison of Methods ............................................................................... 40

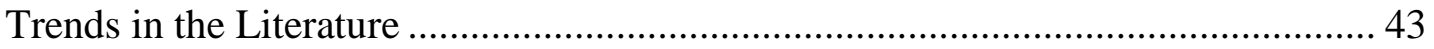

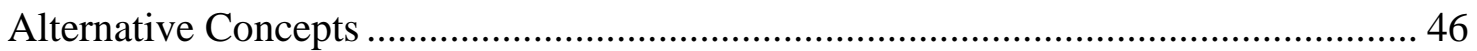

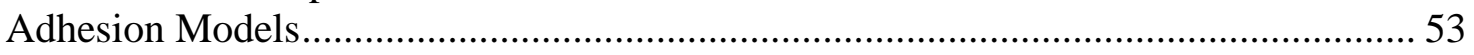

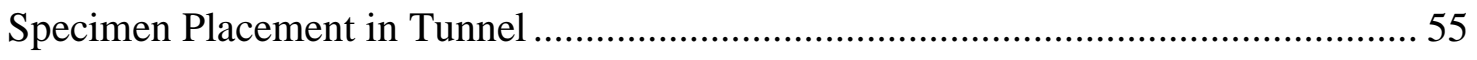

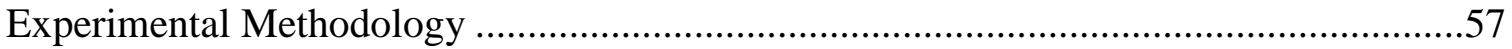

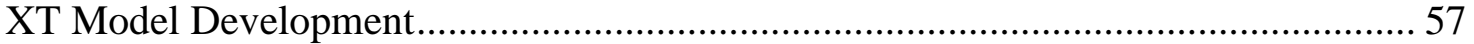

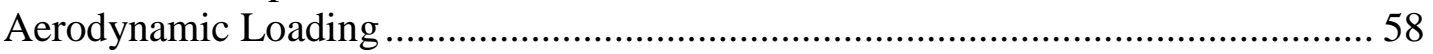

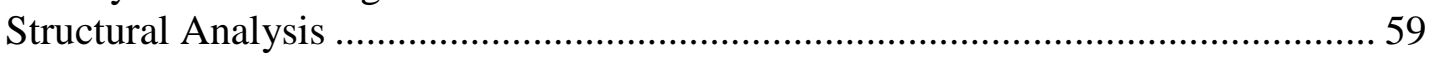

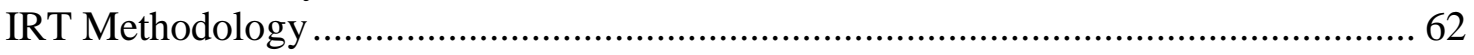

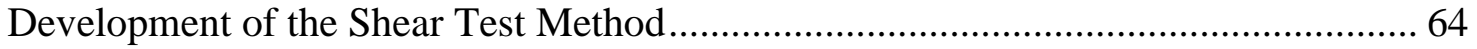

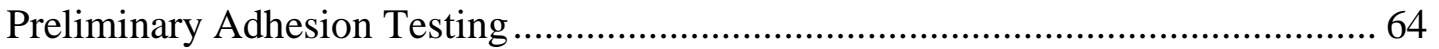

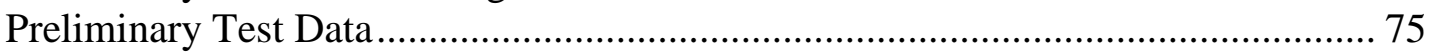

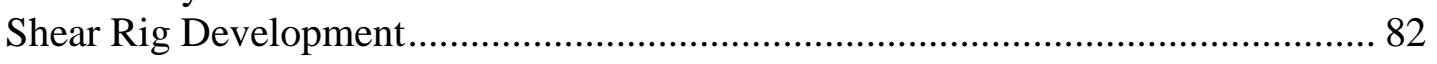

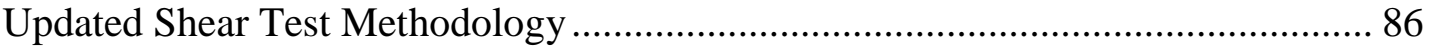

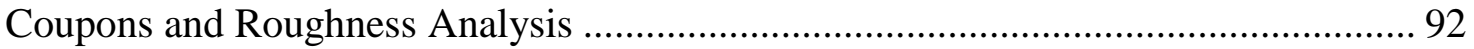

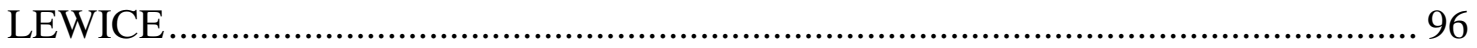




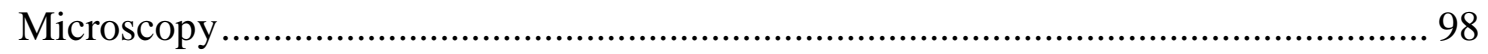

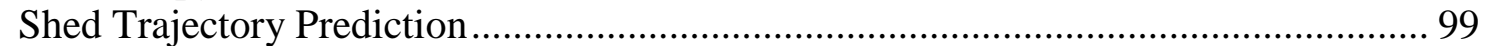

The Shedding Trajectory Analysis Tool............................................................. 100

The Shedding Trajectory Model.......................................................................... 104

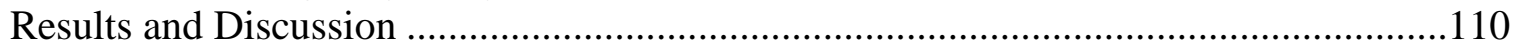

First IRT Test Entry Data and Results ................................................................. 110

First IRT Test Entry Grain Images .................................................................. 120

Second IRT Test Entry Data and Results........................................................ 125

Third IRT Test Entry Data and Results.................................................................. 151

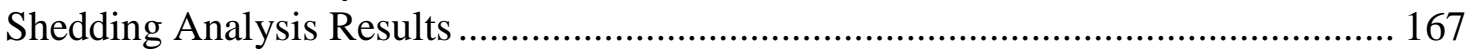

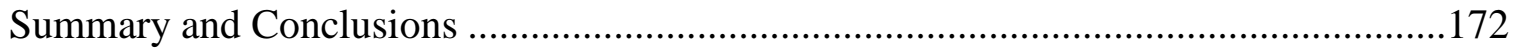

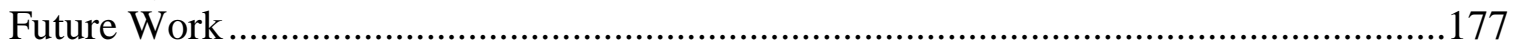

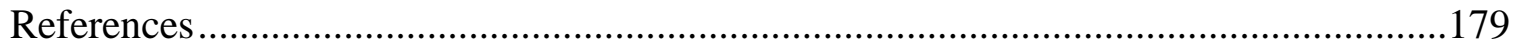

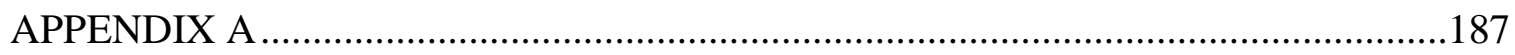

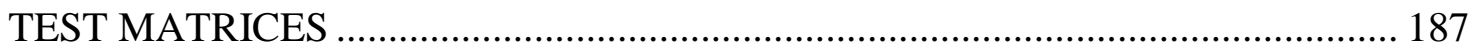

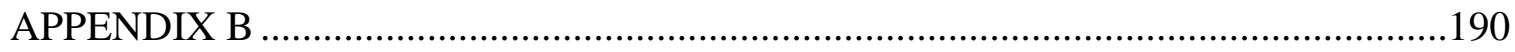

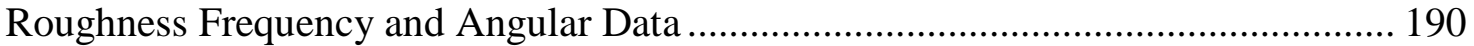

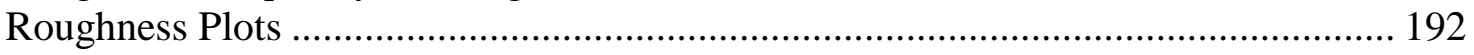

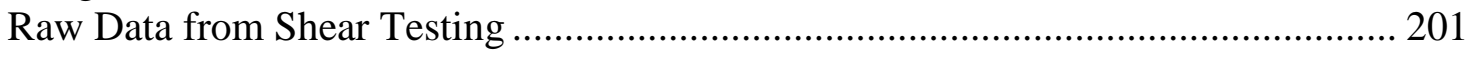

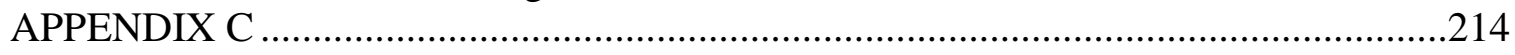

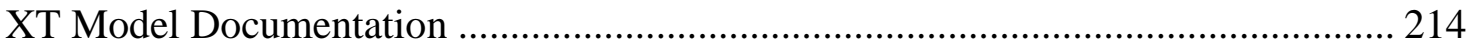

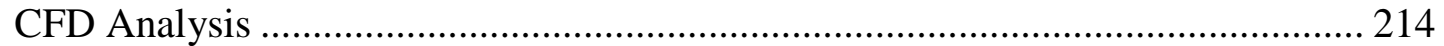

Material Properties for XT Model Simulation ................................................... 220

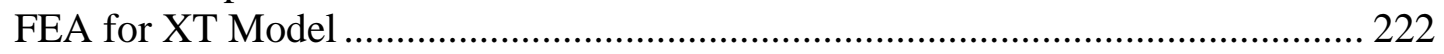

Sample EFAST Analysis .............................................................................. 226

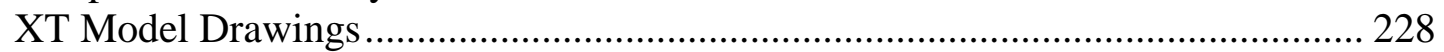

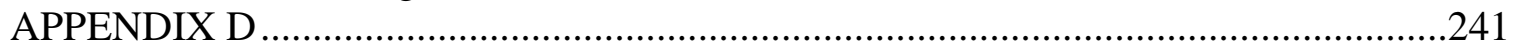

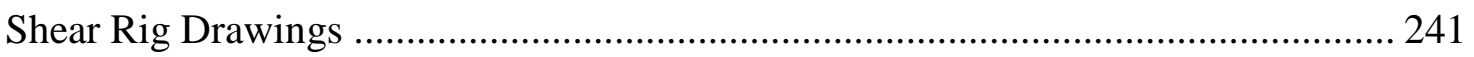

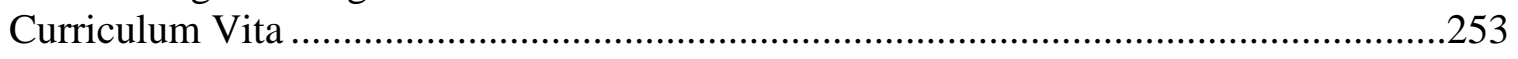

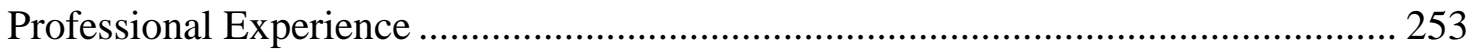

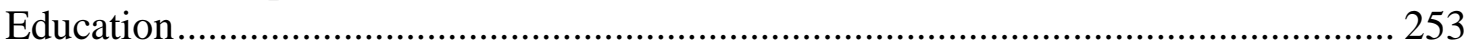

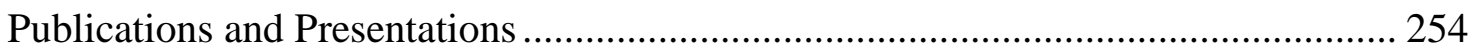




\section{LIST OF TABLES}

Table 1. Default Mechanical Properties of Ice Used...................................................... 27

Table 2. Comparison of Centrifuge and Lap-Jount tests. ............................................. 43

Table 3. Predicted load vs. temperature and ice thickness. .......................................... 59

Table 4. Material properties used for joint analysis. *calculated using von Mises failure

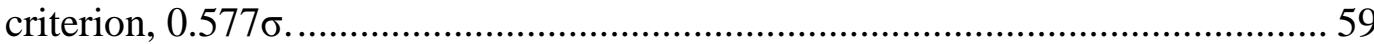

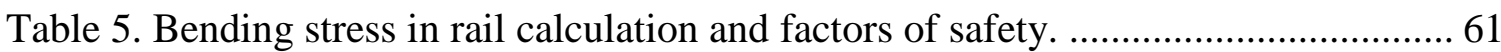

Table 6. Summary of Margin of Safety, and factors of safety for load bearing parts. ..... 62

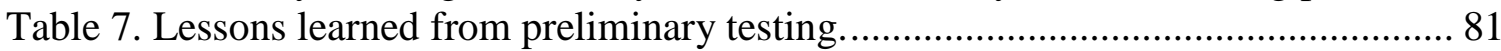

Table 8. Summary of Roughness Data Averaged by Magnification. ............................. 93

Table 9. Frequency and angle data for first five peaks for group 2 scans. ..................... 95

Table 10. Run parameters for LEWICE data. ITIMFL $=0, \mathrm{IFLO}=10, \mathrm{DSMN}=6.0 \mathrm{D}-4$.

Table 11. Standard deviations of data from IRT test entry 2 ..................................... 144

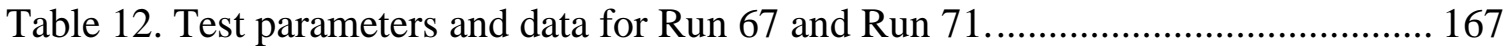

Table 13. Piggyback tests, 5/31/16, 8/15/16 ......................................................... 188

Table 14. IRT testing tunnel parameters, 10/2/17, 11/20/17, 1/17/18. AG: 4 sprays, 10, 20, 30, 60 s. AH: 2 sprays, 30, 120 s. AI: 2 sprays, 30, 150 s. AG, AH, AI: averaged data for first spray only............................................................................. 189

Table 15. Frequency data for the first five extracted points of all roughness scans...... 190

Table 16. Angular data for the first five extracted points of all roughness scans........... 191

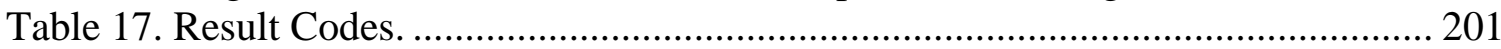

Table 18. Data from the primary IRT runs. ............................................................. 202

Table 19. Properties used for initial simulations, properties in parenthesis used for worstcase simulation. 


\section{LIST OF FIGURES}

Figure 1. Roughness zones demonstrating features that would likely result in different types of failure.

Figure 2. Simplified theoretical expansion of ice in a triangular roughness element. Top left: water in crevice. Top right: water expands into ice with no bonding. Bottom left: no slipping but substrate deforms freely. Bottom right: surface deforms, and stress concentrations form at corners and along surfaces. ................................ 18

Figure 3. The contact angle of water and Young's interfacial energies........................... 20

Figure 4. Wenzel vs. Cassie-Baxter wetting states..................................................... 22

Figure 5. Types of aircraft ice. Left: rime ice (8/19/16 run 3), Middle: glaze ice (8/18/16 run 1), Right: Glaze (8/15/16 run 1). Photographs by Quentin Schwin. .............. 30

Figure 6. Averaged results from push tests in the literature, used from [45]. St is Steel, Al is Aluminum, and Tef is for Teflon. ................................................................. 35

Figure 7. Averaged shear test results from the literature, used from [45]. St is Steel, Al is Aluminum, Poly is for Polyurethane, and Tef is for Teflon. .............................. 36

Figure 8. Averaged centrifuge test data, used from [45]. St is Steel, Al is Aluminum, and AERTS is the Adverse Environment Rotor Test Stand..................................... 39

Figure 9. Averaged aluminum data in the literature, used from [45]. ............................ 44

Figure 10. Averaged steel data in the literature, used from [45]. .................................. 45

Figure 11. A sample SSLJT block (left) and full test (right)...................................... 47

Figure 12. Sample simulation of SSLJT. In lieu of ice properties, aluminum was used for ice, while steel was used for the block and slat. Left: Von Mises stresses at the interface. Right: Strain in the block and ice shape............................................. 48

Figure 13. Preliminary diagram of the RTI method for determining ice adhesion.......... 49

Figure 14. RITA test area close up. Left to right: Test rod, Coupon, Lower Cylinder, and full assembly with Soft Inserts and Upper Cylinder........................................ 50

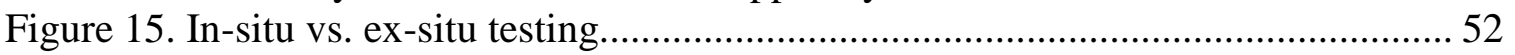

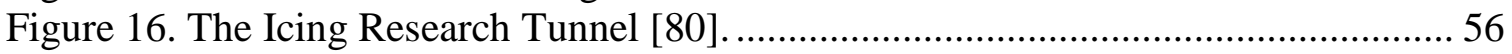

Figure 17. Tinius Olsen universal tester and supporting equipment schematic. .............. 65

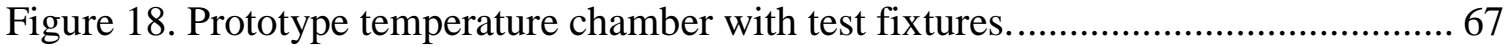

Figure 19. Impact ice samples grown in the IRT. Left: 3-minute run. Right: 20-minute run.

Figure 20. Test coupon mounted in the zipper slot of the IRT, aft of the primary test model.

Figure 21. Ice created using pipette on sub-freezing aluminum sample, 1 "x3"............ 72

Figure 22. The first ice adhesion test run on the Tinius Olsen Universal tester. Sample prepared on warm surface using pipette. Left: Adhered sample. Right: Post-test showing nearly pure adhesive break. ............................................................. 73

Figure 23. Data from second adhesion test. Sample prepared using pipette and sub-freezing

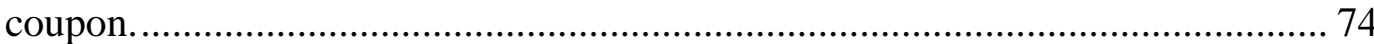


Figure 24. First set of lap-test results from first preliminary IRT test entry.................. 76

Figure 25. Results from second preliminary IRT test entry (8-15-16 - 8/19/16). Legend shows tests marked day \#, run \#, coupon \# (D\#, R\#, C\#) ................................... 78

Figure 26. Adhesion strength vs. coupon RMS roughness........................................ 79

Figure 27. Adhesion strength vs. difference in block temperature (environmental chamber)

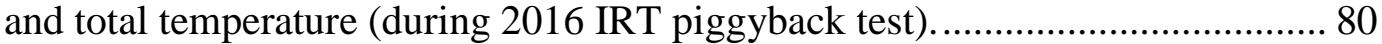

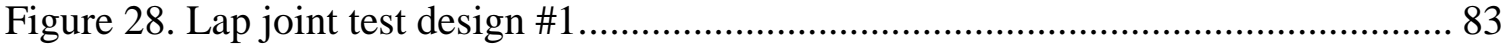

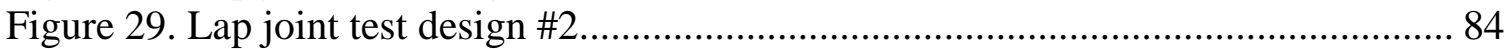

Figure 30. Left: completed shear rig. Right: shear rig installed in test equipment.......... 85

Figure 31. Sample B1. Left: mounted sample pre-break, right: post-break. .................. 87

Figure 32. Shear rig with addition of cooling block and deflectometers........................ 90

Figure 33. Profilometer stage with coupon, middle low-resolution scan. ...................... 93

Figure 34. Sample \#1 Group \#2, top: height data (X direction is along coupon long axis),

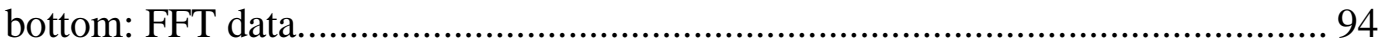

Figure 35. Image of coupon (top) on mount showing inconsistent large radius in corners.

Figure 36. LEWICE runs for first IRT test entry in October 2017. Flow from left to right.

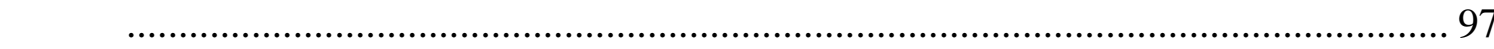

Figure 37. Preliminary images of ice grain structure from IRT ice. Left: glaze ice (8/18/16

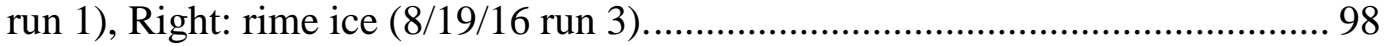

Figure 38. Rotor stand inside test section of IRT in 2013 tests [168]. .......................... 100

Figure 39. Flowchart overview of the STAT script............................................... 101

Figure 40. Top: Original image from Run 71. Bottom: Perspective corrected image.... 102

Figure 41. Superposed processed images from Run 71. Gray circle represents boundary of the rotor disk. Ice outside the rotor disk is binarized..................................... 103

Figure 42. Coordinate system for model. The $\mathrm{X}$ axis rotates with the rotor blade ice is shed from. The ice break starts at $d_{1}$ and ends at $d_{2}$, while the length of the blade ( $L$, not shown) may exceed $d_{2}$.............................................................................. 104

Figure 43. Illustration of $\mathrm{A}$ and $\mathrm{W}$ variables. $\mathrm{A}$ is the cross-sectional area of the ice (light blue area), and W is the length of the red line. Gray area is a cropped NACA 0012

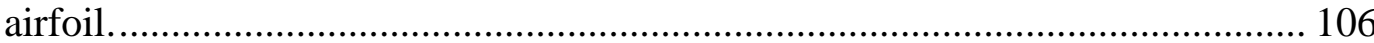

Figure 44. Force-position curves for four typical samples. ................................... 111

Figure 45. Force-time curve for sample H23, constant crosshead speed $0.02 \mathrm{~mm} / \mathrm{min} .112$

Figure 46. Dependence of adhesion strength on time to test, at crosshead speed of 0.2

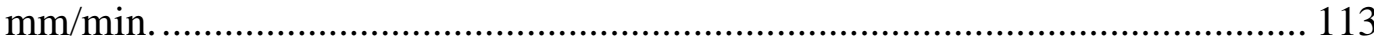

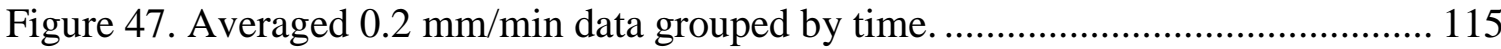
Figure 48. Average Ultimate Strength vs. Crosshead Speed, initial test sets excluded.. 116 Figure 49. Percent standard deviations for adhesion strength vs. crosshead speed, first IRT

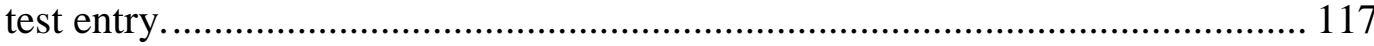

Figure 50. Average Ultimate Strength vs. Wind Speed at $25 \mu \mathrm{m}$ MVD...................... 118

Figure 51. Average Ultimate Strength vs. Mean Volumetric Diameter of droplet size distribution, 150 knots tunnel speed. .......................................................... 119

Figure 52. Stitched grain images for IRT runs A-D. The nominal velocities were 125, 150, 175, and 200 knots, respectively. The nominal MVD was $20 \mu \mathrm{m}$. 120 
Figure 53. Stitched grain images for IRT runs E-J. The nominal MVD was 15, 25, 30, 40, 50 , and $180 \mu \mathrm{m}$, for runs $\mathrm{E}, \mathrm{I}, \mathrm{F}, \mathrm{J}, \mathrm{G}$, and $\mathrm{H}$, respectively. The nominal velocity was 150 knots. 122

Figure 54. Sample E15. Left: image taken immediately after cut. Right: image taken one day later. 124

Figure 55. Left: Test section temperature vs. sample count. Right: Average ice sample thickness vs. sample count......................................................................... 126

Figure 56. Frost on sample K11 (top) and W6 (bottom). Some frost sublimated before pictures could be taken................................................................................. 127

Figure 57. Temperature profile of three coupons in run K during spray...................... 128

Figure 58. Sample W13 showed relative motion between the ice and coupon and was bonded on removal................................................................................. 129

Figure 59. Thermal images of ice melting, with time shown in top left of each frame.. 132 Figure 60. Temperature history for two samples during shear test. Left: sample K23 (40s heat, 10s cool). Right: sample M15 (101s heat, 7s cool). Shared vertical axis. . 133

Figure 61. Force-time plot for selected samples during shear test, raw data................. 135

Figure 62. Depiction of idealized ice-serrated block interface. Left: interface not under compression. Right: interface under compression. Teeth to scale, water layer not to

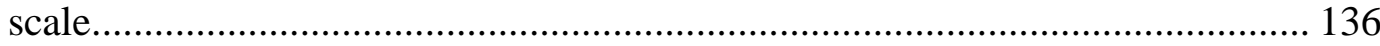

Figure 63. Delta deflectometer and encoder positions for selected shear tests. ............ 138

Figure 64. Stress-strain diagram using delta deflectometer data and encoder data....... 139

Figure 65. Histogram of results from $\mathrm{O}$ and $\mathrm{P}$ runs after being placed in storage for more than 1000 hours at $10^{\circ} \mathrm{C}$

Figure 66. Strength vs. time. Top left: averaged raw data. Top right: averaged data sans outliers $(<\lambda-\sigma)$. Bottom left: averaged data sans outliers $(<$ maximum $-2 \sigma)$. Bottom right: maximum values. ............................................................................ 142

Figure 67. Apparent failure stress of all data with no outliers removed grouped by annealing time. 143

Figure 68. Longest stored data points with trend lines from IRT test entry 2.............. 145

Figure 69. Data taken during the night of the IRT tests for IRT test entry 2................ 146

Figure 70. Parametric analysis of IRT test entry 2 data, using data $>\lambda-\sigma$ for averaged results. Long-term storage results............................................................ 147

Figure 71. Parametric analysis of IRT test entry 2 data, using data $>\lambda-\sigma$ for averaged results. Short-term storage results........................................................ 148

Figure 72. Failure stress vs. sample ice thickness. Top: four conditions with multiple data points. Bottom: Series $\mathrm{O}$ and $\mathrm{P}\left(-7^{\circ} \mathrm{C}, 20 \mu \mathrm{m},<65 \mathrm{~d}\right)$ individual repeats.......... 150

Figure 73. XT model in tunnel after run $\mathrm{AB}$, a glaze condition................................ 152 Figure 74. XT model in test section after run AJ, a rime condition. ............................ 153 Figure 75. Top down view of samples from three runs. From left to right: AB1, AB2, AD1,

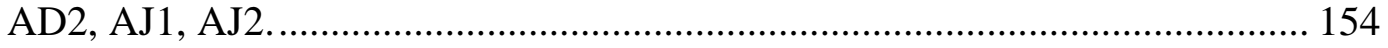

Figure 76. Side and front views of three samples. From left to right: AB5, AD5, AJ5. 155 Figure 77. Sample delaminated during run AD showing marks near either end at icecoupon interface.................................................................................. 155

Figure 78. Sample AB10 post-test showing frost in the middle of the sample. ............. 156

Figure 79. Temperature data for runs AG-AJ with spray on, thermocouple in dummy

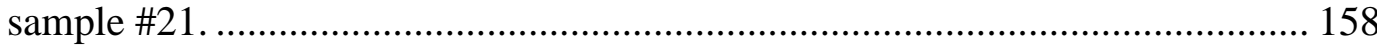


Figure 80. Sample AF17 repeated loading data (excluded from aggregate data analysis).

Figure 81. Surface cohesive break on sample AG15, post-test. ................................. 161

Figure 82. Sample AG9 machined to a cuboid shape, pre-test.................................. 162

Figure 83. Results from runs AB-AF showing variation in adhesion strength with velocity. 163

Figure 84. Results from runs AG-AI. Top: average measured apparent failure stress. Bottom: maximum measured apparent failure stress. Cuboid samples circled in red. 165

Figure 85. Sample thickness vs. average measured failure stress. Cuboid samples circled in red. 166

Figure 86. Unfit model compared to shed in Run 71. $\mathrm{B}=0, \mathrm{~F}=0, \mathrm{c}=0, \theta=0$. Blue, red, and green lines represent first, second, and third pieces of ice respectively. Yellow lines are drawn from centroids in the $5^{\text {th }}$ image to a tangent point on the rotor disk. 168

Figure 87. Left: $\mathrm{F}=0, \mathrm{~B}=60, \mathrm{c}=0.0004, \theta=0$. Right: $\mathrm{F}=0, \mathrm{~B}=60, \mathrm{c}=0, \theta=0$. Dotted cyan line is predicted front for corresponding singular shed event. 169

Figure 88. Left: Shed 1, $F=0, B=60, c=0, \theta=0$. Center: Shed 1, $F=0, B=140, c=0$, $\theta=4$. Right: Shed 2, $\mathrm{F}=0, \mathrm{~B}=60, \mathrm{c}=0, \theta=0$.

Figure 89. Lowest resolution mesh for model without coupon, with plate (A3A dp0). 150,000 nodes.

Figure 90. Resultant forces for case A3A dp3. 216

Figure 91. Grid convergence results for mean and oscillating forces at $155 \mathrm{~m} / \mathrm{s}$ on iced geometries.

Figure 92. Grid convergence results for mean and oscillating forces at $155 \mathrm{~m} / \mathrm{s}$ on clean

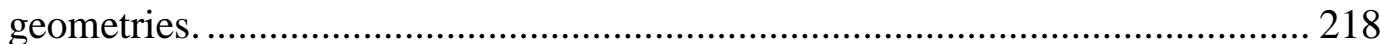

Figure 93. Velocity prediction of FEA code without coupon and rounded edges.......... 219 Figure 94. Sample data from DMA Q800 on polyurethane samples from McMaster-Carr.

Figure 95. Preliminary static results for XT model. 223

Figure 96. Harmonic Response for XT Model using maximum computed loads from CFD. 224

Figure 97. Frequency Response for Y axis stresses (worst case). 225 


\section{INTRODUCTION}

Icing occurs when water droplets in air are deposited on a surface and freeze. This can happen from meteorological conditions, such as rain, or from other sources, such as sea spray. While icing poses problems to power lines, runways, walking paths, and wind turbines, the research focus has been predominately in the areas of aircraft and sea icing. Sea icing on ships predates the existence of aircraft and presents a severe problem to ships travelling in colder climates; it was also more readily sampled than aircraft icing - thus the phenomena has been studied in greater detail. In contrast to sea icing, the mechanical and micro-properties of aircraft icing are poorly understood. Moreover, aircraft are far more sensitive to icing because flight is more sensitive to aerodynamic force and aircraft weight changes than are ships. On aircraft, icing can reduce thrust and lift, increase drag and weight, and foul critical instrumentation such as pitot tubes. The rate, shape, and location at which ice accretes on aircraft are all important factors that critically influence flight safety. Designing aircraft to prevent and survive icing has also been difficult due to weight, power, and cost restrictions. In contrast to sea icing, aircraft icing mitigation has also been problematic. Workers cannot manually deice airplanes mid-flight as in ship icing, and systems designed to deice planes must be light, preserve the aerodynamic performance of the aircraft, be cost-effective, and fit within the energy envelope of the aircraft while being effective at removing ice in a wide variety of conditions. Hence, aircraft icing stands out as having posed a greater challenge through both the technical complexity and the severity of the problem, and care must be taken in treating the differences. 
Despite decades of research, outstanding challenges remain, and new challenges emerge. First, several high profile accidents [1-3] and the recent discovery of engine icing [4] have led to an increasing focus on icing as a hazard to aircraft. The FAA has passed new regulations to deal with Supercooled Large Drop (SLD) icing [5] and is expected to do so for engine icing as well. With the investigation of new SLD conditions, people have realized that conventional deicing systems are not adequate in all conditions [2] and new measures must be taken to prevent SLD icing. Second, with the discovery of engine icing and continued focus in the rotorcraft industry to protect against icing, natural shedding of ice is a phenomenon that must be understood. As ice builds up on rotating equipment, it will reach a critical mass at which point the adhesive forces are not enough to counter the centripetal forces leading to a shed event. The ice can leave a rotor blade and strike other parts of a helicopter or shed off a turbine rotor and fly through the engine, damaging it. Understanding the adhesion of ice is central to dealing with these areas of aircraft icing. State-of-the-art icing simulation software has no capability to accurately predict the shedding of ice $[6,7]$ and new models are required to accurately predict the ice shedding.

Aircraft icing is challenging because it is different from other types of icing for several key reasons. The ice accretion in aircraft icing is due to high speed droplet impact, which introduces several critical mechanisms. As droplets in a cloud approach a wing, they may break up before impact [8], but will ultimately strike the craft at a higher speed than in other forms of icing. This can lead to penetration into surface roughness elements not seen in other forms of icing, potentially transitioning the wetting state of the supercooled liquid from a Cassie-Baxter to a Wenzel wetting state (discussed in the following sections). This causes several problems such as mechanical clamping, and the destruction of 
icephobic surfaces - especially through repeated icing events. In general, the wetting of liquid water on a surface relates directly to ice adhesion [9]. This change in wetting states can, however, make ice adhere very well to hydrophobic surfaces. ${ }^{1}$ Aircraft icing can occur at a variety of altitudes and is highly sensitive to weather conditions, resulting in ice with drastically varying properties.

The study of aircraft icing has largely centered on the prediction of the shape ice will take on an aircraft. NASA currently uses the Icing Research Tunnel (IRT) located at Glenn Research Center (GRC) to perform experiments to record the shape ice will take on different airfoils. The shape of the ice has been recorded and used to validate the predictive code developed by NASA called LEWICE $[6,10]$. Data has also been used to develop scaling laws to allow comparison between ice shapes grown on airfoils of different sizes [11-13]. Historically, NASA has not focused much research on the adhesion of ice to substrates, though work in this area was some of the earliest done by NASA (and its' predecessor NACA) and has received sporadic attention in the IRT [14-18].

\section{The High Cost of Aircraft Icing}

To understand the motivation for studying aircraft icing and the needs of the industry, it is necessary to understand what cost icing exacts upon the aerospace community. Unfortunately, the highest cost has been paid in the lives of crash victims. Between 1982 and 2000, 583 accidents claimed more than 800 lives [19]. Most of these accidents occurred in personal or recreational flights, while only six accidents occurred on large aircraft with more than 10 passengers. Two accidents in particular have provided

\footnotetext{
${ }^{1}$ The effects of liquid water wetting on ice adhesion is discussed in more detail in the Wetting and Interfacial Phase Change section.
} 
sobering reminders that icing is a problem currently not understood sufficiently to ensure the safety of even large aircraft: the 1982 Air Florida Flight 90 in Washington D.C. [19, 20], and the 1994 American Eagle Flight 4184 in Roselawn, Indiana [1, 21]. The Roselawn case was particularly troubling since ice formed behind the pneumatic boots that protected the aircraft, an occurrence caused by SLD icing.

Several studies have been performed to analyze the statistics behind icing accidents, with the best known of these being that by Green [21]. Between 1978 and 2002, Green found 645 accidents and incidents related to icing in the NTSB Accident Database and Synopses and the FAA Accidents/Incidents Data System, and an additional 299 NASA Aviation Safety Reporting System reports [21]. Some misinterpretation was likely to be present in the results due to lack of available knowledge. For example, engine icing wasn't recognized as a significant problem until 2006 [4]. Also, only certain information was available after icing events. Icing conditions are difficult to measure even in controlled environments [22, 23], while human reports are often subjective.

Even in conditions where icing may be mitigated by ice protection systems, icing may still provide the opportunity for disaster. In 2009, icing caused the stall and crash of Colgan Air Flight 3407 in New York. In this case, the pilot responded incorrectly to the stall event by not applying full power to prevent stall [2]. Due to the possibility of human error, even if icing could be completely mitigated, wider safety margins would still be beneficial. A more recent study examining accidents and incidents between 2006 and 2010 found 228 accidents and incidents, with 40 accidents occurring inflight with icing on the wings, fuselage, or control surfaces [24]. Unfortunately, it is likely that aircraft icing will continue to be a major concern well into the future. 
The cost to life was the most obvious cost associated with icing, and this has certainly been the highest cost paid. Icing also impacts the aerospace industry financially in a significant way through mandatory investment into ground deicing, ice protection systems, regulatory requirements, meteorology, expenses related to accidents, delayed flights, and even lost sales due to safety concerns. Recently, engine icing concerns caused Japan Airlines to remove the Boeing 787 from certain routes, a move which has significant financial implications [25]. Delayed flights are a commonplace nuisance to travelers, but to the intricately controlled scheduling that exists in the aerospace industry, it has been a massive problem [26, 27]. Flight disruptions (from all sources) in 1998 are estimated to have cost the top 10 United States airlines over $\$ 1.8$ billion [28], and in 2014 reached $\$ 4.2$ billion while averaging $\$ 2.4$ billion between 2009 and 2014 [29]. This is an inevitable expense as long as aircraft and runways need to be deiced and planes must stay grounded for fear of icing. However, separating the costs due to the potential for in-flight icing and ground deicing, let alone determining the total cost of inflight icing, was most likely impossible.

In response to the threat posed by inflight icing, the FAA regulates the industry. The FAA has outlined atmospheric icing conditions and outlines sets of regulations grouping icing conditions together in envelopes. Conditions traditionally understood to cause in-flight icing are outlined in 14 CFR Part 25 Appendix C [30]. More recently, the FAA updated Part 25 to include Appendix O to address SLD icing [5]. Appendix O also defines the meteorology conditions to define an SLD icing envelope, and requirements on how to deal with this. Part 25 was amended to include Appendix O in 2014, 20 years after 
American Eagle Flight 4184, giving the industry sufficient time to meet the requirements. Currently, the FAA does not have regulations relating to ice-crystal engine icing.

\section{Rotorcraft Icing}

Rotorcraft icing is a subject that has obtained much less attention than fixed-wing icing. This is due to the fact that rotorcraft make up a much smaller portion of the market than fixed-wing aircraft, and primarily serve in utility roles. Unfortunately, rotorcraft accidents caused by icing are not uncommon. A search on the NTSB Aviation Accident Database for the term "icing" for helicopters yields 143 incidents since 1964 [31]: 33 of these accidents were fatal, while 19 involved the destruction of the helicopter involved. It is important to note that the accidents only list a probable cause. Not all of these accidents were exclusively due to icing, but icing contributed in some way. An example case is the 2013 crash in Clear Lake Iowa, where a Bell 407 medical helicopter flew into icing conditions. Evidence at the crash suggests that the engine anti-ice status light was illuminated, and icing conditions were present. The NTSB determination for the probable cause of the accident was "The pilot's inadvertent encounter with localized icing conditions and his subsequent in-flight loss of helicopter control." Icing on rotor blades has been shown to be a significant problem to safety [32-35]. In response, a variety of anti-icing equipment has been utilized and tested, with electro-thermal and pneumatic systems showing the most promise [36]. Still, only large helicopters can utilize anti-icing equipment while small rotorcraft are left unprotected. The expense of certification and testing new equipment has proven problematic to developing better ice protection systems for helicopters. In response, several icing codes have been modified to simulate helicopter icing including LEWICE and FENSAP-ICE [7, 35]. 
Several large-scale efforts have been made to advance the state of the art protection for rotorcraft. In the mid-1980's, a large number of parties worked together on the UH-1H helicopter icing flight test program [37-39]. NASA, the U.S. Army, the U.S. Air Force, the Canadian National Research Council (NRC), BF Goodrich, Bell, and others worked together in a massive effort to study helicopter icing and to test pneumatic boots for use on rotorcraft. These collaborative tests have continued at NASA with Sikorsky well into the 1990's with numerous studies on rotor performance [35, 40-44].

\section{Aircraft Ice Adhesion and Its Challenges}

Adhesion is one of the fundamental problems of icing: if ice didn't stick to the airplane on impact, there would be no problem of icing. Correspondingly, adhesion is a central issue in every type of icing. In spite of this, adhesion has often been overlooked or taken for granted. Ice adhesion can be treated as binary when studying many concerns in icing since ice either sticks or it does not. Much of the effort to account for icing has been to evaluate what the aerodynamic impact of icing was under the worst-case conditions. The primary concern has been the shape of the ice, which was often determined by the duration of icing and the properties of the icing cloud rather than the strength of ice adhesion. While the shedding of ice strongly affects the shape in rotating conditions, the adhesion of ice has been sufficient in icing on fixed wing aircraft that the ice will remain attached on the structure well past aircraft failure in most conditions. Hence adhesion may often be assumed and ignored. However, in rotating environments and in deicing this may not be assumed since the adhesion directly affects the maximum size an ice shape can take.

To understand when ice will shed off a rotor blade, several key pieces of information must fall in place. Perhaps the most difficult piece of information to obtain has 
been the local adhesion between an ice shape and a rotor blade at any point on the interface. Constitutive properties also need to be identified. In performing failure analysis, stresses internal to the ice shape and at the interface must be calculated and compared to these strengths in order to determine when ice will separate from a rotor. The primary focus of this work was the measurement of the adhesion of ice and predicting the shedding of ice in the context of helicopter rotors, laying the foundations for the development of an adhesion model as a tool to predict shedding events.

Little data has been taken on the adhesion strength of aircraft ice and the data that was available was of poor quality due to the technical challenges encountered during measurement [45]. This is problematic for three reasons. First, adhesion data is critical to understanding the removal of ice, which is of critical importance to aircraft flying in icing conditions. Second, understanding ice adhesion is also critical for the development of new ice prevention techniques. For example, the study of icephobics is currently expanding as researchers try to develop surfaces that will prevent ice from adhering to surfaces, a goal which, if realized, would solve the icing problem. ${ }^{2}$ Better adhesion data is required to make this effort fruitful, and to use the adhesion data effectively the mechanisms behind adhesion need to be better understood. Third, reliable adhesion data is required to develop shedding models to predict shedding accurately.

A great amount of work has been performed to understand the material properties of non-aircraft ice relevant to adhesion. Early work focused on refrigerated ice and glacier ice cores [46-49]. More recent work has been increasingly specialized in a variety of areas,

\footnotetext{
${ }^{2}$ This is likely impossible since ice adhesion cannot be eliminated but only reduced, and icephobic coatings must be both affordable and durable. The reduction of adhesion would allow for reductions in the energy required to remove the ice, enhancing existing equipment.
} 
including river and lake ice [50-53], sea ice [54-58], and glacier ice [59]. The microstructure $^{3}$ of aircraft ice must have different characteristics from non-aircraft ice and, like adhesion, has typically not been considered to be directly relevant to aerodynamic studies. Due to the wide range of variables encountered in aircraft icing insufficient study of the micro-physics of ice has been performed in aircraft icing [60]. For example, it was impossible to identify the average grain size of aircraft ice at flight speeds from the literature, let alone in all icing conditions likely to be encountered in nature. Current work to investigate the material properties of in-flight aircraft ice was largely related to icephobic surface design [18, 61-69], adhesion [9, 60, 63, 70-78], and deicing [72, 79]. The science has progressed in this manner since the microphysics of ice are unnecessary when studying the rate, shape, location, and aerodynamic impact of ice growth - it is only when ice is removed that the material properties need to be studied. The diameters of impacting droplets in sea icing and aircraft icing can be in similar ranges [23, 80, 81], but the thermodynamics of accretion are very different, especially in aircraft icing since droplets are typically supercooled. This leads to drastically different ice structure since the growth rates are different. Ice crystal icing (the type of icing most relevant to engine icing) forms from an entirely different mechanism still. Thus, the study of material properties of inflight aircraft icing lag behind that of other types of ice, and these properties are poorly understood. For aircraft ice that has annealed, it may be possible to use material data from polycrystalline ice in the literature (such as in Hobbs [82]), though testing is required before using the data since the effect of the accretion process on the microstructure is poorly understood for in-flight icing.

\footnotetext{
${ }^{3}$ The grain structure and orientation, crystalline defects, the voids within the ice (such as air in Rime ice), and strain frozen into the ice through the freezing process are included in the term "microstructure".
} 
Furthermore, the interaction of ice with surface roughness is poorly understood. Most studies investigate the effect of the average roughness height but ignore the effects of individual roughness elements. Few roughness adhesion models exist, and those aren't useful in understanding the mechanisms affecting adhesion strength. ${ }^{4}$ Test data presents large scatter, and since the mechanisms of ice adhesion are poorly understood [9], skepticism of the applicability of existing data is prudent. A new method to measure ice adhesion is required to study the underlying mechanisms of adhesion to allow meaningful interpretation of the data. Without such a method, the effects of clamping, capillary forces during wetting, the grain structure of ice, and geometric variations in impact velocity (droplets impacting at high speed at the leading edge and at low speed at the impingement limits) cannot be studied or accounted for.

Additionally, the large number of inherent variables involved makes adhesion a difficult problem. The following three categories of variables play important roles in determining adhesion:

- Bulk properties of the ice, including elastic modulus, tensile strength, fracture properties, and creep properties.

- Substrate properties, including the roughness, surface chemistry and wetting, geometry, and possibly the elastic modulus.

- Accretion parameters: droplet size distribution, the airspeed, collection efficiency, the relevant temperatures, and the humidity. The accretion parameters exist in a wide number of envelopes, many of which are difficult to test.

\footnotetext{
${ }^{4}$ See Adhesion Models section.
} 
Again, many aspects of impact ice are poorly understood, and others are difficult to control. The effects of a single variable on icing adhesion are difficult to quantify since they are all interconnected. For example, varying the roughness height changes the apparent wettability of the surface, and can reduce adhesion low-speed impact accretions and raise adhesion in high-speed impact accretions, and will likely have other unknown effects. This is discussed in more detail in the Roughness and Clamping section.

\section{Deicing Methods}

There are a number of methods to protect aircraft from icing, such as pneumatic boots, electro-impulsive and -expulsive systems, shape memory alloys, and ultrasound methods [79]. These methods and thermal methods use a large amount of energy and are expensive to implement. Critical to designing these systems is knowing how much energy and force it will take to break the interface, and the best way to apply these forces. Systems that use less energy and require less force will provide better economy for aircraft through lower weight and power requirements. On the other hand, knowing the requirements can also prevent the use of under-powered systems that are ineffective. Adhesion is not the only factor to consider. The location of ice accretion and the tradeoffs associated with leaving some ice behind or how long to wait to remove ice are also critical in the design process.

Besides these deicing techniques, a new technical area is developing to provide passive protection via carefully engineered surfaces. Icephobic materials are being developed to reduce adhesion and even prevent icing. These are termed 'icephobics' as they are designed to prevent the accretion of ice and reduce its adhesion to the surface of interest. Icephobics have the potential to drastically reduce the weight and energy required 
by active deicers, which is of particular interest on smaller aircraft such as drones. However, these surfaces further complicate adhesion testing since they rely on several creative mechanisms to reduce adhesion, such as changing the wetting characteristics of the surface through patterned surfaces, changing the solid-ice interface to a liquid-ice interface (SLIPS surfaces), or changing the chemistry of the surface to minimize chemical bonding. Adhesion testing through many use-cycles is important since many otherwise effective surfaces fail durability testing. ${ }^{5}$

Ice Shedding in a Rotating Environment

As previously mentioned, there are two primary areas in icing where ice may selfshed on a macroscopic scale: rotorcraft icing and engine icing. ${ }^{6}$ Unlike airframe icing, rotorcraft icing, and engine icing have high enough centripetal forces to remove ice from the surface, breaking adhesion. Adhesive or cohesive failure of the ice then controls how frequently and how much ice will shed, controlling the size of ice chunks leaving the surface and potentially re-impinging critical areas, such as a tail rotor or subsequent stages in a turbine engine. In fixed-wing icing, even after adhesion is broken aerodynamic forces often hold ice in place [16]. The rotating forces also modify the accretion in other ways, such as changing the film dynamics in glaze conditions; the implications of these changes are poorly understood with regards to the material properties of the ice.

The shedding of ice from helicopter rotors presents a significant problem to helicopter safety [83-86]. Ice may re-impinge on the tail rotor, fly into the engine, or strike the body of the rotorcraft. The shedding from helicopter blades has much in common with

\footnotetext{
${ }^{5}$ This also includes rain erosion, sand erosion, bug strike, UV exposure, and chemical testing.

${ }^{6}$ On a smaller scale, small features of large shapes of ice, such as 'feathers', are known to self-shed under aerodynamic loading; this was observed in testing in the IRT on airfoils during piggyback testing.
} 
the shedding of ice in turbine engines [87-89] and on windmills [90-92]. As ice mass grows on blades, ice will typically shed in a rotating environment when the centrifugal force, which is proportional to ice mass, exceeds the adhesion forces which are proportional to contact area. Since the ice mass/volume can grow long past the point at which the contact area becomes limited, the adhesion forces will likely be surpassed by centrifugal forces, and self-shedding is probable. This principle holds in any rotating environment, hence the similarity to engine and windmill icing.

The adhesion strength, then, largely dictates how long ice will grow on a surface before shedding off. This controls the maximum amount of aerodynamic effect the ice can have, how large chunks of ice can become as projectiles, and how effective deicing equipment will be. Experiments on rotors to capture deicing can provide highly beneficial data but are extremely expensive. In response, some effort has been made to develop codes to model the breakup and shedding of ice on rotor blades. The only current code with such models known to exist to the author is FENSAP-ICE [7, 93, 94]. The model incorporated into FENSAP-ICE meshes the ice shape and tracks crack growth through the ice shape. Such a model requires costly 3D modeling. LEWICE currently has no shedding model for helicopter rotors or for deicing purposes, which provides much of the motivation for the this work $[6,95]$. A model to predict the location and time of an ice-shedding that requires little resources would provide new capability to ice-growth prediction codes like LEWICE without adding the extra cost of a full 3D simulation between accretion steps.

The prediction of shedding is particularly reliant on accurate adhesion data. Several authors in the literature provide a model predicting when ice will shed off of a rotor [86, 96]. Both methods balance the centrifugal force on the ice and the cohesive and adhesive 
forces holding the ice in place. Itagaki's method was simplistic and just performed a simple balance. Fortin's method was more nuanced and, similar to the work of Scavuzzo and Chu [97], used stress distributions in the sample to improve the predicted adhesive break. These models rely on the current state-of-the-art adhesion data, which still limits their applicability as will be discussed in the following sections.

Disordered Interface

In 1859, Faraday first published regarding the layer of liquid on the surface of ice [98]. Faraday's demonstration showed the regelation (freezing together) of two pieces of ice and postulated that this was due to the presence of a liquid layer at the surface. The problem was neglected for a long period of time, but recently has become the focus of much research. The Disordered Interface has also been called the Quasi-Liquid Layer, or the Liquid-Like Layer. The naming of this interfacial effect has been the subject of some controversy since the layer has properties differing from liquid water (as well as ice) [99], and because the terms Quasi-Liquid Layer and Liquid-Like Layer suggest a continuous layer while the interfacial region is not homogeneous [100]. This work will refer to the phenomena as the Disordered Interface (DI) since this most accurately reflects the current understanding of the problem. The terms are, as used herein, interchangeable.

The DI exists at the interface of ice and decreases in thickness with decreasing temperature. The DI becomes detectable in the range of -35 to $-3{ }^{\circ} \mathrm{C}$ [99], depending on the method used. There are a wide variety of techniques used to measure the DI thickness, and data shows that the methods used disagree by several orders of magnitude. BartelsRausch et al. compiled a useful comparison of different techniques used to evaluate the DI [99]. 
The DI exists at the ice-air interface, but also at the ice-substrate interface. Interestingly, the properties of the water in the DI do not match liquid water or solid ice. For example, the viscosity of the DI has been shown to be much higher than that of supercooled water [101], and the density smaller than both supercooled water and ice [102]. The diffusivity in the DI is also different than in bulk supercooled water [99]. The mechanical properties, such elasticity and cohesive strength, exhibited by the DI are largely unknown. Even the thickness of the layer is currently the focus of a debate in the literature. Other properties, such as viscosity, density, and surface energy will also be central in developing a new adhesion model.

Since these properties are central to adhesion, the DI poses a significant challenge to the modeling of adhesion. The properties of this layer will likely have a significant effect on the adhesive strength of the interface. Increasing viscosity will likely strengthen the interface under rapid loading. Increased cohesion and surface tension will likely increase the strength of the interface at all conditions. The elastic properties, viscosity, and the thickness of the DI will facilitate or impede the dissipation of stresses at the interface, effecting the likelihood of cohesive breaks at the interface. Perhaps the most promising route to answering these questions is the method of molecular simulation [102]. However, it was outside of the scope of this paper to answer these questions, necessitating either the liberal use of assumptions regarding the DI or the neglect of the DI when developing a model.

Roughness and Clamping

Surface roughness interacts with ice adhesion in several important ways. It increases the surface area over which ice bonds to the surface and creates a mechanical 
advantage to adhesion. As an example, when ice is sheared off the surface of a wing, the presence of roughness necessarily includes non-flat local geometry relative to the macroscopic shear plane. When the ice is sheared, it will press against this geometry at an angle such that the roughness will inhibit shearing. This effect can be quite complicated since at some angles the required shear force to break the local adhesion is greater than the force required to cause a cohesive break near the roughness element.

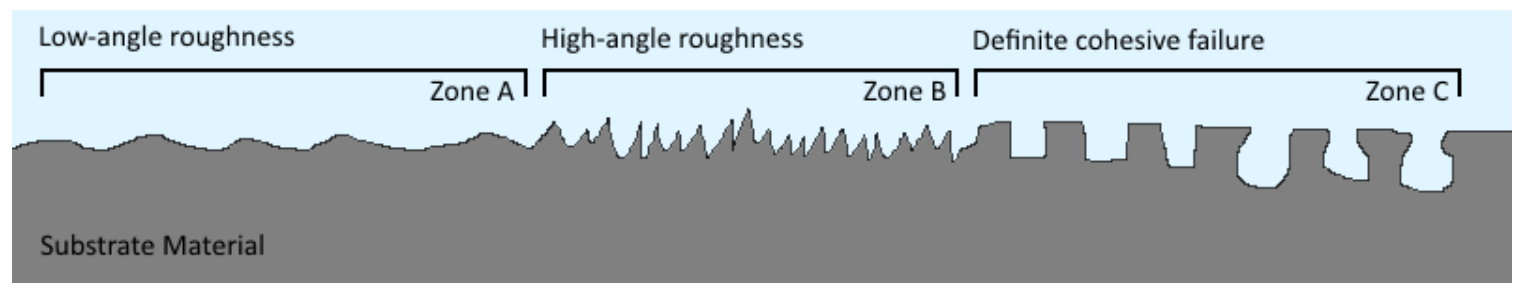

Figure 1. Roughness zones demonstrating features that would likely result in different types of failure.

If adhesive strength is lower than cohesive strength (the tensile or the shear strength of the ice) on an ideally flat surface, pure shear loading should always result in a pure adhesive failure. An adhesive break is one in which the interface between the ice and the substrate is broken directly, such that no ice is left adhered to the substrate. A cohesive break is one where the ice-substrate interface is left intact and the fracture occurs in the ice. On surfaces with low-angle roughness features, as in Zone A of Figure 1, the shearing force must push the ice against an inclined plane, providing a mechanical advantage to increase the adhesion strength. This mechanical advantage will be significant but perhaps not enough to induce cohesive breaking. In Zone B, cohesive failure is likely due the angle of the shear force to the roughness-element surface normals. Zone $\mathrm{C}$ exhibits roughness features that will ensure cohesive failure due to vertical features. In this case, shear forces on the ice will create a normal force at this vertical interface, and an adhesive mode- 2 
(shear) failure is conceptually impossible. On the right side of Zone C, cavities exist that would prohibit a purely-adhesional mode-1 failure.

Clamping may also occur inside roughness elements such that supercooled water enters the roughness elements, freezes, and expands. In Zone A of Figure 1, this may actually be advantageous in reducing adhesion since expansion would stress the interface and aid in delamination. In Zone B, this is unlikely to be the case and clamping will contribute to the onset of cohesive failure at the interface. In Zone C, the exaggerated geometry contains a number of corners around which stress concentrations will be significant. This clamping creates local stresses, but also interacts in unknown ways with the ice at the interface and may potentially increase or decrease the force of adhesion through various mechanisms (such as increased friction forces, changes to the Disordered Interface, etcetera). This is demonstrated through the idealized roughness element in Figure 2 , where the water is assumed to freeze by instantaneously solidifying and then undergoing an isometric expansion. 


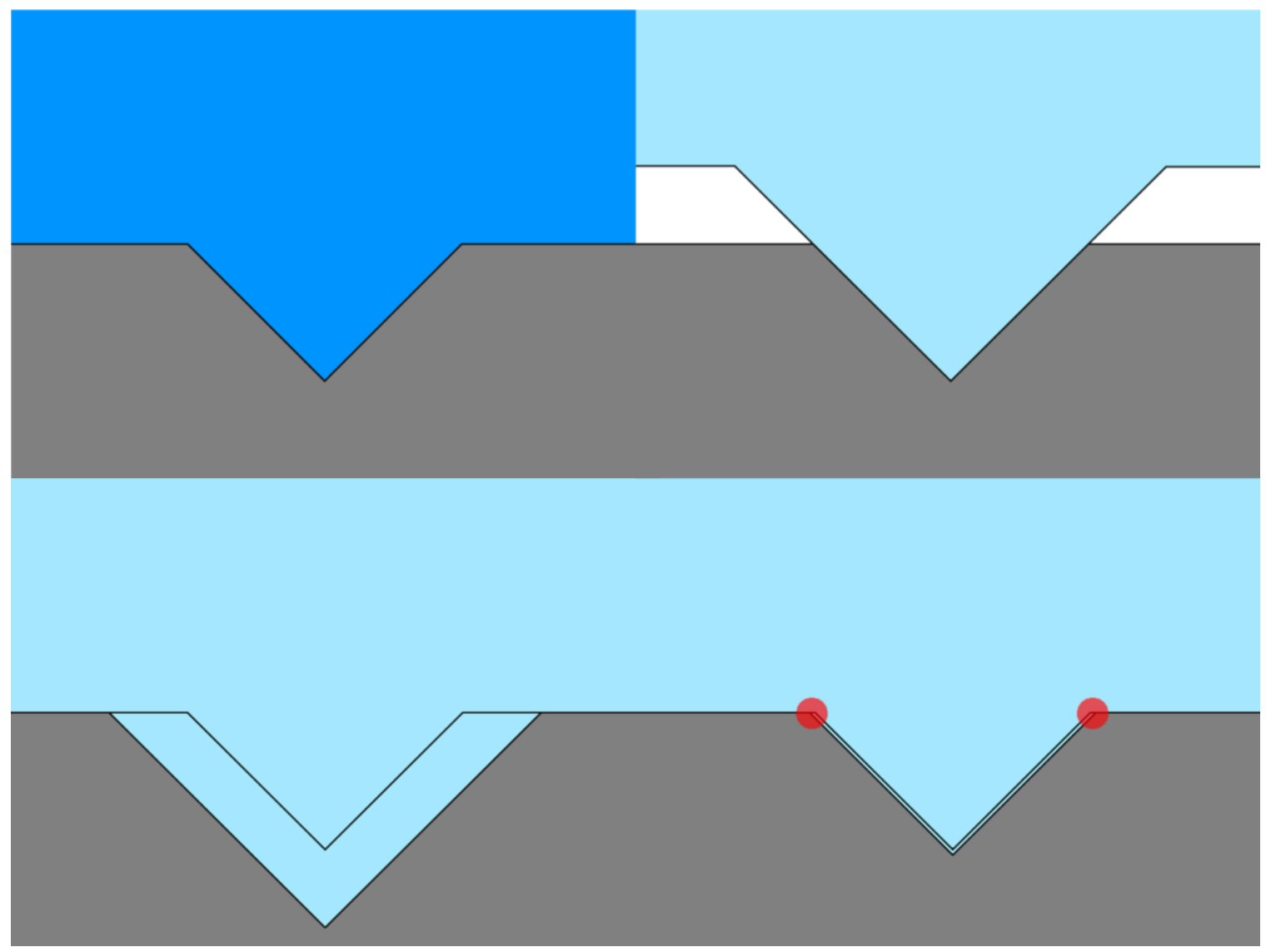

Figure 2. Simplified theoretical expansion of ice in a triangular roughness element. Top left: water in crevice. Top right: water expands into ice with no bonding. Bottom left: no slipping but substrate deforms freely. Bottom right: surface deforms, and stress concentrations form at corners and along surfaces.

If the substrate (gray region in Figure 2) does not adhere to the ice, the triangular section of water would expand away from the interface as in the top right of Figure 2. If the surface offered no resistance to the ice expanding (i.e. the substrate had zero stiffness), the ice would expand into the surface as in the bottom right. In the bottom right the substrate offers resistance to expansion and a complicated stress field is formed as adhesive forces compete against expansion to prevent delamination at the interface. Since the ice tends to push out of the roughness element and adhesive bonding along the interface inside the roughness element tends to pin the corners down, shear stress is expected at the corners inside the roughness element. Normal forces are expected to drive deformation of the 
substrate. Since the ice is pushing out of the roughness element, it tends to push the bulk ice away from the horizontal interface (top right). Since the ice adheres to the interface and substrates do not freely deform, a complex stress-field develops inside the newly formed ice and a complex stress field forming around the corners is expected (bottom right, red circles). In reality the ice does not freeze in such a simplistic way - a crystal is nucleated at some point and grows rapidly, but not instantaneously, and water expands as it freezes. Latent heat is released, and unfrozen water is driven away. The residual stresses are not expected to be simple. Since the freezing and expanding process is currently poorly understood, interfacial stresses and cracking are impossible to quantify. The potential for trapped air beneath the ice adds another layer of complexity to this problem.

Several authors have made progress into investigating these effects. One experimental study in particular stands out. Penn State University researchers tested exceptionally flat epoxy surfaces, and then roughness by adding channels by ablating epoxy away with a laser [103]. Unfortunately, this work only produced preliminary data, and the analysis provided was not rigorous. ${ }^{7}$ Theoretical work has been performed investigating clamping [70], but as of yet no experimental work has been performed that gives insight into these problems.

Roughness can interact in the wetting of the surface by modifying the apparent contact angle of the liquid phase and creating a capillary force at the interface. A suitably hydrophobic surface could repel supercooled water before it has the opportunity to freeze [69]. Even though this has only been demonstrated at low impact speeds, the geometry of the surface roughness strongly effects the wetting state prior to freezing. Some surface

\footnotetext{
${ }^{7}$ See the Adhesion Models section.
} 
design has been focused on reducing the residence time of liquid water on a surface during impact conditions, which is to act as an icephobic. This is discussed in more detail in the Icephobics and Wetting and Interfacial Phase Change sections.

Wetting and Interfacial Phase Change

The substrate wettability affects the strength of adhesion of ice $[9,68,104-107]$; therefore, a large part of designing icephobic surfaces has been centered on designing hydrophobic surfaces. Because of this, it is important to understand what the contact angle is, and how it effects the adhesion of ice to a substrate. This background information serves several purposes. First, it will show the limits of icephobic surfaces based on hydrophobicity. Second, it will lead to a better understanding of the physics behind accretion and the interface created between the ice and substrate. Third, it will also provide background information on the chemical adhesion of ice to the surface. The contact angle is depicted in Figure 3.

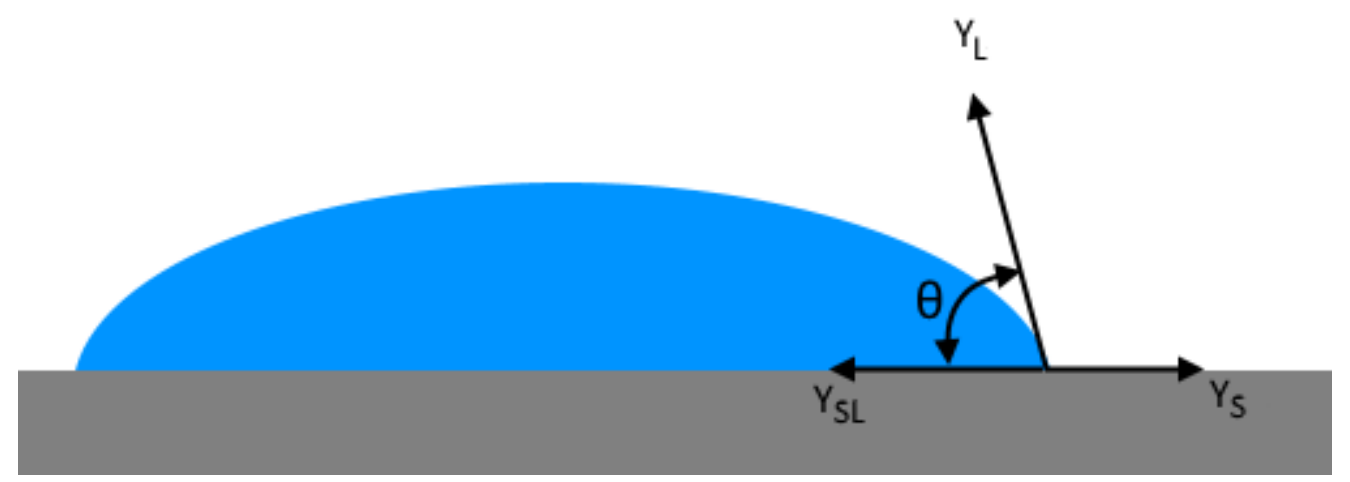

Figure 3. The contact angle of water and Young's interfacial energies.

The knowledge of contact angles with respect to wetting primarily started with the work of Young and Dupre [108, 109]. It follows that the most basic understanding of contact angle physics comes in the form of Young's relation, 


$$
\gamma_{s}-\gamma_{s l}=\gamma_{l} \cos (\theta)
$$

where $\gamma_{s}$ is the solid-air interfacial tension, $\gamma_{s l}$ is the solid-liquid interfacial tension, $\gamma_{l}$ is the liquid-air interfacial tension, and $\theta$ is the contact angle. This equation was not written directly by Young but described in words. The work of adhesion of two condensed phases a and $\mathrm{b}$ was given by Dupre:

$$
W_{a b}=\gamma_{a}+\gamma_{b}-\gamma_{a b}
$$

This is combined with (1) to provide the work of adhesion, which is given by the famous Young-Dupre equation [110-112]:

$$
W_{a b}=\gamma_{l}(1+\cos (\theta))
$$

where $W_{a b}$ is the work of adhesion between condensed materials a and $b$. Interestingly, the interfacial energy of ice and liquid water are approximately the same near the freezing point [113]. Because of this, it has been assumed that the contact angle of water on a particular substrate can be used to give the work of adhesion for ice for that substrate [9].

One problem with Equation 1 is that the equation is for flat and chemically homogeneous surfaces, which do not exist. It has been demonstrated that hydrophobicity is a poor indicator of icephobicity on several occasions in the past $[18,76,114]$. Low speed data does exist to the contrary, showing a strong inverse correlation between hydrophobicity and adhesion strength [73]. The relationship between hydrophobicity and icephobicity in general is poorly understood, and existing data is difficult to interpret due to most adhesion tests using poorly characterized interfacial conditions [9]. 


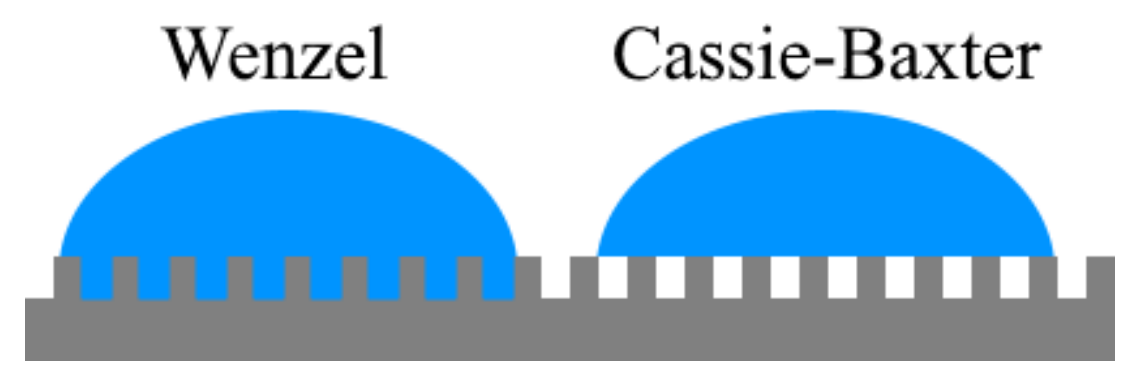

Figure 4. Wenzel vs. Cassie-Baxter wetting states.

Surface roughness provides a particular problem for the adhesion of ice to aircraft. There are two possible states of wetting for rough surfaces (shown in Figure 4): the Wenzel state $[115,116]$, and the Cassie-Baxter state $[116,117]$. The Wenzel state is defined by complete wetting of the surface - water penetrates the surface roughness leaving nothing between the liquid and solid at their interface. The Cassie-Baxter state is defined by partial wetting at the surface; the liquid does not fully penetrate the surface roughness and typically air is trapped at the interface. The transition from the Wenzel state to the CassieBaxter state is marked by increasing hydrophobicity [118-120]. The transition between the two states has proven useful to control droplet motion [121] and reduce contact time on a surface [69], but is also effected by kinetic effects, such as vibration [122] or impact. It is highly desirable to reduce the contact time of water on a surface in order to reduce the probability for the droplet to ice and adhere to the surface, an approach that shows promising results at lower speeds $[66,69]$. Hydrophobic surfaces that take advantage of the Cassie-Baxter wetting state typically fail at reducing the adhesion of ice at high speeds, however, since high speed impacts common to aircraft icing force water into the surface elements. This increases the contact area for adhesion, increasing adhesion forces [77], and leads to clamping which further increases adhesion $[9,70,123]$. One such example is 
Teflon, which exhibits hydrophobicity in general use, but at high speeds performs poorly at reducing ice adhesion $[18,76]$.

Another problem with equation 1 is that it does not account for plastic deformation. As the interface is broken, force must be transferred to the interface via the ice and the substrate materials. When subjected to loading, these materials will deform. For the purposes of removing ice, the energy spent on plastic or even elastic deformation is nonrecoverable. The measurable work to remove ice is then larger than that predicted by (3). For example, in the case of ice on steel, (3) predicts a value for the work of adhesion of $0.09 \mathrm{~J} / \mathrm{m}^{2}$ [9], while a value of $1.1 \mathrm{~J} / \mathrm{m}^{2}$ has been found experimentally [124]. While (3) doesn't account for the work to deform the materials making up the interface, it should be expected to be a good trend indicator of the total work to separate the ice since deformation will depend on the force required to break the interface $[125,126]$.

The wetting state of the surface was expected to directly affect mechanical clamping, strengthening or weakening the interfacial adhesion. The thermodynamics and hydrodynamics associated with phase change at the interface are poorly understood, so properties of the resultant ice-substrate interface are poorly understood. As liquid water freezes and expands, it does so partially inside surface roughness elements (depending on the wetting state). This will undoubtedly cause stress concentrations; however, to knowledge of the author, there was no current work investigating phase change inside surface roughness elements in detail. Understanding the phase change inside rough surface is critical to adhesion since it affects clamping at the interface [70, 123], and, depending on the dynamics of phase change, the expansion could pre-crack the interface or weaken surface adhesion. Further still, it was unknown how creep in the ice will affect these 
unknown stresses over time. These concerns aren't new: Merkle and others have expressed concerns over surface stress concentrations, interfacial bubbles, and ice crystalline structure at the interface in 1968 [18].

Icephobics

"Icephobic" refers to surfaces that can either prevent the accretion of ice (including frost) or reduce the adhesion of ice once it has accreted. Some sources make a distinction between preventing the accretion of ice and reducing the adhesion of ice; herein the term "icephobic" is used in the general sense. There are several types of icephobic surfaces for aircraft icing. One type includes hydrophobic surfaces, which attempt to reduce the adhesion of ice by reducing the adhesion of liquid water and rely on the similar characteristics between liquid and solid water. These aren't necessarily patterned surface with fixed geometry; as an example greased surfaces have shown promise [63, 127], as well as SLIPS surfaces [128]. Another type is micro- and nano-textured surfaces which attempt to prevent the accretion of ice by repelling water from the surface before it solidifies and adheres, but also attempt to maintain a Cassie-Baxter wetting state to reduce adhesion $[69,129,130]$. Other types can disrupt the Disordered Interface [67, 102].

Icephobics designed to mitigate adhesion using textured surfaces are prone to suffering from the same problem Teflon suffers from: penetration into surface elements can clamp ice to the surface and increase adhesion. They are then sensitive to the impact speed of the droplets and the pressure tending to push water into roughness elements. Surfaces that are capable of reaching higher speeds have a smaller autocorrelation length for their roughness features, providing larger capillary forces to counteract this pressure 
[130]. Current research has shown effectiveness only at very limited speeds, too slow for application against in-flight icing.

Surfaces that provide high levels of adhesion reduction often exhibit poor wear resistance [131]. These surfaces typically require grease or oil coatings, or finely textured surfaces with poor wear characteristics. Clamping can destroy textured surfaces, and coatings can erode over time. Frost formation can also bypass certain icephobic features [77]. No coatings exist with perfect icephobic characteristics, and no such coating is likely to ever exist.

In spite of these challenges, icephobics remains a promising area of research. While it is likely that no coating will ever solve the icing problem, the potential benefits to icephobics that are within reach are critical. Coatings exist that can reduce adhesion by more than an order of magnitude [63], though the task of making these coatings durable isn't straight forward. Even much smaller reductions in adhesion would greatly aid the aerospace industry by enabling the use of lighter and more efficient deicing equipment. Making deicing technology lighter, more power efficient, and more affordable is critical to equipping smaller craft with deicing equipment. Reductions in adhesion may be enough to solve icing problems on rotating equipment since ice may self-shed before becoming problematic. These payoffs coupled with the promising results that are becoming all the more common ensure that the study of icephobics will become an increasingly important topic in decades to come.

\section{Mechanical Properties of Impact Ice}

Unfortunately, there is very little data on the mechanical properties of impact ice in the literature. The most relevant data comes from the work of Scavuzzo and Chu $[17,83$, 
$97,132,133]$ who published a series of papers with co-authors providing experimental data for ice adhesion, elastic modulus, and tensile strength. Several of their papers, including their paper that contains data on the elastic modulus of impact ice, was unavailable for use in this work as the articles were not available. Their work represents the only known published tensile measurements of ice generated in the IRT at the time of writing.

Chu and Scavuzzo have presented results documenting the tensile strength of impact ice, but many important parameters are undocumented - such as the cloud properties and wind speed [133]. They report values for the ultimate tensile strength of ice in the range of 120.6 to $170.0 \mathrm{psi}$, or 0.83 to $1.17 \mathrm{MPa}$. These values fall well within the range of tensile strengths for ice in a review by Petrovic and correspond to ice with a $1 \mathrm{~mm}$ grain size at approximately $-10^{\circ} \mathrm{C}[134]$. Petrovic provides a range of ice tensile strength of 0.7 to $3.1 \mathrm{MPa}$ in the temperature range of -10 to $-20^{\circ} \mathrm{C}$. Petrovic also reports a Young's modulus in the range of 9.7 to $11.2 \mathrm{GPa}$, and a Poisson's ratio of 0.29 to 0.32 . This agrees with values given by Schulson, where Schulson reports a value of 9.0 GPa and 0.33 for the Young's modulus and Poisson's ratio, respectively, for polycrystalline ice at $-5{ }^{\circ} \mathrm{C}$ [135]. Schulson reports that polycrystalline ice behaves in a brittle fashion up to its melting point at higher strain rates since anisotropy in individual grains will cause stress concentrations leading to cracks. At lower speeds, these stresses have time to dissipate, and ice will flow more plastically. The inclusion of brine also reduces internal stresses and aides in plastic flow. It is thus suspected that included air-pockets in rime-ice will have a similar effect. In lieu of data pertaining directly to impact ice with pertinent details of the ice formation, the properties in will be used for calculation at $-10{ }^{\circ} \mathrm{C}$. The ice will also be assumed to fail in 
a brittle mode since high-strain rate applications are the primary interest in this work, and to be isotropic due to the polycrystalline nature of impact ice.

Table 1. Default Mechanical Properties of Ice Used.

\begin{tabular}{|c|c|}
\hline Ice Property & Value \\
\hline Young's modulus, E $(\mathrm{GPa})$ & 9.7 \\
\hline Poisson's ratio, $\mu$ & 0.33 \\
\hline Ultimate tensile strength, $(\mathrm{MPa})$ & 1.17 \\
\hline Density, $\rho\left(\mathrm{g} / \mathrm{cm}^{3}\right)$ & 0.92 \\
\hline Shear Modulus, $\mathrm{G}(\mathrm{GPa})$ & $3.6^{8}$ \\
\hline
\end{tabular}

A second set of tensile strength data is present in the work of Itagaki [86] who performed a series of centrifuge tests to determine the tensile strength of the ice (as well as the adhesive shear strength) using the thickness of the ice, the release point, and the rotational speed. Itagaki's model assumed that the ice had a constant cross-section. Their data contains a high degree of scatter but must be taken into consideration given the lack of availability of alternative data. They found tensile strengths between $0.26 \mathrm{kPa}$ and 2.19 MPa and reported densities between 0.90 and $0.92 \mathrm{~g} / \mathrm{cm}^{3}$. A subset of these tests were with impact ice, and contained only three data points, each on a different surface. Values for tensile strength in these three tests were 1064, 1680, and $975 \mathrm{kPa}$ (for run 9, 10, and 11, respectively). Density was $0.9 \mathrm{~g} / \mathrm{cm}^{3}$ for all three of these data points. For a review of the mechanical properties of non-impact ice, see $[59,82,135]$.

${ }^{8}$ Calculated using $G=\frac{E}{2(\mu+1)}$ 


\section{Creep and Fracture}

Creep is the plastic flow of a solid material over time, which can act in response to internal stresses. In ice, creep motion is largely dependent on defects in the ice's crystalline structure. The presence of grains affects creep since motion can occur along grains, and grains are a source of dislocations. However, since the grain structure of impact ice was largely unknown, and the local stresses at the interface are largely unknown, it was impossible to predict how much of an effect creep will have on the adhesion of ice. It is important to realize that creep is a potential mechanism for time-dependent change in adhesion, and that the time between ice formation and testing needs to be controlled. Aircraft icing occurs between -30 and $0{ }^{\circ} \mathrm{C}$, though is more common at warmer temperatures. The corresponding homologous temperature range is $0.89-1.00$, suggesting that creep is likely to be significant during an icing event.

During the process of delamination, the breaking of adhesion can be treated as a fracture mechanics problem $[93,114,135]$. The formation of a crack followed by the propagation of the crack leads to total adhesive, cohesive, or mixed failure of the interface, allowing ice to break free of a surface. Though exact strain rates depend on the specific application, delamination is typically a high strain-rate failure since ice is removed quickly during shedding or deicing applications. The onset of crack formation likely leads to some scatter in the available data, while the propagation of the crack is poorly understood and difficult to model. Shiping et al. were able to complete a 3D analysis of ice shapes on rotors and follow crack propagation through an ice shape [93]. The same group also simulated crack propagation through airframe icing [94]. In their simulations, cracks formed and 
propagated when local values of adhesion or cohesion strength were exceeded using a Von Mises stress criterion.

Ice Structure

While ice is popularly defined as the common solidified form of water, solidified water can vary significantly in structure. When ice is mentioned herein, it is in reference to ice $\mathrm{I}_{\mathrm{h}}-$ which is also known as ice I. This form of ice possesses a hexagonal structure at the molecular level and exists over the complete set of temperatures and pressures known to occur in aircraft icing $[5,30,82,136]$. Within this type of ice there are more distinctions commonly made separating samples by the method of their formation and their grain structure. Lake ice grows on lakes, glacier ice is found in glaciers, impact ice is accreted through the impact of small droplets, frost forms from humidity in the air on cold surfaces, etcetera $[17,59,82]$. These distinct types of ice are not distinct because of their crystalline structure, but because of the grain structure of the ice. Lake ice commonly has tall, columnar grains developed as ice forms at the top of a lake and the liquid-ice interface grows downward into the lake; sea ice is similar (when grown in similar fashion). Glacier ice varies significantly with depth since pressure strongly influences sintering in glaciers [59]. The distinctions made in this manner can be further subdivided, and often overlap. Aircraft icing mostly includes impact ice but also can include frost formation (which is significant with relation to icephobic surfaces [77]). Glaze ice and rime ice, or some mixture of the two, are the types of impact ice encountered in aircraft icing. These types of ice are shown on coupons during IRT testing in Figure 5, where glaze ice is seen to be clear while rime is milky-white. Ice may also grow from sea spray, which has a significantly 
different grain structure than the sea ice previously referred to [54, 137]. Thus, it is important to be unambiguous when discussing the type of ice being dealt with.

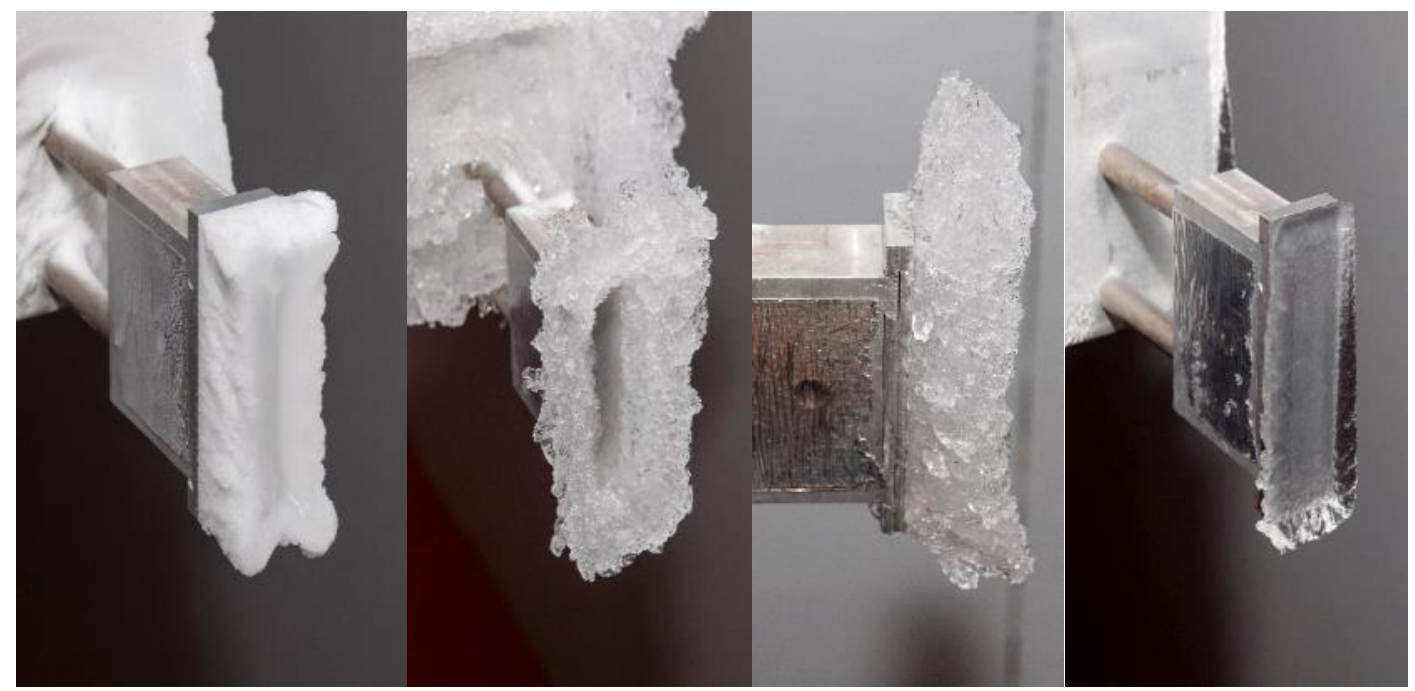

Figure 5. Types of aircraft ice. Left: rime ice (8/19/16 run 3), Middle: glaze ice (8/18/16 run 1), Right: Glaze (8/15/16 run 1). Photographs by Quentin Schwin.

The micro-structure of ice determines the constitutive properties of ice in several ways. Ice can typically be treated as isotropic since most common forms of ice is polycrystalline where there is no preferred orientation in the grains. But this is not always the case - single crystals of ice are anisotropic and certain accretions can have anisotropy when grains have preferred orientations; lake ice, for example, possesses a preferred orientation and exhibits anisotropic constitutive properties [59]. Grain boundaries weaken ice by disrupting the crystalline structure and can act as a source and sink for defects in ice, increasing defect mobility. Grains can also slide. Thus, the grain structure affects the strength and plastic properties of ice.

The grain structure of aircraft icing was largely unknown, but some work has been done to investigate it in more detail [60]. Large variations in the grain structure of ice have been observed corresponding to variance in several key parameters. The change from Rime to Glaze ice conditions marks a transition from finer to coarser grain sizes $[138,139]$. This 
is also shown in Figure 37. The size of impacting drops as well as the thermodynamic state of the droplets affect how quickly droplets freeze and will likely affect the grain size. Additionally, the speed of the droplets and the aerodynamics specific to each application, among other properties, will determine the breakup of droplets before and after impact and hence potentially affect grain size. Druez. et al. [60] found that the grain size in aircraft ice tended to decrease with increasing velocity, and also decrease with decreasing temperature. This suggests that at higher speeds, droplet breakup may play a key role in determining the grain structure of ice, and its mechanical properties. Despite this work it was still unknown what grain structure to expect at speeds more relevant to FAA icing envelopes. ${ }^{9}$

A potential issue for storing samples is that the properties of ice may change as the ice ages. Storage could be beneficial since it would provide annealing, allowing residual stresses to flow out and the adhesion test to be run on a stress-free sample. On the other hand, it may be problematic since the stored ice may no longer be equivalent to ice during a flight condition. One source reports that the grain size of polycrystalline ice will increase over several days of storage at $-1{ }^{\circ} \mathrm{C}$ and $-10{ }^{\circ} \mathrm{C}$, but that the change is much slower further from the melting point [140].

\section{Adhesion Testing}

Adhesion tests on aircraft ice have been published as early as 1939 [141]. In 1946 Loughborough and Haas found values of adhesion to deicer surfaces showing a linear relationship with temperature [142]. They found a value of $1 \mathrm{MPa}$ at $-25^{\circ} \mathrm{C}$, and zero at 0 ${ }^{\circ} \mathrm{C}$. As shown by some of the data discussed in the following sections, this agrees poorly

\footnotetext{
${ }^{9}$ This may be seen online in the Electronic Code of Federal Regulations (e-CFR) in Title 14, Chaper 1, Subchapter C, Part 25, Appendix C [30] and Appendix O [5], at https://www.ecfr.gov/cgi$\underline{\text { bin/retrieveECFR } ? \mathrm{~g} p=\& \mathrm{SID}=1 \mathrm{e} 75806 \mathrm{e} 224857 \mathrm{f} 96 \mathrm{~b} 292 \mathrm{~d} 08639263 \mathrm{cf} \& \mathrm{mc}=\text { true } \& \mathrm{n}=\mathrm{pt} 14.1 .25 \& \mathrm{r}=\mathrm{PART}}$ \&ty=HTML\#sp14.1.25.i.
} 
with more recent testing. The focus of Loughborough's paper, as well as the focus of current testing today, is to find the adhesive strength of aircraft ice, and to develop deicers. They suggested the use of a silicone film as an icephobic, which they suggested would reduce the adhesive strength of ice by $90 \%$.

There are a wide variety of tests that have been performed to measure the adhesion of ice since Loughborough and Haas's paper. Some of these tests are more traditional adhesion tests, such as pusher-type shear tests and lap tests. Other tests are more specialized to use with ice, such as centrifuge tests. Each of the many types of ice tests have critical flaws, but also possess certain strengths. These strengths and weaknesses relate to the manner in which the test must be run, and the stress loading created at the interface and in the body of the ice specimen. Results are discussed in the context of how the ice was formed. ${ }^{10}$

In general, there are two categories of tests: controlled and uncontrolled displacement tests. Tests like centrifuge tests do not control or measure the displacement of the ice but provide a single data point for adhesion. Controlled displacement tests, like certain pusher tests and lap-joint shear tests, introduce strain into the sample in a controlled manner, and can track the displacement allowing the strain to be estimated. This delineation is important since the results obtained from each type of test are fundamentally different.

Pusher Tests

Pusher tests involve creating shear force on a sample by pushing or pulling them from one side or the other. Since no sample is perfectly stiff, these tests create non-uniform shear at the interface, typically with a stress concentration near the pusher. Numerous

${ }^{10} \mathrm{~A}$ more thorough review of the literature was completed as part of this project, see [45]. 
authors have used this type of test $[18,60,73,76,143-145]$. These tests accounted for a wide variety of parameters and are the most popular type of test to obtain adhesion data on ice.

In 1967, Merkle tested a variety of surfaces in the IRT at NASA Glenn on a modified DC-9 wing tip [18]. Merkle accreted ice on the specimen, and then cut samples out of the ice shape using a steam knife with a suction line. He would then measure the sample area and line up a pusher plate to push the sample off of the test article. For uncoated aluminum, Merkle found values of adhesion ranging from 55 to $210 \mathrm{kPa}$, showing a wide degree of scatter. Merkle attributed the scatter to a variety of problems such as variation in the shape of the ice sample being pushed and sample preparation.

In 1979, Druez et al. tested impact ice on cylindrical aluminum conductors using strain gages in a custom-build load cell $[60,143]$. They showed a dependence on ambient temperature, wind tunnel velocity, and surface roughness. Testing at impact speeds up to approximately $23.5 \mathrm{~m} / \mathrm{s}$, they showed an increase in adhesion strength with increasing velocity. Interestingly, they also imaged the grain structure of the ice at several speeds and showed that grain size varied with velocity and temperature as well. They found a mean adhesion strength of $300 \mathrm{kPa}$, and maximum values over $400 \mathrm{kPa}$. These papers also present a useful list of studies on adhesion as background research that is not recreated here.

In 1991, Chu and Scavuzzo published a set of results obtained from testing in the IRT [17]. These tests are particularly interesting since they are relatively well documented and have similarities to the tests proposed in this work. A set of cylinders were placed inside the IRT test section and rotated to accrete ice. An outer cylinder was used to push 
ice off the inner cylinder, on which ice was accreted. Despite a significant effort to increase repeatability, there is unfortunately a large amount of scatter in their data. Samples were cleaned with acetone before accretion and not touched with skin to prevent contamination. While it was possible that variability was produced by crack nucleation or water penetration between the cylinders, much of the scatter was due to data processing since they combined data irrespective of many of their test conditions [45]. They did take a statistically significant number of samples and provided average variation to fit lines and compared adhesive strength to a number of properties including droplet momentum, temperature, wind velocity, and ice thickness. They obtained values ranging from 0 to 1 $\mathrm{MPa}$, and show a linear decrease with temperature above $-10{ }^{\circ} \mathrm{C}$. While this lines up well with the data from Loughborough, they show the linear trend stops at $-10{ }^{\circ} \mathrm{C}$ and $1 \mathrm{MPa}$ is much higher than typical results. They show similar values of adhesion between stainless steel and aluminum and increasing trends in adhesion with increasing wind velocity and droplet momentum. No relationship was established between substrate thickness and adhesion strength.

In 2010, Meuler et al. performed push-type tests on a wide variety of surfaces that did not include aluminum [73]. A large number of tests were performed and the number of tests with complete adhesive failure were documented. These results were particularly interesting for examining the relationship of the contact angle to adhesion. Extensive surface characterization was carried out using a variety of techniques including interferometry, profilometry (using a $10 \mu \mathrm{m}$ stylus), a scanning electron microscope (SEM), and an atomic force microscope (AFM). Water was poured onto a surface in cuvettes and frozen. The cuvette was left in place and pushed off the surface using a force 
transducer. A Peltier plate was used to cool the samples to $-10{ }^{\circ} \mathrm{C}$. Care was taken to obtain repeatable results by grinding the cuvettes with 1200 grit sand paper, treating them to reduce their surface energy, and bolting them to the test substrate to ensure a flush mount. It was reported that leaking was not present when the edges were ground and the surface treated. Still, a large degree of scatter was present in the data; the average uncertainty was $20.5 \%$, with maximum and minimum values at $33.5 \%$ and $10.5 \%$, respectively. Variability in crack nucleation is suspected to cause the scatter in their data.

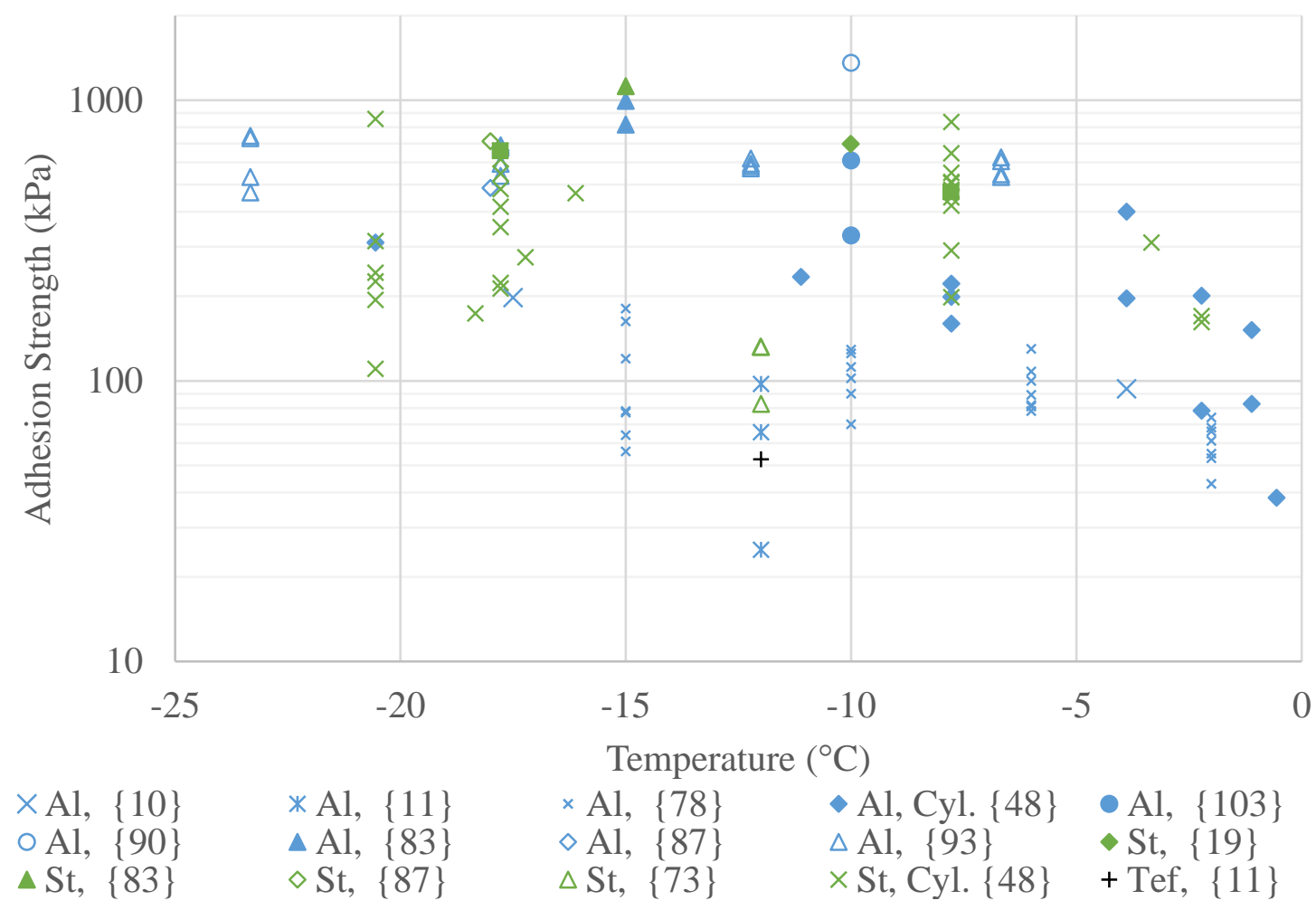

Figure 6. Averaged results from push tests in the literature, used from [45]. St is Steel, Al is Aluminum, and Tef is for Teflon.

Averaged data from the literature is shown in Figure 6. The data for aluminum and steel show little apparent difference, though the data spans nearly two orders of magnitude and apparently starts to collapse above $\sim 7^{\circ} \mathrm{C}$. Much of the data was from Scavuzzo and $\mathrm{Chu}$, and so the trends they discuss in their paper tend to dominate the other data. The data 
appeared flat below $-10{ }^{\circ} \mathrm{C}$. The average standard deviation was $23.9 \%$ for aluminum and $15.1 \%$ for steel across all of the papers surveyed.

\section{Shear Tests}

Similar to push tests, shear tests allow displacement recording and provide more uniform stress fields in the ice. Much less data is available from shear tests in the literature, but several test geometries have been used on ice. Most prominently lap-joint tests have been used, and the zero degree cone test has been used. The data in the literature for these tests is shown in Figure 7.

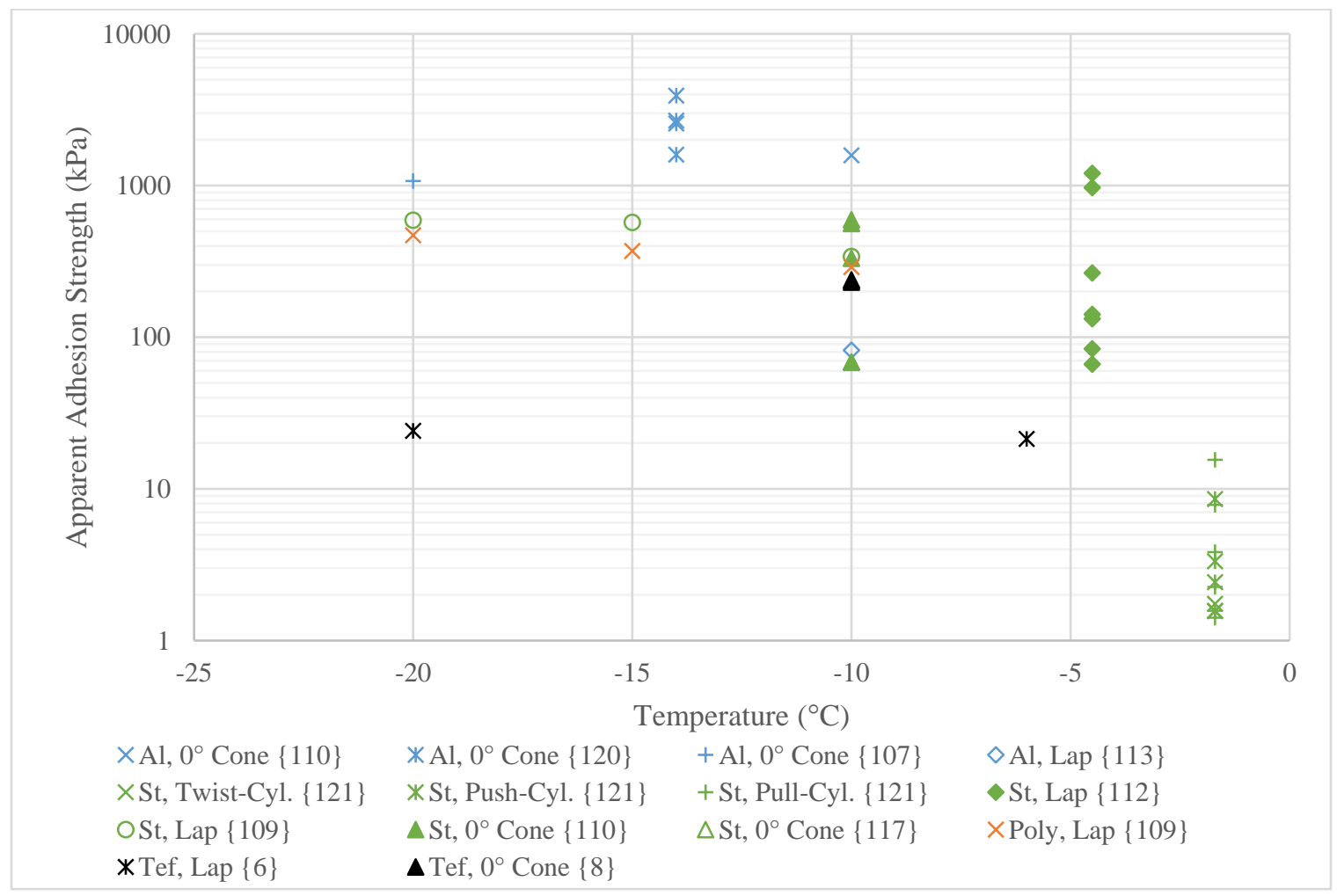

Figure 7. Averaged shear test results from the literature, used from [45]. St is Steel, Al is Aluminum, Poly is for Polyurethane, and Tef is for Teflon.

The data for shear tests drops at temperatures above $-5^{\circ} \mathrm{C}$ but appears flat at lower temperatures. Stresses peak at higher values than in other tests, suggesting lower stress concentrations than in centrifuge tests. Interestingly, the average reported standard 
deviation was lower for aluminum than for steel, with values of $11.8 \%$ and $22.1 \%$, respectively.

\section{Centrifuge Tests}

Centrifuge tests involve spinning ice to measure the adhesive force between the ice and a substrate. Samples can be prepared and then moved to the centrifuge or prepared on the centrifuge directly. Centrifuges were used to measure the adhesion of ice at least as early as 1946 [142]. Another early use of a centrifuge method to measure the adhesive strength of a sample was by Beams et al. [146], where a thin films were used on rotors. Later, Raraty and Tabor used the same method to measure the adhesive strength of ice, and showed a connection between frictional and adhesive forces [147].

One of the most important works on the use of centrifuge tests to obtain data for ice adhesion is that by Itagaki at the US Army Corps of Engineers Cold Regions Research and Engineering Laboratory (CRREL) [86]. Itagaki developed relationships to predict when ice would shed and where (discussed in the following sections) and used test data to back out the material properties of ice using previously obtained data [148]. His method assumed a constant cross-section of ice and had a high degree of uncertainty. Itagaki found values for adhesion ranging between 27.3 and $157.3 \mathrm{kPa}$, showed no correlation between adhesion strength and temperature, and showed a poor correlation between accretion speed and adhesion strength with stronger adhesion at higher accretion rates.

Several groups currently run adhesion tests on impact ice using centrifuges. The two primarily groups are PSU (Penn State University) [149-151] and AMIL (Anti-icing Materials International Laboratory) [63, 71], though there are others [68]. PSU uses a 10 ft. diameter rotor stand capable of reaching 1500 RPM, on which ice is directly accreted. 
The sample section is placed at the end of the rotor and attached via a load cell to obtain force data. The sample section is shaped in the form of an airfoil and stands off from the main rotor. AMIL, by contrast, typically accretes ice on small, flat samples and attaches them to their centrifuge adhesion test (CAT) $[63,64,72]$. AMIL's CAT uses a $34 \mathrm{~cm}$ diameter rotor, and accelerates samples in a climate-controlled centrifuge chamber at constant rate of $300 \mathrm{rpm} / \mathrm{s}$. AMIL also uses a Spinning Rotor Blade test (SRB), in which they placed a $0.78 \mathrm{~m}$ diameter rotor into their low speed icing tunnel, where they could achieve tip velocities of $130 \mathrm{~m} / \mathrm{s}$, wind speed up to $37 \mathrm{~m} / \mathrm{s}$, and repeatability below $30 \%$ [96]. (AMIL also tests using tensile, torsion, and bending tests more suitable for power line deicing $[72,152])$.

The high throughput capability AMIL possesses is highlighted by the number of samples they have run. AMIL has tested icephobics on their CAT for over 10 years and has found a wide variety of performance with coatings performing up to 1000 times better than aluminum, and some performing half as well. As AMIL points out, wear is a major problem for many of these coatings [64]. In spite of potential issues with testing as mentioned above, AMIL is able to demonstrate repeatability with standard deviations of $\pm 15 \%[64,153]$.

PSU has reported similar standard deviations [131]. PSU has also provided some data on custom made surfaces, such as epoxy with roughness varied in a very controlled manner [103]. An important difference in capability to point out is that the impact speed attainable by PSU is much higher than AMIL since PSU can produce impacts using the speed of their rotor tip, which can reach approximately $240 \mathrm{~m} / \mathrm{s}$. AMIL has not traditionally focused on aircraft icing, but on power-line icing [154]. This icing still involves the 
accretion of ice via droplet impact, and so the group's work has generally centered on adhesion testing for low-speed impact ice.

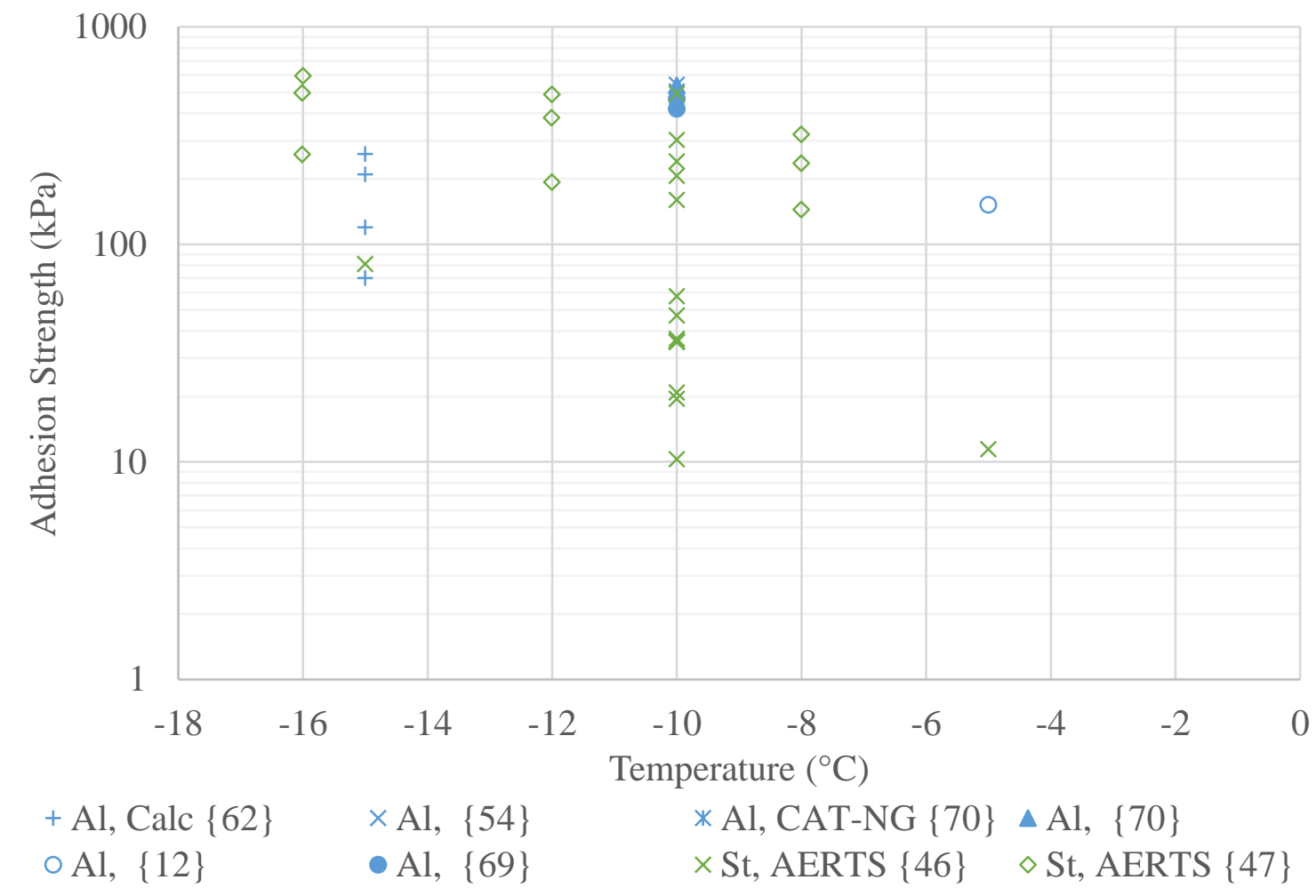

Figure 8. Averaged centrifuge test data, used from [45]. St is Steel, $\mathrm{Al}$ is Aluminum, and AERTS is the Adverse Environment Rotor Test Stand.

Centrifuge test data taken from the literature are shown in Figure 8. Similar to the push test data, the centrifuge data spans nearly two orders of magnitude in range. Most of the data was taken at $-10{ }^{\circ} \mathrm{C}$, and no strong trends showed up in the data. The data range for centrifuge data is lower than that for the push tests, suggesting that consistently higher stress concentrations were present in these tests, or that there were other complications in the induced stress fields during the test leading to the discrepancy. The average standard deviation was $21.2 \%$ for aluminum, and $14.5 \%$ for steel. The lower standard deviation for steel suggests that some property of steel samples lends to more repeatable testing. 


\section{Other Tests}

A variety of other kinds of tests have been performed. The Blister test involves leaving an open space under an ice accretion and using air pressure to delaminate the ice sample. At least two groups have used this with ice with good results [78, 125, 126, 130]. To investigate the delamination of ice on power lines, AMIL has investigated a variety of other methods where the substrate is deformed to delaminate ice $[72,152]$. These methods and a variety of other methods are discussed in much greater detail in [45].

Comparison of Methods

Centrifugal tests involve moving the specimen to produce force at the interface. This allows the test to use a single interface, meaning that nothing must be attached to ice samples post-accumulation to test the ice. Tests that fall into this category are referred to as "single-sided". The force distribution inherent to centrifuge tests is also very uniform under particular conditions. If the ice centroid is relatively close to the interface, the moment created on the ice is negligible. Assuming the length of the ice sample in the radial direction is small compared to the radius of rotation, the variation in centrifugal forces is also negligible. To estimate the adhesion force, these tests rely on increasing the forces linearly by accelerating rotation. The event of shedding is recorded and the speed at which a shed occurred is used to back out the shear force at the interface. The contact area between the ice sample and substrate must be measured before and after (in the event of a mixedmode failure) the shed event. Assuming a predominantly adhesive break, an estimate of the adhesion strength in shear is obtained by dividing the shear force by the change in contact area. This method obtains a single data point. Potential issues include sources of unknown loads, including aerodynamic and vibrational loading. This method is speculated to include 
greater variation due to variability in substrates that will affect the onset of crack propagation. Some variations, most notably the stand used by Penn State University, mount the ice on a force transducer to obtain force data directly, meeting the need for the specimen to be weighed. Since the specimen is moving, it is nearly impossible to use an extensometer to measure the strain in the specimen. Rotating equipment is well understood and designing a test rig to fit the IRT should be straightforward. Stress concentrations in the ice are also a significant concern. The use of a centrifuge would likely prohibit the shaping of the ice, and regardless of the geometry sharp stress concentrations are produced near the edges [155]. The variability in the geometry must only compound this problem. The investigation of other unknown effects in the ice, such as thermal contraction or the amount of ice left in surface roughness elements, would also be prohibitively difficult.

Controlled displacement methods do not require specimens to be rotated at high speeds, and thus have greater flexibility in testing geometry and in making measurements. It is straightforward to use extensometers to make strain measurements for these tests. Since the displacement is controlled, it is possible to make multiple measurements during crack propagation. It is also possible to obtain similarly uniform stress distributions through the use of lap-joint tests which are also less dependent on the thickness of ice than centrifuge methods. The disadvantage of using a lap-joint test is that the ice must be adhered to two substrates. If less-uniform surface shear is not prohibitive, a variety of test orientations are possible to measure shear that allow for single-sided testing. To understand the stress distribution in any test and develop the theory of operation, simulation is necessary. Single-sided adhesion tests that may be used in the IRT test section require new design and testing to validate their use. 
The centrifugal method is thought to provide useful data with much less design work and development of theory and can measure the adhesion strength of ice with the cloud still on. A centrifugal method may also provide direct validation for shedding models. Unfortunately, the centrifugal method will require ballistic shields limiting visibility during the test. The data from these tests will be of lower quality due to the issues including the stress concentration, variable geometry, the inability to preserve the specimen, and the inability to analyze stress-strain data. Each specimen will require direct attention before testing, such as for the measurement of mass and surface area, likely requiring the tunnel to be powered down before each test. Lap-joint tests will have no such requirement and should not be affected by aerodynamic forces while vibrational forces should be negligible. A comparison is shown in Table 2. 
Table 2. Comparison of Centrifuge and Lap-Jount tests.

\begin{tabular}{|c|c|c|}
\hline $\begin{array}{c}\text { Interfacial Stress } \\
\text { Distribution }\end{array}$ & Non-uniform & Lap-Joint \\
\hline $\begin{array}{c}\text { Unaccounted } \\
\text { Forces }\end{array}$ & $\begin{array}{c}\text { Aerodynamic, Vibrational, } \\
\text { Residual }\end{array}$ & Ron-uniform \\
\hline $\begin{array}{c}\text { Potential for In- } \\
\text { Situ IRT Stand }\end{array}$ & $\begin{array}{c}\text { Requires expensive test stand, Handling } \\
\text { ballistic panels, dedicated test }\end{array}$ & $\begin{array}{c}\text { Can be built into existing } \\
\text { models or into a piggyback test }\end{array}$ \\
\hline $\begin{array}{c}\text { Measurement } \\
\text { Data }\end{array}$ & $\begin{array}{c}\text { Mode 2 adhesive strength, } \\
\text { potential for tensile strength }\end{array}$ & $\begin{array}{c}\text { Mode 1, 2, and mixed mode } \\
\text { adhesive strength (compression } \\
\text { optional mode 2 only), Elastic } \\
\text { Modulus, friction }\end{array}$ \\
\hline $\begin{array}{c}\text { Sample } \\
\text { Preserving }\end{array}$ & No & Yes \\
\hline $\begin{array}{c}\text { Potential to Detect } \\
\text { Ice Residue }\end{array}$ & No & Limited \\
\hline
\end{tabular}

In summary, lap-joint tests should provide higher quality/lower noise data that yields much more information. Lap-joint tests should be less expensive in the long run and more straightforward to test. Due to the complicated stress distribution in lap-joint tests (as in any test), the method will require FEA to interpret the results quantitatively. Confidence will be obtainable through the number of data points acquired per sample, allowing for curve-fits of the data.

Trends in the Literature

For many working in the icing field, it is commonly heard that adhesion strength increases as temperature decreases. This largely stems from a 1958 report by Raraty and Tabor which showed linear trends with temperature in some of their data sets [147]. While this trend shows up in some newer publications [156], it is hardly universal. It doesn't show 
up in some of Raraty and Tabor's results, and also doesn't show up in Scavuzzo and Chu's results. Raraty and tabor suggested that it may have been due to a ductile-to-brittle transition [147]. The data is plotted as a function of temperature in Figure 9 and Figure 10, respectively.

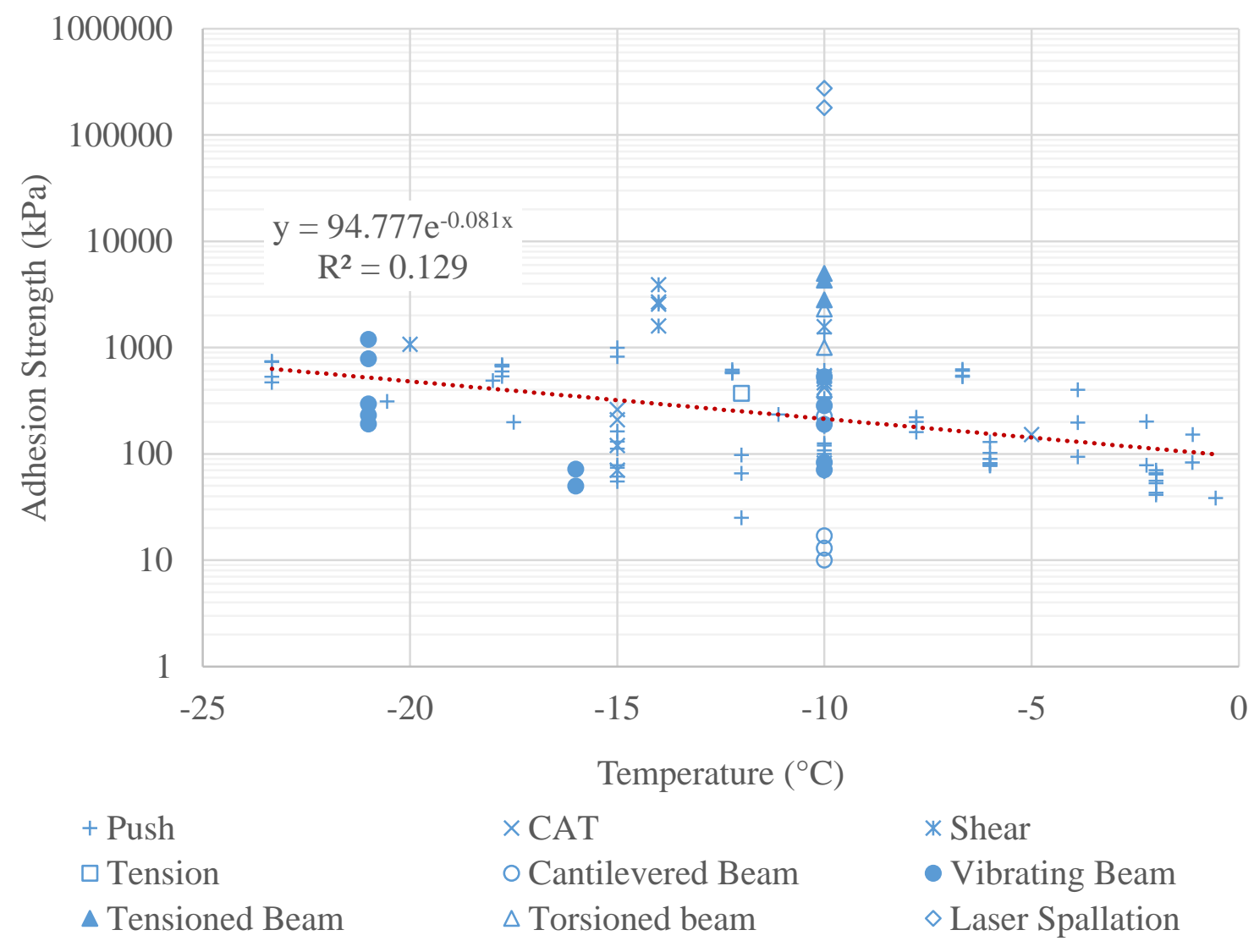

Figure 9. Averaged aluminum data in the literature, used from [45]. 


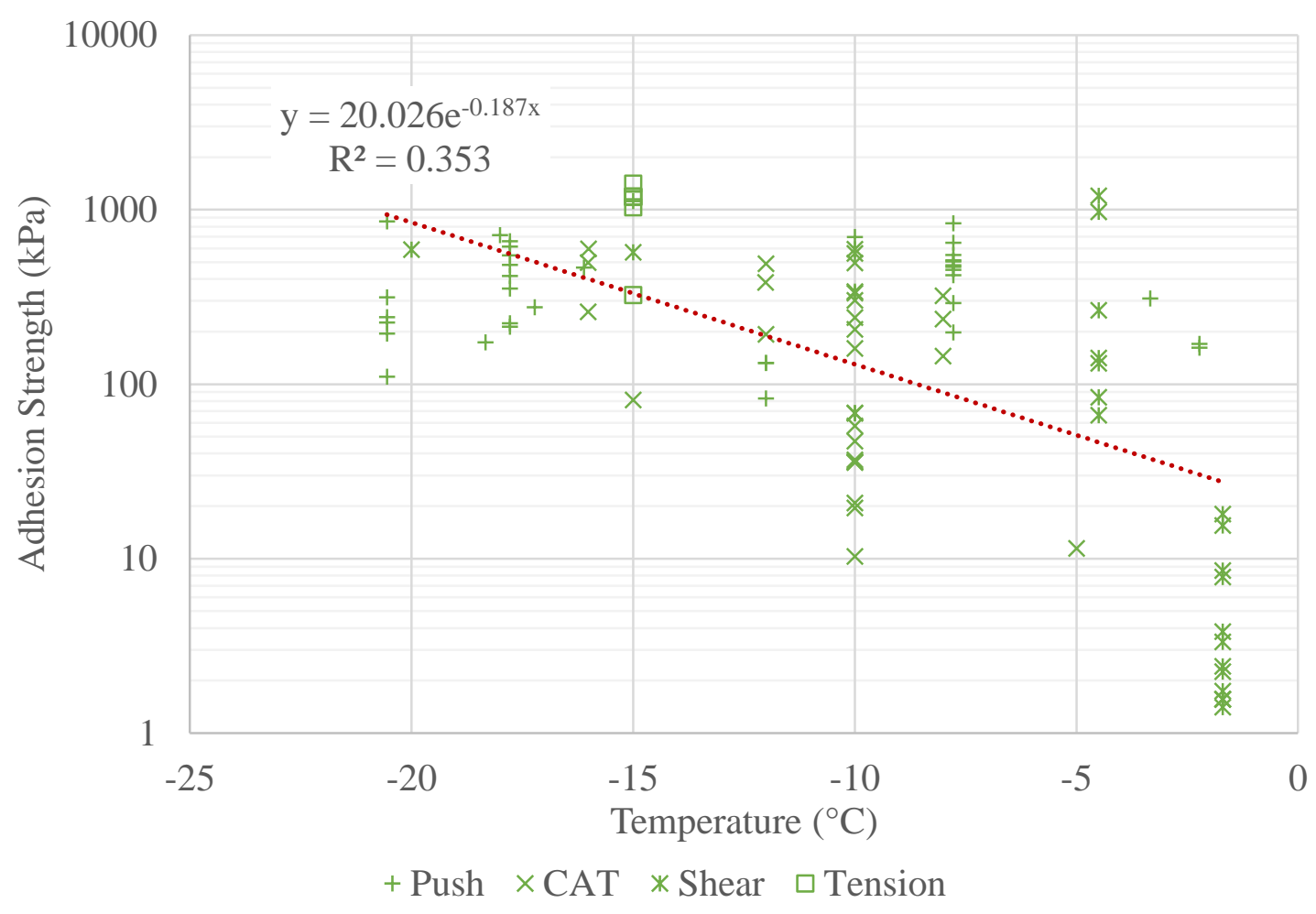

Figure 10. Averaged steel data in the literature, used from [45].

In both sets of data, a small upward trend is shown on average, but the fit to the data is poor. Linear fits provided lower $\mathrm{R}^{2}$ values. The data for both appears flat below -10 ${ }^{\circ} \mathrm{C}$, and most of the trend appears to be at warmer temperatures. In some data, this may be due to unaccounted for factors such as variable stress concentrations. For example, glaze shapes have much larger overhang on some centrifuge tests than rime conditions, which likely skewed the data to show the trend more strongly $[123,156]$.

Few papers investigated the effect of strain rate on the adhesion strength of the ice; only two were found to do so $[157,158]$. In these papers, the data showed an increase in the measured adhesion strength with increasing strain rate or displacement. While strong trends do not show up in the literature for impact speed for aluminum or steel, impact has been shown to make a difference in adhesion strength through the testing of Teflon, though little data was found to be suitable for comparison. 


\begin{abstract}
Alternative Concepts
Very few sources provide in-situ adhesion data, and existing methods have critical flaws that are not currently accounted for. Of the data in the literature, often too little information is reported to repeat the tests, and even if it were possible the flaws in many tests cannot be corrected. Stress concentrations in the ice, thermal contraction, and poor data reporting indicate the need for a new test method.

Three new methods for testing adhesion have been conceptualized. These methods utilize similar geometry to a single cover plate lap joint test and will possess several advantages over other test methods. Both use open-faced geometry such that ice may accrete on two surfaces that may be moved independently so that the samples will not require handling for testing. The first devised test was termed the Single-Sided Lap-Joint Test (SSLJT). The SSLJT utilizes two components for a test coupon: a block, and a slat. A representation of sample block is shown in Figure 11.
\end{abstract}




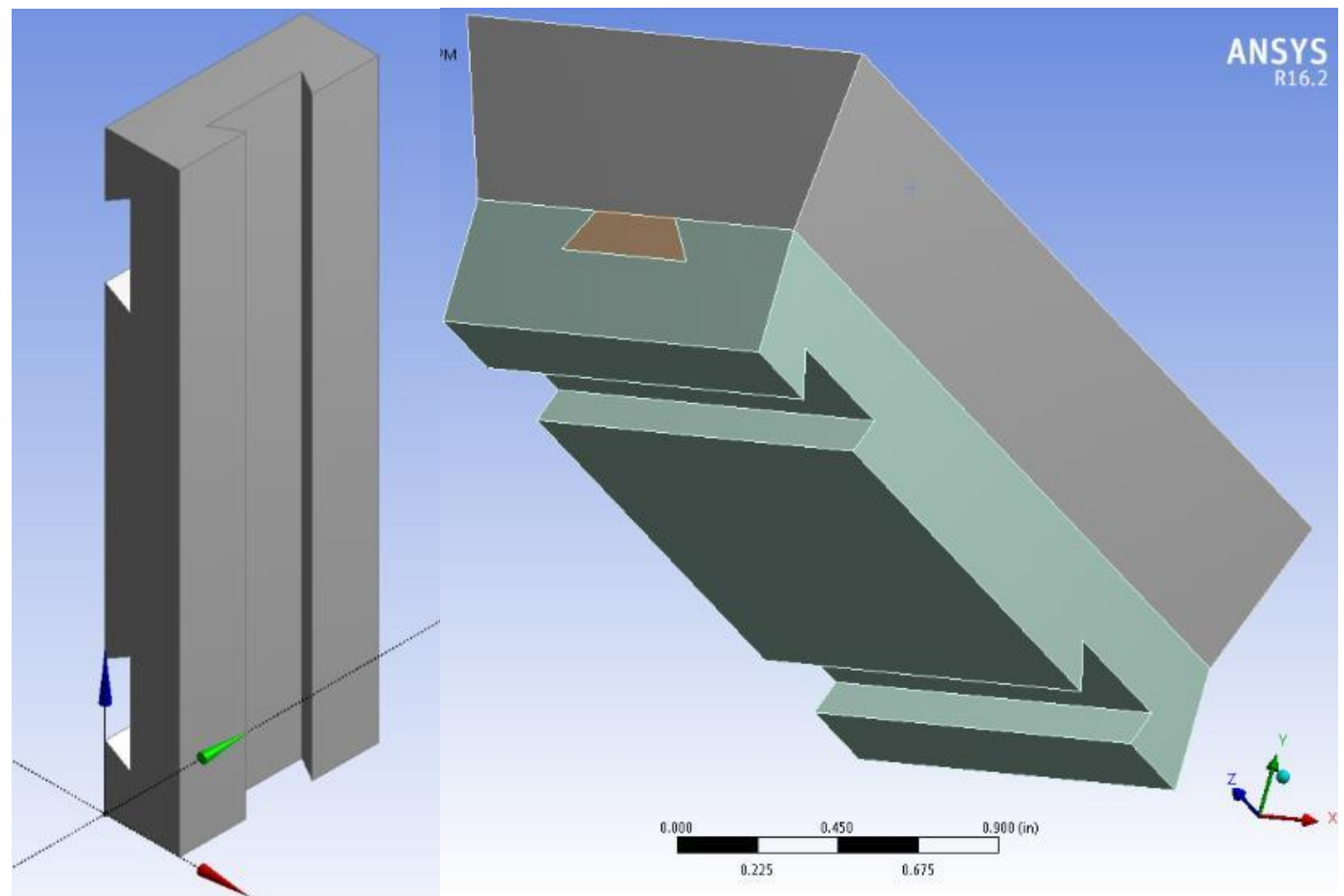

Figure 11. A sample SSLJT block (left) and full test (right).

The SSLJT block features a dovetail groove down its centerline. Inside the groove is placed a slat, pictured in orange on the right side of Figure 11. The two dovetails on the back side of the block will be used to hold the block in place on a fixture, similar to the fixtures in Figure 18. The gray shape on the right side of Figure 11 represents an ice sample. A preliminary simulation was run on this configuration to give an idea as to what sort of stress concentrations would be created by testing with an SSLJT. The results of this preliminary simulation are shown in Figure 12. 


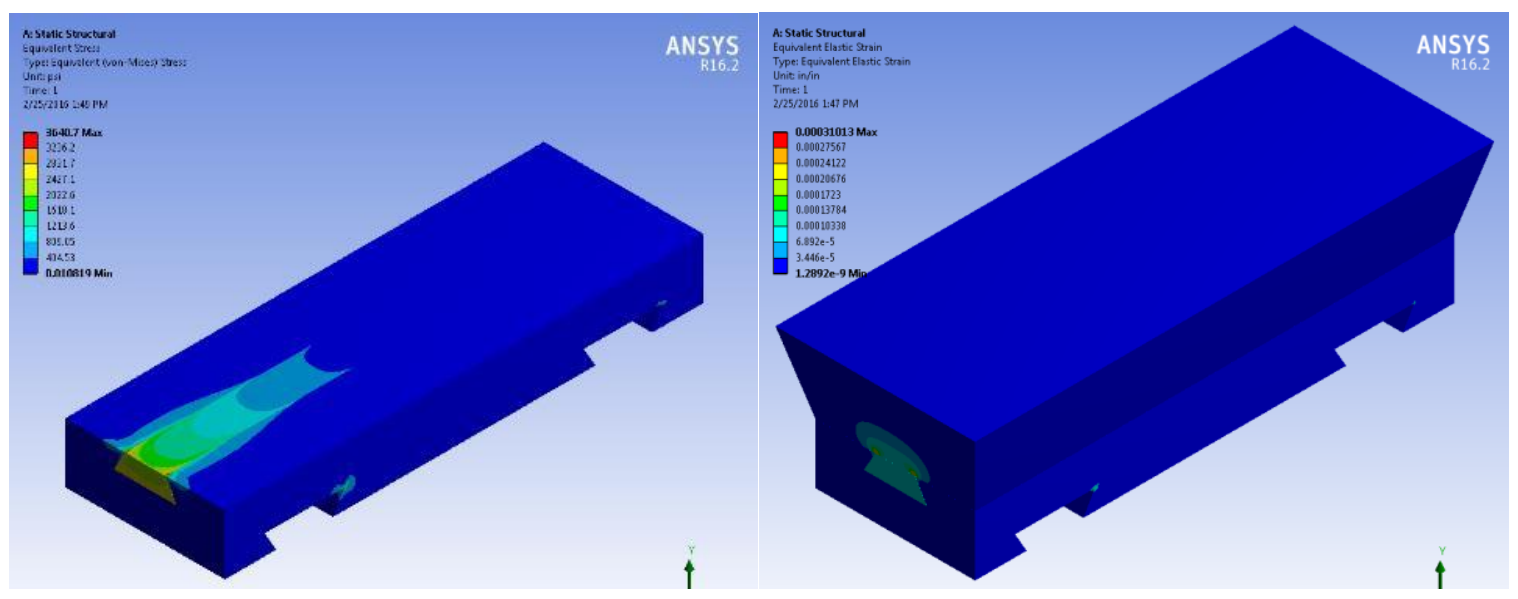

Figure 12. Sample simulation of SSLJT. In lieu of ice properties, aluminum was used for ice, while steel was used for the block and slat. Left: Von Mises stresses at the interface. Right: Strain in the block and ice shape.

The preliminary simulation of SSLJT used aluminum for the ice shape and steel as the material for the block and slat since suitable properties for ice hadn't yet been identified. The SSLJT configuration has several drawbacks; primarily it creates strong stress concentrations localized at the corners of the slat. It also will require tight tolerances and lubrication between the slat and block. The lubrication will also need to serve the dual purpose of keeping ice from growing inside the space between the block and the slat.

The upsides of the SSLJT are important, however. The SSLJT has simple geometry and can be inserted into small or large geometries, such as airfoils, that can be placed in the IRT. This means that the test can be built into a small airfoil placed to the side of the chamber, allowing for piggyback testing. Or, the test could be built into large airfoils that span the entire section of the IRT. This would help prevent ice from reaching the edges of the test fixtures. Piggyback tests have the distinct advantage of being free, but conditions cannot be controlled since they are dictated by the primary test, and test time must be made available. 
A second test method proposed is being developed with the same advantages of the SSLJT test but with less complicated geometry, reducing the cost and increasing the ability of the test to measure adhesion in multiple locations. This test is the Rotating or Translating Interface test (RIT). The RTI method is depicted in Figure 13 for a rotating configuration.
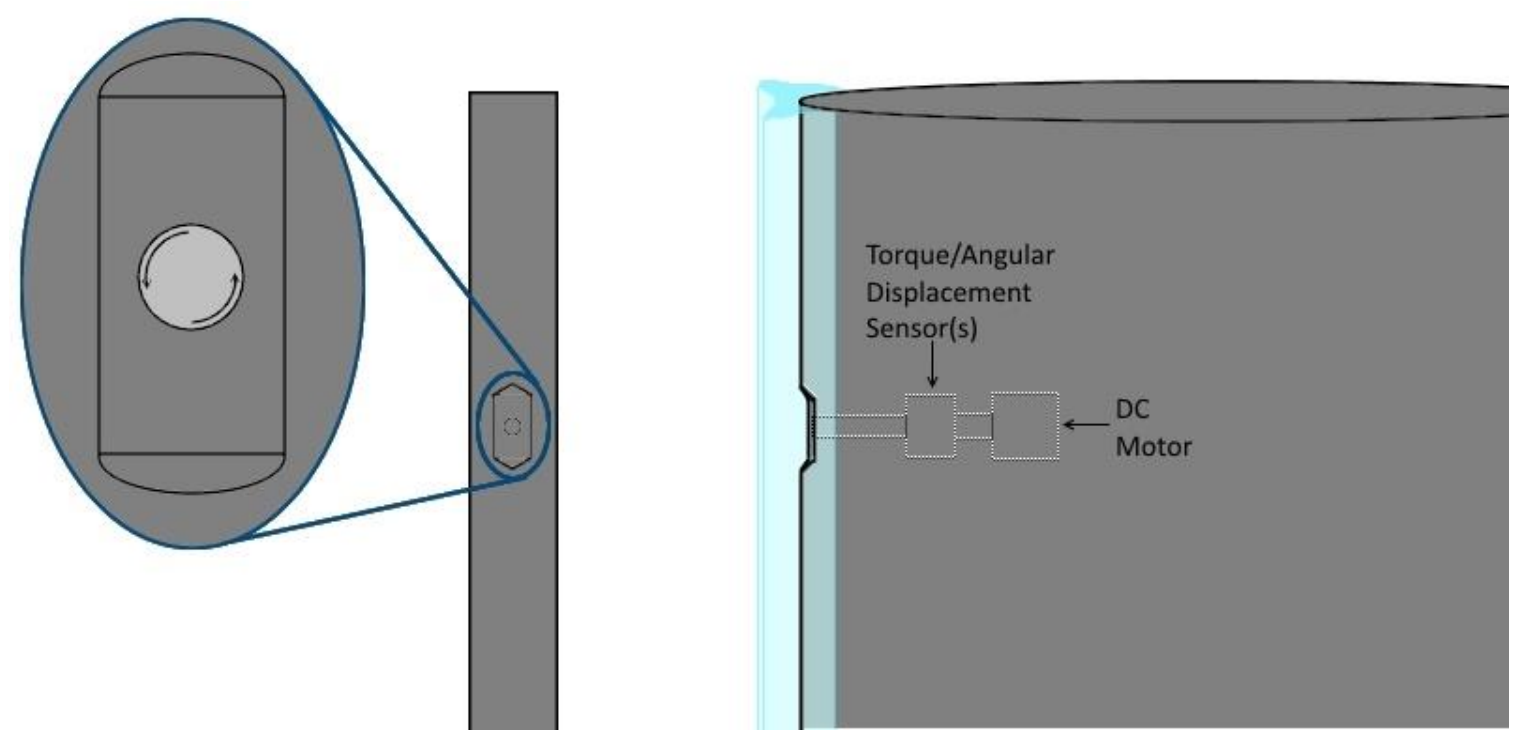

Figure 13. Preliminary diagram of the RTI method for determining ice adhesion.

The RTI method rotates a small section of the interface under the accreted ice and measures the force required to do so in order to determine the adhesion strength. This idea requires the use of simulations to relate the maximum torque measured to the adhesion strength at the interface given the inherently complicated loading conditions (as compared to a simple lap-joint test). Similar to the SSLJT test, stress near the border of the moving surface will diverge as the clearance between the moving and static surfaces goes to zero. A translating mode could be used that wouldn't require a flat to be ground into the airfoil, and soft rubber inserts could be used to control the stress concentration and reduce the need for excessively tight tolerances.

The final concept presented is the Rotational In-situ Test for Adhesion (RITA). The RITA uses a rotating cylindrical test article to accrete and test the ice. The coupon is a 
smaller cylindrical piece mounted at the center of the test surrounded by rubber inserts, and when the test is run the coupon is translated about the axis of the assembly to break the ice. A depiction of the test is shown in Figure 14.
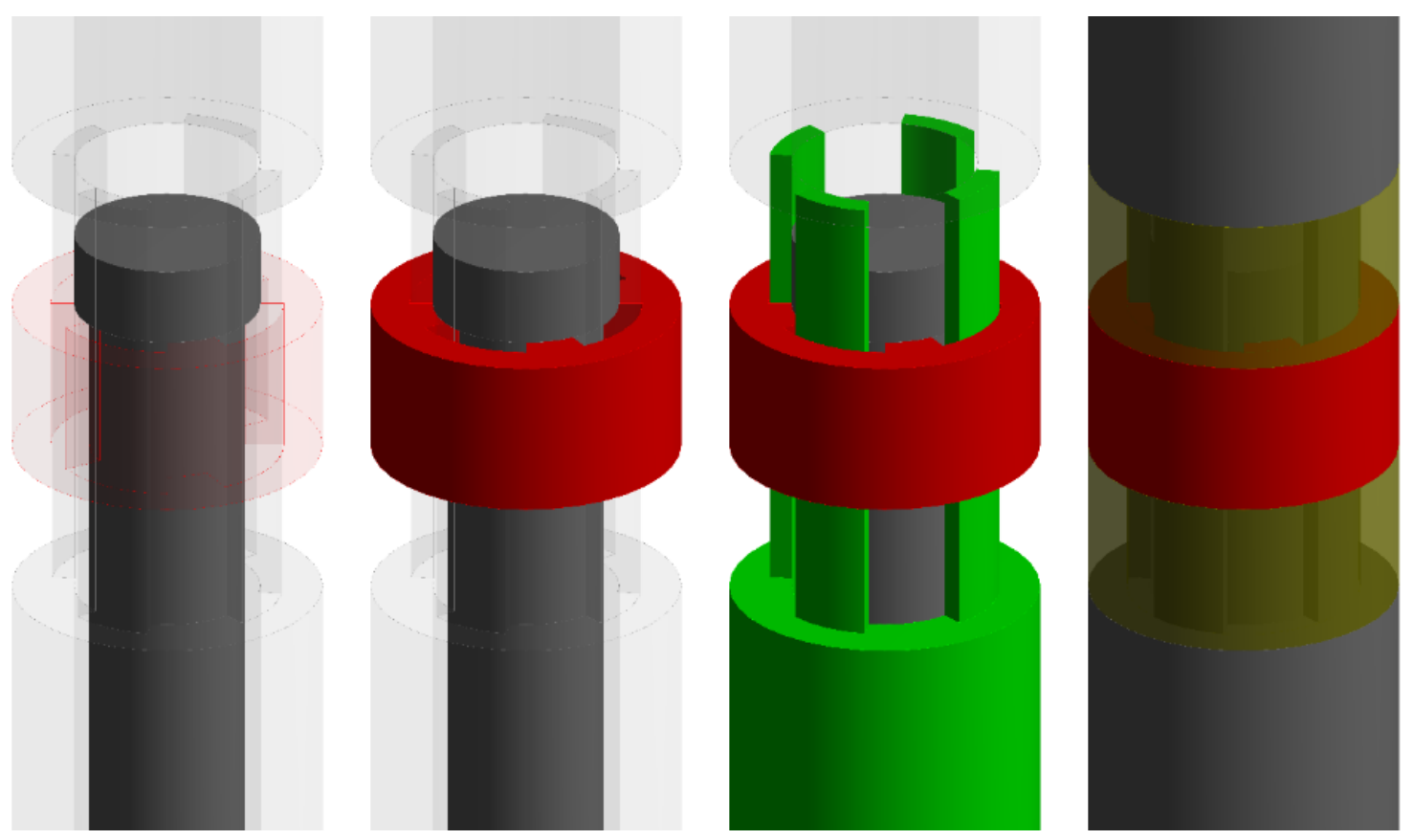

Figure 14. RITA test area close up. Left to right: Test rod, Coupon, Lower Cylinder, and full assembly with Soft Inserts and Upper Cylinder.

The RITA concept possesses a number of strengths. The stress concentration would be controlled through the length of rubber inserts on either side, which could easily be customized. Once an adhesion test is finished, a tensile test could be run on the remaining ice, and Young's modulus data could be acquired. Most importantly, geometric effects are minimized since the ice shape would be uniform at the interface (which would also be true for the RTI method), meaning randomized geometry in the icing process would have a reduced or eliminated effect on results. The method would also be adaptable to pre-cracked testing, and the cylinders could be instrumented to obtain strain data due to the accretion of ice. 
Small-scale tests are desirable due to cost. Large-scale tests in the center of the IRT will be expensive since a day of IRT test time currently costs in excess of $\$ 50,000$. Large scale tests using the RTI method could potentially test a large number of samples at a variety of locations on the surface of a realistic model, all within the best calibrated region of the tunnel. Two such models have been acquired for this purpose, a NACA 0012 with a 6' chord and a NACA 65-415 with a 3' chord. Both models span the entire height of the IRT. Alternatively, NACA 0012 and a NACA 23012 airfoils have been identified that have removable leading edges. The RTI test is suitable to be built into the removable leadingedge inserts. The RITA test would require a new model to be built from scratch.

These methods would allow data to be taken inside the IRT or other facilities. This is critical to obtaining better adhesion data since merely turning the cloud off in an icing tunnel changes the environmental conditions [159]. Traditionally, test coupons must be handled, carried around, and go through various temperature changes. The lap-joint test using the tensile tester shown in Figure 17 requires test samples from the IRT pass through the following workflow. 


\section{Ex-Situ Test Coupon Process}

In-Situ Test Coupon Process
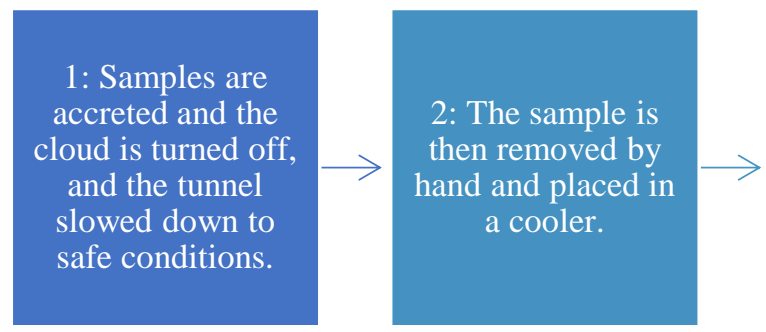

3: The cooler is

then taken to the

facility where the

test equipment is

$$
\text { located. }
$$
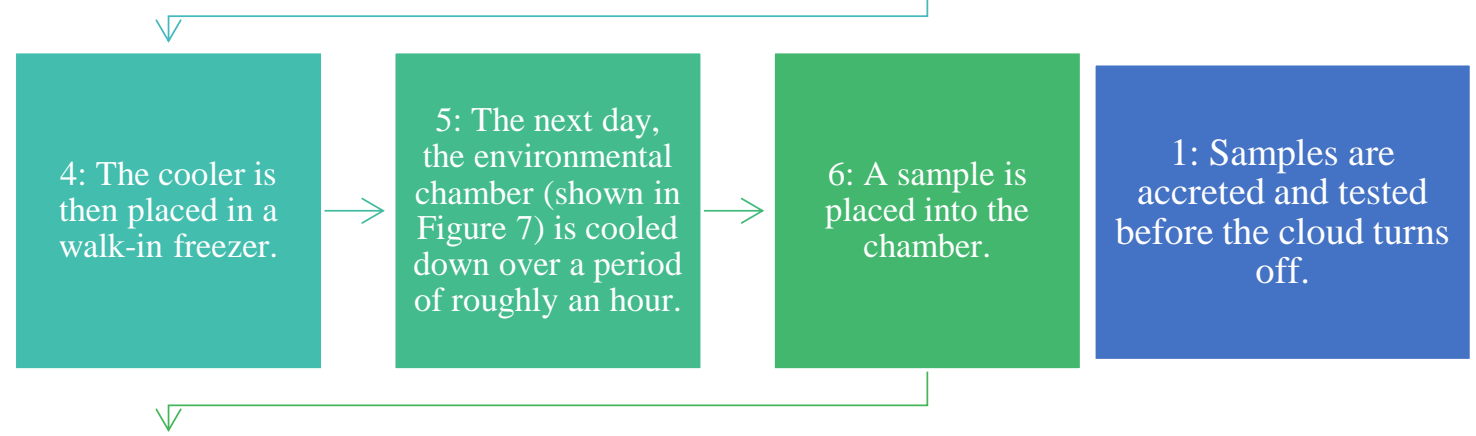

7: The serrated

block is adhered to

the ice sample by

placing water at

equilibrium and

allowing it to

freeze the two

surfaces together.

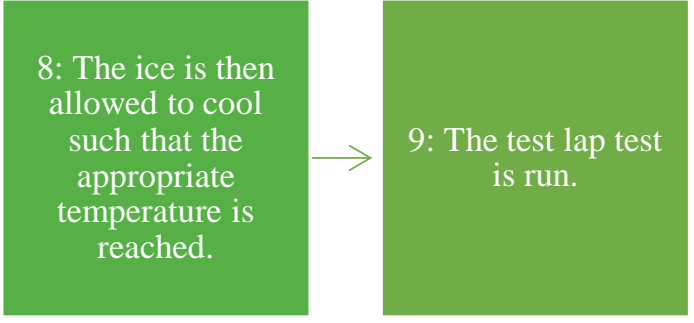

Figure 15. In-situ vs. ex-situ testing.

In the recently completed August 2016 tests, steps $4-8$ were replaced by carrying the samples up a flight of stairs, improving the test by bringing the test equipment closer to the IRT, but the difference cannot be eliminated without an in-situ test. In-situ tests remove time effects like creep and changing temperature history from consideration. The single-sided tests also would provide a curve of data, unlike centrifuge tests, where more than one material property could be measured at a time.

Unfortunately, none of the methods identified fit into the project budget, and a more traditional method had to be selected. A modified lap-joint test was utilized for the test 
since it provided the most flexibility in data acquisition and parameter control of the existing techniques.

Adhesion Models

An adhesion model for ice should predict the apparent adhesive strength of the ice to a substrate given the topology of the substrate and properties of both the ice and the substrate. Such a model would allow for the predictive capability of current hypotheses regarding the mechanisms of ice adhesion. With the necessary data, this will also allow the accurate prediction of adhesion strength of a surface based on the topology, providing insight useful for surface design and modeling. Currently, very few such models exist. There are three that will be discussed in this paper.

The earliest model discussed here is that developed by Fortin and Perron [70]. Their model contains a number of important effects and ultimately more detail than other models. This model uses the RMS roughness to predict adhesion strength. Unfortunately, they note that their model cannot accurately predict adhesion strength, and that more fundamental studies are needed to investigate the effects of roughness. Interestingly, they predict increasing adhesion strength with decreasing roughness. In their words:

At the present time, the proposed model cannot predict with good accuracy the ice adhesion shear stress because of a lack of knowledge of the substrate surface roughness and the parameters and constants involved in the model. Furthermore, the mechanical locking terms and the ice strength model are not yet validated. To improve the model, more fundamental studies are needed. First the use of the RMS roughness to characterize the substrate roughness has to be validated. Second, the ice strength model has to be better understood: how the porosity of rime and small grain ice affects the critical grain boundary sliding displacement; and how the temperature and the related cooling rate affect grain size. [70]

The intent of this work is to provide baseline data to develop a more simplistic model to predict shedding. To engage in the study suggested by Fortin and Perron, a more 
thorough investigation of the role of roughness answering whether or not RMS roughness can be used to characterize a substrate is needed. This would need to be done by using an average value for bond strength and investigating the mechanical interlocking effects of the roughness on the ice assuming negligible deformation prior to delamination. The author has not found an existing test method suitable for providing this data.

Guerin et al. developed a model of ice adhesion based on mechanics of the Disordered Interface (Liquid-Like Layer) [71]. The Disordered Interface is a poorly understood area of ice physics that is undoubtedly important to ice adhesion. However, Guerin et al. make a number of unwarranted assumptions regarding the properties of this layer, and calculate the capillary forces created by the disordered interface as if it were a pure liquid with known properties. Similar assumptions were made regarding the other properties of the layer. This was done because these properties cannot be measured. This model uses average roughness and the mean spacing of irregularities. Unfortunately, until properties of the Disordered Interface are known better, this model will not provide accurate adhesion data.

The final model for ice adhesion is that of Knuth [103]. This model attempts to predict the adhesion strength based on the angle of the surface roughness elements. Knuth's model uses a 2D force balance of individual roughness elements. Unfortunately, this model fails several critical checks. First, the model does not allow for cohesive breaking. Second, it predicts finite values of shear stress for adhesive failure in cases where pure adhesive failure is impossible (such as that shown in Figure 1, zone C). The experimental data used to validate the model contained such surfaces, suggesting that the cohesive strength of the ice was similar to the predicted adhesive strength, or possibly that the ice did not penetrate 
the elements fully. As such, the author was unable to find any current adhesive model by which the roughness of substrates could be studied.

The difficulty of modeling adhesion lies in obtaining useful data. Given so much spread in the data and so many relevant mechanisms, it is difficult to correlate available data to a model. Features such as mechanical clamping, the DI, and the grain structure of the ice cannot be separated such that their individual effects can be studied independently. The difficulty of working with impact ice also makes it difficult to study the interface posttest to obtain evidence on the failure mechanisms.

\section{Specimen Placement in Tunnel}

No icing tunnel can produce a perfectly uniform cloud. The Liquid Water Content (LWC) in the tunnel can vary significantly across the cross section. Some tunnels also produce ice crystals, where the parameter corresponding to the LWC is the Icing Water Content (IWC). Each of these parameters are typically measured in the units of $\mathrm{g} / \mathrm{m}^{3}$, the mas of water per cubic meter of air in the tunnel. The combination of the IWC and LWC is the Total Water Content (TWC). The cloud is made up of droplets of water, and the size distribution of these droplets is commonly discussed in terms of the Mean Volumetric Diameter (MVD). By definition, half of the liquid water resides in droplets with diameters below the MVD and the other half in droplets with diameters above the MVD. The distribution and MVD of the droplets can vary across the tunnel cross-section. Extensive calibrations of the IRT were performed regularly using the best experimental methods available. This process was documented extensively in the literature $[23,80]$. The work on tunnel calibration in the IRT provides the data necessary to understand the variation in these parameters in this work. What the data shows is that the best calibrated region of the 
tunnel is in the center. Larger droplets tend to produce shorter clouds since the cloud begins to fall away from the ceiling. There are several locations in the tunnel with a non-uniform cloud, as well. Typically, measurements on the geometry of ice in the IRT were taken at the center of the tunnel since this area was the best calibrated $[160,161]$.

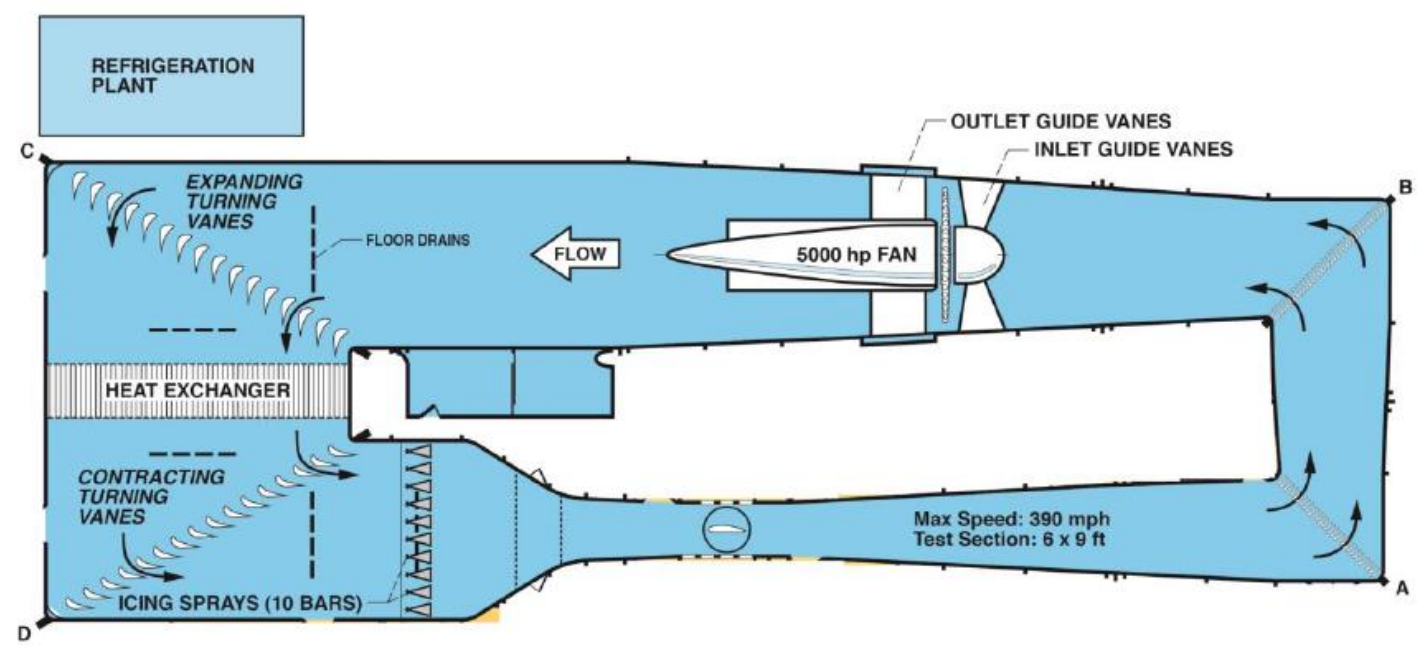

Figure 16. The Icing Research Tunnel [80].

During piggyback tests, ice samples were acquired on small coupons in the tunnel's zipper slot aft of the primary test model. The samples were mounted on a block with two dovetail rails with a set screw, offset from a steel bar in the test section. The test section of the IRT is 6' tall and 9' wide. The top edge of the coupon closest to the outside wall of the tunnel (the wall at the bottom of the test section in Figure 16) was approximately 20" from the wall and 49" from the floor of the test section. The center of the sample was then approximately 48 " from the floor and 33 " from the center of the tunnel, just inside the calibrated cloud in the horizontal direction. Vertically, the coupon leaves the calibrated cloud when the MVD reaches $100 \mu \mathrm{m}$. At larger droplet diameters gravity can create appreciable velocity differences between the droplets and the surrounding air; this can cause the droplet distribution to vary along the vertical direction of the test chamber. 


\section{EXPERIMENTAL METHODOLOGY}

In order to provide realistic data for the adhesion strength of ice to aircraft components, a new adhesion testing methodology has been developed. A new IRT wind tunnel model (the XT Model) was designed to hold samples during accretion. Coupons were produced; samples were then collected, stored, and tested. To test the adhesion strength of the samples, a new shear rig was developed with the special ability to run a variety of tests. Finally, a method was developed to shave down samples for microscopy to record the grain structure of the ice.

\section{XT Model Development}

A new model for the IRT was required to obtain shear test samples of aircraft ice in the IRT, the Materials Testing Model (XT model). The model was required to mount either 24 or 48 coupons in the IRT on dovetail mounts. In order to meet the requirements specified in the IRT Manual, the following design effort was undertaken. It was attempted to determine the aerodynamic loading using ANSYS Fluent on rigid geometries, including a clean geometry and a geometry with ice attached. In order to perform a dynamic analysis, it was attempted to obtain an ice shape with LEWICE - but a shape could not be obtained with any confidence. A flat plate with rounded edges was used as a worst-case scenario, creating a T-beam geometry. Using the $\mathrm{X}$ - and $\mathrm{Y}$-axis loading from the CFD analysis, a structural analysis was performed using ANSYS Mechanical to provide an estimate of maximum model vibration. The results from a static analysis were fed into a modal 
simulation, and then into a harmonic simulation where the force amplitude of the Von Karman effect was applied to the model at the modal's natural frequencies below $4000 \mathrm{~Hz}$. However, since dynamic loads could not be determined the FEA results are for reference only. ${ }^{11}$ Ultimately it was determined that it was not feasible to calculate the dynamic loading on the model and so a static analysis was performed using a known coefficient of drag from literature.

Aerodynamic Loading

The static load in the drag direction was determined using a coefficient of drag $\left(C_{d}\right)$ of 1.8 for a 2D T-beam geometry [162]. The total cross sectional area $\left(\right.$ in. $\left.^{2}\right)$ and estimated Ice Length (IL, the perimeter exposed to ice, in.) for one clean model was counted as follows: 21.6 (43.2 IL, qty. 5x) for each tie rod assembly (sleeves, end caps, nuts, washers, and tie rods), 50.85 (135.6 IL, qty. 4x) for each vertical rail, 1.8 (3.025 IL, qty. 16x) for each block and matching insulator, 17.25 (13.3 IL, qty. 2x) for each end plate, and 28 (7 IL, qty. 2x) for each adapter plate. Bolts and nuts were neglected. The total clean crosssectional area (per 4-rail model) is $\mathrm{A}_{\mathrm{c}}=430.7 \mathrm{in}^{2}\left(0.2779 \mathrm{~m}^{2}\right)$, and the total length to use for added area from ice is $L_{i}=847.4$ in $(21.52 \mathrm{~m})$. The total area was calculated using

$$
A=A_{c}+w_{i} L_{i}
$$

where $\mathrm{w}_{\mathrm{i}}$ is the width of the ice. The force on the model was calculated using

$$
F_{d}=\left[\frac{C_{d} \rho u^{2} A}{2}\right]
$$

where $u$ is the maximum speed of the tunnel ( 300 knots, or $155 \mathrm{~m} / \mathrm{s}$ ), and $\rho$ is the density of air at the tunnel minimum temperature of $-40{ }^{\circ} \mathrm{C}\left(1.514 \mathrm{~kg} / \mathrm{m}^{3}\right)$. At the worst-

${ }^{11}$ The CFD and FEA results are documented in APPENDIX C. 
case scenario, the force on a single clean model was $2045 \mathrm{lb}_{\mathrm{f}}(9097 \mathrm{~N})$. Table 3 shows the calculated force vs. ice thickness and temperature.

Table 3. Predicted load vs. temperature and ice thickness.

\begin{tabular}{|c|c|cccccc|}
\hline Temp & Density & \multicolumn{7}{|c|}{ Thickness (in) } \\
$\left({ }^{\circ} \mathrm{C}\right)$ & $\left(\mathrm{kg} / \mathrm{m}^{3}\right)$ & 0 & 0.10 & 0.20 & 0.30 & 0.39 & 0.49 \\
\cline { 3 - 8 }-40 & 1.514 & 2045 & 2441 & 2837 & 3233 & 3629 & 4025 \\
-20 & 1.395 & 1884 & 2249 & 2614 & 2979 & 3344 & 3709 \\
0 & 1.293 & 1747 & 2085 & 2423 & 2761 & 3099 & 3437 \\
10 & 1.247 & 1685 & 2011 & 2337 & 2663 & 2989 & 3315 \\
20 & 1.204 & 1626 & 1941 & 2256 & 2571 & 2886 & 3201 \\
& & \multicolumn{7}{|c}{ Load $\left(\mathrm{lb}_{\mathrm{f}}\right)$} \\
\hline
\end{tabular}

Structural Analysis

Once the aerodynamic loads were available, a structural analysis was performed to ensure that the model and the tunnel wouldn't be damaged during testing. The properties used for analysis of design critical components are shown in Table 4.

Table 4. Material properties used for joint analysis. *calculated using von Mises failure criterion, $0.577 \sigma$.

\begin{tabular}{|l|l|l|l|}
\hline & $17-4$ PH H900 & $\mathbf{1 8 - 8}$ & A286 \\
\hline Young's modulus (ksi) & $28,500[163]$ & $28,000[164]$ & 29,000 \\
\hline Ultimate Shear Strength (ksi) & $110^{*}$ & $43^{*}$ & $75^{*}$ \\
\hline Yield Shear Strength (ksi) & $98^{*}$ & $15[165]$ & $49 *$ \\
\hline Ultimate strength (ksi) & $190[163]$ & $75[165]$ & $130[166]$ \\
\hline Yield strength (ksi) & $170[163]$ & $30[165]$ & $85[166]$ \\
\hline
\end{tabular}

The structural connections considered for analysis are the vertical rails to the end plates (rail to blocks, blocks to end plates), the end plate to the tunnel adapter plate, and the tunnel adapter plate to the tunnel. A design load of $4025 \mathrm{lb}_{\mathrm{f}}$. was assumed to be distributed evenly over the rails for analysis of all connections. Shear of the tie rods was also considered using proportional loading on a single sleeve, with the tie rod in double shear. Bending of the rails was considered as a beam with simple supports at both ends. The analysis for this is shown in Table 5. Each rail was treated as a simply supported 
cantilever beams for the sake of modal analysis. ${ }^{12}$ Struts were added to hit the peak amplitudes along the rails of the model for the first four mode shapes and angled to prevent common modes of vibration from developing between each rail and to maximize stiffness under static loading for the model.

Each tie rod has a diameter of $0.5 \mathrm{in}$, corresponding to an area of $0.196 \mathrm{in}^{2}$, and a yield shear strength of $98.1 \mathrm{ksi}$. Each sleeve had an iced area of $11.25 \mathrm{in}^{2}$, corresponding to a load of $53.4 \mathrm{lb}_{\mathrm{f}}$, and a factor of safety of approximately 720 (805 for ultimate shear). Shear loads on the bolts were determined simply by dividing the design load proportionally for each joint (by 8 for bolts in the blocks, by 2 for bolts in the end plates), and again by the number of bolts in each joint. The tension force was determined from summing the moments around the back edge of each joint with reaction forces at the bolt location, assuming only half of the bolts were in tension. These loads as well as the tightening torque for each fastener were used in EFAST. ${ }^{13}$

\footnotetext{
${ }^{12}$ This was qualitatively verified using modal analysis. Modal analysis with struts performed in ANSYS Mechanical is presented in APPENDIX C.

${ }^{13}$ EFAST is a joint analysis design tool developed internally at NASA GRC, a sample analysis is shown in APPENDIX C.
} 
Table 5. Bending stress in rail calculation and factors of safety.

\begin{tabular}{|c|c|c|c|c|}
\hline Variable & Equation & Value & Unit & Description \\
\hline $\mathrm{b}$ & Input & 0.75 & in & Box width \\
\hline $\mathrm{h}$ & Input & 3.25 & in & Box height \\
\hline $\mathrm{L}$ & Input & 66.88 & in & Rail length \\
\hline $\mathrm{W}_{\text {tot }}$ & Input & 4025 & $\mathrm{lb}_{\mathrm{f}}$ & Total force over all 4 rails \\
\hline E & Input & $28,500,000$ & psi & 17-4 PH H900 modulus of elasticity \\
\hline$\sigma_{\mathrm{y}}$ & Input & 170000 & psi & 17-4 PH H900 min. yield strength \\
\hline$\sigma_{\mathrm{u}}$ & Input & 190000 & psi & 17-4 PH H900 min. ultimate strength \\
\hline K & Input & 3 & & Stress concentration from holes \\
\hline $\mathrm{r}$ & $=\mathrm{h} / 2$ & 0.375 & in & Radius \\
\hline$r_{c}$ & $=4 \mathrm{r} /(3 \pi)$ & 0.159 & in & Center of semicircle from edge \\
\hline $\mathrm{d}$ & $=\mathrm{h} / 2+\mathrm{r}_{\mathrm{c}}$ & 1.78 & in & Distance from center to circle center \\
\hline$A_{c}$ & $=\pi \mathrm{r}^{2}$ & 0.221 & in $^{2}$ & Area of semicircle \\
\hline$I_{x c c}$ & $=(\pi / 8-8 /(9 \pi)) \mathrm{r}^{4}$ & 0.00217 & in $^{4}$ & $\begin{array}{l}\text { Moment of single semicircle about its } \\
\text { center }\end{array}$ \\
\hline $\mathrm{I}_{\mathrm{xc}}$ & $=\mathrm{I}_{\mathrm{xcc}}+\mathrm{A}_{\mathrm{c}} \mathrm{d}^{2}$ & 0.705 & in $^{4}$ & Moment of single semicircle about center \\
\hline $\mathrm{I}_{\mathrm{xb}}$ & $=\mathrm{bh}^{3} / 12$ & 2.15 & in $^{4}$ & Moment of rectangle \\
\hline $\mathrm{I}_{\mathrm{x}}$ & $=\mathrm{I}_{\mathrm{xc}}+\mathrm{I}_{\mathrm{xb}}$ & 3.56 & in $^{4}$ & Total X moment \\
\hline $\mathrm{W}$ & $=\mathrm{W}_{\text {tot }} /(4 \mathrm{~L})$ & 15.0 & $\mathrm{lb}_{\mathrm{f}} / \mathrm{in}$ & Distributed load, lbf/in of rail \\
\hline $\mathrm{M}_{\max }$ & $=\mathrm{WL}^{2} / 8$ & 8412 & $\operatorname{lb}_{f}$ in & Maximum moment (in center) \\
\hline $\mathrm{D}_{\max }$ & $=5 \mathrm{WL}^{4} /\left(384 \mathrm{EI}_{\mathrm{x}}\right)$ & 0.0387 & in & Maximum deflection \\
\hline$\sigma_{\max }$ & $=\mathrm{M}(\mathrm{h} / 2+\mathrm{r}) / \mathrm{I}_{\mathrm{x}}$ & 4731 & psi & Maximum stress (no concentration) \\
\hline$\sigma_{\max , \mathrm{a}}$ & $=\sigma_{\max } \mathrm{K}$ & 14193 & psi & Maximum stress (with concentration) \\
\hline $\mathrm{F}_{\mathrm{y}}$ & $=\sigma_{\mathrm{y}} / \sigma_{\mathrm{max}, \mathrm{a}}$ & 12.0 & & Yield factor of Safety \\
\hline $\mathrm{F}_{\mathrm{u}}$ & $=\sigma_{\mathrm{u}} / \sigma_{\max , \mathrm{a}}$ & 13.4 & & Ultimate factor of Safety \\
\hline
\end{tabular}

The remainder of the critical components requiring analysis were fasteners, and the corresponding analysis is shown in APPENDIX C. A summary of the factors of safety for load bearing parts are presented in Table 6 . 
Table 6. Summary of Margin of Safety, and factors of safety for load bearing parts.

\begin{tabular}{|l|l|l|l|}
\hline Part & MS & Fsu & Fsy \\
\hline 7,8 & & 12.0 & 13.4 \\
\hline 5 & & 805 & 720 \\
\hline 12 & 0.68 & 8.4 & 5.5 \\
\hline 13 & 1.28 & 11.4 & 7.4 \\
\hline 16 & 2.69 & 18.5 & 12.1 \\
\hline $1 / 2-20$ Tunnel Bolt & 0.23 & 6.2 & 3.4 \\
\hline 3/8-16 Tunnel Bolt & 0.46 & 7.3 & 4.1 \\
\hline
\end{tabular}

All parts have a suitable factor of safety, and the design was limited by the bolts connecting the model to the tunnel. Larger samples with increased drag could be used provided that additional bolts were used to fasten the model to the IRT test section. The above analysis is conservative since the tunnel will almost certainly never be used at -40 ${ }^{\circ} \mathrm{C}$ with this model since the IRT is not calibrated at that condition, and the tunnel is unlikely to be able to sustain 300 knots test section velocity with both models in place.

The model was ultimately placed in the tunnel in the 24-sample configuration since a suitable number of test coupons were not available to use the 48 -sample configuration. The model was first run on 10/2/17 and passed aerodynamic checkout and has since been run in the 24-sample configuration for six nights of testing.

\section{IRT Methodology}

After a series of preliminary data was taken in piggyback tests, a series of three tests were conducted in the IRT. Each test was two days long. The first of these tests was conducted on 10/2/17-10/3/17, the second on 11/20/17-11/21/17, and the third on $1 / 17 / 18$ $1 / 18 / 18$. The purpose of each test was originally the same: to collect samples across a range of velocities and droplet size distributions at a particular temperature. However, during the 
second test and third tests unexpected problems were encountered and the test plan was modified.

In each test, samples were cleaned using SF-77 cleaner (diluted 10:1), mounted on the XT model, and then cleaned again using isopropyl alcohol. After the last cleaning, care was taken not to touch the samples until they were removed. Samples were placed into labeled bags and stored either in portable freezers or coolers and transported to a walk-in freezer in the Fundamentals of Adhesion and Shedding Testing for Icing Laboratory (FASTI Lab). Air-tight Whirl-Pak sample bags were used to store the iced samples. The procedure to store the samples varied with each test. In the first test, samples were carried to the FASTI Lab at the end of the night and stored in the portable freezers inside the walkin freezer. In the second test, they were carried over at the end of each test, so that shear tests could be run shortly after the cloud was turned off. Samples were placed in grocery bags and stored in boxes. It was observed that samples placed outside of insulated containers would frost over on the inside, indicating that internal sublimation and deposition cycles were taking place. This was observed to a much lesser extent in the samples stored in the portable freezers, indicating that proper insulation could prevent the cycle from occurring. In the third test, each run had a dedicated cooler that was pre-cooled in a freezer and carried to the FASTI Lab for immediate testing.

In the second and third tests, two researchers were stationed in the FASTI Lab. The first was to run shear tests on samples with the goal of obtaining five per test before the end of the following test. The second was to cut and image samples as detailed in the Microscopy section. Run conditions for each of the three tests are shown in APPENDIX A. 


\section{Development of the Shear Test Method}

To measure the adhesion strength of ice, a shear test was developed in two large steps. First, using the equipment freely available, a prototype adhesion test was developed, and a small number of samples were tested. Second, new equipment was specified and purchased for an updated test methodology. Using the new equipment, hundreds of samples from the IRT were tested.

Preliminary Adhesion Testing

Several methods were considered to perform adhesion tests. For this project, a lapjoint test was selected since it is a common method to test the adhesive strength of substances and was expected to provide a nearly uniform force distribution at the interface. Samples were collected in the IRT by mounting samples vertically in the tunnel on a dovetail mount with a set screw at the back. The samples were removed post-test and placed in a cooler, and then into cold storage. Two sets of piggyback testing have been performed. In the first set of testing, samples were stored long-term and tested in the Icing FASTI Lab at NASA GRC. In the second set of testing, the test equipment was moved above the tunnel and were stored for less than 10 minutes. The samples were then mounted in the test chamber on another dovetail block. This block was attached to the tensile tester frame (or load frame). A second block (termed "serrated block") was attached to the ice directly. This was done by heating the block to melt the ice and allowing it to refreeze. At this point, the tensile tester was activated to pull the serrated block up, breaking adhesion between the coupon and the ice.

To test the adhesion of ice using a lap-joint method, it was necessary to construct a new fixture. A prototype lap-joint shear fixture was designed, which is depicted in Figure 
18. To support the test, a Tinius Olsen 10,000 series universal tester was acquired including $100 \mathrm{lb}_{\mathrm{f}}$ and $1000 \mathrm{lb}_{\mathrm{f}}$ load cells. The frame included a built-in signal conditioning system and digital readouts and provides analog out in the form of a $\pm 1 \mathrm{~V}$ signal. The output extension and force readings were conditioned using a Krohn-Hite 3342 signal conditioner with a $20 \mathrm{~dB}$ gain and a low pass filter set to half the recording frequency. The force and extension voltages were then measured using an ioTech DaqBook 2020. A LabVIEW script was created to control both the universal tester and the DaqBook. The script was set such that the motion command to the universal tester was sent after the DaqBook began recording such that triggering was not necessary. The system was professionally calibrated in compliance with ASTM E4-15 and had an uncertainty of less than $0.23 \%$ at a $95 \%$ confidence level. The system is shown in Figure 17.

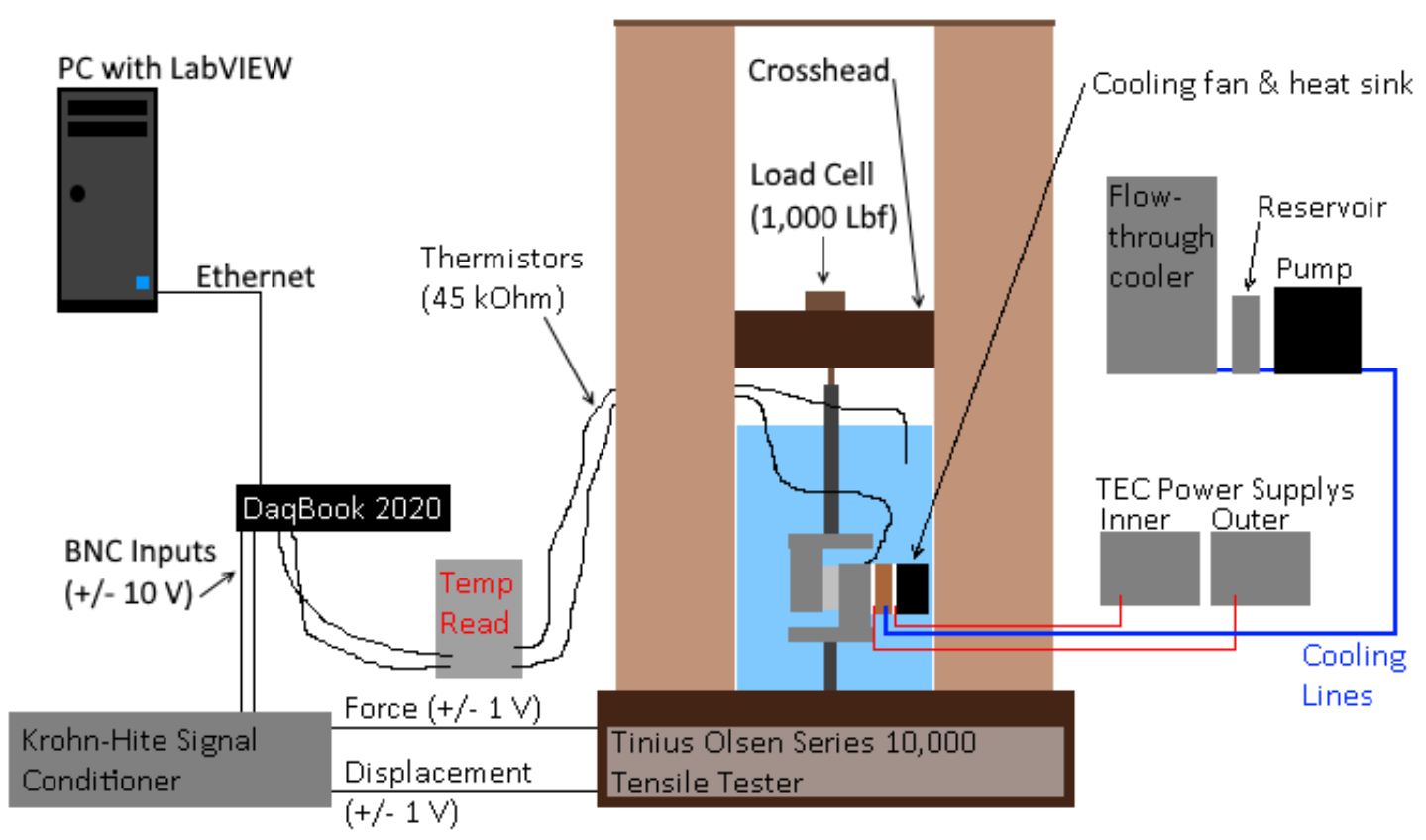

Figure 17. Tinius Olsen universal tester and supporting equipment schematic.

A refrigerated chamber was created from Styrofoam panels, and was cooled using an FTC-350 flow-through cooler with an external pump and cold stage. The cold stage was 
sandwiched between two sets of Peltier coolers. On one side, the coolers were in contact with a CPU cooler with a $12 \mathrm{~V}$ fan. This provided cold air to the chamber. A thermistor was placed in the mounting fixture, hooked up to a Deban 4300 digital thermometer (two of these were in a single box, labeled "Temp Read" in Figure 17). This provided a voltage output read into the DaqBook and recorded by the LabVIEW script. On the other side of the cold stage, the Peltier coolers contacted the mounting fixture used to hold the coupon for the test. The thermocouple for the temperature controller and a second thermistor were inserted into the fixture to measure and record the temperature. To facilitate thermal conduction between these components, Artic Silver 5 thermal paste was used. The cold chamber is shown mounted inside the universal tester in Figure 18. 


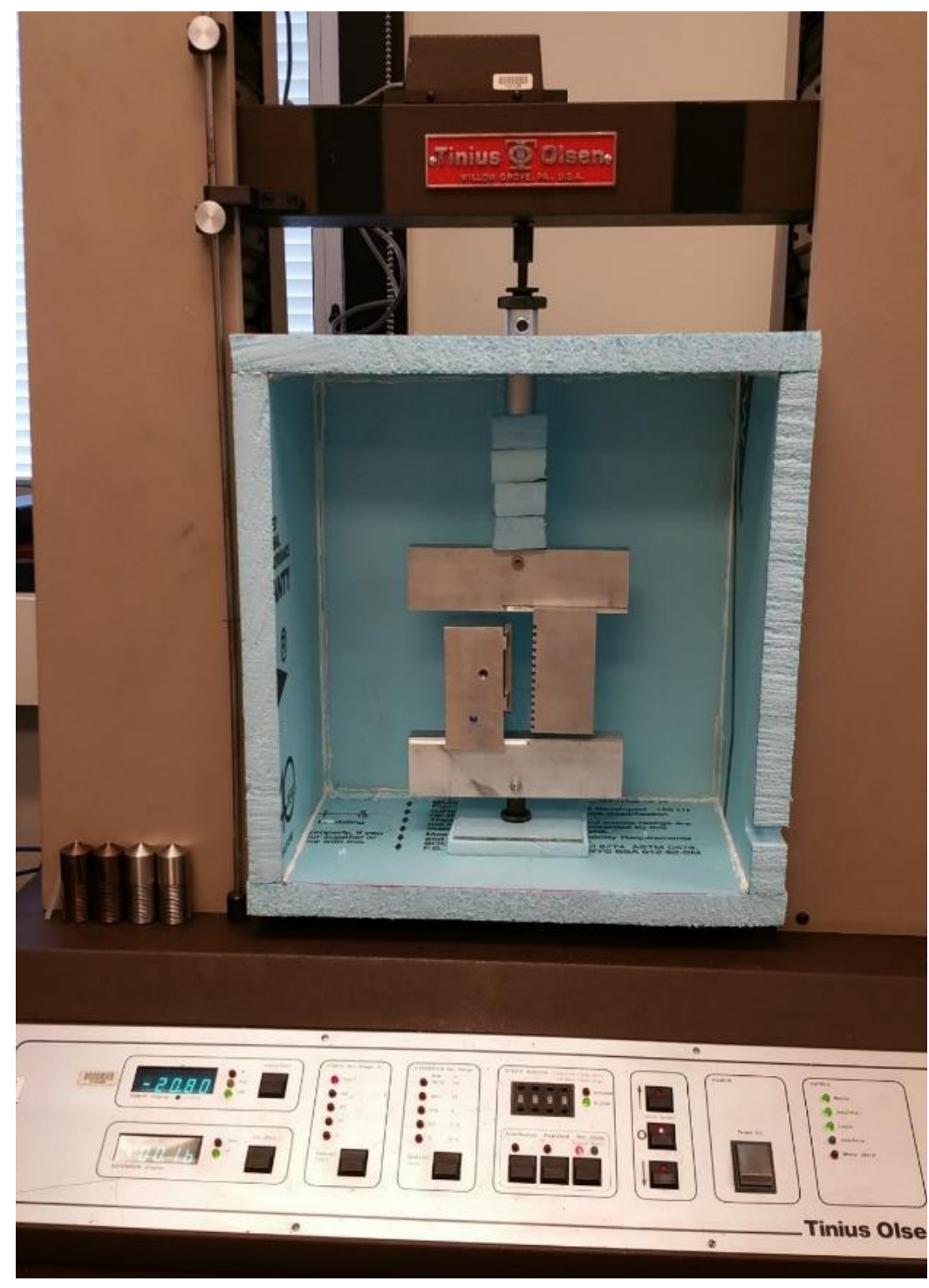

Figure 18. Prototype temperature chamber with test fixtures.

Test fixtures were designed to perform a lap-joint shear test on collected ice samples. A sample coupon is shown mounted on the left upright block in Figure 18. A set screw at the back of the sample presses the sample against two dovetail rails. The fixture was designed such that a dovetail rail at the bottom allows the interface to be aligned with the center-axis of the tensile tester, allowing out-of-plane forces at the interface to be minimized. The dovetail rail at the top allows the serrated block (on the right side) to be adjusted, allowing ice samples of various thickness. In testing, the ice sample was placed 
in the chamber on the mounting block and tightened with the set screw. The serrated block was slid into place and one of several methods was used to attach the serrated block to the ice. In the first method, water at equilibrium was added to the new interface and allowed to freeze. The second method involved dipping the serrated block into boiling water and sliding it into contact with the ice to melt and refreeze it. The third method utilized Peltier coolers embedded into the serrated block (not shown in Figure 17) to heat and cool the interface to melt and refreeze the ice. The third method was found to work best of the three options since it allowed for a more controlled interfacial bond, was much faster to perform, and likely left fewer residual stresses in the ice.

A set of preliminary samples of impact ice were acquired in the IRT as a practice set, and to qualitatively inspect the samples to ensure useful samples could be acquired. Samples were acquired ranging up to 20-minute run times on 1"x3" 6061 Aluminum test coupons. Two typical samples are shown in Figure 19. These samples were acquired on thicker aluminum coupons in September 2015 and were not tested. New samples have been taken and tested on different coupons, as discussed in the following sections. 

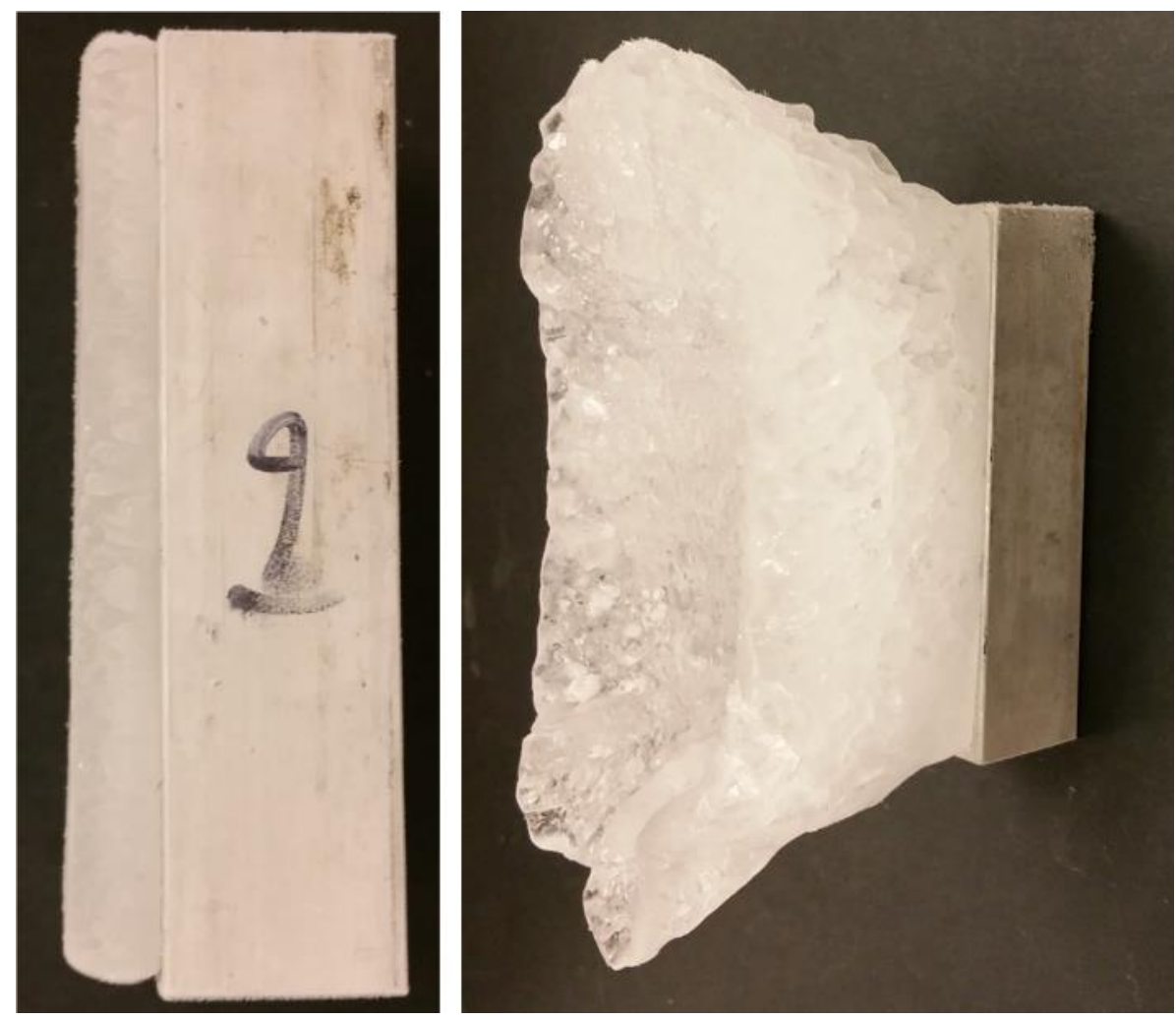

Figure 19. Impact ice samples grown in the IRT. Left: 3-minute run. Right: 20-minute run.

The sample on the left was typical of shorter runs where a nearly uniform layer of ice was accreted over the sample surface. The sample on the left was accreted over 20 minutes and formed a ridge around the edges. This ridge formed due to flow around the sharp edges of the sample where the surface had a higher collection efficiency. Droplets in the flow are less able to follow the flow around sharper corners and so more collect at the edges than in the center. This shows that the ice will tend to form a cup shape over longer runs, similar to the horn patterns seen on many airfoils. Interestingly, on both samples, the ice does not neatly reach the edge. In the left sample, there is a small lip around the edge. In the sample to the right, the edge of the ice tends to neck inwards above the surface. The samples pictured were allowed to sit over several months since test equipment was not available at the time of the test and sublimated. After sitting for 10 months, the samples 
lost most of their fine features and changed shape. The temperature history of the ice was also not tracked, and the samples and substrate changed temperatures multiple times. The coefficient of thermal expansion for ice and aluminum are not matched, so changes in temperature stress the interface, and may possibly cause delamination or residual stresses.

The coupons used originally were $3 / 4$ " thick, in order to allow for sufficient depth for threaded holes to be placed in the back. This design was found inadequate on several counts - primarily, screwing the sample to a mount in the IRT resulted in much jarring of the sample as it was mounted and dismounted. The coupon was also thicker than desiredthinner samples were expected to reduce unwanted stresses in the fixtures, reducing flexure and undesirable stresses at the interface. The sample is shown mounted in the IRT in Figure 20. The new coupons utilized a dovetail mount that was mounted in the IRT similarly to the coupons in Figure 20. The piggyback test is run such that a primary model is mounted in the center of the tunnel upstream (to the left) of the sample shown in Figure 20. The test sample is mounted through a zipper slot in the IRT ceiling, just inside the calibrated cloud. 


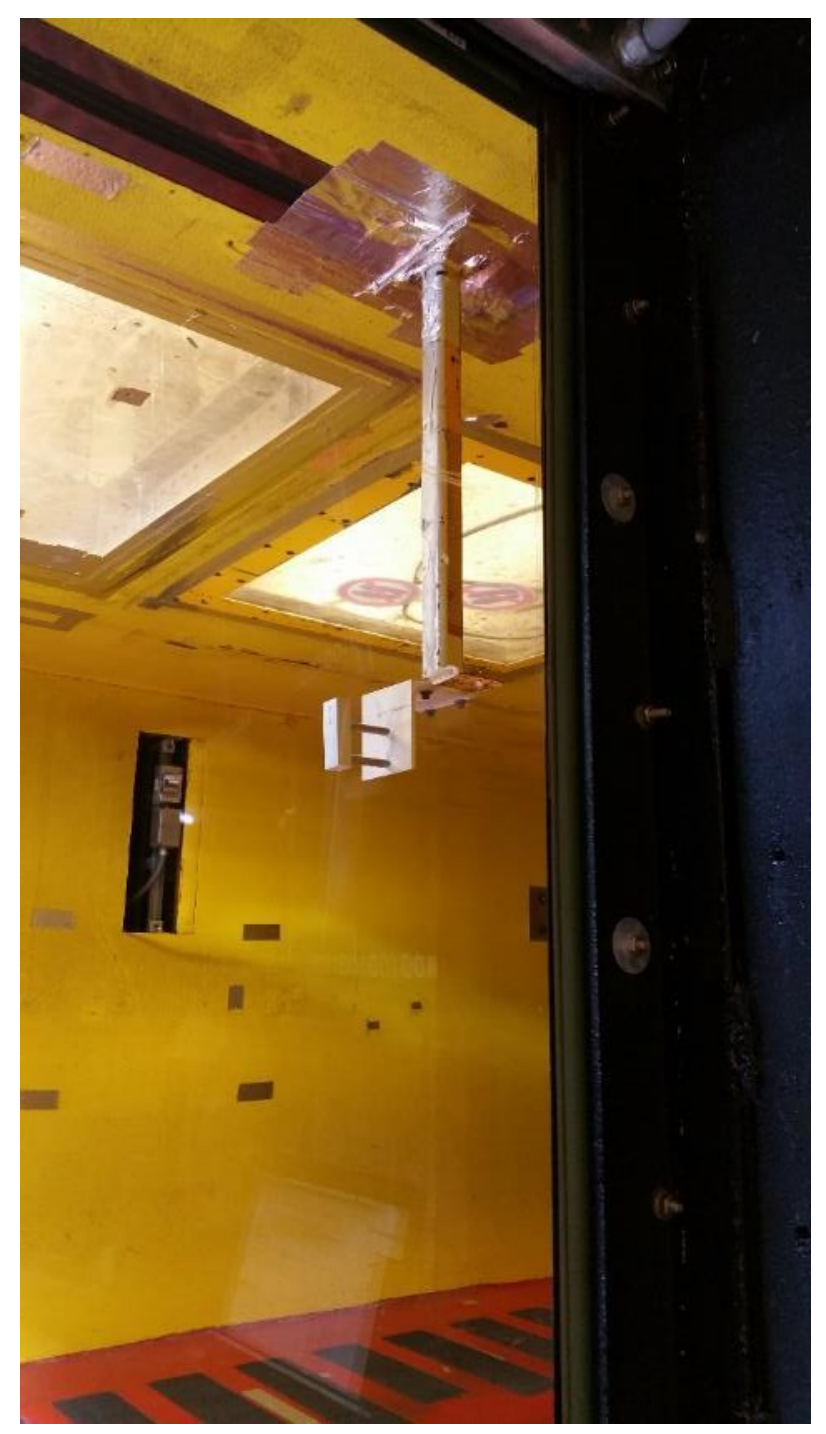

Figure 20. Test coupon mounted in the zipper slot of the IRT, aft of the primary test model.

A new method of mounting coupons was devised using dovetail railings and a set screw. Samples can be slid into and out of place, minimizing jarring to the sample, and fastened only with a set screw pressed against the back of the sample. These test coupons were $1 / 4$ " thick and were made to mount to the fixtures shown in Figure 18. These fixtures still possess most of the same problems as the first design. Some handling is still required between accretion and testing. The coupons cannot be utilized to run an in-situ test, so samples must be stored. 
IRT-grown impact ice will be unavailable in the needed quantities for most of the duration of this project, however, due to the expense of the testing time and model creation. To continue to develop new adhesion testing methods, ice has been grown in a laboratory environment to evaluate and compare new test methods before using them inside the IRT. These early attempts of creating ice samples to test have revealed several key problems besides the mechanical differences between impact and lab ice. One-inch by three-inch samples were prepared using two methods. The first method was to use a pipette to pour water onto a sample surface, which was then allowed to freeze (some samples were prepared inside a vacuum chamber). The second method involved building sidewalls around the sample and pouring water to fill the created container, letting it freeze, and then removing the sidewalls.

For the first method, it was found that the surface temperature of the substrate played a significant role. If the surface was below freezing, water would freeze on contact up to a point creating regions with visibly different characteristics. These regions can be seen in Figure 21.

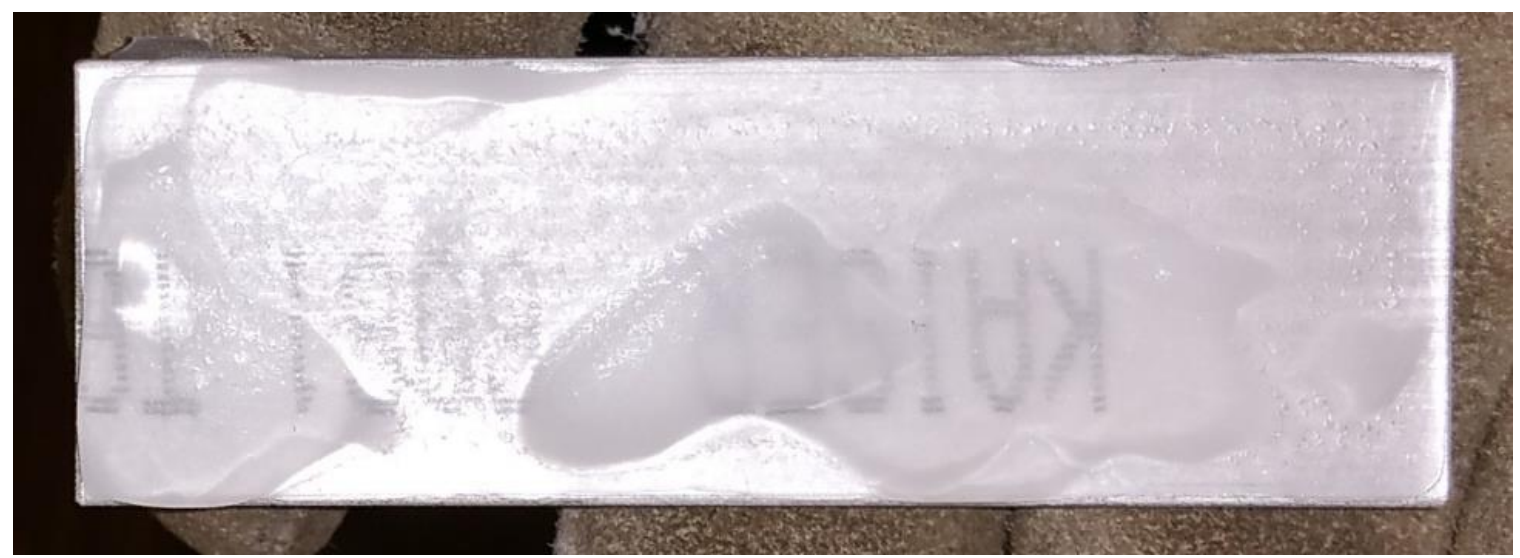

Figure 21. Ice created using pipette on sub-freezing aluminum sample, 1"x3".

The surface in Figure 21 was prepared as follows. An aluminum sample was placed in a freezer at approximately $-30{ }^{\circ} \mathrm{C}$. Through the open freezer door, water at room 
temperature was dropped on the surface using a pipette. The water first placed on the surface froze quickly and made up the darker regions of ice shown above. Since the water spread poorly on the surface, the lighter regions were formed afterwards to cover the surface. The process was repeated using a sample at room temperature, which was coated with water and then frozen. The ice sample prepared on the warmer substrate cracked while freezing. Both ice samples fit the edges of the substrate poorly, indicating this technique as unsuitable for preparing useful samples.

Each of the samples mentioned above was tested using the Tinius Olsen universal tester. Both were placed into the chamber and tightened in place using a set screw. Then, each sample was adhered to the serrated block using a pipette and an ice-water bath. The adhered sample ready for test is shown in Figure 22.
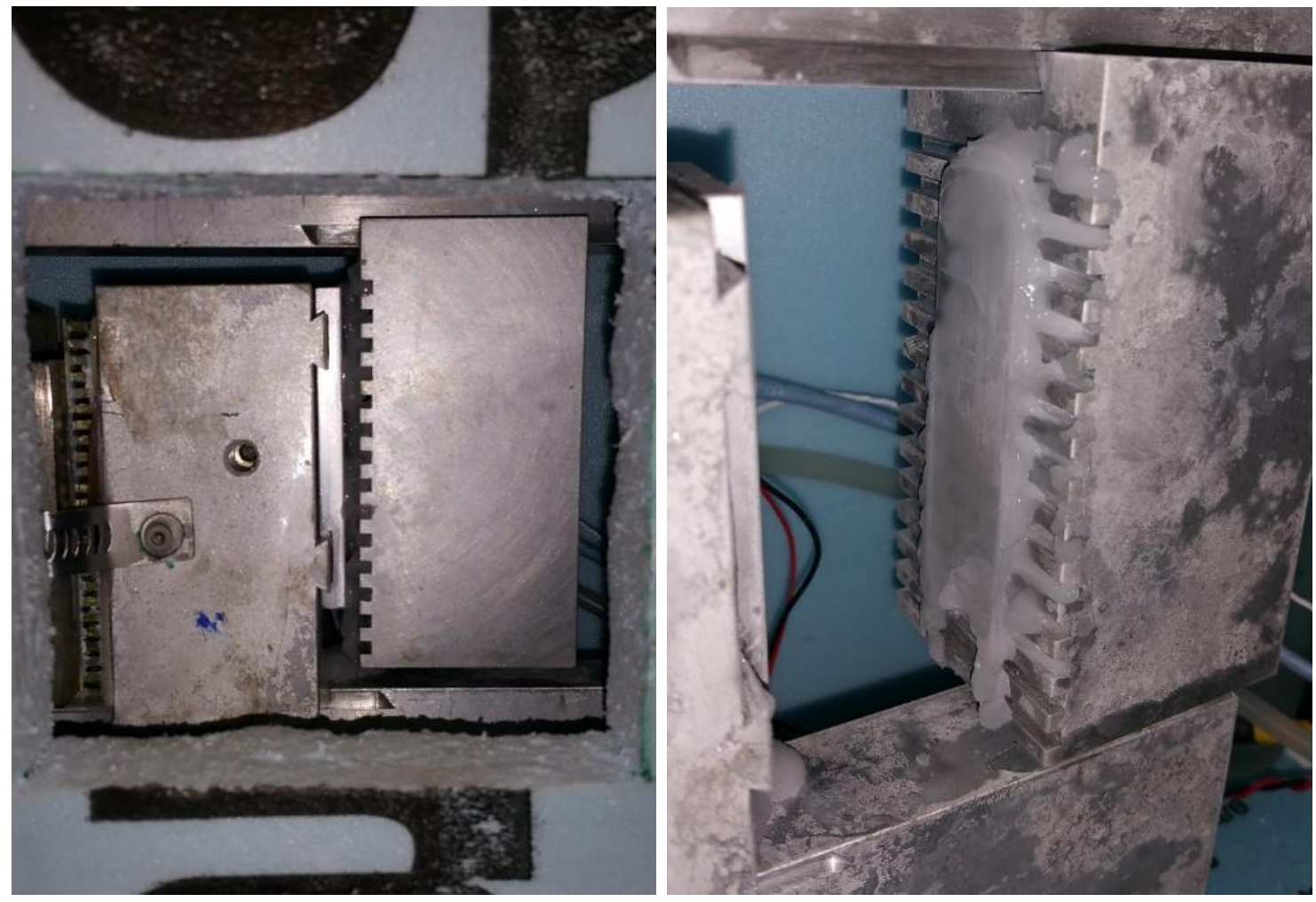

Figure 22. The first ice adhesion test run on the Tinius Olsen Universal tester. Sample prepared on warm surface using pipette. Left: Adhered sample. Right: Post-test showing nearly pure adhesive break. 
The force data from this test contained a number of false peaks due to loose fixturing. This was fixed before the subsequent test. The pre-test crack in the specimen can be seen in the post-test image near the top of the ice. Also, important to note is that the forces near the sample will be difficult to model accurately since the method of connecting the ice to the serrated block creates random geometry. The second adhesion test used the sample created with a sub-freezing surface. Data for the second test is shown in Figure 23.

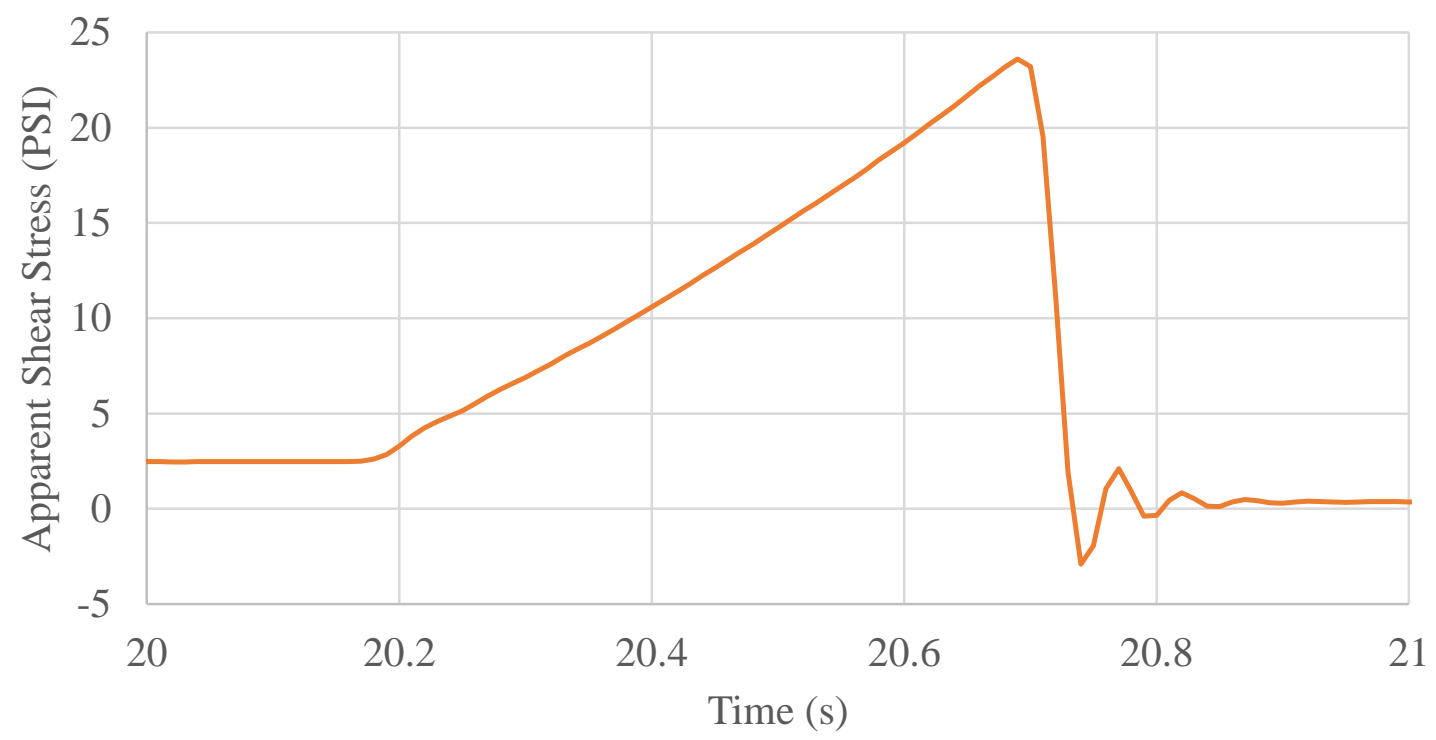

Figure 23. Data from second adhesion test. Sample prepared using pipette and subfreezing coupon.

The data in Figure 23 was intentionally not force-balanced after placing the coupon in the chamber (before adhering to the serrated block) to show another problem. When freezing the sample to the serrated block by adding water with a pipette, expansion of the ice can create local and bulk forces on the sample, in this case at approximately $34 \mathrm{~N}$ along the direction of the load cell. The unit was calibrated after the fact and data was corrected using calibration data. The test was run at a crosshead speed of $20 \mathrm{~mm} / \mathrm{min}$ and started about 20 seconds into taking data. A peak force of $70.8 \mathrm{lb}_{\mathrm{f}}$ was obtained, yielding an adhesion strength of approximately $23.6 \mathrm{psi}(163 \mathrm{kPa})$. 
Samples prepared using the method of building sidewalls around a coupon have been attempted and using current methods create unrepeatable geometries since the sidewall material was not rigid. This was required when depositing ice onto the substrate directly to allow for easy removal of the sidewall material, which reduces the risk of damaging the sample. To create repeatable samples, ice was then formed in commercially available molds (ice-cube trays). Initial attempts were marked by irregular deviation from the mold shape. This was due to the outer water freezing and forming a shell in the mold. As the inner water froze, it broke the shell - typically in the region of interest. To improve the process, distilled water was used, and the water was frozen slowly by reducing the freezer temperature from $-30{ }^{\circ} \mathrm{C}$ to $-10{ }^{\circ} \mathrm{C}$. A container was created to insulate the top and sides of the tray to prevent the formation of a closed shell. This was successful in creating samples with repeatable outer geometry, but samples were found to have air trapped in random orientations inside the ice. The water was deaerated to correct this, which did improve the problem but not fix it completely. A summer student was given the task of performing tests using this ice to build a base data set.

Preliminary Test Data

Three days of testing were completed between May $31^{\text {st }}$ and June $2^{\text {nd }}, 2016$ in the IRT. During these tests a dovetail mount was placed inside the tunnel in the zipper slot position shown in Figure 20. During testing, a wide variety of spray conditions were run. ${ }^{14}$ All but 8 of the samples were destroyed in handling or were too thin to test using the current method. The first set of results are shown in Figure 24.

\footnotetext{
${ }^{14}$ These are shown in the test matrices in APPENDIX A.
} 


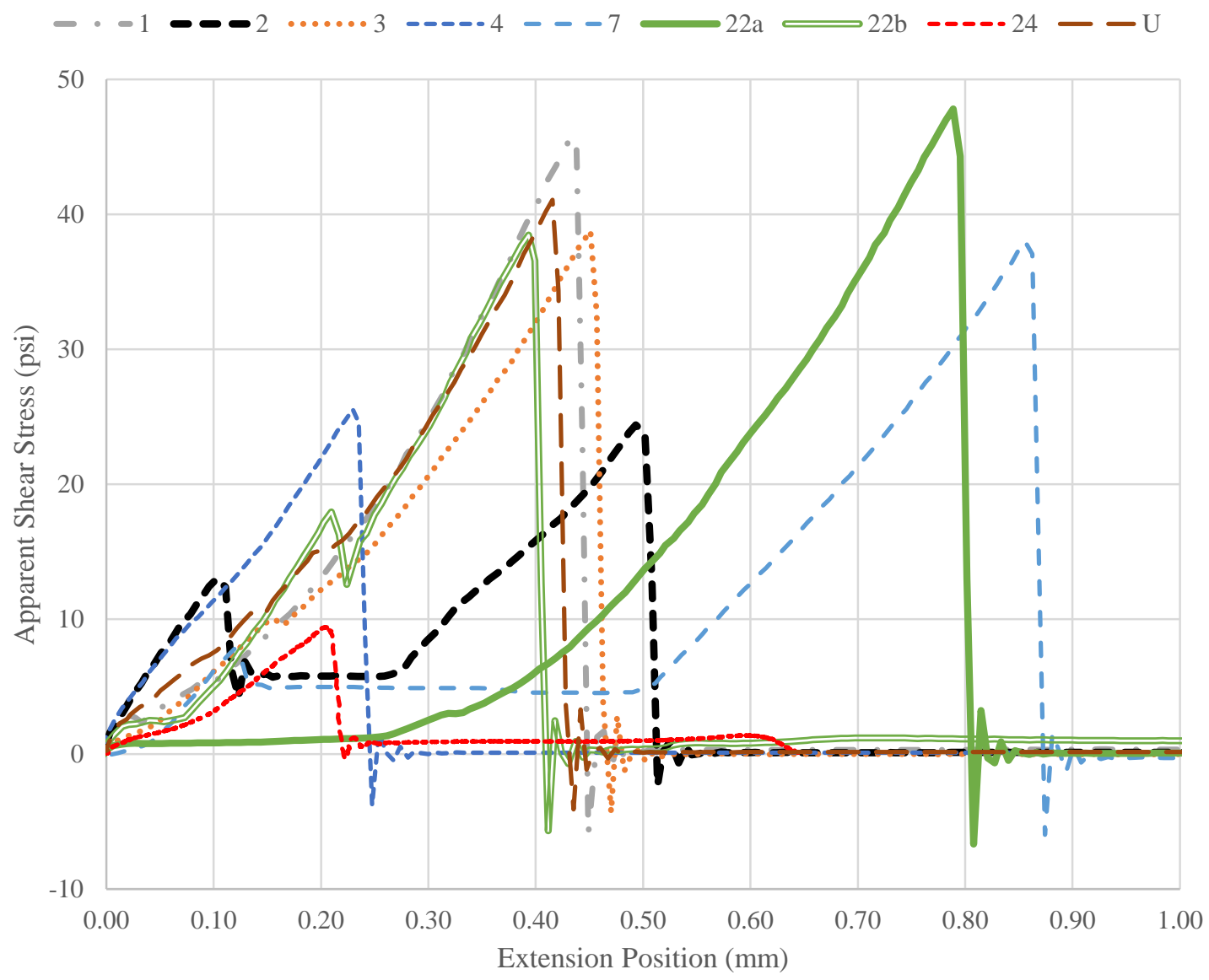

Figure 24. First set of lap-test results from first preliminary IRT test entry.

First, while several coupons appear to have a very tight grouping of peak adhesion strength $(3,7,22 \mathrm{~b}$, and $\mathrm{U}$, separately 2 and 4$)$, there was little apparent reason for these two groupings. Second, several tests have multiple peaks $(2,7,22 b)$. In these cases, it was believed that the fixture slipped either at the lower mount point or at the dovetail. Third, each test shows oscillation after the break. While the fixture may not be as stiff as desired since it was made from aluminum with poor tolerancing, the cause of this oscillation was likely the load cell used. The load cell was an S-type cell with an attached cantilever, and visibly had a large amount of travel. These problems were fixed in the final set of tests by the design and manufacture of a new fixture with tighter tolerances and collars that took 
up any slack, and the use of a new load frame with a pancake load cell and two deflectometers to measure the position of each block.

It was also of interest in the results is the testing of coupon 22 twice. The first test, 22a, exhibited a more noticeable non-linear stress-strain curve. After the adhesive break, the ice sample was checked and found to still be adhered to the coupon and was tested a second time. The second test produced a curve more similar to the results from the other tests. ${ }^{15}$ The adhesive strength obtained was in good agreement with previously published data but was higher in most cases than the refrigerated ice tested (shown in Figure 23). This was unexpected since the test data shown in Figure 24 was with polished surfaces, where the data in Figure 23 was taken from a sample with a stock finish. The stock finish did appear to have a pattern running along the direction of the test. A more detailed comparison requires data from the optical profilometer, which has been taken but not analyzed yet.

Five more days of testing in the IRT were completed between August $15^{\text {th }}$ and $19^{\text {th }}$ 2016. These tests were also conducted in a piggyback mode. In this test, the test equipment was moved above the test section to determine what difference time effects may have on the ice. Only coupons from the first test were used. Due to preliminary failure (handling and melting) and testing errors, only six samples yielded data.

\footnotetext{
15 The run log is shown in APPENDIX A.
} 


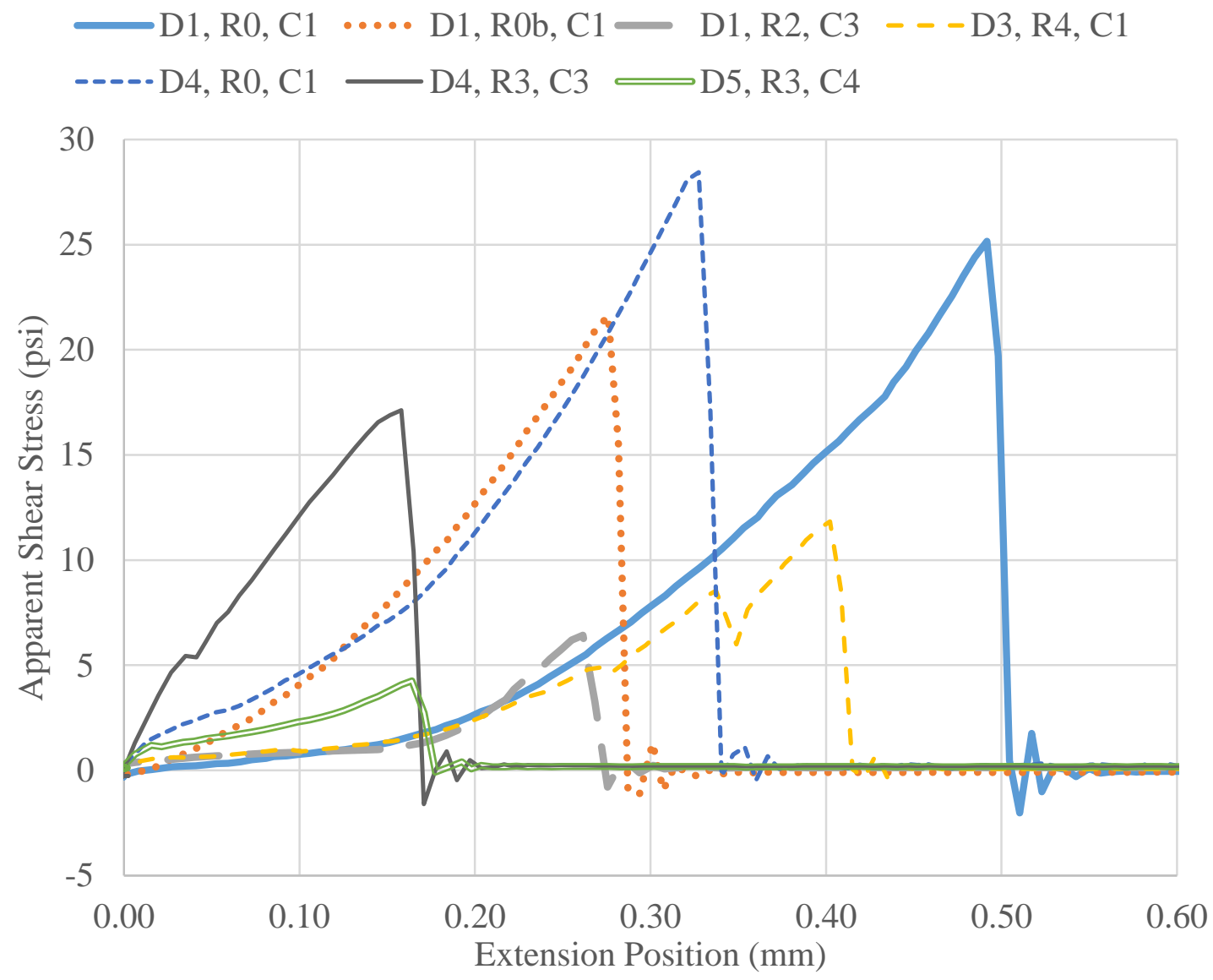

Figure 25. Results from second preliminary IRT test entry (8-15-16 - 8/19/16). Legend shows tests marked day \#, run \#, coupon \# (D\#, R\#, C\#).

The first sample tested in the 8/15/16-8/19/16 tests was a dummy spray (shown in Figure 25 as D1, R0, C1) was tested twice. After the initial test, the ice re-adhered and was tested a second time. The second test had lower adhesion than the first. This was similar to the sample from the May-June tests that was repeat tested. No other samples re-adhered after a test. The new results showed much lower values of adhesion strength on the sample coupons. There were not enough samples or repeats for an exact comparison, but the trend indicates an effect from a difference in the testing method. This was likely due to the change in time between ice accretion and testing. Stresses frozen into the interface had less 
time to flow out due to creep in the second set of testing since samples were tested within 15 minutes of the end of accretion, as opposed to approximately 3 weeks.

A problem with both sets of test data was the changing temperatures. Each run used a different temperature, but samples were all placed into a cooler that had been sitting in a freezer at a constant temperature, and then placed into an environmental chamber with varying temperature. Frequently after colder tests, ice would fall off the samples on dismounting them. This was suspected to be due to the increasing temperature in the tunnel post-test where thermal strain caused the interface to delaminate. ${ }^{16}$

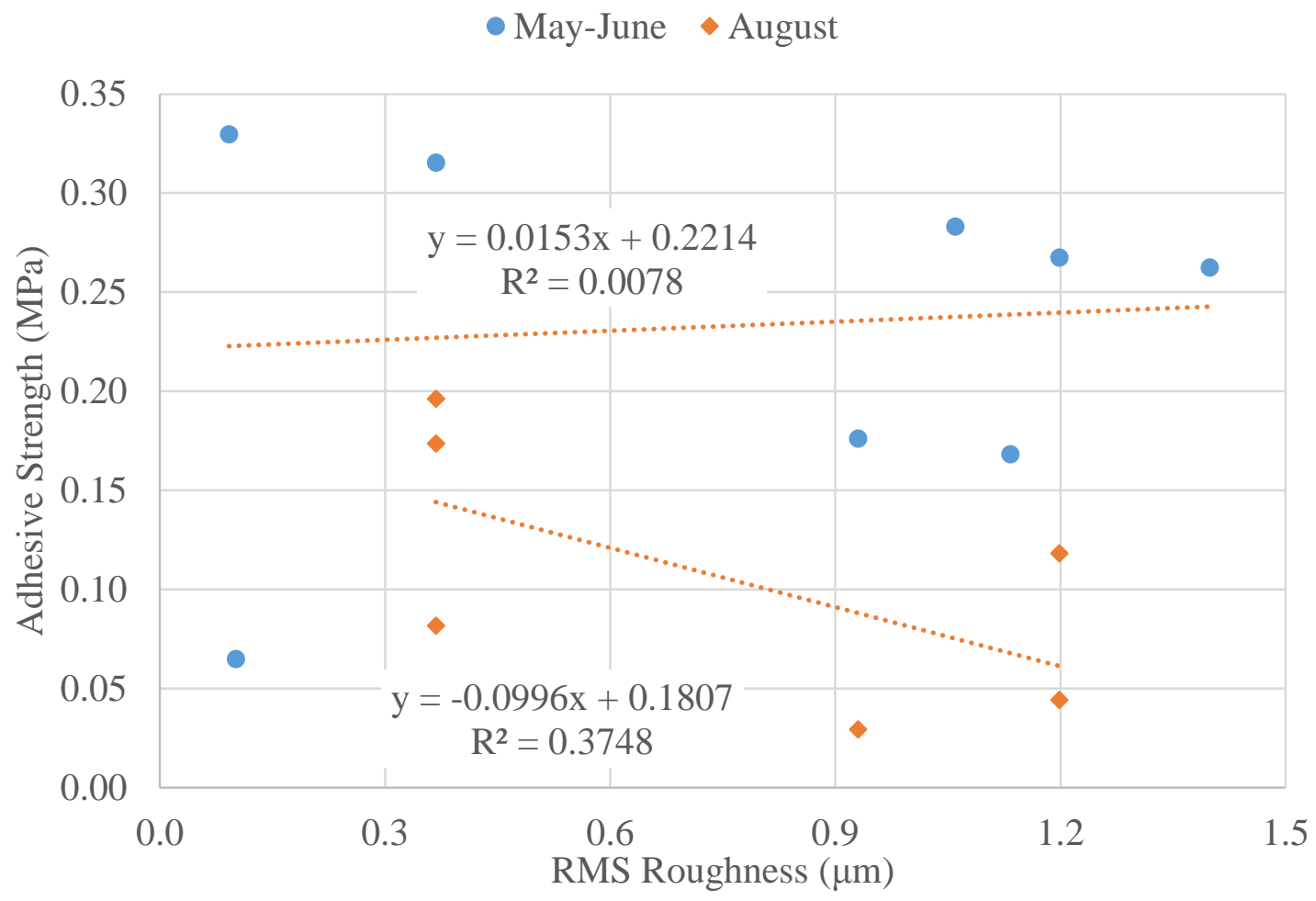

Figure 26. Adhesion strength vs. coupon RMS roughness.

\footnotetext{
${ }^{16}$ All run conditions for the second set of testing are shown in APPENDIX A.
} 


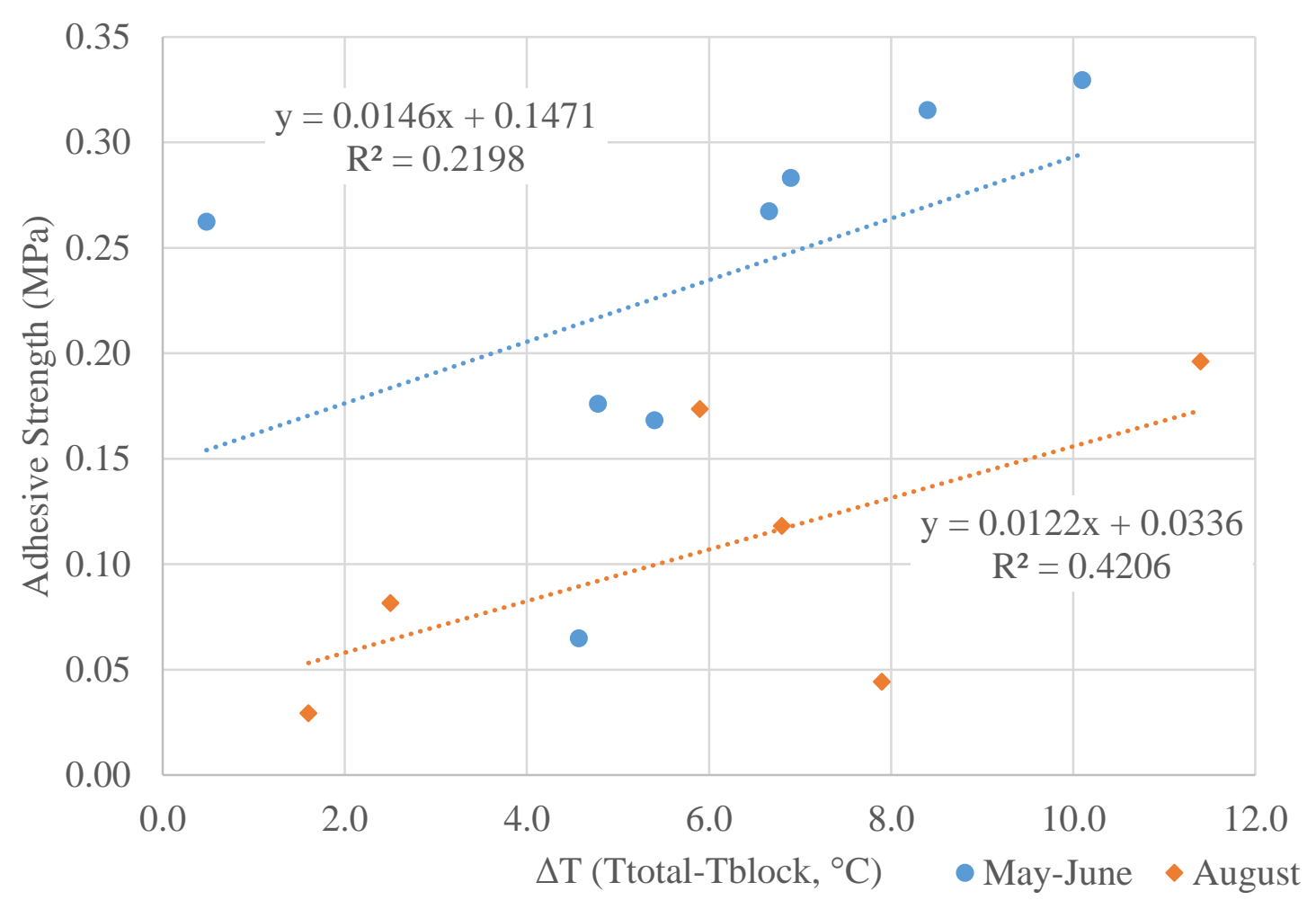

Figure 27. Adhesion strength vs. difference in block temperature (environmental chamber) and total temperature (during 2016 IRT piggyback test).

Weak correlations exist between adhesion strength, roughness, and temperature difference. An insufficient amount of data was taken for a statistically significant comparison. No other trends were found, and it was not possible to conduct repeat tests while piggyback testing. Lessons learned from the preliminary testing are shown in Table 7. 
Table 7. Lessons learned from preliminary testing.

\section{Problem}

\section{Solutions}

\begin{tabular}{|c|c|}
\hline Melting/cracking during refreezing & $\begin{array}{l}\text { - Use thinner teeth } \\
\text { - Repeatable melting using embedded } \\
\text { heat }\end{array}$ \\
\hline $\begin{array}{l}\text { Sublimation changes coupon } \\
\text { geometry }\end{array}$ & - Use airtight sample bags \\
\hline Temperature history unknown & $\begin{array}{l}\text { - Record temperature during test } \\
\text { - Run at one temperature }\end{array}$ \\
\hline Jumps in stress-strain curves & $\begin{array}{l}\text { - Make fixture to tighter tolerances } \\
\text { - Include locking mechanisms at each } \\
\text { joint }\end{array}$ \\
\hline $\begin{array}{l}\text { Cutting ice for grain structure } \\
\text { imaging }\end{array}$ & - Use microtome to cut ice \\
\hline Fixture alignment poor & - Make fixture to tighter tolerances \\
\hline Sample handling in warm air & - Work with samples in walk-in freezer \\
\hline
\end{tabular}

A new test cell and laboratory equipment were acquired midway through the project, providing opportunity to revise the test method. The advantage of preliminary testing was that many issues were identified in the tests, most of which were directly addressed in redesigning the experiment. However, some issues could not be addressed with this methodology. ${ }^{17}$ A new IRT model was designed and created to enable the collection of a sufficient number of samples, and a new shear rig was created to address the issues identified above.

\footnotetext{
${ }^{17}$ A new methodology addressing all of these issues is proposed in the Alternative Concepts section but was not able to be used in this work due to budgetary restrictions.
} 


\section{Shear Rig Development}

The experiment devised to determine the shear strength of ice is a modification of a lap-joint test method. Two iterations of the shear rig were constructed and used. The first shear tests have been devised to obtain mode 2 strength on coupons as shown in Figure 22. Tests for mode 1 and mixed-mode tests are needed to determine the influence of mixed loading during real world applications, and a capability to test them has been built into the new shear rig.

Testing to obtain cohesion data is important but is currently seen to be outside of the scope of the current project. Available cohesive data on polycrystalline ice is very good for non-impact ices. Values will be used corresponding to ice with similar grain size as the impact ice being considered. Comparison will be made using data from the cross-polarized images obtained during adhesive testing. Cohesive testing will be possible, however, by carefully preparation of samples. Ice samples taken from the IRT would need to be shaped carefully, perhaps by removal of ice through sublimation instead of melting the ice. This would be possible by blowing cold, dry air onto ice. Scans of the shape would be needed to obtain the geometry of the ice, which is possible through the use of a laser scanning system. The IRT recently obtained a laser-scanning system that has been used to measure 3D ice shapes [160, 161]. 

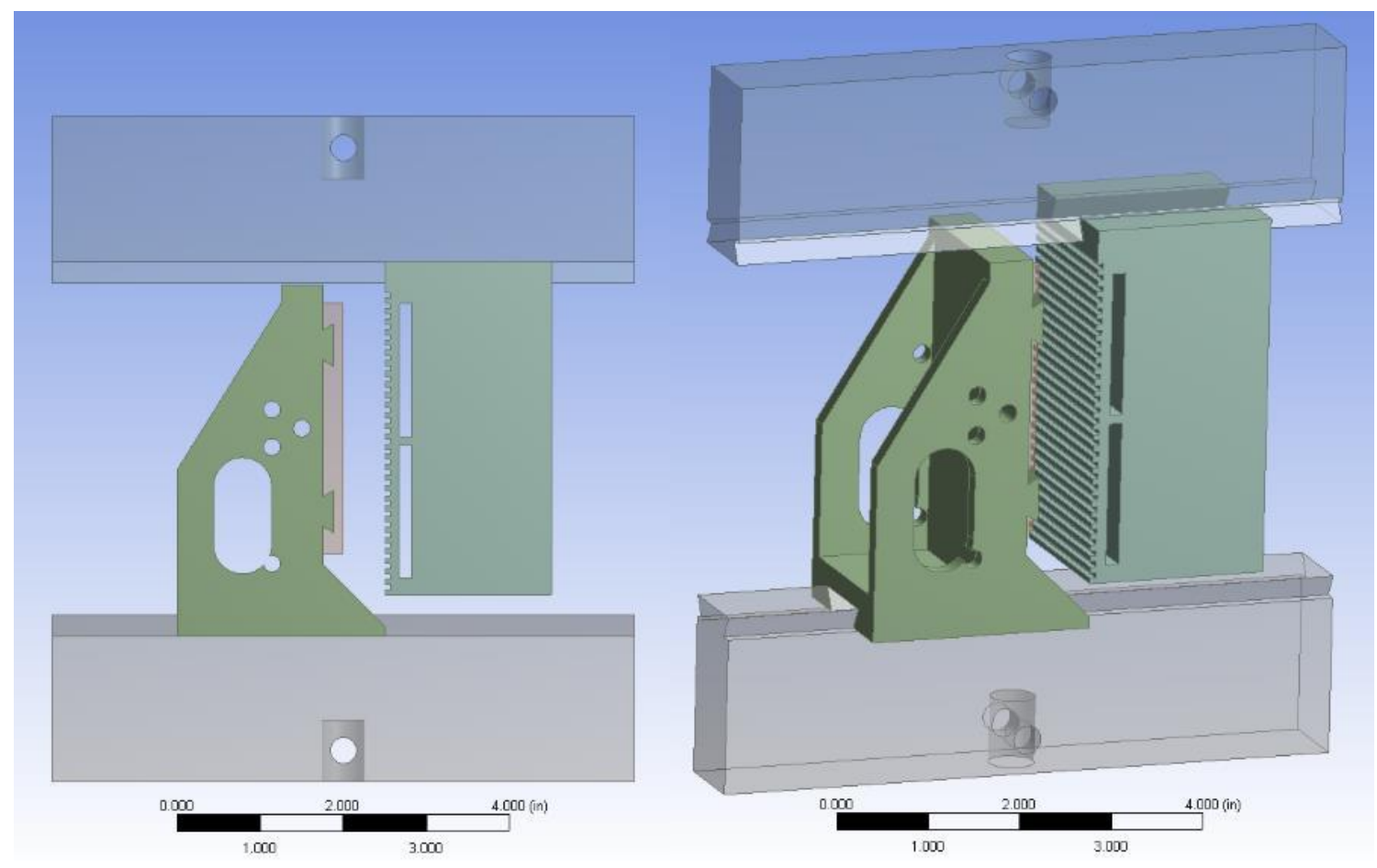

Figure 28. Lap joint test design \#1.

To test ice, two lap joint test fixtures have been designed. The prototype for the first fixture has been built from aluminum and is seen in the test images above. The prototype that was built did not have the required tolerances, and so the fixture would slip often during tests. The new fixture was designed with tighter tolerances and included nuts to tighten pin connections at the mounts. The first design, shown in Figure 28, allowed for the shear testing of samples on coupons. The coupon block is improved to allow for the closer mounting of a cold stage to the samples, reducing insulating effects due to block thickness. It also allows for the cold stage to be bolted directly to the block, improving mounting capability. 


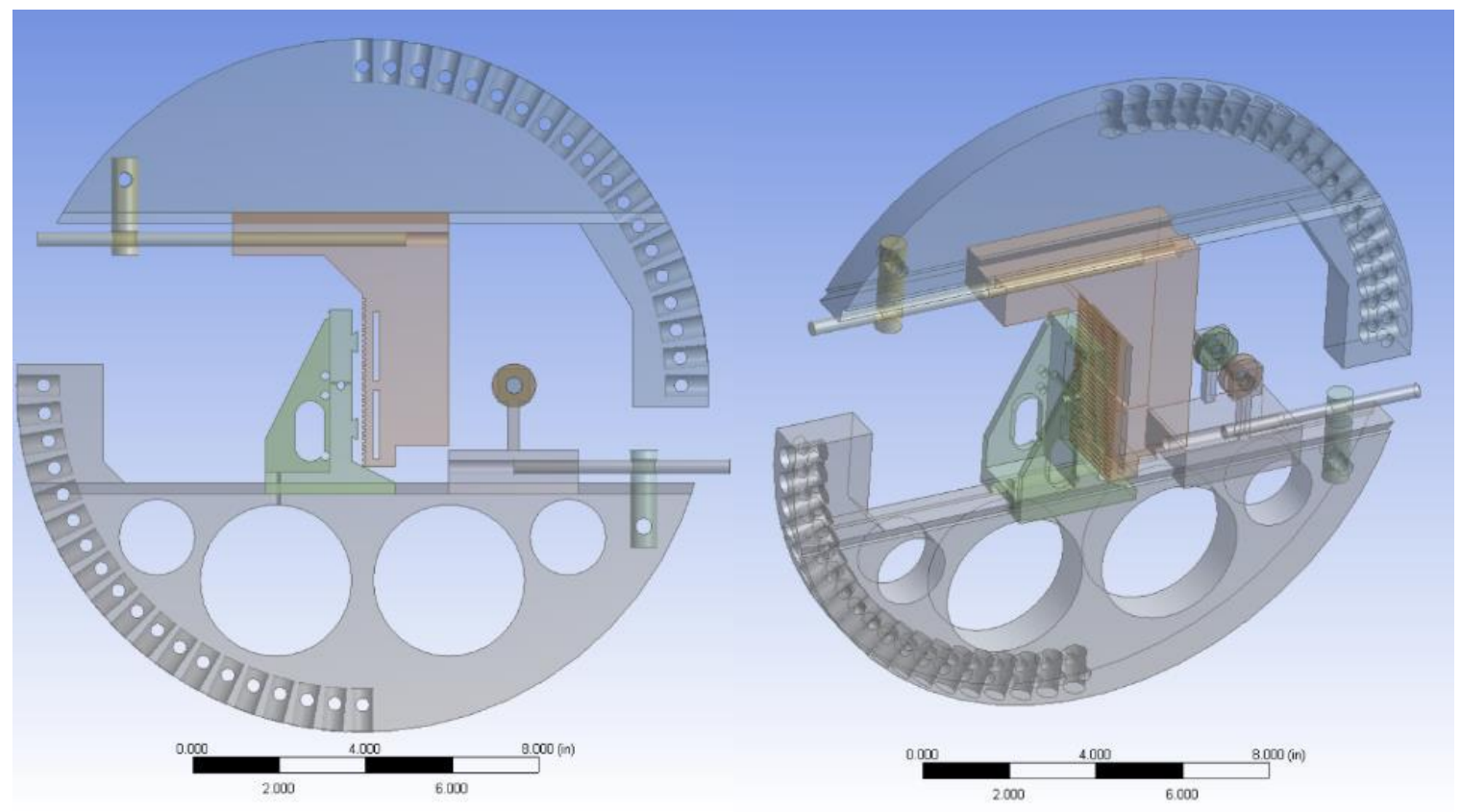

Figure 29. Lap joint test design \#2.

The second design added two important capabilities. The first was the capability to perform mode 1 and mixed-mode testing in addition to the mode 2 testing provided by the prototype. The second was the capability to perform tests under compression. The first capability was to be provided by the mounts in $5^{\circ}$ increments around the perimeter of the top and bottom rails. When testing at an angle, the coupon block can be pinned down and the serrated block can be fastened by the use of a threaded rod (the yellow rod shown in Figure 29). During compression testing, the serrated block could be unfastened from its threaded rod and the compression block used to clamp the serrated block against the ice sample (the serrated block is the rightmost block in Figure 29). The compression block is positioned using a threaded rod. Compression force applied to the serrated block will be taken using strain gages attached to the base of the vertical columns on the serrated block. Ball-bearings will be used to make contact with the serrated block to reduce shear forces from the clamping effect. The deflection of the serrated block and top rail assembly can be 
measured without ice in place to determine its stiffness. The deflection measured during a test will provide the reduction in clamping force seen by the ice sample. In addition to testing adhesion, this will also allow frictional coefficients to be obtained. These new capabilities will aid in answering whether or not clamping effects inside roughness elements on surfaces will increase the adhesion strength of ice. The second design was selected, refined, and machined.

To keep manufacturing costs down, several changes were made. The material was changed to aluminum, and the top and bottom rails were modified to be identical. Mount points were changed to be in $10^{\circ}$ increments, providing a larger flat and less machining. Weight-reducing holes were removed since the parts were much lighter after the changes. The completed shear rig is shown in Figure 30.

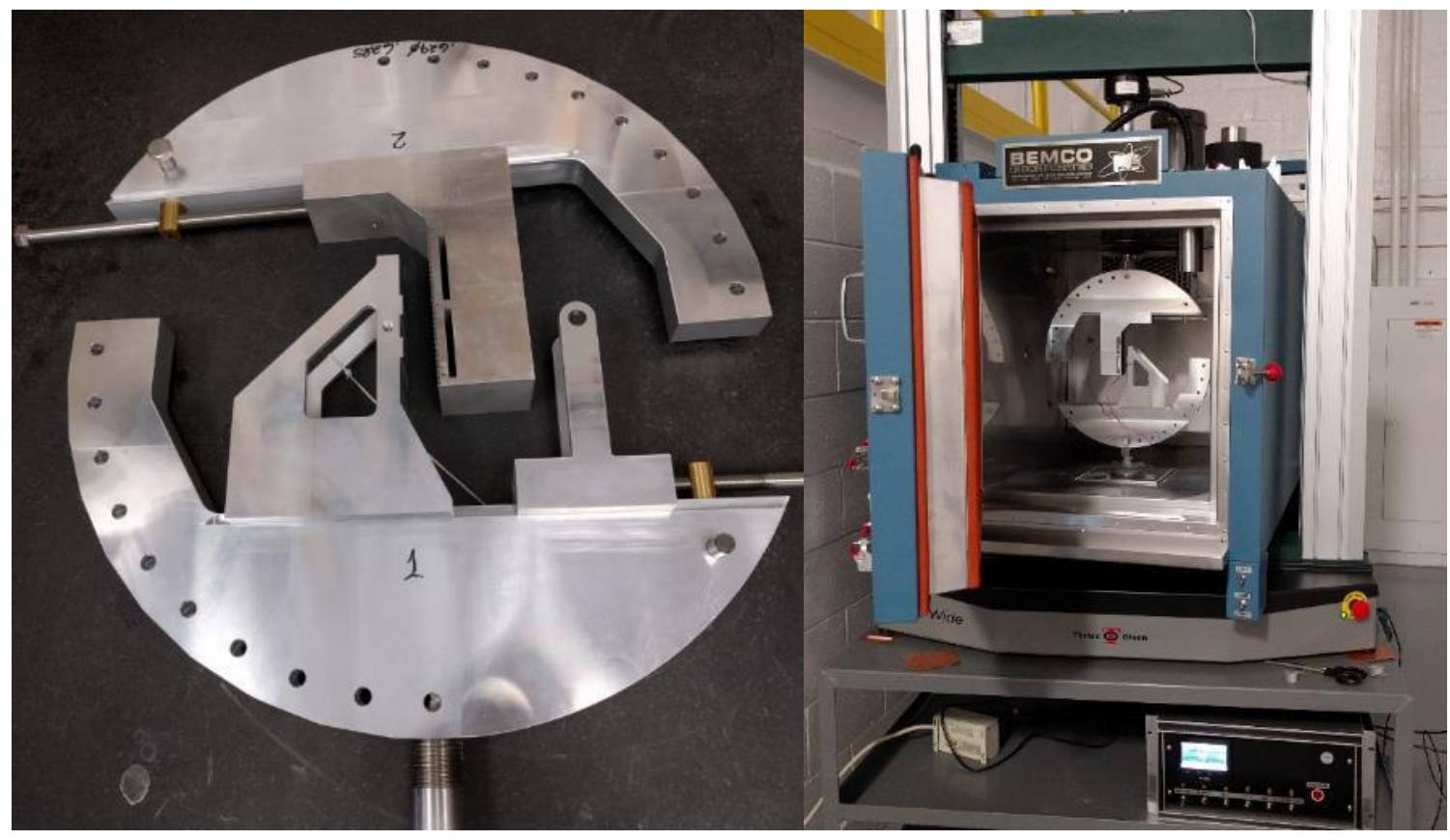

Figure 30. Left: completed shear rig. Right: shear rig installed in test equipment. 
To prevent ice from forming and locking the dovetails in place, vacuum grease was used as a lubricant on the serrated rail ${ }^{18}$. In the first series of tests, the serrated block was used on top with the Peltier wires hanging from it during testing. The bellows at the top were noted to produce forces during use when attached, and when allowed to slide unattached these forces dropped to below $5 \mathrm{~N}$ in all cases. The force was noted to be generally dependent on the crosshead speed, with the most notable exception being when the bellows froze to the extension bar connecting the crosshead to the fixture. The shear rig was aligned on the mounts by rotating the rails and pressing the serrated and coupon blocks into each other before tightening the mounts down.

\section{Updated Shear Test Methodology}

The first methodology (TM1) used to test samples on the final set of equipment evolved over the course of the project to address issues as they were identified. The methodology was used for the samples from the 10-2-17 tests and the first batch of samples from the 11-20-17 tests. Initially, the force was zeroed per batch, but was eventually zeroed per coupon, then zeroed per batch while the crosshead was in motion at the rate prescribed for the test, and finally the test method in the software controlling the tester (Horizon) was modified to zero the load cell at the prescribed rate each test.

Samples were loaded in batches, typically containing between 8 and 16 samples. They were carried from storage to the environmental chamber in a plastic bag, each in their own sample bags. A sample was selected, unbagged in the chamber, and loaded onto the coupon block. The set screw was tightened to hold the coupon in place (the torque was not

\footnotetext{
${ }^{18}$ It was noted that a non-aluminum rail mount using gibs would have been an improvement resulting in lower cost and better functionality since galling was observed, cold-welding the non-lubricated coupon block in place on its final reinstallation. The block was able to be tapped into correct alignment with a hammer.
} 
recorded, finger tightened only). Typically, the serrated block was moved close to the sample without touching, and the zeroing process was completed (uniformly for all samples starting with the 11-20-17 test). Embedded TECs were used to melt and refreeze the ice using a custom-designed circuit and a LabVIEW VI controller. Two $12 \mathrm{~V}$ rated TECs were used in parallel with a $24 \mathrm{~V}$ supply. Melting was controlled by timing the heat. In early tests, it was attempted to cool the samples by reversing the TECs, but this was found to be disadvantageous due to the heating of the block's core temperature ${ }^{19}$. The heating and cooling time was recorded, as was any observed excessive melting of the ice. A mounted sample before and after break is shown in Figure 31.

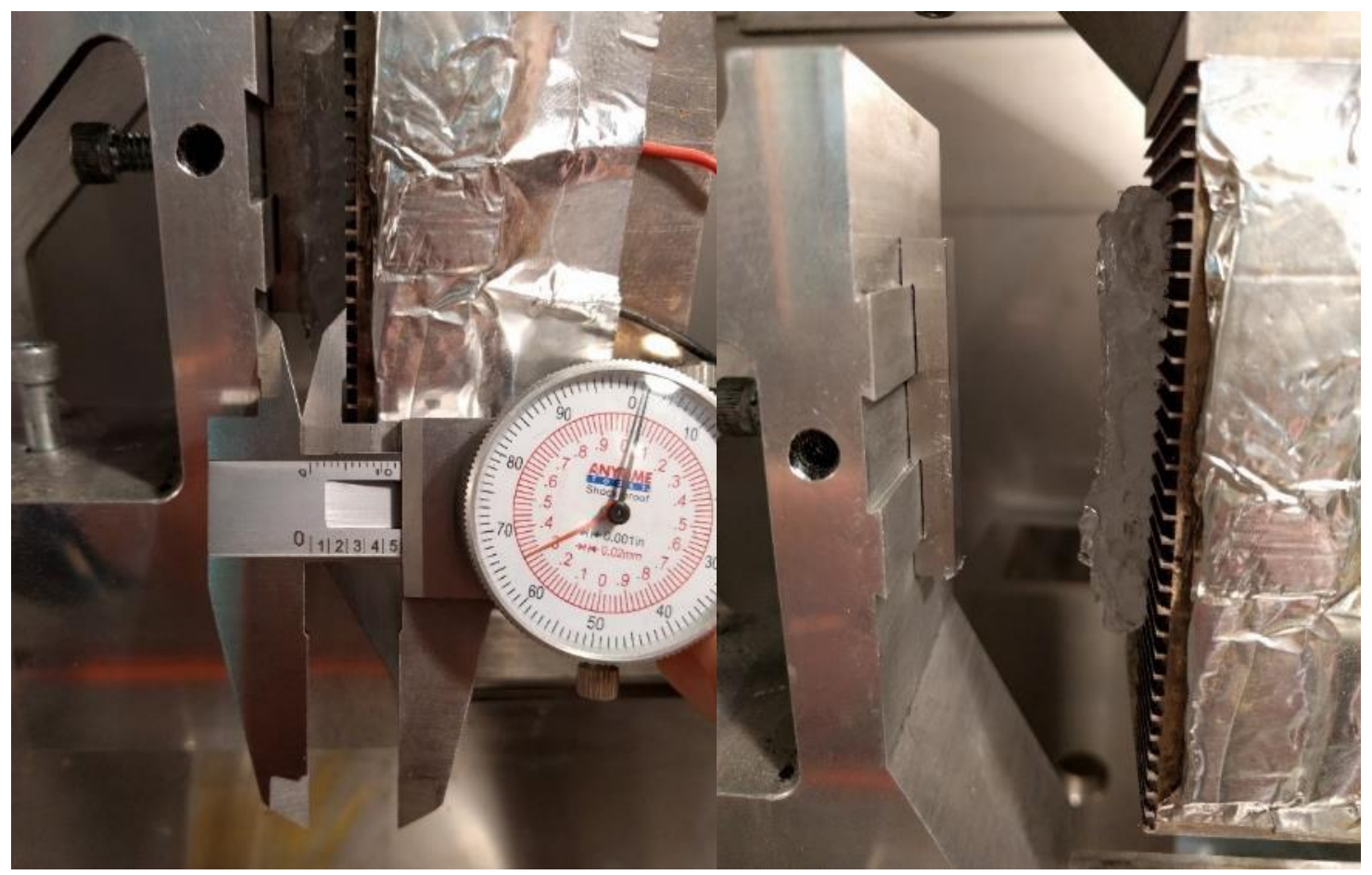

Figure 31. Sample B1. Left: mounted sample pre-break, right: post-break.

${ }^{19}$ A set of TEC controllers will be used in future testing, with one control loop dedicated to controlling the tooth temperature, and the second to controlling the block's core temperature. This was not available for these tests. 
While the TECs were on, the serrated block was gently pressed into the coupon and ice, sinking the teeth into the sample ${ }^{20}$. Once the TECs shut off, the sample quickly refroze, and the serrated block set screw was finger tightened. At this point, a few notes were recorded (early observations, heating time, and sample number), and then the equilibrium time was started. Initially, samples were allowed to equilibrate for 3-5 minutes, timed by the operator using a clock. In the majority of tests using TM1, samples were allowed to equilibrate for 3 minutes, which was programmed into the Horizon test method to ensure repeatable testing ${ }^{21}$.

Once the equilibrium time was completed, the test began, and the crosshead started moving up at the prescribed rate until sample failure. Post-failure, the ice was detached by use of the TECs. Light pressure was maintained on the sample to prevent excessive melting. In initial tests, the width was recorded using an image taken prior to the test. The method was updated to measure the thickness with dial calipers measuring between the coupon block and the serrated block, though due to poor adherence to the specified tolerances during machining, there was variability in the separation between the coupons and the coupon block ${ }^{22}$. Starting with the 11-20-17 samples, the thickness was obtained post-test by measuring the thickness of the detached sample using Vernier micrometers. Care was taken to make the measurement at the thickest part of the sample with the micrometer resting in the flat where the coupon was previously attached on the back side

${ }^{20}$ This was not done uniformly - it was observed early in testing that some samples appeared to have significant frictional forces post-break when sliding, compression due to inconsistent methods used to sink the teeth into the ice was suspected. Further, not all operators were consistent with observing proper methodology. This is discussed in more detail in the Results and Discussion section.

${ }^{21}$ See APPENDIX B for documentation.

${ }^{22}$ For these measurements, an average coupon and gap thickness was obtained by measuring coupons with small and large gaps. This value was subtracted from the measured thickness obtained with dial calipers, giving the thickness of the ice +/- 0.05". A sample measurement is shown in Figure 31. 
of the ice, and on a flat from the back of the teeth on the opposite side. Data was exported by Horizon into CSV files for each sample, and into a summary report including figures and notes on each sample, including result observations.

In the second test methodology (TM2), three cement-on type-K thermocouples were attached to the teeth of the serrated block, and one to the opposite face of the block by the instrumentation group at NASA GRC using a non-electrically-conductive epoxy with relatively high thermal conductivity. The thermocouples were placed as follows. The first thermocouple was attached to the center tooth between the Peltier coolers, in the center of the block. The second and third thermocouples were placed on the respective tooth $15 / 16$ " higher or lower than the initial thermocouple. The thermocouple on the opposite face was positioned to match the first thermocouple mounted to the tooth side of the block. Temperature data was recorded and exported to a CSV file in LabVIEW. Additionally, a FLIR SC640 was used to image samples undergoing the melting and refreezing process. The area near the sample was spray painted flat black, masking the dovetails and threaded holes in this area of the shear rig. Three pieces of black electrical tape were placed on the lower rail, and an additional piece was placed on the coupon block near the coupon location (Figure 32 shows this piece of electrical tape and the paint on the coupon block). The area was masked using a piece of black cardboard with a window cut in the middle. Imaging was performed with the door to the chamber open. ${ }^{23}$ The door was kept closed while images were not being taken in order to minimize the effect of warm air heating the sample and surrounding parts, with the door open for less than five seconds for each image.

\footnotetext{
${ }^{23}$ Six panes of glass are used in the front door window on the environmental chamber, with the innermost one coated with a transparent heater. Reflections through the glass were problematic for all types of imaging, especially infrared.
} 
The block was flipped such that the serrated block was on the bottom due to the additional wiring needed. The addition of these thermocouples added thermal insulation to the teeth at those locations, and noticeably slowed down the melting/refreezing process. The set screw was tightened with a hex key to prevent a loose mechanical connection due to the grease used to lubricate the contact between the serrated block and the rail ${ }^{24}$. A cooling unit and two Epsilon deflectometers were added, with both deflectometers mounted to a steel plate bolted to the bottom rail. These are shown in Figure 32.

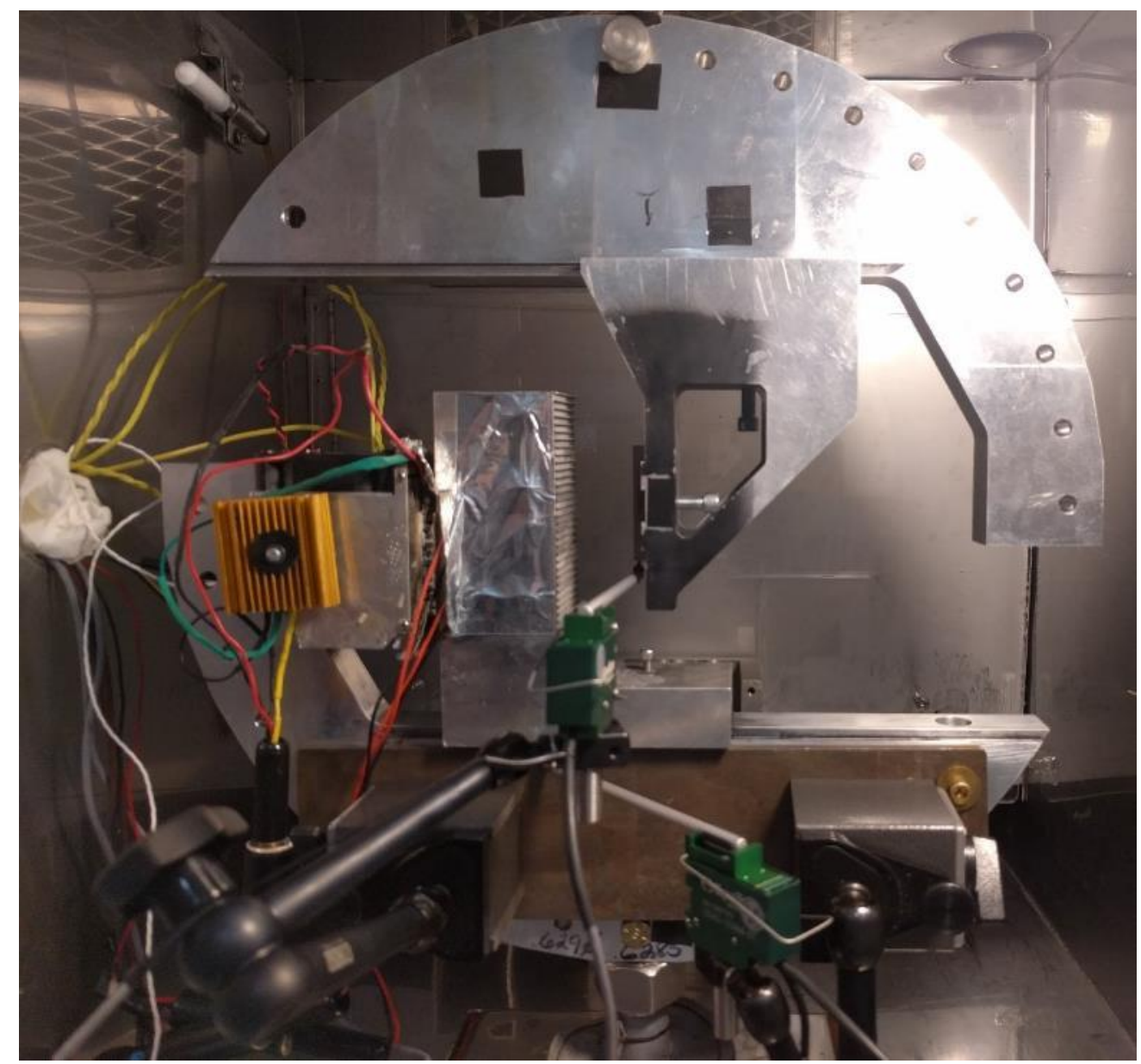

Figure 32. Shear rig with addition of cooling block and deflectometers.

${ }^{24}$ This was unneeded in the TM1 position since gravity helped to settle the serrated block to provide tight contact. 
The deflectometers were the small green boxes with lever arms sticking out of them. A 6-mm deflectometer was used on the bottom to check for deflection on the serrated block. A 12-mm deflectometer was placed on the coupon during each test. After the deflectometers were verified to work, they were repositioned to the back side of the shear fixture to provide space for mounting samples in the chamber. The 12-mm deflectometer was also useful to qualitatively determine the amount of force being applied to the sample during melting and refreezing. The top block would deflect as force was applied, and the deflectometer would not since it was attached to the bottom rail, allowing for more careful mounting of the samples.

A number of samples from the third IRT test entry were cut into cuboid shapes prior to shear testing. These samples were first cut on a microtome to put a flat on the top of the ice such that it could be gripped, and then the sides were cut on the microtome further. In some cases, chipping was observed to remove small pieces of ice, sometimes near the interface. Since the microtome knife couldn't be used to cut the ice straight with the coupon edges without risking damaging the blade or sample, the ice was sanded using a Dremel tool to create a uniform edge geometry, with the sanding motion going along the edge of the interface. Investigation showed no signs of melting on the ice itself, but a small amount of ice refroze to the sandpaper after six samples. This was performed on glaze ice shapes, and no cracking was observed at the interface except small chips flaking off around the edges during the microtome process. The use of the microtome is detailed in the Microscopy section. 


\section{Coupons and Roughness Analysis}

Four hundred coupons were ordered for testing, to be delivered before the first IRT test entry, originally planned for 9/5/17-9/6/17. The plan for this body of work was to use coupons with tightly controlled tolerances and test surfaces with statistically similar roughness characteristics. To hurry the production of samples ahead of testing a specific finish wasn't specified, except through a request that a consistent machining method be used. A detailed analysis of the coupon surface was scheduled prior to testing, but the delivery of coupons was delayed pushing the analysis to after the testing in the IRT. This was unfortunate since the results of the analysis showed that the coupons were not made with consistent methods. While most of the coupons had a pattern of horizontal roughness lines (along the short dimension of the coupon, considered $0^{\circ}$ ), at least one batch had a pattern of lines at an angle of approximately $67.5^{\circ}$ and $90^{\circ}$, and others had prominent scratches in the $90^{\circ}$ though the primary pattern was at $0^{\circ}$. Ten coupons were tested on a Wyko NT-9100 optical profilometer. Two effective magnifications were used at the extremes of what the profilometer was equipped with to attempt to capture low and high frequency noise, providing $\sim 40.5 \mathrm{x}$ and $\sim 2.7 \mathrm{x}$ magnification on a $480 \mathrm{x} 640$ pixel array. The effective resolutions were $244 \mathrm{~nm}$ and $3.66 \mu \mathrm{m}$ for the high- and low-resolution modes, respectively. Four data sets were taken of each sample except the first, which only had one. These four data sets were grouped by location and magnification; images were taken either in the middle of the coupon or off to one end. Data taken in the middle at low resolution were group 1, at the end at low resolution were group 2, at the middle at high resolution were group 3, and at the end at high resolution were group 4 . The coupons were aligned visually with bolts in the stage, shown in Figure 33. 


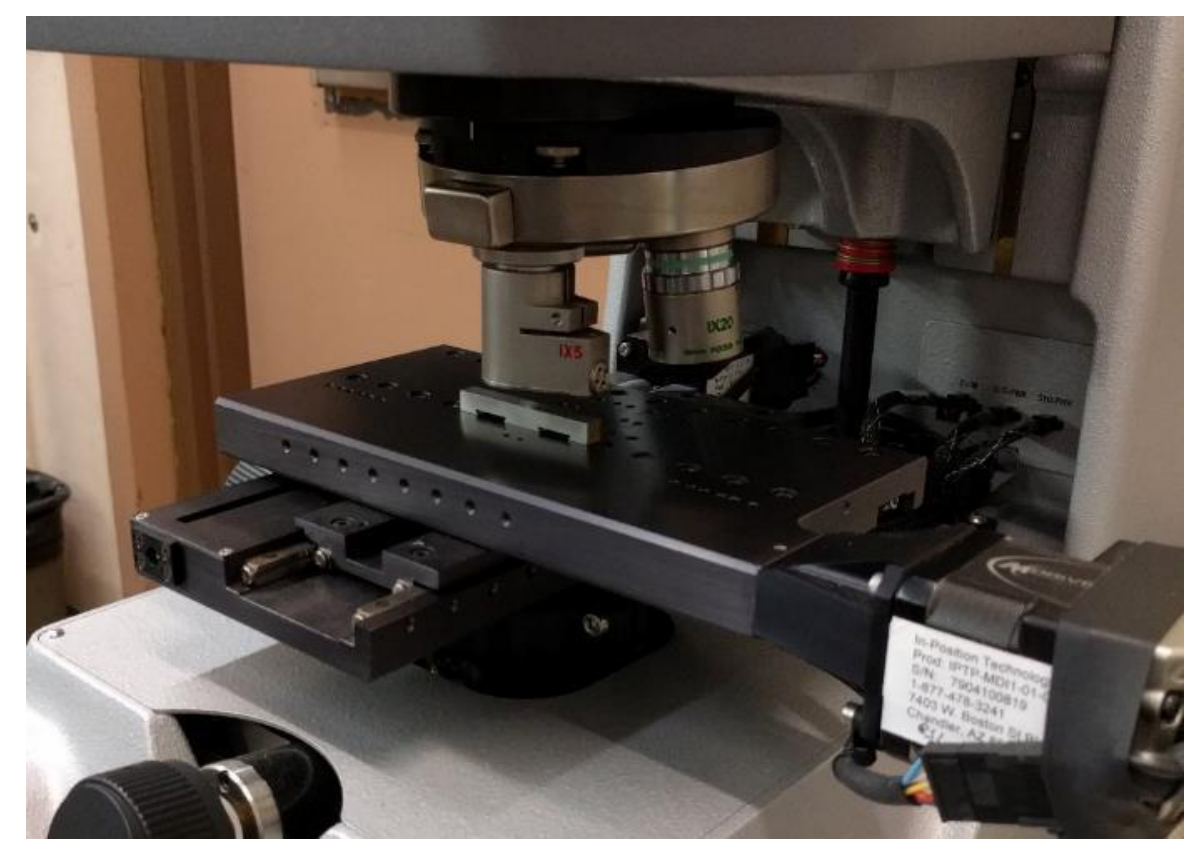

Figure 33. Profilometer stage with coupon, middle low-resolution scan.

While the stage had two degrees of tilt correction, the software was programmed to remove tilt from the data, making precise tilt corrections unnecessary. Alignment with bolt holes was only performed visually and was within $+/-5^{\circ}$.

Table 8. Summary of Roughness Data Averaged by Magnification.

\begin{tabular}{|l|c|c|c|c|c|c|c|c|c|c|}
\hline Sample & $\mathbf{1}$ & $\mathbf{2}$ & $\mathbf{3}$ & $\mathbf{4}$ & $\mathbf{5}$ & $\mathbf{6}$ & $\mathbf{7}$ & $\mathbf{8}$ & $\mathbf{9}$ & $\mathbf{1 0}$ \\
\hline Ra Low & 227 & 202 & 1088 & 290 & 262 & 255 & 253 & 252 & 274 & 202 \\
\hline Ra High & & 197 & 1038 & 229 & 233 & 170 & 280 & 188 & 217 & 184 \\
\hline Rq Low & 289 & 257 & 1452 & 369 & 330 & 333 & 321 & 342 & 348 & 264 \\
\hline Rq High & & 244 & 1284 & 291 & 290 & 248 & 353 & 241 & 279 & 232 \\
\hline
\end{tabular}

Sample $\mathrm{Ra}$ and $\mathrm{Rq}$ roughness are shown in Table 8, where it is shown that the RMS roughness for all samples except \#3 was between 170 and $370 \mathrm{~nm}$, with an Rq Low total average of $317 \mathrm{~nm}$. Sample \#3 was exceptional since it possessed non-orthogonal roughness. A colormap of the scan data from sample \#1 and the corresponding FFT data are shown in Figure $34 .^{25}$

\footnotetext{
${ }^{25}$ Scanned data for samples \#2-\#10 are shown in APPENDIX B.
} 

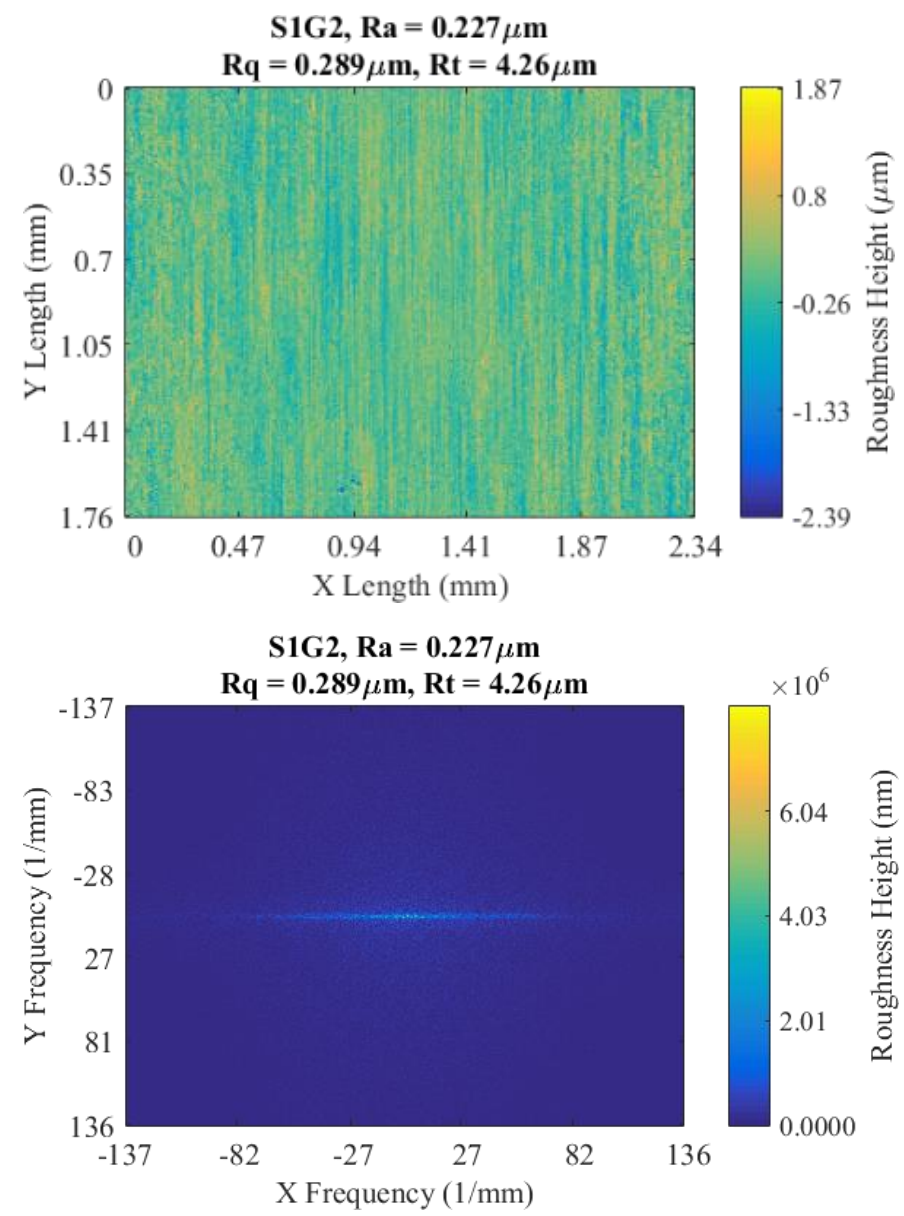

Figure 34. Sample \#1 Group \#2, top: height data (X direction is along coupon long axis), bottom: FFT data.

The data shown for sample \#1 shows roughness aligned in the $0^{\circ}$ direction. The $\mathrm{X}$ axis was aligned with the long axis of the coupon, while the $\mathrm{Y}$ axis was aligned with the short axis of the coupon. The five largest peaks from the FFT data were extracted for each sample and are shown in Table 9 for group 2 data. ${ }^{26}$

${ }^{26}$ Data for all scans are shown in APPENDIX B. 
Table 9. Frequency and angle data for first five peaks for group 2 scans.

\begin{tabular}{|c|c|c|c|c|c|c|c|c|c|c|}
\hline & 1 & 2 & 3 & 4 & 5 & 6 & 7 & 8 & 9 & 10 \\
\hline \multirow{5}{*}{$\begin{array}{l}\text { Frequency } \\
(1 / \mathrm{mm})\end{array}$} & 4.27 & 0.85 & 9.73 & 0.57 & 0.43 & 0.43 & 0.43 & 1.14 & 0.57 & 0.43 \\
\hline & 0.85 & 2.99 & 3.12 & 1.14 & 6.83 & 0.71 & 3.42 & 0.43 & 1.14 & 1.71 \\
\hline & 1.40 & 1.28 & 1.22 & 1.71 & 0.85 & 0.71 & 0.85 & 1.71 & 5.98 & 3.42 \\
\hline & 1.03 & 9.39 & 3.65 & 5.98 & 1.71 & 1.03 & 5.98 & 1.71 & 0.43 & 1.71 \\
\hline & 3.84 & 8.99 & 1.42 & 8.11 & 7.71 & 1.22 & 8.54 & 1.40 & 3.42 & 3.84 \\
\hline \multirow{5}{*}{$\begin{array}{c}\text { Angle } \\
\left({ }^{\circ}\right)\end{array}$} & 0.0 & 0.0 & 69.4 & 90.0 & 0.0 & 0.0 & 0.0 & 90.0 & 90.0 & 0.0 \\
\hline & 0.0 & 0.0 & 65.8 & 90.0 & 0.0 & 53.1 & 0.0 & 0.0 & 90.0 & 90.0 \\
\hline & 24.0 & 0.0 & 69.4 & 0.0 & 0.0 & 126.9 & 0.0 & 90.0 & 0.0 & 0.0 \\
\hline & 33.7 & 0.0 & 69.4 & 0.0 & 0.0 & 33.7 & 0.0 & 41.6 & 0.0 & 0.0 \\
\hline & 0.0 & 3.6 & 53.1 & 0.0 & 4.2 & 110.6 & 0.0 & 24.0 & 0.0 & 0.0 \\
\hline
\end{tabular}

In $60 \%$ of cases, the roughness followed the $0^{\circ}$ for the fundamental frequency component, and in $30 \%$ of cases followed the $90^{\circ}$ component. Several samples had significant frequency content in numerous directions, which was a good indicator of largerscale scratches being present. Visual inspection of the high-resolution scans shows nominal roughness spacing of $\sim 5 \mu \mathrm{m}$, corresponding to a spatial frequency of $2001 / \mathrm{mm}$. This scale roughness, while visibly apparent, was quantitatively small compared to the lower frequencies. In both high and low-resolution scans, the peak values were grouped near the low-frequency end of the plot (for instance, in the center of the FFT data shown in Figure 34). Since the low-resolution scan was more suited to the data, the average $\mathrm{Rq}$ of $431 \mathrm{~nm}$ and $\mathrm{Ra}$ of $330 \mathrm{~nm}$ are taken to be representative of the set of 200 coupons tested.

Another problem with the coupons was the tolerancing. ${ }^{27}$ The coupons used for testing did not meet geometric specifications and the mount designed poorly compensated for unevenness in the machining process. One of the coupons and IRT mounts was placed on an optical comparator, an image of which is shown in Figure 35.

${ }^{27}$ Coupon drawings are shown in APPENDIX D. 


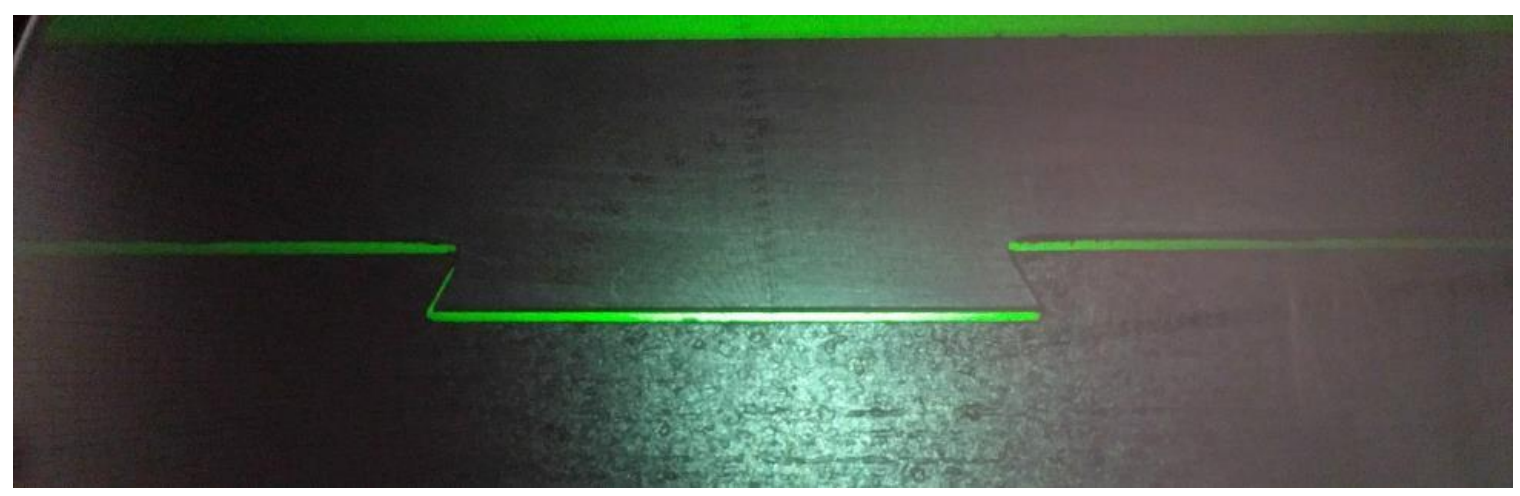

Figure 35. Image of coupon (top) on mount showing inconsistent large radius in corners.

For future work, the mounts should be redesigned to compensate for uneven samples and to distribute stress only on the back of the coupon - preventing strain at the ice/coupon interface. ${ }^{28}$ The coupons were designed to have flat 0.25 "x2" faces to reduce the effect of edges in growing ice shapes with large open sections in the middle. ${ }^{29}$

\section{LEWICE}

In order to estimate the shape that the ice would take, and predict when runback would occur, LEWICE 3.2 was employed. A MATLAB script was written to automate LEWICE using inputs from an Excel file. The goal of this analysis was to allow the prediction of the width of the ice when a necessary thickness had been obtained, and to also allow aerodynamic analysis to be performed. To this end, over 400 cases were run to help determine design loads, and nine cases were run to predict ice shapes for the October 2017 IRT test entry (first entry). The run parameters used in LEWICE are shown in Table 10.

\footnotetext{
${ }^{28}$ A new set of coupons was ordered for the third test but delays in the manufacturing process prohibited their use.

${ }^{29}$ Ice preferentially grows at sharp edges due to higher local collection efficiency, making it more difficult to insure an adhesive failure in testing in some run conditions.
} 
Table 10. Run parameters for LEWICE data. ITIMFL $=0$, IFLO $=10$, DSMN $=6.0 \mathrm{D}-4$.

\begin{tabular}{|c|c|c|c|c|c|c|c|c|}
\hline Case & $\begin{array}{c}\text { LWC } \\
\left(\mathbf{g} / \mathbf{m}^{\mathbf{3}}\right)\end{array}$ & $\begin{array}{c}\text { TSTOP } \\
(\mathbf{s})\end{array}$ & $\begin{array}{c}\text { VINF } \\
(\mathbf{m} / \mathbf{s})\end{array}$ & $\begin{array}{c}\text { TINF } \\
(\mathbf{K})\end{array}$ & $\begin{array}{c}\text { CHORD } \\
(\mathbf{m})\end{array}$ & SLD & FLWC & $\begin{array}{c}\text { DPD } \\
(\boldsymbol{\mu m})\end{array}$ \\
\hline $\mathbf{4 0 1}$ & 1.5 & 300 & 64.3 & 264.0 & 0.0508 & 0 & 1 & 20 \\
\hline $\mathbf{4 0 2}$ & 1.33 & 300 & 77.2 & 263.1 & 0.0508 & 0 & 1 & 20 \\
\hline $\mathbf{4 0 3}$ & 1.21 & 300 & 90.0 & 262.0 & 0.0508 & 0 & 1 & 20 \\
\hline $\mathbf{4 0 4}$ & 1.12 & 300 & 102.9 & 260.8 & 0.0508 & 0 & 1 & 20 \\
\hline $\mathbf{4 1 1}$ & 1.4 & 300 & 77.2 & 263.1 & 0.0508 & 0 & 1 & 15 \\
\hline $\mathbf{4 1 2}$ & 1.4 & 300 & 77.2 & 263.1 & 0.0508 & 0 & 1 & 30 \\
\hline $\mathbf{4 1 3}$ & 1.4 & 300 & 77.2 & 263.1 & 0.0508 & 0 & 1 & 50 \\
\hline $\mathbf{4 1 4}$ & 1.1 & 300 & 77.2 & 263.1 & 0.0508 & 1 & 1 & 180 \\
\hline $\mathbf{4 1 5}$ & 0.99 & 200 & 128.6 & 258.0 & 0.0508 & 0 & 1 & 20 \\
\hline
\end{tabular}

The data was run to simulate one night of testing with a velocity sweep in the tunnel, and a second night with an MVD sweep. The results from LEWICE are shown in Figure 36.
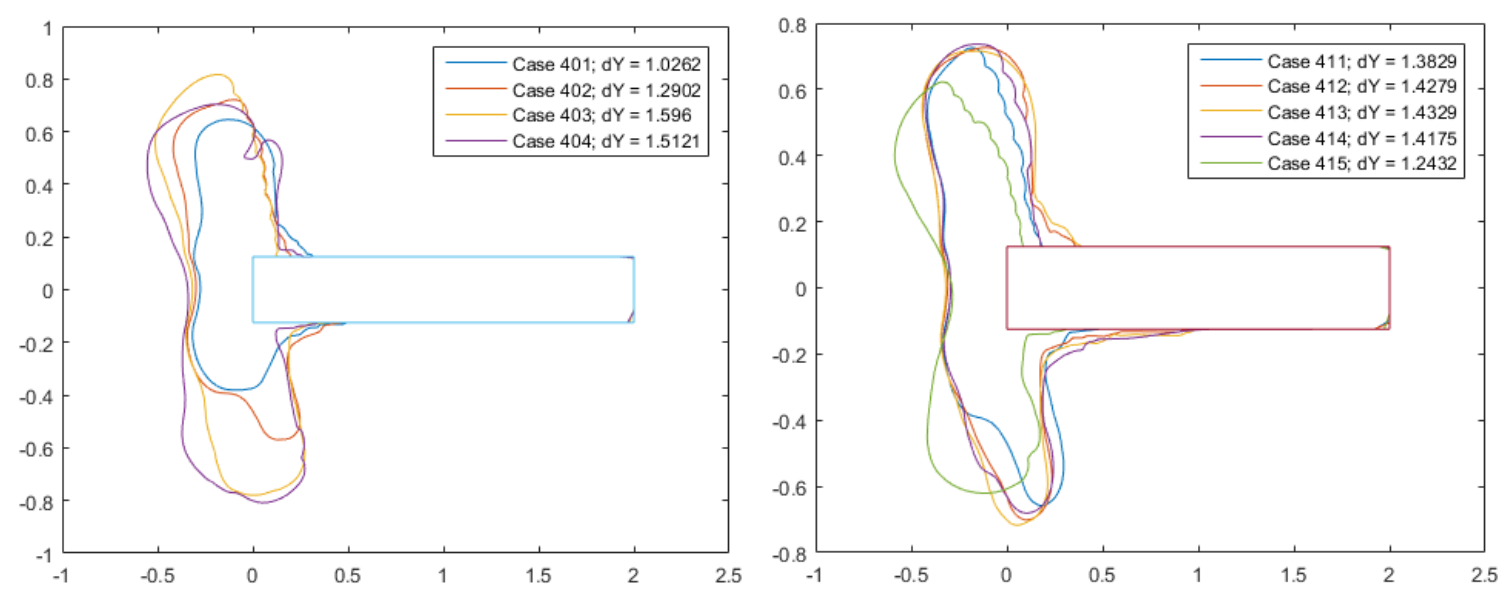

Figure 36. LEWICE runs for first IRT test entry in October 2017. Flow from left to right.

While the prediction of runback was accurate, the shape prediction was not. Ultimately, LEWICE is not designed to run with small geometries with sharp edges and repeatable ice geometries could not be obtained with the coupon shape provided. It was determined that tests must be run with the operator making a visual determination to end the test when the ice reaches the dimensions desired. 


\section{Microscopy}

A Leica DMRX optical microscope was used with cross polaroids to image samples of ice. Samples were imaged in both reflected and transmitted configurations. In preliminary testing, the reflected configuration was used with a mirror backing, sample images are shown in Figure 37.

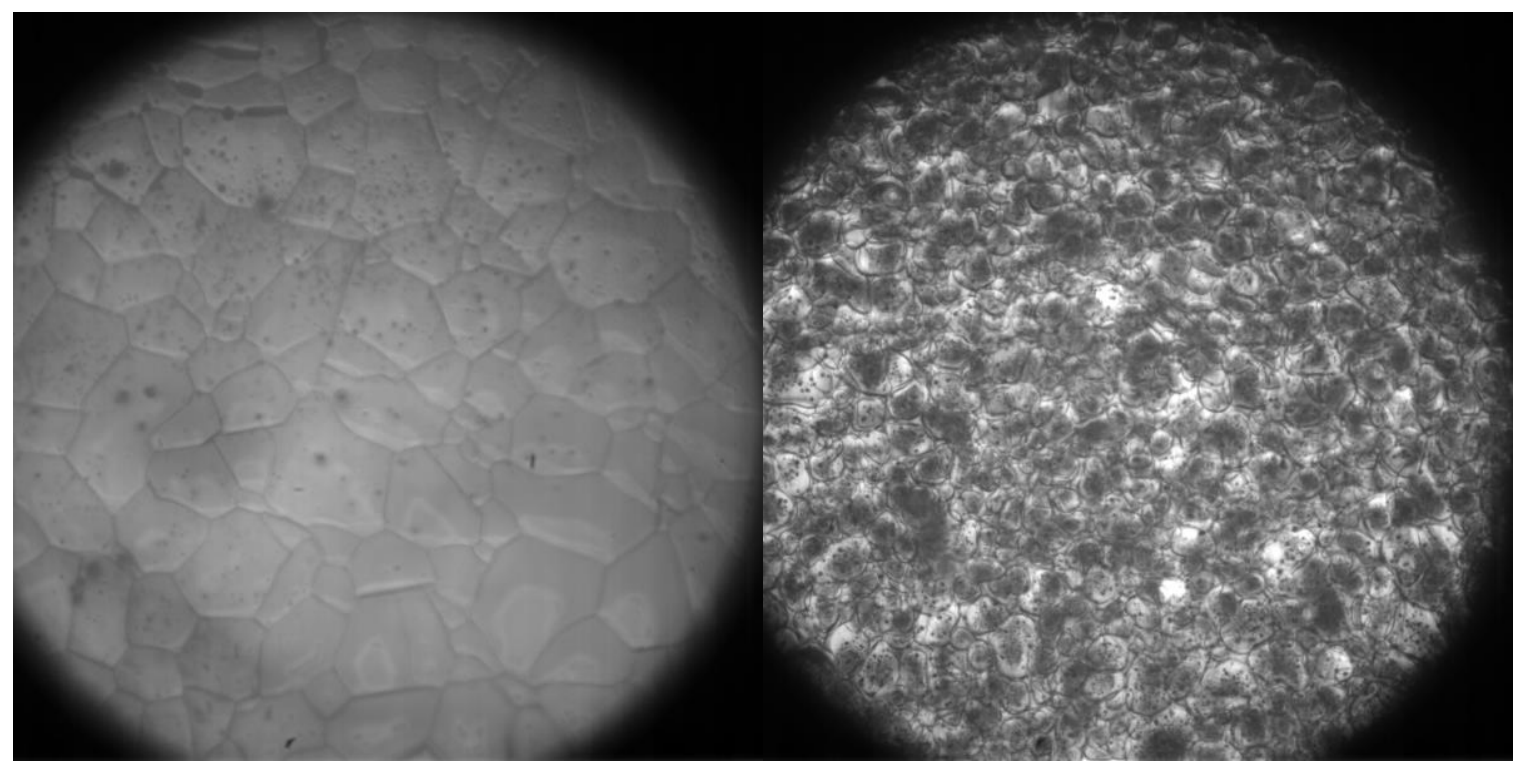

Figure 37. Preliminary images of ice grain structure from IRT ice. Left: glaze ice (8/18/16 run 1$)$, Right: rime ice (8/19/16 run 3$)$.

Preliminary images of the grain structure of ice were taken using a Phantom V10 camera during the 8/19/16 tests. The images were taken with and without cross-polarized linear filters. Since a color camera was not available and the samples were not prepared using a microtome, the cross-polarization made little noticeable difference in the images. Pieces of samples were placed on pieces of aluminum in a freezer and then moved to the microscope to be imaged. The lack of humidity control resulted in surfaces frosting over and clouding up. The glaze ice had a much larger average grain size than the rime ice.

Once equipment was obtained, ice samples were prepared and imaged inside a freezer after being removed from coupons in the adhesion testing procedure. The samples 
were frozen to an aluminum block by use of a pipette and an ice-water bath, and the coupon side was resurfaced using a Bright 8000 sledge microtome. Once resurfaced, the sample was detached and placed on a glass slide. The slide was prepared with a line of vacuum grease, and the newly resurfaced piece of ice was pressed into the vacuum grease carefully to remove air voids. The sample was then frozen by placing equilibrium water in a border around the sample, freezing it to the slide. Vacuum grease prevented water from penetrating into the region of interest. Once frozen, the sample was then shaved down using the microtome. The microtome was equipped with a tungsten carbide blade, and the knife angle set to approximately $15^{\circ}$. Cracks forming was not uncommon during the shaving process and were visible in the imaging. Rime conditions did not need to be shaved and could be imaged directly using reflected light.

\section{Shed Trajectory Prediction}

It was desirable to predict the trajectories of ice when it leaves a rotor blade during a shed in order to obtain a good understanding of the risk potential. To predict the trajectory of ice, it is necessary to develop a mathematical model describing the trajectory. It is also necessary to obtain data to improve and validate the model. A project was undertaken at NASA GRC to achieve these goals, which is documented in the literature [167, 168]. The Vertical Lift Consortium ran a series of tests in the IRT in 2013 that provide data to perform a simple validation of such a model. The tests utilized a tail rotor from a Bell 206 helicopter. The test served several purposes, one of which was to evaluate shedding off of the tail rotor and to compare surface heating techniques. Figure 38 shows the rotor stand in the test section of the IRT. 


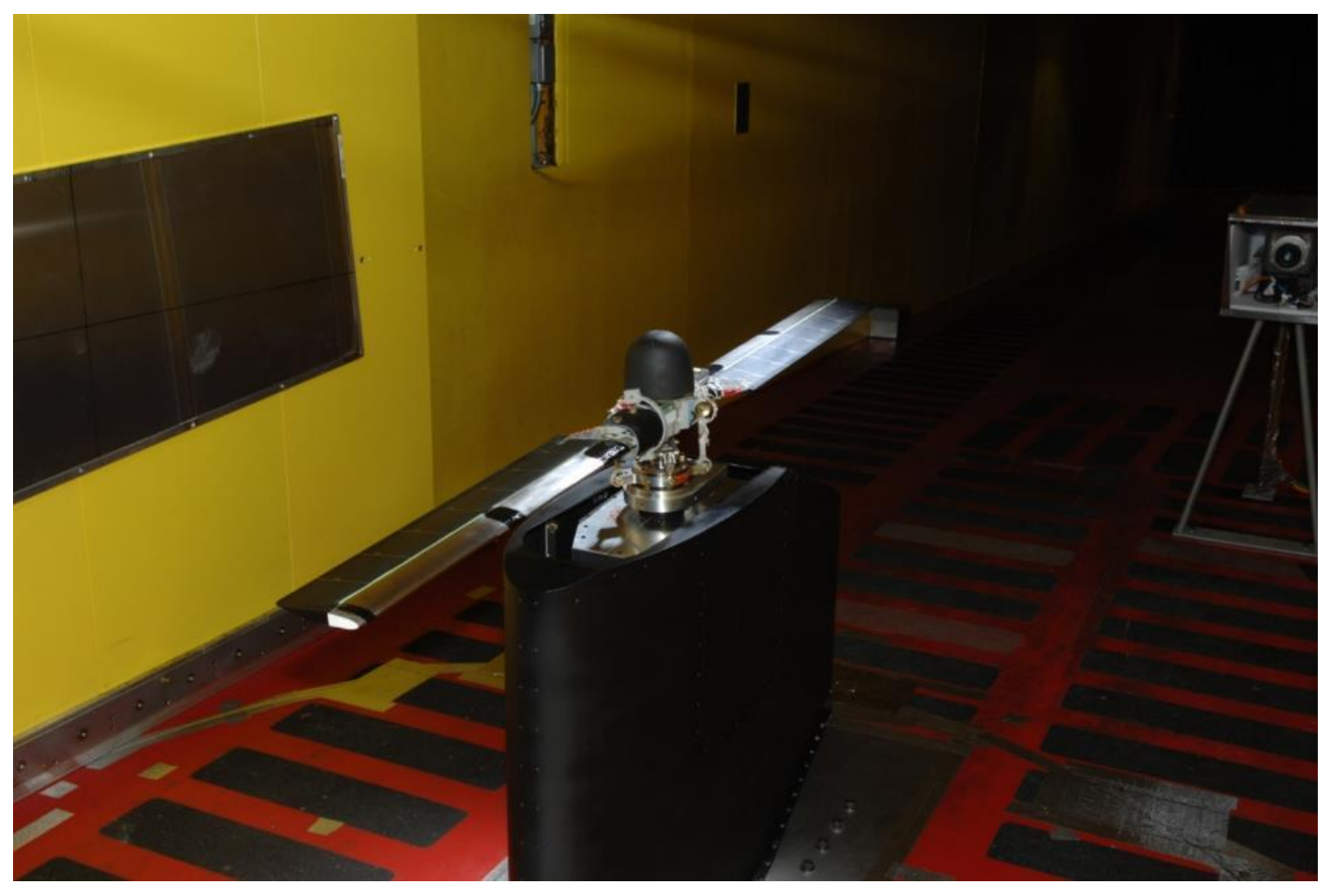

Figure 38. Rotor stand inside test section of IRT in 2013 tests [168].

The rotor collective pitch could be adjusted as well as the mast angle. Typical speeds were 1200 RPM. Surface heating elements were cycled in a chordwise or spanswise manner - i.e. for a chordwise shed a heating element running along the leading edge of the airfoil would be turned on and off, followed by heating elements further back along the chord. Two high speed cameras recorded the tests at frame rates up to 480 frames per second. The top camera captured several shed events, two of which were captured at a high enough resolution for analysis.

The Shedding Trajectory Analysis Tool

To perform the analysis, a Matlab script was developed to post-process images obtained from the tests. The script is the Shedding Trajectory Analysis Tool, or STAT. The tool performed several functions, which are outlined in Figure 39. 


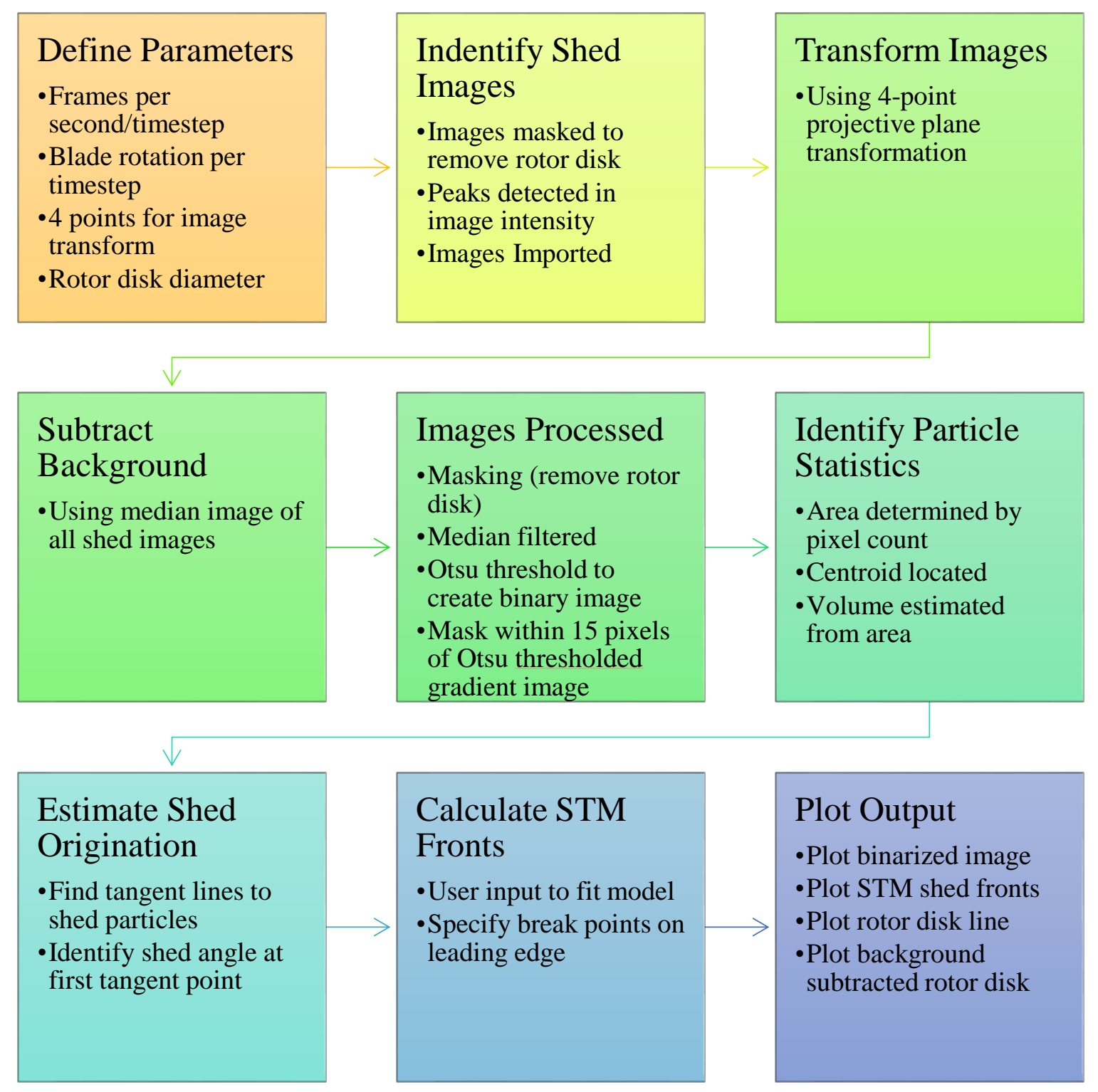

Figure 39. Flowchart overview of the STAT script.

Once the images that contained a shed were identified, a four-point projective plane transformation was required to correct the perspective error of the camera on the ceiling. An unmodified image from Run 71 is shown with a perspective corrected image in Figure 40. The ceiling camera was not mounted normal to the plane of the rotor disk, which appeared to make objects shrink as they moved towards the imaged IRT wall. Points for the transformation were obtained from three images of the rotor in different positions. A 
point was selected at a geometric feature on the end of the rotor in each of the three images, and a fourth was taken at the center of the rotor disk. The final resolution in the plane of the rotor disk was 10 pixels per inch. A median filter was used to isolate the background, and background subtraction was performed. The final processed images for Run 71 are shown superposed with each other in Figure 41.

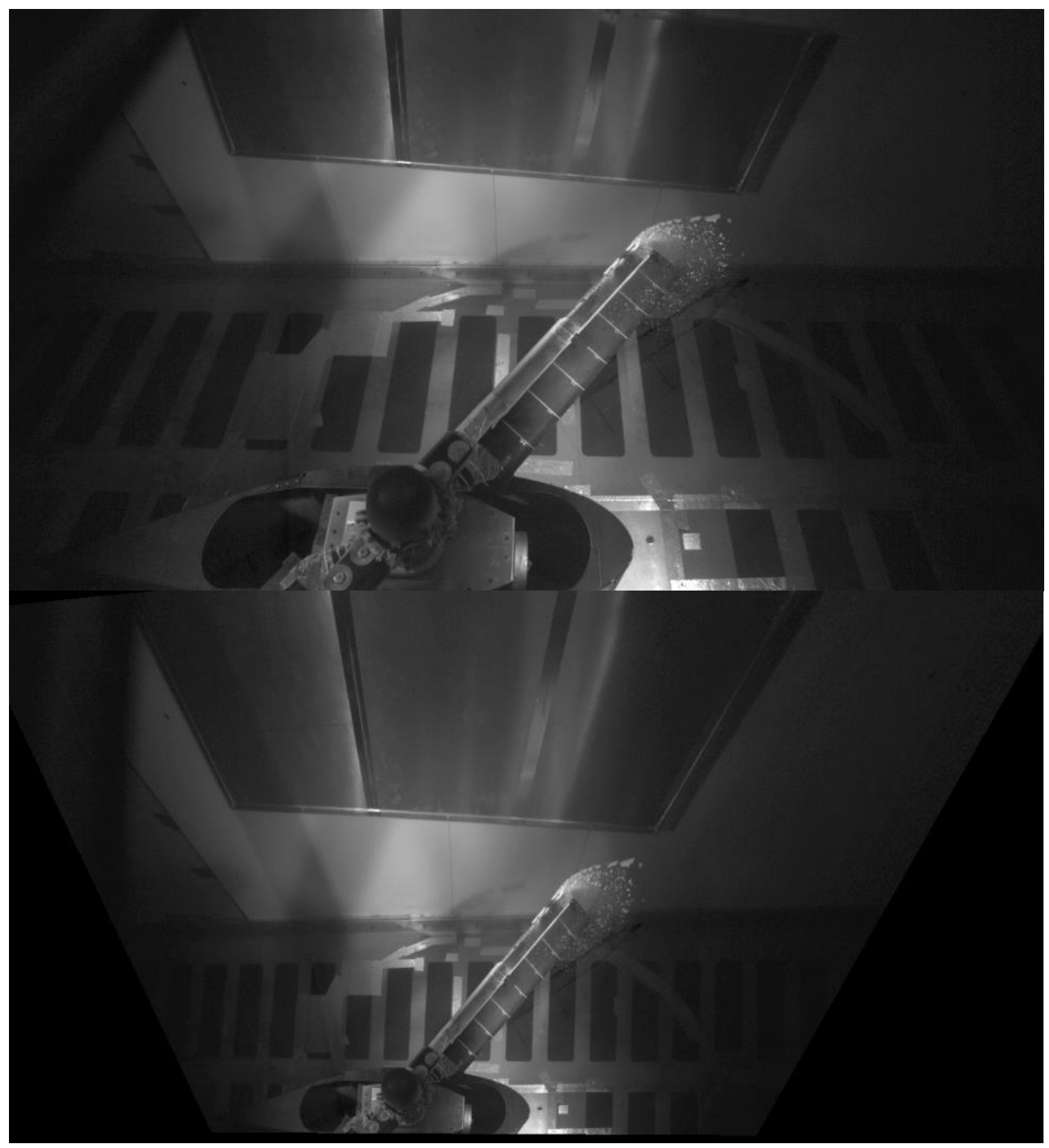

Figure 40. Top: Original image from Run 71. Bottom: Perspective corrected image. 


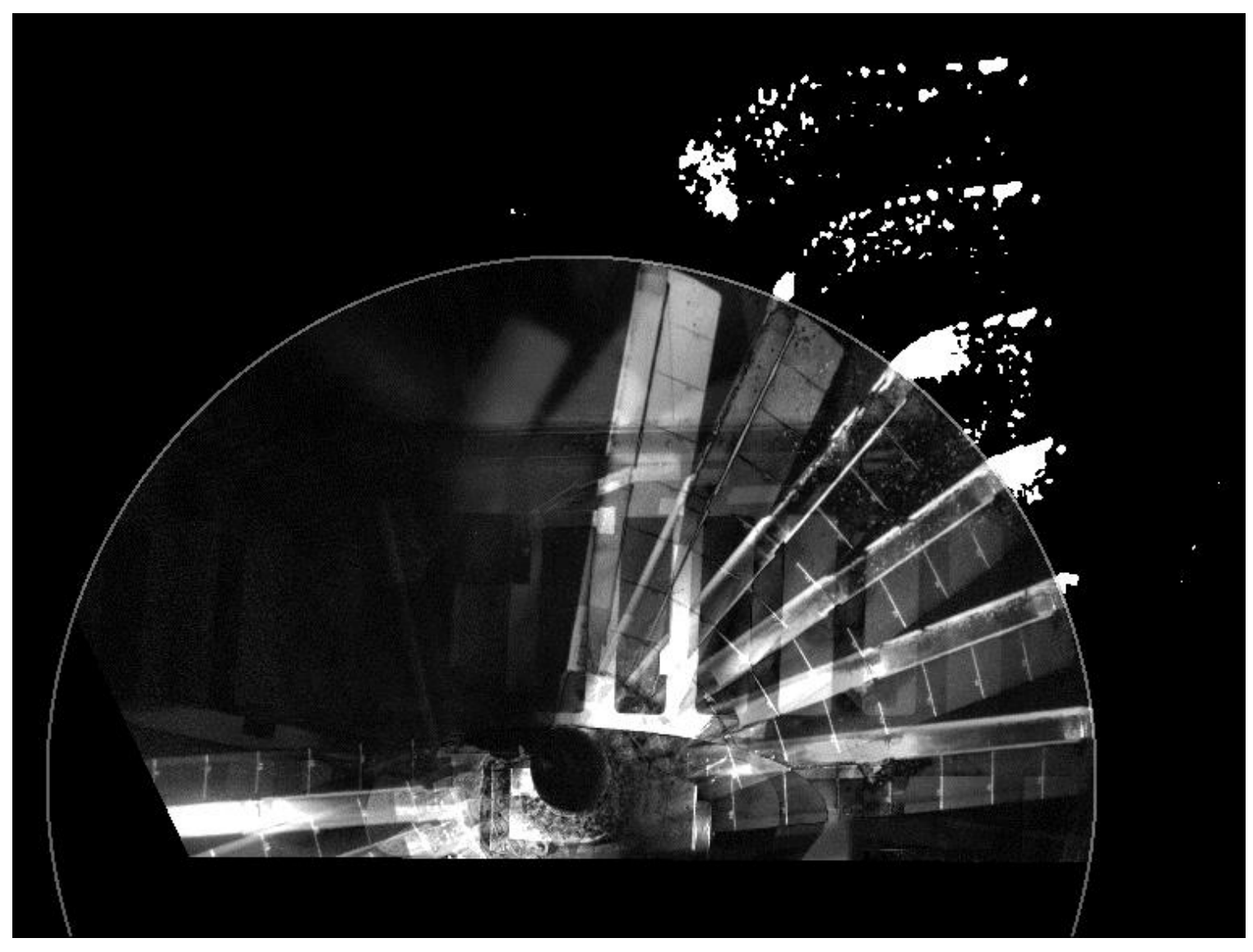

Figure 41. Superposed processed images from Run 71. Gray circle represents boundary of the rotor disk. Ice outside the rotor disk is binarized.

Ice outside of the rotor disk was processed and binarized using the Otsu thresholding method [169]. The final image in the post-processing step shown in Figure 41 was modified further to overlay the predicted shed results from the model developed. Several features in Figure 41 are worth noting in some detail. First, there were several identified pieces of ice which appear to be outliers. These white spots that were obviously outliers were due to a change in lighting in an individual image that was highlighted during background subtraction. The outlier right above and to the left of the top-center of the rotor disk was due to a reflection off background paneling. Second, it is worth noting that the 
edge of the rotor lines up well with the identified rotor disk line over the six images used in this analysis. ${ }^{30}$

The Shedding Trajectory Model

The movement of ice during a shed event is described in two stages, the first while the ice is on the rotor edge, and the second after it has left the rotor edge. In the first stage, it was assumed that the rotor front edge line intersected the center of the rotor disk and rotated in a $2 \mathrm{D}$ plane with constant velocity. The front edge line of the rotor blade did not intersect the center of the rotor disk and was offset by approximately 1.5 inches. The second stage was assumed to be a $2 \mathrm{D}$ ballistics problem with quadratic drag, where the ice broke instantly as it passed the tip of the rotor. Figure 42 shows the coordinate system used for this problem.

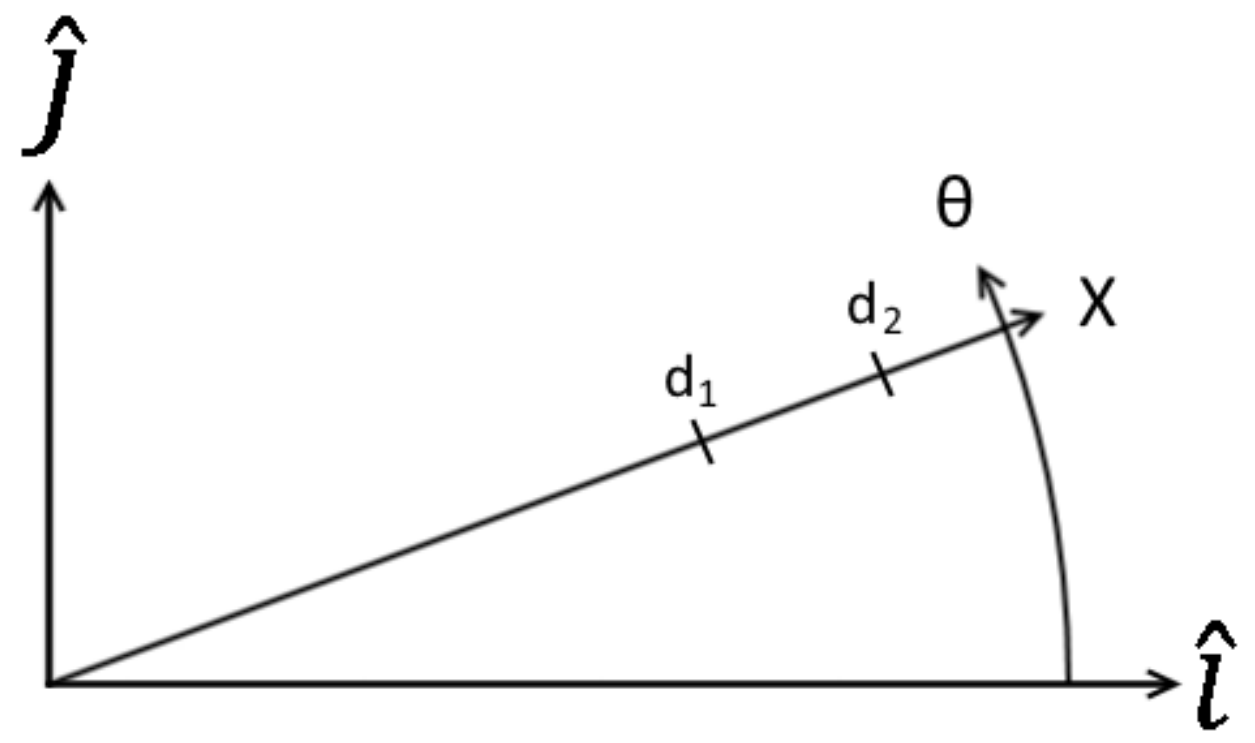

Figure 42. Coordinate system for model. The $\mathrm{X}$ axis rotates with the rotor blade ice is shed from. The ice break starts at $\mathrm{d}_{1}$ and ends at $\mathrm{d}_{2}$, while the length of the blade (L, not shown) may exceed $\mathrm{d}_{2}$.

\footnotetext{
${ }^{30}$ Images from a half-revolution were used to obtain a larger spatial distribution of points for the projective
} transformation. 
The position during the second stage was calculated as follows. The initial position was determined using

$$
x_{i}=L \cos \left(\omega t+\theta_{i}\right) \hat{\imath}+L \sin \left(\omega t+\theta_{i}\right) \hat{\jmath}
$$

where $\omega$ was the rotational speed of the rotor in $\mathrm{rad} / \mathrm{s}, L$ was the length of the rotor blade in inches, and $\theta_{i}$ was the angle when the ice reached the end of the blade, which was the angle the shed began at in the case that the ice began at the edge. The tangential velocity was calculated

$$
V_{t}=\omega L
$$

and the radial velocity, $V_{r}$, was determined from the first stage. This allowed the initial velocity to be calculated

$$
\begin{aligned}
V_{i}=\left(V_{r} \cos (\omega t\right. & \left.\left.+\theta_{i}\right)-V_{t} \sin \left(\omega t+\theta_{i}\right)\right) \hat{\imath} \\
& +\left(V_{r} \sin \left(\omega t+\theta_{i}\right)+V_{t} \cos \left(\omega t+\theta_{i}\right)\right) \hat{\jmath}
\end{aligned}
$$

Assuming a constant coefficient of drag, the position was then calculated

$$
x_{2}(t)=\frac{m}{c} \ln \left(1+\frac{c V_{i} t}{m}\right)
$$

where $c=\frac{c_{d} A_{p} \rho_{a}}{2 m}, c_{d}$ is the coefficient of drag, $\rho_{a}$ is the density of the air, $A_{p}$ is the cross sectional area of a particle, and $\mathrm{m}$ is the mass of a particle. The position function was fit for the entire shed (not individual particles) by varying $c$ as a single term.

The motion of the ice during the first stage was needed to obtain the radial velocity in the second stage. The derivation for the position of the ice in the first stage is as follows. The position along the blade relative to the rotor hub was $x_{s}$. The first stage occurred in two parts (if $d_{2}<L$ ). In the first part, the ice slides as one solid piece until it reaches the blade tip. In the second, the ice continues to slide but is broken off as it passes the tip. The point separating these two parts is when the ice reaches the tip, 


$$
z=L+d_{1}-d_{2}
$$

The force on the ice mass has two terms, that due to centripetal acceleration ( $1^{\text {st }}$ term), and resistive forces (viscous drag while sliding across a liquid layer or friction if poorly lubricated).

$$
\begin{aligned}
& F_{i}\left(x_{s}\right)= \\
& \left\{\begin{array}{c}
\frac{\rho A \omega^{2}}{2}\left(2 x_{s}\left(d_{2}-d_{1}\right)+\left(d_{2}-d_{1}\right)^{2}\right)-\left(d_{2}-d_{1}\right)\left(\dot{x_{s}} b W+F_{f}^{\prime}\right), x_{s}<z \\
\frac{\rho A \omega^{2}}{2}\left(L^{2}-x_{s}^{2}\right)-\left(L-x_{s}\right)\left(\dot{x_{s}} b W+F_{f}^{\prime}\right), x_{s} \geq z
\end{array}\right.
\end{aligned}
$$

where $\rho$ is the density of the ice, $A$ is the cross sectional area of the ice (assumed constant along the length of the rotor blade), $\mathrm{b}$ is the viscous damping coefficient, $\mathrm{W}$ is the width of the ice-blade contact area (along the thickness of the blade), and $F_{f}{ }^{\prime}$ is the frictional force per unit length. The variables A and W are illustrated in Figure 43.

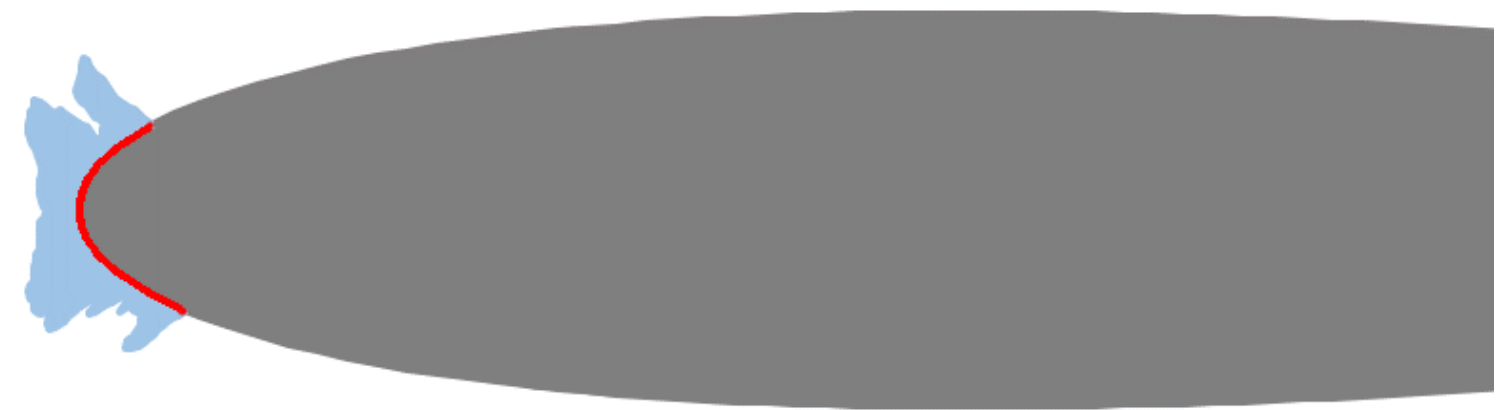

Figure 43. Illustration of A and $\mathrm{W}$ variables. A is the cross-sectional area of the ice (light blue area), and W is the length of the red line. Gray area is a cropped NACA 0012 airfoil.

The viscous damping and frictional force per unit length coefficients represent interfacial forces between the ice and the rotor as the ice slides. Since ice possesses a Liquid-Like Layer and the interface was heated, it was likely that a viscous layer existed between the ice and the rotor. It was possible that frictional forces were present however, so a frictional term was included. The mass was calculated 


$$
M_{i}\left(x_{s}\right)=\left\{\begin{array}{r}
\rho A\left(d_{2}-d_{1}\right), x_{s}<L+d_{1}-d_{2} \\
\rho A\left(L-x_{s}\right), x_{s} \geq L+d_{1}-d_{2}
\end{array}\right.
$$

and the acceleration was calculated

$$
a_{i}\left(x_{s}\right)=\frac{F_{i}\left(x_{s}\right)}{M_{i}\left(x_{s}\right)}=\left\{\begin{array}{c}
\frac{\omega^{2}}{2}\left(2 x_{s}+\left(d_{2}-d_{1}\right)\right)-\frac{\left(\dot{x_{s}} b W+F_{f}^{\prime}\right)}{\rho A}, x_{s}<z \\
\frac{\omega^{2}}{2}\left(L+x_{s}\right)-\frac{\left(\dot{x_{s}} b W+F_{f}^{\prime}\right)}{\rho A}, x_{s} \geq z
\end{array}\right.
$$

At this point it was helpful to make two substitutions, and put the acceleration equation into differential form:

$$
\begin{gathered}
B=\frac{b W}{\rho A} \\
F=\frac{F_{f}^{\prime}}{\rho A \omega^{2}} \\
\left\{\begin{array}{c}
\ddot{x}_{s}+B \dot{x_{s}}-\omega^{2} x_{s}=\frac{w^{2}\left(d_{2}-d_{1}-2 F\right)}{2}, x_{s}<z \\
\ddot{x}_{s}+B \dot{x_{s}}-\frac{\omega^{2}}{2} x_{s}=\frac{w^{2}(L-2 F)}{2}, x_{s} \geq z
\end{array}\right.
\end{gathered}
$$

$\mathrm{B}$ and $\mathrm{F}$ are the coefficients used by the STAT that hold the viscous damping and frictional force per unit length coefficients. It must be stressed that these two coefficients are essentially unknown. While data could be taken to determine the values of these coefficients, no such data currently exists and these coefficients are used as parameters to fit the STM to the data. The differential equations have the particular solution:

$$
x_{p}(t)=\left\{\begin{array}{r}
F-\frac{d_{2}-d_{1}}{2}, x_{s}<z \\
2 F-L, x_{s} \geq z
\end{array}\right.
$$

The characteristic equations for the differential equation were used to solve the complementary solution, and are as follows: 


$$
\left\{\begin{array}{l}
r^{2}+B r-\omega^{2}=0, x_{s}<z \\
r^{2}+B r-\frac{\omega^{2}}{2}=0, x_{s} \geq z
\end{array}\right.
$$

where the roots are

$$
\begin{aligned}
& r_{1,2}=\frac{\left(-B \pm \sqrt{B^{2}+4 \omega^{2}}\right)}{2} \\
& r_{3,4}=\frac{\left(-B \pm \sqrt{B^{2}+2 \omega^{2}}\right)}{2}
\end{aligned}
$$

The complementary solution takes the form

$$
x_{c}(t)=\left\{\begin{array}{l}
c_{1} e^{r_{1} t}+c_{2} e^{r_{2} t}, x_{s}<z \\
c_{3} e^{r_{3} t}+c_{4} e^{r_{4} t}, x_{s} \geq z
\end{array}\right.
$$

and combined with the particular solution, gives the function for the position of the ice along the rotor

$$
x_{s}(t)=\left\{\begin{array}{r}
c_{1} e^{r_{1} t}+c_{2} e^{r_{2} t}+F-\frac{d_{2}-d_{1}}{2}, x_{s}<z \\
c_{3} e^{r_{3} t}+c_{4} e^{r_{4} t}+2 F-L, x_{s} \geq z
\end{array}\right.
$$

The velocity of the ice along the rotor is the derivative of the position function,

$$
\dot{x_{s}}(t)=\left\{\begin{array}{l}
c_{1} r_{1} e^{r_{1} t}+c_{2} r_{2} e^{r_{2} t}, x_{s}<z \\
c_{3} r_{3} e^{r_{3} t}+c_{4} r_{4} e^{r_{4} t}, x_{s} \geq z
\end{array}\right.
$$

The coefficients are solved using the following initial conditions

$$
x_{s}(0)=\left\{\begin{array}{r}
d_{1}, x_{s}<z \\
z, x_{s} \geq z
\end{array} \dot{x_{s}}=\left\{\begin{array}{r}
0, x_{s}<z \\
V_{1 f}, x_{s} \geq z
\end{array}\right.\right.
$$

where $V_{1 f}$ is the velocity at $x_{s}=z$ from the first part. The coefficients are as follows:

$$
\begin{gathered}
c_{1}=\frac{d_{1}+d_{2}-2 F}{2\left(1-\frac{r_{1}}{r_{2}}\right)} \\
c_{2}=\frac{d_{1}+d_{2}-2 F}{2\left(1-\frac{r_{2}}{r_{1}}\right)}
\end{gathered}
$$




$$
\begin{aligned}
& c_{3}=\frac{V_{1 f}-r_{4}\left(2 L-2 F-d_{2}+d_{1}\right)}{r_{3}-r_{4}} \\
& c_{4}=\frac{V_{1 f}-r_{3}\left(2 L-2 F-d_{2}+d_{1}\right)}{r_{4}-r_{3}}
\end{aligned}
$$

The radial velocity was then obtained for a given time after initial shed using $\dot{x}_{s}(t)$. This model provided four terms to fit the data: $\theta_{i}, B, F$, and $c$. The first was set to match the position of the blade when the ice initially broke; this value was obtained automatically but the parameter could be adjusted independently to modify the start position. The friction and damping terms were varied to match the position of the ice as it slid along the blade, and the drag term was set to match the position of the ice in stage 2 . 


\section{RESULTS AND DISCUSSION}

\section{First IRT Test Entry Data and Results}

A total of 193 samples were collected for testing from the first two days of testing in the IRT. Of these samples, 167 were used to produce results with an adhesive failure and no obvious errors in the testing process. Repeat samples were averaged together; grouping was based on MVD, velocity, crosshead speed, and annealing time (time between cloud-off and test). ${ }^{31}$ During the test, the model was noted to 'sing', producing audible tones depending on the wind speed and icing condition. The tones generally disappeared when icing began, but in a few cases throughout all three IRT test entries they were noted to begin with the onset of ice accretion. Speeds where the tones were prevalent were avoided. The acoustic power of the tones was not measured but was considerable: the model could be heard two buildings over during use. ${ }^{32}$ Other discussion generic to each test is included in the Third IRT Test Entry Data and Results section. Four different crosshead speeds were used to test samples; typical force-position recordings are shown in Figure 44.

\footnotetext{
${ }^{31}$ The run conditions for these tests are shown in APPENDIX A, and images of the XT model after a glaze ice spray during the third test are shown in Figure 73, which was similar to run conditions during the first test.

32 This was anticipated during the design process; suitable countermeasures could not be identified. Future tests should include instrumentation to record the induced vibrational loading to the samples.
} 


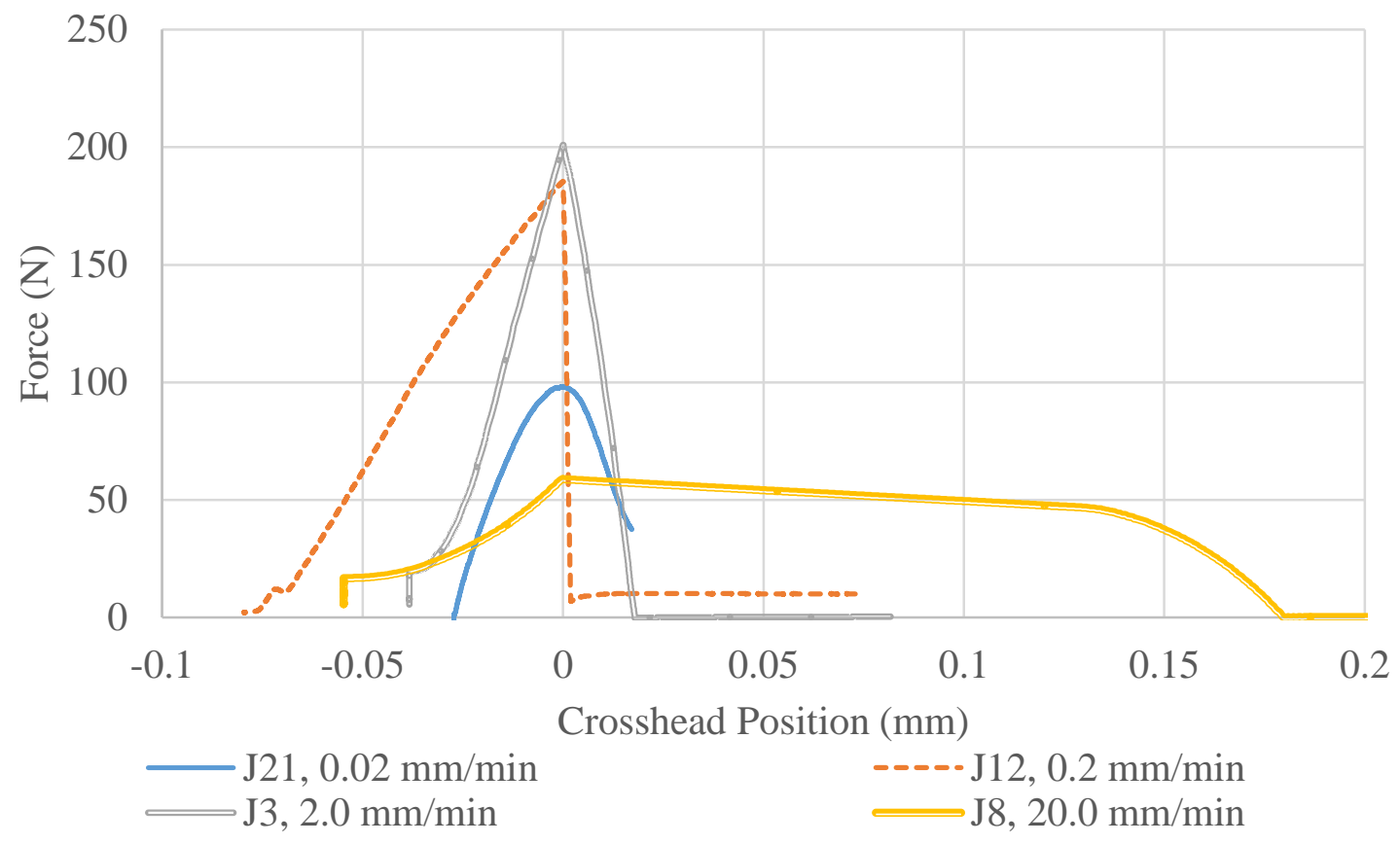

Figure 44. Force-position curves for four typical samples.

The vertical lines at the start of loading were due to a change in the load before the test began. This data was typical of other force-position plots obtained, though in some cases rounded plots were obtained at higher speeds. In some cases, a steady force was left after the break, likely due to the sample being lightly compressed and frictional sliding occurring. The next to slowest speed sample (J12) had a nearly linear response with some offset at the top, possibly indicating some plastic deformation. The $2.0 \mathrm{~mm} / \mathrm{min}$ sample (J3) behaved a little differently, with a rounded section at the beginning. This was possibly due to slipping in the shear rig giving a non-linear response. The drop-off in this instance was also quite slow, possibly due to a steady delamination of the sample, frictional sliding, or inertia in the fixture. For the fastest case, J8, the rounding was much more pronounced. The time from test start to failure was approximately $0.16 \mathrm{~s}$. The force plot shows two different regions after the peak load, a linear region, and a rounded region. The behavior in these two regions was likely due to different mechanics, possibly due to steady 
delamination in the first region, and inertial forces in the second. Generally, the middle two speeds were considered to behave in the least-complicated fashion. The data for the lowest speed (J21) shows a rounded curve where the sample did not finish delaminating during the test. This test ran for approximately 5 minutes, and the rounded plot likely indicates a plastic response and potentially creep flow in the ice. To investigate this further, one sample was run for approximately 40 minutes at this speed. This is shown in Figure 45 .

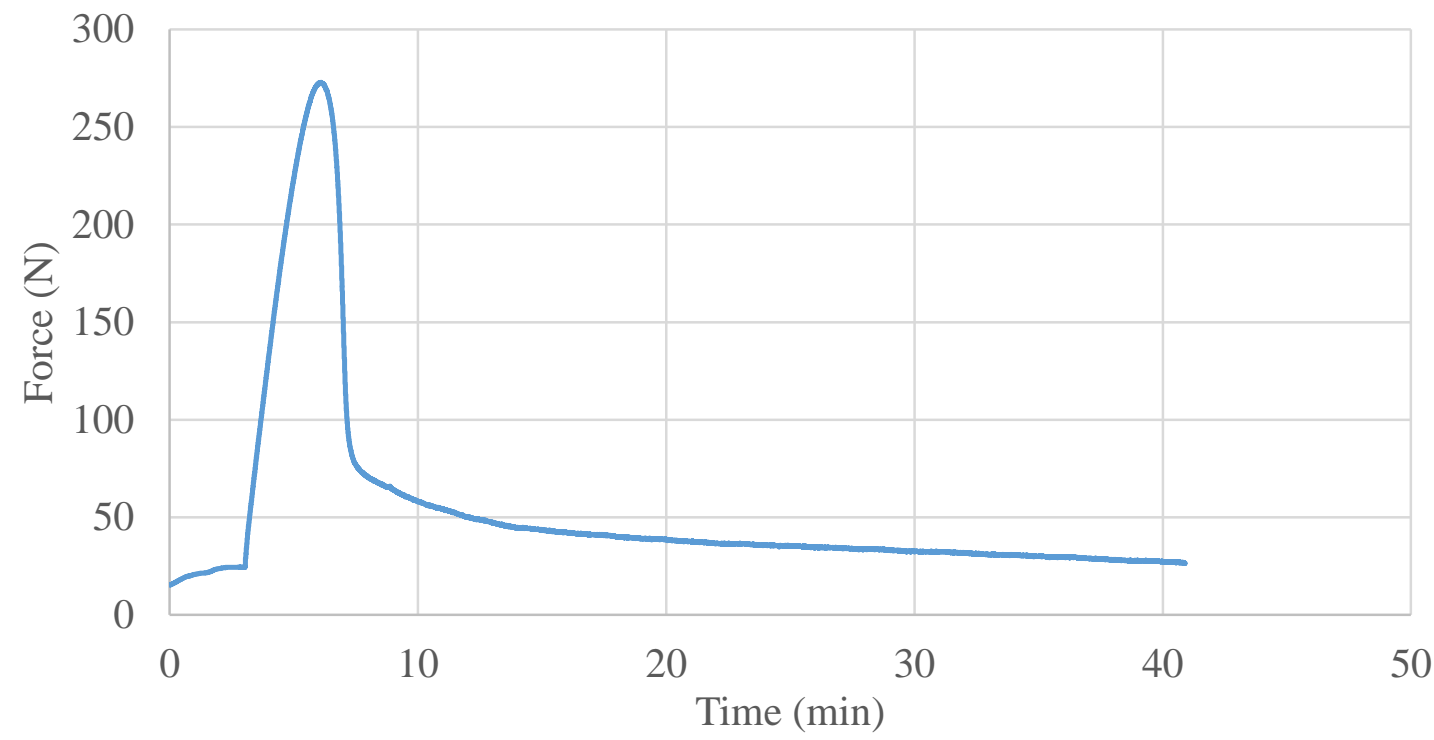

Figure 45. Force-time curve for sample H23, constant crosshead speed $0.02 \mathrm{~mm} / \mathrm{min}$.

The data in the zero to three minute range shows pre-loading prior to the initiation of the test. The $\mathrm{H} 23$ sample was noted to be well attached, with the operator noting that the manual application of approximately $20 \mathrm{lb}_{\mathrm{f}}$ normal force was required to separate the ice from the coupon post-test. ${ }^{33}$ The behavior was likely plastic flow in the sample. It was undetermined if the crystal structure had a preferred orientation in subsequent analysis, though the presence of grains with gray-scale coloring may have indicated that the

${ }^{33}$ This was performed by hand and was not measured; the number was provided to estimate the amount of force to indicate that it would not have been easily mistaken as a smaller force may have been. The lower half of the shear rig was also noted to vibrate on separation, indicating that the sample was still bonded. 
preferred orientation for the c-axis of the grains was normal to the interface. ${ }^{34}$ If this was the case, plastic flow would have been aligned with the basal plane. Results for the analysis of aggregate data are shown in Figure 46, with annealing time grouped by day.

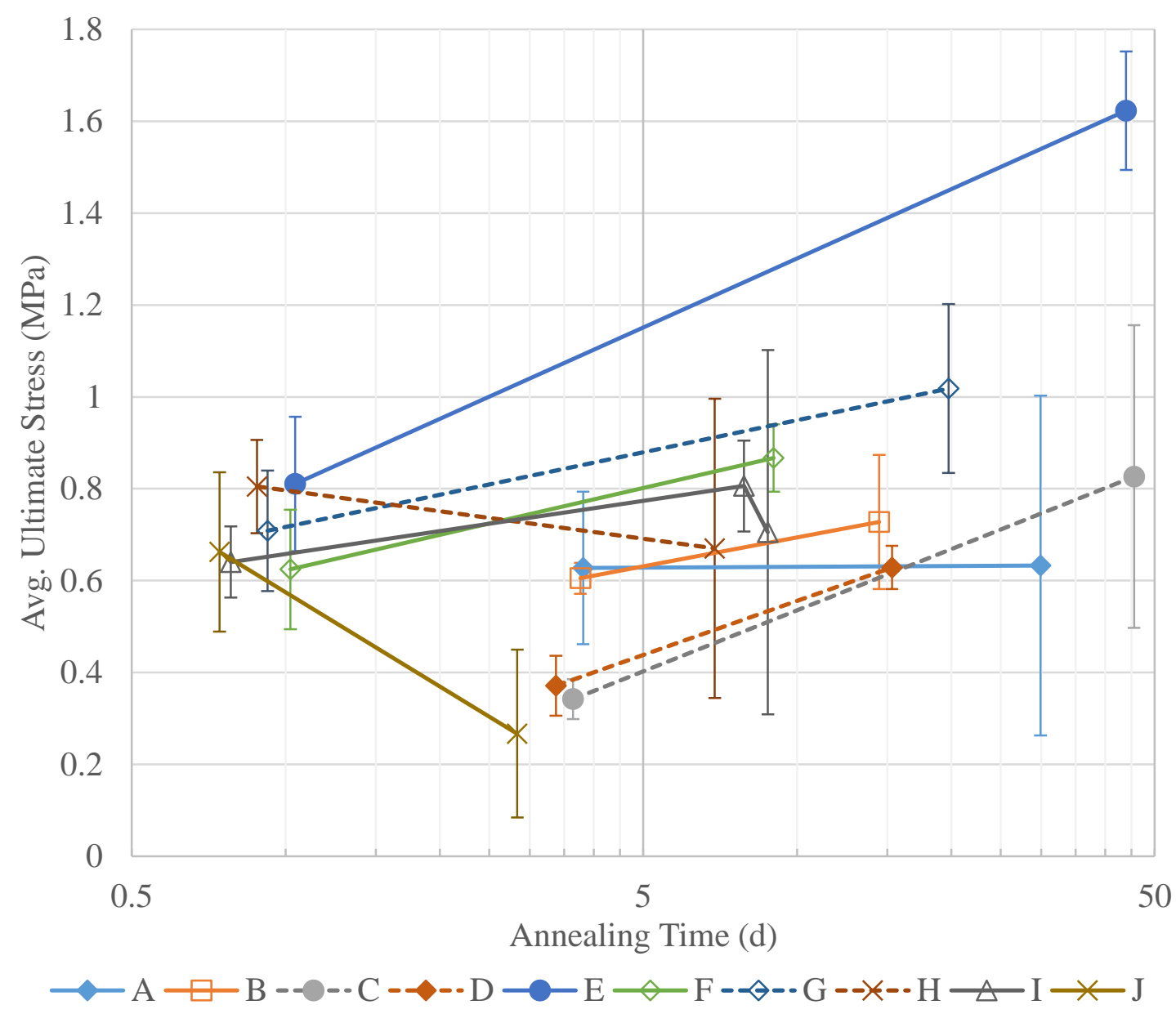

Figure 46. Dependence of adhesion strength on time to test, at crosshead speed of 0.2 $\mathrm{mm} / \mathrm{min}$.

The earliest sample was tested 15.4 hours after cloud-off, while the last sample was tested 1094.7 hours after cloud-off. Runs H and J were tested across more crosshead speeds than other runs, and so a smaller number of samples were used for these runs at each data point, possibly contributing to the downward trend for these two runs. All other runs

\footnotetext{
${ }^{34}$ See Figure 53
} 
showed a flat or upward trend, showing increasing adhesion strength over time. Initially, this was suspected to be due to annealing only. In older samples, it was observed that the samples had evidence of sublimation and deposition inside their storage bags, indicating that there was likely a thermal gradient. No samples were observed to have broken off and re-adhered in this batch of results. Regardless, the samples allowed to sit for over 1,000 hours were observed to double in strength over their earlier test points.

The standard deviation relative to the mean remained relatively consistent for the first batch of tests $(<30$ hours) at $17.8 \%$. The tests that had a downward or flat trend with annealing time tended to have much higher scatter - especially on the tests with higher annealing time; this was most prominent in $\mathrm{A}, \mathrm{H}$, and I. $\mathrm{J}$ also had higher scatter (and a much lower sample count), while $\mathrm{C}$ also showed a significant increase in scatter for the latest batch of tests even with an upward trend. The last point for $\mathrm{J}$, and the first point for the A series both only had two repeats, while the remainder of the points all had three or more. The average standard deviation over the entire set of results from the first IRT test entry was $22.6 \%$. The $0.2 \mathrm{~mm} / \mathrm{min}$ crosshead speed data was grouped for all series and averaged by the annealing time. This set of results is shown in Figure 47. 


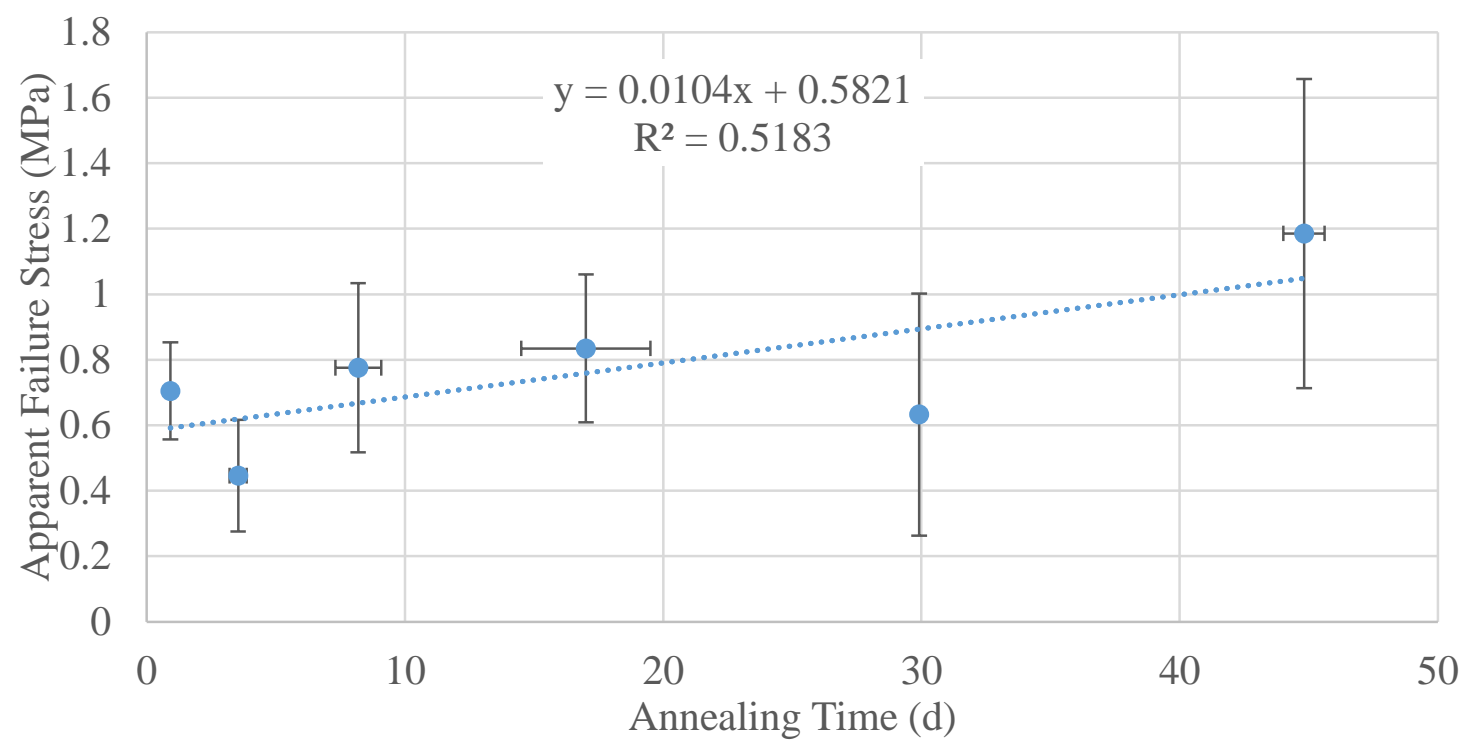

Figure 47. Averaged $0.2 \mathrm{~mm} / \mathrm{min}$ data grouped by time.

The averaged data shows positive correlation between annealing time and strength. The scatter in the apparent failure stress was high since multiple test conditions were grouped irrespective of run conditions.

In the first set of results, the crosshead speed was varied to induce different strain rates. However, these rates were not the same as the strain rates since it will be shown that the relative motion of the coupon and teeth are not the same as the motion of the crosshead due to flexure in the shear rig and connecting rods. Since the flexure of the connecting rods varied with the force exerted on them (following Hooke's Law) the actual strain rate would have varied as samples possessed various stiffness such that stiffer samples were likely subjected to a lower strain rate than more compliant samples. This was not accounted for in the first data set since samples did not have the deflectometers mounted. Data showing the effect of crosshead speed on adhesion strength is shown in Figure 48. 


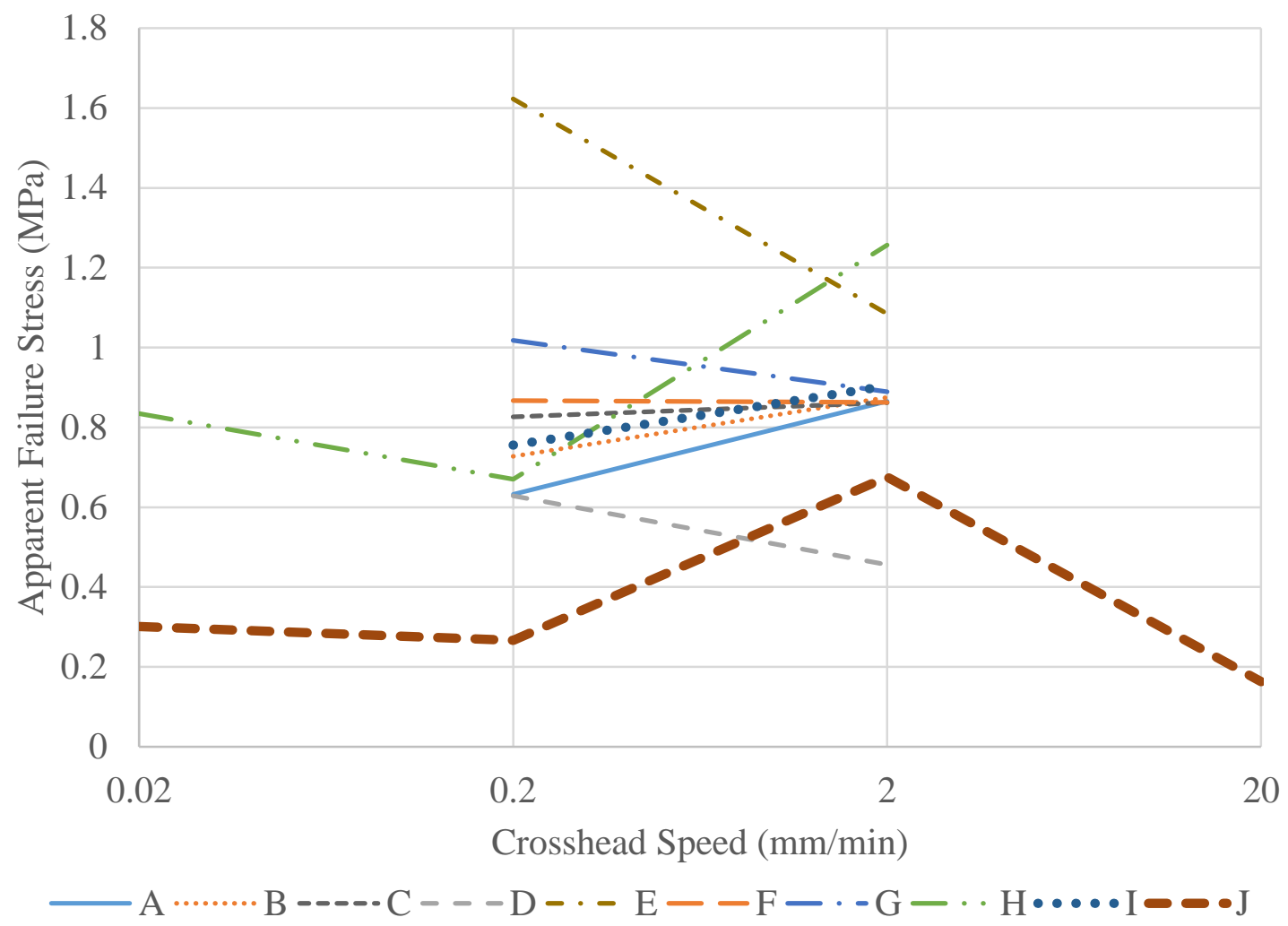

Figure 48. Average Ultimate Strength vs. Crosshead Speed, initial test sets excluded.

Initial test sets were excluded to reduce the effect of annealing time on test results. Speed variation was only performed after the initial set of tests, and each run was grouped together so that each crosshead speed was tested over the same annealing time span. The data did not show strong trends, except that the difference between runs seen at $0.2 \mathrm{~mm} / \mathrm{min}$ mostly disappeared at $2 \mathrm{~mm} / \mathrm{min}$. While most runs showed an upward trend between the two speeds, three runs showed a downward trend. The upward trend was in agreement with the literature. While it was possible that some of the trend was due to sample contamination due to preliminary delamination in some cases, this was thought to be unlikely since no signs of delamination were observed prior to testing. Runs A and E both had significant runback, which artificially increased the adhesion strength. The percent standard deviation for these tests is shown in Figure 49. 


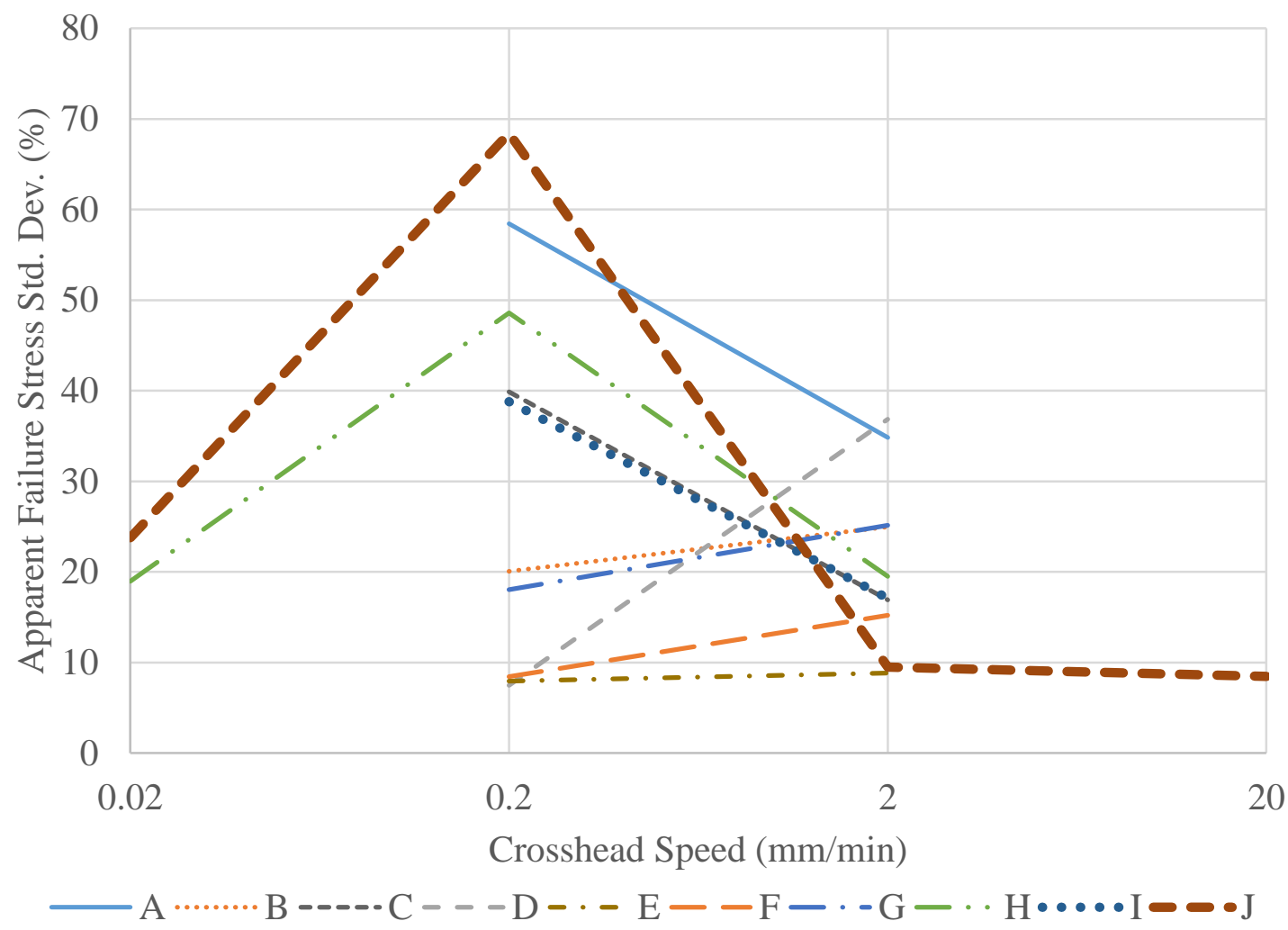

Figure 49. Percent standard deviations for adhesion strength vs. crosshead speed, first IRT test entry.

The standard deviations fit in a larger range for the $0.2 \mathrm{~mm} / \mathrm{min}$ crosshead speed than for the $2 \mathrm{~mm} / \mathrm{min}$ crosshead speed data. The $\mathrm{J}$ test at $0.2 \mathrm{~mm} / \mathrm{min}$ only had two data points, while others had at least three. No obvious trend was identified from the data.

The first day of testing was devoted to acquiring samples over a range of velocities while holding everything else constant. Constant nozzle settings were used for the first day such that the cloud formed from the IRT should have been identical except for velocityrelated effects. The averaged data from this is shown in Figure 50. 


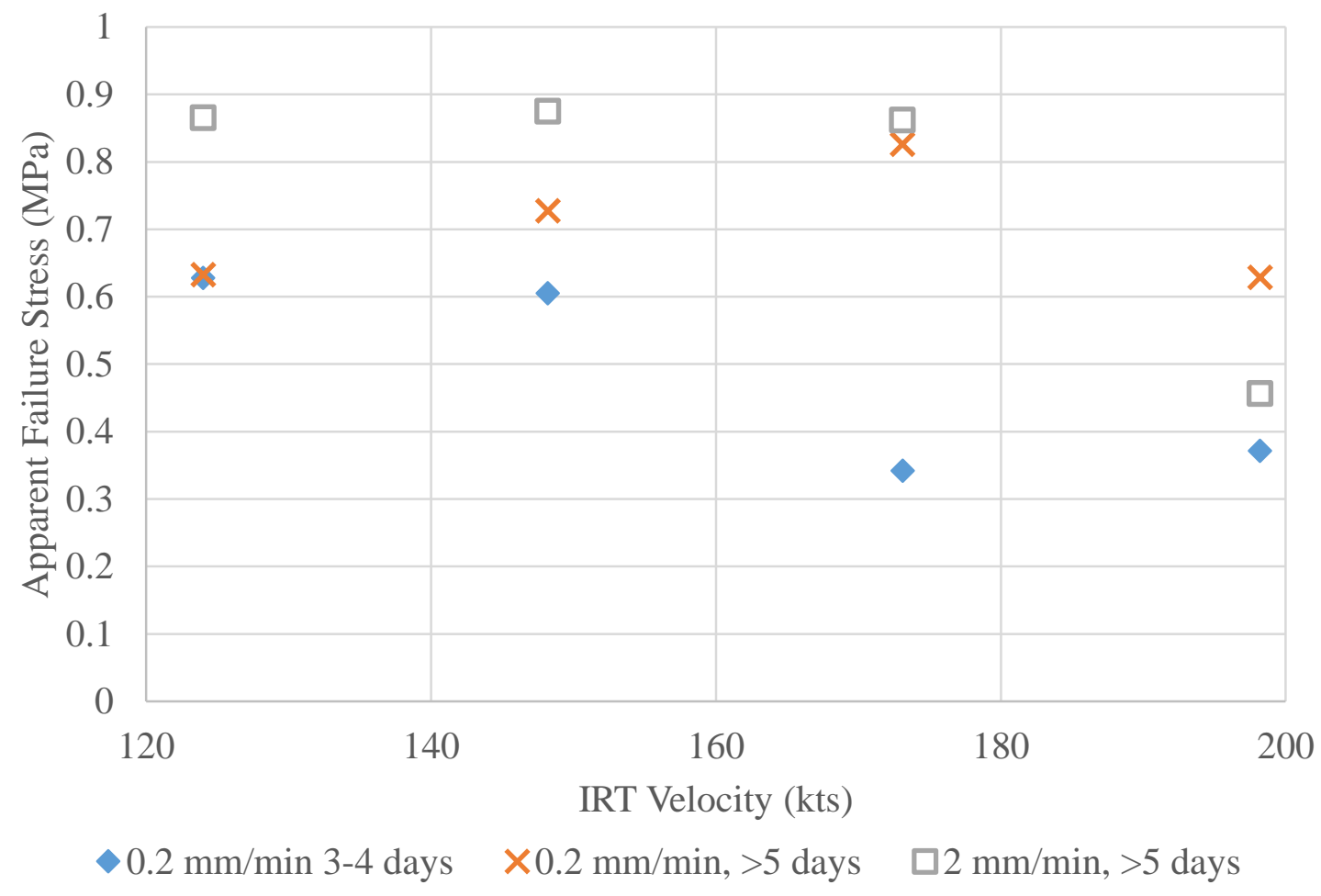

Figure 50. Average Ultimate Strength vs. Wind Speed at $25 \mu \mathrm{m}$ MVD.

While the data was flat in the lower speed region, the adhesion strength at the highest speed was the lowest data point for each group. The data, grouped by age and crosshead speed, show different trends. The $2 \mathrm{~mm} / \mathrm{min}$ data if virtually flat at the lowest three speeds, while the older $0.2 \mathrm{~mm} / \mathrm{min}$ data shows an increasing trend. The data at 125 knots was from test A, which also may have produced higher-than-actual strengths due to runback.

The second day of testing was devoted to changing the MVD of the cloud, holding the velocity constant at 150 knots to determine if the MVD had any effect on the data for the limited set of conditions run. The averaged data is shown in Figure 51. 


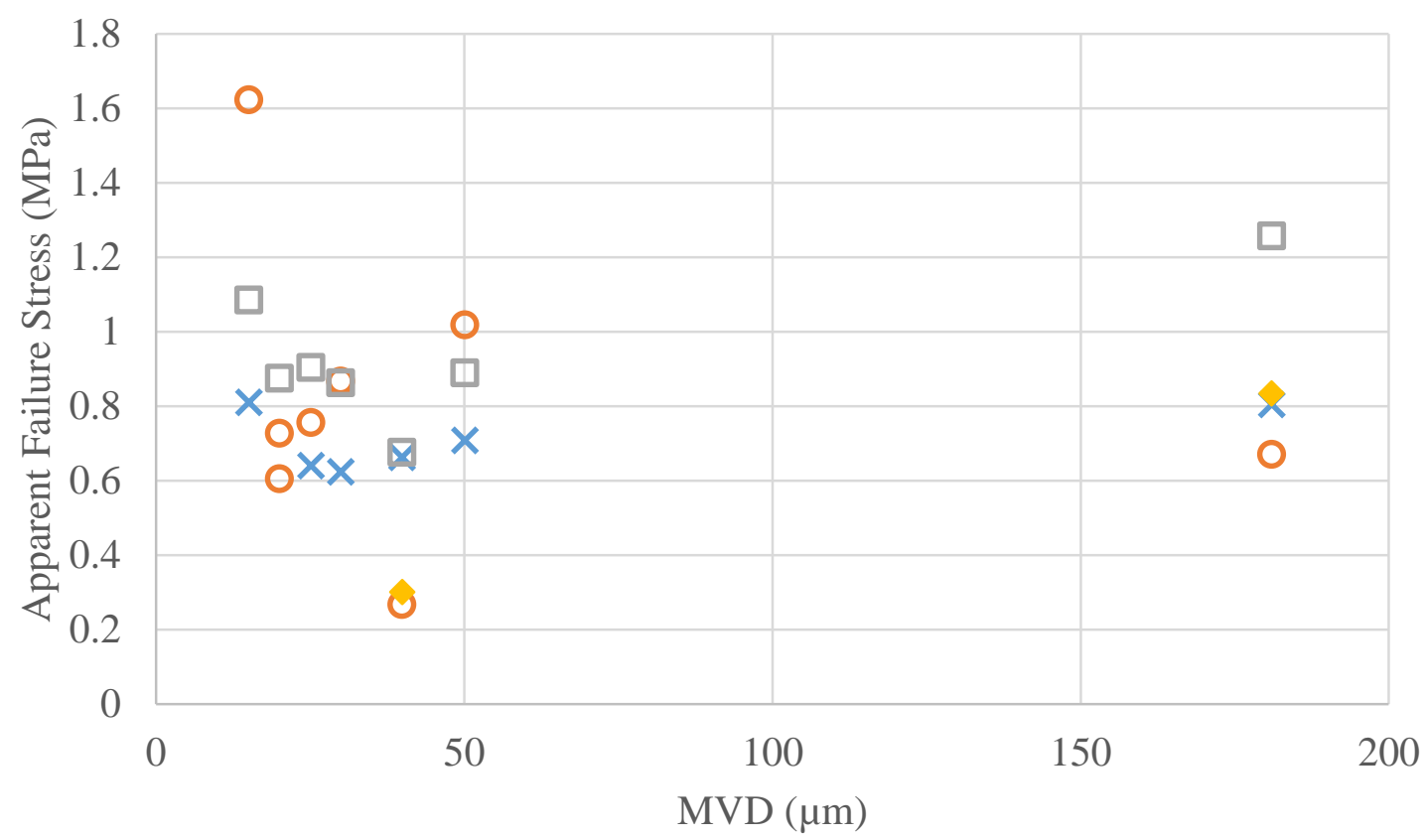

$\times 0.2 \mathrm{~mm} / \mathrm{min}, 0.5-1.5$ days $\quad 00.2 \mathrm{~mm} / \mathrm{min},>2.5$ days

$\square 2 \mathrm{~mm} / \mathrm{min},>2.5$ days $\quad 0.02 \mathrm{~mm} / \mathrm{min}>2.5$ days

Figure 51. Average Ultimate Strength vs. Mean Volumetric Diameter of droplet size distribution, 150 knots tunnel speed.

Little effect was visible from the change in MVD in the data. In lower MVD data, a slight downward trend existed due to a spike in the lowest MVD across several tests, but no obvious trend was apparent from analysis of the data. The $15 \mu \mathrm{m}$ data was from the $\mathrm{E}$ test, and likely showed a spike in strength due to excessive runback on the samples. The $180 \mu \mathrm{m}$ test was an SLD case and samples varied based on vertical position, since the largest droplets tended to miss the last two rows. Omitting the results from $\mathrm{E}$ and $\mathrm{H}$ tended to produce a small upward trend in the data.

It was desired to compare the strengths based on coupon position in the IRT, however there was not enough data for a reliable comparison since most tests produced less than 20 samples. Of the three tests producing 20 or 21 samples (F, G, I), the samples were spread across two different displacement rates randomly, eliminating the possibility 
of direct comparison. Several samples were damaged due to handling, and many runs were completed with less than a complete number of samples since an insufficient number of samples were available for complete runs. The average thickness for samples run from the first test was $5.85 \mathrm{~mm}$, after the refreezing process.

First IRT Test Entry Grain Images

Grain images from the first IRT test entry were taken two months after the test date, a few days prior to the second IRT test entry. The samples were stored at a constant $-10{ }^{\circ} \mathrm{C}$ over this time, insuring that the samples were well annealed. This likely resulted in an increase in the average size of the grains [140]. Images showing the grain structure over the velocity sweep data set are shown in Figure 52.

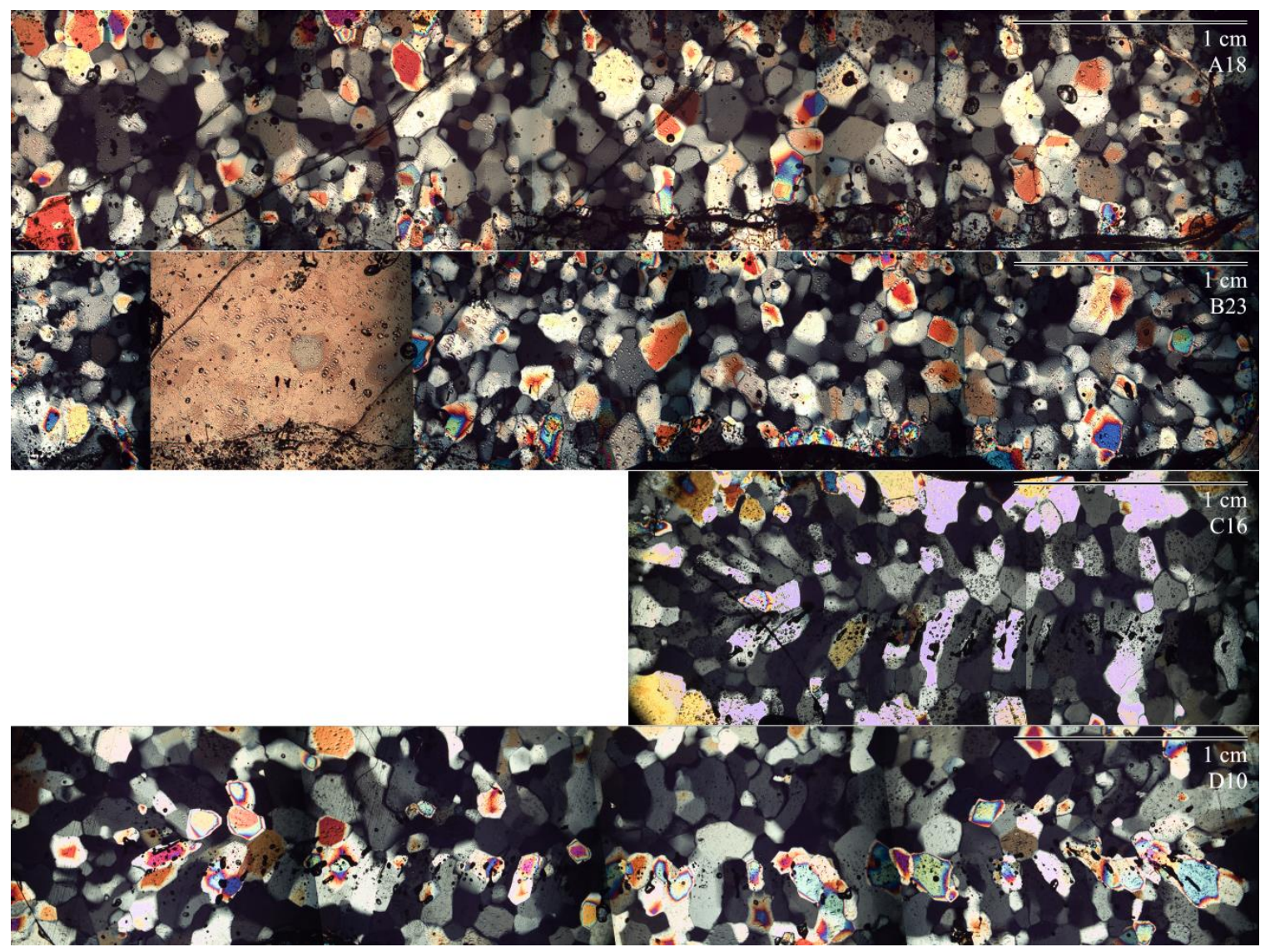

Figure 52. Stitched grain images for IRT runs A-D. The nominal velocities were 125 , 150,175 , and 200 knots, respectively. The nominal MVD was $20 \mu \mathrm{m}$. 
Lab-poured ice was used to freeze the samples in place and can be seen around the borders of the images in most cases, while the region of the IRT ice sample corresponding to the metal coupon was roughly centered in each image. While the same camera and lens was used for each image, different software was used providing very different color palettes when using the built in white balance, so images were modified in Adobe Lightroom to make them more consistent with what was observed visually in the microscope. ${ }^{35}$ Some differences in the structure were observed over the velocity sweep. At lower velocities grains appeared to be larger and equiaxed, though this could not be quantified in this set of results. At higher velocities, grains became more oblong, tending to follow the flow path over the coupon. In all cases, larger, rounder grains tended to locate in the middle while smaller grains were found near the edges of the sample. This may have been due to either of two different effects. Stresses concentrate on the edges and could have resulted in smaller grains, though it was also possible that the smaller grains were a result of a higher collection efficiency at the edges. The collection efficiency did not have a simplistic effect on the growth of the ice since it affected the mass flow rate to the surface, and the thermodynamics at the surface, and the fluid dynamics as well. The elongated grains were indicative that anisotropic properties may be expected. The large grain sizes observed may also have implications for analysis since they may not be small enough to justify a continuum analysis - though the grains likely grew while in storage. Grain images from samples with varying MVD are shown in Figure 53.

\footnotetext{
35 This was most noticeable in C and D. The microscope was not color-calibrated prior to imaging. Some vignetting was observed, particularly on the right side of images. The software used to acquire images, Infinity Capture and Infinity Analyze, were supplied with the camera.
} 


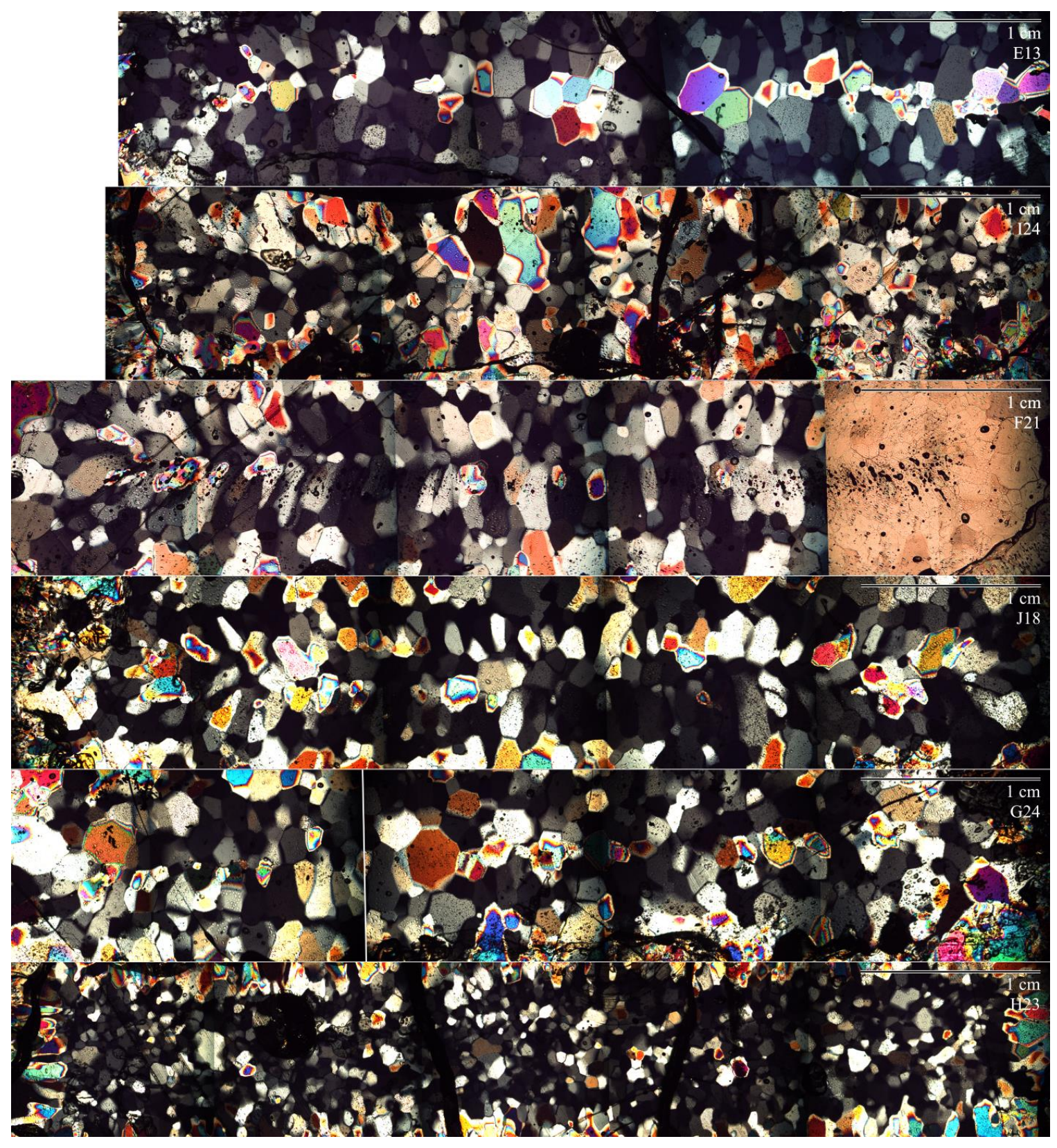

Figure 53. Stitched grain images for IRT runs E-J. The nominal MVD was 15, 25, 30, 40, 50 , and $180 \mu \mathrm{m}$, for runs $\mathrm{E}, \mathrm{I}, \mathrm{F}, \mathrm{J}, \mathrm{G}$, and $\mathrm{H}$, respectively. The nominal velocity was 150 knots.

Trends were less obvious in the MVD sweep. The structure in E and G were similar, even though they were far apart in MVD (15 vs $50 \mu \mathrm{m}$, respectively), while F and J show elongated grains similar to the high velocity runs $\mathrm{C}$ and $\mathrm{D}$. The grains in run I appeared to have some elongation, but not to the extent of that in $\mathrm{F}$ and $\mathrm{J}$. Run $\mathrm{H}$ had an interesting 
grain structure. The image was taken from a sample at the bottom to insure coverage with SLD droplets, since these tended to fall and miss the top two rows of coupons by the time the cloud reached the test section. The grain sizes were much smaller than in other cases, and with no obvious elongation. This suggests that SLD icing may have significantly different thermodynamics during the formation process, resulting in different but likely isotropic properties. This was similar to observation from rime ice cases, such as that shown in the Microscopy section.

The data could not be quantified since individual grains could not be discerned. This wasn't observed until the data was analyzed, after it was too late to retake the photographs. The grains were highlighted by the cross-polarization, but neighbor grains with similar orientations had similar coloring, and boundaries weren't readily visible. Several techniques were attempted to visualize the boundaries, but these failed as well. One sample was left out open in the freezer for a day and imaged as an after picture to see how the ice changed. The before and after images are shown in Figure 54. 


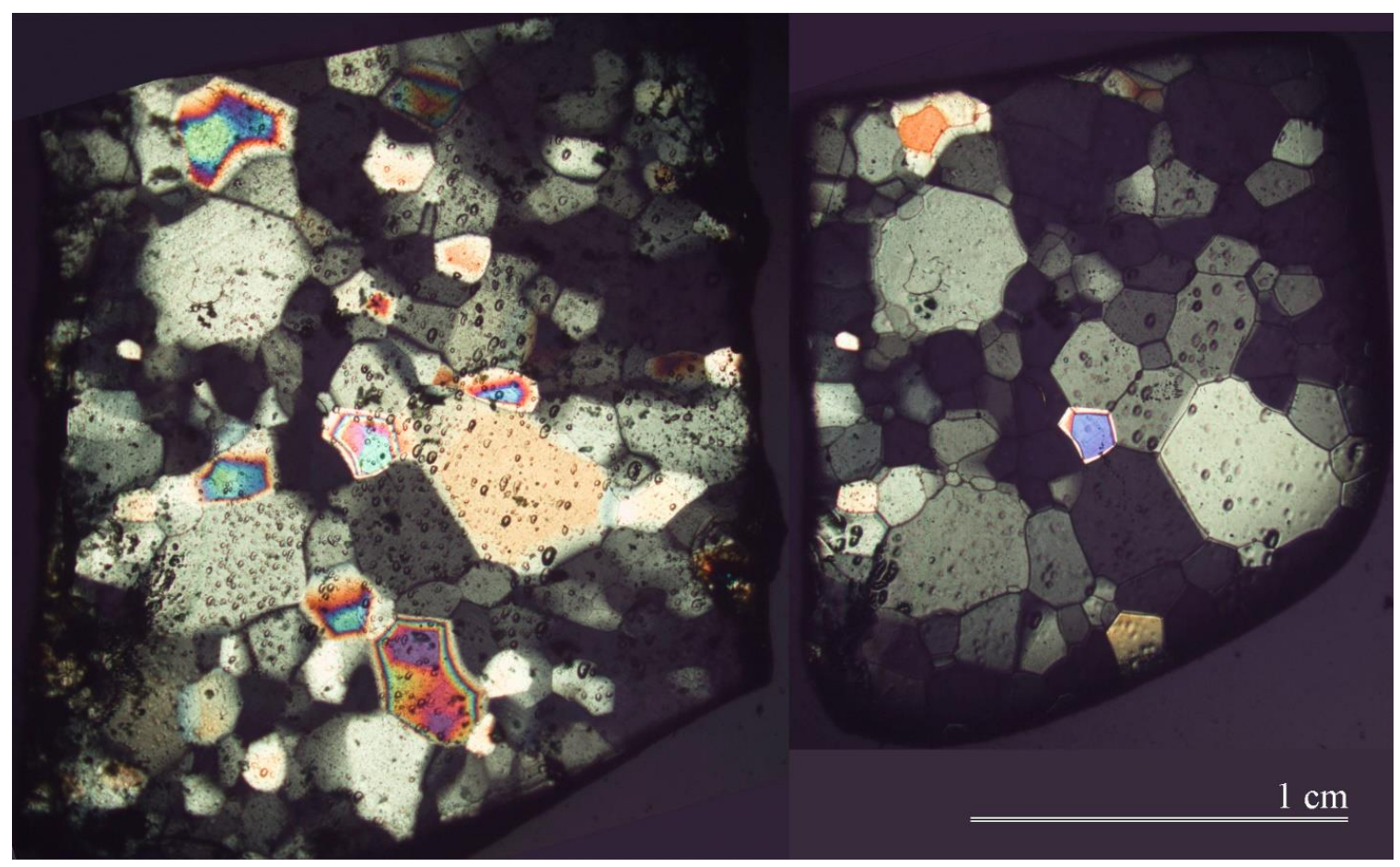

Figure 54. Sample E15. Left: image taken immediately after cut. Right: image taken one day later.

The vapor pressure in the freezer stays below equilibrium, which resulted in sublimation when ice was left out. The E15 sample was an early sample cut without using vacuum grease and left out on a counter exposed to the open air. The sample became thinner and shrunk from the edges. The grains appeared to stay approximately the same size, but some appeared to shift with relation to each other likely due to the non-vertical grain boundaries. Smaller grains either were formed or were uncovered by the process, as can be seen around the larger grains in Sample E15 in the one day later image. The image also cleared up since the sample got thinner since many of the bubbles clouding the image were in the ice that sublimated away. The color in the grains were also much less vibrant after the sample sat for a day and varied less over each grain. The large grain at the top of the sample also shows a small U-shaped crack, which can be seen in both images showing that the crack was fairly deep and stable enough to not grow significantly during the 
sublimation that the sample experienced. It was possible that the grains formed and broke into smaller grains under stress shortly after the freezing process, which would explain why the shade of many grains was similar, having similar orientations. The grain boundaries also were highlighted by the sublimation process since the higher-energy ice at the grain boundaries sublimated at a faster rate than the ice in the middle of a crystal. Obtaining an average grain size for most samples was impossible since the grain boundaries were not easily identified; this result suggests that a better method for future work would be to allow the samples to sublimate for a short time prior to imaging.

\section{Second IRT Test Entry Data and Results}

The second dedicated IRT test entry was completed on 11/20/17 and 11/21/17. One researcher was assigned to run shear tests as samples were collected to minimize the time from the tunnel being turned off to the start of the shear test for a given run. A second researcher was assigned to shave ice and record grain images and assist the PI in transporting samples to the lab for testing. Samples were stored in cardboard boxes, grouped inside plastic bags ${ }^{36}$. Individual samples were stored in air-tight Whirl-Pak ${ }^{\circledR}$ bags. The objective for the second test was to repeat the first test at a lower temperature, although on the first night of testing it became immediately apparent that there was an unconsidered effect in the adhesion process when samples began to fall off on removal. Trends in the literature suggest that adhesion should be stronger for ice, impact or non-impact, as the temperature decreases - but samples were observed to fall off at the slightest disturbance

\footnotetext{
${ }^{36}$ Samples from the last run, $\mathrm{X}$, were left in the portable freezer used to transport samples, which provided better insulation. Markedly less ice crystals were observed in this series after storage than in the other sample sets.
} 
where identical runs at warmer temperatures produced robust samples. Data on the number of samples surviving into the testing process is shown in Figure 55.
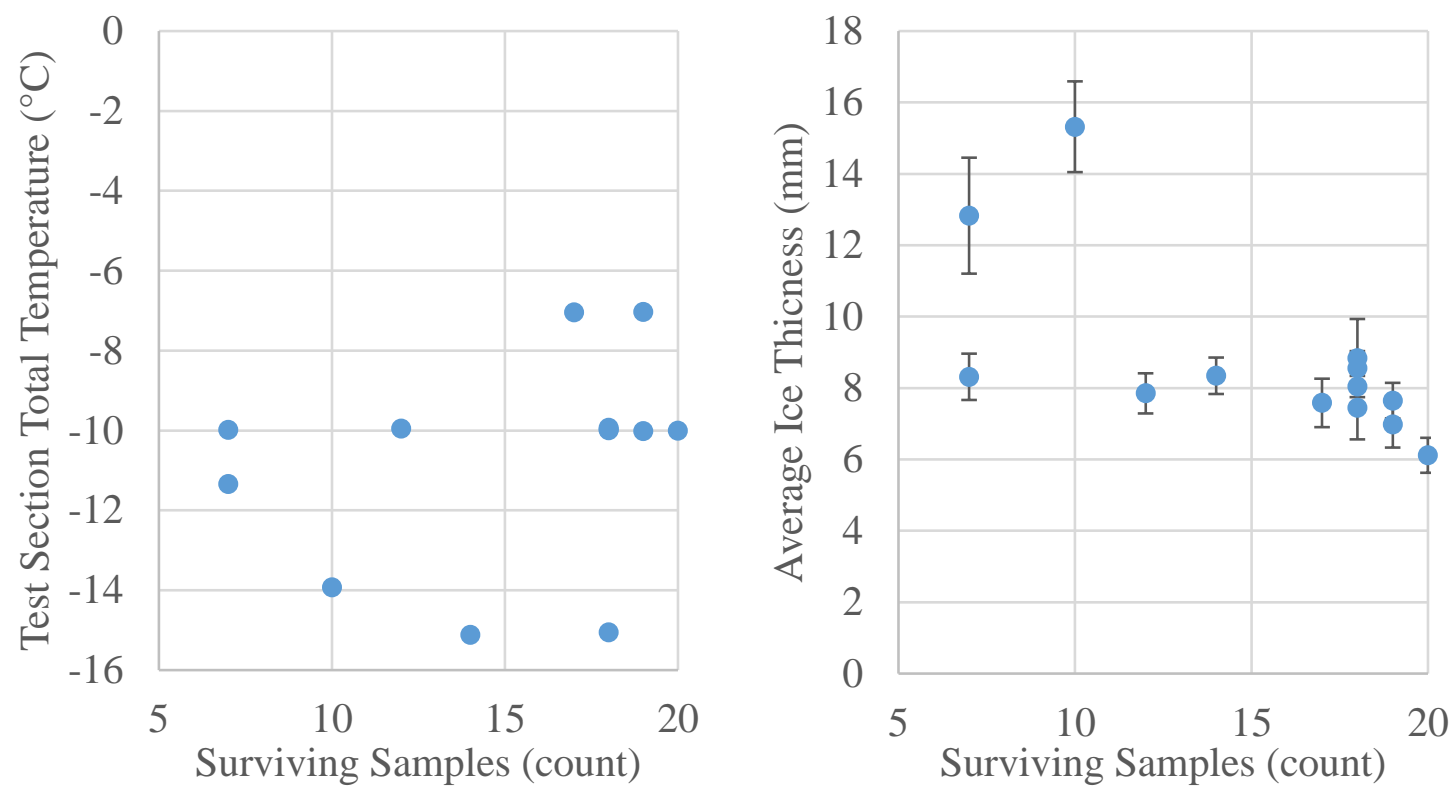

Figure 55. Left: Test section temperature vs. sample count. Right: Average ice sample thickness vs. sample count.

Several samples did not survive into testing due to operator errors (such as dropping samples on the test section floor ${ }^{37}$ ). The data shows some effect with temperature, where colder temperatures resulted in more broken samples. The data shows a stronger trend with thickness, where thinner samples were more likely to survive. It was hypothesized during the test that thermal stresses were expanding or contracting the ice since vibrational loading and bending of the coupons from the set screw configuration did not change significantly from the first test. It was unintentional that thicker samples were obtained during the second test, where average thickness for samples run from the second IRT test entry was $8.29 \mathrm{~mm}$, a $41.7 \%$ increase from the first test. Thicker samples likely resulted in stronger residual

\footnotetext{
${ }^{37}$ The first two IRT test entries were run with the fan on idle, which made handling samples difficult due to the wind and low temperature, while thicker gloves lowered finger dexterity.
} 
stresses, and a stiffer specimen increasing stresses from bending of the coupons during removal. It was noted that many of the broken samples had a pattern on the interface showing lines near the edges. ${ }^{38}$ This pattern matched a pattern of frost formed on the samples which was observed post-test, an example of which is shown in Figure 56.

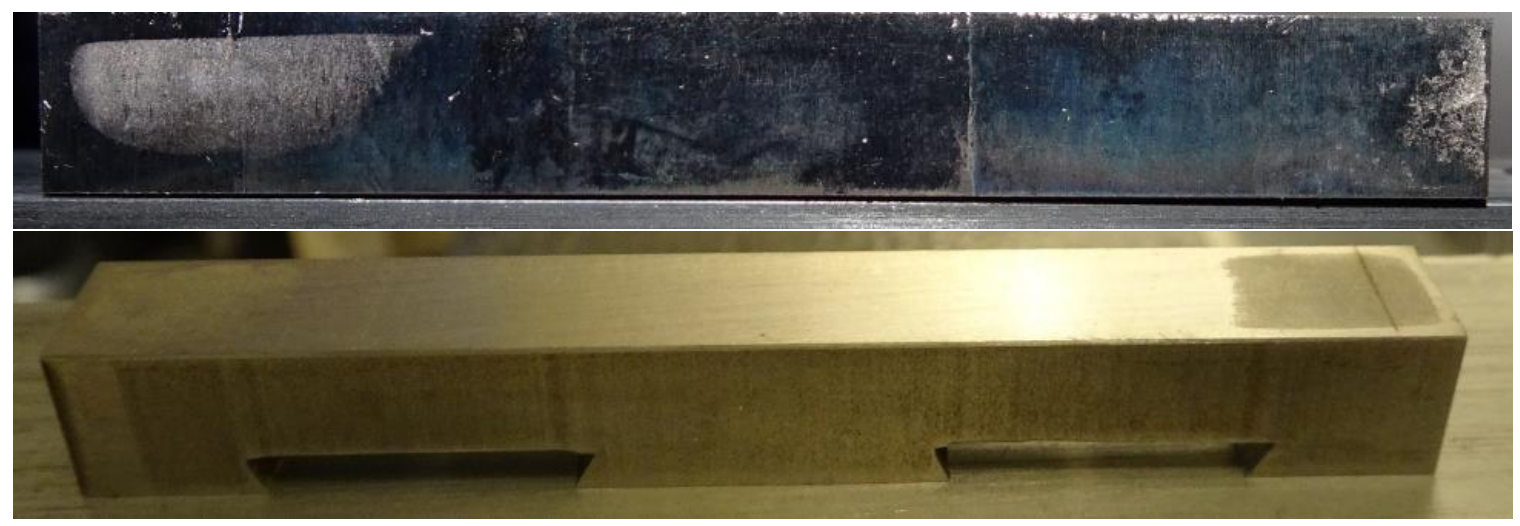

Figure 56. Frost on sample K11 (top) and W6 (bottom). Some frost sublimated before pictures could be taken.

These patterns were likely due to a sublimation and frost cycle which occurred on a delaminated section of ice. Sublimation was observed to occur in sample bags in storage since small particulates of ice would form detached from the sample. This effect was observed to be reduced by insulation, though not eliminated. Frost patterns were observed on the $\mathrm{X}$ series samples more prominently than in other tests, which was well insulated while stored. These frost patterns were also observed during testing on the first night of testing in the third test campaign, before storage in the freezer. This pattern was not consistent with bending of the sample, since the ice would form with the set screw in place, and when it was removed, the flexure of the coupon would pull the middle of the coupon away from the ice - tending to delaminate the ice in the middle. It was possible that frost only formed in the IRT and was removed in some cases from the sublimation-frost cycle

\footnotetext{
${ }^{38}$ Images were taken but the quality of the images were too poor to document the shape on the ice.
} 
in storage. This would make sense considering the temperatures of the samples and of the coupons. The coupon temperature during spray for run $\mathrm{K}$ is shown in Figure 57.

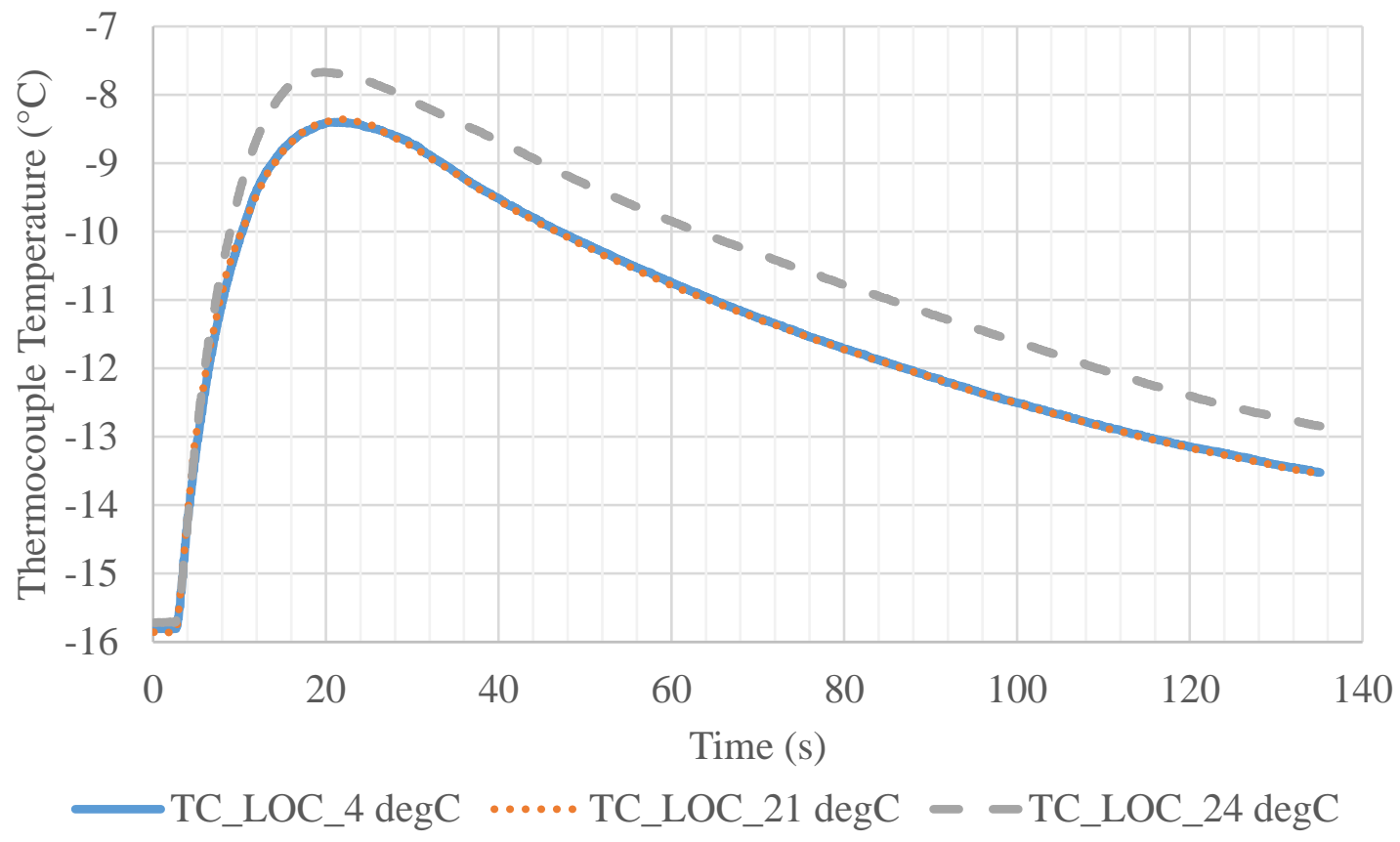

Figure 57. Temperature profile of three coupons in run K during spray.

The three thermocouples used in each IRT test entry were placed in coupon slots 4 , 21 , and $24 .{ }^{39}$ In all cases, it was observed that the maximum temperature recorded occurred shortly after the onset of the spray and was always approximately half of the difference in the initial coupon temperature and the equilibrium temperature. This was likely a coincidence due to the placement of the thermocouple. ${ }^{40}$ The thermocouple embedded in the coupons was not representative of the ice temperature. All conditions run for the first two IRT test entries were glaze conditions, where the impinging droplets form a liquid film that freezes to form the ice. In glaze conditions, the latent heat release from formation is not sufficient to completely freeze the water on impact, which results in the film. The

\footnotetext{
${ }^{39}$ Slots were labelled left to right, top to bottom in numerical order, such that the top row consisted of slots $1,2,3,4$, and the second row of $5,6,7,8$, and so on.

40 Thermocouples were embedded $1 / 16$ " beneath the center of the front face of the coupons.
} 
temperature of the ice on its outermost layer was thus almost certainly $0{ }^{\circ} \mathrm{C}$, and as new ice formed over the initial layer at the interface, the interfacial temperature started to cool back to ambient conditions. If ice partially delaminated during this process, the temperature across the delaminated region would not be continuous, with the ice side warmer than the coupon. Local mass transfer could then occur between the ice and the coupon, leaving marks on the ice and frost on the coupon.

Even if the scenario described above was what occurred in the tunnel, it was observed that samples could reattach to the surface after a sufficient amount of time. At least three samples showed relative motion between the ice and coupon while in storage and were taken out of storage bonded together. An example of this is shown in Figure 58.

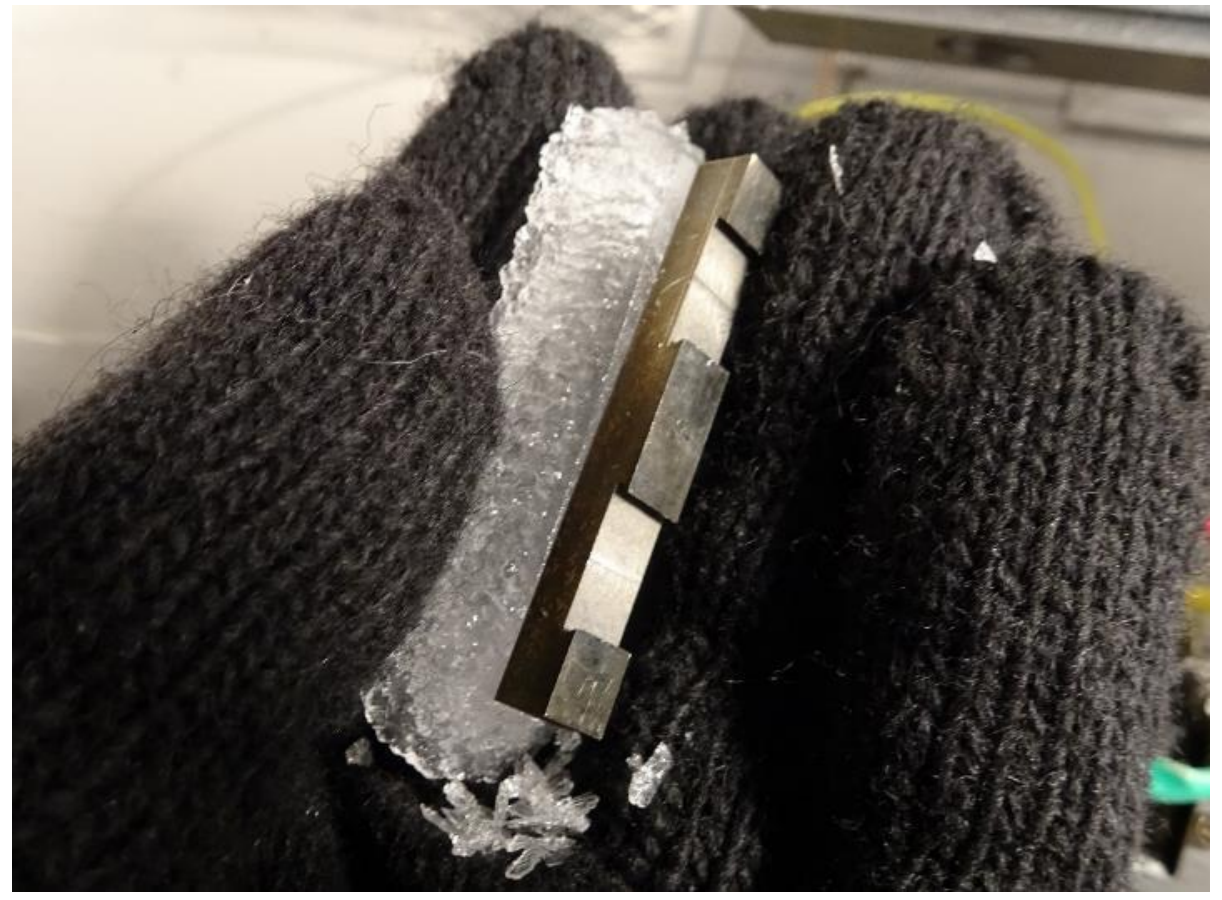

Figure 58. Sample W13 showed relative motion between the ice and coupon and was bonded on removal.

This was observed in two other samples to a similar extent and may have occurred to a lesser extent in other samples. It was unknown whether the ice stayed attached during 
the process, or if it slid. It was possible that the mechanism for reattachment was the same as observed in the preliminary testing. ${ }^{41}$ Two samples that had a large dislocation all showed significant amounts of sublimation, however this was observed to a small extent in an X series sample which had only small amounts sublimation. All samples had sat for nearly two months before this was observed. Some of this frost for the W13 sample can be seen in the glove in Figure 58 beneath the sample. While W13 was tested, samples that had large dislocations were deemed questionable and excluded from the bulk of the analysis. W13 recorded as a good break with an ultimate force of 83.1 N. S19 also had a large amount of sliding (good break, 71.1 N), as well as light sliding in X16 (good break, $198 \mathrm{~N})$. The values for S19 and W13 were low for their groups, while that for X16 was near the peak value recorded for the $\mathrm{X}$ series. Most of the $\mathrm{X}$ series results had visible frost, and X16 was included in the analysis. Frost was also observed in the K, L, S, and W tests to a lesser extent. It was possible that frost occurred but was not observed on tests before 5 PM 1/15/17 since operators were not aware to check for it.

On the second night of testing, rough-cut acrylic samples were tested to help determine the cause of the preliminary delamination. Acrylic has a larger coefficient of thermal expansion than ice or steel, and a lower modulus of elasticity than either. All acrylic samples were placed in coupon slot \#3. Only three were successfully tested; these were samples R3a, U3a, and V3a, which had ultimate stresses of $0.58,0.065$, and 0.105 $\mathrm{MPa}$, respectively. The average failure strengths for the stainless-steel samples of the R, $\mathrm{U}$, and $\mathrm{V}$ sets tested on the same day as the acrylic samples were $0.14,0.27$, and 0.079 $\mathrm{MPa}$, respectively. R3a and $\mathrm{V} 3 \mathrm{a}$ were tested on 11/21/17, while U3a was tested on

${ }^{41}$ See Figure 24 and surrounding discussion. 
11/28/17. The data, while likely not statistically significant, implies that the adhesion strength was higher on the first day for the acrylic samples than for the steel samples, but lower a week into storage. Regardless, several acrylic-ice samples were broken in transit, similarly to the stainless-steel samples. This indicated that a mismatch between the samples coefficient of thermal expansion was not a likely cause of the preliminary delamination. The set screw force was also likely to be lower on the acrylic samples since they were softer and could deform more freely - operators tended to put less torque on the set screws and less torque was needed since the screws tended to dig in more than with the stainlesssteel coupons. However, it was observed that some samples were stronger than others, and many fell off after the set screw was loosened. Since only one sample fell off with the tunnel running (in the cloud-off condition) out of 13 runs, each with 24 samples ( 3 dummy samples for the thermocouples), it was regarded as unlikely that vibrational loading was significant; however, it is planned to instrument the XT structure with accelerometers in future testing to verify the loads. Thermal imaging was performed in an attempt to measure the delay between the refreezing cycle on the shear rig and the return to equilibrium. One series of images is shown in Figure 59, with Time $=0 \mathrm{~s}$ within $10 \mathrm{~s}$ of the end of melting. 


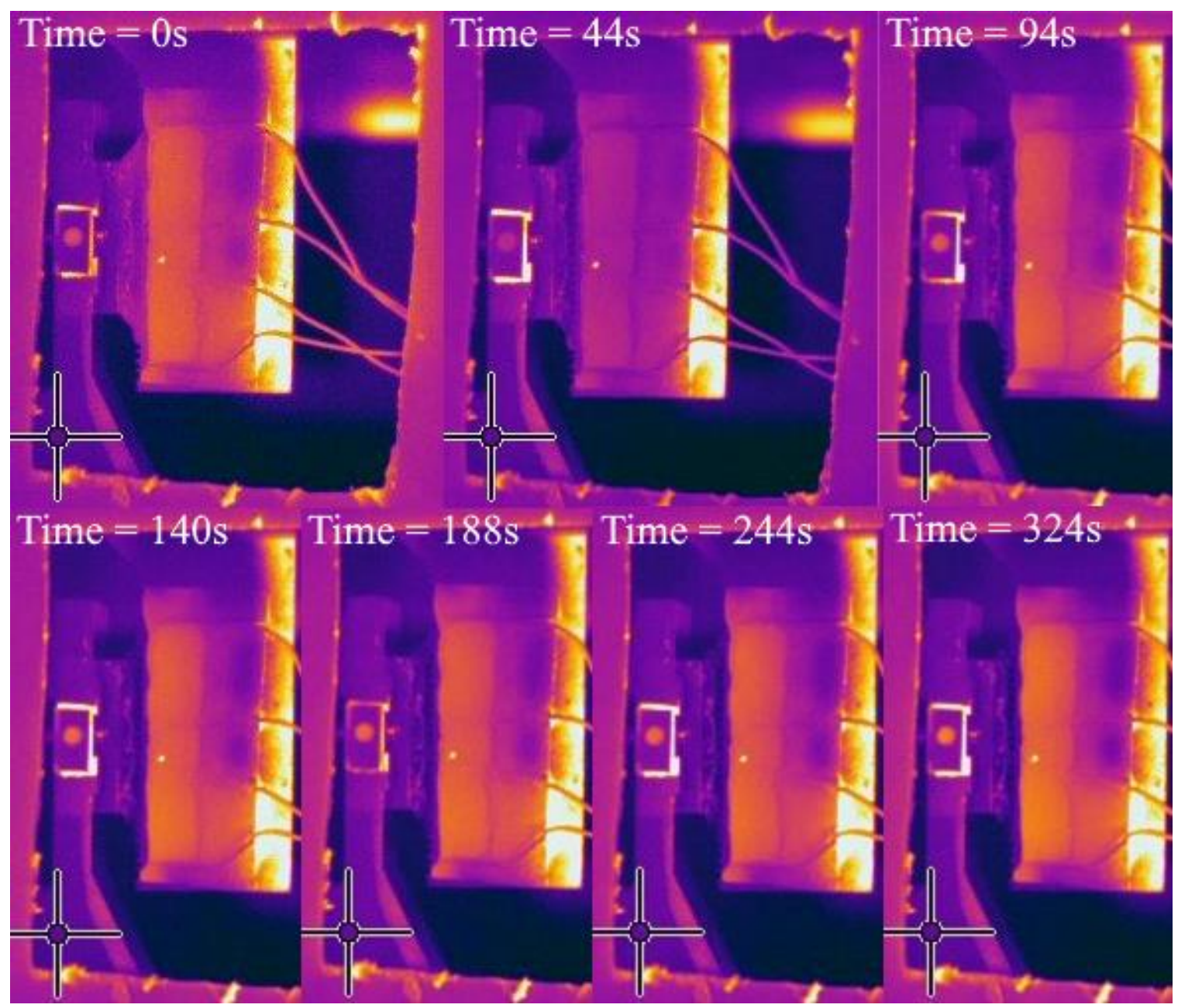

Figure 59. Thermal images of ice melting, with time shown in top left of each frame.

Several issues were observed in the imaging. Spurious temperatures were observed, possibly because the spray paint and tape were not thick enough to fully absorb radiation from the aluminum bodies they were applied to. Evidence can be seen in the tape on the coupon block, the small rectangle with the bright circle in the middle - where the circle outlines a threaded hole in the block. Separate temperature readings indicated that the block temperatures were warmer than the air temperature. While flat black paint was used, it was possible that reflections were visible on the paint or signal was transmitted through the paint. Regardless, the ice showed visible heating in the first frame (time $=0 \mathrm{~s}$ ), and returned to near equilibrium prior to $140 \mathrm{~s}$. To measure the temperature more directly, several 
surface mount probes were mounted to the teeth of the serrated block before continuing testing on the remaining samples from the second test. These probes are present in the image of Figure 32. Two example sets of data from these probes are shown in Figure 60.
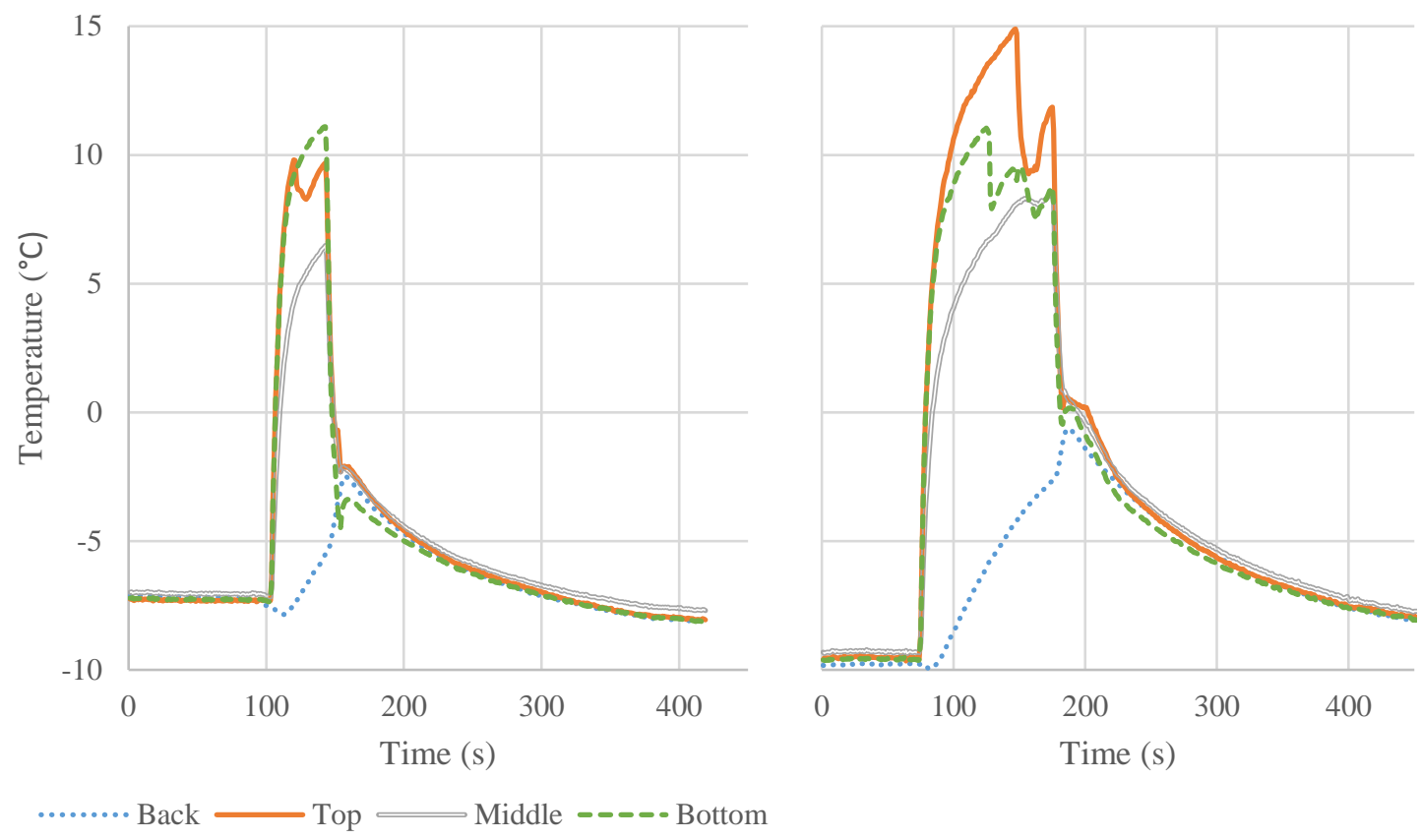

Figure 60. Temperature history for two samples during shear test. Left: sample K23 (40s heat, 10s cool). Right: sample M15 (101s heat, 7s cool). Shared vertical axis.

The average required heating time prior to the installation of the thermocouples was $24.8 \mathrm{~s}$, while the average heating time after the installation rose to $65.9 \mathrm{~s}$ after the installation of the thermocouples, showing that they increased thermal resistance by a significant margin. Higher heating times resulted in longer required equilibrium times. The process was modified with the probes in place to start the test once a set temperature was passed. These equilibrium temperatures were between -6.8 and $-7.8 \mathrm{C}$. Warmer temperatures were used earlier in testing before it was discovered that the warmer equilibrium temperature was due to the cyclical opening of the glove ports in the chamber. The process was modified to keep the ports closed more frequently, and colder temperatures were used to start the test. This was recorded where applicable in the comments for the data set. 
The temperature plots also show that the middle probe typically registered cooler temperatures during heating than the other probes. This was due to the presence of a strut connecting the front plate to the main block, allowing heat transfer between the teeth and the block. The bottom temperature also was lower than the top since it was connected more directly to the rail, giving lower thermal resistance between the body of the block and the teeth than at the top probe.

Several side effects likely were present from increasing the heating time of the block. The selection of aluminum for the serrated block was made due to cost, however using aluminum for the teeth was likely problematic since aluminum has a relatively high coefficient of thermal expansion, and when the teeth cool they likely compress the ice along the vertical axis. The possibility for this to not occur was if the water at the teeth did not refreeze prior to the teeth reaching equilibrium. The water freezing to hold the ice to the serrated block was observed to lag behind the temperature of the teeth since the loading reported from the load cell was nearly zero until the ice froze to lock the serrated block in place. Once locked in place, the sample would load due to thermal contraction of the fixture. Increasing the heating increased this larger contraction and slowed the cooling process once the teeth temperature dropped below $0{ }^{\circ} \mathrm{C}$, as can be seen in Figure 60 . Slowing this likely increased the clamping on the ice since the ice was likely locked in place for a larger teeth-temperature shift.

The tensile tester was generally run in the position control mode, where the position of the crosshead was controlled directly. Drift was observed in the data in the first data set but was low compared to the values observed in the second data set. The time history of the force plot for three selected samples is shown in Figure 61. 


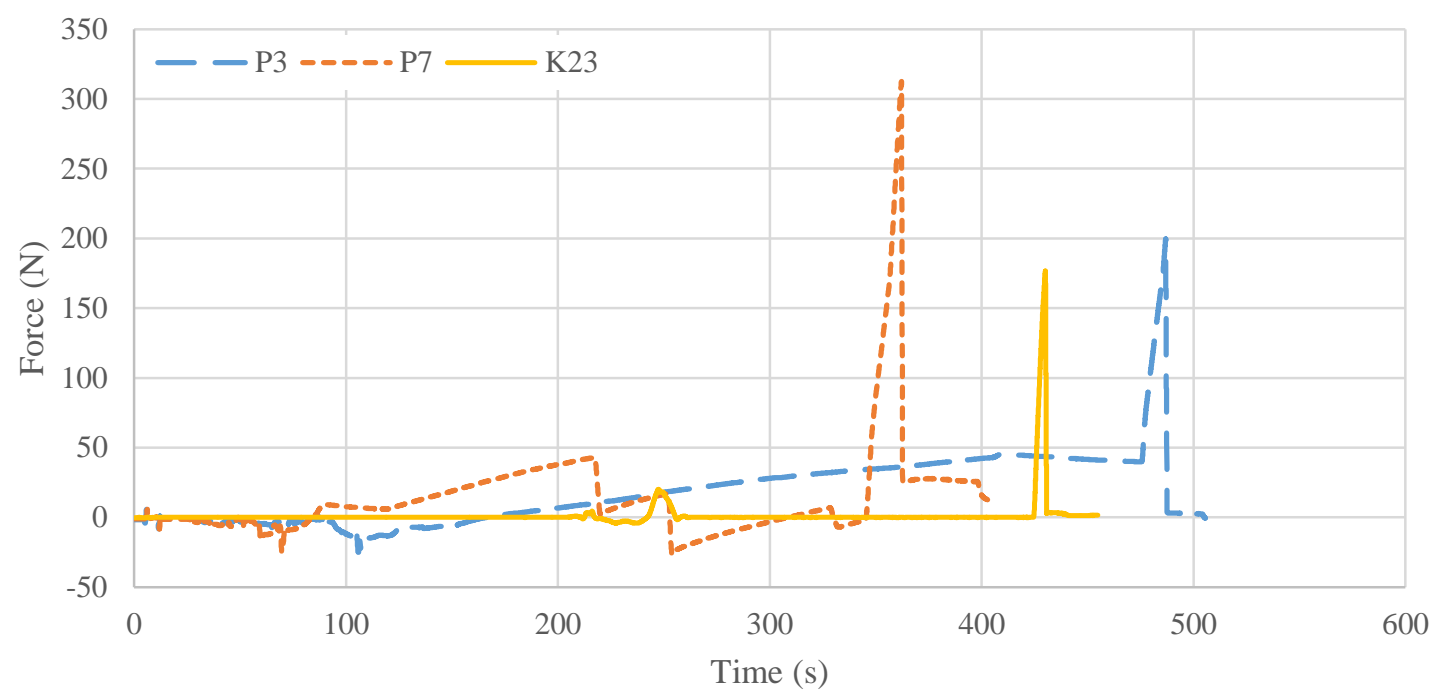

Figure 61. Force-time plot for selected samples during shear test, raw data.

Several important observations from the force-time plots are that drift was observed in the test prior to loading, likely due to thermal contraction, and some samples possessed frictional-like loading post-break. The P-series samples shown did not use force control. P3 was tested first, where the pre-load was approximately $25 \%$ of the total load. In P7, the crosshead was manually controlled to reduce the load - this shows up as sharp, nearly linear decreases in the force as the crosshead was moved. With K23, force control was used. The regions with erratic force (before $\sim 120$ s in P3, 90s in P7, and 260s in K23) show the forces during sample loading, melting, and refreezing. The P-series tests showed slowly increasing force after the sample refroze, while K23 did not. Different behavior in this region was observed across tests, where some cases without force control showed similar trends to K23 - a linear, near zero force while the sample was equilibrating. The lack of thermal expansion in some instances was possibly to be due to slipping in the couponcoupon block connection, and is discussed in the following sections. Many samples would show a constant approximately zero force after handling was finished in the mounting process. This varied with mounting procedure, where if the upper fixture was allowed to 
relax with the teeth still warm the force would go to zero with hands off of the fixture. In most cases, the operator pushed the serrated block into the ice, deflecting the extension rods to put force on the teeth and ice to accelerate the sinking process. If this was the case, the zero force was not observed, and the force also did not drop to near-zero post-break. This was observed in the data for sample P7 where the post-break force hovered around 25 $\mathrm{N}$ while the crosshead was in motion. The drop at the end of the P7 curve was when the crosshead was stopped right before data collection ended. Samples P3 and K23 were probably not put under lasting compression as a result of the melting and refreezing process. Allowing the fixture to relax prior to the test prevented compression of the sample along the horizontal axis and indicated the presence of water around the teeth since a direct mechanical connection would have resulted in a non-zero force. This also showed that the liquid water near the teeth could reduce the effect of the contraction of the fixture along the vertical axis until solidification took place, showing a potential method for reducing the effect of local contraction of the teeth on the ice. A schematic depicting the theorized geometry of these two scenarios is depicted in Figure 62.

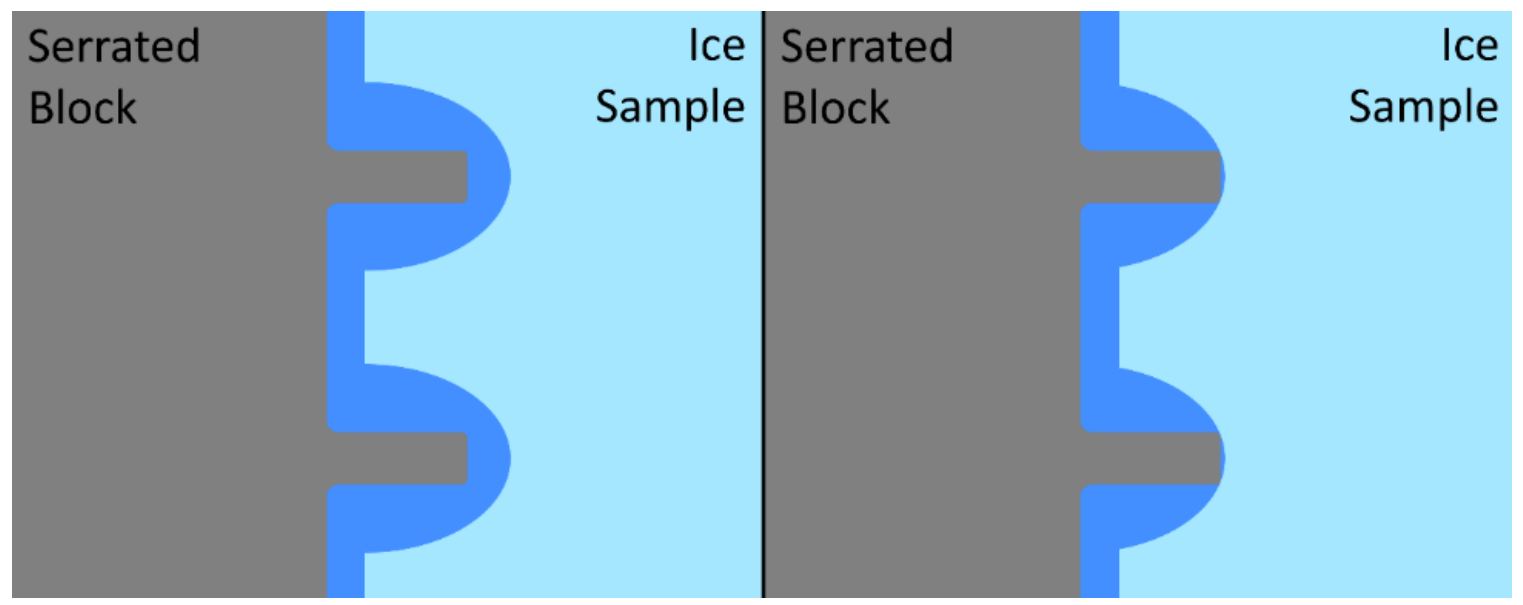

Figure 62. Depiction of idealized ice-serrated block interface. Left: interface not under compression. Right: interface under compression. Teeth to scale, water layer not to scale. 
When the interface was not under compression and a liquid boundary layer was maintained, the relative motion between the serrated block and ice sample did not load the sample. If the sample was under compression, the large-scale liquid layer could not be maintained, and relative motion loaded the sample. The method that the operator used to attach the ice to the serrated block could not be tracked for these tests. To minimize loading of the sample, a repeatable method should be used to produce minimal compressive forces and a repeatable liquid layer. Minimizing the time required to cool the serrated block back to equilibrium temperature would also produce better results. The teeth should be thermally isolated from the body of the block to reduce heat loss and increase temperature uniformity on the teeth, and probes should be embedded to decrease the time to sink the teeth into the ice. Copper would likely be a more suitable material for the teeth due to higher thermal conductivity, a lower coefficient of thermal expansion, and higher stiffness - which would also serve to reduce flexure of the teeth. Position data corresponding to the three tests shown in Figure 61 are shown in Figure 63. 


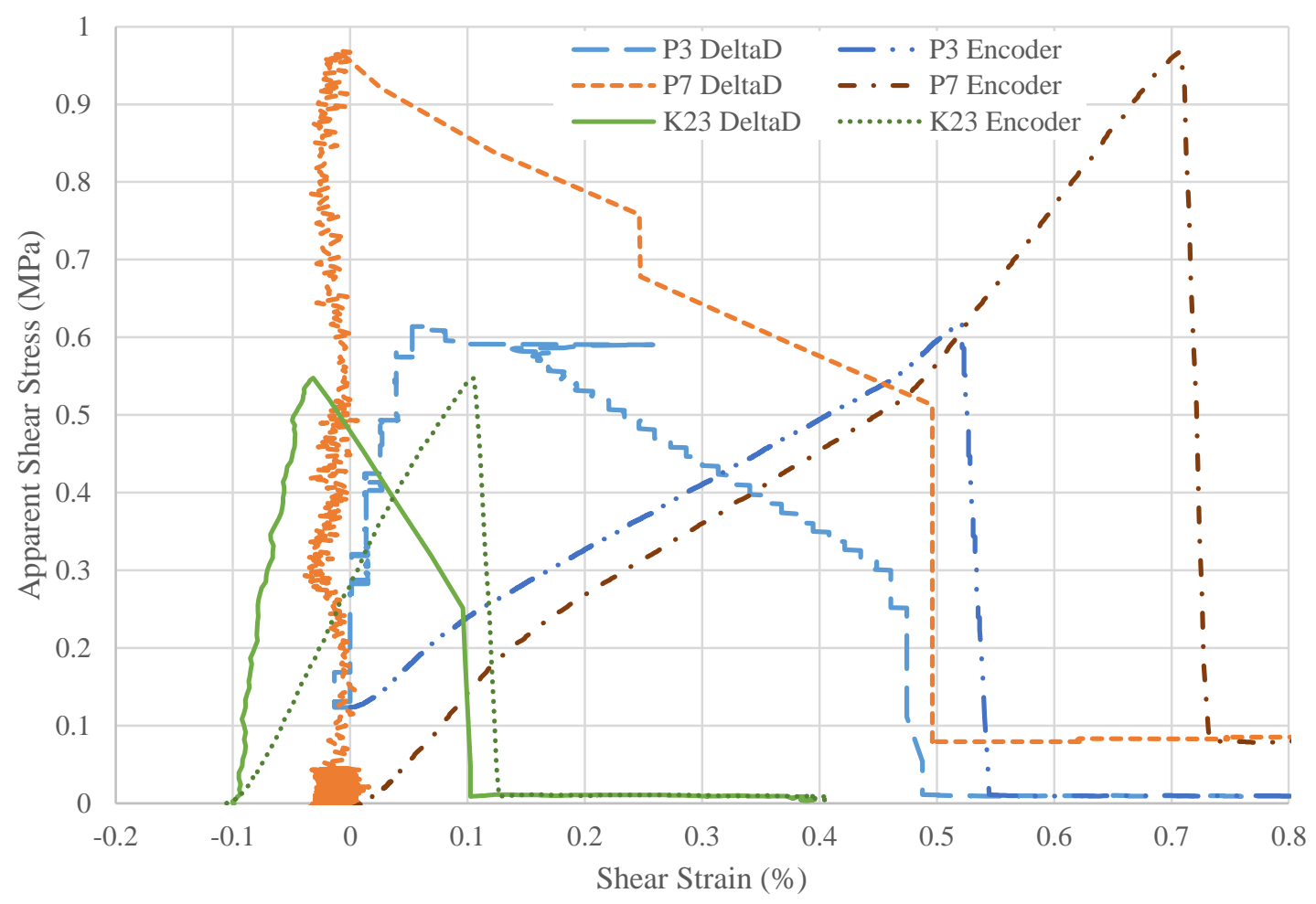

Figure 63. Delta deflectometer and encoder positions for selected shear tests.

The delta deflectometer (DeltaD) position data was calculated by taking the difference in the coupon and serrated block deflectometer readings. The data going off the charts shows when the deflectometer was being moved prior to the melting and refreezing process the $\mathrm{P} 3$ test, where other jumps in the data show the effects of handling during refreezing process. The segment of the encoder data with the linear increase shows the motion of the crosshead during the test, and the jump in the DeltaD data show when the break occurred. The difference in the two lines prior to the break would ideally capture the deflection of the rig, which was not accomplished in the P7 test since a flat line was obtained prior to the test. Before the test starts the encoder position for the K23 test shows the position change due to the force control, and the small difference between the encoder and delta deflectometer data showed that most of the thermal expansion was between the deflectometers. Some tests, such as P3, show a more significant difference in the two 
position sources, showing some expansion or contraction in the test fixture outside of the two rods. The stress-strain curves for sample K23 is shown in Figure 64.

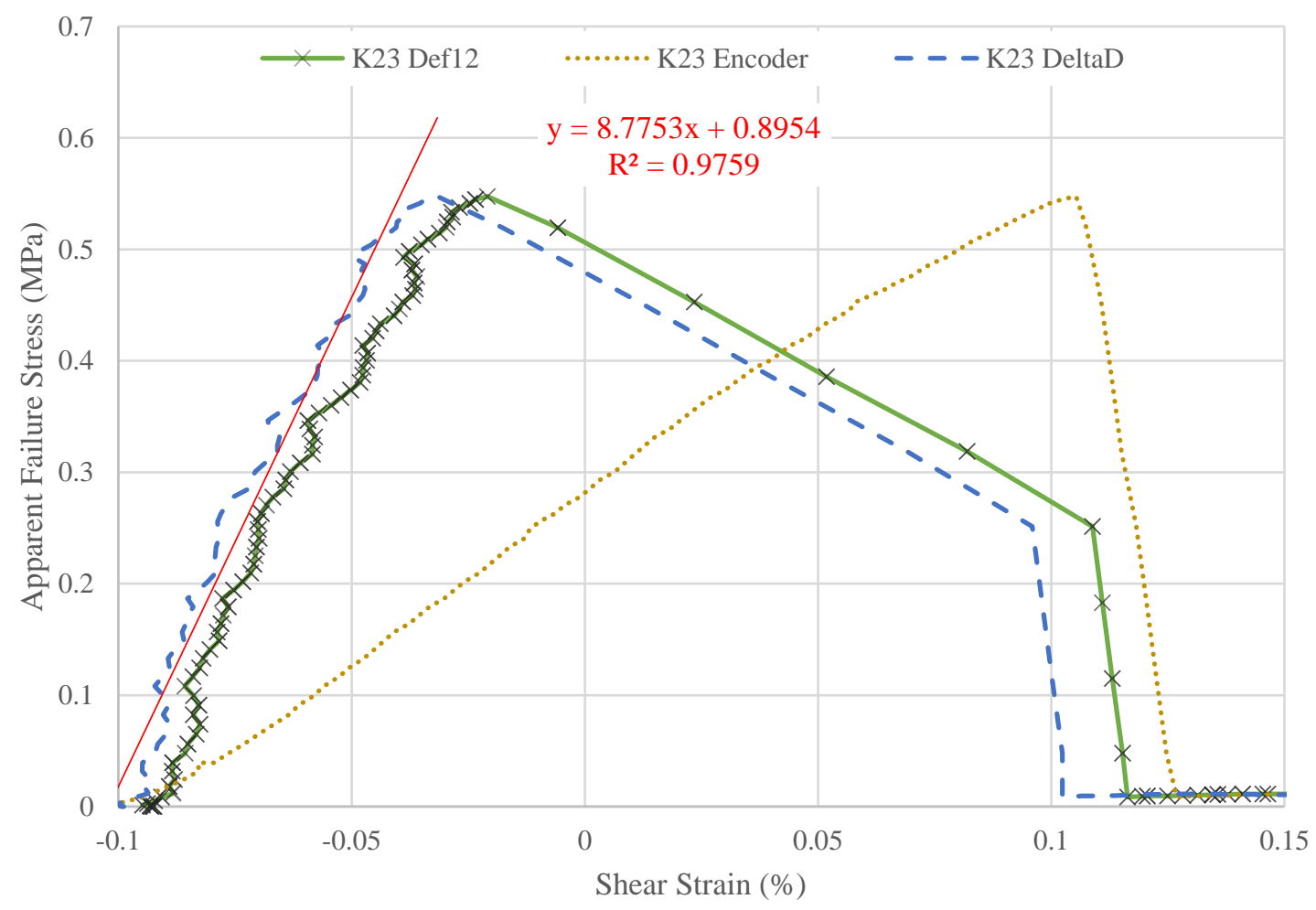

Figure 64. Stress-strain diagram using delta deflectometer data and encoder data.

The difference between the coupon deflectometer data (K23 Def12) was nearly a steady offset from the serrated block deflectometer. The encoder data follows a much shallower slope, showing that the fixture and extension rods flex substantially during the test, offsetting the apparent shear modulus. The first linear segment showing a drop in stress on the deflectometer data corresponds to the fixture relaxing post-break and occurred over approximately 0.37 seconds. The data from the deflectometers was particularly noisy, and in some cases the deflectometers got stuck and didn't track the sample (such as in the sample P7 shear test). The deflectometers still were removed from the sample, capturing the relative deflection of the serrated block and the coupon with respect to the lower rail. The shear modulus calculation was problematic for this reason, but for sample K23 the 
calculated shear modulus, G, was $0.88 \mathrm{MPa}$. This was high compared to most values calculated in Horizon, but only $24 \%$ of what was found in the literature ${ }^{42}$ - suggesting that flexure of the teeth, coupon, and serrated block collectively account for most of the deflection observed in the tests. Since better deflection data was not obtainable from the bulk of the tests, further consideration of the shear modulus was omitted from the analysis of the stress-strain diagrams obtained from Horizon. Further, the data system available exhibited issues where intermittently the maximum test speed was not available ${ }^{43}$ and some peculiar results were observed - such as in K23 stress-strain curve where on the downward slope immediately following sample break the data system recorded repeat values in groups of four.

No data sets from the first test had enough data points to reasonably perform more detailed statistical analysis from the first set of data. To compensate for this in the second IRT test entry, two repeat runs in the tunnel were run $(\mathrm{P}$ was a repeat of $\mathrm{O}$, and $\mathrm{U}$ was a repeat of S). Also, only one crosshead speed was run $(0.2 \mathrm{~mm} / \mathrm{min})$. Still, few data sets were available with enough samples for a statistical analysis. The $\mathrm{O}$ and $\mathrm{P}$ test conditions were identical, and combined series results contained 26 samples. Other sets of data with consistent run parameters only had 13 samples or less. A histogram of this set is shown in Figure 65.

\footnotetext{
${ }^{42}$ See Table 1.

${ }^{43}$ For K23, the system recorded at approximately $50 \mathrm{~Hz}$ even though settings specified $1 \mathrm{kHz}$ and were not changed between tests. Most tests recorded at the specified $1 \mathrm{kHz}$. This was most likely due to a malfunction of the equipment and was observed intermittently.
} 


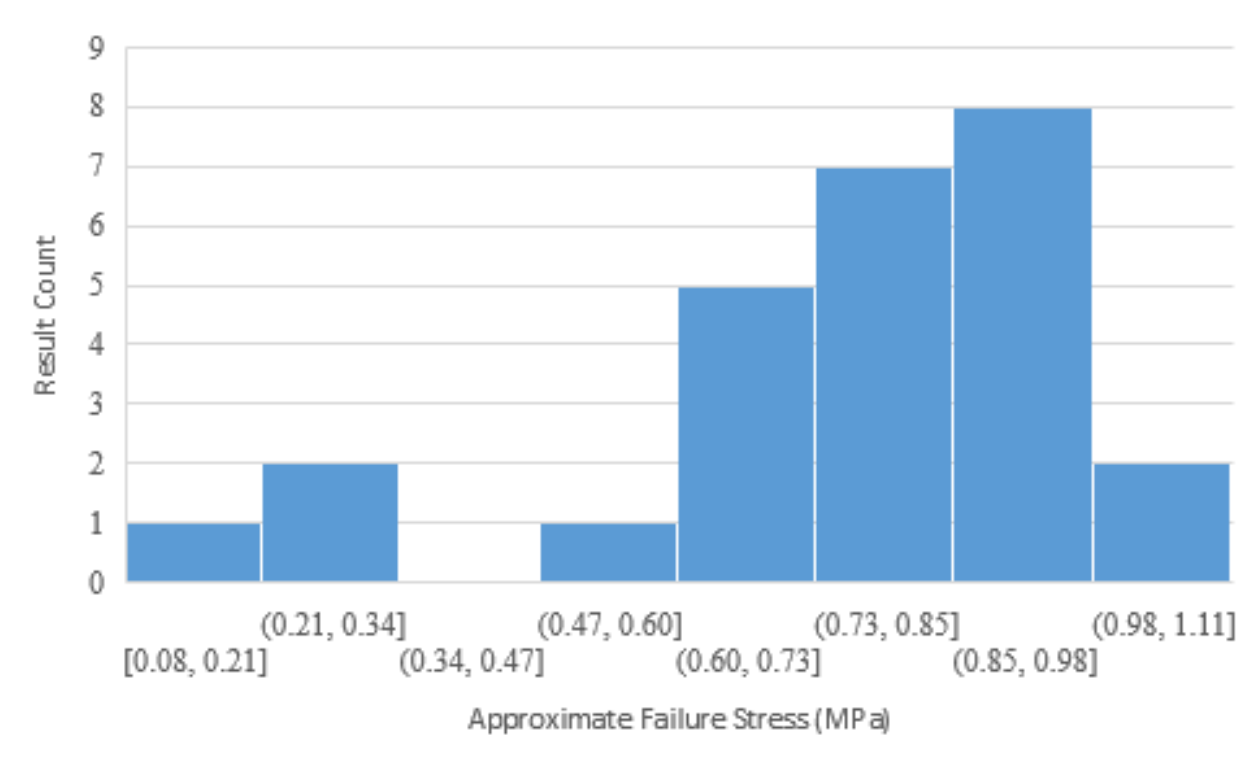

Figure 65. Histogram of results from $\mathrm{O}$ and $\mathrm{P}$ runs after being placed in storage for more than 1000 hours at $10^{\circ} \mathrm{C}$.

The lowest three values in the $\mathrm{O}$ and $\mathrm{P}$ data sets are likely erroneous. Something likely occurred during the test process to damage the samples, which was unaccounted for. Damaged in samples would almost certainly manifest as erroneously low values of adhesion strength, and so the strongest samples in the group should best represent actual values of adhesion strength assuming that the flaws are not inherent to the icing process. This was almost certainly not the case since samples were observed to be damaged in-situ but may serve as a reasonable approximation for most applications where damage is less likely to occur during the icing process. Samples from both $\mathrm{O}$ and $\mathrm{P}$ had moderate runback, as well as the $\mathrm{R}$ set of samples.

To compensate for this in the analysis, data from the second IRT test entry was reduced in several ways. Repeat data points were averaged, and a standard deviation was taken for the repeat points. The process was repeated removing low-end outliers from the data set in two manners, removing all data one standard deviation beneath the mean $(<\lambda$ $\sigma)$, and removing all data two standard deviations from the maximum value obtained 
$(<\operatorname{maximum}-2 \sigma)$. Maximum values were also compared. Data was grouped by the time to test with data taken in less than 5 hours after the test as the first group, data taken in more than 5 hours but less than 200 in the second group, and data taken in more than 800 hours as the third group. Data was not available at intermediate times due to downtime with the test stand and scheduling conflicts. The data is shown in Figure 66.
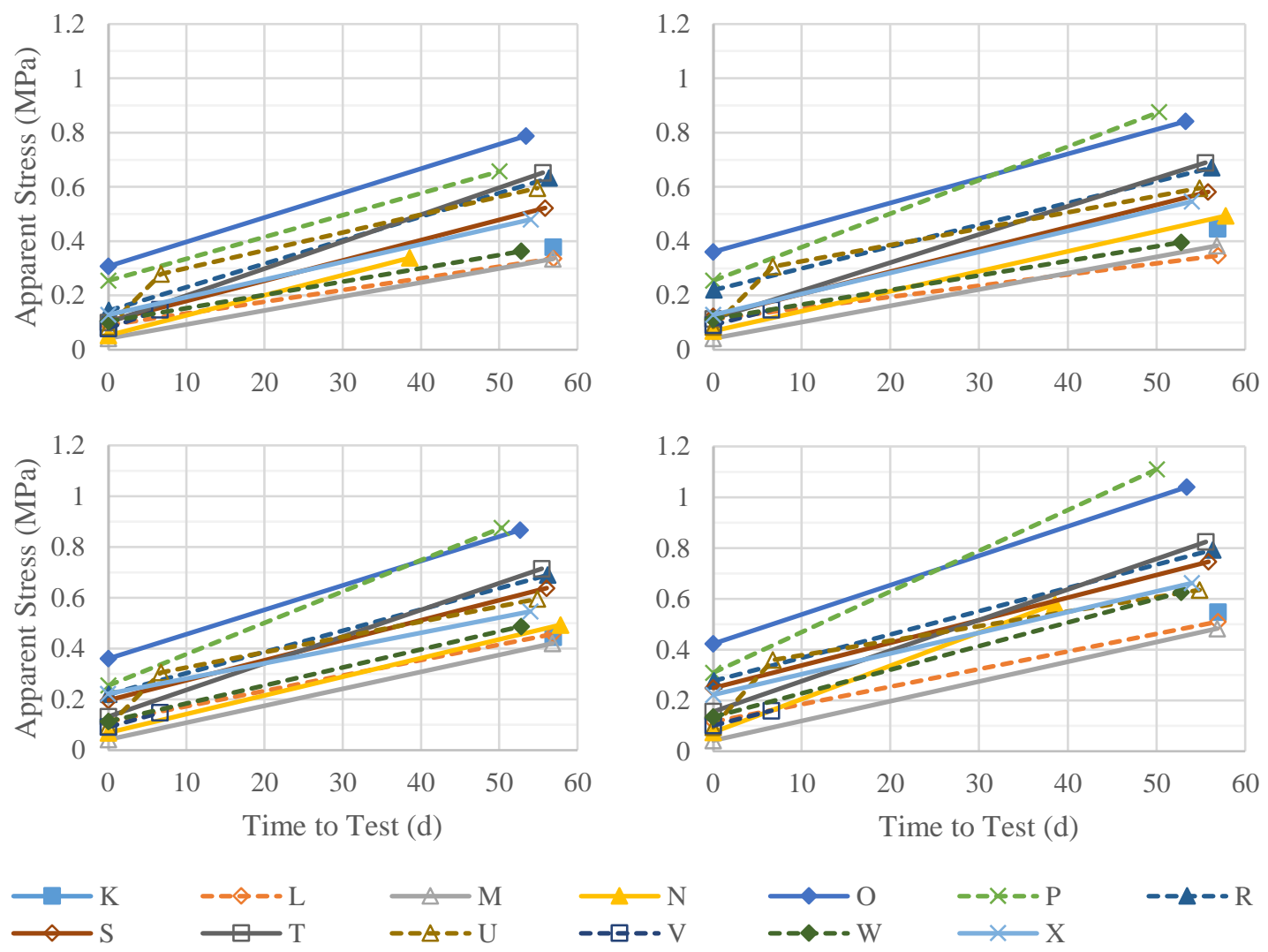

Figure 66. Strength vs. time. Top left: averaged raw data. Top right: averaged data sans outliers $(<\lambda-\sigma)$. Bottom left: averaged data sans outliers (<maximum-2 $\sigma)$. Bottom right: maximum values.

The data increases in magnitude as more low-end values are excluded from the average. Excluding values more than two standard deviations below the maximum value almost always excluded as many or more values than excluding values more than one standard deviation below the average. Low-end outliers shifted the data considerably in some cases to cross trends. Removing outliers results in $\mathrm{O}$ and $\mathrm{P}$ having increasingly close 
values (top two lines in Figure 66) until only the maximum values are taken. The full data set grouped and averaged by annealing time is shown in Figure 67.

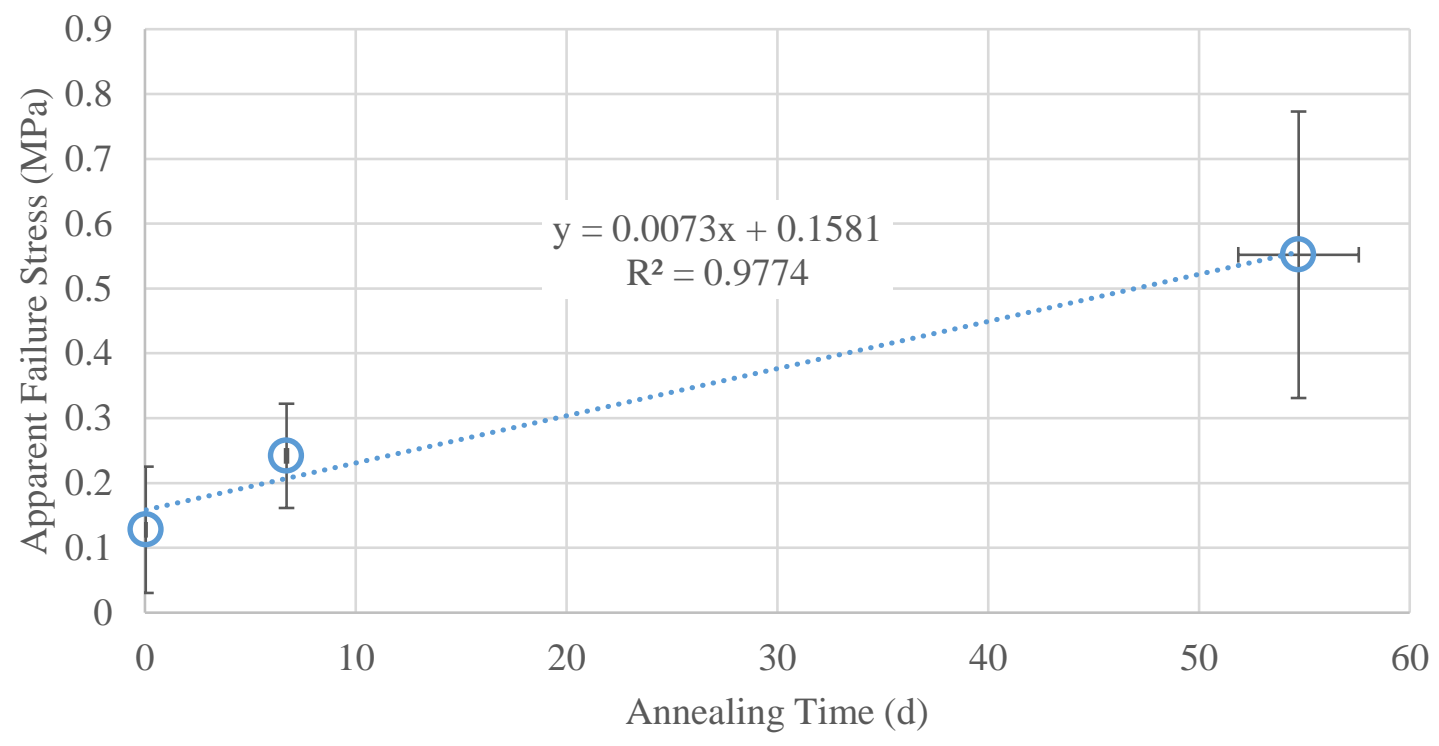

Figure 67. Apparent failure stress of all data with no outliers removed grouped by annealing time.

The trend of increasing adhesion strength with storage time was universal, in comparison with data from the first IRT test entry where several runs had opposite or weak trends. Both showed similar increasing trends, with the data from the first test having a slope of $0.0104 \mathrm{MPa} / \mathrm{day}$, while the data from the second test had a lower slope of 0.0073 $\mathrm{MPa} /$ day. From series $\mathrm{U}$, it appeared that the bulk of the change in adhesion strength happened within the first 200 hours of storage, and that the data likely reaches a plateau at longer intervals. Standard deviation values for each method of reducing the data are shown in Table 11. 
Table 11. Standard deviations of data from IRT test entry 2.

\begin{tabular}{|c|c|c|c|c|c|}
\hline Test & $\lambda_{\text {time }}(\mathrm{h})$ & Count & $\sum_{\text {raw }}(\%)$ & $\sum_{\lambda-\sigma}(\%)$ & $\sum_{\mathrm{m}-\mathrm{\sigma}}(\%)$ \\
\hline $\mathrm{L}$ & 2.22 & 3 & 33.8 & 3.1 & 3.1 \\
\hline $\mathrm{M}$ & 0.74 & 1 & & & \\
\hline $\mathrm{N}$ & 0.85 & 3 & 43.8 & 8.4 & 8.4 \\
\hline $\mathrm{O}$ & 0.87 & 5 & 39.1 & 17.4 & 17.4 \\
\hline$P$ & 0.64 & 2 & 21.4 & 21.4 & 21.4 \\
\hline $\mathrm{R}$ & 2.51 & 5 & 72.6 & 28.2 & 28.2 \\
\hline$S$ & 1.07 & 6 & 72.8 & 56.6 & 26.7 \\
\hline $\mathrm{T}$ & 1.14 & 5 & 41.6 & 30.8 & 13.9 \\
\hline $\mathrm{U}$ & 0.78 & 3 & 8.8 & 8.8 & \\
\hline $\mathrm{V}$ & 0.85 & 4 & 29.9 & 13.7 & 13.7 \\
\hline $\mathrm{W}$ & 0.64 & 5 & 21.2 & 13.1 & 13.1 \\
\hline $\mathrm{X}$ & 0.65 & 5 & 37.2 & 37.2 & \\
\hline $\mathrm{U}$ & 162.21 & 5 & 22.6 & 13 & 13 \\
\hline V & 158.64 & 2 & 8.5 & 8.5 & 8.5 \\
\hline $\mathrm{K}$ & 1366.07 & 9 & 29.4 & 15 & 15 \\
\hline $\mathrm{L}$ & 1366.44 & 10 & 21.1 & 19.3 & 11.6 \\
\hline$M$ & 1364.39 & 6 & 27.6 & 19.7 & 10.9 \\
\hline $\mathrm{N}$ & 925.62 & 3 & 68 & 17.5 & 17.5 \\
\hline $\mathrm{O}$ & 1281.72 & 13 & 15.8 & 9.9 & 8.2 \\
\hline $\mathrm{P}$ & 1200.91 & 15 & 51.3 & 17.6 & 17.6 \\
\hline $\mathrm{R}$ & 1352.69 & 11 & 17.6 & 12.3 & 9.7 \\
\hline$S$ & 1340.81 & 11 & 33.6 & 23.2 & 14.7 \\
\hline $\mathrm{T}$ & 1333.63 & 13 & 17.8 & 11.6 & 9.2 \\
\hline $\mathrm{U}$ & 1316.71 & 2 & 6.6 & 6.6 & 6.6 \\
\hline $\mathrm{W}$ & 1267.39 & 8 & 39.8 & 30.9 & 16.6 \\
\hline$X$ & 1296.23 & 13 & 29.8 & 15.5 & 15.5 \\
\hline Average & & 6.46 & 32.47 & 18.37 & 13.94 \\
\hline
\end{tabular}

The values of standard deviation for the raw data rose $44 \%$ to $32.5 \%$ from the first test, which was likely due to several factors. Samples in the second test were obviously damaged in-situ with partial delamination occurring, and thicknesses in the second test were more inconsistent, but also much larger on average. Larger data sets were used in the second test which may better represent the scatter inherent to the problem. To determine whether the samples were done aging, the individual data points used for the last groups 
were plotted in tests (with no outliers removed) with trend lines fit. Only series with data points spanning multiple days of testing were used; this is shown in Figure 68.

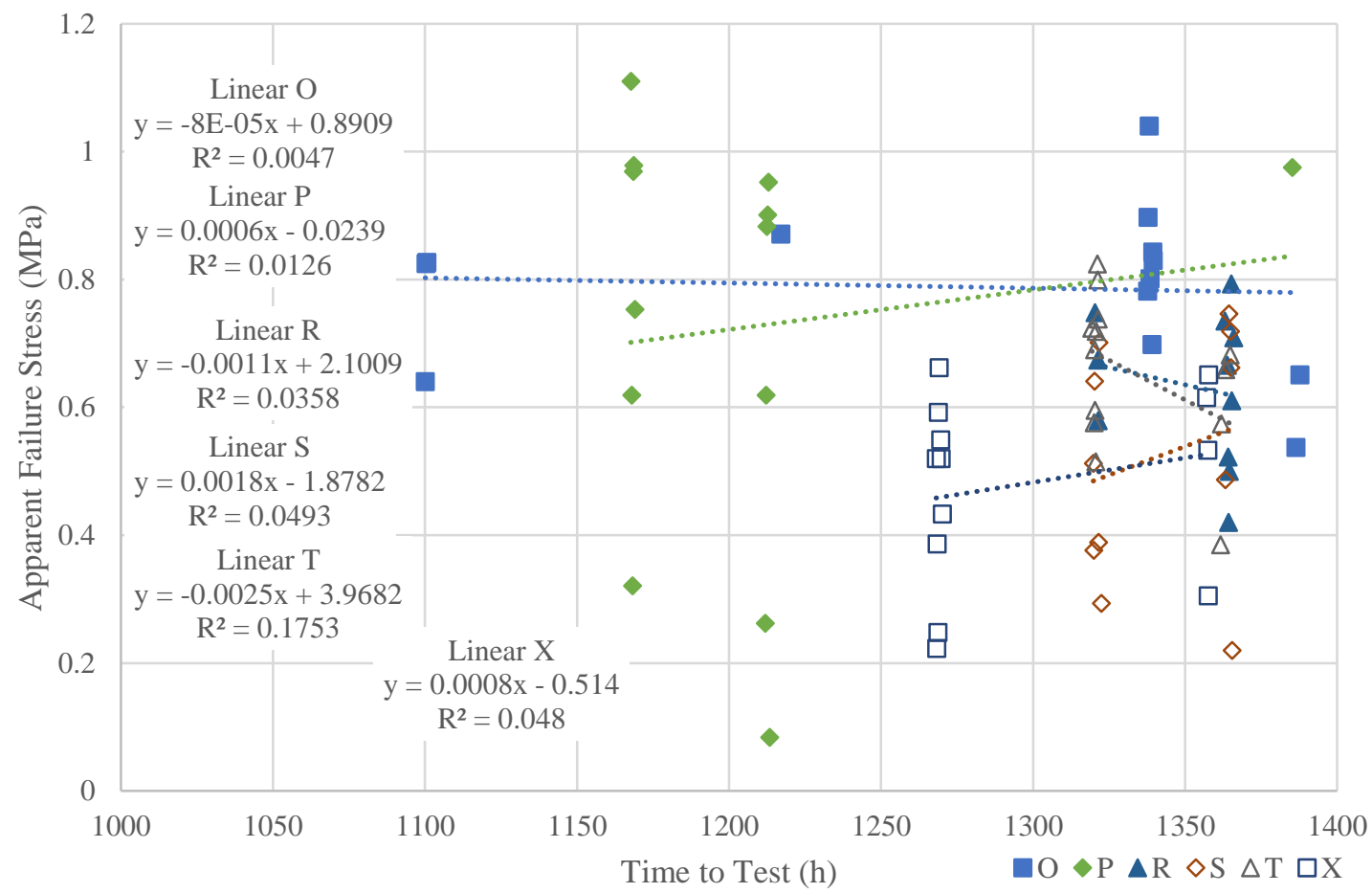

Figure 68. Longest stored data points with trend lines from IRT test entry 2.

The trend lines all have very low slope (and poor fit), but the ones with the longest span in test time have the flattest trends. This leads to the conclusion that samples were not significantly changing while aging after 1000 hours of storage. The data tested the same night of the test is shown in Figure 69. 


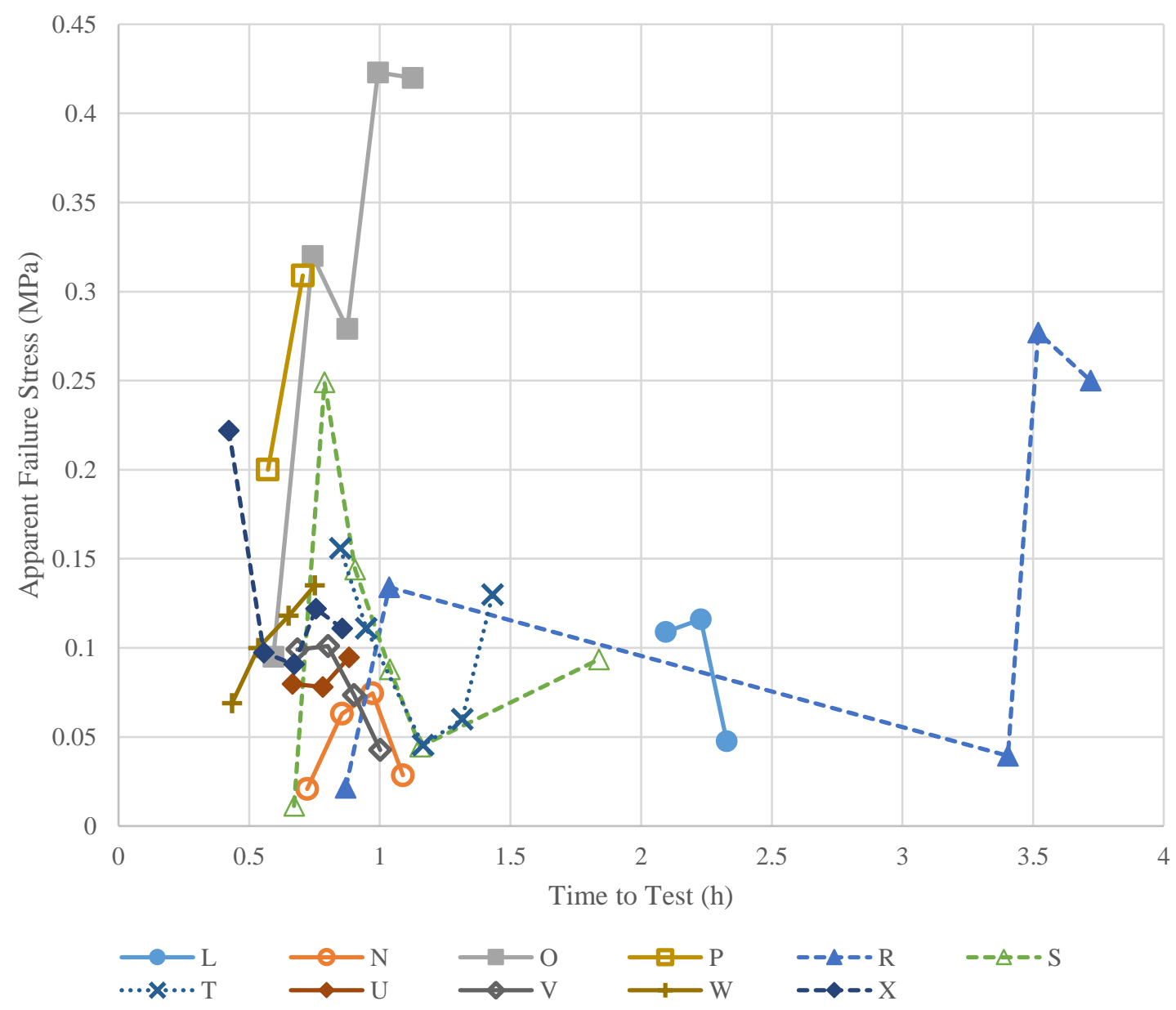

Figure 69. Data taken during the night of the IRT tests for IRT test entry 2.

Weak trends were seen in the earliest data, while many series appear to have an upward trend, some evidently have a downward trend. The long-term trend was likely present in the short-term data but buried by noise in the test. A parametric plot of the averaged data removing outliers more than one standard deviation below the mean is shown in Figure 70. 


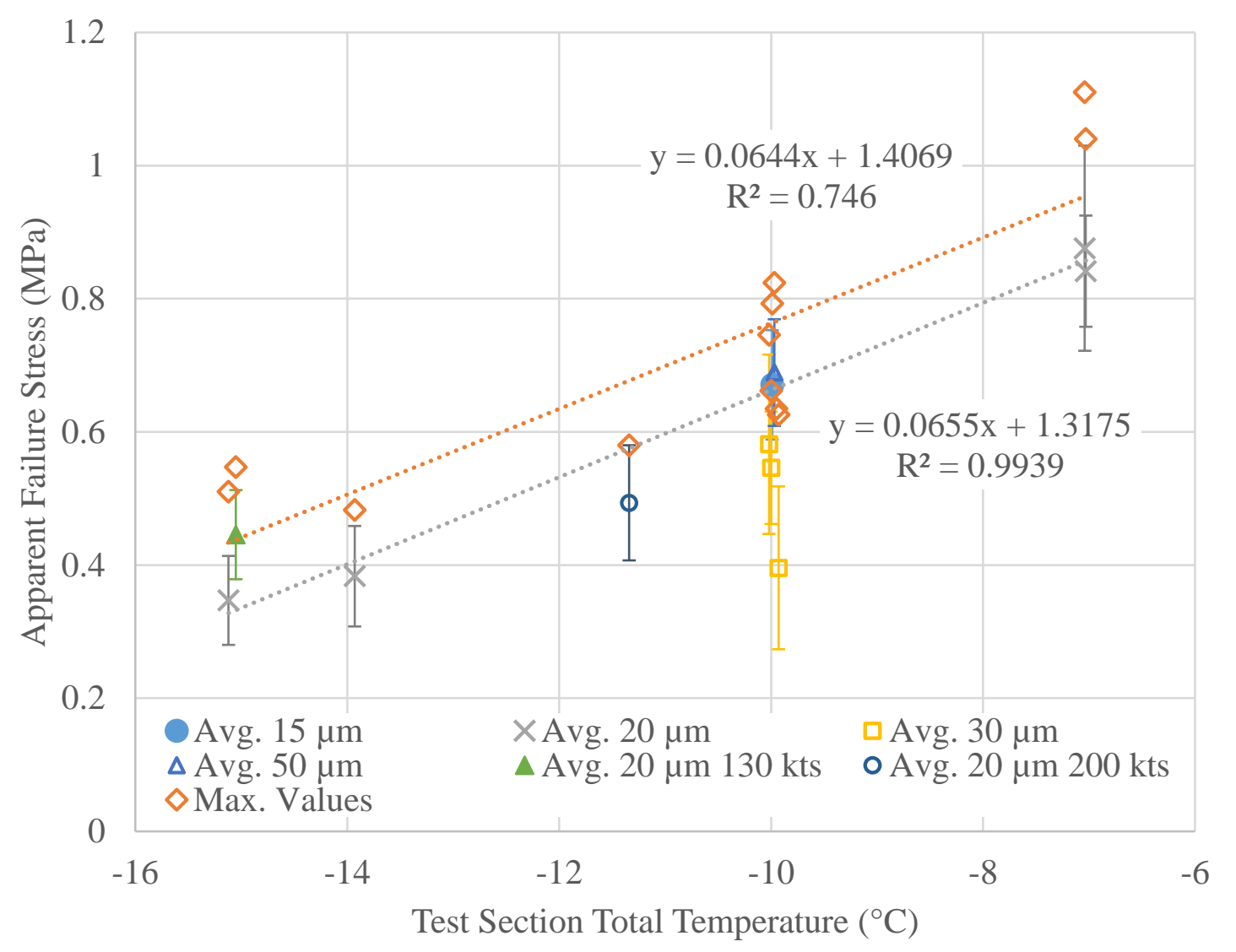

Figure 70. Parametric analysis of IRT test entry 2 data, using data $>\lambda-\sigma$ for averaged results. Long-term storage results.

Data with less than 900 hours of storage time was excluded from parametric analysis, as was data with less than 4 repeats (except from the maximum values data set). The original intent was to run an MVD sweep and a velocity sweep, but a temperature sweep was performed to help troubleshoot the problem of samples falling from coupons. The data showed a strong correlation with the test section temperature, possibly due to increased preliminary delamination. The highest points were from tests $\mathrm{O}$ and $\mathrm{P}$, which exhibited moderate runback and likely artificially raised the observed adhesion strength and also probably prevented the possibility for preliminary delamination. The $\mathrm{R}$ run also had runback, which was the solitary $15 \mu \mathrm{m}$ data point. Too few points were taken to obtain a strong correlation from velocity, but the singular 130 knot test point was above the 150 
knot trend, and the singular 200 knot test point was below the 150 knot trend suggesting a downward trend with increasing velocity. MVD appeared to have no effect on the data since the single $15 \mu \mathrm{m}$ and $50 \mu \mathrm{m}$ test points fell within a standard deviation of the $20 \mu \mathrm{m}$ trend, which was consistent with the data from the first IRT test. The data from samples stored for less than 900 hours (nominally on the same night as the corresponding IRT tests) is shown in Figure 71.

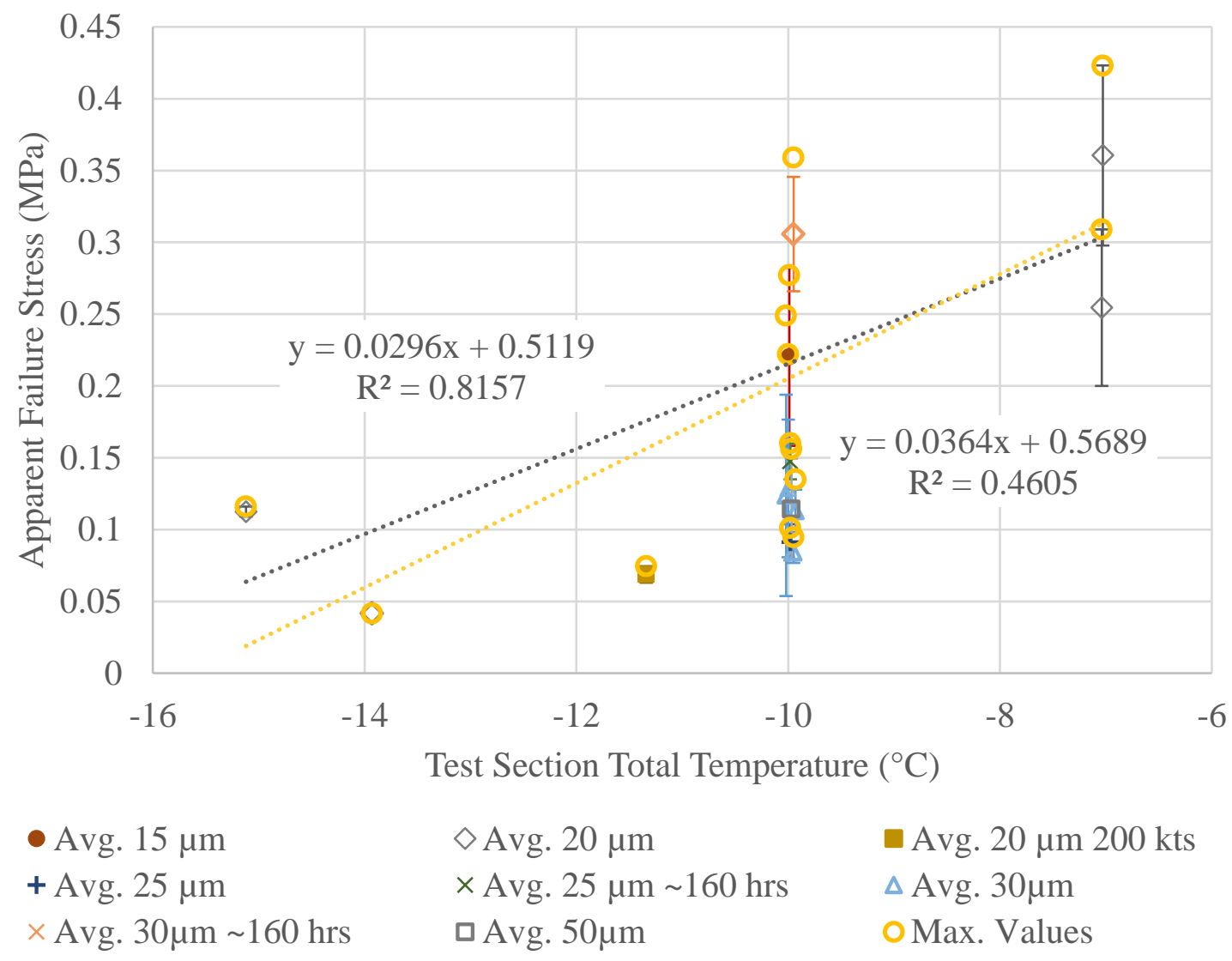

Figure 71. Parametric analysis of IRT test entry 2 data, using data $>\lambda-\sigma$ for averaged results. Short-term storage results.

The same trends present in the long-term storage data are present in the short-term data. The one case with enough data points for a trend was the $20 \mu \mathrm{m}$ MVD case, which showed a weaker trend with a lower slope than the long-term storage equivalent data. The maximum values showed a similar trend, also with a smaller slope than the long-term data. 
Both trends were poorer fits than the long-term data fits as well. The $30 \mu \mathrm{m}$ MVD data ranged from lower to higher values of shear stress at failure from the $\mathrm{U}, \mathrm{W}, \mathrm{S}$, and $\mathrm{X}$ cases, respectively. $\mathrm{W}$ and $\mathrm{X}$ were pulsed cases, with $\mathrm{X}$ possessing a smaller pulse time. Ice in both W and X IRT runs were notably more difficult to remove from coupons while they were still mounted in the IRT test section but fell very close to the values from the $S$ run in initial testing. Standard deviations covered a much larger range in the early set of tests, with the warmer temperature data exhibiting higher scatter. The trend obtained in the data was the opposite of what was expected from the literature, not accounting for residual stresses in the ice. The difference was thought to be due to the difference in geometry used for the test, where the flat geometry used in this sample possibly resulted in samples delaminating partially from the edges. In the data in the literature where an opposite trend was seen for impact ice, curved airfoil surfaces were generally used. The difference in geometry and the residual stresses theorized would cause pealing in these tests and clamping in those showing an opposite trend, explaining the difference. Finally, the effect of sample thickness on the adhesion strength was investigated; the data used in this analysis is shown in Figure 72. 

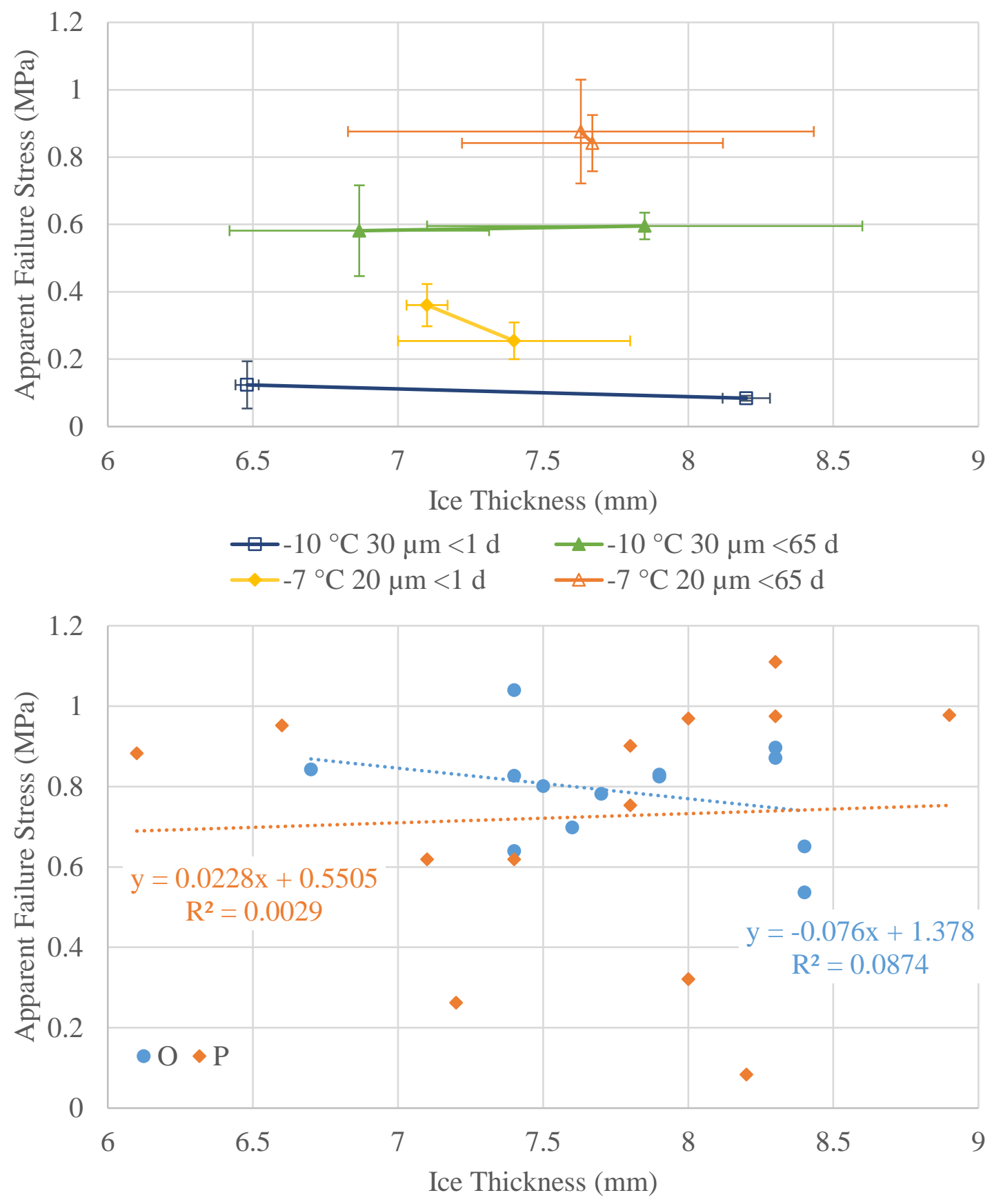

Figure 72. Failure stress vs. sample ice thickness. Top: four conditions with multiple data points. Bottom: Series $\mathrm{O}$ and $\mathrm{P}\left(-7^{\circ} \mathrm{C}, 20 \mu \mathrm{m},<65 \mathrm{~d}\right)$ individual repeats.

Thicker samples should experience a lower shear rate at a fixed crosshead speed, and a fixed crosshead speed of $0.2 \mathrm{~mm} / \mathrm{min}$ was used for all samples in processing the results from the second IRT test entry. The data shows little effect due to the increase in 
thickness, except a slight downward trend. This wasn't universal, and the trend was likely small compared to the noise in the test. From the literature, a lower strain rate should result in lower adhesion, but the effect should be observable only on the scale of orders of magnitude difference. Further, the change in geometry resulted in different stress concentrations, an effect which was not accounted for which could show a false trend. The slight downward trend may also have been caused by variable partial delamination, where thicker samples were more likely to delaminate further resulting in a false trend in the data.

\section{Third IRT Test Entry Data and Results}

The third IRT test entry was conducted similarly to the first two, with the XT model in the center-mount configuration, and with the same coupons. The same issues were observed in this test as were in the previous two: the model produced audible tones, and the ice samples would fall off of the coupons while handling them as in the second test. This was unexpected since the third test was run at warmer temperatures than the second test. In the first run, AA, the total temperature was $-5^{\circ} \mathrm{C}$. Samples did not fall off at this temperature, and were robust during handling, but runback was a problem. The temperature was changed to $-8{ }^{\circ} \mathrm{C}$ to produce samples without runback, and to avoid the issue of samples delaminating as in the second test. The XT model after the run AB spray is shown in Figure 73. 


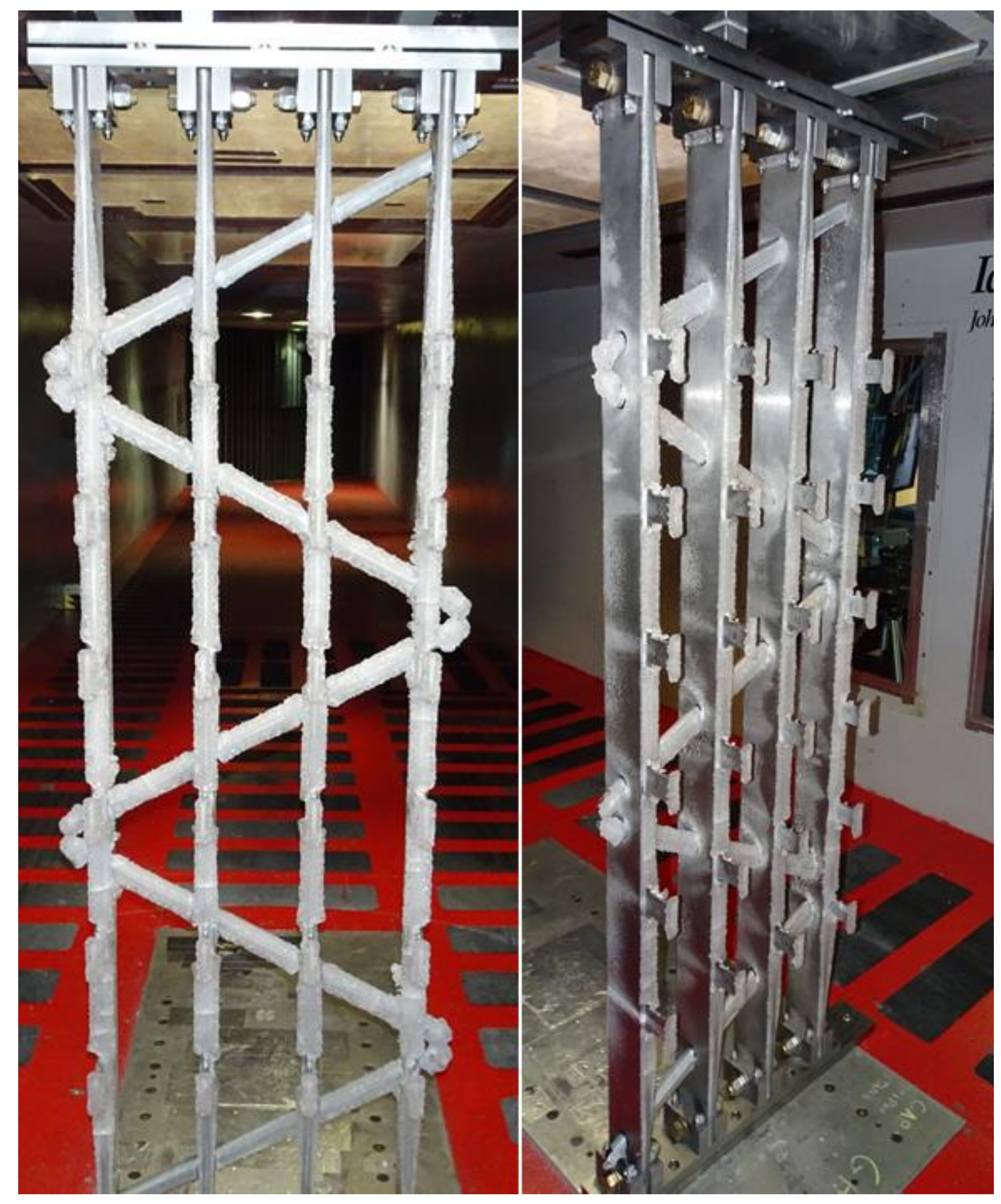

Figure 73. XT model in tunnel after run AB, a glaze condition.

As in previous tests, no damage was observed on the model. The ice showed a number of cracks between the sample mounts which are visible in the left image in Figure 73. The location of these cracks appeared to be random and were possibly caused by 
aerodynamically induced vibration or residual stresses in the ice. The last run for the series of tests was a rime condition, shown in Figure 74.

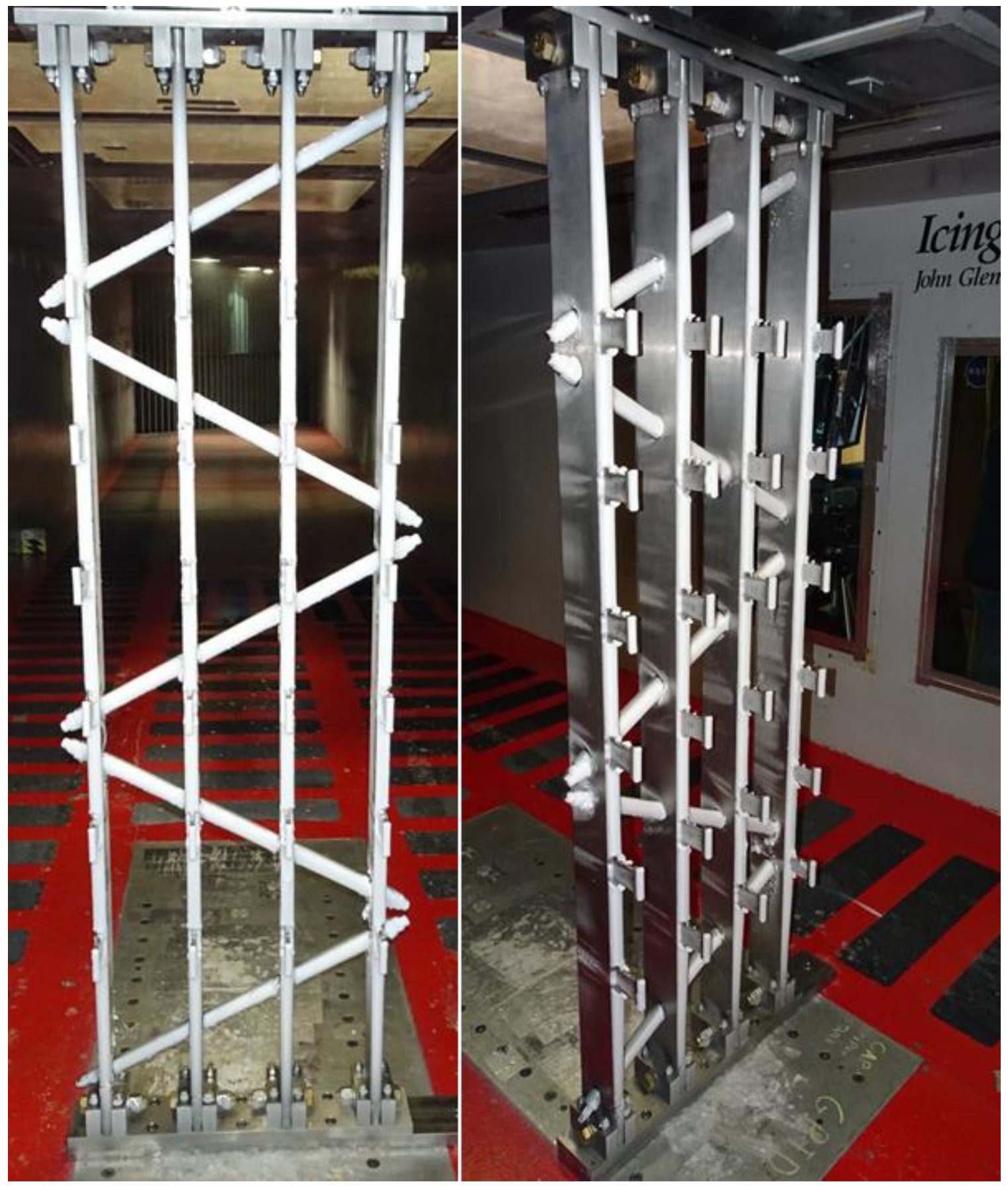

Figure 74. XT model in test section after run $\mathrm{AJ}$, a rime condition.

Disturbances in the flow downstream of the mounts were made visible by the accretion of the ice on the vertical rails. Ice attached to the instrumented dummy-coupons 
in positions 4,20 , and 24 , were removed manually. The ice was noted to be much stronger and the samples were robust during handling. No marks were observed on the back of the ice suggesting preliminary partial delamination. The position of the samples on the rig also influenced the shape of the ice. Interior samples (on the middle two rails) tended to be more symmetric, while the outer rails tended to tilt inward. This was observed during all three tests, suggesting test-section blockage was a factor in the growth of the ice. Top-down images taken during three runs are shown in Figure 75.

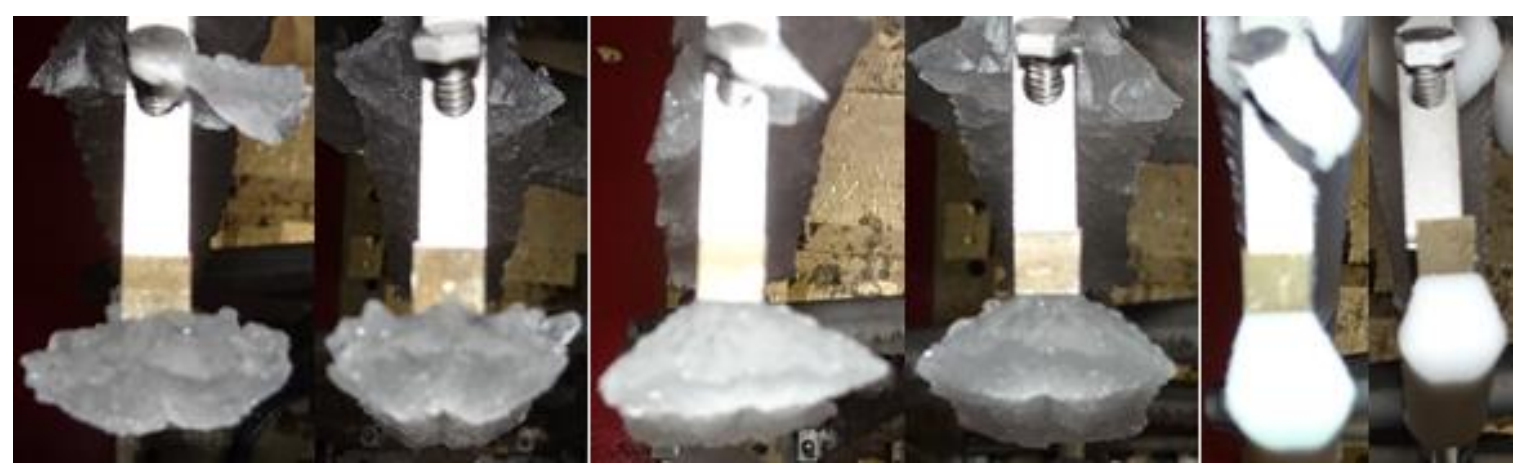

Figure 75. Top down view of samples from three runs. From left to right: $A B 1, A B 2$, $\mathrm{AD} 1, \mathrm{AD} 2, \mathrm{AJ} 1, \mathrm{AJ} 2$.

The effect of the variation in geometry was not evident in the test results, except that samples with a large degree of asymmetry had to be melted longer to sink the teeth suitably in testing on the sheer rig. Notably, not all conditions showed strong asymmetry, for example AB1 was not obviously asymmetric while AD1 was. This effect showed up in the rime case, indicating this was likely a result of droplet impingement angle and collection efficiency as opposed to being solely due to film dynamics. The front and side views of the samples on mount \#5 are shown in Figure 76. 


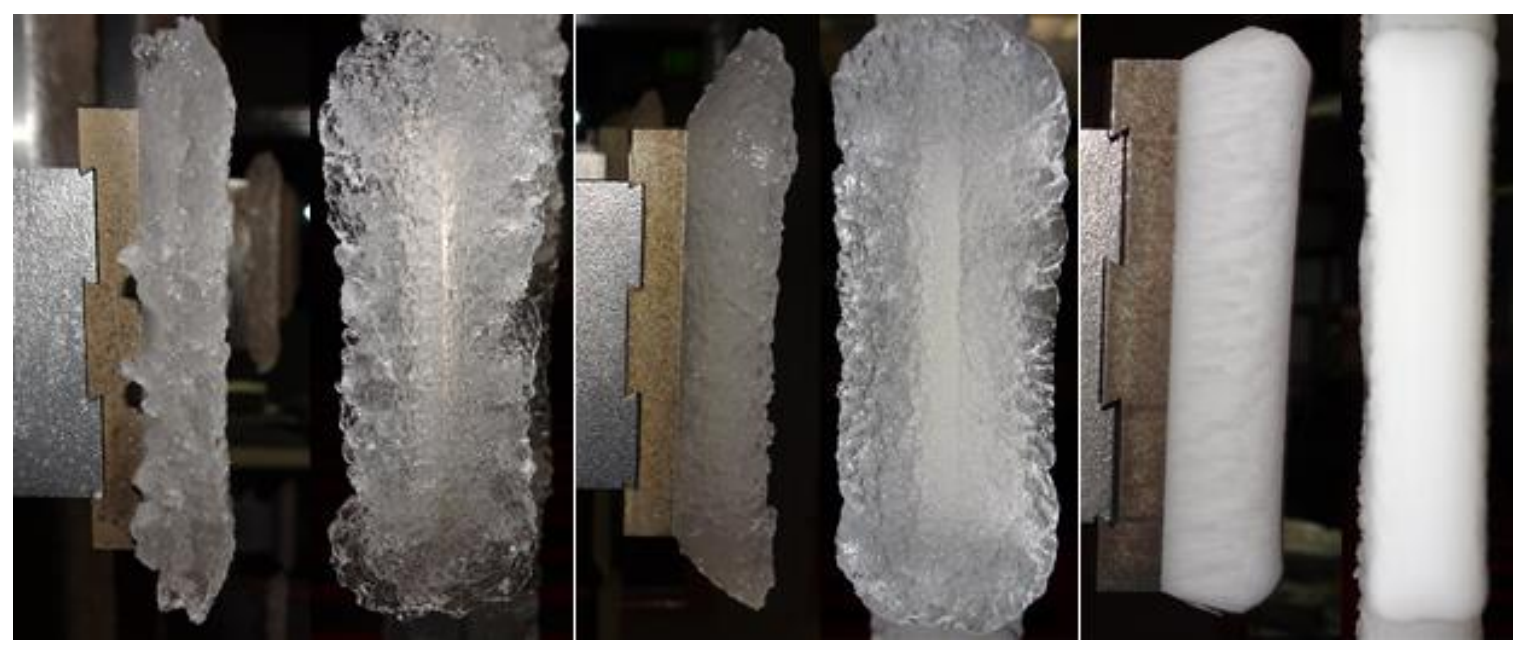

Figure 76. Side and front views of three samples. From left to right: AB5, AD5, AJ5.

The glaze shapes tended to form with a valley down the middle, which was not observed in the rime case. The angle of the ice coming off the coupon was observed to change between tests. This likely resulted in different stress concentrations between tests and was likely responsible for some of the trends observed in the data; resources were not available to correct the data for this effect. Angles were generally symmetric in the vertical direction. When removing dummy samples, marks were observed on the ice near the edges of the coupon-ice interface. Such marks are shown in Figure 77.

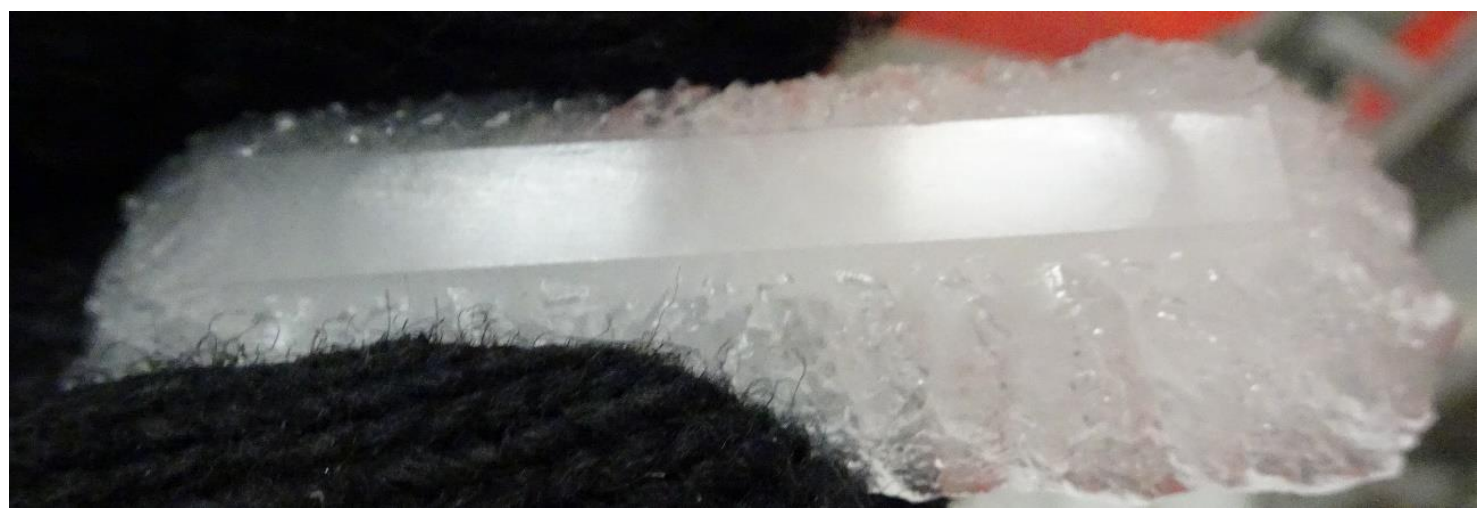

Figure 77. Sample delaminated during run AD showing marks near either end at icecoupon interface.

The marks shown were likely due to preliminary, thermally induced delamination. Contraction or expansion of the ice would tend to concentrate stresses at the edges, 
particularly at the corners. The shape of the marks observed suggest that delamination initiated at the corners and proceeded down the long edges until an equilibrium state was induced. A temperature differential across the ice-steel interface at the air gap likely resulted in mass transfer via sublimation and deposition to the coupon, leaving evidence of the delamination on both the coupon and the ice. The marks were consistent in appearance with sublimation observed on post-test samples being manipulated for microscopy, except that grain boundaries were not readily visible as a result of the process. While these marks were almost exclusively observed at the ends, frost was observed in the middle of a single sample, shown in Figure 78.

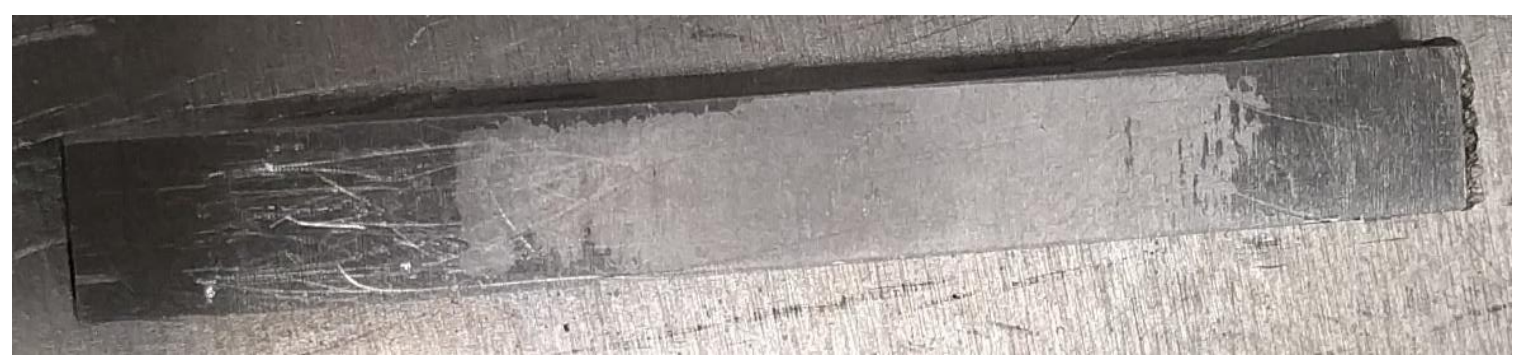

Figure 78. Sample AB10 post-test showing frost in the middle of the sample.

These markings were not consistent with the theory that ice contracts or expands concentrating stress at the ends of the sample. Technicians noted that, while removing and bagging samples, the ice was most likely to detach after loosening the set screw. The set screw tended to load the coupon in a three-point bending configuration and loosening the set screw after the accretion of the ice would tend to un-bow the sample, putting the interface in tension in the center of the sample. The lack of a second observation of this was likely due to the rarity of nucleating a stable crack when loosening the set screw on a sample with no thermally-induced delamination. This was likely the case for sample AB10, which was observed to have a small amount of runback on the right edge as shown in Figure 78. Runback was likely on the left edge as well but removed because of the shear 
test. The runback likely prevented the thermally induced delamination and prevented the sample from fully delaminating when the set screw was loosened. Runback was avoided as much as possible to avoid or minimize cohesive failures during the shear test, so this was likely a rare event. Once it was realized during the test that bending in the coupon was likely responsible for delamination of the samples, shorter sprays were used to produce thinner samples. This would also explain the higher incidence of failure in the second IRT test entry since longer sprays were used than in the first and third tests. Samples still delaminated, however, and so the cloud was again run in a variable manner on the second night in an attempt to reduce residual thermal stresses. The first run of the second night, run $A G$, was run by using an extremely short spray, stopping the tunnel, and inspecting the samples. This was repeated in run AG with a total of four sprays, with spray times of 10 , 20, 30, and 60 seconds, respectively. In between runs, the samples were investigated and the ice from a dummy sample was removed. After the first spray, the ice on dummy sample \#4 was removed and the edges tended to pop off violently but appeared to be well attached. In the second spray, the \#4 and \#24 samples were removed. After the third, all three dummy samples were removed. Edge marks were not observed, though in the first two sprays the ice was too thin to preserve while removing. In the following two runs, AH and AI, the cloud was run at the test condition for a 30 second spray. The test section was then warmed to $-4{ }^{\circ} \mathrm{C}$ total temperature and held for approximately 15 minutes, and then a longer spray was used to put a thicker layer of ice down. For run $\mathrm{AH}$, this second spray was 1.5 minutes, and for AI the second spray was 2 minutes. The same nozzle parameters were used. In the fourth and final run of the night, a rime spray was used to test the hypothesis that a thermally-induced stress would decrease for a hard rime condition. This was postulated 
since a rime condition would not support a liquid film holding the outer layer at equilibrium, reducing the effect of latent heat. After the spray, the ice was removed from the dummy coupons and observed to be relatively much stronger than samples. While the run $\mathrm{AJ}$ was conducted at $-30{ }^{\circ} \mathrm{C}$, the samples were stored at $-10{ }^{\circ} \mathrm{C}$ and tested at $-20{ }^{\circ} \mathrm{C}$. The single sample successfully tested, AJ18, had remarkably high ultimate force for a same-day test at $212 \mathrm{~N}$, and was excluded from aggregate data analysis due to a lack of repeats and the poor temperature control of the sample. While not conclusive evidence, this was a good indicator that the relationship to temperature should not be expected to be linear over the entire range of temperatures relevant to aircraft icing. The IRT temperature data for dummy sample \#21 is shown in Figure 79.

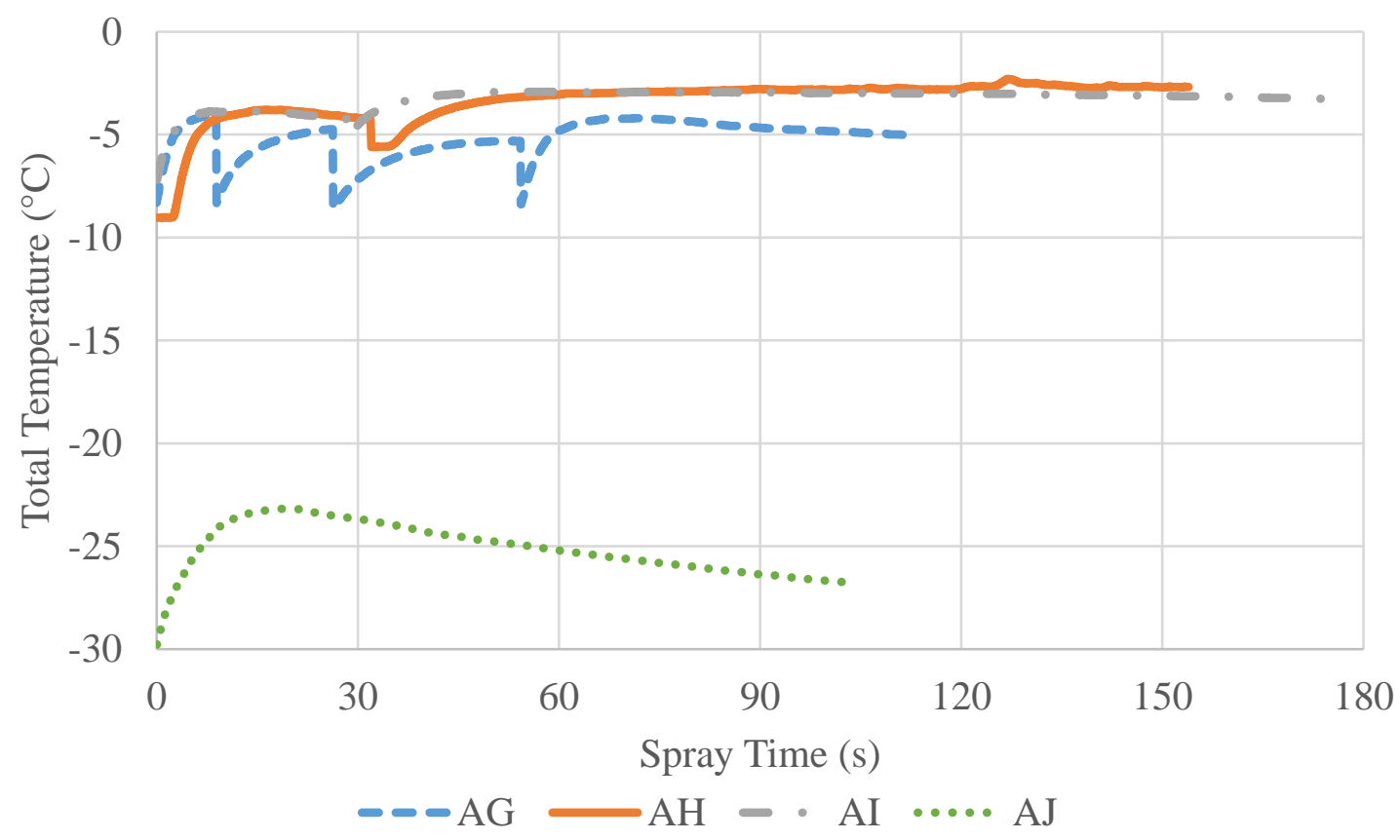

Figure 79. Temperature data for runs AG-AJ with spray on, thermocouple in dummy sample \#21.

The temperature jump in runs AG-AJ was between 5 and $8^{\circ} \mathrm{C}$. The last spray in the AG test was run with the ice removed from dummy sample \#21, which explains why the 
temperature runs higher than in the previous two sprays. The second sprays in $\mathrm{AH}$ and $\mathrm{AI}$ resulted in a nearly flat temperature profile after the 60 second mark, while other tests tended to drop back towards the total temperature at the start of the first spray.

To further explore the issue of delaminating samples, a sample was loaded and allowed to sit in repeated fashion. The resulting force-position data is shown in Figure 80.

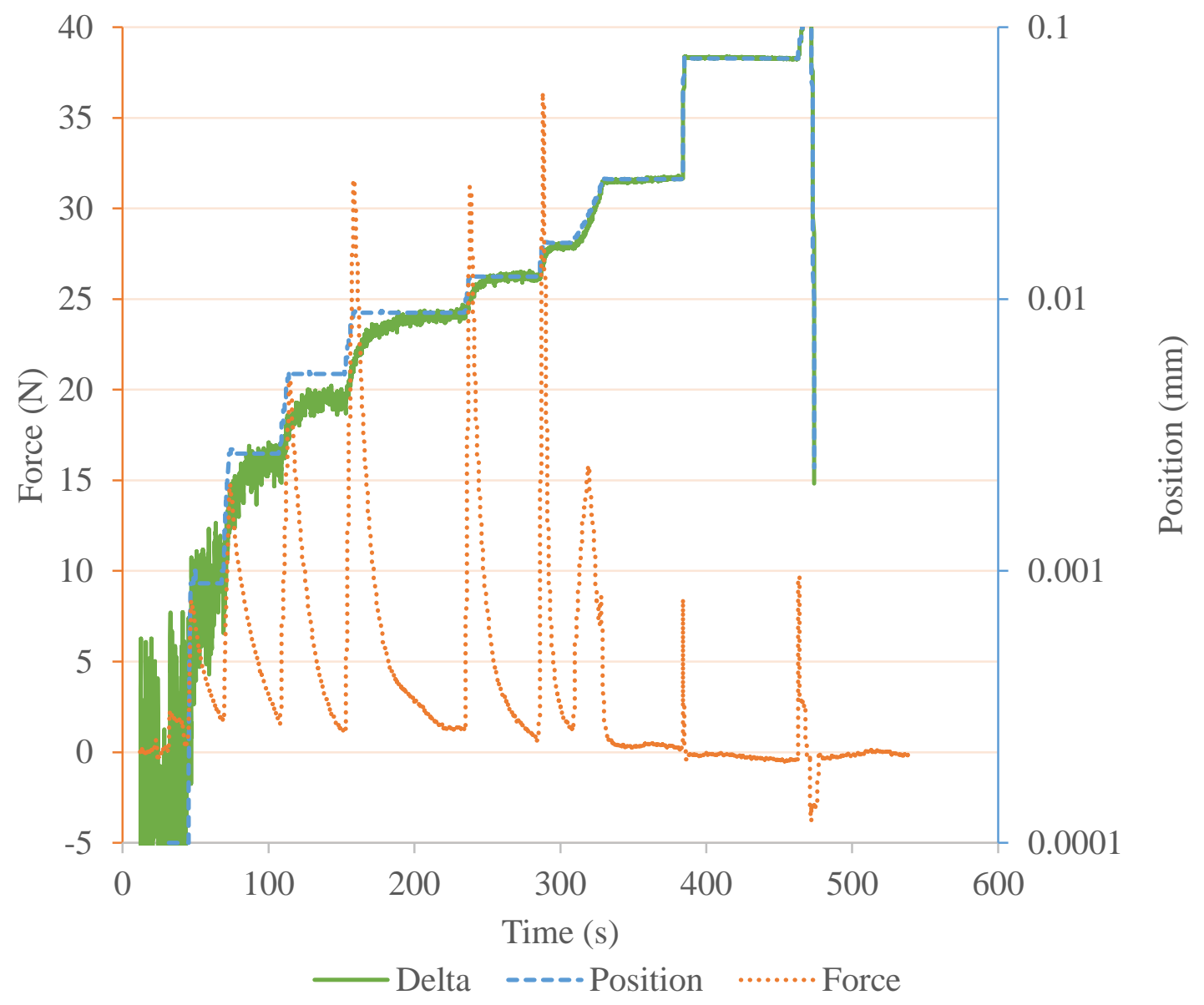

Figure 80. Sample AF17 repeated loading data (excluded from aggregate data analysis).

Sample AF17 was repeatedly loaded and allowed to relax, and the load was observed to steadily drop after each instance until a failure occurred after the $300 \mathrm{~s}$ mark. The Delta data in the figure shows the difference between the two deflectometers used in the test, while the Position data shows the crosshead position of the load frame as 
determined by the built-in optical encoder. A difference between the two showed up when a load was induced due to the flexure of the shear rig. This likely did not account for all of the difference since only one deflectometer could be placed close to the sample. The drop in the force was likely due to stead crack growth and not due to plastic flow in the ice. Many samples were observed to be preloaded to loads up to $50 \mathrm{~N}$ in the first test results since force control was not used. In response, several methods are suggested to determine whether the sample was partially delaminated prior to the onset of the test. First, preloading the sample and insuring that a preload could be held at time scales similar to that shown in the AF17 test would likely indicate the lack of crack growth. Second, shear modulus data would likely be affected by the crack growth since the relaxation of the sample would reduce the slope of the stress-strain curve. At lower strain rates, this effect would likely be significant and could be identified in post-processing. In the data presented herein, the position measurement was not suitable for such analysis. Third, samples presenting exceptionally low adhesion values with respect to the group were likely delaminated prior to the onset of the test. Some data was presented in the second IRT test entry results excluding low-outlier data points on this basis. Finally, a more difficult solution would be to use advanced methods to track the crack. Digital Image Correlation could be used to determine the location of the crack based on the strain in the sample; alternatively, acoustic emission testing could be used to determine the location of the front. The presence of a preliminary delamination likely produces a rate-dependent effect on the apparent strength of the ice. As demonstrated by the AF17 shear test, a small load was used to fully delaminate the ice when the crack was given time to grow. The ultimate failure strength of $36.4 \mathrm{~N}$ was considerably lower than the average of 54.7 for the samples tested the day prior, 
with a maximum force of $96.3 \mathrm{~N}$ for sample AF12. AF17 was tested the following day, which should have increased the apparent adhesion strength as shown in the results for the first two IRT test entries. This did fall within the scatter of the AF series results, however.

Several samples did not fail in a predominately adhesive mode, and left ice on the coupon. This occurred in one of two ways. Samples may have left a large chunk of ice with a partial adhesive failure or left a thin layer of ice on the surface as depicted in Figure 81.

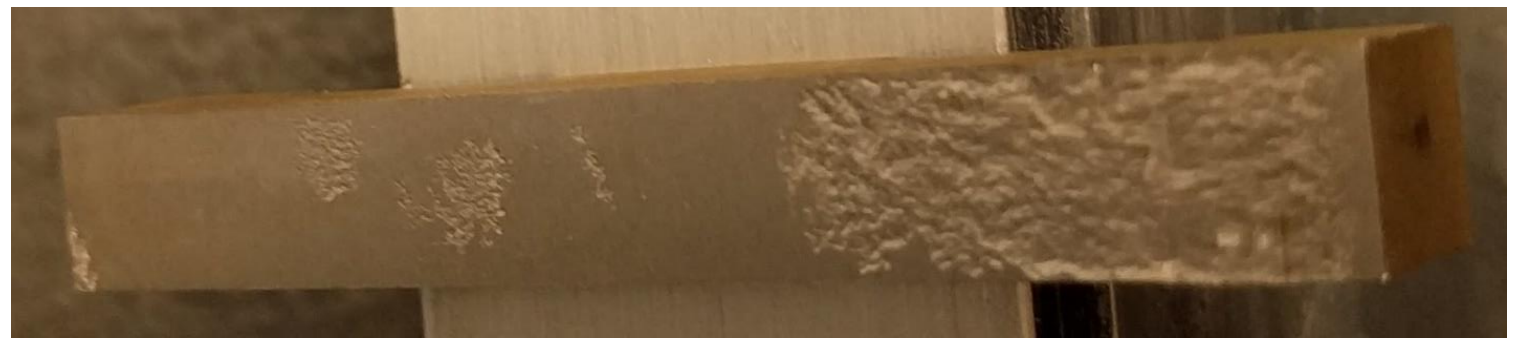

Figure 81. Surface cohesive break on sample AG15, post-test.

Several samples with apparent pure adhesive failure ${ }^{44}$ also exhibited interfacial cracking near one end, similar to the pattern left in the surface cohesive break of sample AG15. Most samples exhibited good breaks where no visible ice was left on the surface, and some had minor pieces of ice left on the short edges that likely slightly reduced the measured adhesion strength proportional to the area differential $(<2 \%)$ yielding a small conservative bias from those samples. Samples with large-scale cohesive failure (near surface or not) were excluded from aggregate data analysis in all results.

It was desired to test cuboid samples to reduce the effects of geometric variability on the test. Samples from the AG and AH run were machined using the microtome and sanded using a Dremel to produce cuboid geometries. This was done at the storage

\footnotetext{
${ }^{44}$ All good break samples had apparent adhesive failure where no or extremely little visible ice was left on the coupon. This was referred to as apparent adhesive failure since micro-scale pieces of ice may have been left in the sample roughness elements, where cohesive failure would have occurred on the micro-scale. The samples could not be observed under a microscope quickly enough to check for this phenomenon.
} 
temperature of $-10{ }^{\circ} \mathrm{C}$. The ice behaved in a brittle manner and chipped very easily during cutting, meaning great care had to be taken to reduce chips near the interface on the final product. A cuboid sample is shown in Figure 82.
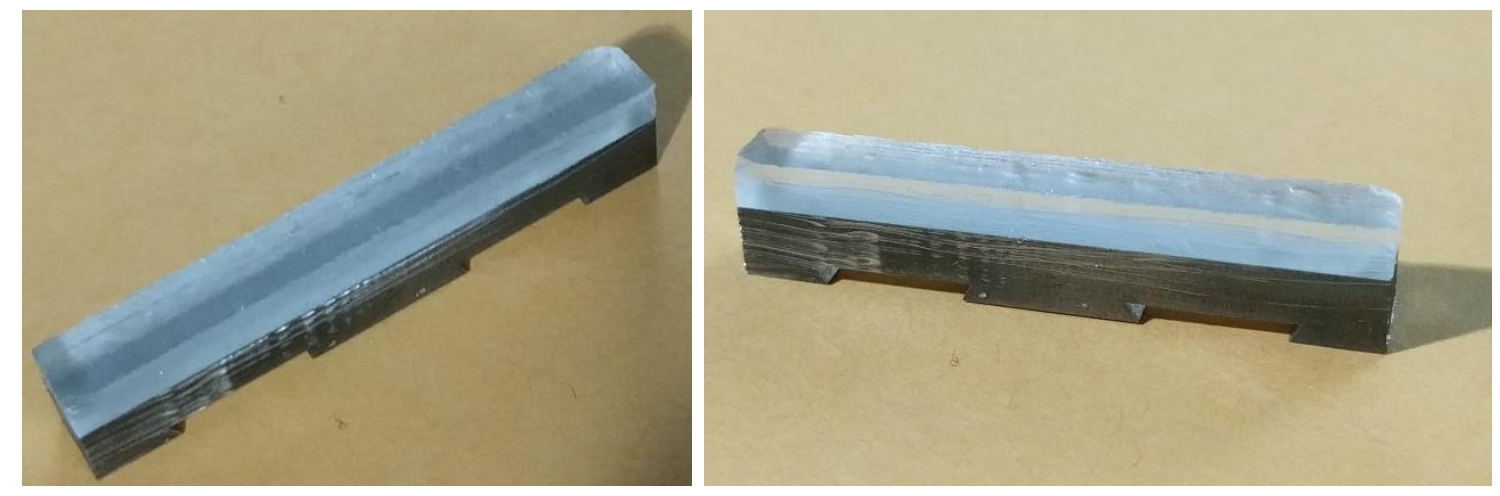

Figure 82. Sample AG9 machined to a cuboid shape, pre-test.

Sample AG9 machined particularly well, and relatively sharp corners were maintained. In other cases, small chips would be removed from the corners near the interface. Complete delamination was induced in one sample, and it was noted that delamination was difficult to observe through the ice even though the ice was very clear. Producing cuboid shapes was also pursued to enable performing 2D Digital Image Correlation (DIC) on the samples.

A limited number of samples were used for testing from the third test. The available samples were tested prior to 2-9-18, when the shear rig components began to seize up due to galling. A fix was designed and implemented, but additional results were not produced in time for inclusion in this work. The results at $-8{ }^{\circ} \mathrm{C}$ from the first night are shown in Figure 83, where the averaged data was an average of repeat runs excluding data one deviation or more below the average of the unmodified data set. 


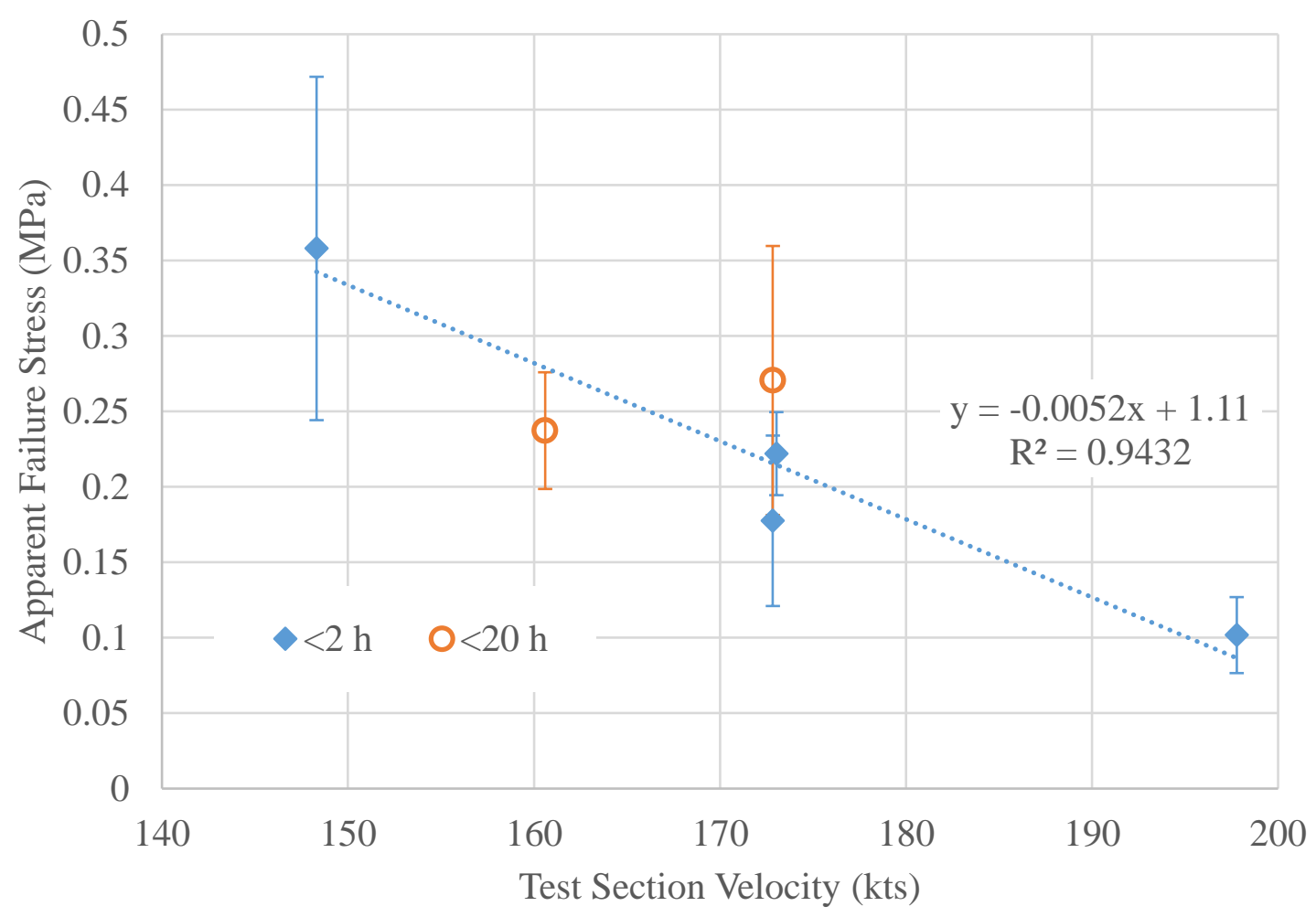

Figure 83. Results from runs AB-AF showing variation in adhesion strength with velocity.

Unlike prior tests, the results show a strong correlation to velocity, where the observed strength decreased with increasing velocity. The cause for this trend may have been that the increased heat transfer caused stronger residual forces in the ice or increased aerodynamic forces may have propagated cracks further reducing the apparent strength. Effects may have been visible in this set of results since the residual stresses were high enough to delaminate samples prematurely, where in the first IRT test entry the samples may not have had cracks initiated. It was unlikely that modifications to the test were likely for the change since the updates between the first and third IRT test entries reduced noise, improved heat transfer to the stand, and worsened the heat transfer from the teeth to the 
ice. ${ }^{45}$ None of the changes were expected to have resulted in the unmasking of a trend or the creation of a false trend in the data. Perhaps the most plausible explanation was that the trend was caused by residual stresses which were annealed out of the first test results since the velocity sweep samples were not tested until days after accretion in the IRT. The data may have been biased by geometric differences in the ice, but this would have been true of the data from the first IRT test entry as well, and almost certainly did not account for the full trend observed here. Scatter was improved on the results obtained on the first night of testing with an average standard deviation of $26 \%$, and results from the second night had an average standard deviation of $25 \%$ (omitting cuboid samples). Results from the second night of testing are shown in Figure 84, where outliers more than one standard deviation below the average of the unmodified data were omitted.

${ }^{45}$ The heat transfer was worsened by the surface mount thermocouples, which had the tradeoff of allowing the temperature to be measured close to the ice-teeth interface. 

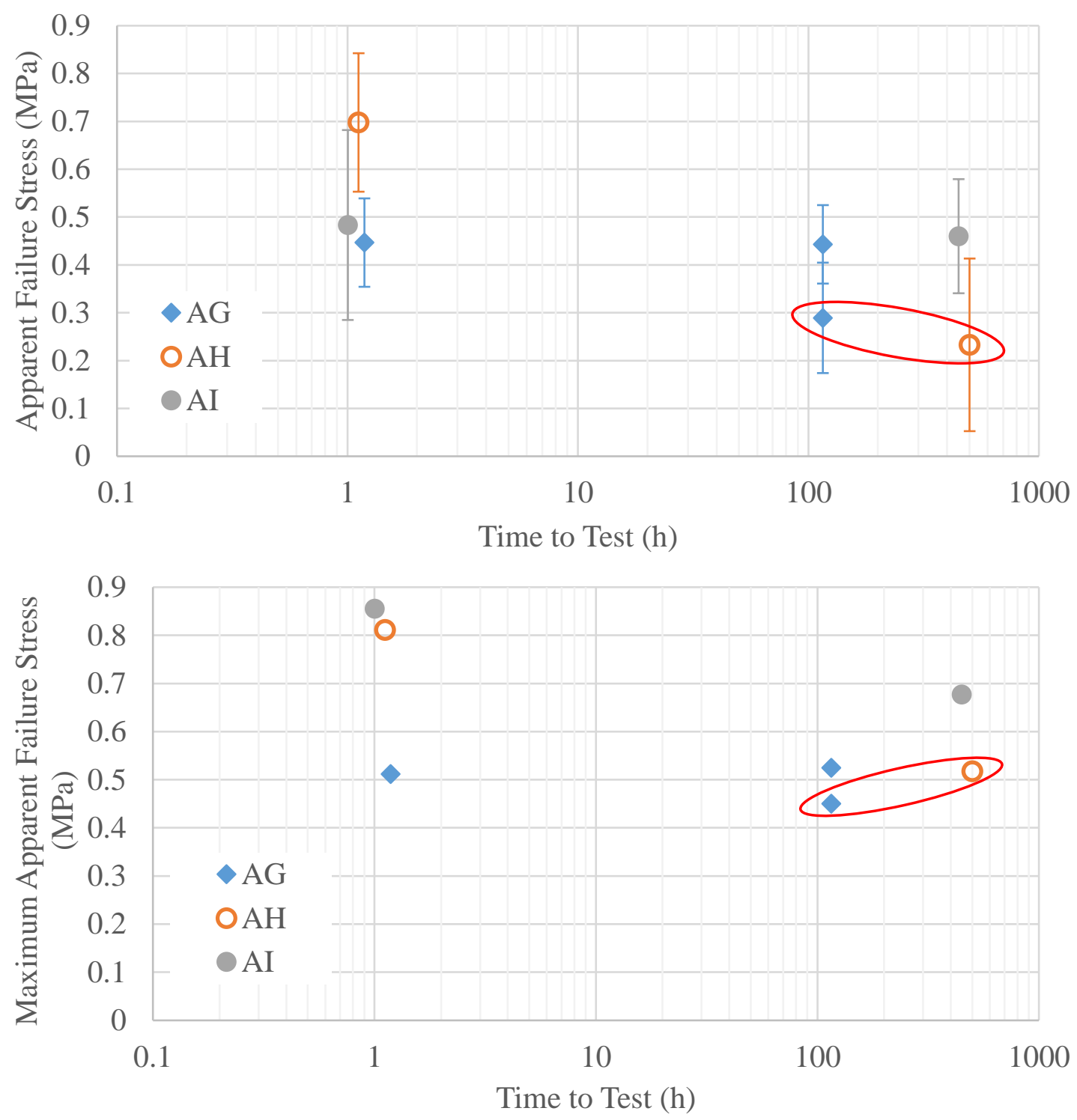

Figure 84. Results from runs AG-AI. Top: average measured apparent failure stress. Bottom: maximum measured apparent failure stress. Cuboid samples circled in red.

The results for the second night were less amenable to parametric analysis since something different was attempted with the use of multiple sprays. Test AG had comparatively low adhesion, when the test was run at one temperature with four sprays. The following two tests, $\mathrm{AH}$ and AI, were easier to handle in the IRT without destroying the samples, but still exhibited high scatter. Cuboid samples were cut out of the AG and AH samples, which reduced the strength of the samples. This was due to damaging the 
samples by initiating cracks, stronger stress concentrations due to the modified geometry, or both. The spread in these results was higher than in the first test since a number of the cuboid samples had extremely low values of adhesion that were not omitted due to too few repeats being measured. The maximum data points for the cuboid samples were still lower than for the uncut samples but fell within the standard deviation of the uncut samples. The data for the second night of testing compared to mean sample thickness is shown in Figure 85.

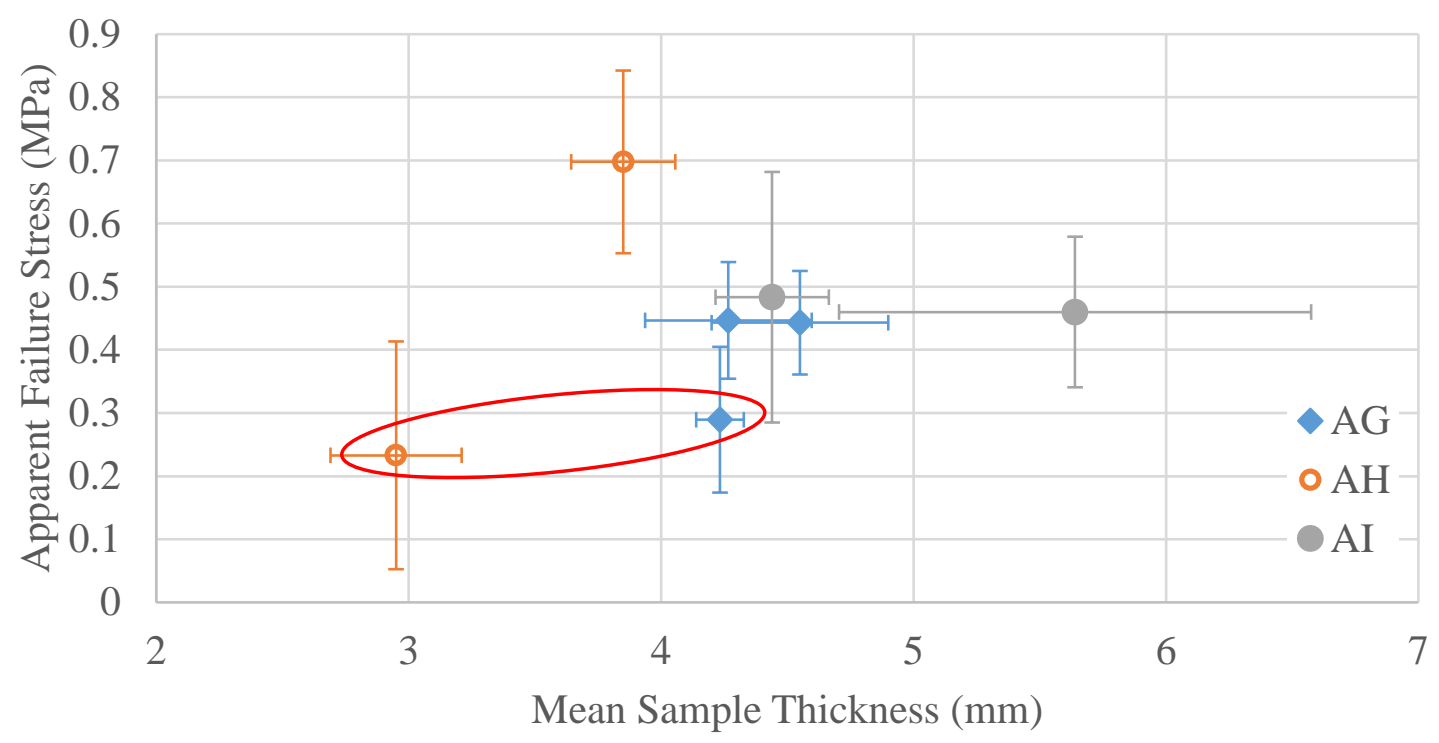

Figure 85. Sample thickness vs. average measured failure stress. Cuboid samples circled in red.

Sample thickness data shows that the AG cuboid samples did not require much shaved off the top to produce a flat sample, while the AH samples lost nearly $1 \mathrm{~mm}$ on average. AI samples tested more than 1 day after the $3^{\text {rd }}$ IRT test entry varied widely in thickness (0.94 $\mathrm{mm}$ std. dev.), compared to the average variation $(0.34 \mathrm{~mm})$. No significant trend was observed with increasing thickness.

The AA run was excluded from this analysis since the total temperature was higher $\left(-5^{\circ} \mathrm{C}\right)$, and significant runback was observed. All other runs on the first day produced 
samples where frost was observed (AB-AF). Samples from runs AA and AG-AJ did not produce frost, suggesting that pulsing the cloud was an effective means to reduce or eliminate preliminary delamination.

Shedding Analysis Results

Two test runs were identified that had suitable sheds. The first was Run 67, which contained two shed events, the second was Run 71 (shown in Figure 41). The test settings for each run are shown in Table 12. The second shed in Run 67 must be used with caution since the rotor stand began shaking after the first shed.

Table 12. Test parameters and data for Run 67 and Run 71.

\begin{tabular}{|l|l|l|}
\hline Parameter & Run 67 & Run 71 \\
\hline Heating Scheme & Chordwise & Spanwise \\
\hline RPM & 1200 & 1200 \\
\hline Tunnel Velocity (kts) & 30.9 & 30.9 \\
\hline Collective Angle $\left(^{\circ}\right)$ & 10 & 5 \\
\hline Tstatic ('F) & -4 & -4 \\
\hline tspray (mm:ss) & $06: 49$ & $05: 25$ \\
\hline LWC (g/m3) & 0.5 & 0.5 \\
\hline MVD ( (um) & 15 & 15 \\
\hline Frame Rate (fps) & 320 & 480 \\
\hline Number of Sheds & 2 & 1 \\
\hline Pieces of ice per shed & 1 & 3 \\
\hline
\end{tabular}

The shed event in Run 71 was particularly interesting since it contained three breaks of ice in a single shed event; the ice had two interstitial breaks before leaving the rotor. It was suspected that this was the case since Run 71 was a Spanwise heating case, where the 
spanwise variation in heat may have contributed to the multiple breaks. Both sheds in Run 67 contained a singular break where the chordwise heating should have provided uniform heating in the spanwise direction. The STM prediction for Run 71, with all fit parameters set to zero, is shown in Figure 86.

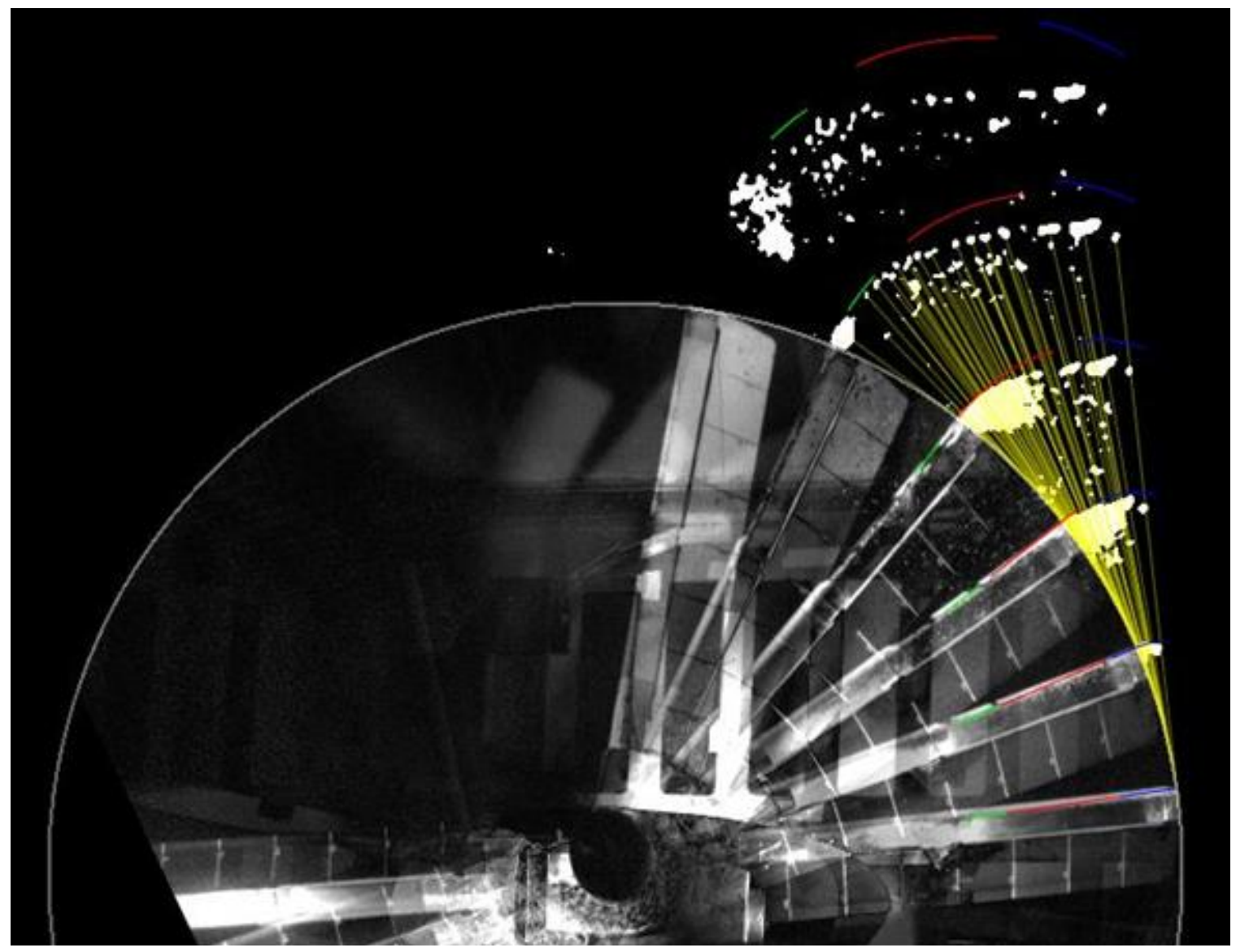

Figure 86. Unfit model compared to shed in Run 71. $\mathrm{B}=0, \mathrm{~F}=0, \mathrm{c}=0, \theta=0$. Blue, red, and green lines represent first, second, and third pieces of ice respectively. Yellow lines are drawn from centroids in the $5^{\text {th }}$ image to a tangent point on the rotor disk.

The unfit prediction for Run 71 had several flaws. The ice accelerated too quickly off the edge of the rotor, as can be seen by comparison of the green line to the last piece of ice in the background picture. This resulted in too high of a radial velocity. The predicted fronts also did not decelerate since c was set to zero, meaning the tangential velocity was too high. To correct for this, the fit parameters were modified and set of terms providing 
good fit were visually identified. The fit prediction is shown in Figure 87, next to a fit with drag set to zero and a fit assuming a single piece of shed ice (no interstitial breaks).
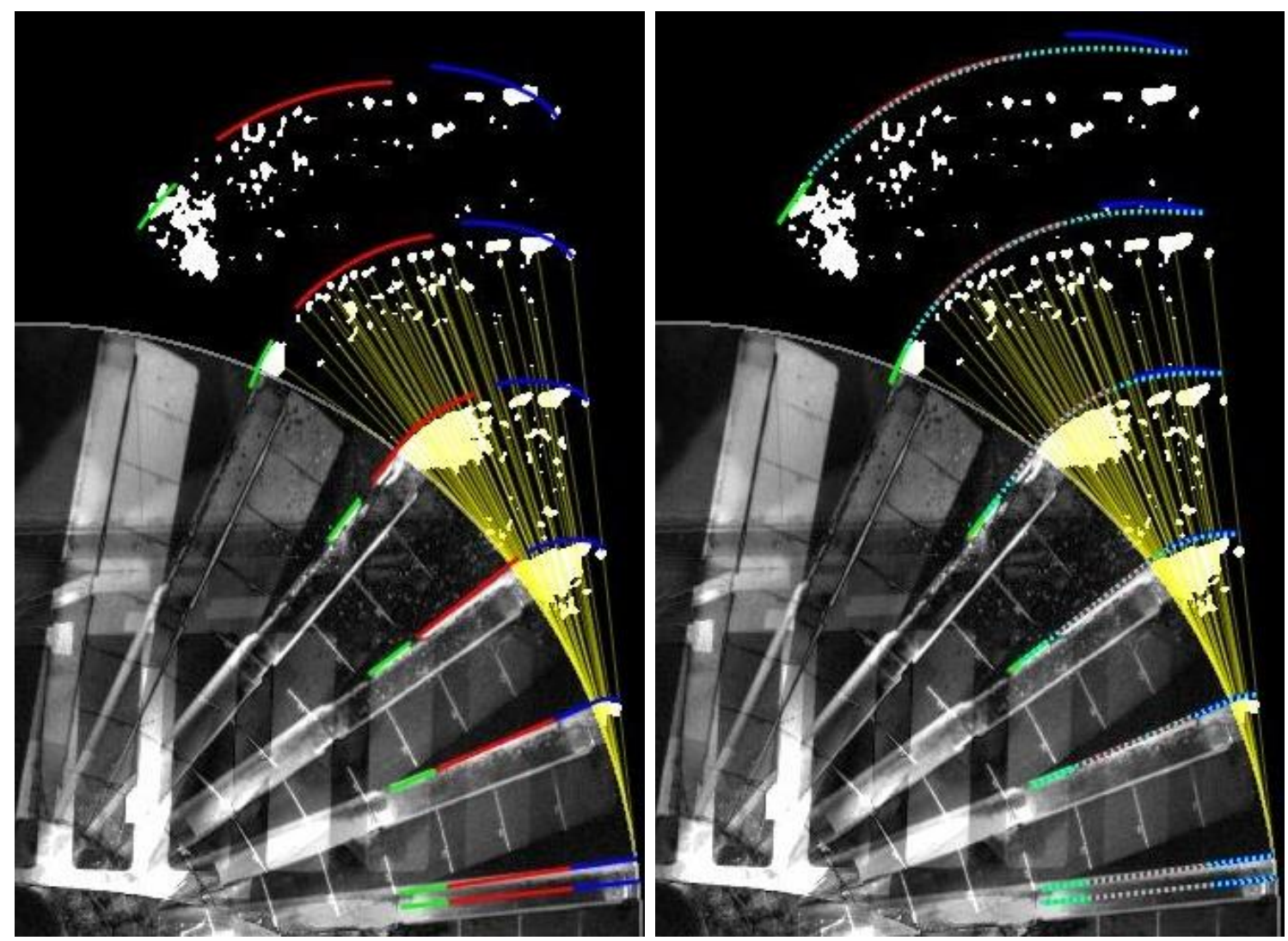

Figure 87. Left: $\mathrm{F}=0, \mathrm{~B}=60, \mathrm{c}=0.0004, \theta=0$. Right: $\mathrm{F}=0, \mathrm{~B}=60, \mathrm{c}=0, \theta=0$.

Dotted cyan line is predicted front for corresponding singular shed event.

The fit predicted shed front on the left side of Figure 87 matched the position on the rotor well and matched the lengths and positions of the shed front relatively well. The blue line doesn't match the shape of the front well; it was believed that this was due to the assumption that ice breaks continuously as it reaches the rotor edge. The first piece of ice broke primarily into three pieces of ice, which was poorly represented by the continuous front assumption. Solid breaks also allow more acceleration in the radial and tangential direction, since pieces can be accelerated faster than the tip speed of the rotor. On the right side of Figure 87, air drag was removed for comparison, showing how the predicted front 
tended to run away from the experimental shed front. The dotted cyan line represents the predicted front for a shed with no interstitial breaks, or a singular shed. The singular shed provided a shorter front than in the multi-break shed since the would-be second and third pieces of ice were pulled forward with the first piece of ice as a solid piece. Fit predicted shed fronts for both sheds in Run 67 are shown in Figure 88.

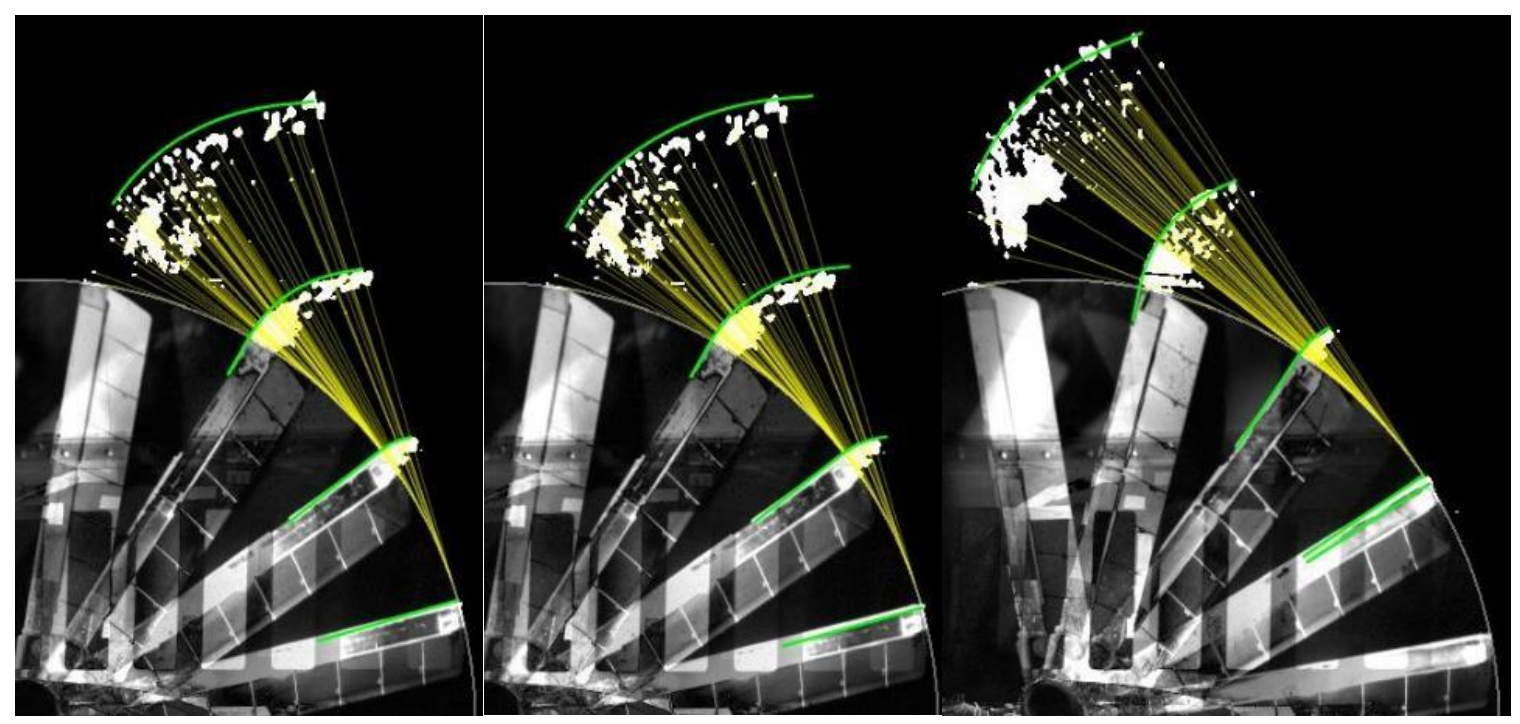

Figure 88. Left: Shed 1, F $=0, B=60, c=0, \theta=0$. Center: Shed 1, F =0, B = 140, $\mathrm{c}=0$, $\theta=4$. Right: Shed 2, $F=0, B=60, c=0, \theta=0$.

The predicted shed front for Shed 1 in Run 67 fits the length well and matches the front. It was interesting that the predicted front did not run further ahead of the shed ice since drag was set to zero. This was the case in both shed events for Run 67 . The center image in Figure 88 shows a modified fit with B set to 140 to demonstrate how the B and F parameters were used to fit the shed event. Both viscous and frictional forces at the interface slowed the motion of the ice on the rotor, creating a longer shed front. Viscous and frictional forces create different velocity profiles since viscous forces would slow the ice proportional to its current speed while frictional forces would create a constant resistive 
force. The right most image shows Shed 2 from Run 67. Shed 2 occurred after Shed 1 while the rotor stand was shaking from the imbalance created from the first shed.

It was shown that the model predicts trajectories qualitatively similar to shed trajectories captured in the 2013 rotor tests. The model was capable of being fit to the data allowing for reasonable modeling. Viscous damping forces at the interface best represented the motion of the ice, capturing the leading and trailing edges of the ice better while on and off of the rotor. More data was necessary to obtain statistically significant data to fit the model, and currently provided fit terms should only be used as an initial guess. 


\section{SUMMARY AND CONCLUSIONS}

A critical review of the methods identified a number of unaccounted-for physical phenomena in the test methods used in the literature. These phenomena were expected to produce orders of magnitude of disagreement. From the literature review, it was observed that the many methods used to measure the adhesion of ice demonstrate poor agreement, with scatter in the data exceeding five orders of magnitude at the most commonly tested temperature. Of the large data sets in the literature, data reporting was relatively poor making a detailed analysis and comparison impossible. It was determined that the data in the literature did not account for strain rate, and stress concentrations in the respective tests (which were variable depending on icing condition in some cases). Other parameters, such as surface roughness, were poorly documented in the literature though general agreement was expressed that increasing roughness lead to increased adhesion strength. Trends in the data also disagreed, with some authors showing strong linear trends for adhesion strength and temperature, while others showed a piecewise trend that was flat in some areas and linear in others. Most authors did not use impact ice, and most data sets provided extremely few repeats (three or less) for individual data points.

A new test method was developed to measure the adhesion of ice that was a modified version of a lap-joint shear test. A new technique was developed to reattach the ice that allowed the lap-joint test to be used to measure the adhesion strength of impact ice. The new test methodology provided several improvements over existing test methods used for impact ice, including the ability to record a stress-strain curve in a test with a more 
uniform stress distribution than found in pusher-type tests. The fixture designed for the method was capable of running in mixed-mode configurations and compressing the ice during mode 2 testing. A new wind tunnel model was designed allowing the simultaneous collection of up to 48 samples of ice in a single IRT run. This model was successfully used to complete three test campaigns in the IRT collecting approximately 600 samples over 6 nights of testing in the center-mount (24-coupon) configuration. The temperature profile of the shear rig was characterized during the melting cycle and the temperature at the melting point was measured directly.

A comparatively large data set measuring the apparent adhesion of ice was taken for the purpose of allowing more detailed analysis into the phenomena of ice adhesion, and the methods used to measure it. The new data set included detailed information on the roughness of the samples used for measurement, including frequency data. Sample preparation methodology and sample composition were documented. The displacement rate was controlled across the data set and four crosshead speeds were used in testing the samples, each an order of magnitude apart. Flexure in the shear fixture was documented. The variations in the stress-strain curve of the data was discussed in the context of the strain rate the ice was subjected to, showing the effects of plasticity on the data at low speeds, and dynamic concerns at high speeds. Stainless steel samples were used to allow direct comparison to data in the literature, where the results were relatively high compared to the steel data in the literature.

A strong trend with velocity was identified in the pre-annealed ice from the third IRT test. This was not observed in the data from the first IRT test entry, where data produced from samples stored over long periods showed a decrease in adhesion strength at 
the highest speeds. While data in the literature tends to show increasing adhesion strength with decreasing temperature, a strong trend was identified showing increasing adhesion strength with increasing temperature - likely due to residual stresses from the formation process. These thermal stresses in the formation process were completely neglected in the literature and was likely hidden due to geometric effects. Rounded coupons used in the literature likely experienced clamping with the ice contracting on the curved surface, while flat samples experienced a stress concentration at the edges tending to reduce the apparent adhesion strength. Many samples were delaminated prior to testing, which was likely due to these residual stresses and flexure in the test coupons during unmounting. Running tests in multiple sprays and with warmer secondary sprays eliminated the observed delamination, supporting the conclusion that thermal residual stresses were observed and are a significant and time-dependent factor in the adhesion of aircraft ice. The opposite trend observed demonstrates that the simplistic methods used to measure the adhesion of ice may impart false trends to the "true value" of adhesion strength observed in nature.

The data presented herein likely was biased to lower-than-realistic values since stress concentrations and residual stresses were not accounted for. False trends may have been observed due to the delamination of some samples and the shape of the ice which varied from run to run. The data from the single rime condition run at $-30{ }^{\circ} \mathrm{C}$ suggested that variation of the actual adhesion strength was not linear with temperature. Samples were observed to demonstrate reattachment of the ice to the coupon, indicating that other time dependent effects were present and unaccounted for that may have increased the adhesion strength over time. While either due to these unknown effects or to annealing, the adhesion strength of the ice was observed to increase over time, asymptotically approaching a final 
value after approximately 40 days of storage. Nearly all cases over doubled in strength after being stored for 40 days. The ice in the samples was observed to sublimate and deposit new crystals of ice elsewhere in the air-tight bags used to store the samples. This effect was observed to decrease when samples were thermally insulated in storage. Data may also have been biased to lower-than-realistic values due to contraction of the serrated block after refreezing the ice. Vibrational loading and loading from handling were unaccounted for. With the exception of time-dependent effects, all of the physics unaccounted for in testing would tend to bias the data to lower values. Because of this the data should be interpreted as a minimum value as a function of time, with trends particular to the test method and not to ice in nature. These identified physics explain the discrepancy between the data presented in the literature, at least in part.

The grain structure of the long-term-storage ice was shown to change significantly with different test conditions, though the data could not be quantified. Tests were run with droplet MVDs between 15 and $50 \mu \mathrm{m}$, with one SLD case at $180 \mu \mathrm{m}$. The one SLD condition run had particularly small grains compared to the other conditions. The lowvelocity ice had round grains with larger grains concentrating in the center of the sample, while higher-velocity ice had oblong grains following the streamline of the surrounding flow. The oblong grains formed a pattern around the edges of the sample suggesting possible anisotropy in the ice. The ice was allowed to anneal for approximately two months, and the largest grains were over $2 \mathrm{~mm}$ in diameter.

A number of models exist in the literature to predict the adhesion strength of the ice, but all fail to take into account a number of relevant physics. The combination of the complexity of the problem and poor data in the literature likely mean that producing such 
an analytical or pseudo-analytical model was impossible. The data in the literature was not suitable for validation and the test techniques currently available are incapable of accounting for all the relevant physics.

A high-speed imaging data set from an icing tail rotor test was analyzed. The analysis performed allowed the development of a mathematical model to predict the trajectory of ice shed from a rotor. The model was successfully fit to the data available and adapted to fit single- or multi-break shed events. The model accounted for viscous and frictional sliding forces in the ice travelling across the rotor and drag forces when the ice left the tip of the rotor. This allowed the prediction of trajectories of the shed ice useful for risk assessment of shedding events. 


\section{FUTURE WORK}

In moving forward in the short term, the third set of data needs to be completed and analyzed to investigate the effect of storage on the trends observed in the data. The fixture needs to be reworked to include a removable copper tooth plate in place of the existing aluminum on the serrated block to better thermally isolate the teeth, reduce thermal contraction, and allow testing with multiple teeth configurations. The entire shear rig needs to be anodized, like the lower rail was, and gibs need to be added to the coupon and compression blocks. The coupon mounts on the XT model and on the coupon block in the shear rig need to be redesigned to minimize the loading of the coupons that leads to bending. Coupons need to be redesigned to prevent the preliminary delamination of the ice.

2D and/or 3D digital image correlation needs to be made to work to investigate the growth of cracks and the strain distribution during testing. This needs to be used to validate the use of properties for ice available in the literature; if this cannot be established then a series of materials tests will need to be run in order to obtain bulk properties for use in FEA. FEA will then need to be run to determine more accurately the distribution of stresses in the samples to perform failure analysis. In future tests, the XT model needs to be instrumented with accelerometers to document the vibrational loading on the model and the samples. The lap-shear test will then need to be run to collect much more data, running mode 1, 2, and mixed mode configurations.

The time dependence of the ice also needs to be investigated more thoroughly, including a redesign of the storage used for the samples. Next, appropriate temperatures 
for storage need to be determined, and the temperature sweeps repeated. Investigation of the surface post-test must be performed to determine the degree to which ice is left on the surface, which will be required to obtain a fruitful understanding of the physics behind the interaction of roughness with the apparent adhesion strength.

Ultimately, a new in-situ method is required to solve the problems experience by conventional testing. Development of the RITA test proposed in the Alternative Concepts section would likely be the best course of action to take. Such a test would allow testing with the cloud still on and isolate the complex geometry of the ice from the coupon while still providing a stress strain curve. Residual stresses from the ice would need to be recorded in such a test, likely by measuring the hoop stresses with embedded strain gages in the RITA cylinder. 


\section{REFERENCES}

1. Yeoman, K.E., AMA 99-0495 Shortcomings Of The ATR-72 Accident Investigation And Disposition Of The Case. 1999.

2. Mosher, F.R., et al., Analysis of Causes of Icing Conditions which Contributed to the Crash of Continental Flight 3407. 2010.

3. Stoklosa, J.H., The Air Florida and Pan American Accidents-A Further Look. 1983, SAE Technical Paper.

4. Mason, J.G., J.W. Strapp, and P. Chow. The ice particle threat to engines in flight. in 44th AIAA Aerospace Sciences Meeting, Reno, Nevada. 2006.

5. Appendix $O$, in 14 C.F.R. $\$ 25$. U.S.A.

6. Wright, W., User's Manual for LEWICE Version 3.2. 2008.

7. Aubé, M.S., et al., Application of FENSAP-ICE-Unsteady to helicopter icing. 2007, SAE Technical Paper.

8. Feo, A., M. Vargas, and A. Sor, Rotating Rig Development for Droplet Deformation/Breakup and Impact Induced by Aerodynamic Surfaces. 2012.

9. Makkonen, L., Ice adhesion-theory, measurements and countermeasures. Journal of Adhesion Science and Technology, 2012. 26(4-5): p. 413-445.

10. Wright, W.B., User Manual for the NASA Glenn Ice Accretion Code LEWICE. Version 2.2. 2. 2002.

11. Anderson, D.N. and J.-C. Tsao, Additional results of ice-accretion scaling at SLD conditions. 41st Aerospace Sciences and Exhibition, 2003.

12. Tsao, J.-C. and R.E. Kreeger, Evaluation of scaling methods for rotorcraft icing. 2010.

13. Tsao, J.-C. and S. Lee, Evaluation of Icing Scaling on Swept NACA 0012 Airfoil Models. 2012.

14. Geer, W.C. and M. Scott, The Prevention of the Ice Hazard on Airplanes. 1930.

15. Knight, M. and W.C. Clay, Refrigerated Wind Tunnel Tests on Surface Coatings for Preventing Ice Formation. 1930.

16. Anderson, D.N. and A.D. Reich, Tests of the performance of coatings for low ice adhesion. 1997: National Aeronautics and Space Administration.

17. Chu, M. and R. Scavuzzo, Adhesive shear strength of impact ice. AIAA journal, 1991. 29(11): p. 1921-1926.

18. Merkle, E. Icing tunnel tests of icephobic coatings. in Proc. 8th Natl. Conf. on Environmental Effects of Aircraft and Propulsion Systems. 1968.

19. Petty, K.R. and C.D. Floyd. A statistical review of aviation airframe icing accidents in the US. in Proceedings of the 11th Conference on Aviation, Range, and Aerospace Hyannis. 2004.

20. Feeler, R.A., De-icing and anti-icing are major safety factors in winter operations. Aviation Mechanics Bulletin, 1991.

21. Green, S.D. A study of US inflight icing accidents and incidents, 1978 to 2002. in 44th AIAA Aerospace Sciences Meeting and Exhibit. 2006.

22. Lawson, R.P., et al., Improved measurements of the drop size distribution of a freezing drizzle event. Atmospheric research, 1998. 47: p. 181-191. 
23. Steen, L.E., R.F. Ide, and J.F. Van Zante, An Assessment of the SEA Multi-Element Sensor for Liquid Water Content Calibration of the NASA GRC Icing Research Tunnel. 2015.

24. Appiah-Kubi, P., US Inflight Icing Accidents and Incidents, 2006 to 2010. 2011.

25. Norris, G. JAL Surprises Boeing, GE With 787 Withdrawals. 2013 [cited 2016 2/9/2016]; Available from: http://aviationweek.com/awin/jal-surprises-boeing-ge-787-withdrawals.

26. Jarrah, A.I., et al., A decision support framework for airline flight cancellations and delays. Transportation Science, 1993. 27(3): p. 266-280.

27. Bratu, S. and C. Barnhart, Flight operations recovery: New approaches considering passenger recovery. Journal of Scheduling, 2006. 9(3): p. 279-298.

28. Shavell, Z., The effect of schedule disruptions on the economics of airline operations. 2000: ATM Seminar.

29. Schoen, J.W. Tab for winter flight cancellations: \$2.4 billion. [News Article] 2015 3/3/2016 2/9/2016]; Available from: http://www.cnbc.com/2015/03/02/tab-for-winterflight-cancellations-24-billion.html.

30. Appendix C, in 14 C.F.R. $\$ 25$. U.S.A.

31. Aviation Database. 2016, NTSB.

32. Cao, Y., G. Li, and J. Sheridan, Airflow hazard prediction for helicopter flight in icing condition. Proceedings of the Institution of Mechanical Engineers, Part G: Journal of Aerospace Engineering, 2012: p. 0954410012469764.

33. Cao, Y., G. Li, and G. Zhong, Tandem helicopter trim and flight characteristics in the icing condition. Journal of Aircraft, 2010. 47(5): p. 1559-1569.

34. Zanazzi, G., et al., Ice accretion prediction on helicopter rotor blade in hover flight. 2007, SAE Technical Paper.

35. Flemming, R.J., R.K. Britton, and T.H. Bond, Role of wind tunnels and computer codes in the certification and qualification of rotorcraft for flight in forecast icing. 1994.

36. Coffman, H., Helicopter rotor icing protection methods. Journal of the American Helicopter Society, 1987. 32(2): p. 34-39.

37. Shaw, R.J. and G.P. Richter, The UH-1H helicopter icing flight test program: An overview. 1985.

38. Korkan, K., L. Dadone, and R. Shaw, Helicopter rotor performance degradation in natural icing encounter. Journal of Aircraft, 1984. 21(1): p. 84-85.

39. Leary, W.M., "We Freeze to Please": A History of NASA's Icing Research Tunnel and the Quest for Flight Safety. 2002.

40. Bond, T.H., R.J. Flemming, and R.K. Britton, Icing tests of a model main rotor. 1990.

41. Britton, R.K., T.H. Bond, and R.J. Flemming, An overview of a model rotor icing test in the NASA Lewis Icing Research Tunnel. 1994.

42. Flemming, R.J., R.K. Britton, and T.H. Bond, Model rotor icing tests in the NASA Lewis Icing Research Tunnel. 1991.

43. Robert J. Flemming, T.H.B., Randall K. Britton, Results of a Sub-Scale Model Rotor Icing Test. 1991.

44. Korkan, K., Experimental study of performance degradation of a rotating system in the NASA Lewis RC icing tunnel. 1992.

45. Work, A. and Y. Lian, A Critical Review of the Measurement of Ice Adhesion to Solid Substrates. Progress in Aerospace Sciences, 2018.

46. Gold, L.W., The cracking activity in ice during creep. Canadian Journal of physics, 1960. 38(9): p. 1137-1148.

47. Higuchi, K., A new method for recording the grain structure of ice. J. of Glaciology, 1957. 3: p. 131-132.

48. Penner, E., Ice-grain structure and crystal orientation in an ice lens from Leda clay. Geological Society of America Bulletin, 1961. 72(10): p. 1575-1577. 
49. Quincke, G., The formation of ice and the grained structure of glaciers. Proceedings of the Royal Society of London. Series A, Containing Papers of a Mathematical and Physical Character, 1905. 76(512): p. 431-439.

50. Ashton, G.D., River and lake ice engineering. 1986: Water Resources Publication.

51. Iliescu, D. and I. Baker, The structure and mechanical properties of river and lake ice. Cold regions science and technology, 2007. 48(3): p. 202-217.

52. Michel, B. and R. Ramseier, Classification of river and lake ice. Canadian Geotechnical Journal, 1971. 8(1): p. 36-45.

53. Dempsey, J., et al., Scale effects on the in-situ tensile strength and fracture of ice. Part I: Large grained freshwater ice at Spray Lakes Reservoir, Alberta, in Fracture Scaling. 1999, Springer. p. 325-345.

54. Makkonen, L., Salinity and growth rate of ice formed by sea spray. Cold Regions Science and Technology, 1987. 14(2): p. 163-171.

55. Perovich, D.K., C.S. Roesler, and W.S. Pegau, Variability in Arctic sea ice optical properties. Journal of Geophysical Research: Oceans (1978-2012), 1998. 103(C1): p. 1193-1208.

56. Sinha, N., Technique for studying structure of sea ice. Journal of Glaciology, 1977. 18(79): p. 315-323.

57. Timco, G. and W. Weeks, A review of the engineering properties of sea ice. Cold Regions Science and Technology, 2010. 60(2): p. 107-129.

58. Weeks, W.F. and S.F. Ackley, The growth, structure, and properties of sea ice. 1986: Springer.

59. Schulson, E.M. and P. Duval, Creep and fracture of ice. 2009: Cambridge University Press Cambridge.

60. Druez, J., et al., The adhesion of glaze and rime on aluminum electrical conductors. Transactions of the Canadian Society for Mechanical Engineering, 1979. 5(4): p. 215-220.

61. Fortin, G., Considerations on the Use of Hydrophobic, Superhydrophobic or Icephobic Coatings as a Part of the Aircraft Ice Protection System. 2013, SAE Technical Paper.

62. Koivuluoto, H., et al., Research on icing behavior and ice adhesion testing of icephobic surfaces. IWAIS XVI, June, 2015: p. 6.

63. Laforte, C. and A. Beisswenger. Icephobic material centrifuge adhesion test. in Proc 11th International Workshop on Atmospheric Icing of Structures, Montréal. 2005.

64. Laforte, C., C. Blackburn, and J. Perron, A Review of Icephobic Coating Performances over the Last Decade. 2015, SAE Technical Paper.

65. Baker, H., W.D. Bascom, and C. Singleterry, The adhesion of ice to lubricated surfaces. Journal of colloid science, 1962. 17(5): p. 477-491.

66. Antonini, C., et al., Understanding the effect of superhydrophobic coatings on energy reduction in anti-icing systems. Cold regions science and technology, 2011. 67(1): p. 5867.

67. Farhadi, S., M. Farzaneh, and S. Kulinich, Anti-icing performance of superhydrophobic surfaces. Applied Surface Science, 2011. 257(14): p. 6264-6269.

68. Kulinich, S. and M. Farzaneh, Ice adhesion on super-hydrophobic surfaces. Applied Surface Science, 2009. 255(18): p. 8153-8157.

69. Liu, Y., et al., Controlling drop bouncing using surfaces with gradient features. Applied Physics Letters, 2015. 107(5): p. 051604.

70. Fortin, G. and J. Perron, Ice adhesion models to predict shear stress at shedding. Journal of adhesion science and technology, 2012. 26(4-5): p. 523-553.

71. Guerin, F., et al., Analytical model based on experimental data of centrifuge ice adhesion tests with different substrates. Cold Regions Science and Technology, 2016. 121: p. 93-99.

72. Laforte, C. and J.-L. Laforte, Deicing strains and stresses of iced substrates. Journal of Adhesion Science and Technology, 2012. 26(4-5): p. 603-620. 
73. Meuler, A.J., et al., Relationships between water wettability and ice adhesion. ACS Applied Materials \& Interfaces, 2010. 2(11): p. 3100-3110.

74. Palacios, A.M., J.L. Palacios, and L. Sánchez, Eliciting a human understandable model of ice adhesion strength for rotor blade leading edge materials from uncertain experimental data. Expert Systems with Applications, 2012. 39(11): p. 10212-10225.

75. Phan, C.L., P. McComber, and A. Mansiaux, Adhesion of rime and glaze on conductors protected by various materials. Transactions of the Canadian Society for Mechanical Engineering, 1977. 4(4): p. 204-208.

76. Stallabrass, J. and R. Price, On the adhesion of ice to various materials. 1962, National Research Council Canada.

77. Varanasi, K.K., et al., Frost formation and ice adhesion on superhydrophobic surfaces. Applied Physics Letters, 2010. 97(23): p. 234102.

78. Yeong, Y., et al. Ice adhesion strength on hydrophobic and superhydrophobic coatings. in Proceedings of the 6th AIAA Atmospheric and Space Environments Conference, Atlanta, GA, USA. 2014.

79. Goraj, Z. An overview of the deicing and anti-icing technologies with prospects for the future. in 24th International Congress of the Aeronautical Sciences. 2004.

80. Steen, L.E., et al., NASA Glenn Icing Research Tunnel: 2014 and 2015 Cloud Calibration Procedures and Results. 2015.

81. Kulyakhtin, A., Numerical modelling and experiments on sea spray icing. 2014.

82. Hobbs, P.V., Ice physics. Oxford: Clarendon Press, 1974, 1974. 1.

83. Scavuzzo, R., M. Chu, and V. Ananthaswamy, Influence of aerodynamic forces in ice shedding. Journal of Aircraft, 1994. 31(3): p. 526-530.

84. Beaugendre, H., et al., Computation of ice shedding trajectories using cartesian grids, penalization, and level sets. Modelling and Simulation in Engineering, 2011. 2011: p. 3.

85. Morency, F., H. Beaugendre, and F. Gallizio, Aerodynamic force evaluation for ice shedding phenomenon using vortex in cell scheme, penalisation and level set approaches. International Journal of Computational Fluid Dynamics, 2012. 26(9-10): p. 435-450.

86. Itagaki, K., Mechanical Ice Release Processes. I. Self-Shedding from High-Speed Rotors. 1983, DTIC Document.

87. Chen, Y., et al. Numerical Simulation of Ice Shedding From a Fan Blade. in ASME Turbo Expo 2015: Turbine Technical Conference and Exposition. 2015. American Society of Mechanical Engineers.

88. Chen, Y. and L. Fu, Numerical Simulation of Helicopter Blade Ice Shedding using a Bilinear Cohesive Zone Model. 2015, SAE Technical Paper.

89. Chen, Y., et al. Numerical Study on the Adhesion Strength Between Ice and Aluminium Based on a Cohesive Zone Model. in ASME Turbo Expo 2014: Turbine Technical Conference and Exposition. 2014. American Society of Mechanical Engineers.

90. Libergren, P., De-icing and/or anti-icing of a wind turbine component by vibrating a piezoelectric material. 2011, Google Patents.

91. Jasinski, W.J., et al., Wind turbine performance under icing conditions. Journal of Solar Energy Engineering, 1998. 120(1): p. 60-65.

92. Morgan, C., E. Bossanyi, and H. Seifert. Assessment of safety risks arising from wind turbine icing. in EWEC-CONFERENCE-. 1997. BOOKSHOP FOR SCIENTIFIC PUBLICATIONS.

93. Shiping, Z., et al. Computational modeling of ice cracking and break-up from helicopter blades. in 4th AIAA Atmospheric and Space Environments Conference 2012. 2012.

94. Zhang, S., et al., FEM analysis of in-flight ice break-up. Finite elements in analysis and design, 2012. 57: p. 55-66.

95. Wright, W.B., Further refinement of the LEWICE SLD model. 2006. 
96. Fortin, G. and J. Perron, Spinning rotor blade tests in icing wind tunnel. American Institute of Aeronautics and Astronautics, 1th Atmospheric and Space Environment, San Antonio, Texas, USA, 2009.

97. Scavuzzo, R., M. Chu, and C. Kellackey, Impact ice stresses in rotating airfoils. Journal of Aircraft, 1991. 28(7): p. 450-455.

98. Faraday, P., XXIV. On regelation, and on the conservation of force. The London, Edinburgh, and Dublin Philosophical Magazine and Journal of Science, 1859. 17(113): p. 162-169.

99. Bartels-Rausch, T., et al., A review of air-ice chemical and physical interactions (AICI): liquids, quasi-liquids, and solids in snow. Atmospheric Chemistry and Physics, 2014. 14(3): p. 1587-1633.

100. Domine, F., et al., Can we model snow photochemistry? Problems with the current approaches. The Journal of Physical Chemistry A, 2013. 117(23): p. 4733-4749.

101. Pittenger, B., et al., Premelting at ice-solid interfaces studied via velocity-dependent indentation with force microscope tips. Physical Review B, 2001. 63(13): p. 134102.

102. Smith Jr, J.G., et al., Hydrogen-Bonding Surfaces for Ice Mitigation. 2014.

103. Knuth, T.D., Ice Adhesion Strength Modeling Based on Surface Morphology Variations. 2015, The Pennsylvania State University.

104. Bateup, B.O., Surface chemistry and adhesion. International Journal of Adhesion and Adhesives, 1981. 1(5): p. 233-239.

105. Comyn, J., Contact angles and adhesive bonding. International Journal of Adhesion and Adhesives, 1992. 12(3): p. 145-149.

106. Momen, G., R. Jafari, and M. Farzaneh, Ice repellency behaviour of superhydrophobic surfaces: Effects of atmospheric icing conditions and surface roughness. Applied Surface Science, 2015. 349: p. 211-218.

107. Dotan, A., et al., The relationship between water wetting and ice adhesion. Journal of Adhesion Science and Technology, 2009. 23(15): p. 1907-1915.

108. Dupré, A. and P. Dupré, Théorie mécanique de la chaleur. 1869: Gauthier-Villars.

109. Young, T., An essay on the cohesion of fluids. Philosophical Transactions of the Royal Society of London, 1805: p. 65-87.

110. Schrader, M.E., Young-dupre revisited. Langmuir, 1995. 11(9): p. 3585-3589.

111. Pockels, A., Über Randwinkel und Ausbreitung von Flüssigkeiten auf festen Körpern. Phys. Z, 1914. 15: p. 39-46.

112. Adam, N.K. and G. Jessop, CCL.-Angles of contact and polarity of solid surfaces. Journal of the Chemical Society, Transactions, 1925. 127: p. 1863-1868.

113. Makkonen, L., Surface melting of ice. The Journal of Physical Chemistry B, 1997. 101(32): p. 6196-6200.

114. Bascom, W., R. Cottington, and C. Singleterry, Ice adhesion to hydrophilic and hydrophobic surfaces. The Journal of Adhesion, 1969. 1(4): p. 246-263.

115. Wenzel, R.N., Resistance of solid surfaces to wetting by water. Industrial \& Engineering Chemistry, 1936. 28(8): p. 988-994.

116. Quéré, D., Wetting and roughness. Annu. Rev. Mater. Res., 2008. 38: p. 71-99.

117. Baxter, S. and A. Cassie, 8-The water repellency of fabrics and a new water repellency test. Journal of the Textile Institute Transactions, 1945. 36(4): p. T67-T90.

118. Koishi, T., et al., Coexistence and transition between Cassie and Wenzel state on pillared hydrophobic surface. Proceedings of the National Academy of Sciences, 2009. 106(21): p. 8435-8440.

119. Ran, C., et al., Wetting on nanoporous alumina surface: transition between Wenzel and Cassie states controlled by surface structure. Langmuir, 2008. 24(18): p. 9952-9955. 
120. Bormashenko, E., et al., Why do pigeon feathers repel water? Hydrophobicity of pennae, Cassie-Baxter wetting hypothesis and Cassie-Wenzel capillarity-induced wetting transition. Journal of colloid and interface science, 2007. 311(1): p. 212-216.

121. Liu, G., et al., Water droplet motion control on superhydrophobic surfaces: exploiting the Wenzel-to-Cassie transition. Langmuir, 2011. 27(6): p. 2595-2600.

122. Bormashenko, E., et al., Vibration-induced Cassie-Wenzel wetting transition on rough surfaces. Applied physics letters, 2007. 90(20): p. 201917.

123. Soltis, J., et al., Ice Adhesion Mechanisms of Erosion-Resistant Coatings. AIAA Journal, 2014. 53(3): p. 654-662.

124. Wei, Y., R. Adamson, and J. Dempsey, Ice/metal interfaces: fracture energy and fractography. Journal of materials science, 1996. 31(4): p. 943-947.

125. Jiang, K. and L. Penn, Use of the Blister Test to Study the Adhesion of Brittle Materials. Part I. Test Modification and Validation. The Journal of Adhesion, 1990. 32(4): p. 203216.

126. Jiang, K. and L. Penn, Use of the Blister Test to Study the Adhesion of Brittle Materials. Part II. Application. The Journal of Adhesion, 1990. 32(4): p. 217-226.

127. Gohardani, O., The exploration of icephobic materials and their future prospects in aircraft icing applications. Journal of Aeronautics \& Aerospace Engineering, 2013. 2012.

128. Stone, H.A., Ice-phobic surfaces that are wet. ACS nano, 2012. 6(8): p. 6536-6540.

129. Mishchenko, L., et al., Design of ice-free nanostructured surfaces based on repulsion of impacting water droplets. ACS nano, 2010. 4(12): p. 7699-7707.

130. Yeong, Y.H., et al., Atmospheric Ice Adhesion on Water-Repellent Coatings: Wetting and Surface Topology Effects. Langmuir, 2015. 31(48): p. 13107-13116.

131. Tarquini, S., et al., Investigation of ice shedding properties of superhydrophobic coatings on helicopter blades. Cold regions science and technology, 2014. 100: p. 50-58.

132. Scavuzzo, R. and M.L. Chu, Structural properties of impact ices accreted on aircraft structures. 1987.

133. Xian, X., et al., An experimental evaluation of the tensile strength of impact ice. Journal of materials science letters, 1989. 8(10): p. 1205-1208.

134. Petrovic, J., Review mechanical properties of ice and snow. Journal of materials science, 2003. 38(1): p. 1-6.

135. Schulson, E.M., The structure and mechanical behavior of ice. JOM, 1999. 51(2): p. 2127.

136. Lobban, C., J.L. Finney, and W.F. Kuhs, The structure of a new phase of ice. Nature, 1998. 391(6664): p. 268-270.

137. Cole, D.M., The microstructure of ice and its influence on mechanical properties. Engineering Fracture Mechanics, 2001. 68(17): p. 1797-1822.

138. Myers, T. and D. Hammond, Ice and water film growth from incoming supercooled droplets. International Journal of Heat and Mass Transfer, 1999. 42(12): p. 2233-2242.

139. Myers, T.G., Extension to the Messinger model for aircraft icing. AIAA journal, 2001. 39(2): p. 211-218.

140. Wilson, C., Texture and grain growth during the annealing of ice. Texture, Stress, and Microstructure, 1982. 5(1): p. 19-31.

141. Rothrick, A. and R. Selden, Adhesion of Ice in Its Relation to the De-icing of Airplanes. 1939.

142. Loughborough, D.L. and E.G. Haas, Reduction of the adhesion of ice to de-icer surfaces. Journal of the Aeronautical Sciences, 1946.

143. Druez, J., D. Nguyen, and Y. Lavoie, Mechanical properties of atmospheric ice. Cold Regions Science and Technology, 1986. 13(1): p. 67-74.

144. Zou, M., et al., Effects of surface roughness and energy on ice adhesion strength. Applied Surface Science, 2011. 257(8): p. 3786-3792. 
145. Petrenko, V. and S. Peng, Reduction of ice adhesion to metal by using self-assembling monolayers (SAMs). Canadian journal of physics, 2003. 81(1-2): p. 387-393.

146. Beams, J., J. Breazeale, and W. Bart, Mechanical strength of thin films of metals. Physical Review, 1955. 100(6): p. 1657.

147. Raraty, L. and D. Tabor. The adhesion and strength properties of ice. in Proceedings of the Royal Society of London A: Mathematical, Physical and Engineering Sciences. 1958. The Royal Society.

148. Ackley, S., et al., Laboratory experiments on icing of rotating blades. Transportation Research Board Special Report, 1979(185).

149. Han, Y., J. Palacios, and S. Schmitz, Scaled ice accretion experiments on a rotating wind turbine blade. Journal of Wind Engineering and Industrial Aerodynamics, 2012. 109: p. 55-67.

150. Han, Y., J.L. Palacios, and E.C. Smith, An experimental correlation between rotor test and wind tunnel ice shapes on NACA 0012 airfoils. 2011, SAE Technical Paper.

151. Overmeyer, A., et al., Rotating testing of a low-power, non-thermal ultrasonic de-icing system for helicopter rotor blades. 2011, SAE Technical Paper.

152. Laforte, C. and J.-L. Laforte. Tensile, Torsional and Bending Strain at the Adhesive Rupture of an Iced Substrate. in ASME 2009 28th International Conference on Ocean, Offshore and Arctic Engineering. 2009. American Society of Mechanical Engineers.

153. Beisswenger, A., G. Fortin, and C. Laforte, Advances in Ice Adherence and Accumulation Reduction Testing at the Anti-Icing Materials International Laboratory (AMIL). Future De-Icing Technologies, Berlin. 2010.

154. Laforte, J., M. Allaire, and J. Laflamme, State-of-the-art on power line de-icing. Atmospheric Research, 1998. 46(1): p. 143-158.

155. Schulz, M. and M. Sinapius, Evaluation of Different Ice Adhesion Tests for Mechanical Deicing Systems. 2015, SAE Technical Paper.

156. Soltis, J., et al., Evaluation of Ice-Adhesion Strength on Erosion-Resistant Materials. AIAA Journal, 2014. 53(7): p. 1825-1835.

157. Oksanen, P., Friction and adhesion of ice. 1983.

158. Haehnel, R. and N. Mulherin. The bond strength of an ice-solid interface loaded in shear. in Ice in Surface Waters, Proceedings of the 14th International Symposium on Ice. 1998.

159. Reinmann, J., R. Shaw, and W.A. Olsen Jr, Aircraft icing research at NASA. 1982.

160. Lee, S., et al., Development of 3D Ice Accretion Measurement Method. AIAA Paper, 2012. 2938: p. 2012.

161. Lee, S., et al. Implementation and Validation of 3-D Ice Accretion Measurement Methodology. in 6th AIAA Atmospheric and Space Environments Conference, June. 2014.

162. Munson, B.R., D.F. Young, and T.H. Okiishi, Fundamentals of fluid mechanics. New York, 1990. 3(4).

163. Steel, A. 17-4 PH Stainless Steel Product Data Bulletin. 2007 [cited 2017 5/9/17]; Available from: http://www.aksteel.com/pdf/markets_products/stainless/precipitation/174_ph_data_bulletin.pdf.

164. Steel, A., 304/304L Stainless Steel Product Data Sheet. 2007.

165. SSIC Stainless Steel Fasteners. 2017.

166. ASTM, Standard Specification for Precipitation Hardening Iron Base Superalloy Bars, Forgings, and Forging Stock for High-Temperature Service. 2013, ASTM.

167. Kreeger, R.E., et al., Analysis and Prediction of Ice Shedding for a Full-Scale Heated Tail Rotor. 2016.

168. Kreeger, R.E. and J.-C. Tsao. Ice Shapes on a Tail Rotor. in 6th AIAA Atmospheric and Space Environments Conference, June. 2014.

169. Otsu, N., A threshold selection method from gray-level histograms. Automatica, 1975. 11(285-296): p. 23-27. 
170. Tropea, C., A.L. Yarin, and J.F. Foss, Springer handbook of experimental fluid mechanics. Vol. 1. 2007: Springer Science \& Business Media.

171. Qi, H. and M. Boyce, Stress-strain behavior of thermoplastic polyurethanes. Mechanics of Materials, 2005. 37(8): p. 817-839.

172. Raefsky, M., Fatigue Properties of 17-4 Ph and 15-5 Ph Steel in the H-900 AND H-1050 Condition. 1968, DTIC Document. 


\section{APPENDIX A}

TEST MATRICES 
Table 13. Piggyback tests, 5/31/16, 8/15/16

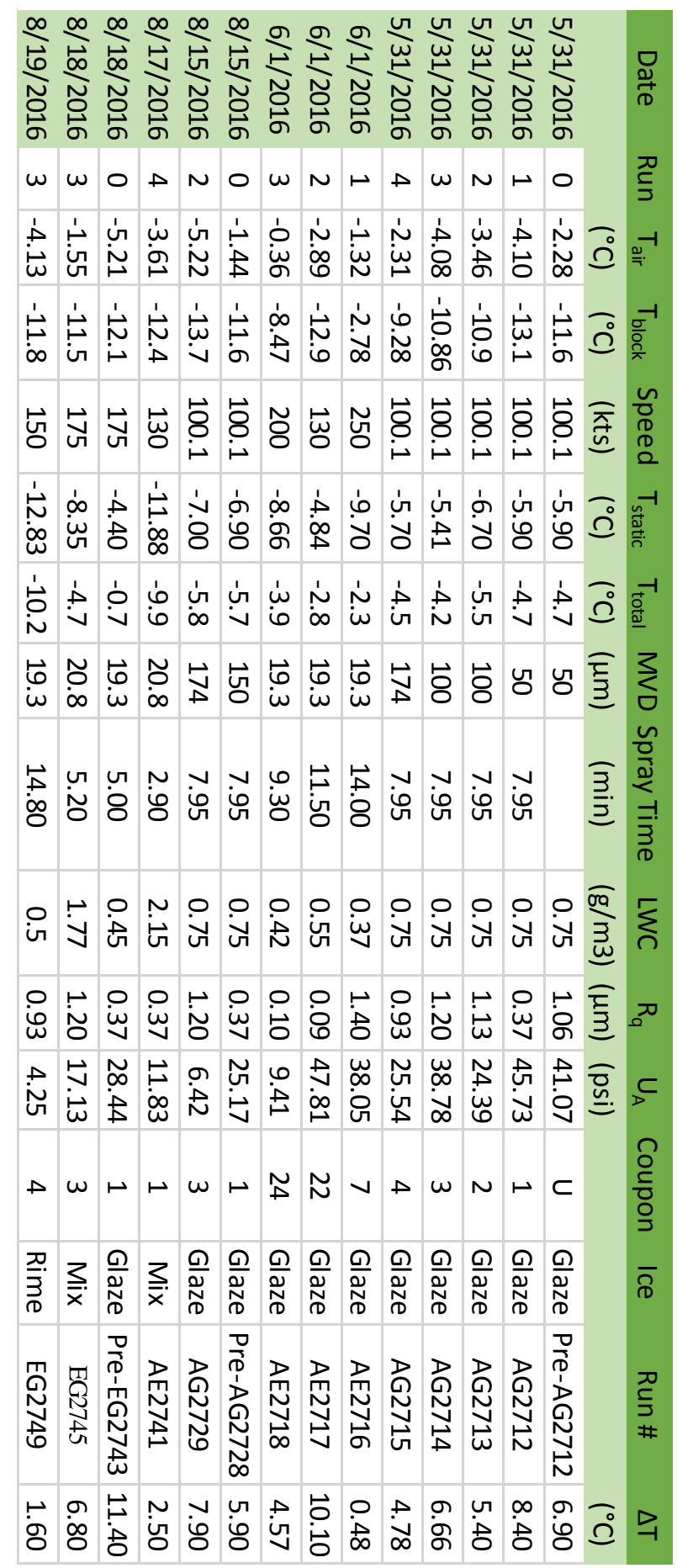


Table 14. IRT testing tunnel parameters, 10/2/17, 11/20/17, 1/17/18. AG: 4 sprays, 10, 20, 30, 60 s. AH: 2 sprays, 30, 120 s. AI: 2 sprays, 30, 150 s. AG, AH, AI: averaged data for first spray only.

\begin{tabular}{|c|c|c|c|c|c|c|c|c|c|}
\hline Date & Test & $\begin{array}{l}\text { Cloud } \\
\text { On }\end{array}$ & $\begin{array}{l}\text { Cloud } \\
\text { Off }\end{array}$ & $\begin{array}{c}\text { Avg. } \\
\text { Velocity }\end{array}$ & $\begin{array}{l}\text { Avg. } \\
T_{\text {total }}\end{array}$ & $\begin{array}{l}\text { Avg. } \\
T_{\text {static }}\end{array}$ & $\begin{array}{l}\text { Avg. } \\
\text { LWC }\end{array}$ & $\begin{array}{l}\text { Avg. } \\
\text { MVD }\end{array}$ & Pulse \\
\hline & & & & (knots) & $\left({ }^{\circ} \mathrm{C}\right)$ & $\left({ }^{\circ} \mathrm{C}\right)$ & $\left(\mathrm{g} / \mathrm{m}^{3}\right)$ & $(\mu \mathrm{M})$ & \\
\hline $10 / 2 / 17$ & $A$ & $17: 50: 45$ & $17: 53: 30$ & 124.0 & -7.075 & -9.102 & 1.519 & 24.06 & \\
\hline $10 / 2 / 17$ & B & $19: 21: 48$ & $19: 24: 22$ & 148.2 & -7.072 & -9.966 & 1.348 & 20.05 & \\
\hline $10 / 2 / 17$ & C & $20: 27: 18$ & $20: 29: 55$ & 173.1 & -7.066 & -11.01 & 1.222 & 20.05 & \\
\hline $10 / 2 / 17$ & D & 21:09:56 & $21: 12: 32$ & 198.2 & -7.037 & -12.21 & 1.125 & 20.02 & \\
\hline $10 / 3 / 17$ & $E$ & $16: 10: 58$ & $16: 13: 02$ & 148.4 & -7.13 & -10.03 & 1.415 & 15.06 & \\
\hline $10 / 3 / 17$ & $\mathrm{~F}$ & 17:01:01 & $17: 04: 02$ & 148.1 & -7.079 & -9.968 & 1.41 & 29.93 & \\
\hline $10 / 3 / 17$ & G & $17: 57: 21$ & $18: 00: 21$ & 148.8 & -7.087 & -10.00 & 1.408 & 50.08 & \\
\hline $10 / 3 / 17$ & $\mathrm{H}$ & $18: 52: 01$ & $18: 55: 00$ & 149.9 & -7.072 & -10.03 & 1.003 & 181.0 & \\
\hline $10 / 3 / 17$ & 1 & $19: 37: 00$ & $19: 40: 00$ & 149.7 & -7.053 & -10.00 & 1.413 & 25.13 & \\
\hline $10 / 3 / 17$ & J & $0: 20: 42$ & $20: 45: 41$ & 147.1 & -7.062 & -9.915 & 1.417 & 39.90 & \\
\hline $11 / 20 / 17$ & K & $16: 25: 25$ & $16: 27: 37$ & 131.1 & -15.05 & -17.49 & 1.416 & 19.95 & \\
\hline $11 / 20 / 17$ & $\mathrm{~L}$ & $17: 23: 19$ & $17: 25: 20$ & 149.3 & -15.12 & -18.06 & 1.336 & 20.00 & \\
\hline $11 / 20 / 17$ & $M$ & $18: 34: 05$ & $18: 38: 22$ & 148.7 & -13.93 & -16.85 & 1.341 & 20.01 & \\
\hline $11 / 20 / 17$ & $\mathrm{~N}$ & $19: 25: 20$ & $19: 28: 41$ & 198.8 & -11.34 & -16.54 & 1.121 & 20.00 & \\
\hline $11 / 20 / 17$ & 0 & $20: 29: 07$ & $20: 32: 27$ & 148.3 & -7.030 & -9.930 & 1.344 & 20.02 & \\
\hline $11 / 20 / 17$ & $P$ & 21:21:06 & $21: 24: 41$ & 148.2 & -7.040 & -9.930 & 1.342 & 20.00 & \\
\hline $11 / 21 / 17$ & $R$ & $16: 08: 12$ & $16: 10: 49$ & 148.3 & -9.990 & -12.89 & 1.408 & 15.04 & \\
\hline $11 / 21 / 17$ & $S$ & $16: 53: 09$ & $16: 55: 42$ & 147.8 & -10.02 & -12.90 & 1.412 & 29.93 & \\
\hline $11 / 21 / 17$ & $\mathrm{~T}$ & 17:39:07 & $17: 42: 06$ & 148.7 & -9.970 & -12.90 & 1.408 & 50.06 & \\
\hline $11 / 21 / 17$ & $U$ & $18: 32: 07$ & $18: 35: 05$ & 148.1 & -9.950 & -12.80 & 1.41 & 29.94 & \\
\hline $11 / 21 / 17$ & V & $19: 23: 56$ & $19: 26: 55$ & 148.6 & -9.980 & -12.90 & 1.409 & 24.94 & \\
\hline $11 / 21 / 17$ & W & $20: 17: 43$ & $20: 26: 57$ & 150.3 & -9.930 & -12.90 & 1.397 & 29.85 & $x$ \\
\hline $11 / 21 / 17$ & $x$ & 21:07:05 & $21: 15: 38$ & 150.2 & -10.00 & -13.00 & 1.398 & 29.89 & $x$ \\
\hline $1 / 17 / 18$ & AA & $16: 25: 37$ & $16: 28: 36$ & 136.2 & -4.988 & -7.431 & 1.416 & 19.96 & \\
\hline $1 / 17 / 18$ & $A B$ & $17: 30: 04$ & $17: 33: 03$ & 148.3 & -8.010 & -10.91 & 1.343 & 20.01 & \\
\hline $1 / 17 / 18$ & $A C$ & $18: 20: 55$ & $18: 23: 53$ & 173.0 & -7.931 & -11.87 & 1.221 & 20.03 & \\
\hline $1 / 17 / 18$ & $A D$ & 19:10:46 & $19: 13: 45$ & 197.8 & -8.003 & -13.16 & 1.126 & 20.02 & \\
\hline $1 / 17 / 18$ & $\mathrm{AE}$ & $20: 19: 12$ & $20: 20: 50$ & 172.8 & -8.019 & -11.95 & 1.221 & 20.03 & \\
\hline $1 / 17 / 18$ & $\mathrm{AF}$ & 21:01:59 & 21:03:57 & 160.6 & -8.015 & -11.41 & 1.280 & 20.04 & \\
\hline $1 / 18 / 18$ & $A G$ & $16: 07: 14$ & $17: 22: 58$ & 151.0 & -8.021 & -11.02 & 1.393 & 29.86 & $x$ \\
\hline $1 / 18 / 18$ & $\mathrm{AH}$ & 18:08:19 & 18:38:11 & 147.5 & -8.066 & -10.93 & 1.414 & 29.92 & $x$ \\
\hline $1 / 18 / 18$ & $\mathrm{Al}$ & $19: 55: 19$ & $20: 20: 48$ & 148.3 & -8.036 & -10.93 & 1.410 & 49.98 & $x$ \\
\hline $1 / 18 / 18$ & AJ & $21: 15: 40$ & $21: 17: 25$ & 149.8 & -30.10 & -33.05 & 1.405 & 25.01 & \\
\hline
\end{tabular}




\section{APPENDIX B}

Roughness Frequency and Angular Data

Table 15. Frequency data for the first five extracted points of all roughness scans.

\begin{tabular}{|c|c|c|c|c|c|c|c|c|c|c|}
\hline \multicolumn{11}{|c|}{ Frequency $(1 / \mathrm{mm})$} \\
\hline & S1 & S2 & S3 & S4 & S5 & S6 & S7 & S8 & S9 & S10 \\
\hline \multirow[t]{5}{*}{ G1 } & & 1.28 & 0.71 & 6.41 & 0.43 & 0.57 & 4.27 & 0.43 & 6.41 & 0.43 \\
\hline & & 6.86 & 1.14 & 5.98 & 1.71 & 0.85 & 11.97 & 1.28 & 1.03 & 1.28 \\
\hline & & 14.09 & 4.19 & 8.11 & 4.27 & 0.43 & 0.85 & 6.83 & 0.85 & 8.54 \\
\hline & & 9.82 & 4.34 & 0.43 & 9.39 & 3.42 & 6.83 & 1.71 & 8.11 & 8.97 \\
\hline & & 8.11 & 3.12 & 1.71 & 2.21 & 10.82 & 0.43 & 1.40 & 1.71 & 1.80 \\
\hline \multirow[t]{5}{*}{ G2 } & 4.27 & 0.85 & 9.73 & 0.57 & 0.43 & 0.43 & 0.43 & 1.14 & 0.57 & 0.43 \\
\hline & 0.85 & 2.99 & 3.12 & 1.14 & 6.83 & 0.71 & 3.42 & 0.43 & 1.14 & 1.71 \\
\hline & 1.40 & 1.28 & 1.22 & 1.71 & 0.85 & 0.71 & 0.85 & 1.71 & 5.98 & 3.42 \\
\hline & 1.03 & 9.39 & 3.65 & 5.98 & 1.71 & 1.03 & 5.98 & 1.71 & 0.43 & 1.71 \\
\hline & 3.84 & 8.99 & 1.42 & 8.11 & 7.71 & 1.22 & 8.54 & 1.40 & 3.42 & 3.84 \\
\hline \multirow[t]{5}{*}{ G3 } & & 12.81 & 18.24 & 64.04 & 6.40 & 6.40 & 6.40 & 44.83 & 25.61 & 12.81 \\
\hline & & 19.21 & 10.67 & 32.02 & 12.81 & 19.21 & 12.81 & 76.84 & 83.25 & 6.40 \\
\hline & & 25.61 & 8.54 & 38.42 & 25.61 & 25.61 & 64.04 & 19.21 & 70.44 & 32.02 \\
\hline & & 115.27 & 44.57 & 44.83 & 51.23 & 32.02 & 76.84 & 32.02 & 108.86 & 44.83 \\
\hline & & 96.06 & 36.48 & 57.63 & 32.02 & 12.81 & 44.83 & 115.27 & 89.65 & 25.61 \\
\hline \multirow[t]{5}{*}{ G4 } & & 76.84 & 18.24 & 12.81 & 6.40 & 17.08 & 19.21 & 6.40 & 38.42 & 19.21 \\
\hline & & 96.06 & 26.40 & 6.40 & 12.81 & 6.40 & 12.81 & 26.40 & 19.21 & 51.23 \\
\hline & & 12.81 & 28.64 & 19.21 & 25.61 & 8.54 & 6.40 & 18.24 & 12.81 & 115.27 \\
\hline & & 57.63 & 10.67 & 57.63 & 32.02 & 12.81 & 64.04 & 25.61 & 25.61 & 76.84 \\
\hline & & 32.02 & 10.67 & 134.48 & 57.63 & 25.61 & 32.02 & 19.21 & 76.84 & 96.06 \\
\hline
\end{tabular}


Table 16. Angular data for the first five extracted points of all roughness scans.

\begin{tabular}{|c|c|c|c|c|c|c|c|c|c|c|}
\hline \multicolumn{11}{|c|}{ Angle (rad) } \\
\hline Group & S1 & S2 & S3 & S4 & S5 & S6 & S7 & S8 & S9 & S10 \\
\hline \multirow[t]{5}{*}{ G1 } & & 0.00 & 0.93 & 0.00 & 0.00 & 1.57 & 0.00 & 0.00 & 0.00 & 0.00 \\
\hline & & 3.06 & 1.57 & 0.00 & 0.00 & 0.00 & 0.05 & 0.00 & 2.55 & 0.00 \\
\hline & & 0.00 & 1.26 & 0.00 & 0.00 & 0.00 & 0.00 & 0.00 & 0.00 & 0.00 \\
\hline & & 0.00 & 1.17 & 0.00 & 0.00 & 1.57 & 0.00 & 0.00 & 0.00 & 0.00 \\
\hline & & 0.00 & 1.15 & 0.00 & 0.26 & 1.57 & 0.00 & 2.72 & 0.00 & 2.82 \\
\hline \multirow[t]{5}{*}{ G2 } & 0.00 & 0.00 & 1.21 & 1.57 & 0.00 & 0.00 & 0.00 & 1.57 & 1.57 & 0.00 \\
\hline & 0.00 & 0.00 & 1.15 & 1.57 & 0.00 & 0.93 & 0.00 & 0.00 & 1.57 & 1.57 \\
\hline & 0.42 & 0.00 & 1.21 & 0.00 & 0.00 & 2.21 & 0.00 & 1.57 & 0.00 & 0.00 \\
\hline & 0.59 & 0.00 & 1.21 & 0.00 & 0.00 & 0.59 & 0.00 & 0.73 & 0.00 & 0.00 \\
\hline & 0.00 & 0.06 & 0.93 & 0.00 & 0.07 & 1.93 & 0.00 & 0.42 & 0.00 & 0.00 \\
\hline \multirow[t]{5}{*}{ G3 } & & 0.00 & 1.21 & 0.00 & 0.00 & 0.00 & 0.00 & 0.00 & 0.00 & 0.00 \\
\hline & & 0.00 & 0.93 & 0.00 & 0.00 & 0.00 & 0.00 & 0.00 & 0.00 & 0.00 \\
\hline & & 0.00 & 1.57 & 0.00 & 0.00 & 0.00 & 0.00 & 0.00 & 0.00 & 0.00 \\
\hline & & 0.00 & 1.28 & 0.00 & 0.00 & 0.00 & 0.00 & 0.00 & 0.00 & 0.00 \\
\hline & & 0.00 & 1.21 & 0.00 & 0.00 & 0.00 & 0.00 & 0.00 & 0.00 & 0.00 \\
\hline \multirow[t]{5}{*}{ G4 } & & 0.00 & 1.21 & 0.00 & 0.00 & 1.57 & 0.00 & 0.00 & 0.00 & 0.00 \\
\hline & & 0.00 & 1.33 & 0.00 & 0.00 & 0.00 & 0.00 & 1.82 & 0.00 & 0.00 \\
\hline & & 0.00 & 1.11 & 0.00 & 0.00 & 1.57 & 0.00 & 1.93 & 0.00 & 0.00 \\
\hline & & 0.00 & 2.21 & 0.00 & 0.00 & 0.00 & 0.00 & 1.57 & 0.00 & 0.00 \\
\hline & & 0.00 & 0.93 & 0.00 & 0.00 & 1.57 & 0.00 & 0.00 & 0.00 & 0.00 \\
\hline
\end{tabular}




\section{Roughness Plots}
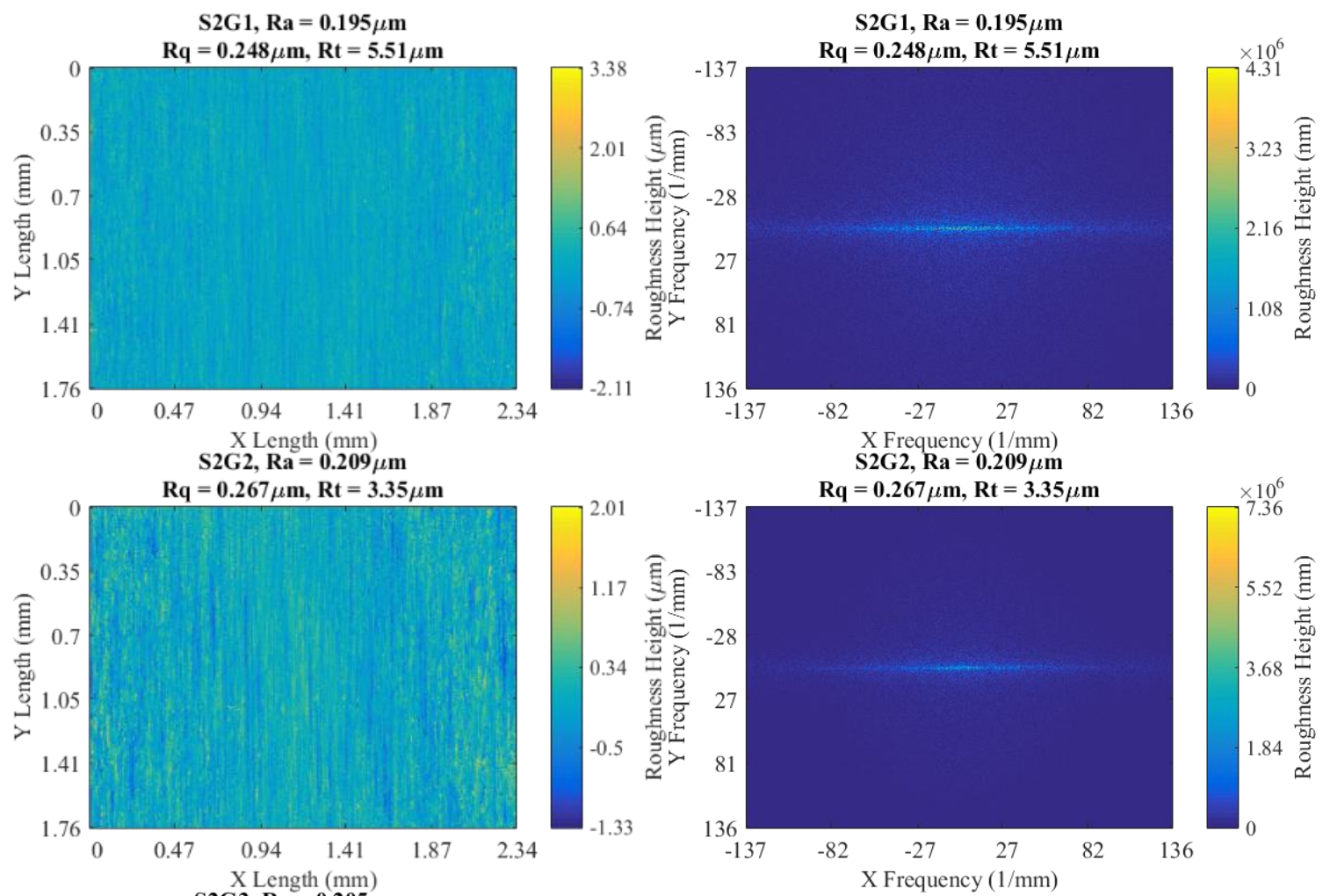

$\mathbf{S 2 G} 2, \mathbf{R a}=0.209 \mu \mathrm{m}$
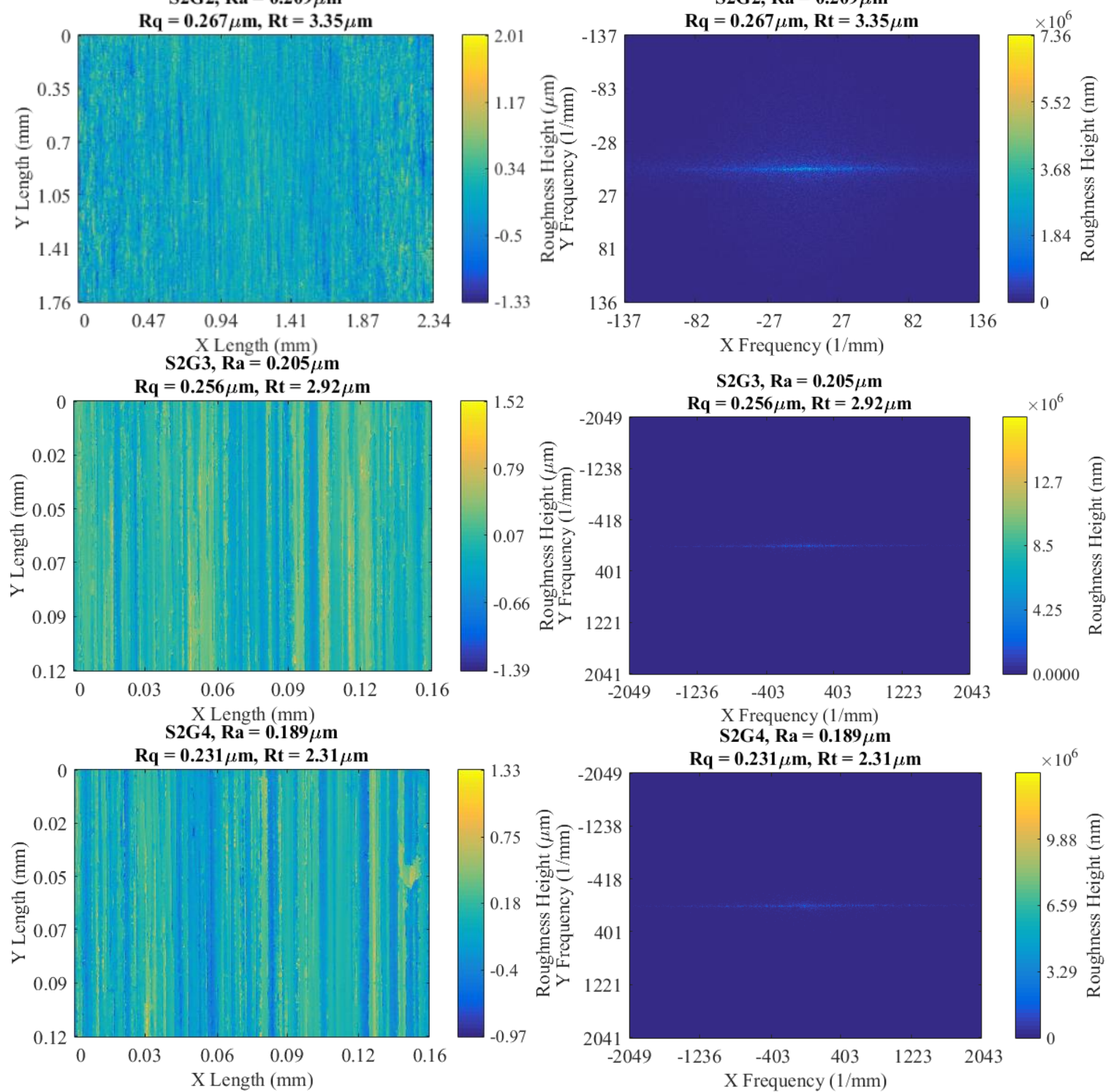

$\mathrm{S} 2 \mathrm{G} 4, \mathrm{Ra}=0.189 \mu \mathrm{m}$

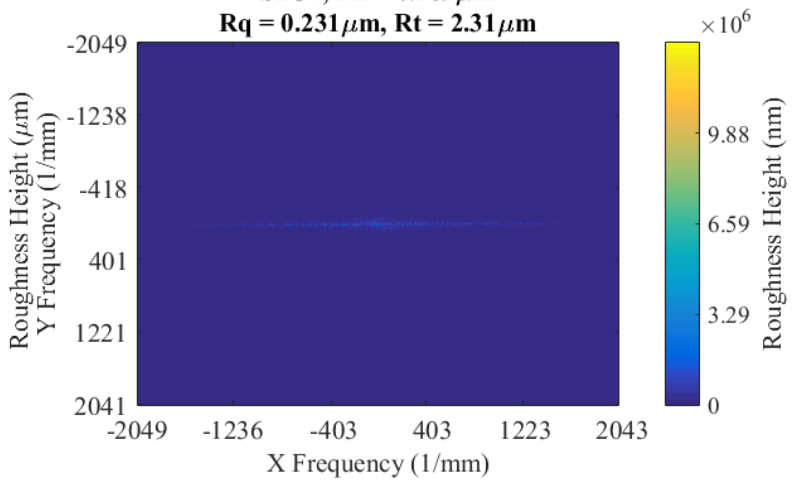



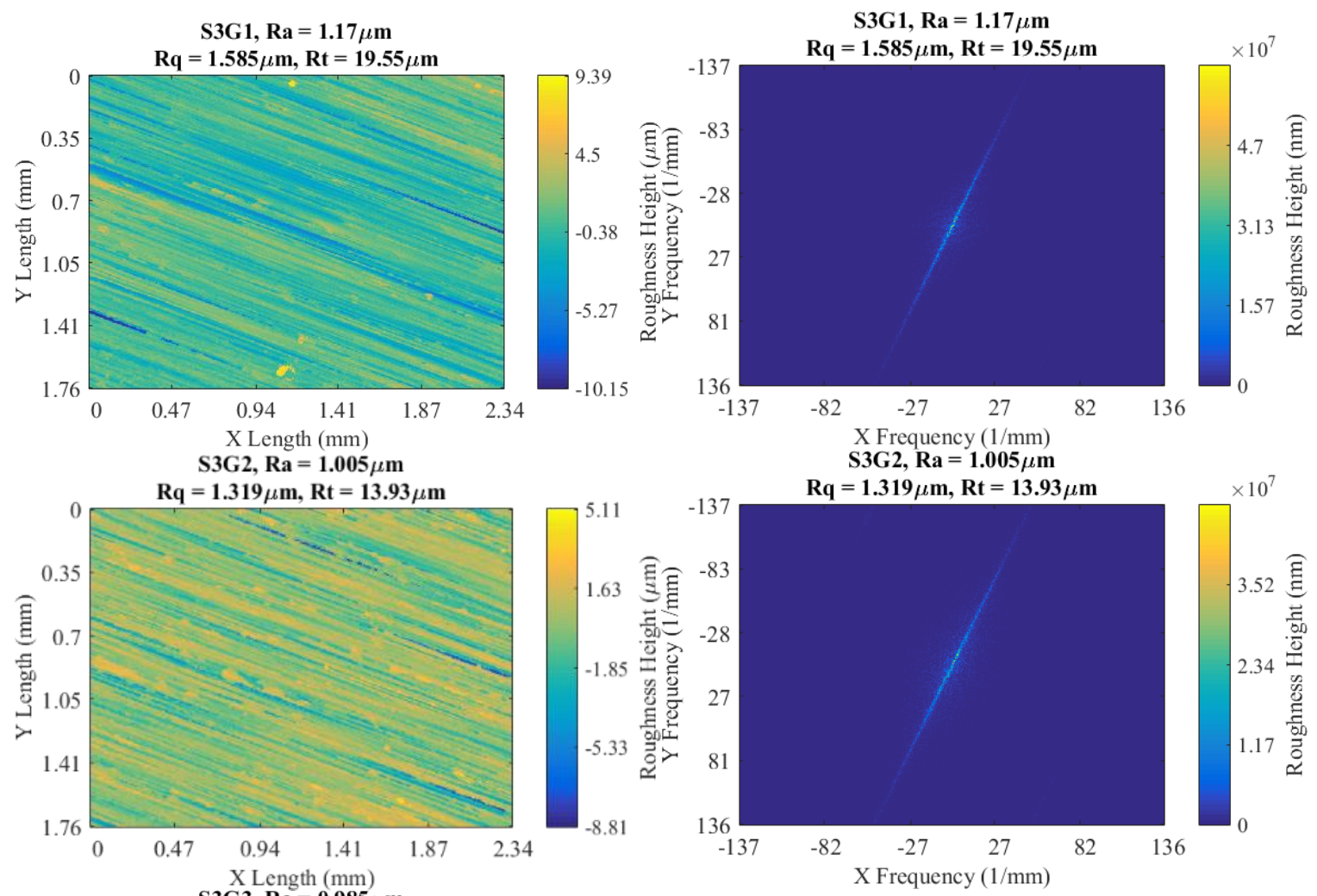

S3G $2, \mathbf{R a}=1.005 \mu \mathrm{m}$

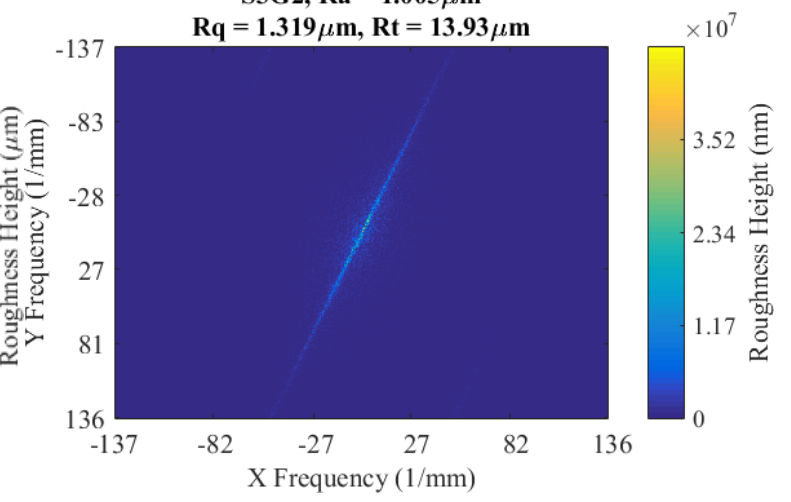

S3G3, Ra $=0.985 \mu \mathrm{m}$

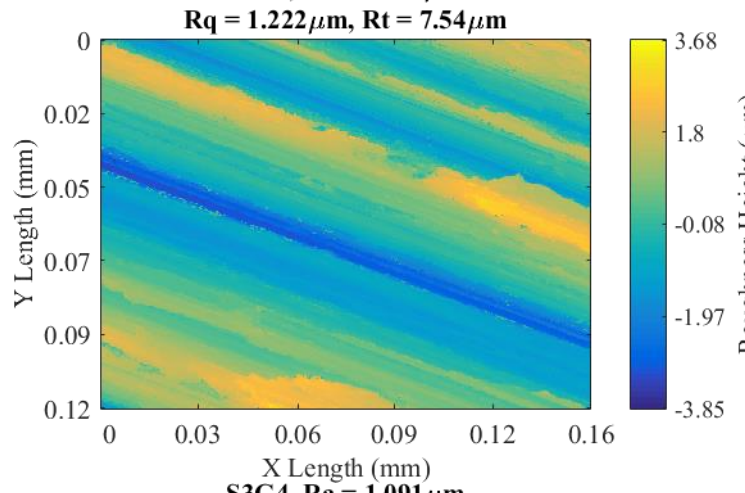

3G3, $\mathrm{Ra}=0.985 \mu \mathrm{m}$
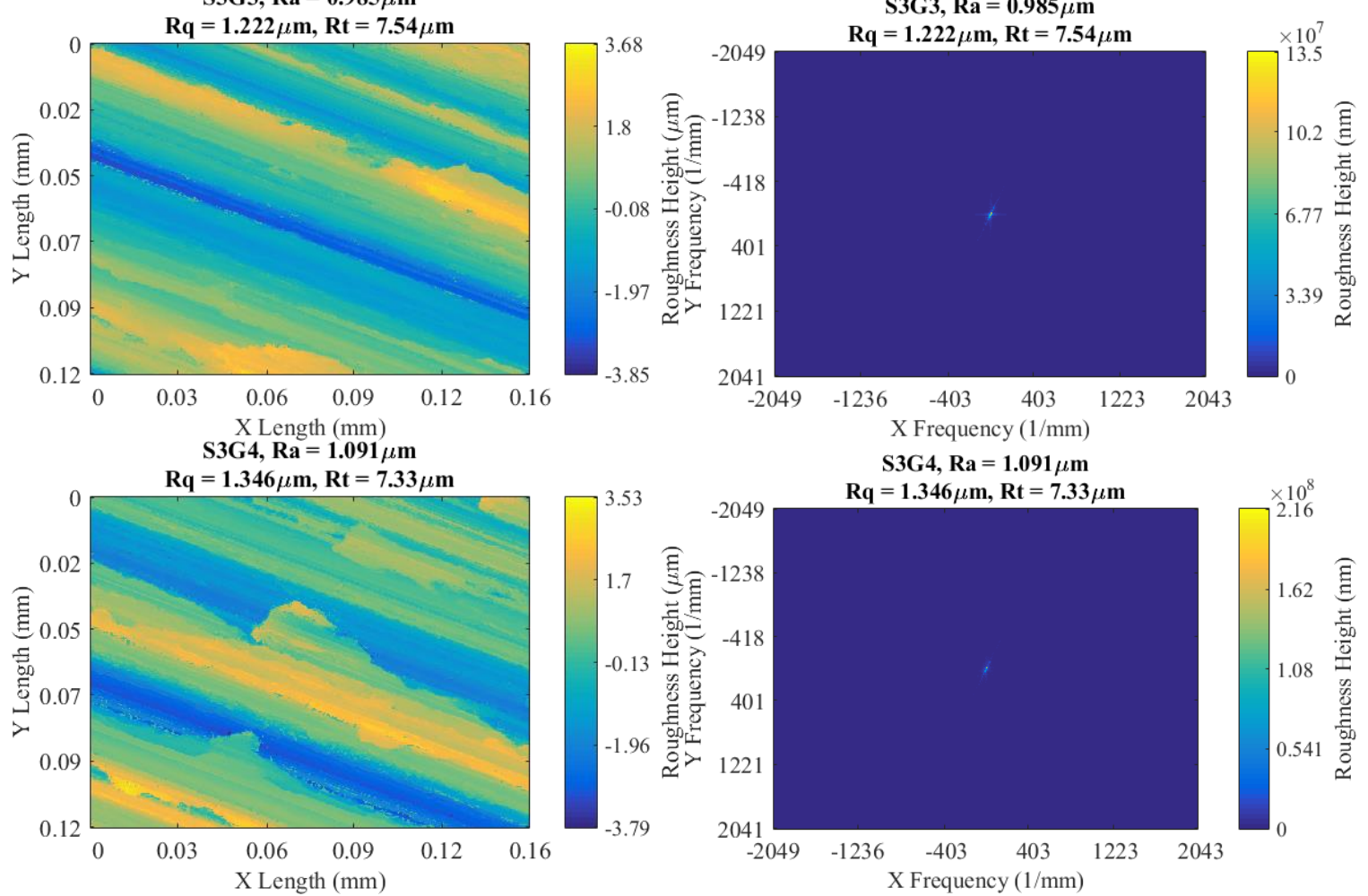

S3G4, Ra $=1.091 \mu \mathrm{m}$

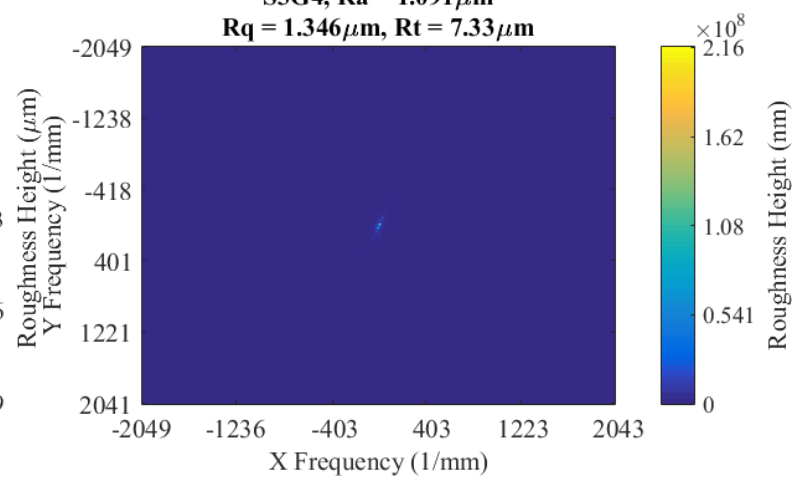



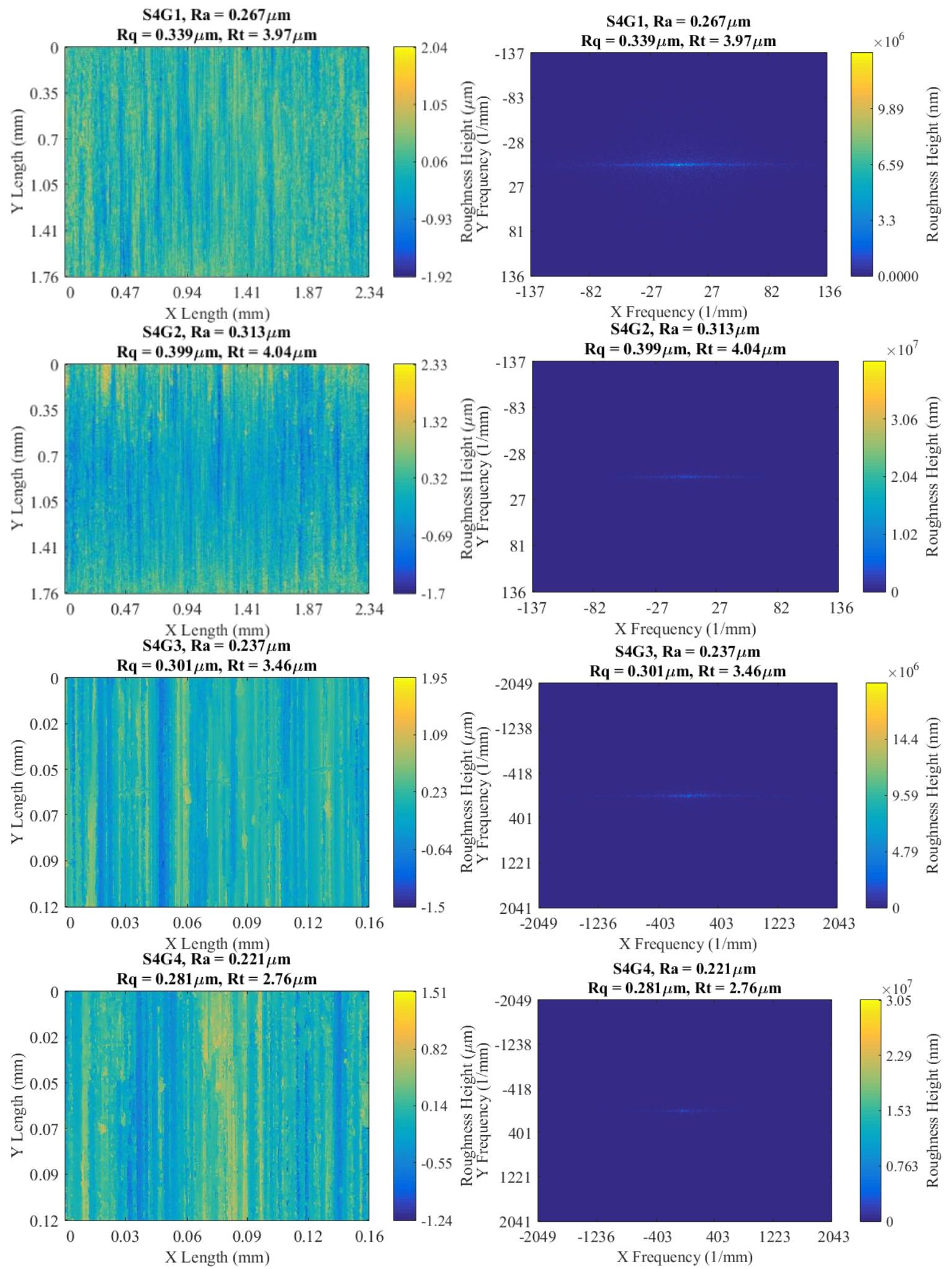

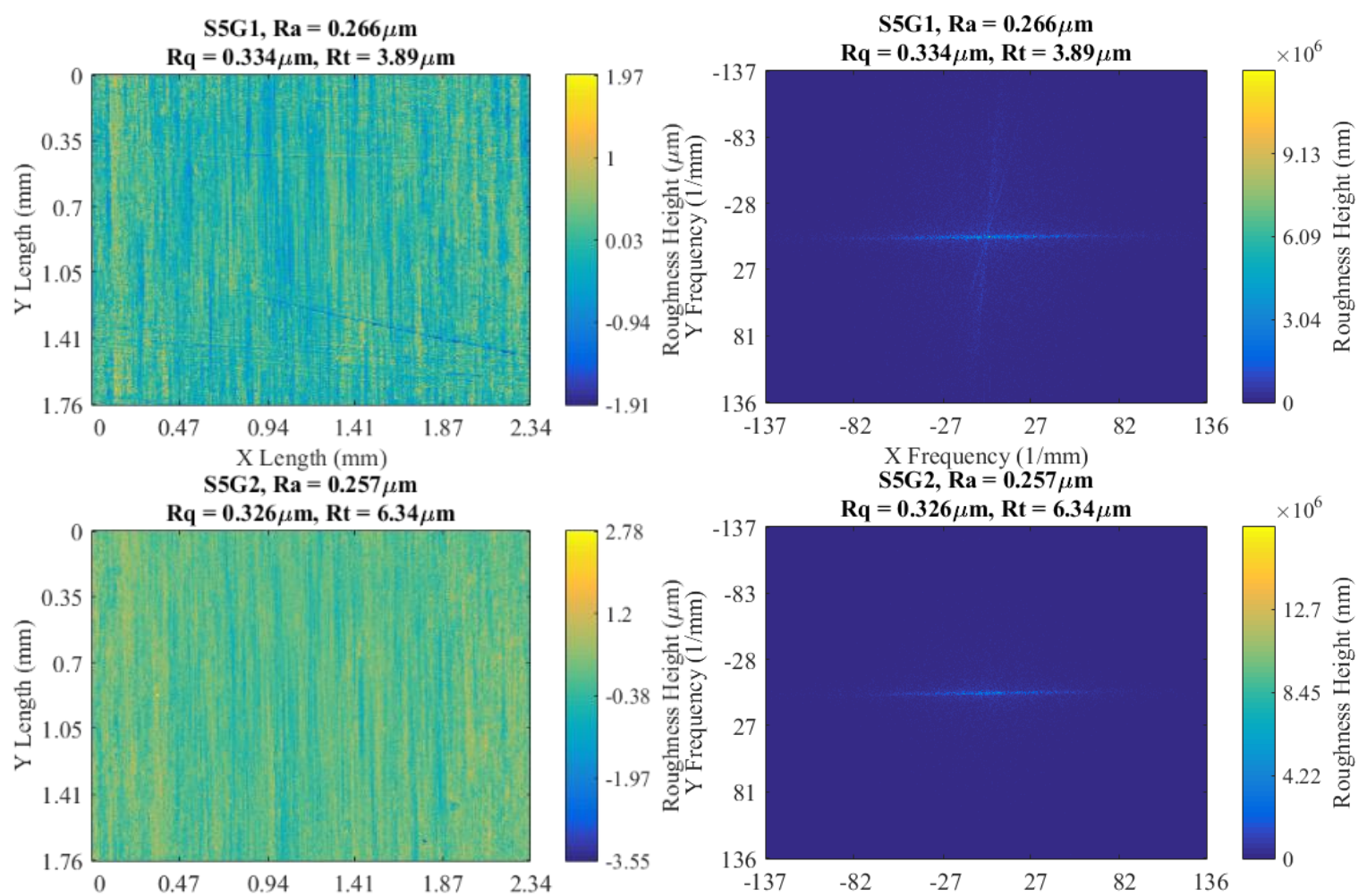

55 G2,

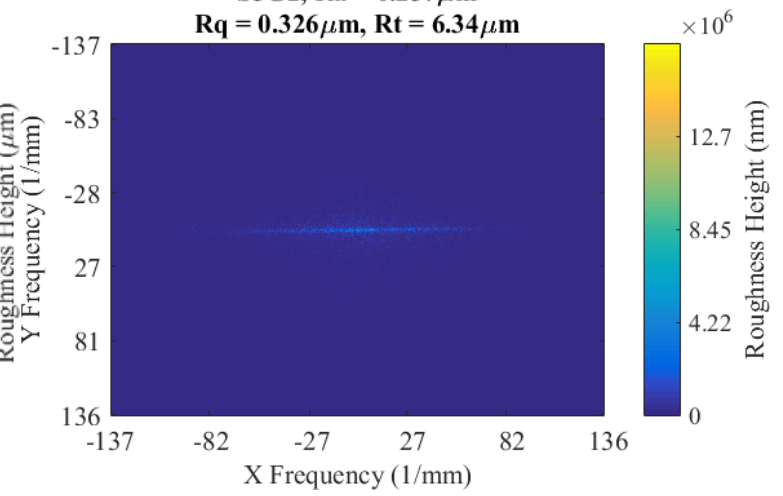

X Length $(\mathrm{mm})$
S5G3, Ra $=\mathbf{0 . 2 2 5} \mu \mathbf{m}$

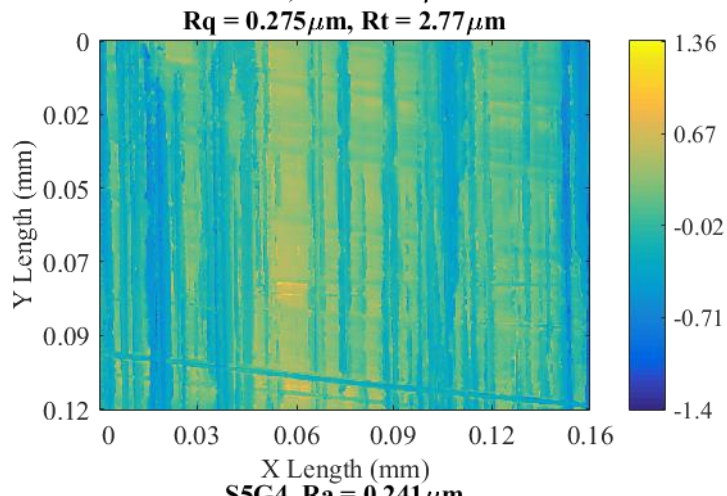

S5G3, Ra $=0.225 \mu \mathrm{m}$

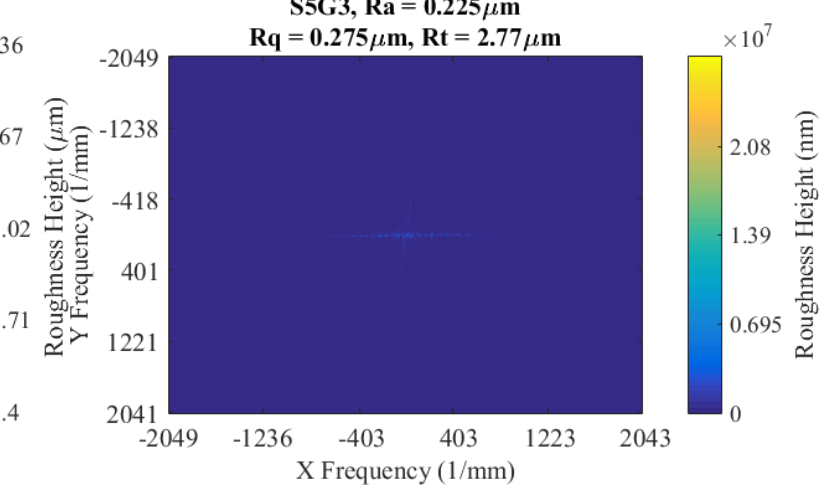

S5G4, Ra $=0.241 \mu \mathrm{m}$

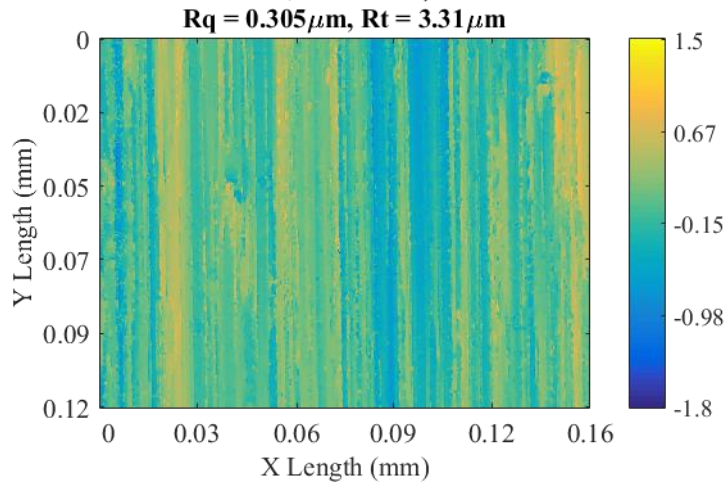

S5G4, Ra $=0.241 \mu \mathrm{m}$

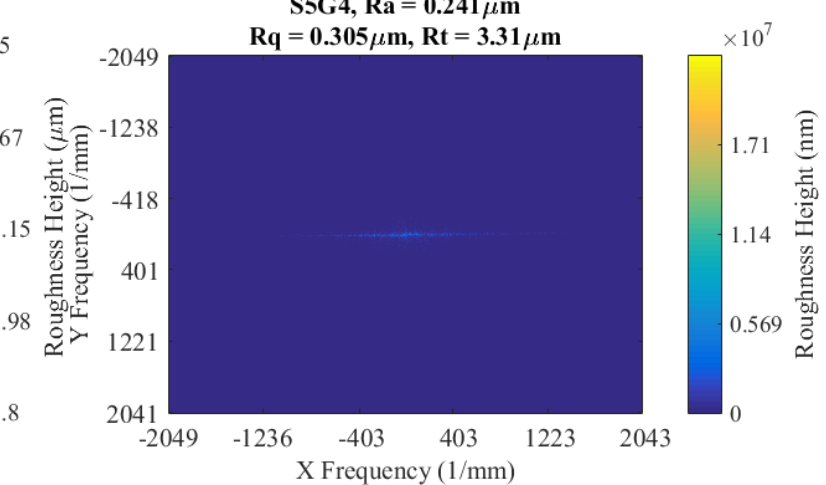



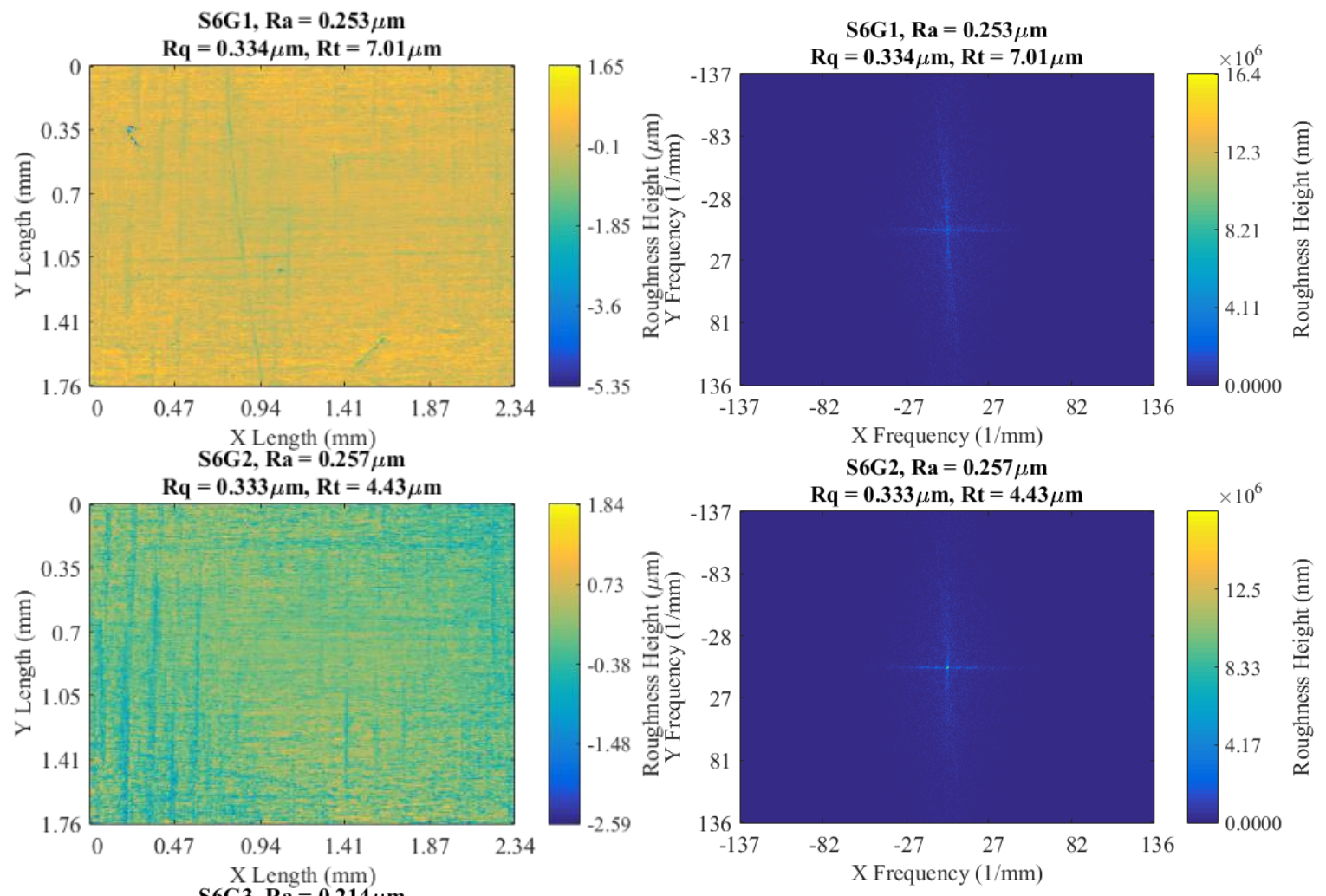

S6G2, Ra $=0.257 \mu \mathrm{m}$

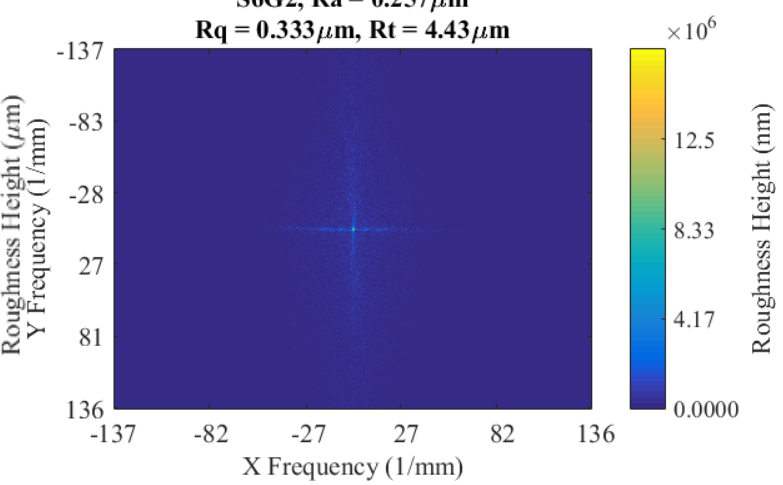

$\mathrm{S6G3}, \mathrm{Ra}=0.214 \mu \mathrm{m}$
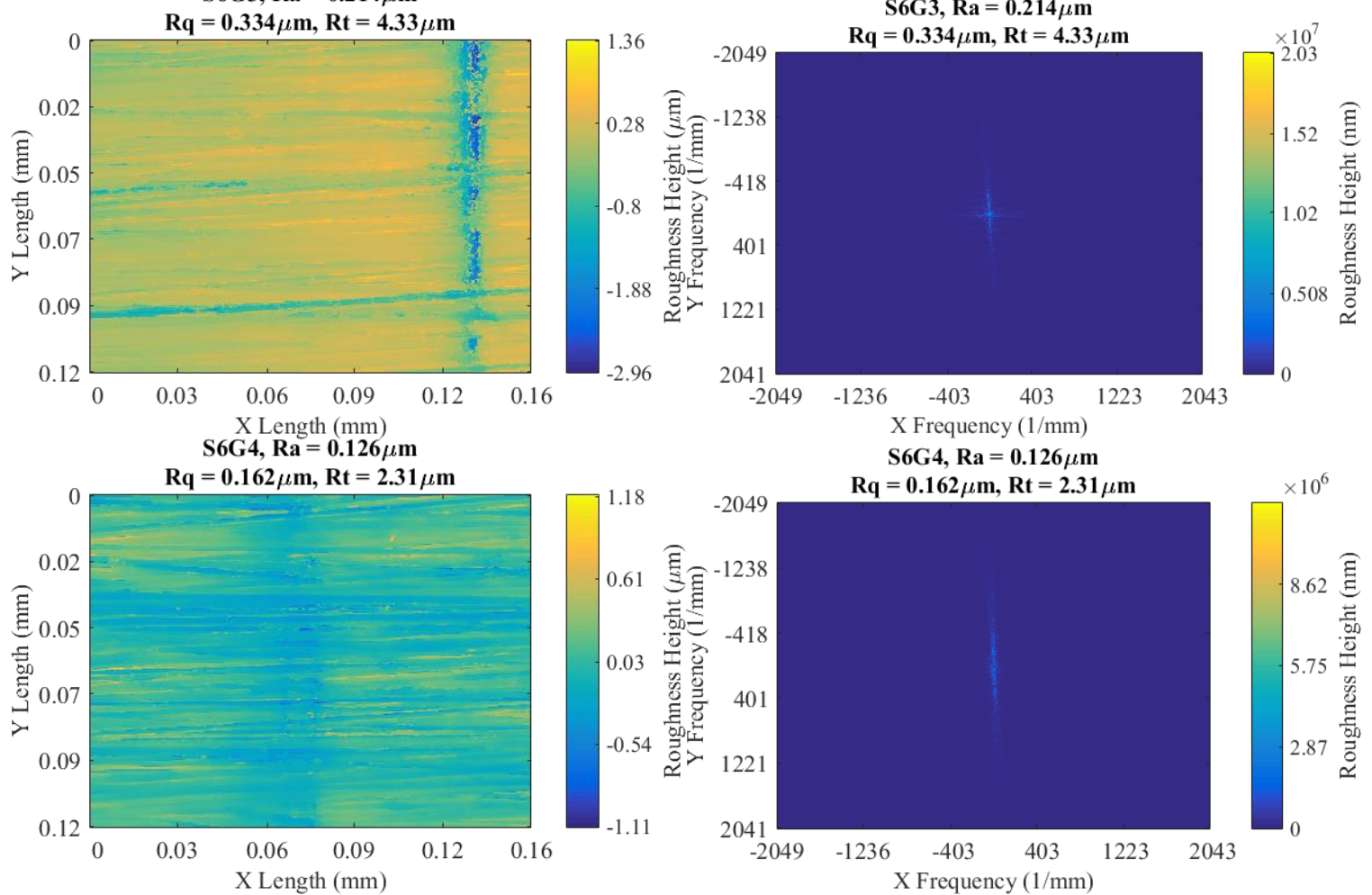

S6G4, Ra $=0.126 \mu \mathrm{m}$

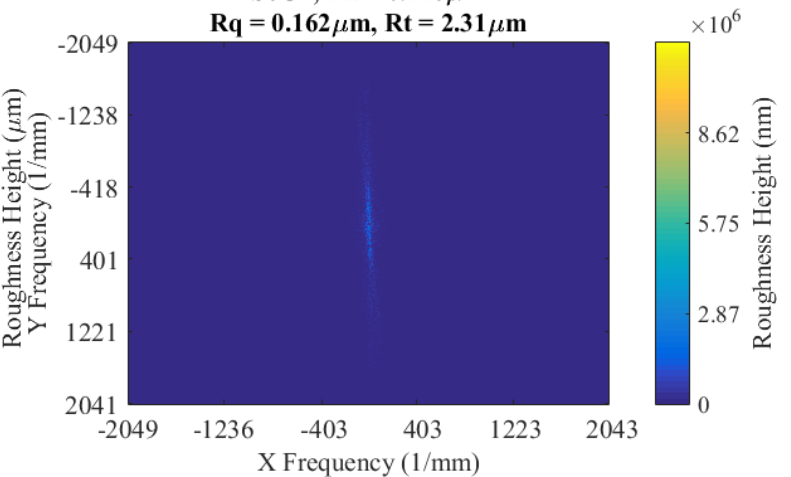



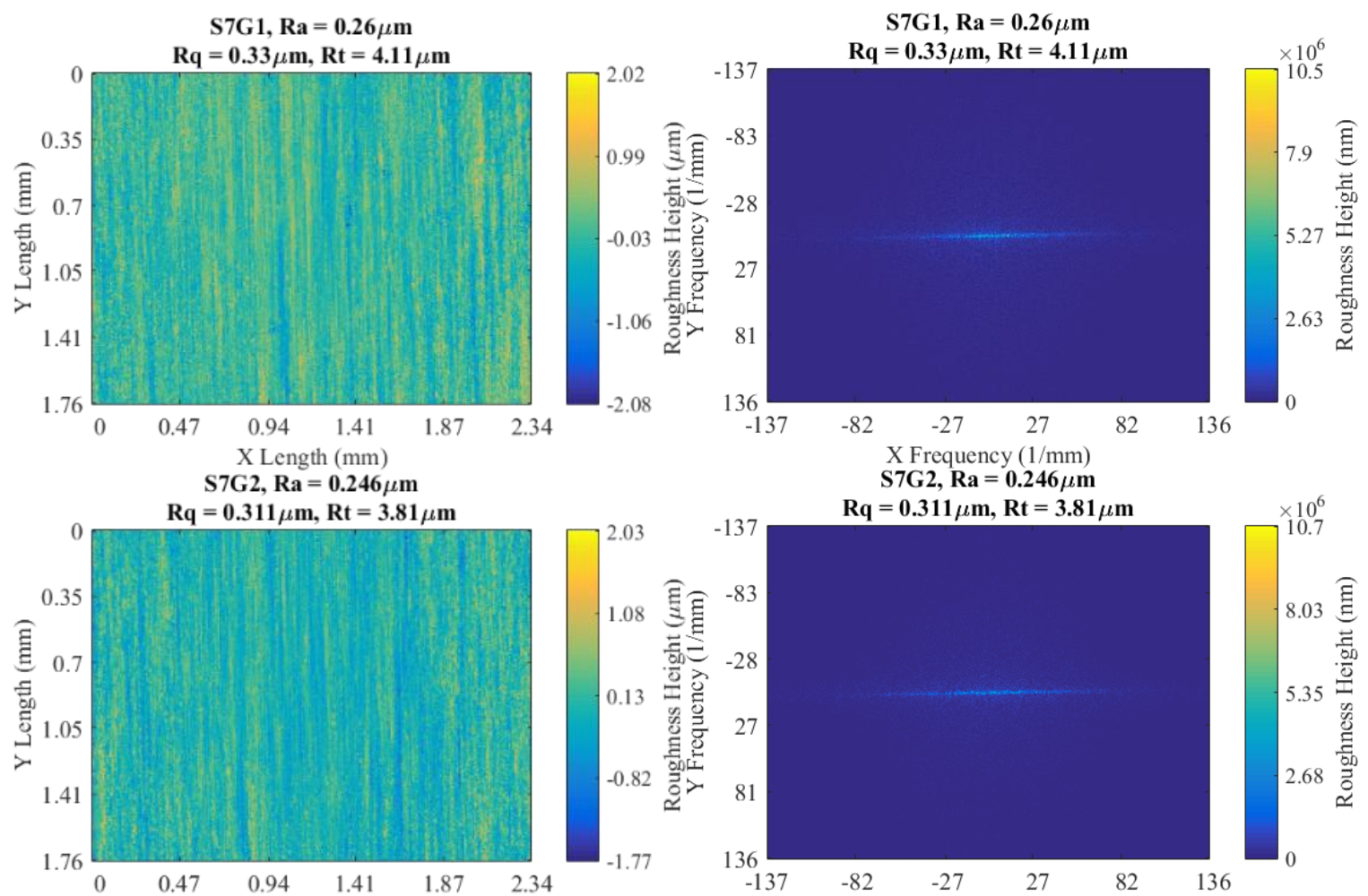

X7G2,

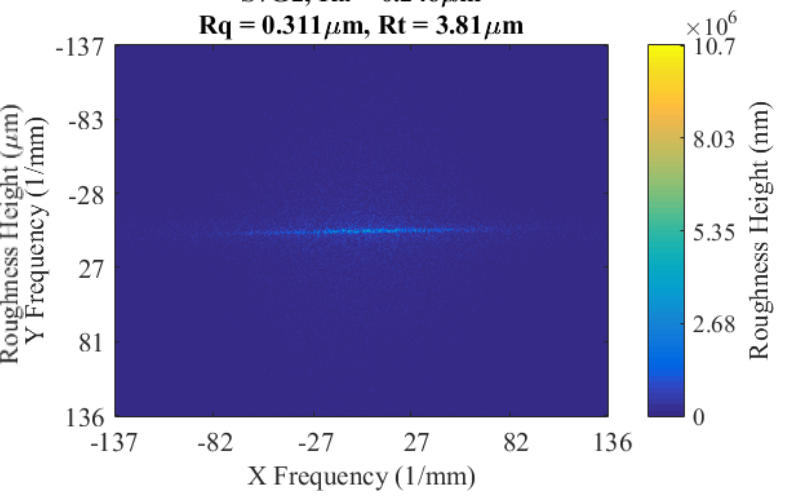

X Length $(\mathrm{mm})$
S7G3, Ra $=\mathbf{0 . 3 2 5} \mu \mathbf{m}$

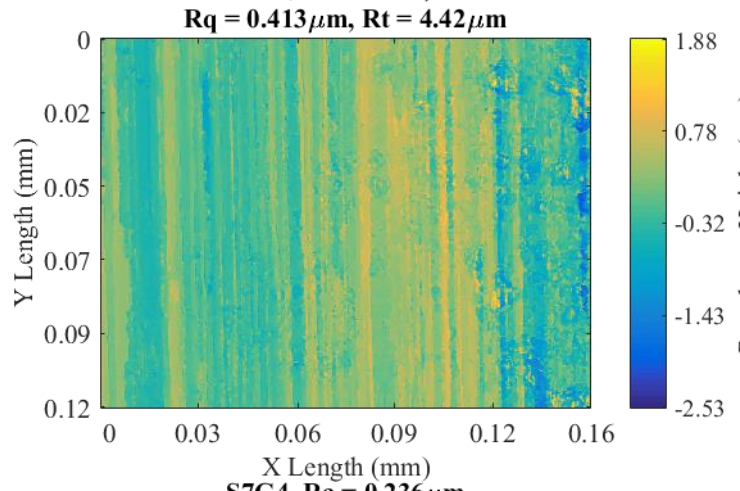

S7G3, Ra $=0.325 \mu \mathrm{m}$
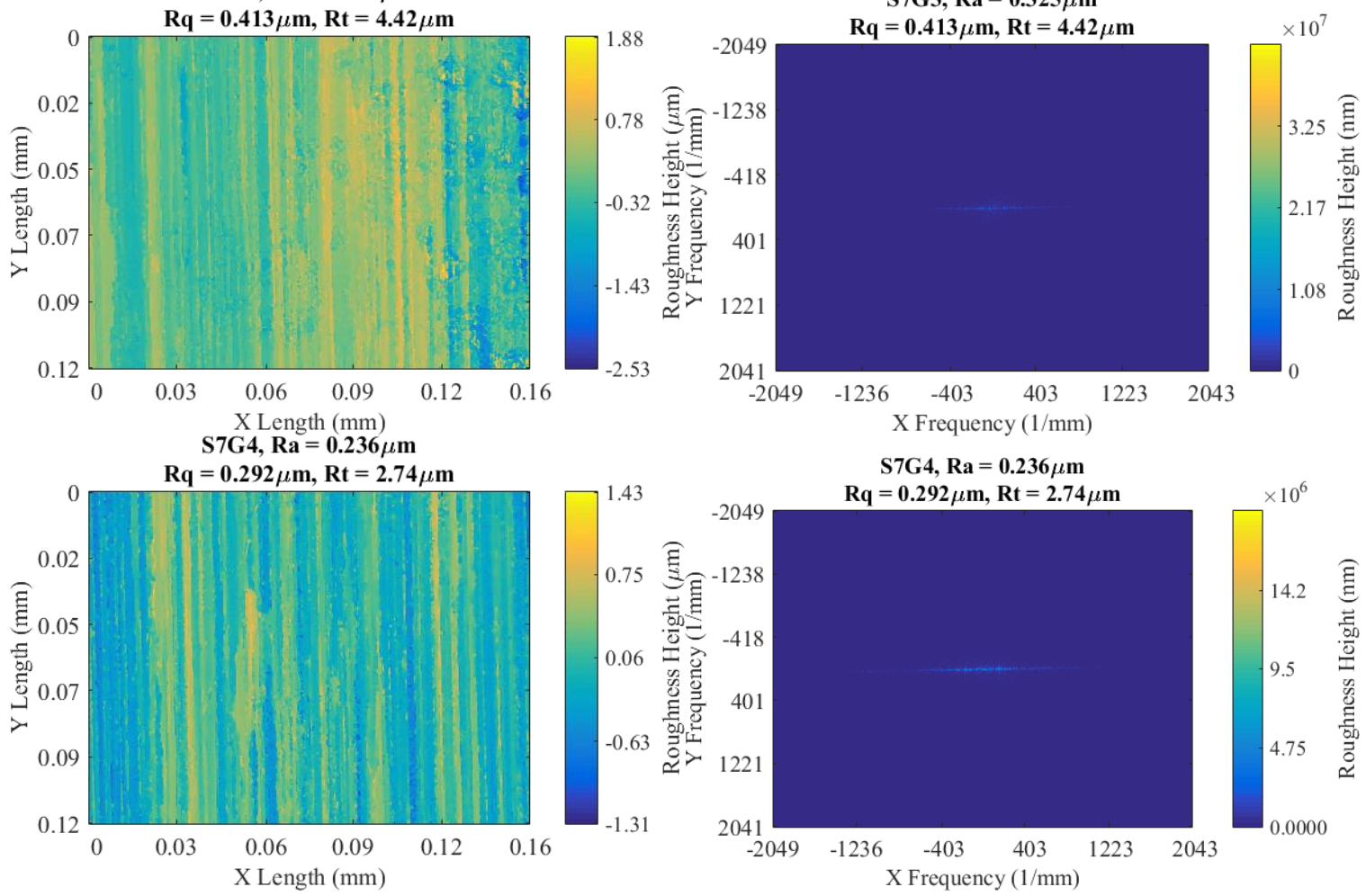

$\mathrm{S} 7 \mathrm{G} 4, \mathrm{Ra}=0.236 \mu \mathrm{m}$

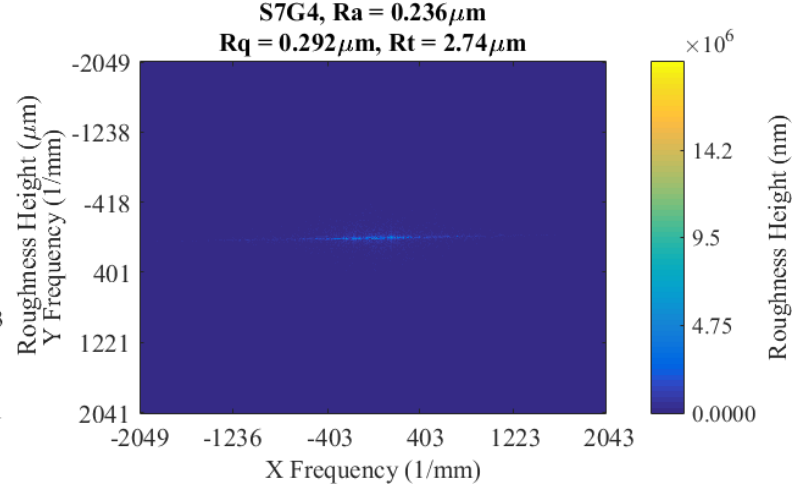



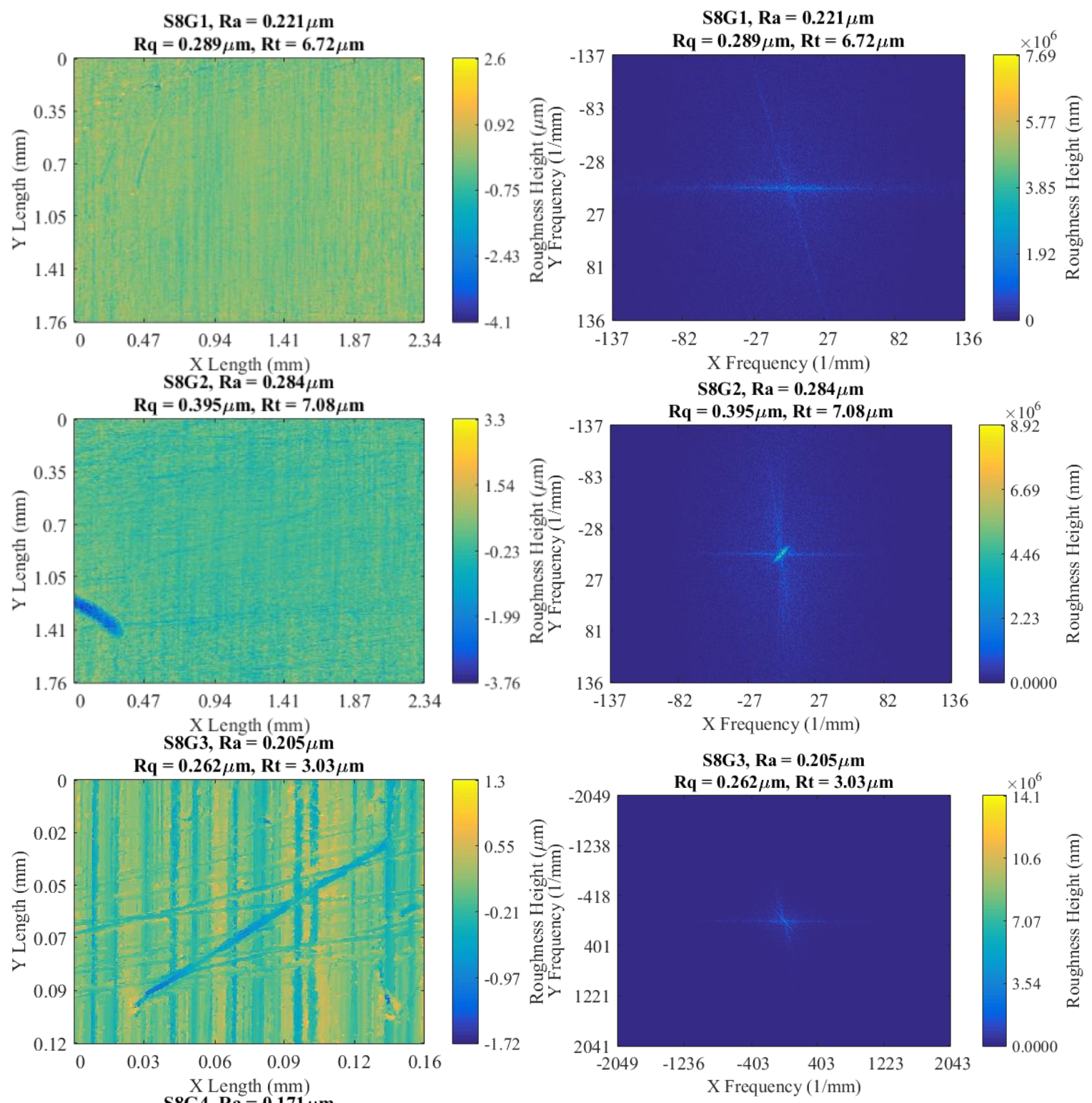

S8G4, Ra $=0.171 \mu \mathrm{m}$

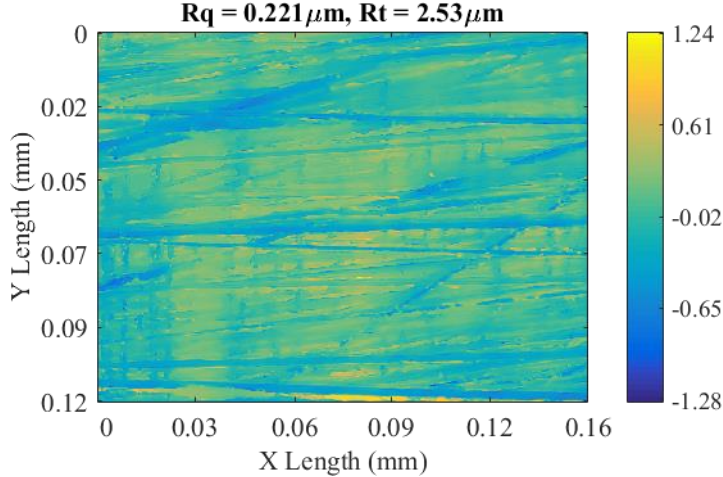

S8G4, Ra $=0.171 \mu \mathrm{m}$

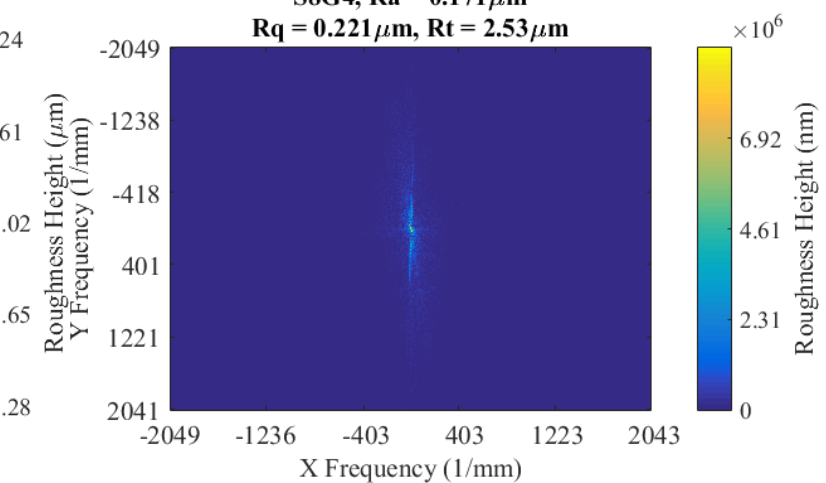



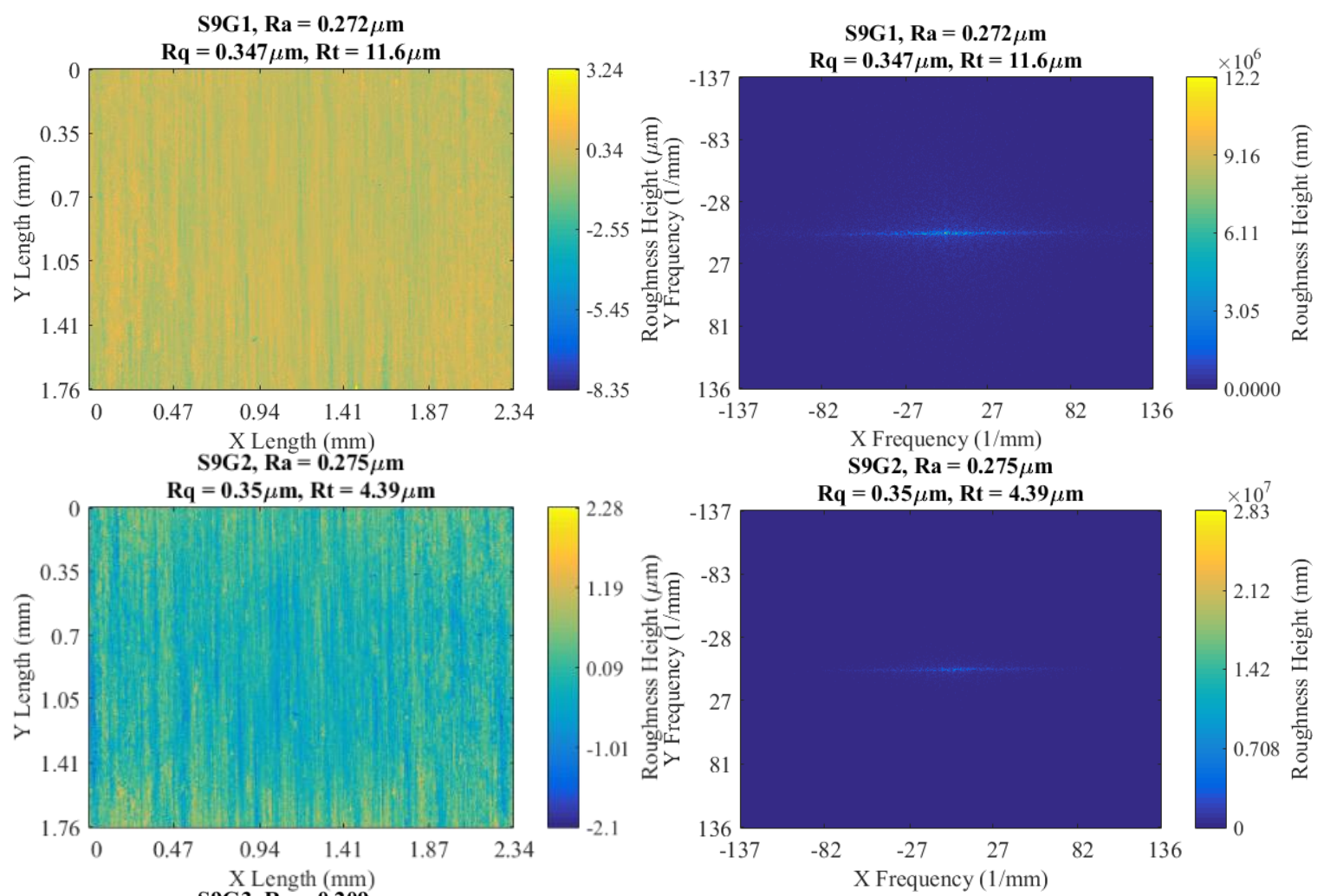

S9G2, Ra $=0.275 \mu \mathrm{m}$

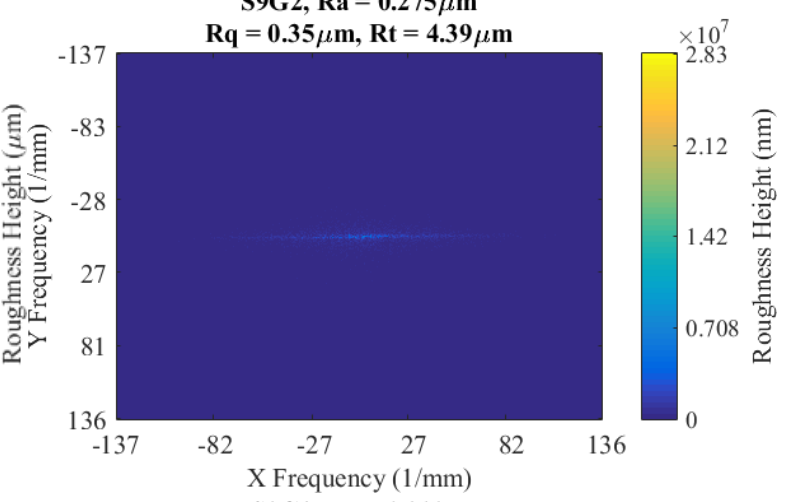

S9G3, Ra $=0.209 \mu \mathrm{m}$

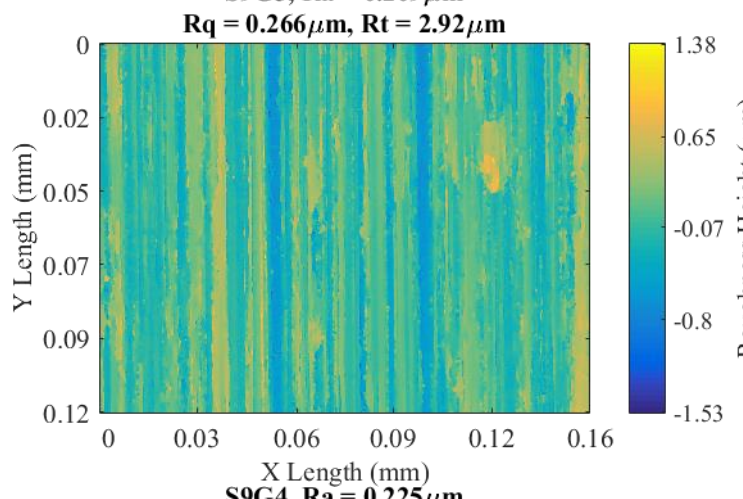

S9G3, Ra $=0.209 \mu \mathrm{m}$
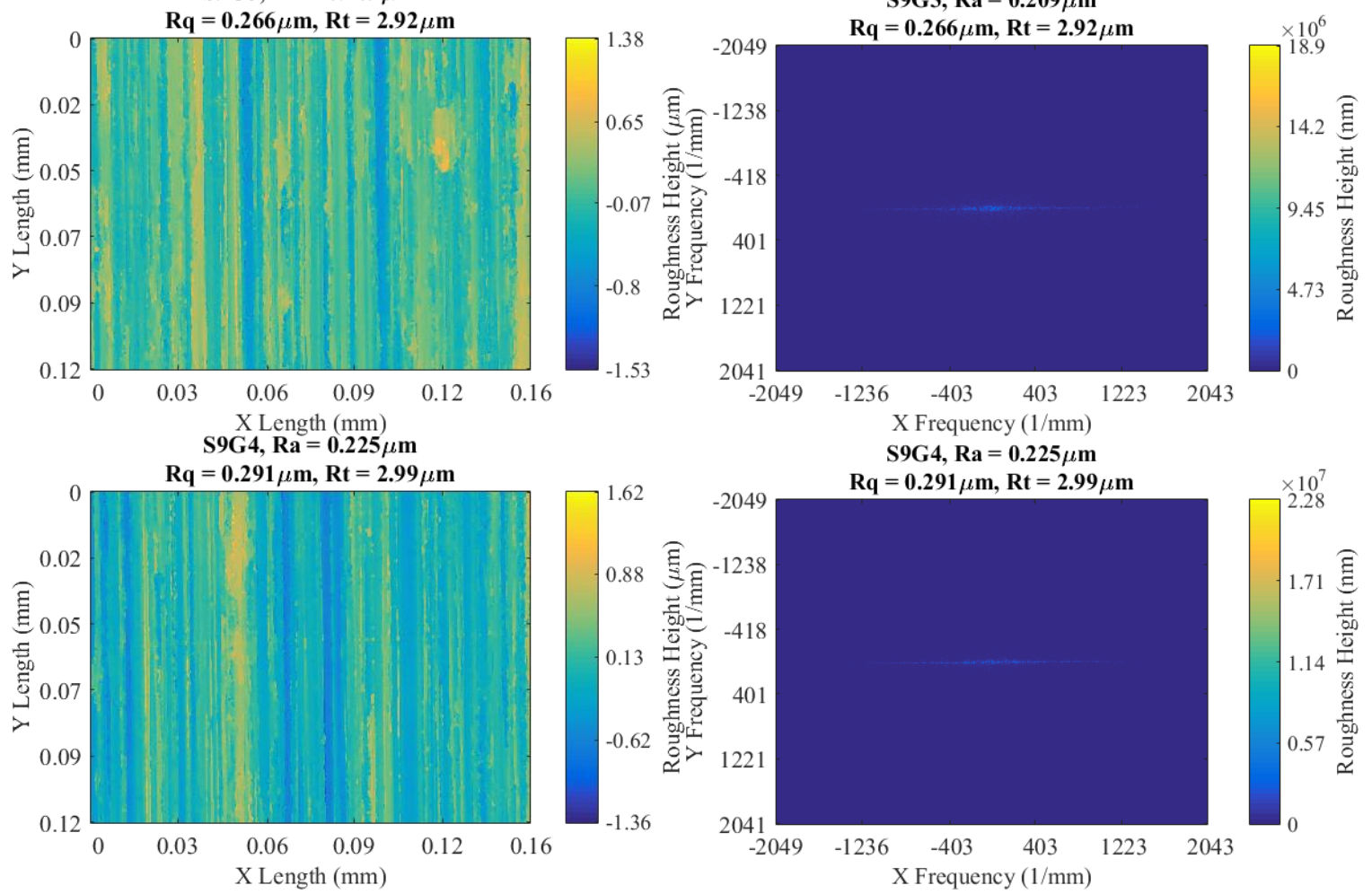

S9G4, Ra $=0.225 \mu \mathrm{m}$

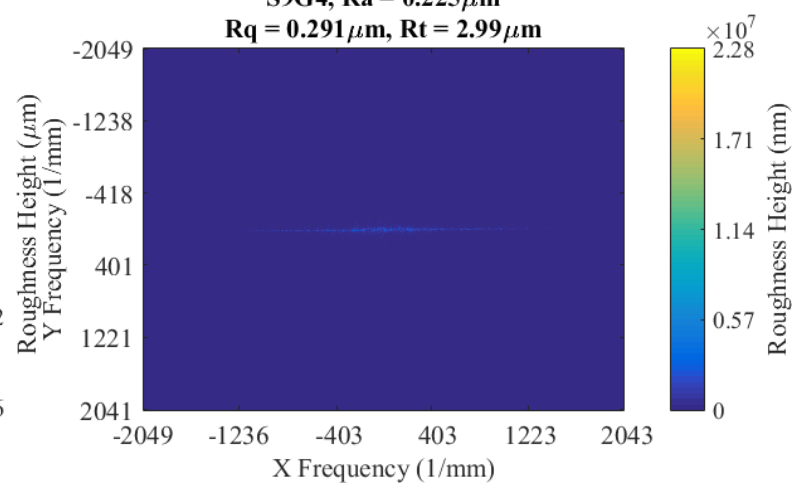



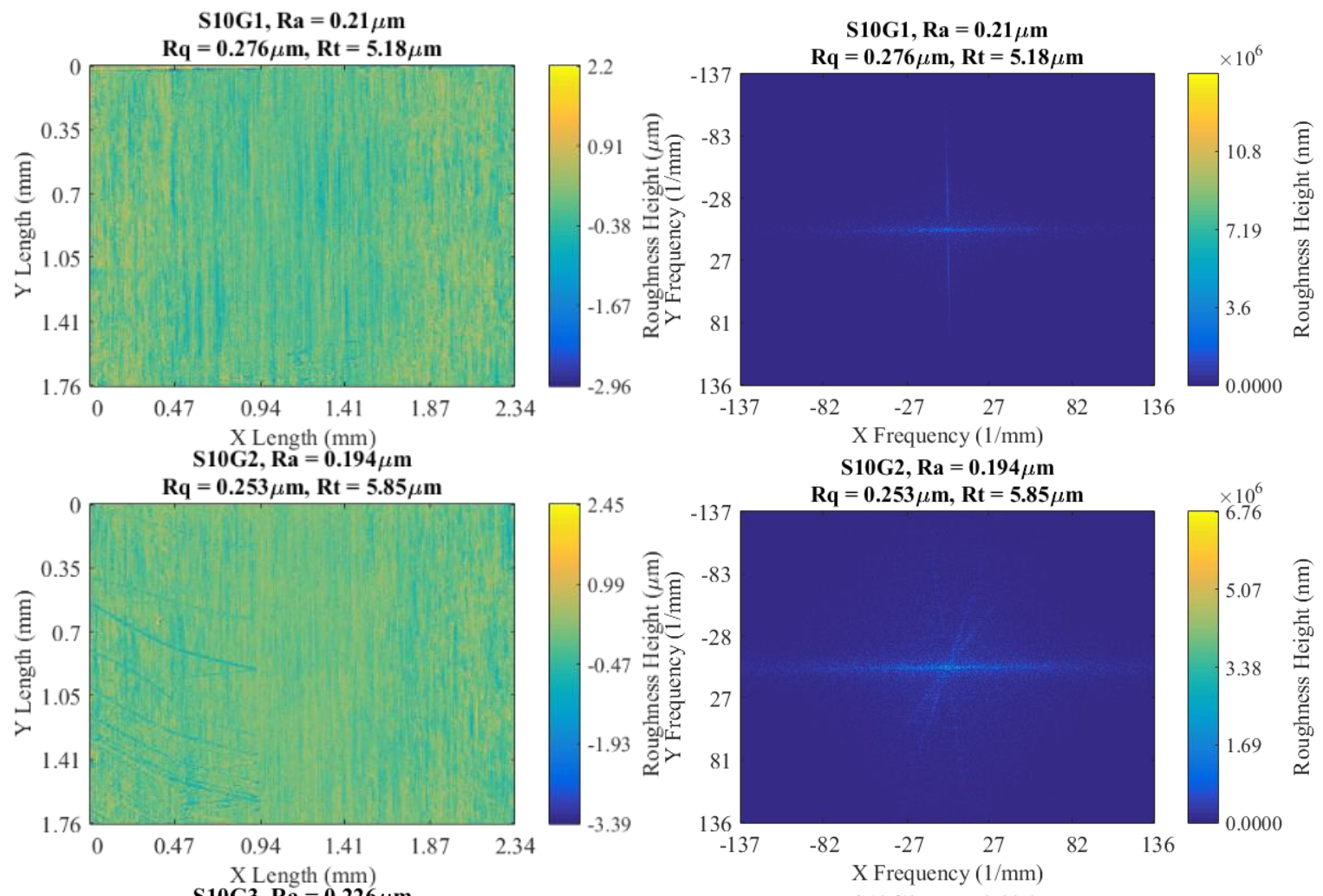

S10G2, Ra $=0.194 \mu \mathrm{m}$

$\mathrm{S} 10 \mathrm{G} 3, \mathrm{Ra}=0.226 \mu \mathrm{m}$

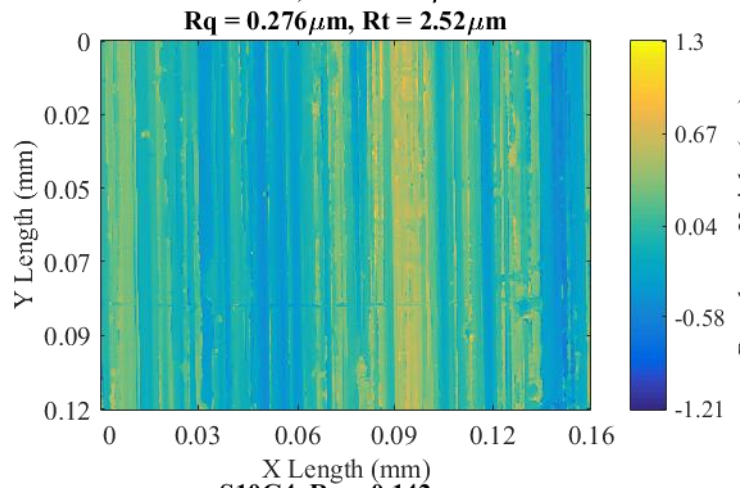

S10G4, Ra $=0.142 \mu \mathrm{m}$
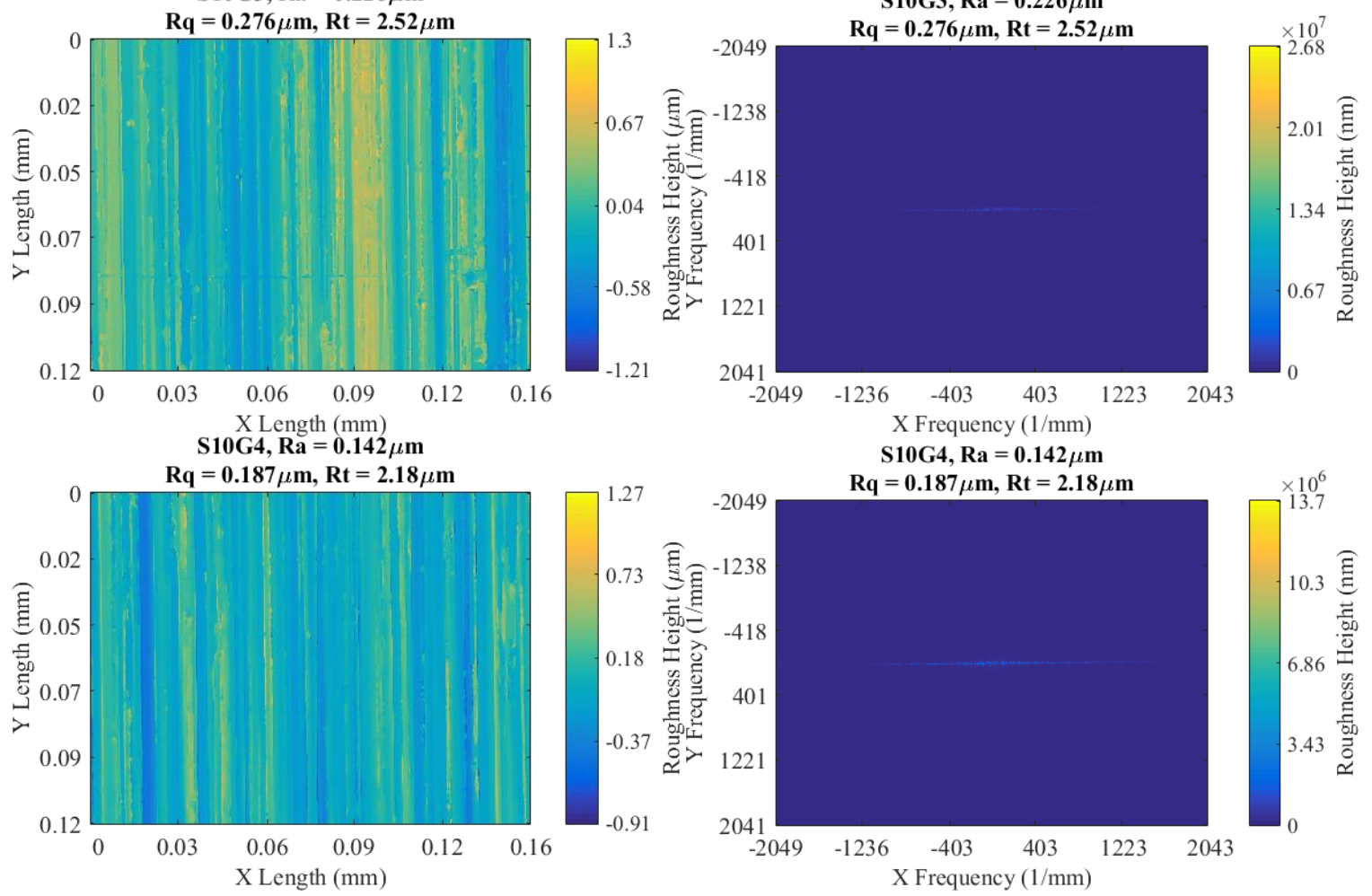

S10G4, Ra $=0.142 \mu \mathrm{m}$

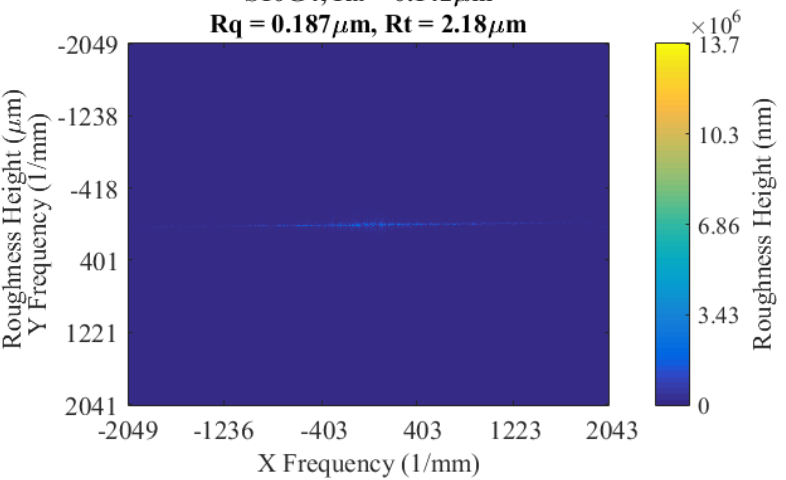




\section{Raw Data from Shear Testing}

$\mathrm{SN}$ is Sample Number, Qu is Questionable, $\mathrm{Cu}$ is Cuboid, He is heating time (s), Co is cooling time (s), CS is crosshead speed ( $\mathrm{mm} / \mathrm{min}$ ), Eq is equilibrium time (min), Op is Operator, Time is Time between cloud off and shear test (h), Thick is sample thickness (mm), RC is result code, and $\tau \max$ is the observed shear strength. Result codes are shown in Table 17. Data is shown in Table 18.

Table 17. Result Codes.

\begin{tabular}{|c|c|}
\hline $\begin{array}{c}\text { Break } \\
\text { Code }\end{array}$ & Description \\
\hline $\mathrm{gb}$ & Good Break \\
\hline $\mathrm{eb}$ & Edge Break - light ice in chunk at edge, $<2 \%$ of area as visually determined \\
\hline $\mathrm{CF}$ & Cohesive Failure - large scale failure with crack splitting sample through \\
bulk
\end{tabular}


Table 18. Data from the primary IRT runs.

\begin{tabular}{|c|c|c|c|c|c|c|c|c|c|c|c|}
\hline SN & Qu & $\mathrm{Cu}$ & $\mathrm{He}$ & Co & CS & $\mathrm{Eq}$ & Op & Time & Thick & $\mathbf{R C}$ & $\tau_{\max }$ \\
\hline A5 & & & 10 & & 0.2 & 3 & $\mathrm{AW}$ & 91.4 & 6.14 & & 0.794 \\
\hline A24 & & & 10 & & 0.2 & 3 & AW & 91.7 & 5.55 & & 0.462 \\
\hline A20 & $X$ & & 10 & & 0.2 & 3 & AW & 91.9 & 5.64 & & 0.623 \\
\hline A9 & $X$ & & 10 & & 0.2 & 3 & AW & 92.1 & 6.09 & & 0.747 \\
\hline A16 & & & 10 & & 0.2 & 3 & $\mathrm{AG}$ & 717.0 & 6.03 & eb & 0.831 \\
\hline A3 & $X$ & & 10 & & 2 & 3 & $\mathrm{AG}$ & 717.3 & 4.32 & $\mathrm{ob}$ & 1.029 \\
\hline A8 & & & 10 & & 0.2 & 3 & AG & 717.5 & 4.57 & $\mathrm{gb}$ & 0.583 \\
\hline A1 & & & 10 & & 0.2 & 3 & $\mathrm{AG}$ & 717.7 & 5.33 & $\mathrm{gb}$ & 1.234 \\
\hline A19 & & & 10 & & 2 & 3 & $\mathrm{AG}$ & 718.0 & 5.46 & $\mathrm{gb}$ & 0.722 \\
\hline A23 & $X$ & & 10 & & 2 & 3 & $A G$ & 718.2 & 5.33 & $o b$ & 0.797 \\
\hline A2 & $X$ & & 10 & & 0.2 & 3 & $\mathrm{AG}$ & 718.4 & 5.08 & $o b$ & 1.163 \\
\hline A13 & & & 10 & & 0.2 & 3 & $\mathrm{AG}$ & 718.6 & 4.83 & $\mathrm{gb}$ & 0.253 \\
\hline A11 & & & 10 & & 0.2 & 3 & $\mathrm{AG}$ & 718.7 & 5.84 & $\mathrm{gb}$ & 0.263 \\
\hline A6 & & & 10 & & 2 & 3 & $\mathrm{AG}$ & 718.9 & 5.84 & $\mathrm{gb}$ & 1.510 \\
\hline A17 & & & 10 & & 2 & 3 & AG & 719.0 & 5.33 & eb & 0.657 \\
\hline A10 & & & 10 & & 2 & 3 & $\mathrm{AG}$ & 719.2 & 5.59 & eb & 0.719 \\
\hline A12 & & & 10 & & 2 & 3 & $\mathrm{AG}$ & 719.4 & 5.84 & $\mathrm{gb}$ & 0.924 \\
\hline A18 & & & 10 & & 2 & 3 & $\mathrm{AG}$ & 719.5 & 5.08 & $\mathrm{gb}$ & 0.660 \\
\hline B8 & & & 10 & & 0.2 & 3 & AW & 89.7 & 6.01 & $\mathrm{gbc}$ & 0.564 \\
\hline B10 & & & 10 & & 0.2 & 3 & AW & 90.3 & 5.75 & & 0.654 \\
\hline B23 & & & 10 & & 0.2 & 3 & AW & 90.8 & 4.89 & & 0.617 \\
\hline B16 & & & 10 & & 0.2 & 3 & $\mathrm{AW}$ & 90.9 & 7.28 & & 0.586 \\
\hline B3 & & & 10 & & 0.2 & 3 & AW & 331.2 & 5.59 & & 0.927 \\
\hline B20 & & & 10 & & 0.2 & 3 & AW & 332.8 & 6.60 & & 0.670 \\
\hline B13 & X & & 10 & & 0.2 & 3 & $\mathrm{AG}$ & 352.2 & 5.59 & & 0.849 \\
\hline B9 & X & & 10 & & 0.2 & 3 & $\mathrm{AG}$ & 352.6 & 5.08 & $\mathrm{gb}$ & 0.209 \\
\hline B6 & X & & 10 & & 0.2 & 3 & $\mathrm{AG}$ & 353.1 & 5.08 & $\mathrm{gb}$ & 0.977 \\
\hline B19 & $X$ & & 10 & & 0.2 & 3 & $\mathrm{AG}$ & 353.3 & 5.08 & $\mathrm{gb}$ & 0.338 \\
\hline B18 & & & 16 & & 0.2 & 3 & $\mathrm{AG}$ & 354.1 & 5.59 & $\mathrm{gb}$ & 0.555 \\
\hline B1 & & & 10 & & 0.2 & 3 & AG & 354.4 & 6.10 & $\mathrm{gb}$ & 0.924 \\
\hline B17 & & & 10 & & 0.2 & 3 & AG & 354.6 & 5.84 & $\mathrm{gb}$ & 0.682 \\
\hline B15 & & & 10 & & 0.2 & 3 & $\mathrm{AG}$ & 354.8 & 5.33 & $\mathrm{gb}$ & 0.608 \\
\hline B5 & & & 10 & & 2 & 3 & $\mathrm{AW}$ & 330.1 & 5.59 & & 0.834 \\
\hline B11 & & & 10 & & 2 & 3 & $\mathrm{AW}$ & 330.3 & 5.59 & & 1.302 \\
\hline B22 & & & 10 & & 2 & 3 & AW & 330.4 & 5.59 & & 0.698 \\
\hline B2 & & & 10 & & 2 & 3 & AW & 330.5 & 5.33 & & 1.085 \\
\hline B24 & & & 10 & & 2 & 3 & $\mathrm{AW}$ & 330.6 & 5.84 & & 0.719 \\
\hline B12 & & & 10 & & 2 & 3 & AW & 330.8 & 6.10 & & 0.834 \\
\hline B21 & & & 10 & & 2 & 3 & AW & 330.9 & 5.33 & & 0.654 \\
\hline
\end{tabular}




\begin{tabular}{|c|c|c|c|c|c|c|c|c|c|c|c|}
\hline SN & Qu & $\mathrm{Cu}$ & $\mathrm{He}$ & Co & CS & $\mathrm{Eq}$ & Op & Time & Thick & $\mathbf{R C}$ & $\tau_{\max }$ \\
\hline C19 & & & 15 & & 0.2 & 3 & AW & 87.1 & 5.58 & & 0.295 \\
\hline C16 & & & 10 & & 0.2 & 3 & AW & 87.4 & 7.35 & & 0.326 \\
\hline C18 & & & 10 & & 0.2 & 3 & AW & 87.7 & 5.74 & & 0.335 \\
\hline C3 & & & 10 & & 0.2 & 3 & AW & 88.0 & 6.20 & & 0.412 \\
\hline C1 & & & 10 & & 0.2 & 3 & DD & 1092.1 & 6.84 & $\mathrm{gb}$ & 0.284 \\
\hline D11 & $X$ & & 10 & & 0.2 & 3 & AW & 84.5 & 6.79 & & 0.197 \\
\hline C15 & & & 10 & & 0.2 & 3 & DD & 1092.9 & 6.02 & $\mathrm{gb}$ & 0.995 \\
\hline C11 & & & 10 & & 2 & 3 & DD & 1093.2 & 5.95 & $\mathrm{gb}$ & 0.946 \\
\hline C13 & & & 10 & & 0.2 & 3 & DD & 1093.3 & 5.92 & $\mathrm{gb}$ & 0.592 \\
\hline D8 & $X$ & & 10 & & 0.2 & 3 & AW & 363.4 & 7.11 & $\mathrm{gb}$ & 0.614 \\
\hline C8 & & & 10 & & 2 & 3 & DD & 1093.7 & 6.26 & $\mathrm{gb}$ & 0.763 \\
\hline C6 & & & 10 & & 0.2 & 3 & DD & 1093.8 & 5.67 & $\mathrm{gb}$ & 1.318 \\
\hline C5 & & & 10 & & 2 & 3 & DD & 1094.0 & 6.14 & $\mathrm{gb}$ & 1.054 \\
\hline $\mathrm{C} 2$ & & & 10 & & 0.2 & 3 & DD & 1094.1 & 5.73 & $\mathrm{gb}$ & 0.772 \\
\hline C10 & & & 30 & & 2 & 3 & DD & 1094.3 & 6.07 & $\mathrm{gb}$ & 0.685 \\
\hline C12 & & & 10 & & 0.2 & 3 & DD & 1094.7 & 6.67 & $\mathrm{gb}$ & 0.998 \\
\hline D13 & & & 20 & & 0.2 & 3 & AW & 82.2 & 6.67 & & 0.391 \\
\hline D1 & & & 10 & & 0.2 & 3 & AW & 86.8 & 7.83 & & 0.440 \\
\hline D9 & & & 10 & & 0.2 & 3 & $\mathrm{AW}$ & 74.1 & 7.58 & & 0.284 \\
\hline D19 & & & 10 & & 0.2 & 3 & AW & 366.3 & 6.10 & $\mathrm{gb}$ & 0.636 \\
\hline D16 & & & 10 & & 0.2 & 3 & AW & 368.5 & 7.87 & & 0.701 \\
\hline D15 & & & 10 & & 0.2 & 3 & AW & 369.7 & 7.37 & $\mathrm{gbc}$ & 0.574 \\
\hline D2 & & & 10 & & 0.2 & 3 & AW & 365.8 & 5.84 & & 0.605 \\
\hline D3 & & & 10 & & 2 & 3 & AW & 363.8 & 6.35 & $\mathrm{gb}$ & 0.651 \\
\hline D10 & & & 10 & & 2 & 3 & AW & 373.9 & 6.60 & $\mathrm{gb}$ & 0.437 \\
\hline D15 & & & 10 & & 2 & 3 & AW & 370.0 & 6.60 & $\mathrm{gb}$ & 0.561 \\
\hline D18 & & & 10 & & 2 & 3 & AW & 367.1 & 5.84 & $\mathrm{gb}$ & 0.154 \\
\hline D12 & & & 20 & & 2 & 3 & AW & 372.2 & 7.11 & $\mathrm{gb}$ & 0.477 \\
\hline D6 & & & 10 & & 0.2 & 3 & $\mathrm{DD}$ & 1092.4 & 6.09 & $\mathrm{gb}$ & 0.716 \\
\hline E15 & & & 10 & & 0.2 & 3 & AW & 24.8 & 5.77 & & 0.825 \\
\hline E16 & & & 10 & & 0.2 & 3 & AW & 24.9 & 6.39 & & 0.902 \\
\hline E20 & & & 10 & & 0.2 & 3 & AW & 25.0 & 6.47 & & 0.989 \\
\hline E18 & & & 10 & & 0.2 & 3 & AW & 25.1 & 5.79 & & 0.781 \\
\hline E22 & & & 10 & & 0.2 & 3 & AW & 25.4 & 6.01 & gbc & 0.555 \\
\hline E12 & & & 30 & & 0.2 & 3 & $\mathrm{AG} / \mathrm{JS}$ & 1053.9 & 4.95 & $\mathrm{eb}$ & 1.603 \\
\hline E13 & & & 10 & & 0.2 & 3 & AG/JS & 1054.2 & 5.60 & $\mathrm{gb}$ & 1.659 \\
\hline E9 & & & 10 & & 2 & 3 & AG/JS & 1054.4 & 5.50 & eb & 0.952 \\
\hline E19 & & & 10 & & 2 & 3 & $\mathrm{AG} / \mathrm{JS}$ & 1054.6 & 5.80 & eb & 1.178 \\
\hline E2 & & & 10 & & 2 & 3 & AG/JS & 1054.8 & 5.80 & eb & 1.008 \\
\hline E10 & & & 10 & & 2 & 3 & AG/JS & 1055.0 & 5.84 & eb & 1.070 \\
\hline
\end{tabular}




\begin{tabular}{|c|c|c|c|c|c|c|c|c|c|c|c|}
\hline SN & Qu & $\mathrm{Cu}$ & $\mathrm{He}$ & Co & CS & $\mathrm{Eq}$ & Op & Time & Thick & $\mathbf{R C}$ & $\tau_{\max }$ \\
\hline E6 & & & 10 & & 2 & 3 & AG/JS & 1055.1 & 5.68 & eb & 1.066 \\
\hline E23 & & & 10 & & 0.2 & 3 & AG/JS & 1055.3 & 5.76 & eb & 1.386 \\
\hline E1 & & & 10 & & 0.2 & 3 & AG/JS & 1055.5 & 5.80 & $e b$ & 1.717 \\
\hline E11 & & & 10 & & 0.2 & 3 & $\mathrm{AG} / \mathrm{JS}$ & 1055.7 & 5.79 & eb & 1.798 \\
\hline E24 & & & 10 & & 0.2 & 3 & $\mathrm{AG} / \mathrm{JS}$ & 1055.8 & 5.30 & $\mathrm{gb}$ & 1.575 \\
\hline E3 & & & 10 & & 2 & 3 & AG/JS & 1056.2 & 5.75 & eb & 1.234 \\
\hline F6 & & & 10 & & 0.2 & 3 & AW & 23.8 & 6.81 & & 0.713 \\
\hline F17 & & & 10 & & 0.2 & 3 & AW & 24.4 & 6.86 & & 0.403 \\
\hline F20 & & & 10 & & 0.2 & 3 & AW & 24.6 & 6.70 & & 0.552 \\
\hline F1 & & & 10 & & 0.2 & 3 & AW & 24.7 & 6.32 & & 0.698 \\
\hline F24 & & & 10 & & 0.2 & 3 & AW & 24.8 & 6.61 & & 0.756 \\
\hline F5 & & & 10 & & 0.2 & 3 & AW & 215.5 & 5.84 & & 0.778 \\
\hline F12 & & & 10 & & 0.2 & 3 & AW & 215.6 & 6.60 & & 0.973 \\
\hline F13 & & & 10 & & 0.2 & 3 & AW & 215.7 & 6.60 & & 0.930 \\
\hline F15 & & & 10 & & 0.2 & 3 & AW & 215.9 & 6.35 & & 0.781 \\
\hline F11 & & & 10 & & 0.2 & 3 & AW & 216.0 & 5.59 & & 0.871 \\
\hline F10 & & & 10 & & 0.2 & 3 & AW & 216.2 & 5.59 & & 0.927 \\
\hline F18 & & & 10 & & 0.2 & 3 & AW & 216.3 & 6.10 & & 0.809 \\
\hline F19 & & & 15 & & 2 & 3 & AW & 214.5 & 6.10 & & 0.946 \\
\hline F23 & & & 15 & & 2 & 3 & AW & 214.7 & 6.10 & & 0.648 \\
\hline F8 & & & 15 & & 2 & 3 & AW & 214.8 & 6.86 & & 0.840 \\
\hline F21 & & & 10 & & 2 & 3 & AW & 214.9 & 6.60 & & 1.054 \\
\hline F16 & & & 10 & & 2 & 3 & AW & 215.1 & 6.86 & & 0.970 \\
\hline F22 & & & 10 & & 2 & 3 & AW & 215.2 & 6.10 & & 0.856 \\
\hline F9 & & & 10 & & 2 & 3 & AW & 215.3 & 5.84 & & 0.725 \\
\hline H12 & X & & 10 & & 0.02 & 3 & AW & 162.8 & 4.03 & $\mathrm{CF}$ & 0.360 \\
\hline H8 & $X$ & & 10 & & 0.02 & 3 & AW & 163.1 & 5.04 & $\mathrm{CF}$ & 0.760 \\
\hline F3 & & & 10 & & 2 & 3 & DD & 1076.7 & 5.89 & $\mathrm{gb}$ & 1.333 \\
\hline G5 & & & 10 & & 0.2 & 3 & AW & 21.4 & 6.40 & $\mathrm{gb}$ & 0.822 \\
\hline H18 & $X$ & & 10 & & 0.2 & 3 & AW & 20.7 & 4.80 & gbli & 1.073 \\
\hline H16 & $X$ & & 10 & & 0.2 & 3 & AW & 21.0 & 4.63 & & 0.332 \\
\hline G18 & & & 10 & & 0.2 & 3 & AW & 21.8 & 5.75 & & 0.583 \\
\hline G15 & & & 10 & & 0.2 & 3 & AW & 22.2 & 5.75 & & 0.608 \\
\hline G8 & & & 10 & & 0.2 & 3 & AW & 22.5 & 6.19 & & 0.908 \\
\hline G17 & & & 10 & & 0.2 & 3 & AW & 22.7 & 5.92 & & 0.623 \\
\hline G20 & & & 10 & & 0.2 & 3 & AW & 473.4 & 6.60 & gbli & 1.101 \\
\hline G22 & & & 10 & & 0.2 & 3 & AW & 473.8 & 6.35 & & 0.747 \\
\hline G13 & & & 10 & & 0.2 & 3 & AW & 474.1 & 5.84 & & 0.831 \\
\hline G9 & & & 10 & & 0.2 & 3 & AW & 474.2 & 5.59 & & 0.992 \\
\hline H3 & X & & 10 & & 2 & 3 & AW & 164.8 & 3.02 & & 0.162 \\
\hline
\end{tabular}




\begin{tabular}{|c|c|c|c|c|c|c|c|c|c|c|c|}
\hline SN & Qu & $\mathrm{Cu}$ & $\mathrm{He}$ & Co & CS & Eq & Op & Time & Thick & $\mathbf{R C}$ & $\tau_{\max }$ \\
\hline G24 & & & 10 & & 0.2 & 3 & $\mathrm{AW}$ & 474.5 & 6.60 & gbli & 0.862 \\
\hline G12 & & & 10 & & 0.2 & 3 & AW & 474.6 & 5.84 & gbli & 1.231 \\
\hline G6 & & & 10 & & 0.2 & 3 & AW & 474.7 & 6.10 & gbli & 1.302 \\
\hline G3 & & & 10 & & 0.2 & 3 & $\mathrm{AW}$ & 475.1 & 5.33 & $\mathrm{gb}$ & 1.079 \\
\hline G2 & & & 10 & & 2 & 3 & AW & 473.6 & 5.84 & gbli & 1.125 \\
\hline G16 & & & 10 & & 2 & 3 & AW & 473.7 & 6.10 & gbli & 0.797 \\
\hline G10 & & & 10 & & 2 & 3 & AW & 474.0 & 5.59 & & 0.961 \\
\hline G19 & & & 10 & & 2 & 3 & $\mathrm{AW}$ & 474.3 & 6.10 & & 0.688 \\
\hline G23 & & & 10 & & 2 & 3 & AW & 474.4 & 6.35 & & 0.800 \\
\hline G1 & & & 10 & & 2 & 3 & AW & 474.9 & 5.33 & $\mathrm{gb}$ & 1.274 \\
\hline G11 & & & 10 & & 2 & 3 & AW & 475.0 & 5.59 & $\mathrm{gb}$ & 0.942 \\
\hline G21 & & & 10 & & 2 & 3 & AW & 475.2 & 6.60 & $\mathrm{gb}$ & 0.527 \\
\hline H21 & & & 15 & & 0.02 & 3 & $\mathrm{AW}$ & 161.4 & 4.50 & & 1.023 \\
\hline H23 & & & 10 & & 0.02 & 3 & AW & 161.9 & 4.50 & & 0.846 \\
\hline H11 & & & 10 & & 0.02 & 3 & $\mathrm{AW}$ & 163.3 & 4.08 & & 0.636 \\
\hline H9 & & & 10 & & 0.2 & 3 & $\mathrm{AW}$ & 20.6 & 4.70 & $\mathrm{gb}$ & 0.772 \\
\hline H10 & & & 10 & & 0.2 & 3 & $\mathrm{AW}$ & 21.1 & 4.91 & & 0.942 \\
\hline H24 & & & 10 & & 0.2 & 3 & AW & 21.5 & 4.65 & & 0.701 \\
\hline H15 & & & 10 & & 0.2 & 3 & $\mathrm{AW}$ & 165.3 & 5.88 & & 0.750 \\
\hline $\mathrm{H} 2$ & & & 10 & & 0.2 & 3 & AW & 165.4 & 4.02 & & 0.412 \\
\hline H5 & & & 10 & & 0.2 & 3 & AW & 165.6 & 4.84 & & 1.249 \\
\hline H1 & & & 10 & & 0.2 & 3 & AW & 165.9 & 3.81 & & 0.614 \\
\hline I20 & $\mathrm{x}$ & & 20 & & 2 & 3 & $\mathrm{AW}$ & 209.5 & 5.59 & $\mathrm{gb}$ & 0.120 \\
\hline H6 & & & 20 & & 2 & 3 & AW & 164.2 & 4.97 & & 0.961 \\
\hline H17 & & & 10 & & 2 & 3 & $\mathrm{AW}$ & 164.4 & 4.49 & gbli & 1.652 \\
\hline H20 & & & 10 & & 2 & 3 & $\mathrm{AW}$ & 165.1 & 5.08 & & 1.035 \\
\hline H22 & & & 10 & & & 3 & $\mathrm{AW}$ & 164.7 & 4.52 & & 1.327 \\
\hline H19 & & & 10 & & & 3 & AW & 165.0 & 4.81 & & 1.308 \\
\hline H13 & & & 10 & & & 3 & AW & 165.7 & 4.85 & & 0.326 \\
\hline J6 B! & $X$ & & & & 0.02 & & $\mathrm{AW}$ & 51.2 & & & 0.086 \\
\hline J12 & $X$ & & 40 & 5 & 0.2 & 5 & $\mathrm{AW}$ & 15.4 & & & 0.574 \\
\hline I1 & & & 20 & 5 & 0.2 & 5 & AW & 17.2 & 7.20 & & 0.772 \\
\hline I15 & & & 15 & 5 & 0.2 & 5 & AW & 18.7 & 6.20 & $\mathrm{gb}$ & 0.639 \\
\hline $\mathbf{J} 20$ & $X$ & & 15 & 5 & 0.2 & 3 & $\mathrm{AW}$ & 18.0 & 5.96 & & 0.223 \\
\hline I13 & & & 15 & 5 & 0.2 & 5 & AW & 18.9 & 6.89 & $\mathrm{gb}$ & 0.558 \\
\hline J24 & $X$ & & 10 & & 0.2 & 3 & AW & 65.9 & 5.08 & & 0.108 \\
\hline J17 & $X$ & & 10 & & 0.2 & 3 & AW & 66.0 & 6.47 & & 0.099 \\
\hline I16 & & & 10 & & 0.2 & 3 & $\mathrm{AW}$ & 19.4 & 7.85 & $\mathrm{gb}$ & 0.667 \\
\hline I17 & & & 10 & & 0.2 & 3 & AW & 19.5 & 7.00 & & 0.567 \\
\hline I3 & & & 20 & & 0.2 & 3 & $\mathrm{AW}$ & 188.6 & 6.04 & & 0.933 \\
\hline
\end{tabular}




\begin{tabular}{|c|c|c|c|c|c|c|c|c|c|c|c|}
\hline SN & Qu & $\mathrm{Cu}$ & He & Co & CS & $\mathrm{Eq}$ & Op & Time & Thick & $\mathbf{R C}$ & $\tau_{\max }$ \\
\hline I10 & & & 20 & & 0.2 & 3 & AW & 188.7 & 5.43 & & 0.794 \\
\hline I18 & & & 10 & & 0.2 & 3 & AW & 189.0 & 5.24 & & 0.691 \\
\hline I11 & & & 20 & & 0.2 & 3 & AW & 210.1 & 6.10 & & 1.066 \\
\hline $\mathrm{J} 22$ & $X$ & & 10 & & 20 & 3 & AW & 65.2 & 6.95 & & 0.151 \\
\hline I19 & & & 20 & & 0.2 & 3 & AW & 210.4 & 5.59 & & 0.154 \\
\hline I22 & & & 10 & & 0.2 & 3 & AW & 210.5 & 6.35 & & 0.896 \\
\hline I9 & & & 10 & & 2 & 3 & AW & 184.1 & 6.56 & & 1.032 \\
\hline E17 & $X$ & & 10 & & 0.2 & 3 & $\mathrm{AG} / \mathrm{JS}$ & 1053.6 & 5.00 & $\mathrm{ob}$ & 1.380 \\
\hline I6 & & & 10 & & 2 & 3 & $\mathrm{AW}$ & 184.2 & 6.57 & & 0.791 \\
\hline I21 & & & 10 & & 2 & 3 & AW & 184.3 & 7.42 & & 0.564 \\
\hline I2 & & & 10 & & 2 & 3 & AW & 184.4 & 5.79 & & 0.800 \\
\hline I5 & & & 20 & & 2 & 3 & AW & 188.2 & 5.15 & $\mathrm{gb}$ & 0.998 \\
\hline I12 & & & 20 & & 2 & 3 & AW & 188.4 & 5.87 & & 0.992 \\
\hline I24 & & & 40 & & 2 & 3 & AW & 209.3 & 6.60 & & 0.908 \\
\hline I8 & & & 10 & & 2 & 3 & AW & 209.6 & 6.35 & & 1.107 \\
\hline I23 & & & 10 & & 2 & 3 & AW & 209.8 & 6.60 & & 0.942 \\
\hline J6 & & & 20 & & 0.02 & 3 & AW & 66.0 & 5.67 & & 0.270 \\
\hline J13 & & & 10 & & 0.02 & 3 & AW & 66.2 & 5.71 & & 0.217 \\
\hline J21 & & & 10 & & 0.02 & 3 & AW & 66.4 & 6.20 & & 0.304 \\
\hline E5 & & & 10 & & 0.2 & 3 & $\mathrm{AG} / \mathrm{JS}$ & 1056.1 & 5.85 & $\mathrm{scb}$ & 1.519 \\
\hline J10 & & & 10 & & 0.02 & 3 & AW & 66.5 & 5.04 & & 0.412 \\
\hline J1 & & & 25 & 5 & 0.2 & 5 & AW & 17.4 & 5.00 & & 0.809 \\
\hline J19 & & & 15 & 5 & 0.2 & 3 & AW & 17.9 & 5.86 & & 0.419 \\
\hline J11 & & & 12 & & 0.2 & 3 & AW & 18.1 & 5.46 & $\mathrm{gb}$ & 0.760 \\
\hline J15 & & & 10 & & 0.2 & 3 & AW & 68.0 & 6.53 & & 0.450 \\
\hline J9 & & & 10 & & 0.2 & 3 & AW & 68.1 & 5.59 & & 0.084 \\
\hline $\mathrm{J} 2$ & & & 10 & & 2 & 3 & $\mathrm{AW}$ & 66.7 & 5.23 & & 0.760 \\
\hline J5 & & & 10 & & 2 & 3 & AW & 66.8 & 5.92 & & 0.716 \\
\hline J23 & & & 10 & & 2 & 3 & AW & 66.9 & 6.26 & & 0.605 \\
\hline J3 & & & 10 & & 2 & 3 & AW & 67.0 & 5.74 & & 0.623 \\
\hline J8 & & & 10 & & 20 & 3 & AW & 67.2 & 6.75 & & 0.183 \\
\hline J16 & & & 10 & & 20 & 3 & AW & 67.3 & 6.40 & & 0.152 \\
\hline J18 & & & 10 & & 20 & 3 & AW & 67.4 & 5.55 & & 0.155 \\
\hline F2 & $X$ & & 10 & & 2 & 3 & DD & 1077.4 & 6.30 & $\mathrm{gb}$ & 1.119 \\
\hline K1 & & & 60 & 3 & 0.2 & & AW/JS & 1368.9 & 9.30 & $\mathrm{gb}$ & 0.337 \\
\hline K10 & $\mathrm{x}$ & & 20 & 0 & 0.2 & & AG & 0.9 & 8.27 & $\mathrm{gb}$ & 0.070 \\
\hline K11 & & & 66 & 11 & 0.2 & & AW/JS & 1367.9 & 8.60 & $\mathrm{scbFc}$ & 0.432 \\
\hline K12 & & & 66 & 14 & 0.2 & & AW/JS & 1363.0 & 8.30 & $\mathrm{gbFc}$ & 0.452 \\
\hline K13 & $\mathrm{x}$ & & 30 & 0 & 0.2 & & $\mathrm{AG}$ & 0.6 & 8.62 & $\mathrm{gb}$ & 0.062 \\
\hline K14 & & & 49 & 8 & 0.2 & & AW/JS & 1370.2 & 8.10 & scbc & 0.580 \\
\hline
\end{tabular}




\begin{tabular}{|c|c|c|c|c|c|c|c|c|c|c|c|}
\hline SN & Qu & $\mathrm{Cu}$ & $\mathrm{He}$ & Co & CS & $\mathrm{Eq}$ & Op & Time & Thick & $\mathbf{R C}$ & $\tau_{\max }$ \\
\hline K15 & & & 44 & 9 & 0.2 & & AW/JS & 1362.7 & 9.60 & gb & 0.204 \\
\hline K17 & & & 51 & 10 & 0.2 & & AW/JS & 1369.2 & 8.70 & $\mathrm{gb}$ & 0.262 \\
\hline K19 & $\mathrm{X}$ & & & & 0.2 & & AW/JS & 1362.4 & 8.50 & $\mathrm{gb}$ & 0.361 \\
\hline K2 & & & 48 & 0 & 0.2 & & AW/JS & 1363.8 & 8.20 & $\mathrm{gb}$ & 0.390 \\
\hline K20 & $\mathrm{X}$ & & 64 & 10 & 0.2 & & AW/JS & 1362.3 & 8.60 & gbF! & 0.337 \\
\hline K22 & & & 57 & 10 & 0.2 & & AW/JS & 1368.8 & 8.30 & $\mathrm{gb}$ & 0.481 \\
\hline K23 & & & 40 & 10 & 0.2 & & AW/JS & 1361.9 & 9.30 & $\mathrm{gb}$ & 0.547 \\
\hline K5 & & & 86 & 0 & 0.2 & & AW/JS & 1367.4 & 7.70 & $\mathrm{gbc}$ & 0.264 \\
\hline K6 & & & 57 & 12 & 0.2 & & AW/JS & 1369.1 & 8.60 & $\mathrm{gb}$ & 0.467 \\
\hline K9 & $\mathrm{x}$ & & 20 & 0 & 0.2 & & $\mathrm{AG}$ & 1.2 & 8.27 & $\mathrm{gb}$ & 0.014 \\
\hline L1 & & & 56 & 0 & 0.2 & & AW/JS & 1369.4 & 9.20 & $\mathrm{gb}$ & 0.269 \\
\hline L11 & & & 68 & 0 & 0.2 & & AW/JS & 1369.5 & 8.00 & $\mathrm{gbF}$ & 0.348 \\
\hline L12 & & & 25 & 0 & 0.2 & & AG & 2.2 & 8.80 & gbla & 0.116 \\
\hline L13 & & & 55 & 0 & 0.2 & & AW/JS & 1364.5 & 8.40 & $\mathrm{gb}$ & 0.321 \\
\hline L15 & & & 25 & 0 & 0.2 & & $\mathrm{AG}$ & 2.3 & 8.40 & gbla & 0.048 \\
\hline L16 & & & 63 & 0 & 0.2 & & AW/JS & 1369.7 & 8.80 & $\mathrm{gbcF}$ & 0.316 \\
\hline L18 & & & 38 & 0 & 0.2 & & AW/JS & 1363.1 & 8.60 & $\mathrm{gb}$ & 0.510 \\
\hline L19 & $\mathrm{X}$ & & & & 0.2 & & AW/JS & 1363.4 & 8.40 & $\mathrm{gb} !$ & 0.350 \\
\hline L2 & & & 114 & 0 & 0.2 & & AW/JS & 1364.0 & 7.50 & $\mathrm{gb}$ & 0.240 \\
\hline L22 & & & 112 & 1 & 0.2 & & AW/JS & 1363.7 & 7.60 & $\mathrm{gb}$ & 0.325 \\
\hline L23 & & & & & 0.2 & & AW/JS & 1365.3 & 7.60 & $\mathrm{gbC}$ & 0.317 \\
\hline L3 & & & 71 & 0 & 0.2 & & AW/JS & 1369.1 & 8.00 & $\mathrm{gbF}$ & 0.312 \\
\hline L5 & & & & & 0.2 & & $\mathrm{AW} / \mathrm{JS}$ & 1366.1 & 8.60 & $\mathrm{gbc}$ & 0.404 \\
\hline L8 & & & 30 & 0 & 0.2 & & AG & 2.1 & 8.90 & gbla & 0.109 \\
\hline M11 & $\mathrm{x}$ & & 35 & 0 & 0.2 & & $\mathrm{AG}$ & 0.6 & 16.20 & $\mathrm{gb}$ & 0.035 \\
\hline M12 & $\mathrm{x}$ & & 25 & 0 & 0.2 & & $\mathrm{AG}$ & 0.5 & 17.20 & $\mathrm{gb}$ & 0.012 \\
\hline M14 & & & & & 0.2 & & AW/JS & 1364.6 & 14.30 & $\mathrm{gbC}$ & 0.375 \\
\hline M15 & & & 107 & 7 & 0.2 & & AW/JS & 1366.2 & 14.10 & $\mathrm{gbFc}$ & 0.239 \\
\hline M22 & & & 66 & 11 & 0.2 & & AW/JS & 1366.4 & 14.70 & $\mathrm{gb}$ & 0.236 \\
\hline M23 & & & & & 0.2 & & $\mathrm{AW} / \mathrm{JS}$ & 1364.4 & 15.30 & $\mathrm{gb}$ & 0.272 \\
\hline M3 & & & & & 0.2 & & AW/JS & 1363.6 & 14.20 & $\mathrm{gb}$ & 0.483 \\
\hline M5 & & & 122 & 11 & 0.2 & & AW/JS & 1361.2 & 14.90 & $\mathrm{gb}$ & 0.403 \\
\hline M7 & $\mathrm{x}$ & & 70 & 9 & 0.2 & & AW/JS & 1367.2 & 14.40 & gbF! & 0.095 \\
\hline M8 & & & 45 & 0 & 0.2 & & $\mathrm{AG}$ & 0.7 & 17.90 & gbla & 0.042 \\
\hline N10 & & & 30 & 0 & 0.2 & & $\mathrm{AG}$ & 0.7 & 14.50 & gbla & 0.021 \\
\hline N13 & & & 25 & 0 & 0.2 & & $\mathrm{AG}$ & 1.0 & 14.20 & gbla & 0.075 \\
\hline N15 & & & 44 & 0 & 0.2 & & AW & 1387.6 & 10.70 & $\mathrm{gb}$ & 0.580 \\
\hline N17 & & & 35 & 0 & 0.2 & & $\mathrm{AG}$ & 0.9 & 14.60 & gbla & 0.063 \\
\hline N19 & & & 51 & 0 & 0.2 & & AW & 1388.2 & 11.00 & $\mathrm{gbc}$ & 0.407 \\
\hline N23 & & & 25 & 0 & 0.2 & & $\mathrm{AG}$ & 1.1 & 13.50 & gbla & 0.029 \\
\hline
\end{tabular}




\begin{tabular}{|c|c|c|c|c|c|c|c|c|c|c|c|}
\hline SN & Qu & $\mathrm{Cu}$ & $\mathrm{He}$ & Co & CS & $\mathrm{Eq}$ & Op & Time & Thick & $\mathbf{R C}$ & $\tau_{\max }$ \\
\hline N3 & $\mathrm{x}$ & & 58 & 0 & 0.2 & & $\mathrm{AW}$ & 1388.5 & 11.30 & gbc! & 0.110 \\
\hline 01 & & & 20 & 0 & 0.2 & & $\mathrm{AG}$ & 1.1 & 7.10 & $\mathrm{gb}$ & 0.420 \\
\hline 010 & & & 56 & 11 & 0.2 & & AW & 1337.8 & 8.30 & $\mathrm{gb}$ & 0.897 \\
\hline 011 & & & 49 & 0 & 0.2 & & AW & 1386.4 & 8.40 & gbli & 0.537 \\
\hline 012 & & & 85 & 10 & 0.2 & & AW & 1337.6 & 7.70 & $\mathrm{gb}$ & 0.782 \\
\hline 013 & $\mathrm{X}$ & & 65 & 0 & 0.2 & & AW & 1100.9 & 7.90 & $\mathrm{gb}$ & 0.665 \\
\hline 014 & & & 40 & 0 & 0.2 & & AW & 1100.6 & 7.40 & $\mathrm{gb}$ & 0.827 \\
\hline 015 & & & 64 & 16 & 0.2 & & AW & 1338.2 & 7.40 & $\mathrm{gb}$ & 1.040 \\
\hline 016 & & & 75 & 12 & 0.2 & & AW & 1217.1 & 8.30 & $\mathrm{gb}$ & 0.871 \\
\hline 017 & & & & & 0.2 & & AW & 1387.7 & 8.40 & $\mathrm{gb}$ & 0.651 \\
\hline 018 & & & 100 & 39 & 0.2 & & AW & 1339.5 & 7.90 & $\mathrm{gb}$ & 0.830 \\
\hline $\mathbf{O 2}$ & & & 58 & 12 & 0.2 & & AW & 1338.5 & 7.50 & $e b$ & 0.801 \\
\hline O20 & & & 65 & 0 & 0.2 & & AW & 1100.4 & 7.90 & $\mathrm{gb}$ & 0.825 \\
\hline $\mathrm{O} 22$ & & & 62 & 11 & 0.2 & & AW & 1339.1 & 7.60 & $\mathrm{gb}$ & 0.698 \\
\hline $\mathbf{0 3}$ & & & 20 & 0 & 0.2 & & $\mathrm{AG}$ & 0.9 & 7.00 & $\mathrm{gb}$ & 0.279 \\
\hline 05 & & & 20 & 0 & 0.2 & & $\mathrm{AG}$ & 0.6 & 8.10 & $\mathrm{gb}$ & 0.095 \\
\hline 06 & & & 20 & 0 & 0.2 & & $\mathrm{AG}$ & 0.7 & 7.10 & $\mathrm{gb}$ & 0.320 \\
\hline 07 & & & 20 & 0 & 0.2 & & $\mathrm{AG}$ & 1.0 & 7.20 & $\mathrm{gb}$ & 0.423 \\
\hline 08 & & & 35 & 5 & 0.2 & & AW & 1100.1 & 7.40 & $\mathrm{gb}$ & 0.640 \\
\hline O9 & & & 73 & 11 & 0.2 & & AW & 1339.3 & 6.70 & $\mathrm{gb}$ & 0.843 \\
\hline P1 & & & 80 & 11 & 0.2 & & AW & 1168.7 & 8.90 & $\mathrm{gb}$ & 0.978 \\
\hline P10 & & & 91 & 15 & 0.2 & & DD & 1213.0 & 6.60 & $\mathrm{gb}$ & 0.952 \\
\hline P11 & & & 67 & 8 & 0.2 & & AW & 1167.7 & 8.30 & eb & 1.110 \\
\hline P12 & & & 52 & 16 & 0.2 & & DD & 1213.4 & 8.20 & $\mathrm{gb}$ & 0.084 \\
\hline P13 & & & 51 & 47 & 0.2 & & DD & 1211.9 & 7.20 & $\mathrm{gb}$ & 0.262 \\
\hline P16 & & & 92 & 69 & 0.2 & & DD & 1212.1 & 7.10 & $\mathrm{gb}$ & 0.619 \\
\hline P17 & $\mathrm{X}$ & & 91 & 7 & 0.2 & & AW & 1169.1 & 7.80 & $\mathrm{gb}$ & 0.753 \\
\hline P18 & $\mathrm{x}$ & & 54 & 14 & 0.2 & & AW & 1171.0 & 7.20 & $\mathrm{gb}$ & 0.168 \\
\hline P2 & & & 45 & 10 & 0.2 & & AW & 1171.4 & 7.20 & $\mathrm{gb}$ & 0.266 \\
\hline P20 & & & 51 & 0 & 0.2 & & $\mathrm{AW}$ & 1385.3 & 8.30 & gbli & 0.975 \\
\hline P22 & & & 35 & 0 & 0.2 & & $\mathrm{AG}$ & 0.6 & 7.80 & $\mathrm{gb}$ & 0.200 \\
\hline P23 & & & 73 & 22 & 0.2 & & DD & 1212.8 & 7.80 & gbli & 0.901 \\
\hline P3 & & & 104 & 8 & 0.2 & & AW & 1168.0 & 7.40 & $\mathrm{gb}$ & 0.619 \\
\hline P5 & & & 83 & 9 & 0.2 & & $\mathrm{AW}$ & 1168.3 & 8.00 & $\mathrm{gb}$ & 0.321 \\
\hline P6 & & & 20 & 0 & 0.2 & & $\mathrm{AG}$ & 0.7 & 7.00 & $\mathrm{gb}$ & 0.309 \\
\hline P7 & & & 69 & 11 & 0.2 & & AW & 1168.5 & 8.00 & $\mathrm{gb}$ & 0.969 \\
\hline P8 & & & 113 & 26 & 0.2 & & DD & 1212.4 & 6.10 & gbli & 0.883 \\
\hline R1 & & & 75 & 0 & 0.2 & & $\mathrm{AW}$ & 1365.9 & 9.60 & $\mathrm{gb}$ & 0.709 \\
\hline R10 & & & 81 & 0 & 0.2 & & AW & 1363.6 & 8.00 & $\mathrm{CFc}$ & 0.788 \\
\hline R11 & & & 25 & 0 & 0.2 & & AG & 3.7 & 5.20 & $\mathrm{gb}$ & 0.250 \\
\hline
\end{tabular}




\begin{tabular}{|c|c|c|c|c|c|c|c|c|c|c|c|}
\hline SN & Qu & $\mathrm{Cu}$ & He & Co & CS & $\mathrm{Eq}$ & Op & Time & Thick & $\mathbf{R C}$ & $\tau_{\max }$ \\
\hline R13 & & & 62 & 0 & 0.2 & & $\mathrm{AW}$ & 1365.4 & 9.00 & gb & 0.610 \\
\hline R15 & & & 10 & 0 & 0.2 & & $\mathrm{AG}$ & 1.0 & 9.00 & $\mathrm{gb}$ & 0.134 \\
\hline R16 & & & 25 & 0 & 0.2 & & $\mathrm{AG}$ & 3.5 & 9.20 & $\mathrm{gb}$ & 0.277 \\
\hline R17 & & & 25 & 0 & 0.2 & & $\mathrm{AG}$ & 3.4 & 8.50 & $\mathrm{gb}$ & 0.040 \\
\hline R18 & & & 70 & 0 & 0.2 & & AW & 1364.5 & 9.20 & $\mathrm{gb}$ & 0.500 \\
\hline R19 & & & 20 & 0 & 0.2 & & $\mathrm{AG}$ & 0.9 & 7.50 & $\mathrm{gb}$ & 0.022 \\
\hline $\mathbf{R 2}$ & & & 45 & 0 & 0.2 & & AW & 1364.2 & 9.60 & $\mathrm{gb}$ & 0.522 \\
\hline R20 & & & 62 & 11 & 0.2 & & AW & 1321.3 & 9.60 & eb & 0.674 \\
\hline R22 & & & 113 & 16 & 0.2 & & AW & 1320.4 & 8.70 & $\mathrm{gb}$ & 0.748 \\
\hline R23 & & & 71 & 10 & 0.2 & & AW & 1321.4 & 10.30 & $\mathrm{gb}$ & 0.579 \\
\hline R3a & & & 15 & 0 & 0.2 & & AG & 1.2 & 8.90 & $\mathrm{gb}$ & 0.250 \\
\hline R5 & & & 65 & 0 & 0.2 & & AW & 1363.1 & 10.00 & ebc & 0.735 \\
\hline R6 & & & 55 & 0 & 0.2 & & AW & 1364.4 & 9.20 & $\mathrm{gb}$ & 0.420 \\
\hline R7 & & & 45 & 0 & 0.2 & & AW & 1365.3 & 8.70 & $\mathrm{gb}$ & 0.793 \\
\hline R9 & & & 80 & 0 & 0.2 & & $\mathrm{AW}$ & 1363.8 & 8.90 & gbc & 0.666 \\
\hline S1 & & & 25 & 0 & 0.2 & & $\mathrm{AG}$ & 1.2 & 6.50 & $\mathrm{gb}$ & 0.045 \\
\hline S10 & & & 25 & 0 & 0.2 & & $\mathrm{AG}$ & 1.0 & 6.50 & $\mathrm{gb}$ & 0.088 \\
\hline S11 & $\mathrm{X}$ & & 47 & 11 & 0.2 & & AW & 1320.4 & 7.20 & $\mathrm{gb}$ & 0.206 \\
\hline S12 & & & 70 & 0 & 0.2 & & AW & 1365.0 & 6.40 & $\mathrm{gb}$ & 0.662 \\
\hline S13 & & & 62 & 14 & 0.2 & & AW & 1320.2 & 6.70 & $\mathrm{gb}$ & 0.641 \\
\hline S14 & & & 14 & 0 & 0.2 & & $\mathrm{AG}$ & 0.8 & 6.50 & $\mathrm{gb}$ & 0.249 \\
\hline S15 & & & 25 & 11 & 0.2 & & AW & 1319.8 & 7.40 & $\mathrm{gb}$ & 0.512 \\
\hline S16 & & & 106 & 12 & 0.2 & & $\mathrm{AW}$ & 1321.7 & 6.90 & $\mathrm{gb}$ & 0.701 \\
\hline S17 & & & 82 & 16 & 0.2 & & $\mathrm{AW}$ & 1320.0 & 5.90 & gb F & 0.376 \\
\hline S18 & & & 65 & 0 & 0.2 & & AW & 1363.3 & 7.00 & $\mathrm{gb}$ & 0.487 \\
\hline S19 & $\mathrm{x}$ & & 35 & 0 & 0.2 & & $\mathrm{AW}$ & 1365.5 & 7.50 & $\mathrm{gb}$ & 0.220 \\
\hline S2 & & & 55 & 10 & 0.2 & & $\mathrm{AW}$ & 1321.6 & 7.20 & $\mathrm{gb}$ & 0.389 \\
\hline S20 & & & 20 & 0 & 0.2 & & $\mathrm{AG}$ & 0.7 & 9.00 & $\mathrm{gb}$ & 0.011 \\
\hline S23 & & & 64 & 0 & 0.2 & & AW & 1364.9 & 7.00 & $\mathrm{gb}$ & 0.719 \\
\hline S3 & & & 61 & 10 & 0.2 & & $\mathrm{AW}$ & 1322.4 & 7.70 & $\mathrm{gbF}$ & 0.293 \\
\hline S5 & & & 25 & 0 & 0.2 & & $\mathrm{AG}$ & 1.8 & 6.40 & $\mathrm{gb}$ & 0.094 \\
\hline S6 & $\mathrm{x}$ & & 49 & 10 & 0.2 & & AW & 1322.3 & 7.10 & $\mathrm{gbF}$ & 0.157 \\
\hline S7 & & & 47 & 0 & 0.2 & & AW & 1364.4 & 7.30 & $\mathrm{gb}$ & 0.746 \\
\hline S9 & & & 25 & 0 & 0.2 & & $\mathrm{AG}$ & 0.9 & 6.50 & $\mathrm{gb}$ & 0.144 \\
\hline T1 & & & 86 & 0 & 0.2 & & AW & 1361.7 & 5.60 & $\mathrm{gb}$ & 0.385 \\
\hline T10 & & & 59 & 10 & 0.2 & & AW & 1320.2 & 8.00 & $\mathrm{gb}$ & 0.690 \\
\hline T11 & & & 63 & 0 & 0.2 & & AW & 1361.9 & 7.00 & $\mathrm{gb}$ & 0.574 \\
\hline T12 & & & 86 & 10 & 0.2 & & $\mathrm{AW}$ & 1320.1 & 6.00 & $\mathrm{gbF}$ & 0.576 \\
\hline T13 & & & 25 & 0 & 0.2 & & AG & 0.9 & 6.50 & $\mathrm{gb}$ & 0.111 \\
\hline T14 & & & 53 & 10 & 0.2 & & $\mathrm{AW}$ & 1321.4 & 7.50 & $\mathrm{gb}$ & 0.738 \\
\hline
\end{tabular}




\begin{tabular}{|c|c|c|c|c|c|c|c|c|c|c|c|}
\hline SN & Qu & $\mathrm{Cu}$ & $\mathrm{He}$ & Co & CS & $\mathrm{Eq}$ & Op & Time & Thick & $\mathbf{R C}$ & $\tau_{\max }$ \\
\hline T15 & & & 58 & 0 & 0.2 & & $\mathrm{AW}$ & 1363.4 & 8.60 & gb & 0.659 \\
\hline T16 & & & 78 & 10 & 0.2 & & AW & 1320.4 & 7.80 & $\mathrm{gb}$ & 0.595 \\
\hline T17 & & & 54 & 10 & 0.2 & & AW & 1321.2 & 7.20 & $\mathrm{gb}$ & 0.824 \\
\hline T19 & & & 25 & 0 & 0.2 & & $\mathrm{AG}$ & 1.3 & 6.80 & $\mathrm{gb}$ & 0.060 \\
\hline $\mathbf{T} 2$ & & & 46 & 0 & 0.2 & & AW & 1364.9 & 8.20 & $\mathrm{gbF}$ & 0.682 \\
\hline T20 & & & 25 & 0 & 0.2 & & $\mathrm{AG}$ & 1.4 & 8.00 & $\mathrm{gb}$ & 0.130 \\
\hline T22 & & & 60 & 11 & 0.2 & & AW & 1319.3 & 8.80 & $\mathrm{gb}$ & 0.724 \\
\hline T23 & & & 40 & 10 & 0.2 & & AW & 1321.3 & 8.60 & $\mathrm{gb}$ & 0.799 \\
\hline T5 & & & 25 & 0 & 0.2 & & $\mathrm{AG}$ & 1.2 & 6.60 & $\mathrm{gb}$ & 0.045 \\
\hline T6 & & & 55 & 10 & 0.2 & & AW & 1320.7 & 8.20 & $\mathrm{gb}$ & 0.719 \\
\hline T8 & & & 25 & 0 & 0.2 & & AG & 0.8 & 7.50 & $\mathrm{gb}$ & 0.156 \\
\hline T9 & & & 74 & 11 & 0.2 & & AW & 1320.6 & 7.20 & $\mathrm{gb}$ & 0.515 \\
\hline U1 & & & 135 & 5 & 0.2 & & AW & 1316.6 & 7.10 & $\mathrm{gbF}$ & 0.635 \\
\hline U11 & & & 30 & 0 & 0.2 & & AW & 162.2 & 7.70 & $\mathrm{gb}$ & 0.248 \\
\hline U15 & & & 25 & 0 & 0.2 & & $\mathrm{AG}$ & 0.7 & 8.10 & $\mathrm{gb}$ & 0.080 \\
\hline U17 & & & 112 & 9 & 0.2 & & AW & 1316.8 & 8.60 & $\mathrm{gb}$ & 0.556 \\
\hline U18 & & & 25 & 0 & 0.2 & & AW & 161.8 & 8.80 & $\mathrm{gb}$ & 0.299 \\
\hline U19 & & & 25 & 0 & 0.2 & & $\mathrm{AG}$ & 0.8 & 8.20 & $\mathrm{gb}$ & 0.078 \\
\hline U2 & & & 30 & 0 & 0.2 & & AW & 162.5 & 7.10 & $\mathrm{gb}$ & 0.175 \\
\hline U20 & & & 25 & 0 & 0.2 & & AG & 0.9 & 8.30 & $\mathrm{gb}$ & 0.095 \\
\hline U22 & & & 20 & 0 & 0.2 & & AW & 162.6 & 7.40 & $\mathrm{gb}$ & 0.359 \\
\hline U3a & & & 30 & 0 & 0.2 & & AW & 162.3 & 8.00 & $\mathrm{gb}$ & 0.065 \\
\hline U7 & & & 25 & 0 & 0.2 & & $\mathrm{AW}$ & 162.0 & 7.80 & $\mathrm{gb}$ & 0.317 \\
\hline U8 & $\mathrm{x}$ & & 12 & 0 & 0.2 & & AW & 160.6 & 7.10 & $\mathrm{gb}$ & 0.214 \\
\hline V13 & & & 25 & 0 & 0.2 & & $\mathrm{AG}$ & 0.7 & 7.90 & $\mathrm{gb}$ & 0.099 \\
\hline V22 & & & 20 & 0 & 0.2 & & $\mathrm{AW}$ & 158.7 & 9.10 & $\mathrm{gb}$ & 0.160 \\
\hline V23 & & & 30 & 0 & 0.2 & & $\mathrm{AW}$ & 158.6 & 9.00 & gb & 0.135 \\
\hline V3a & & & 25 & 0 & 0.2 & & $\mathrm{AG}$ & 0.6 & 7.50 & $\mathrm{gb}$ & 0.105 \\
\hline V7 & & & 25 & 0 & 0.2 & & $\mathrm{AG}$ & 0.9 & 8.10 & $\mathrm{gb}$ & 0.074 \\
\hline V8 & & & 25 & 0 & 0.2 & & AG & 1.0 & 9.00 & $\mathrm{gb}$ & 0.043 \\
\hline V9 & & & 35 & 0 & 0.2 & & $\mathrm{AG}$ & 0.8 & 7.60 & $\mathrm{gb}$ & 0.101 \\
\hline W10 & & & 25 & 0 & 0.2 & & AG & 0.4 & 7.40 & $\mathrm{gb}$ & 0.099 \\
\hline W11 & & & 42 & 11 & 0.2 & & AW & 1266.9 & 7.90 & $\mathrm{gb}$ & 0.273 \\
\hline W12 & & & 98 & 9 & 0.2 & & $\mathrm{AW}$ & 1268.0 & 6.90 & $\mathrm{gb}$ & 0.268 \\
\hline W13! & $\mathrm{x}$ & & 44 & 10 & 0.2 & & AW & 1267.7 & 8.80 & $\mathrm{gb}$ & 0.258 \\
\hline W14 & & & 47 & 10 & 0.2 & & AW & 1267.1 & 8.50 & $\mathrm{gb}$ & 0.280 \\
\hline W15 & & & 61 & 10 & 0.2 & & AW & 1268.5 & 8.40 & $\mathrm{gb}$ & 0.626 \\
\hline W17 & & & 54 & 9 & 0.2 & & $\mathrm{AW}$ & 1267.5 & 9.10 & $\mathrm{gb}$ & 0.448 \\
\hline W18 & & & 25 & 0 & 0.2 & & AG & 0.7 & 7.50 & $\mathrm{gb}$ & 0.100 \\
\hline W19 & & & 25 & 0 & 0.2 & & AG & 0.9 & 7.90 & $\mathrm{gb}$ & 0.135 \\
\hline
\end{tabular}




\begin{tabular}{|c|c|c|c|c|c|c|c|c|c|c|c|}
\hline SN & Qu & $\mathrm{Cu}$ & $\mathrm{He}$ & Co & CS & $\mathrm{Eq}$ & Op & Time & Thick & $\mathbf{R C}$ & $\tau_{\max }$ \\
\hline W2 & $\mathrm{x}$ & & 39 & 10 & 0.2 & & AW & 1266.0 & 8.70 & $\mathrm{gb!}$ & 0.090 \\
\hline W20 & $\mathrm{X}$ & & 50 & 10 & 0.2 & & AW & 1266.2 & 8.70 & $\mathrm{gb}$ ! & 0.054 \\
\hline W22 & & & 25 & 0 & 0.2 & & AG & 0.5 & 8.00 & $\mathrm{gb}$ & 0.069 \\
\hline W23 & & & 25 & 0 & 0.2 & & $\mathrm{AG}$ & 0.8 & 8.00 & $\mathrm{gb}$ & 0.118 \\
\hline W3 & $\mathrm{x}$ & & 52 & 0 & 0.2 & & AW & 1266.4 & 8.20 & $\mathrm{gb}$ ! & 0.026 \\
\hline W5 & & & 47 & 9 & 0.2 & & AW & 1267.3 & 7.90 & $\mathrm{gb}$ & 0.421 \\
\hline W6 & & & 52 & 11 & 0.2 & & AW & 1268.3 & 6.90 & $\mathrm{gb}$ & 0.130 \\
\hline W7 & $\mathrm{x}$ & & 57 & 10 & 0.2 & & AW & 1266.7 & 8.20 & $\mathrm{gb!}$ & 0.089 \\
\hline W9 & & & 92 & 9 & 0.2 & & AW & 1265.7 & 7.70 & $\mathrm{gb}$ & 0.455 \\
\hline X1 & & & 67 & 10 & 0.2 & & AW & 1269.6 & 6.50 & $\mathrm{gbF}$ & 0.549 \\
\hline X10 & $\mathrm{x}$ & & 69 & 0 & 0.2 & & AW & 1357.3 & 6.00 & $\mathrm{gbF}$ ! & 0.202 \\
\hline X11 & & & 45 & 9 & 0.2 & & AW & 1269.7 & 5.20 & $\mathrm{gbF}$ & 0.520 \\
\hline X12 & & & 25 & 0 & 0.2 & & $\mathrm{AG}$ & 0.9 & 5.90 & $\mathrm{gb}$ & 0.111 \\
\hline X13 & & & 67 & 11 & 0.2 & & AW & 1270.1 & 6.70 & $\mathrm{gbF}$ & 0.433 \\
\hline X14 & & & 25 & 0 & 0.2 & & $\mathrm{AG}$ & 0.6 & 5.90 & $\mathrm{gb}$ & 0.097 \\
\hline X15 & & & 64 & 13 & 0.2 & & AW & 1268.5 & 6.20 & $\mathrm{gbF}$ & 0.386 \\
\hline X16 & & & 68 & 0 & 0.2 & & AW & 1357.1 & 5.90 & $\mathrm{gbF}$ & 0.615 \\
\hline X17 & & & 64 & 0 & 0.2 & & AW & 1357.6 & 6.00 & $\mathrm{gbFc}$ & 0.305 \\
\hline X18 & & & 25 & 0 & 0.2 & & $\mathrm{AG}$ & 0.8 & 5.90 & $\mathrm{gb}$ & 0.122 \\
\hline X19 & & & 25 & 0 & 0.2 & & $\mathrm{AG}$ & 0.7 & 6.00 & $\mathrm{gb}$ & 0.091 \\
\hline $\mathrm{X} 2$ & & & 74 & 9 & 0.2 & & AW & 1268.9 & 6.20 & $\mathrm{gbF}$ & 0.592 \\
\hline X20 & & & 25 & 0 & 0.2 & & AG & 0.4 & 6.00 & $\mathrm{gb}$ & 0.222 \\
\hline X22 & & & 76 & 10 & 0.2 & & AW & 1268.7 & 5.80 & $\mathrm{gbF}$ & 0.248 \\
\hline X23 & & & 73 & 12 & 0.2 & & AW & 1268.1 & 5.60 & $\mathrm{gb}$ & 0.520 \\
\hline X5 & & & 62 & 0 & 0.2 & & AW & 1357.8 & 6.20 & $\mathrm{gb}$ & 0.651 \\
\hline X6 & & & 75 & 0 & 0.2 & & AW & 1357.5 & 6.20 & gbcF & 0.533 \\
\hline X7 & & & 64 & 9 & 0.2 & & AW & 1269.3 & 6.20 & scbF & 0.478 \\
\hline X8 & & & 54 & 10 & 0.2 & & AW & 1268.3 & 7.80 & $\mathrm{gb}$ & 0.223 \\
\hline X9 & & & 66 & 12 & 0.2 & & AW & 1269.1 & 6.10 & $\mathrm{gbF}$ & 0.662 \\
\hline AA12 & & & 75 & & 0.2 & & JS/AW & 0.5 & 6.00 & $\mathrm{ob}$ & 0.770 \\
\hline AA12 & & & & & 0.2 & & JS/AW & 0.7 & 5.60 & $\mathrm{CFc}$ & 1.460 \\
\hline AA11 & & & & & 0.2 & & JS/AW & 0.8 & 5.10 & $\mathrm{gb}$ & 0.802 \\
\hline AA15 & & & & & 0.2 & & JS/AW & 1.1 & 5.30 & $\mathrm{gb}$ & 1.510 \\
\hline AA13 & & & 82 & & 0.2 & & JS/AW & 1.3 & 6.00 & $\mathrm{cf}$ & 0.773 \\
\hline AA10 & & & 63 & & 0.2 & & JS/AW & 1.6 & 5.60 & $\mathrm{CF}$ & 1.080 \\
\hline AB1 & & & & & 0.2 & & JS/AW & 0.9 & 7.10 & $\mathrm{gb}$ & 0.518 \\
\hline AB22 & & & 75 & & 0.2 & & JS/AW & 1.1 & 6.50 & $\mathrm{scb}$ & 0.412 \\
\hline AB10 & & & & & 0.2 & & JS/AW & 2.2 & 6.50 & $\mathrm{gbF}$ & 0.293 \\
\hline AB17 & & & & & 0.2 & & JS/AW & 2.4 & 6.50 & $\mathrm{gbF}$ & 0.263 \\
\hline AB6 & & & & & 0.2 & & JS/AW & 2.6 & 7.20 & $\mathrm{gbF}$ & 0.173 \\
\hline
\end{tabular}




\begin{tabular}{|c|c|c|c|c|c|c|c|c|c|c|c|}
\hline SN & Qu & $\mathrm{Cu}$ & $\mathrm{He}$ & Co & CS & $\mathrm{Eq}$ & Op & Time & Thick & RC & $\tau_{\max }$ \\
\hline AC6 & & & 71 & & 0.2 & & JS/AW & 0.5 & 6.40 & $\mathrm{gbF}$ & 0.235 \\
\hline $\mathrm{AC} 22$ & & & 80 & & 0.2 & & JS/AW & 0.6 & 7.30 & $\mathrm{gb}$ & 0.069 \\
\hline AC20 & & & & & 0.2 & & JS/AW & 0.8 & 7.40 & $\mathrm{gbF}$ & 0.245 \\
\hline $\mathrm{AC8}$ & & & & & 0.2 & & JS/AW & 1.0 & 7.00 & $\mathrm{gb}$ & 0.233 \\
\hline AC10 & & & 74 & & 0.2 & & JS/AW & 1.2 & 6.90 & $\mathrm{gb}$ & 0.175 \\
\hline AD2 & & & & & 0.2 & & JS/AW & 1.1 & 7.80 & $\mathrm{gbF}$ & 0.091 \\
\hline AD18 & & & 49 & & 0.2 & & JS/AW & 1.4 & 7.20 & $\mathrm{gbF}$ & 0.107 \\
\hline AD20 & & & 70 & & 0.2 & & JS/AW & 1.6 & 10.10 & $\mathrm{gbF}$ & 0.052 \\
\hline AD9 & & & 185 & & 0.2 & & JS/AW & 1.8 & 8.50 & $\mathrm{gbF}$ & 0.070 \\
\hline AD15 & & & 70 & & 0.2 & & JS/AW & 2.0 & 7.10 & $\mathrm{gbF}$ & 0.139 \\
\hline AE12 & & & 60 & & 0.2 & & JS/AW & 1.1 & 3.30 & $\mathrm{gbF}$ & 0.234 \\
\hline AE17 & & & 79 & & 0.2 & & JS/AW & 1.5 & 4.20 & $\mathrm{gb}$ & 0.121 \\
\hline AE6 & & & 97 & & 0.2 & & AG/AW & 15.4 & 3.80 & $\mathrm{gb}$ & 0.388 \\
\hline AE7 & & & 67 & & 0.2 & & AG/AW & 20.1 & 4.30 & $\mathrm{gb}$ & 0.223 \\
\hline AE1 & & & 75 & & 0.2 & & AG/AW & 20.2 & 4.30 & $\mathrm{gbF}$ & 0.317 \\
\hline AE19 & & & 30 & & 0.2 & & AG/AW & 20.5 & 4.10 & $\mathrm{gb}$ & 0.079 \\
\hline AE8 & & & 27 & & 0.2 & & AG/AW & 20.6 & 4.20 & $\mathrm{gb}$ & 0.154 \\
\hline AF10 & & & 70 & & 0.2 & & AG/AW & 15.2 & 4.90 & $\mathrm{gbF}$ & 0.236 \\
\hline AF17 & $X$ & & & & 0.2 & & AG/AW & 16.2 & 5.20 & $\mathrm{gb}$ & 0.113 \\
\hline AF9 & & & 80 & & 0.2 & & AG/AW & 17.7 & 5.30 & $\mathrm{gb}$ & 0.220 \\
\hline AF14 & & & 80 & & 0.2 & & AG/AW & 18.3 & 4.10 & $\mathrm{gb}$ & 0.046 \\
\hline AF16 & & & 79 & & 0.2 & & AG/AW & 18.5 & 5.10 & $\mathrm{gb}$ & 0.091 \\
\hline AF13 & & & 80 & & 0.2 & & AG/AW & 18.7 & 5.00 & $\mathrm{gb}$ & 0.194 \\
\hline AF12 & & & 26 & & 0.2 & & AG/AW & 18.9 & 5.10 & $\mathrm{gb}$ & 0.299 \\
\hline AG17 & & $\mathrm{x}$ & 95 & & 0.2 & & AW & 114.2 & 3.40 & $\mathrm{CFc}$ & 0.371 \\
\hline AG9 & & $\mathrm{x}$ & 70 & & 0.2 & & AW & 114.7 & 4.10 & $\mathrm{gbc}$ & 0.450 \\
\hline AG12 & & $\mathrm{x}$ & 144 & & 0.2 & & $\mathrm{AW}$ & 115.7 & 4.30 & $\mathrm{gb}$ & 0.184 \\
\hline AG23 & $X$ & $\mathrm{x}$ & 68 & & 0.2 & & AW & 115.9 & 4.80 & $\mathrm{gb}$ ! & 0.058 \\
\hline AG13 & & $\mathrm{x}$ & 91 & & 0.2 & & $\mathrm{AW}$ & 116.2 & 4.30 & $\mathrm{gb}$ & 0.234 \\
\hline AG5 & & & 100 & & 0.2 & & AG/AW & 0.8 & 3.90 & $\mathrm{gb}$ & 0.512 \\
\hline AG14 & $X$ & & 35 & & 0.2 & & AG/AW & 0.9 & 3.70 & $\mathrm{gb} !$ & 0.010 \\
\hline AG22 & & & 49 & & 0.2 & & AG/AW & 1.2 & 4.20 & $\mathrm{gb}$ & 0.182 \\
\hline AG10 & & & 46 & & 0.2 & & AG/AW & 1.3 & 4.20 & $\mathrm{gb}$ & 0.316 \\
\hline AG20 & & & 48 & & 0.2 & & AG/AW & 1.5 & 4.70 & $\mathrm{gb}$ & 0.512 \\
\hline AG15 & $X$ & & 71 & & 0.2 & & AW & 113.9 & 4.50 & scbc! & 0.993 \\
\hline AG18 & & & 84 & & 0.2 & & AW & 114.5 & 4.20 & $\mathrm{gb}$ & 0.525 \\
\hline AG3 & $X$ & & 89 & & 0.2 & & AW & 115.5 & 4.30 & $\mathrm{gb} !$ & 0.087 \\
\hline AG6 & & & 92 & & 0.2 & & AW & 116.7 & 4.90 & $\mathrm{gb}$ & 0.361 \\
\hline AG19 & & & 70 & & 0.2 & & AW & 117.3 & 4.80 & $\mathrm{gb}$ & 0.227 \\
\hline AH5 & & $\mathrm{x}$ & 40 & & 0.2 & & AG/AW & 500.1 & 2.70 & $\mathrm{gb}$ & 0.079 \\
\hline
\end{tabular}




\begin{tabular}{|c|c|c|c|c|c|c|c|c|c|c|c|}
\hline SN & Qu & $\mathrm{Cu}$ & $\mathrm{He}$ & Co & CS & $\mathbf{E q}$ & Op & Time & Thick & $\mathbf{R C}$ & $\tau_{\max }$ \\
\hline AH23 & & $\mathrm{x}$ & 58 & & 0.2 & & AG/AW & 500.5 & 3.10 & $\mathrm{gb}$ & 0.075 \\
\hline AH1 & & $\mathrm{x}$ & 86 & & 0.2 & & AG/AW & 500.7 & 3.30 & $\mathrm{gb}$ & 0.517 \\
\hline AH6 & & $\mathrm{x}$ & 100 & & 0.2 & & AG/AW & 501.0 & 2.70 & $\mathrm{gb}$ & 0.261 \\
\hline AH22 & & & 48 & & 0.2 & & AG/AW & 0.6 & 3.90 & ebc & 0.749 \\
\hline AH9 & & & 49 & & 0.2 & & AG/AW & 0.7 & 4.00 & gbc & 0.811 \\
\hline AH17 & $X$ & & 60 & & 0.2 & & AG/AW & 1.1 & 3.80 & $\mathrm{gb} !$ & 0.039 \\
\hline AH15 & & & 40 & & 0.2 & & AG/AW & 1.4 & 4.00 & $\mathrm{gbF}$ & 0.781 \\
\hline AH12 & & & 87 & & 0.2 & & AG/AW & 1.8 & 3.50 & $\mathrm{gb}$ & 0.450 \\
\hline AH14 & & & 69 & & 0.2 & & AG/AW & 1.9 & 3.40 & $\mathrm{gb}$ & 0.242 \\
\hline AI17 & & & 100 & & 0.2 & & AG/AW & 0.7 & 4.50 & $\mathrm{gbF}$ & 0.306 \\
\hline AI22 & & & 58 & & 0.2 & & AG/AW & 0.9 & 4.60 & $\mathrm{gb}$ & 0.418 \\
\hline AI23 & & & 61 & & 0.2 & & AG/AW & 1.0 & 4.00 & $\mathrm{gb}$ & 0.855 \\
\hline AI15 & & & 77 & & 0.2 & & AG/AW & 1.2 & 4.60 & $\mathrm{gb}$ & 0.505 \\
\hline AI19 & & & 67 & & 0.2 & & AG/AW & 1.3 & 4.50 & $\mathrm{gb}$ & 0.333 \\
\hline AI7 & & & 136 & & 0.2 & & MV/AG & 447.1 & 4.00 & gbli & 0.393 \\
\hline AI20 & & & 107 & & 0.2 & & $\mathrm{MV} / \mathrm{AG}$ & 448.3 & 6.80 & $\mathrm{gb}$ & 0.499 \\
\hline AI14 & & & 135 & & 0.2 & & $\mathrm{MV} / \mathrm{AG}$ & 448.6 & 5.50 & $\mathrm{gb}$ & 0.375 \\
\hline AI10 & & & 70 & & 0.2 & & $\mathrm{MV} / \mathrm{AG}$ & 448.8 & 5.20 & scb & 0.738 \\
\hline AI16 & & & 86 & & 0.2 & & $\mathrm{MV} / \mathrm{AG}$ & 449.1 & 5.70 & $\mathrm{gb}$ & 0.356 \\
\hline AI12 & & & 81 & & 0.2 & & MV/AG & 449.3 & 6.20 & gbli & 0.677 \\
\hline AJ18 & $X$ & & & & 0.2 & & $\mathrm{MV} / \mathrm{AG}$ & 1.0 & 10.00 & scb! & 0.783 \\
\hline
\end{tabular}




\section{APPENDIX C}

\section{XT Model Documentation}

\section{CFD Analysis}

To obtain an ice shape, over 300 LEWICE cases were run and it was determined that a useful methodology to limit the size of the ice would be running rime conditions after a small layer of ice had formed on the coupon. Using this methodology, a maximum-width ice shape was selected and tested in Fluent in a 3D incompressible simulation and found to have lower drag than a clean geometry due to the ice possessing rounded corners. It was learned that LEWICE could not predict ice shapes on small geometry with sharp corners, which was verified in comparison to a piggyback test in the IRT. To find a more suitable worst-case scenario, a flat plate geometry was used that was 0.25 " thick with a 0.125 " radius applied to the edges. The plate was extended 0.5 " from each side of the coupon. Cases with ice were given the A3 designation, while cases without ice were given the A4 designation. Cases without a coupon, mount, and coupon ice were given a second designation of $\mathrm{A}$, while simulations with a coupon mount and ice (if applicable) were given a second designation of $\mathrm{B}$. Case $\mathrm{A} 3 \mathrm{~A}$ dp0 refers to a flat-place (iced) case without a coupon, design point 0 . 


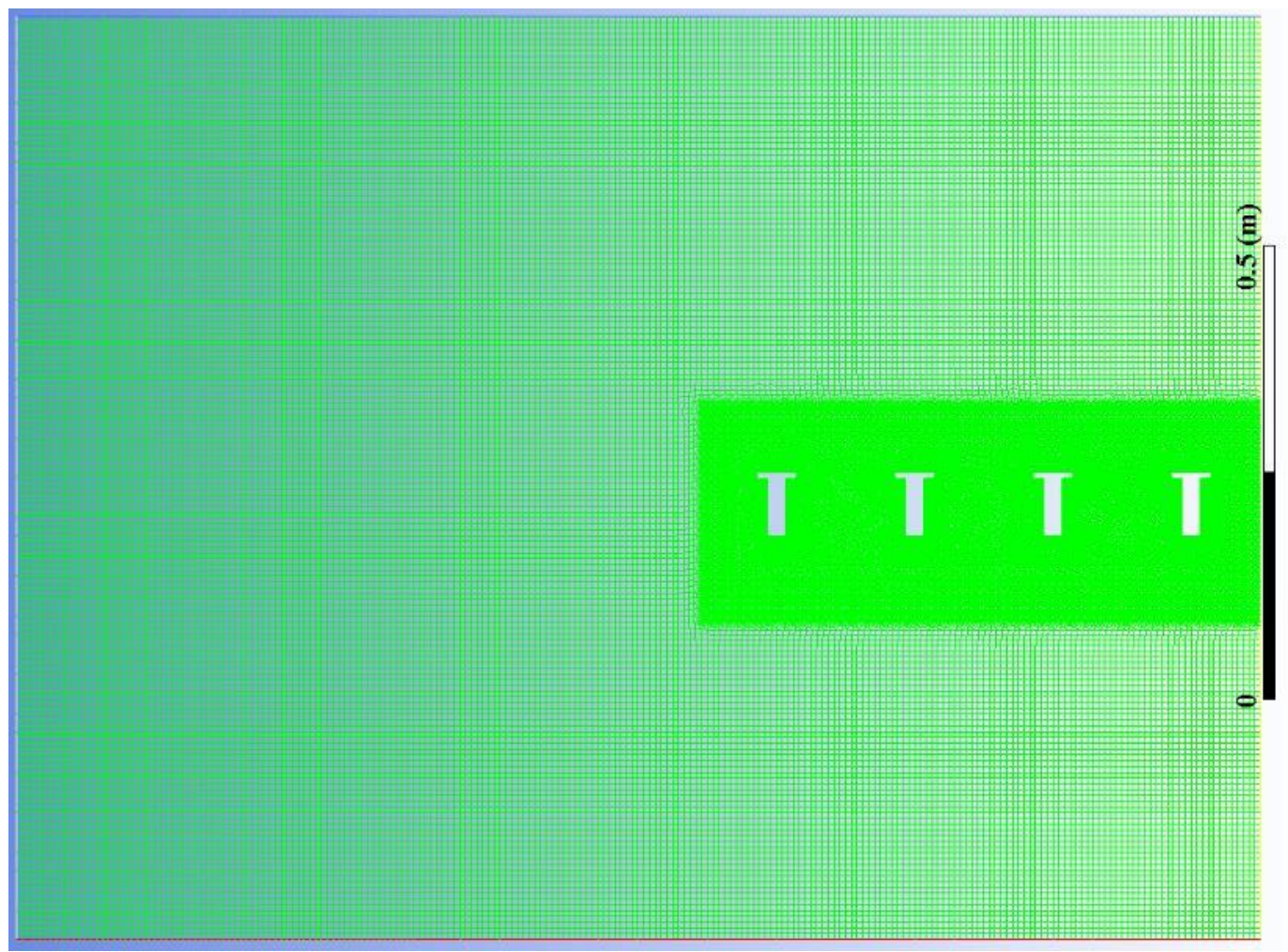

Figure 89. Lowest resolution mesh for model without coupon, with plate (A3A dp0). 150,000 nodes.

Half of the horizontal tunnel cross section was simulated, with the symmetry wall at the right of Figure 89. A velocity inlet was set at the top border, and a pressure outlet at the bottom. The remaining boundaries were set to no slip walls with zero heat flux. The Transition SST turbulence model was used with the curvature correction and viscous heating options turned on, and the energy equation was used. The air was modeled as an ideal gas with power-law viscosity, and temperature set to $253 \mathrm{~K}$. Adaptive time stepping was used with a truncation error tolerance of 0.002 , a minimum time step of $1 \mathrm{e}-6 \mathrm{~s}$, a maximum time step of 1e-4 s, and an end time of $0.06 \mathrm{~s}$. A maximum of 30 iterations per time step were used. Default options were used otherwise. Design points 0-4 increased the mesh density by decreasing the spacing parameters. The relative spacing was $1,0.8,0.6$, $0.5,0.4$, increasing to 670,000 nodes for case A3A dp4. In the case of A4 models, design 
points 3 and 4 repeated the grid size of points 1 and 2, but used a reduced corner radius (0.01" vs. $0.05 ”)$

2D compressible-flow simulations were then run on the clean geometry and the geometry iced with a flat plate with and without the coupon and mount. The drag force in the $\mathrm{X}$ and $\mathrm{Y}$ directions for case A3A dp3 on each rail are shown in Figure 90, where the flow was in the -X direction. Rail 1 is the rail next to the symmetry boundary, while rail 4 is closest to the wall of the IRT.

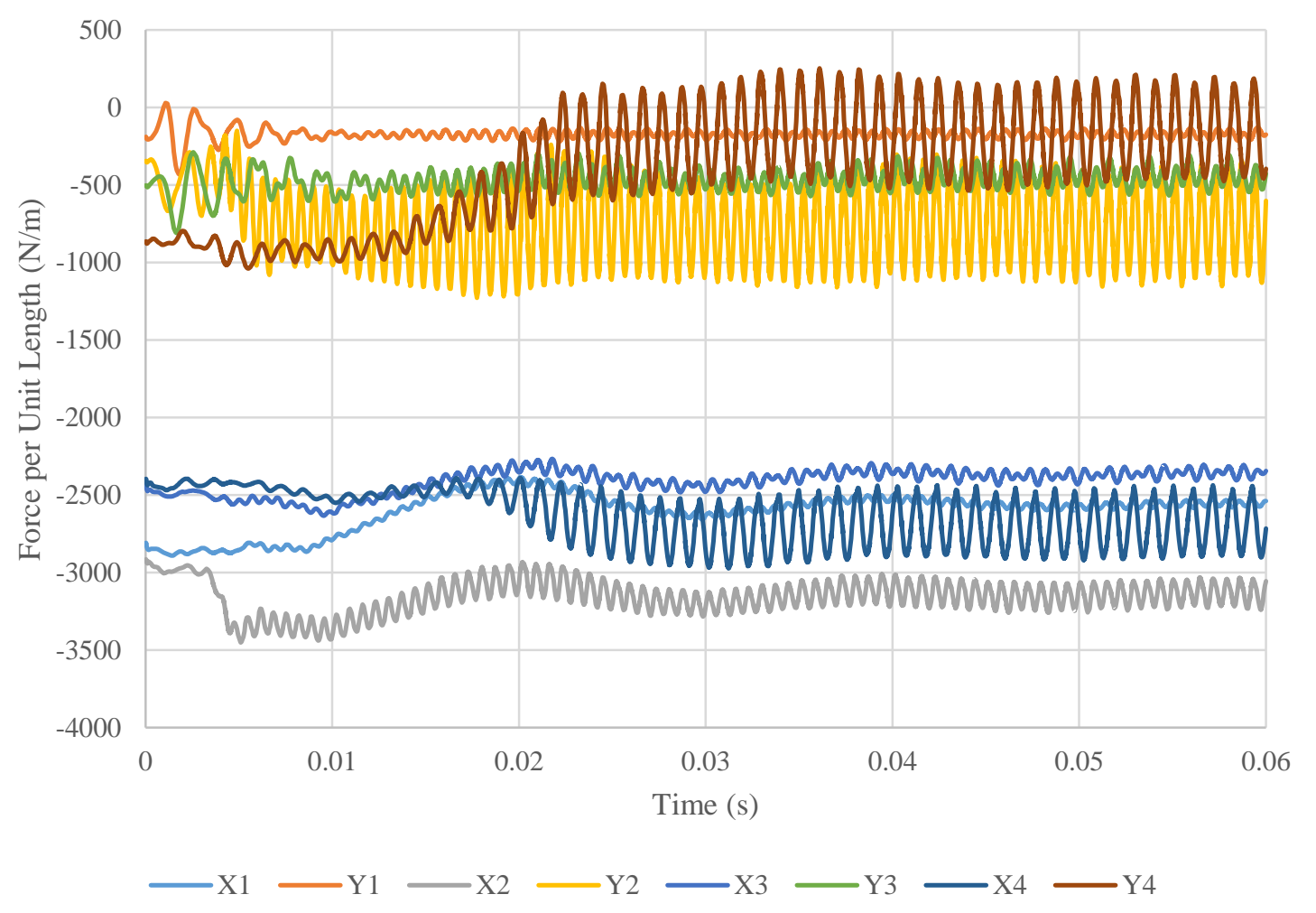

Figure 90. Resultant forces for case A3A dp3.

In all cases, the forces became consistent in average and amplitude before 0.03 seconds, so the last half of data points were used to calculate mean and oscillating forces. The amplitude of oscillating forces was calculated by taking half of the peak-to-peak force. The data was not truncated to an even number of periods for averaging. 

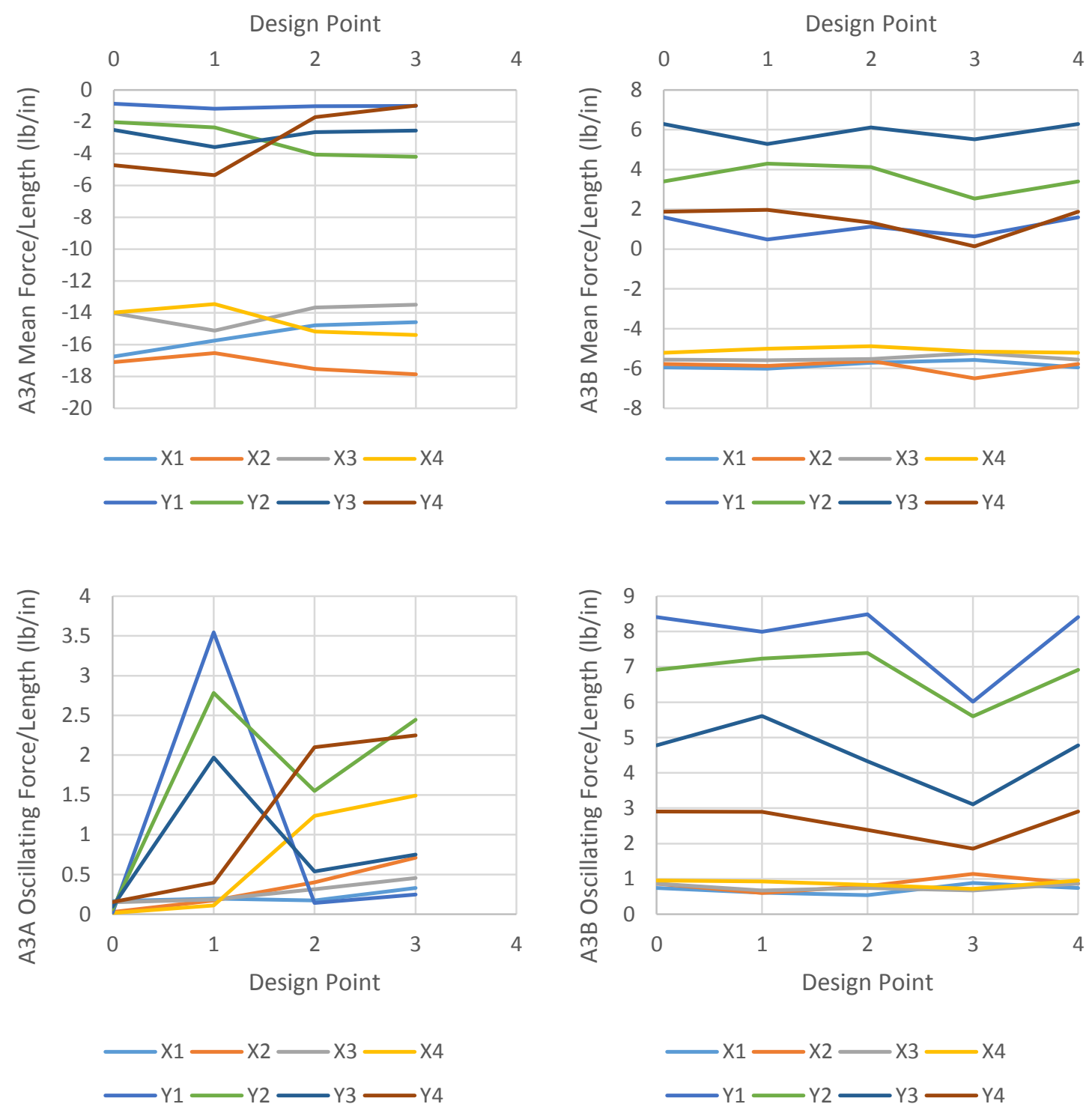

Figure 91. Grid convergence results for mean and oscillating forces at $155 \mathrm{~m} / \mathrm{s}$ on iced geometries.

The peak force for the iced (flat plate) condition in the X direction was $-17.8 \mathrm{Lb}_{f} / \mathrm{in}$, or $4827 \mathrm{lb}$ on the rails, corresponding to a coefficient of drag $\left(C_{d}\right)$ of 4.2 , which is just over double of the published value of 2.0 for a flat plate (and 1.8 for a T-beam), which is nearly constant to a Mach number of $0.7[162,170]$. The shedding frequency is approximately $1000 \mathrm{~Hz}$, corresponding to a Strouhal number just below 0.3. The difference in hand 
calculated values and the CFD does not allow for justification of the CFD results, and so they were ultimately discarded.
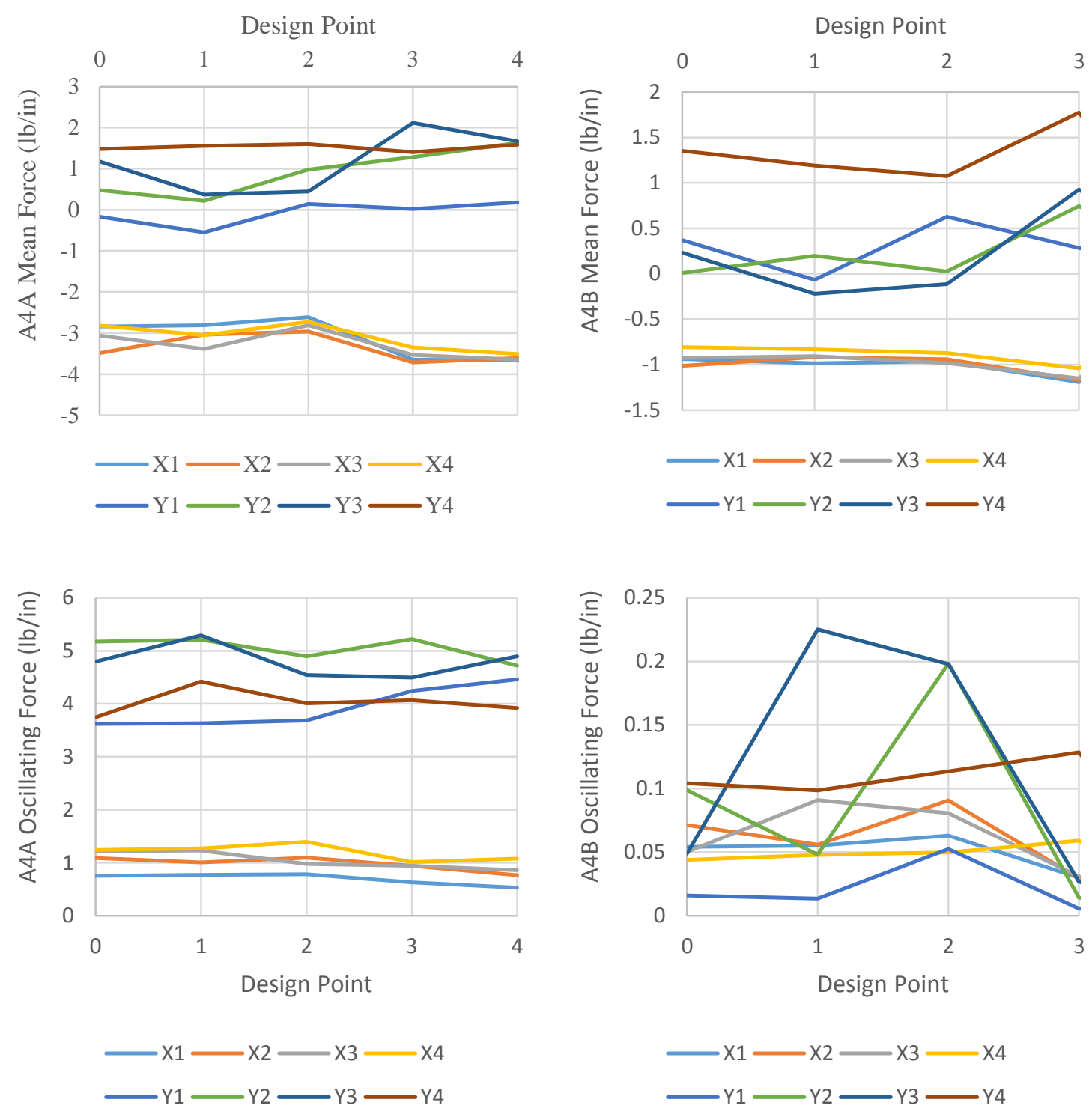

Figure 92. Grid convergence results for mean and oscillating forces at $155 \mathrm{~m} / \mathrm{s}$ on clean geometries.

The reduction in corner radius in design points 3 and 4 (for A4 cases) had little effect on the drag. The worst-case scenario was selected by taking the highest mean and oscillating forces from the three most refined design points in the $\mathrm{X}$ and $\mathrm{Y}$ directions, which resulted in using the flat plate with no coupon mean loads and x-direction oscillating loads. 
The y-component of the oscillating load was taken from the flat plate with coupon case. The clean geometry with no coupon had oscillating forces of similar magnitude to the flat plate case, but much lower mean forces. Scaling loads on the model by cross sectional area can only be done for the flat plate cases, and since the clean geometry oscillating forces are similar only the worst-case can be scaled by cross-sectional area in the static load analysis, not the dynamic analysis. Velocity scaling of aerodynamic forces should be possible in all cases, however. Additional cases were run for the updated geometry using rounded corners in a free-stream simulation, and in the tunnel cross section. Grid independence was not realized for these solutions, and so they are not included. This set of simulations did use a smoothed grid, removing the sharp increase in grid density shown in Figure 89. Shedding frequencies at $155 \mathrm{~m} / \mathrm{s}$ (301 knots) ranged from $900 \mathrm{~Hz}$ for the iced geometry to $2000 \mathrm{~Hz}$ for the clean geometry.

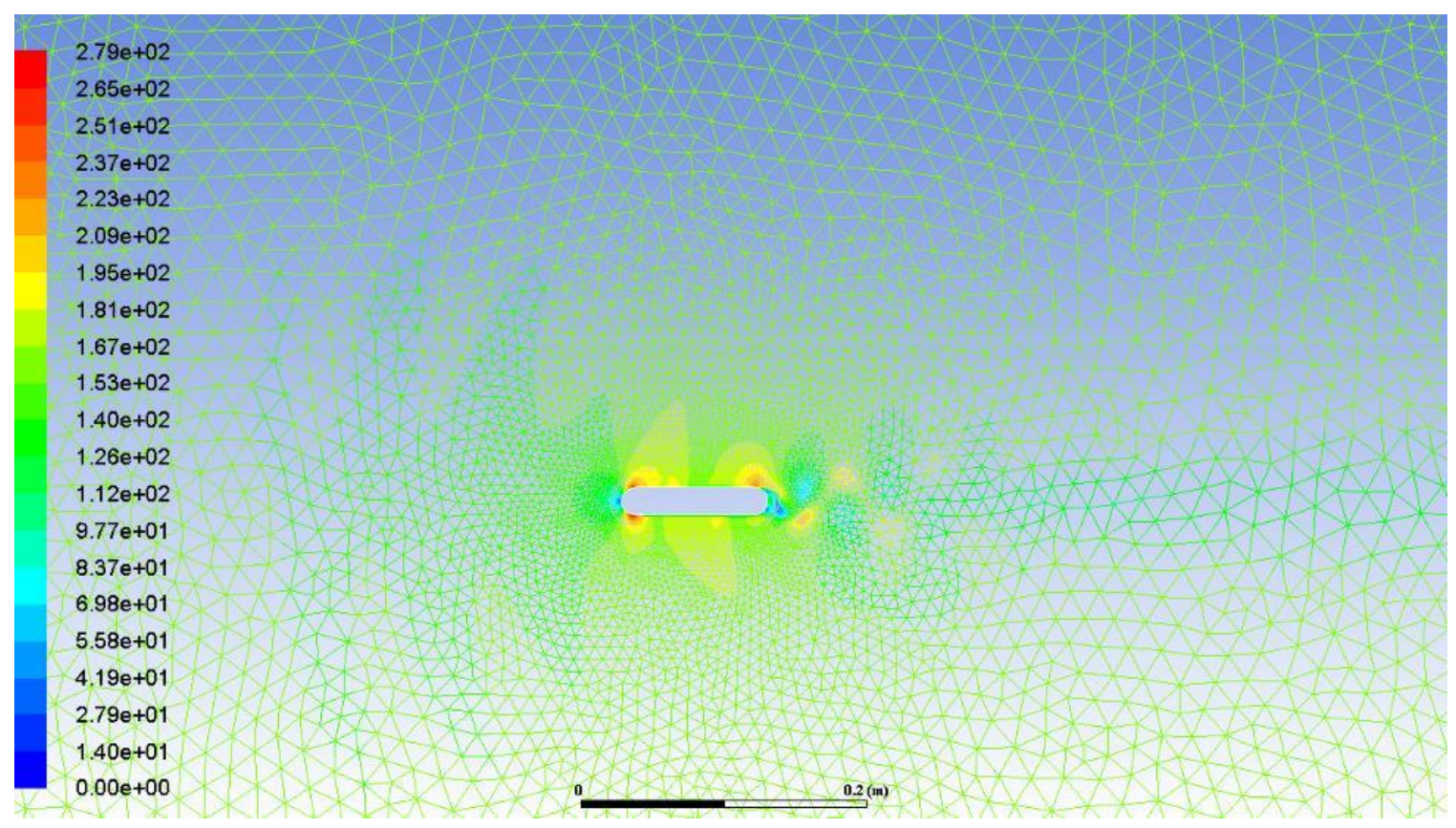

Figure 93. Velocity prediction of FEA code without coupon and rounded edges. 
Material Properties for XT Model Simulation

Three materials were used in the design of the model: 17-4 PH stainless steel, generic aluminum alloy (used as a placeholder for stronger aluminum tubing, such as 2024T3), and polyurethane. An FEA simulation was run to estimate the performance of the model under steady and dynamic loading, but since suitable loads were not obtained through CFD the simulation results are for reference only. There was not suitable information publicly available to simulate polyurethane, so sample material was ordered from McMaster-Carr and tested. Polyurethane tubing and sheet was ordered and visually inspected to insure similar materials were used. Cutouts from the polyurethane sheet were tested in a DMA Q800 tester. Sample data is shown in Figure 94.

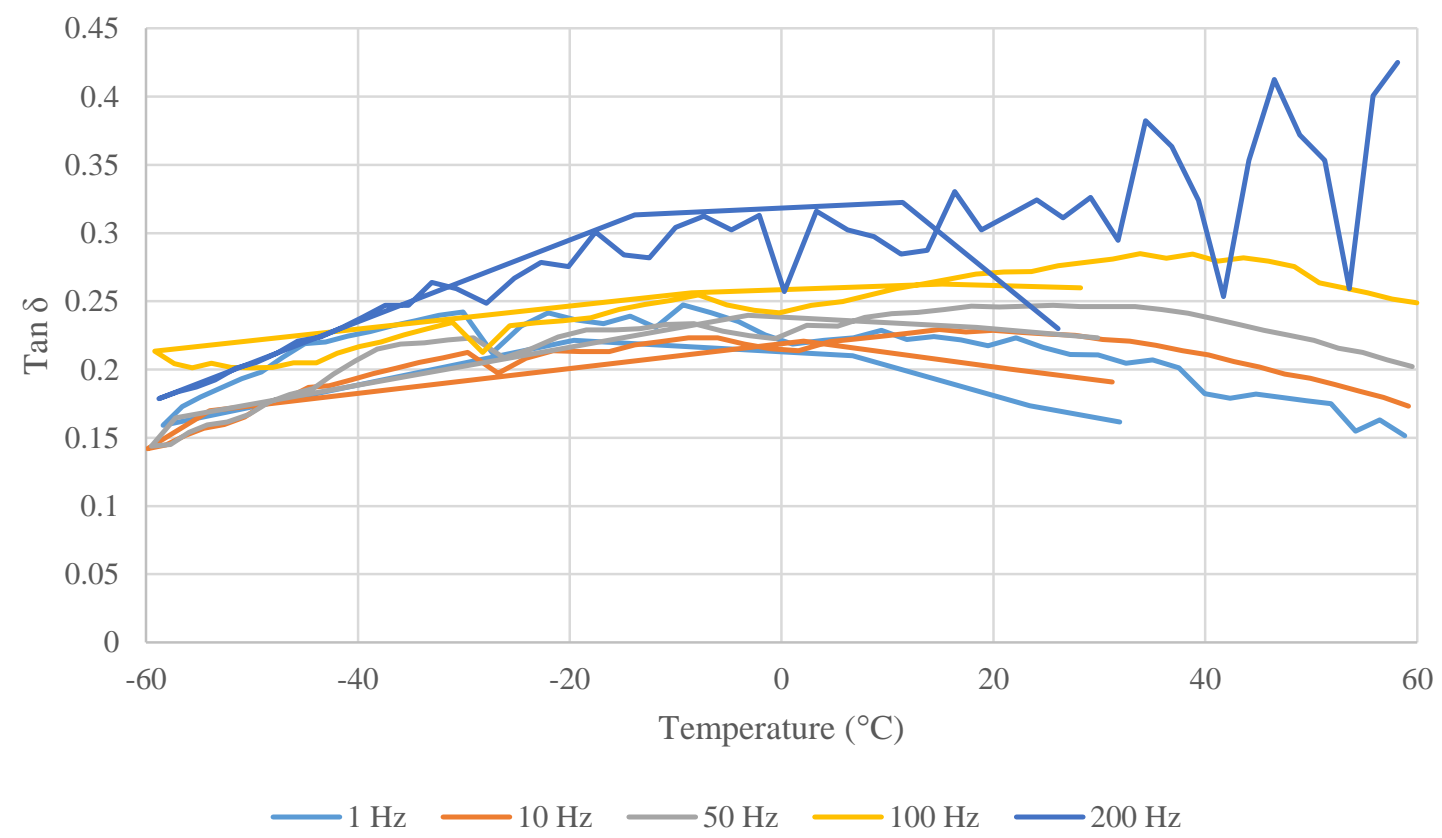

Figure 94. Sample data from DMA Q800 on polyurethane samples from McMaster-Carr.

The data range of interest is between -30 and $20^{\circ} \mathrm{C}$. The data never falls below 0.20 in the -20 to $0{ }^{\circ} \mathrm{C}$ range. The working range of the model is from -20 to $0{ }^{\circ} \mathrm{C}$. Two sets of material properties were used for polyurethane, the measured case and a 'worst case' using 
estimates from publications and higher temperatures. In the case using measured properties, the Young's modulus was set to $13,000 \mathrm{psi}$ - a value obtained from the minimum storage modulus at the lowest frequency tested (the storage modulus approaches Young's modulus as frequency approaches zero). Poisson's ratio was taken as 0.48 [171], and Tan $\delta$ was set to 0.2 . In the worst case, Young's modulus was set to 5,000 psi ([171] presents a value of 8,000 - a lower value was selected to give a wider comparison), Poisson's was set to 0.44 (obtained from testing), and Tan $\delta$ was set to 0.1 (lower than tested values from -40 to $20^{\circ} \mathrm{C}$ to provide a wider comparison).

Properties used for 17-4 PH in initial simulations were taken from generic stainless steel in ANSYS's material library, common to most stainless steels. The properties for aluminum were taken from ANSYS's Aluminum Alloy data, which were conservative compared to published values. A table of properties used for simulation are shown below. Strength values were taken from McMaster Carr as a placeholder for the final material selection.

Table 19. Properties used for initial simulations, properties in parenthesis used for worstcase simulation.

\begin{tabular}{|l|l|l|l|}
\hline & $\begin{array}{l}17-4 \text { PH A } \\
\text { Young's modulus }\end{array}$ & $\begin{array}{l}\text { Aluminum } \\
\text { Alloy }\end{array}$ & Polyurethane \\
\hline $\begin{array}{l}\text { (ksi) } \\
\text { Poisson's ratio }\end{array}$ & 0.3 & 10,300 & $13(5)$ \\
\hline Tan $\delta$ & 0 & 0.33 & $0.48(0.44)$ \\
\hline $\begin{array}{l}\text { Ultimate strength } \\
\text { (ksi) }\end{array}$ & 110 & 0 & $0.2(0.1)$ \\
\hline Yield strength (ksi) & 110 & 40.6 & 5.4 \\
\hline
\end{tabular}

The strength of polyurethane was set to lower values than provided by McMaster Carr since McMaster did not specify ultimate or yield when supplying the tensile strength 
of 5,600 psi. The tensile strength was used for compressive strength as well to provide a conservative estimate. The strength parameters should be interchangeable.

The infinite life strength of 17-4PH H925 and H1050 stainless steel is 95 and 91 ksi, respectively, compared to ultimate tensile strengths of approximately 195 and $162 \mathrm{ksi}$ [172], and the infinite life of $\mathrm{H} 900$ is expected to be higher than H925.

FEA for XT Model

Static, Modal, and Harmonic simulations were run in ANSYS Mechanical. The process was abandoned since accurate dynamic loads could not be determined in favor of hand calculations. Preliminary results are included to provide a visual distribution of the estimated forces. 

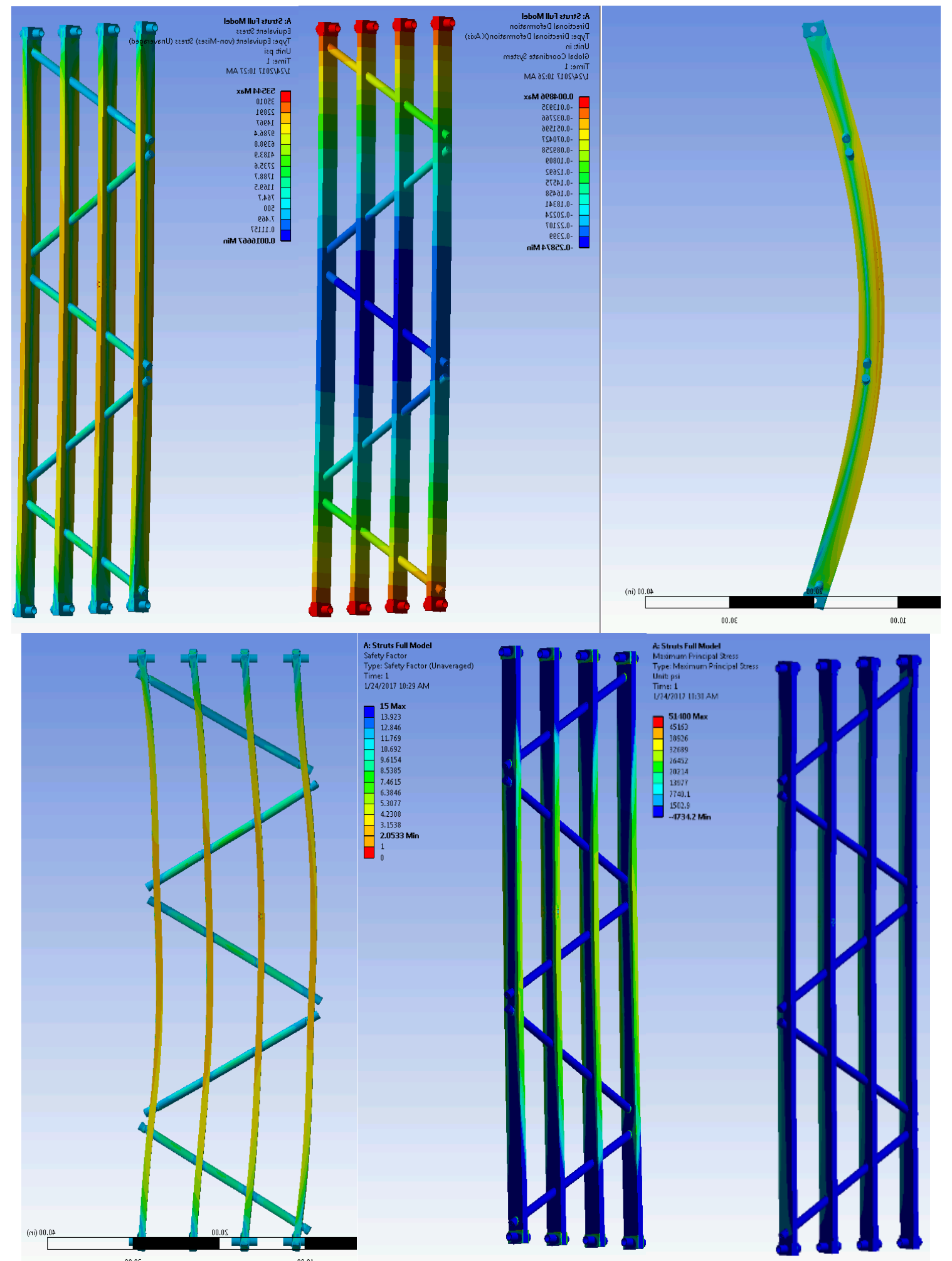

Figure 95. Preliminary static results for XT model. 


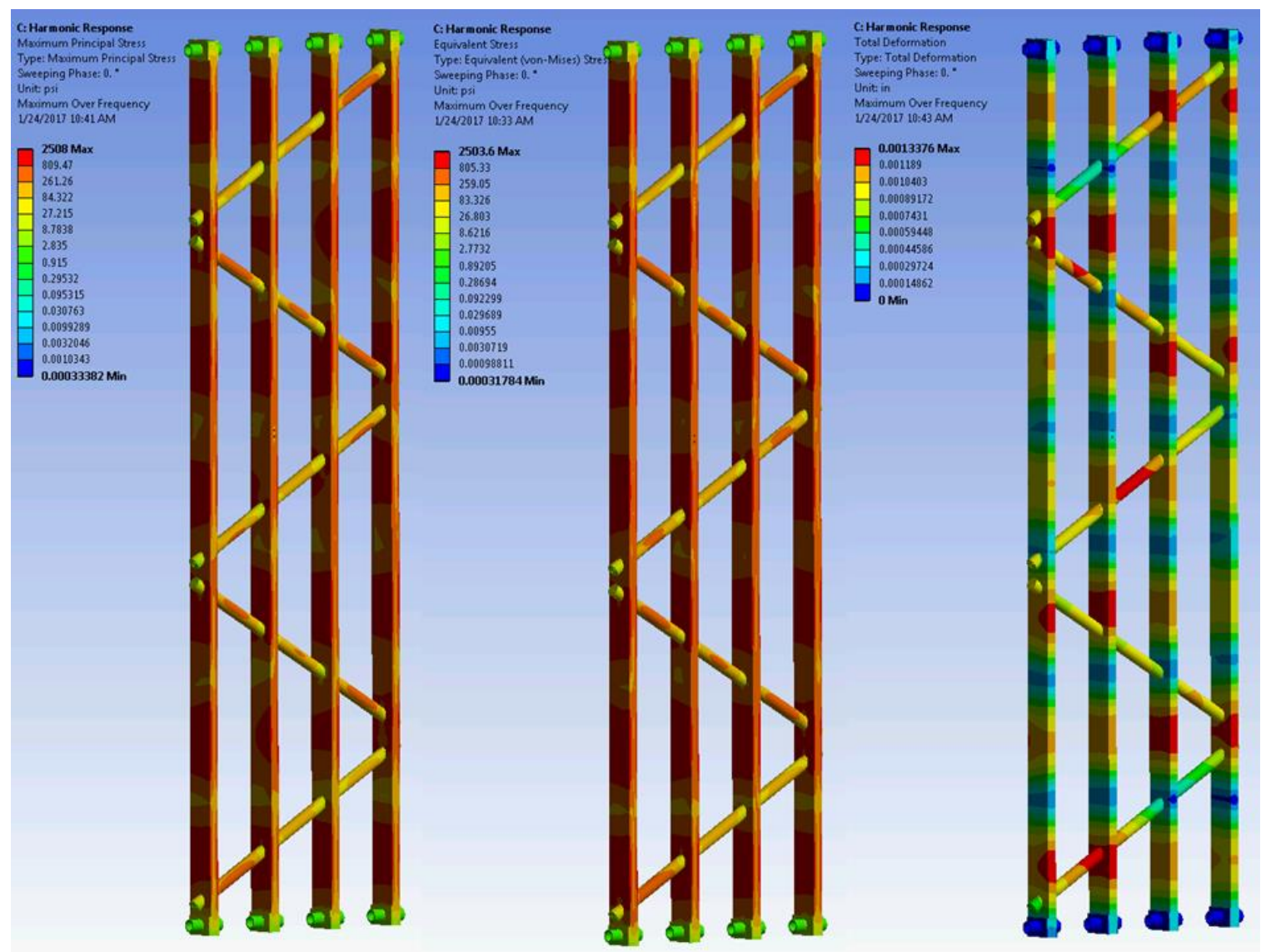

Figure 96. Harmonic Response for XT Model using maximum computed loads from CFD. 
FIGURE 30

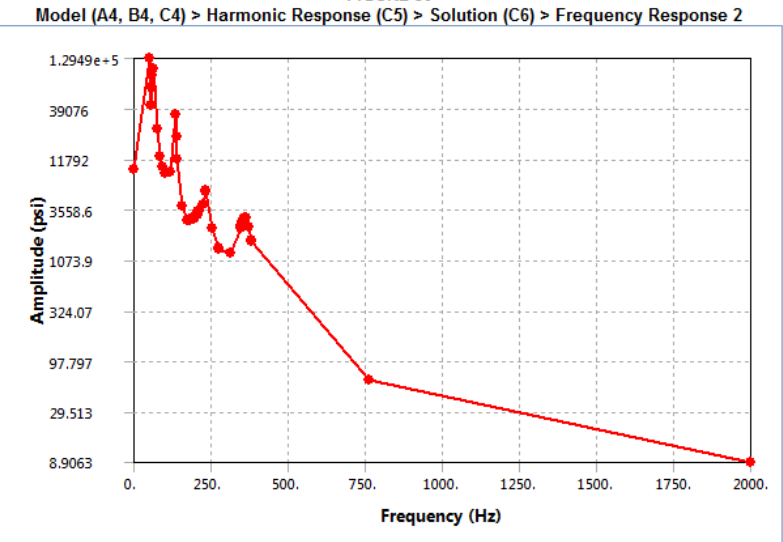

FIGURE 30
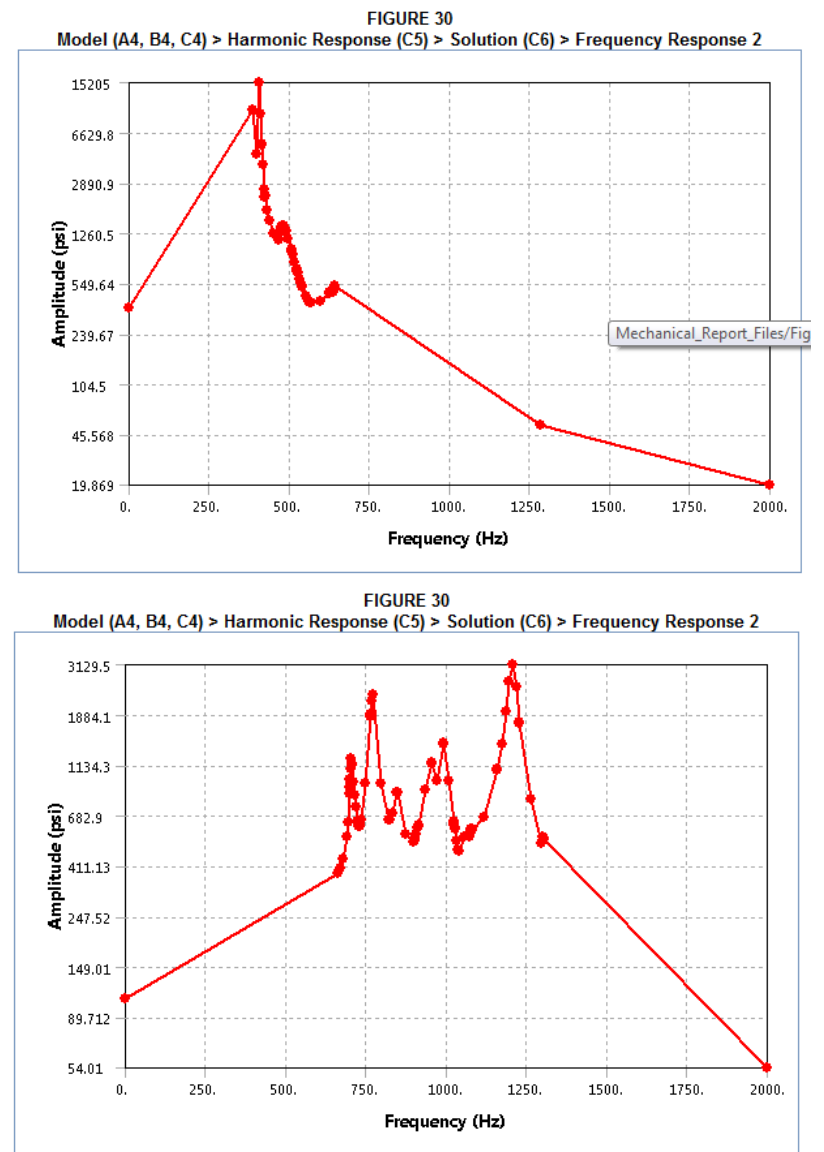

Figure 97. Frequency Response for Y axis stresses (worst case). 
Sample EFAST Analysis

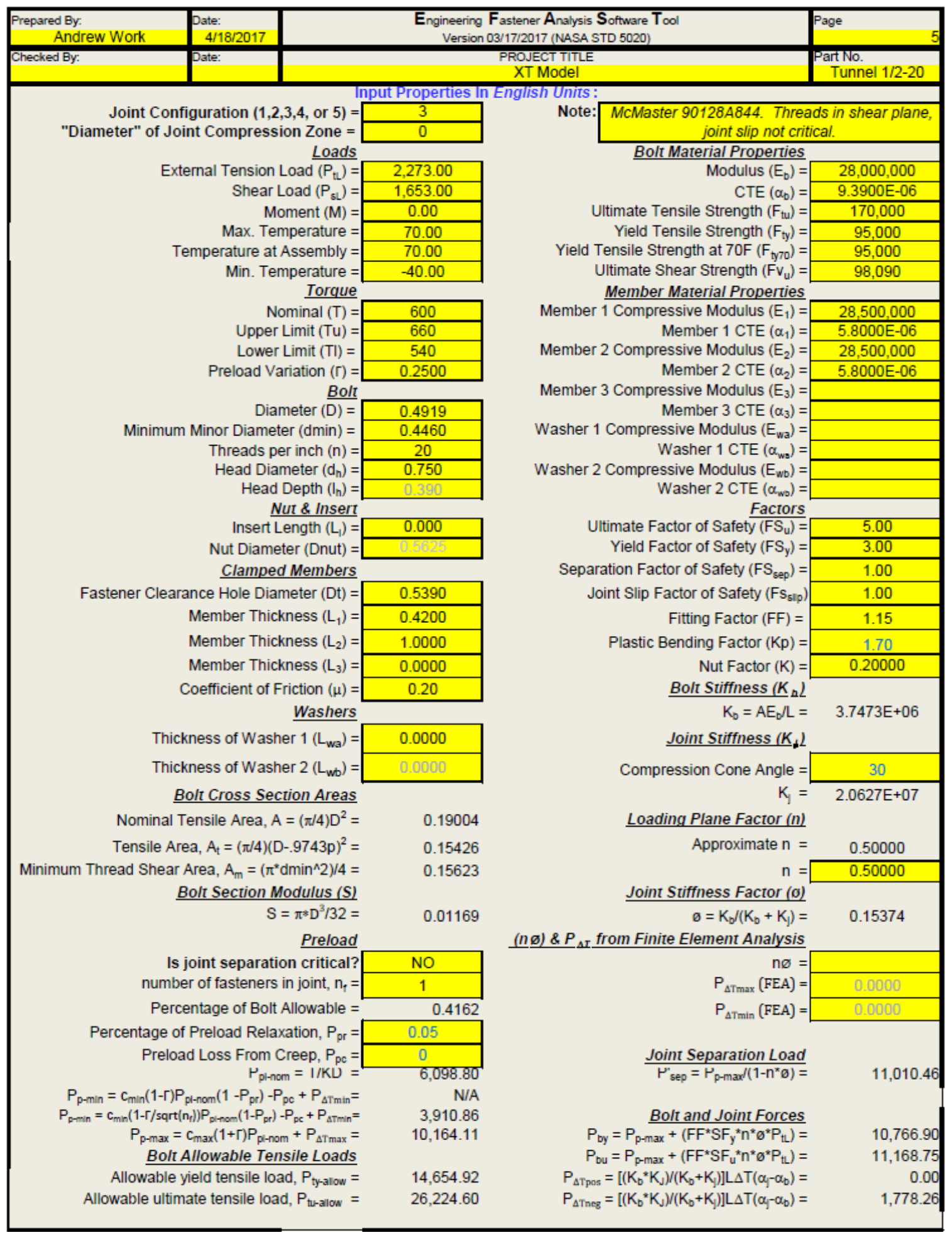




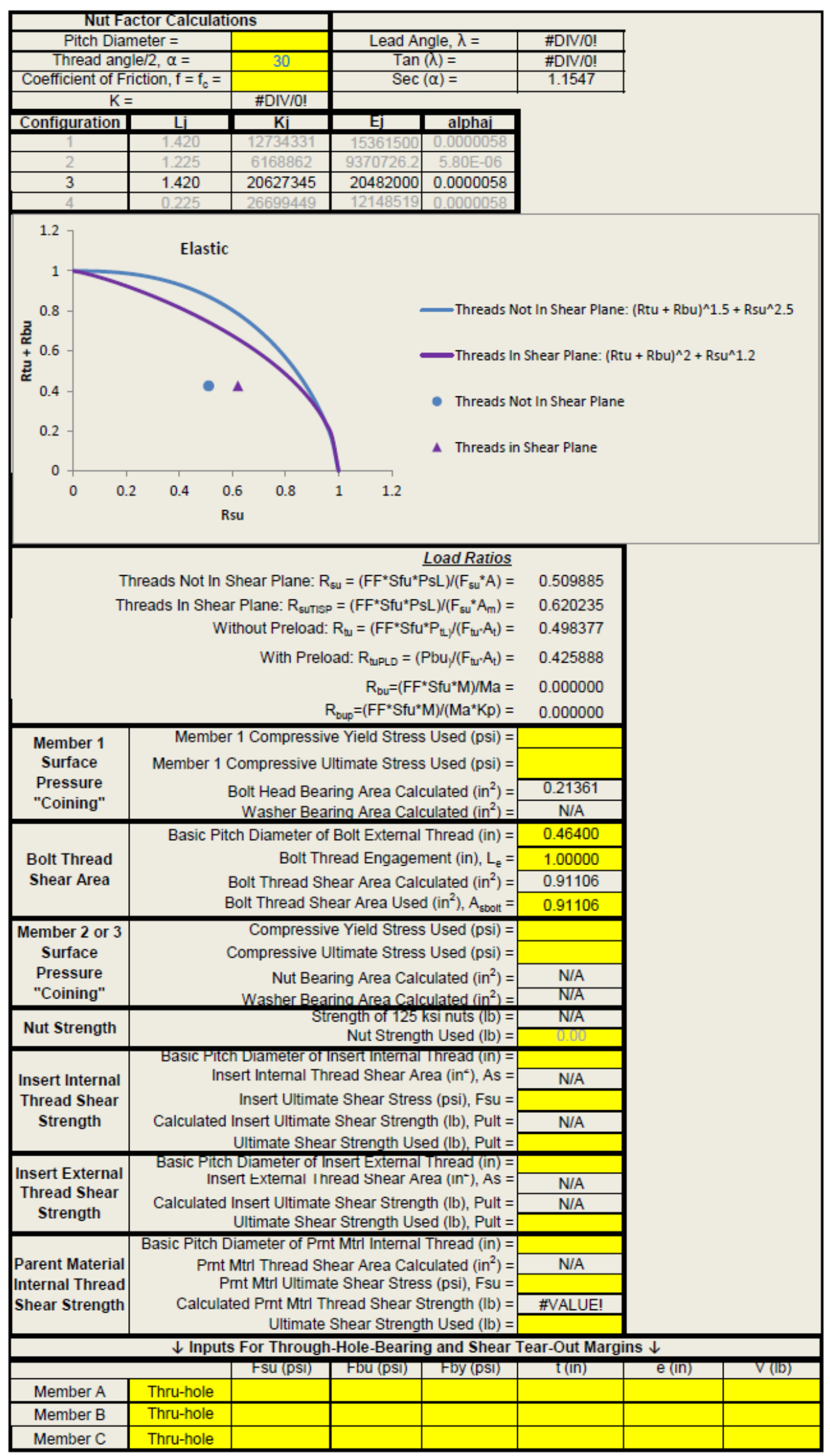




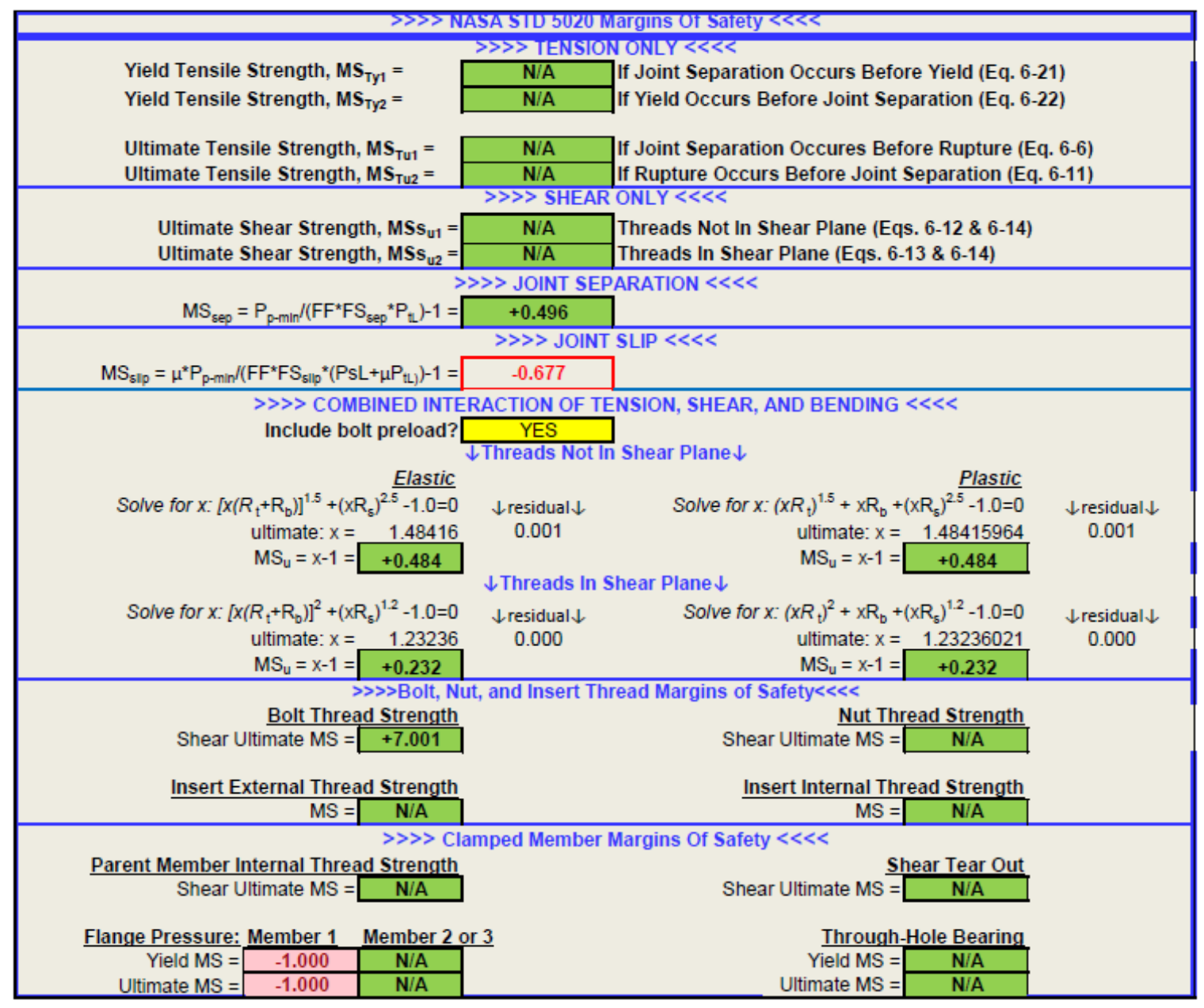

\section{XT Model Drawings}




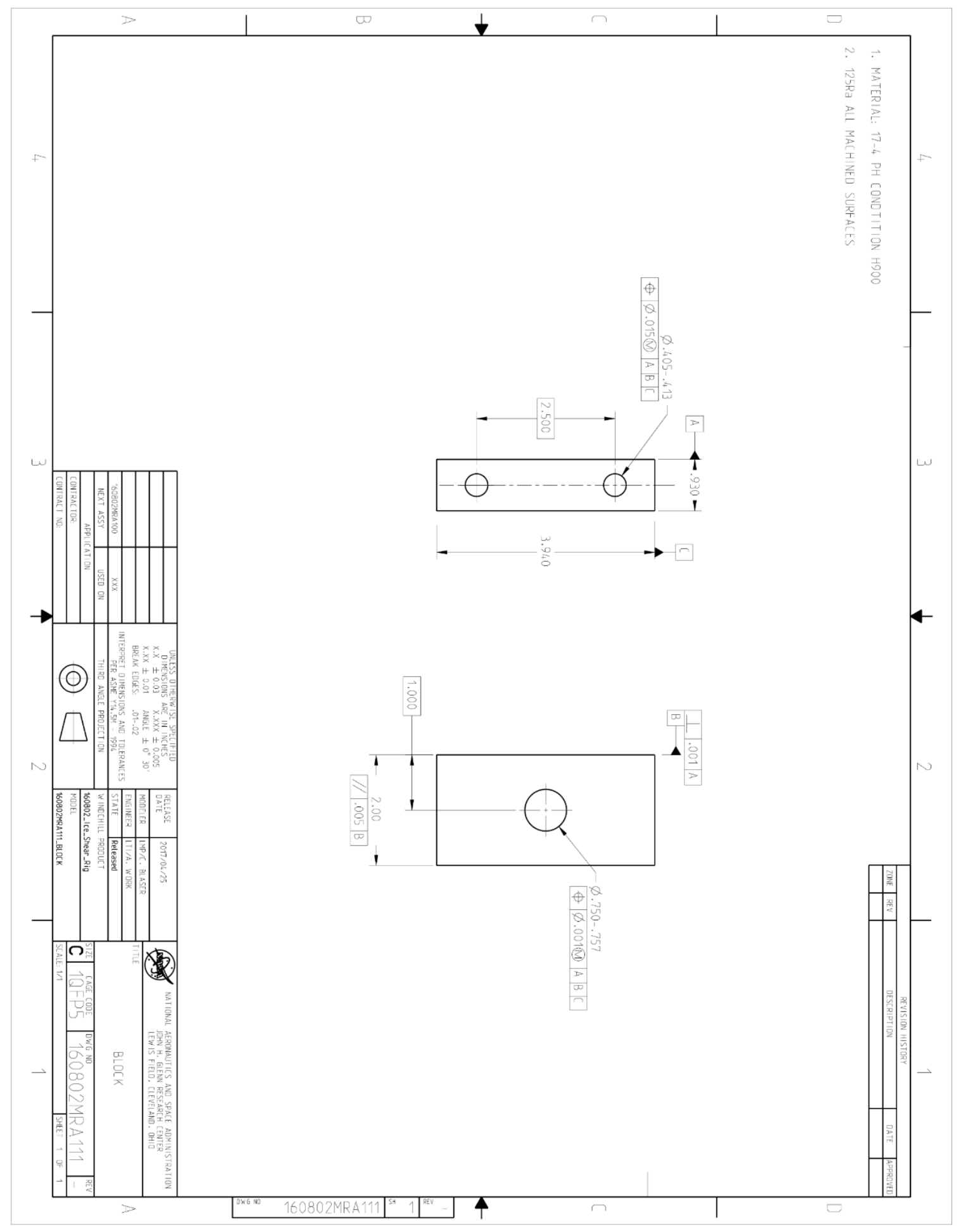




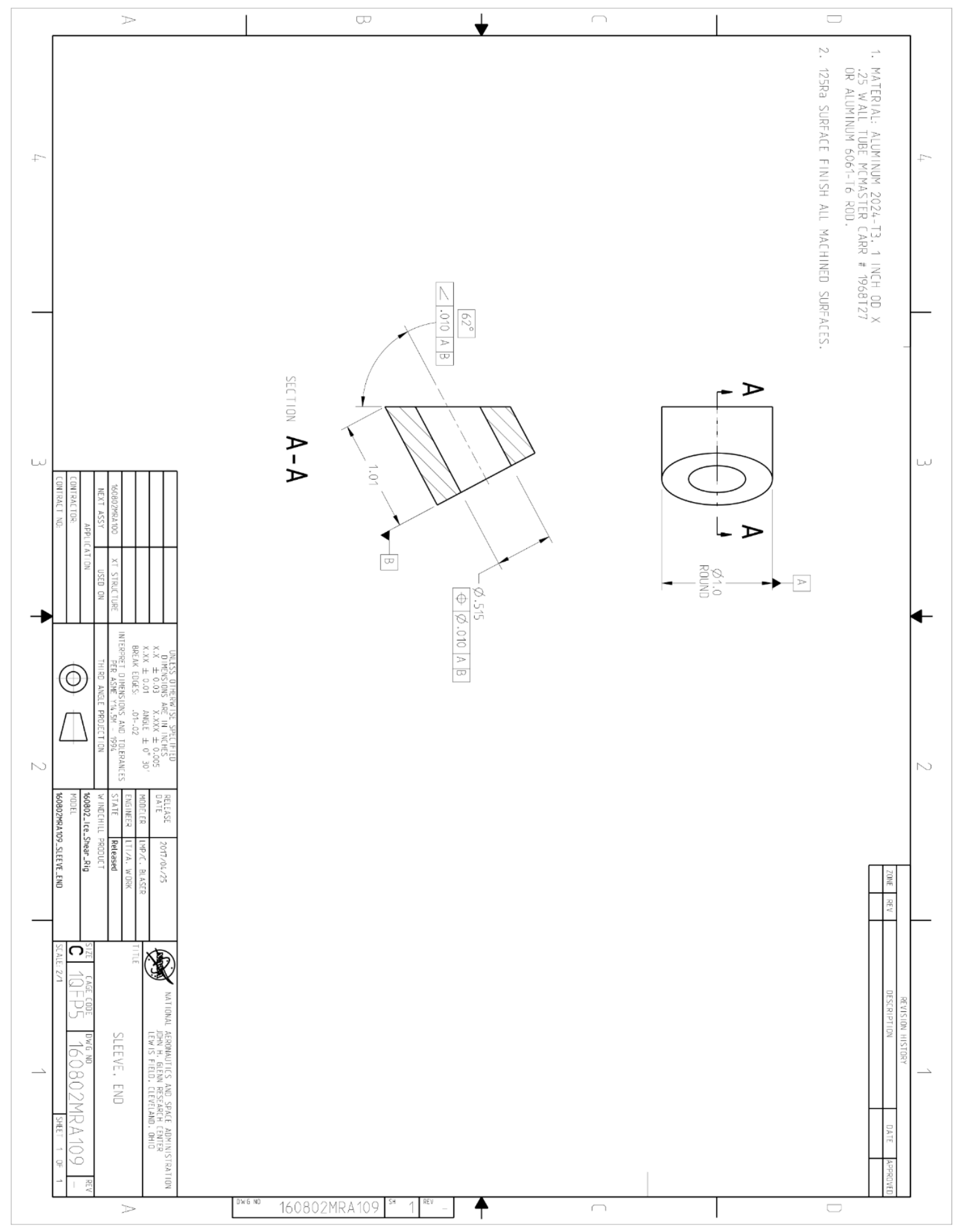




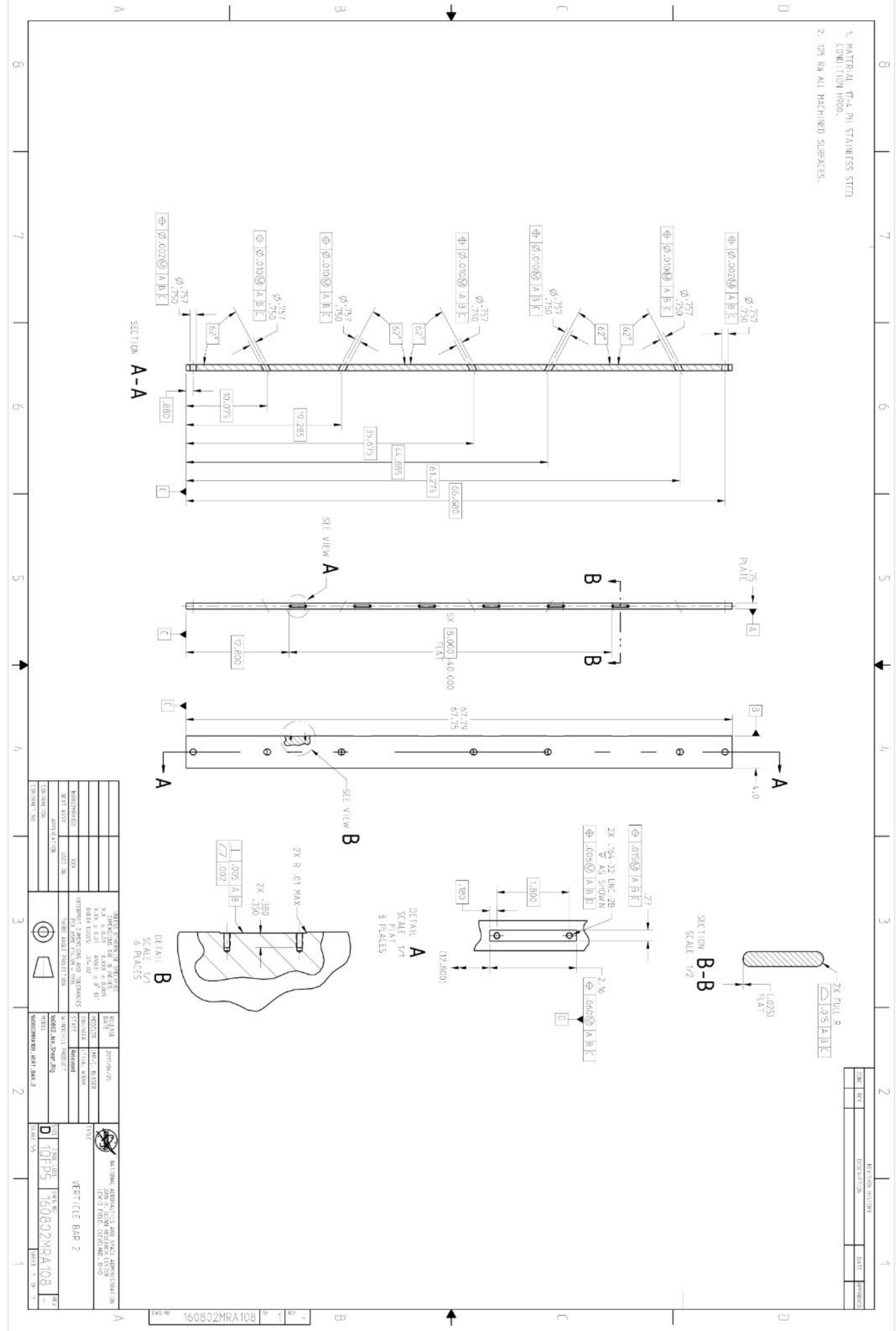




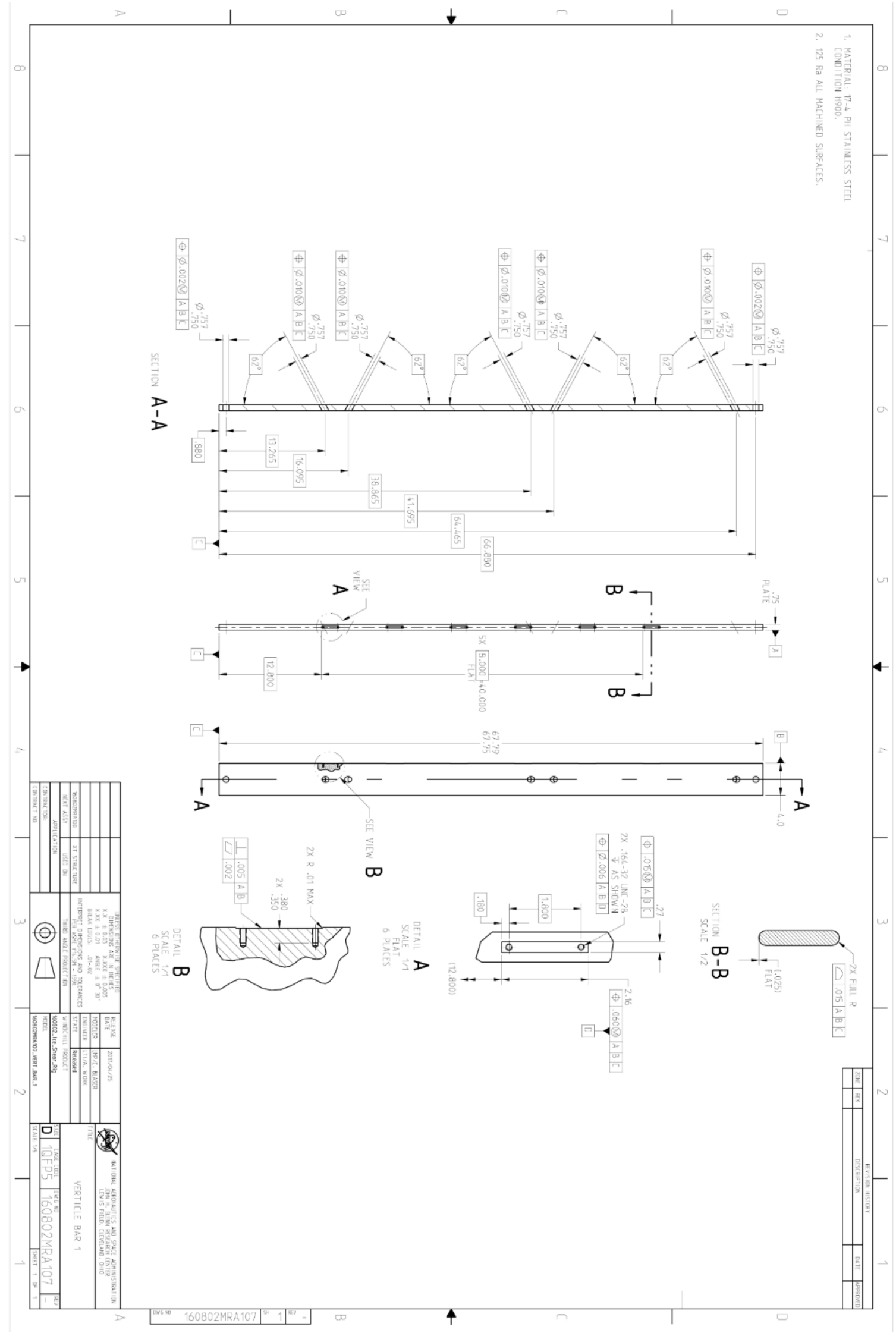




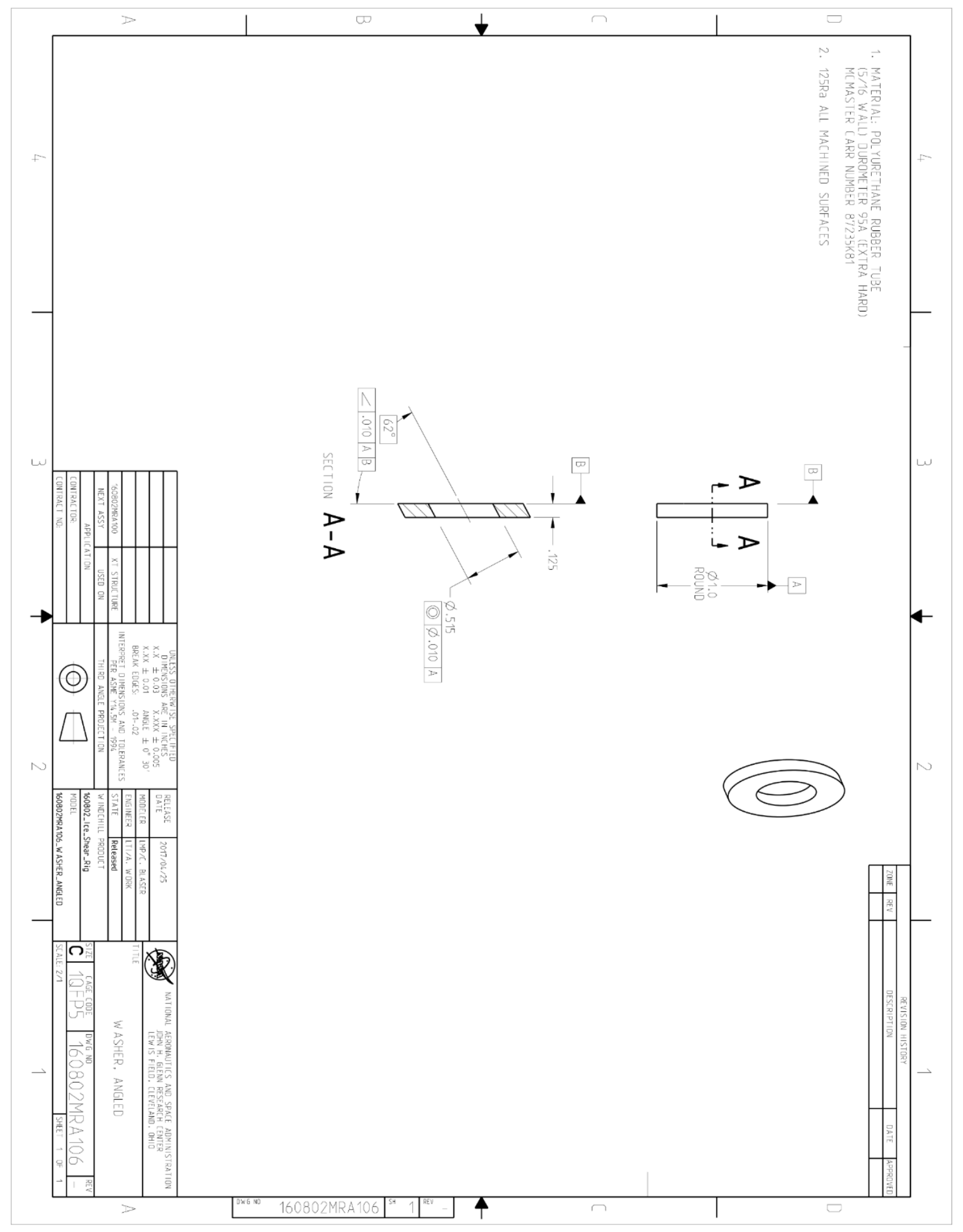




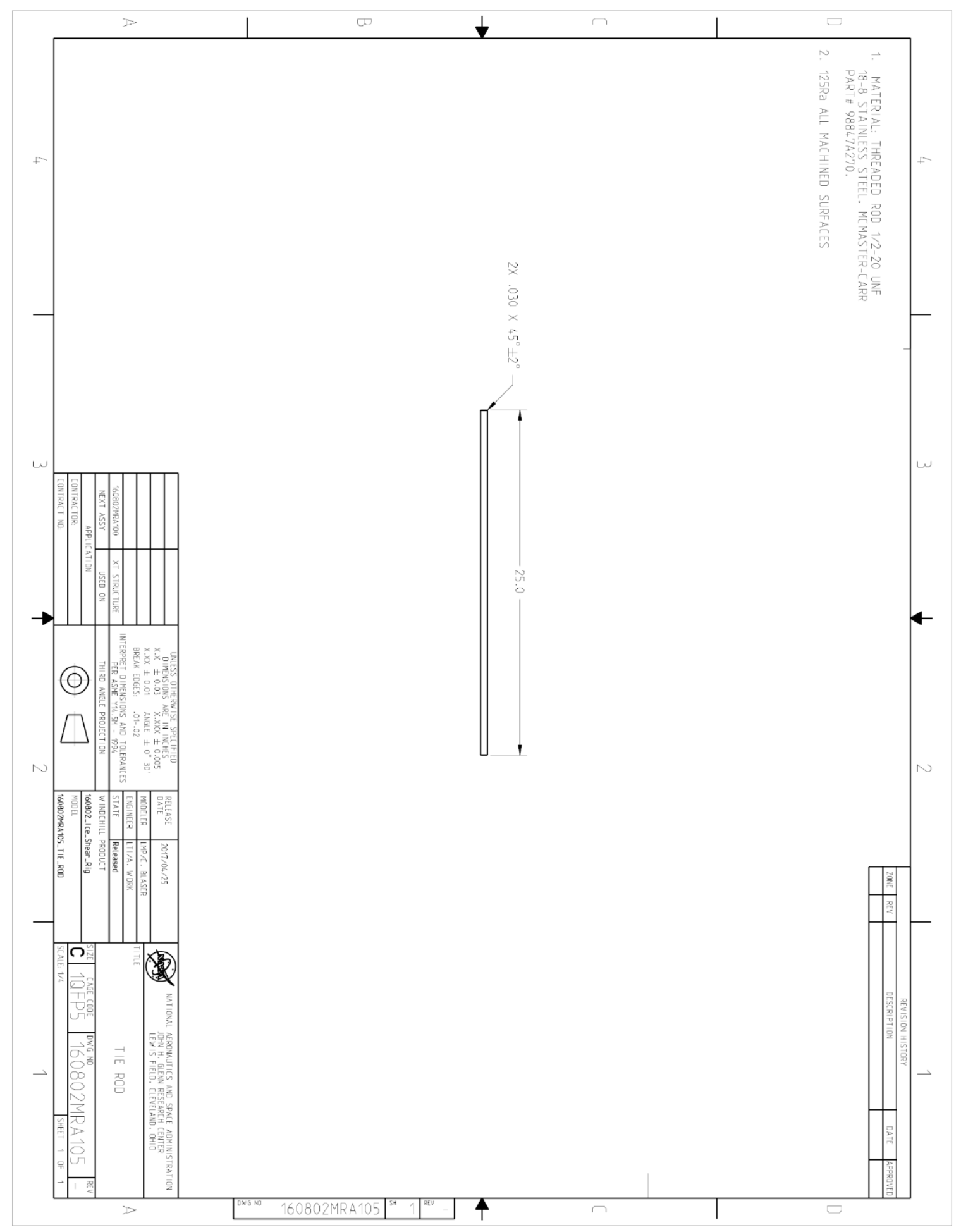




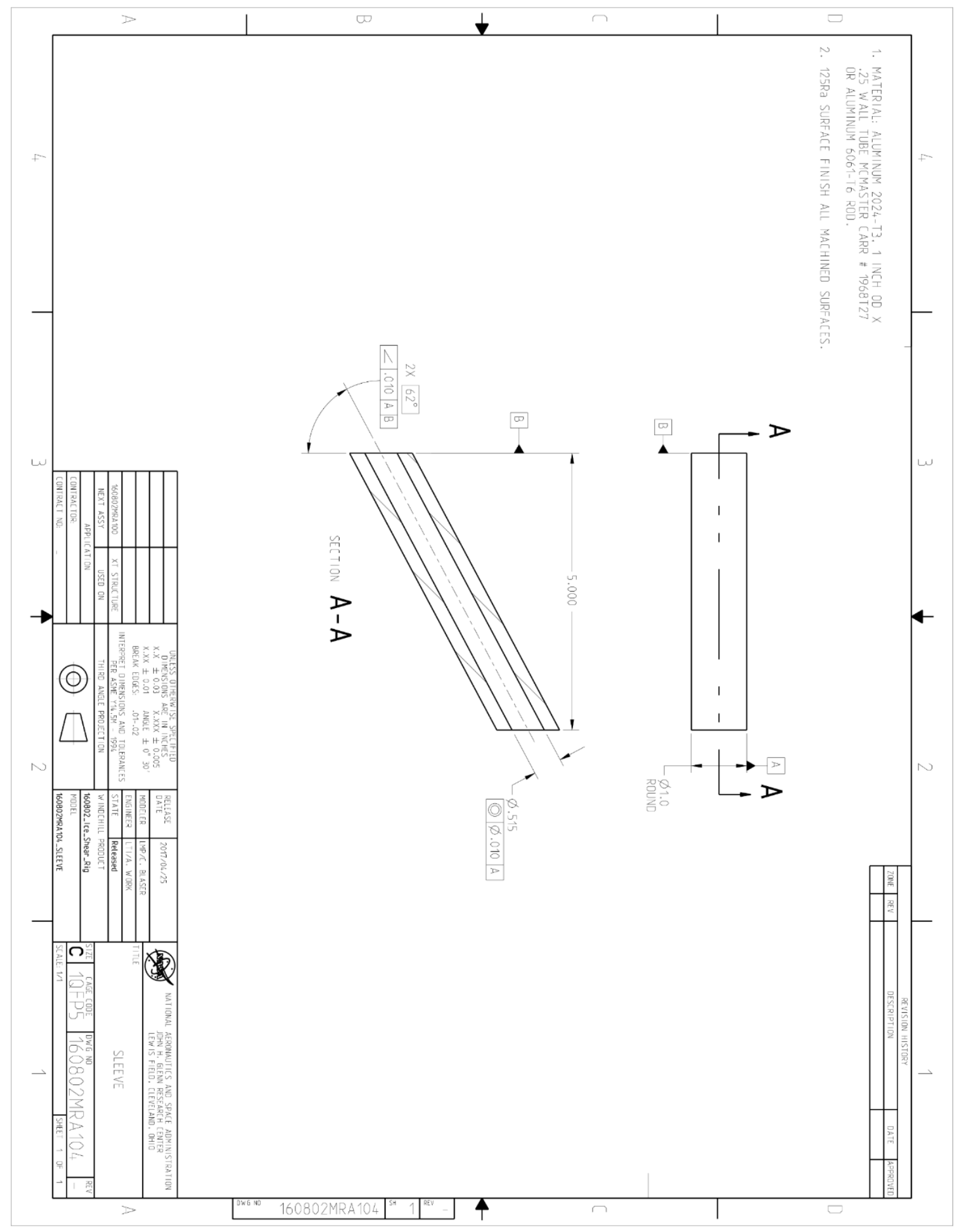




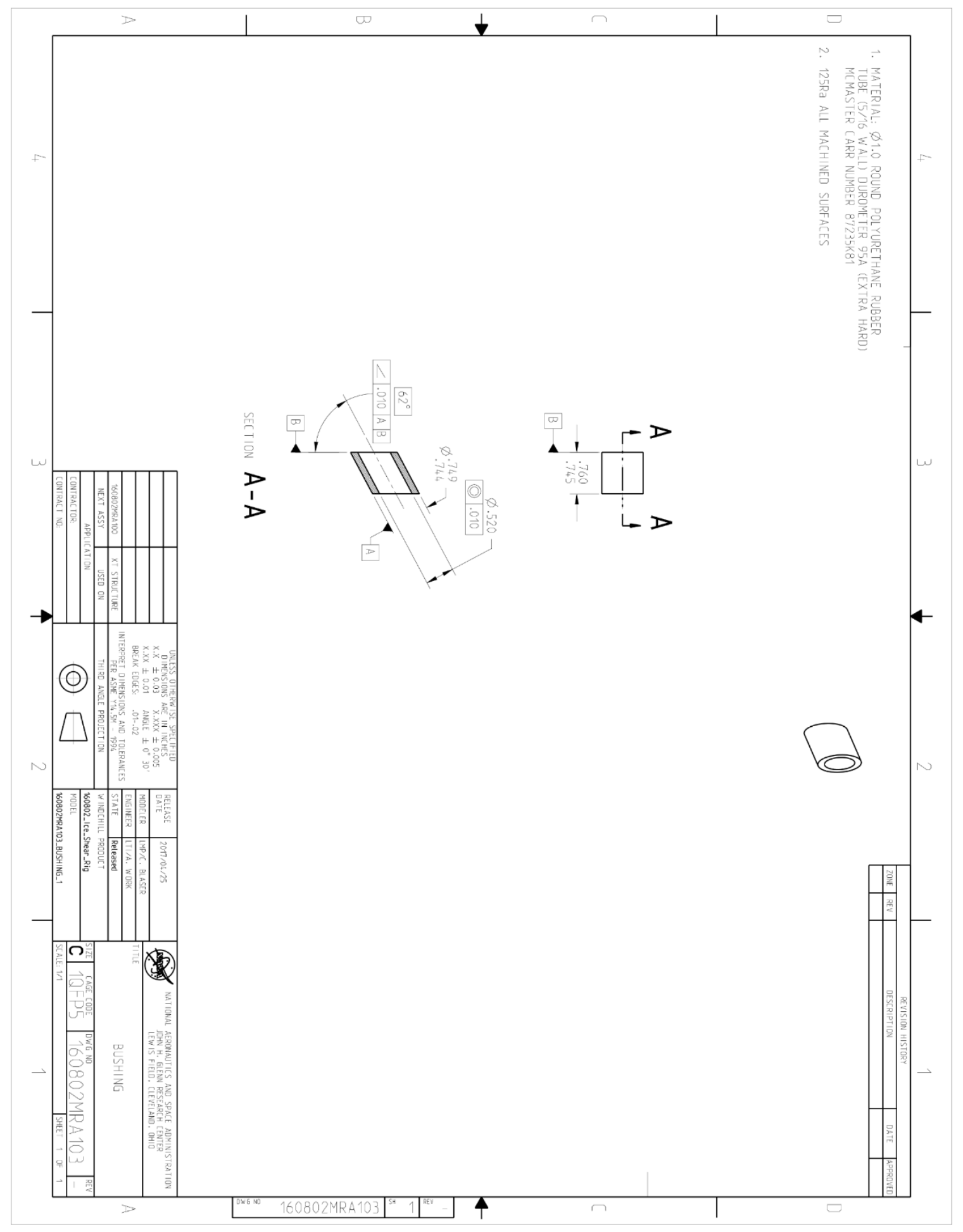




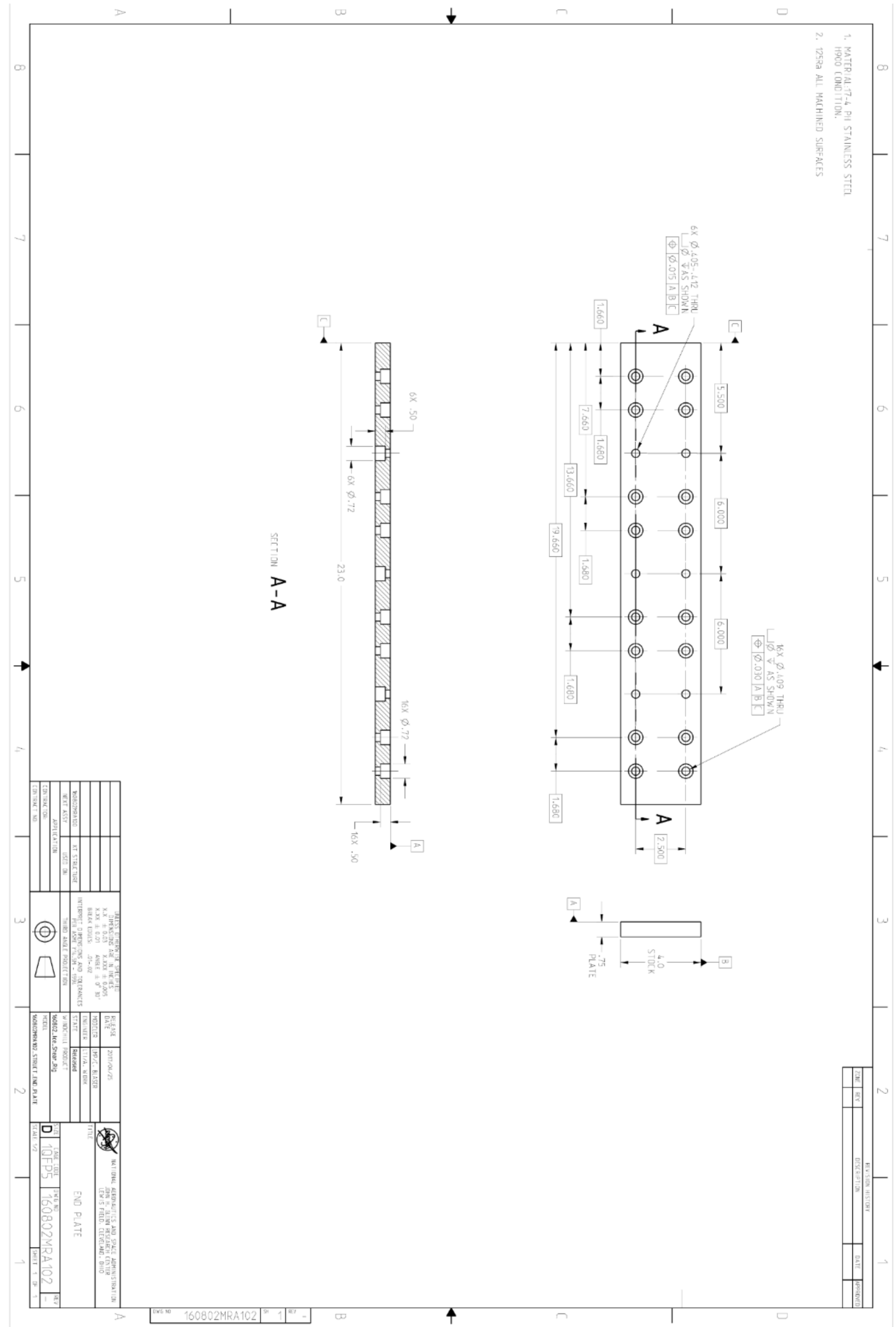




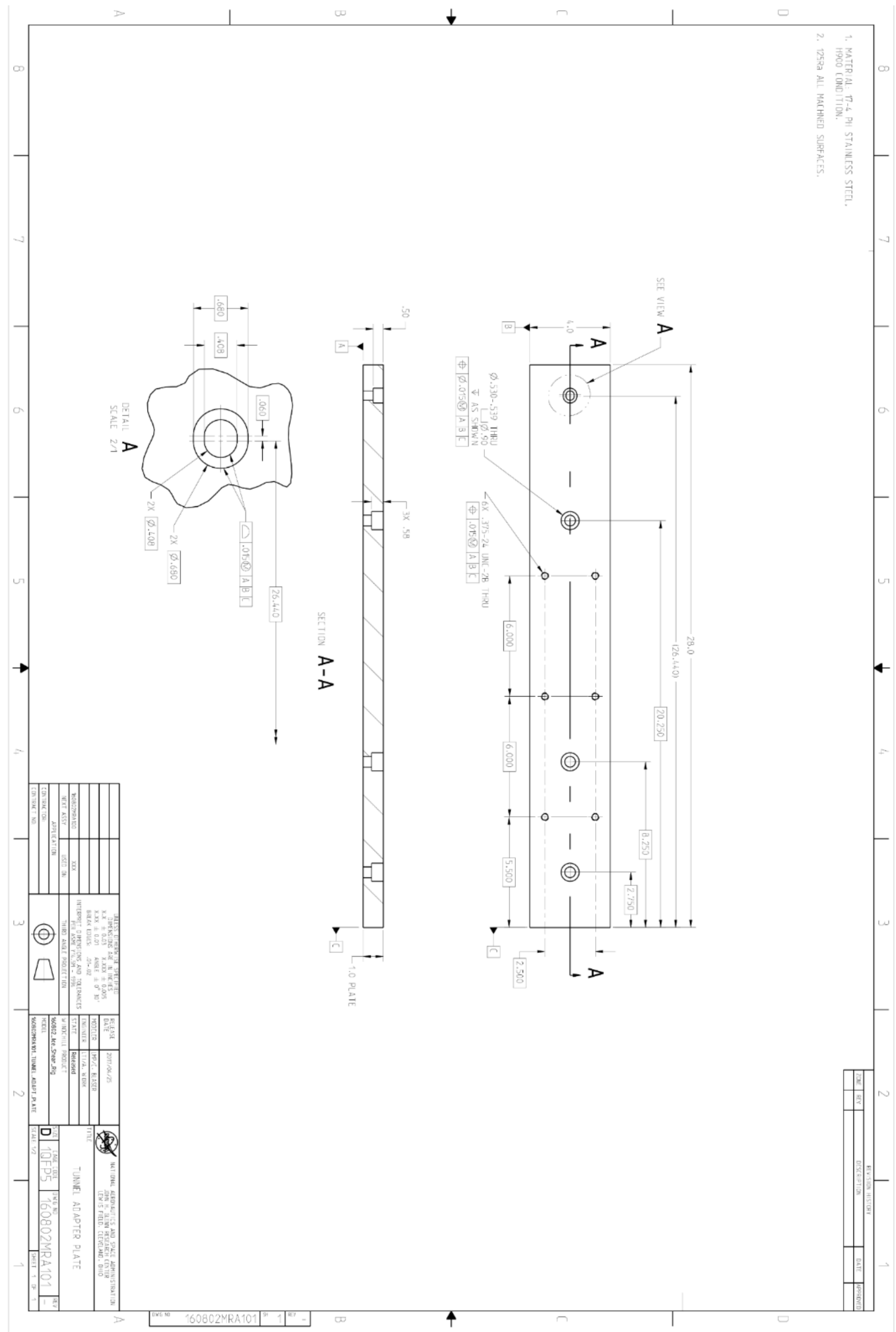




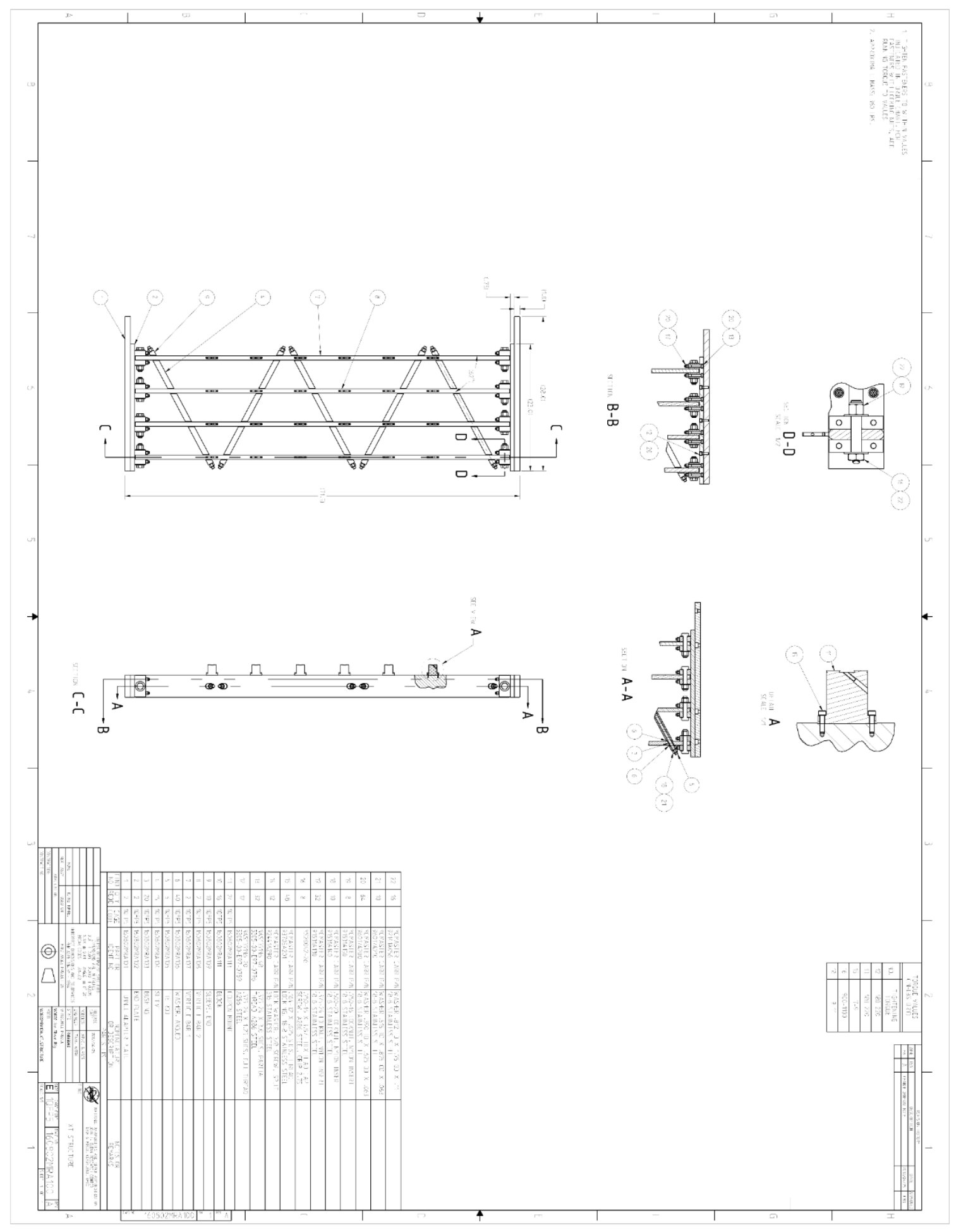




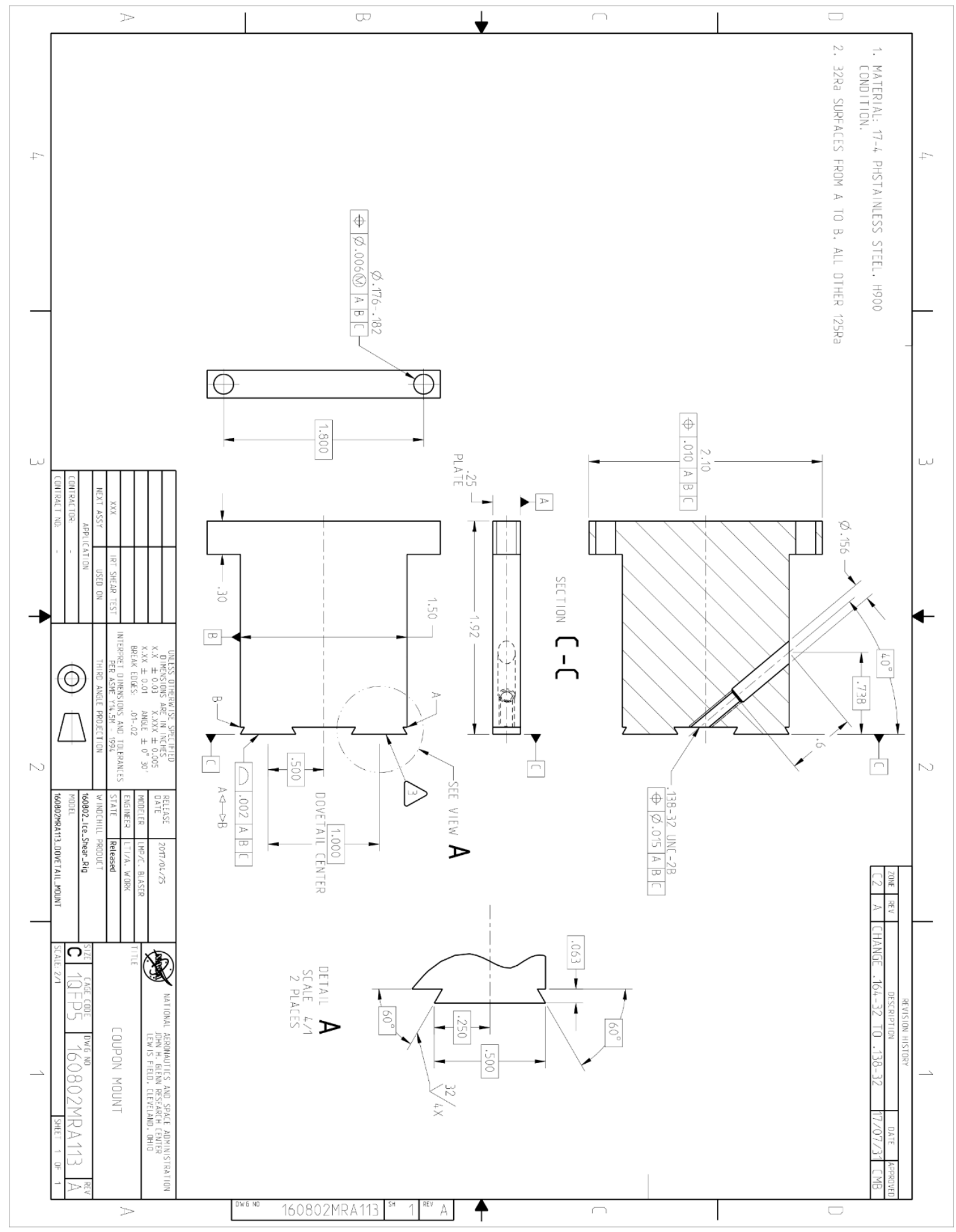




\section{APPENDIX D}

Shear Rig Drawings 


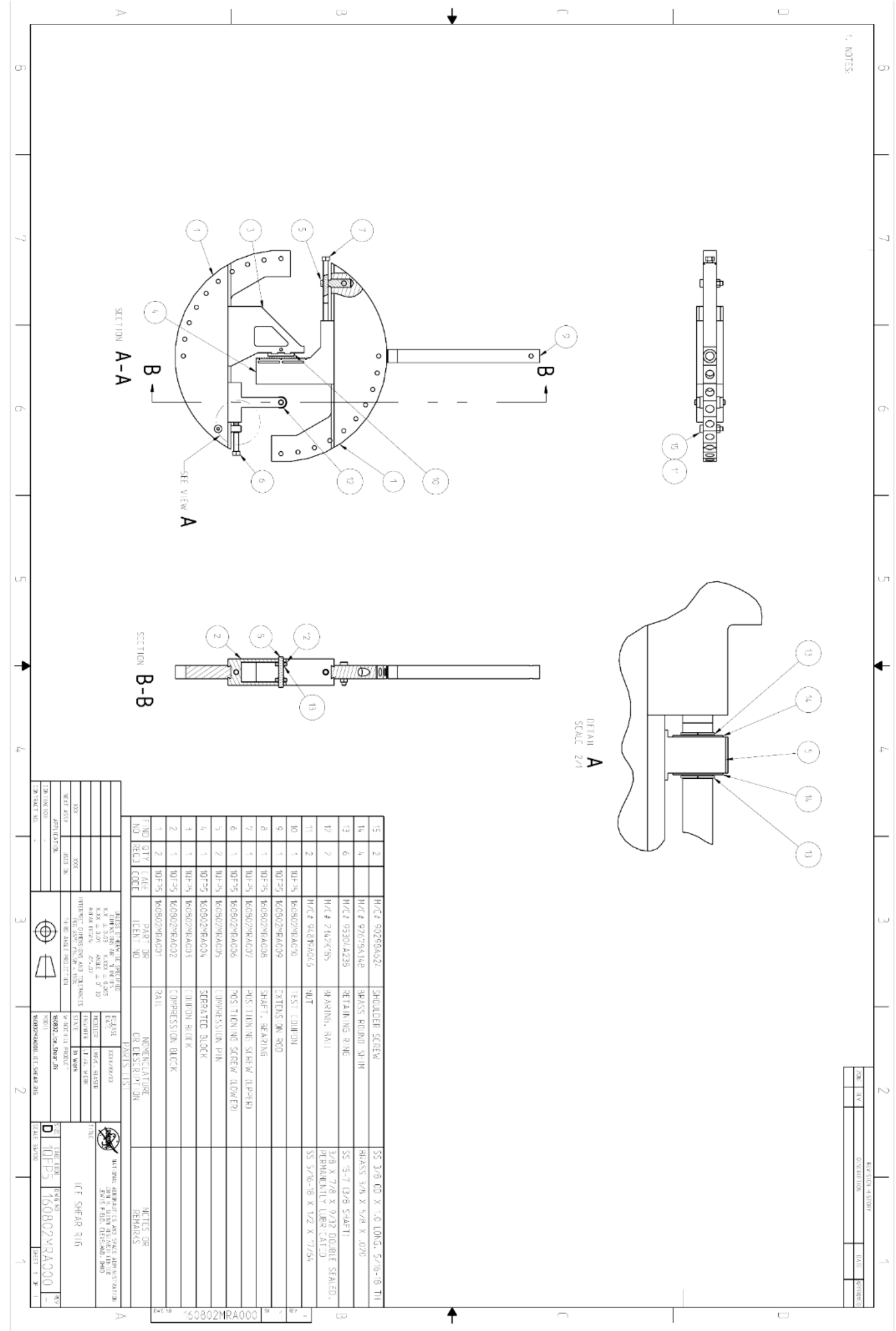




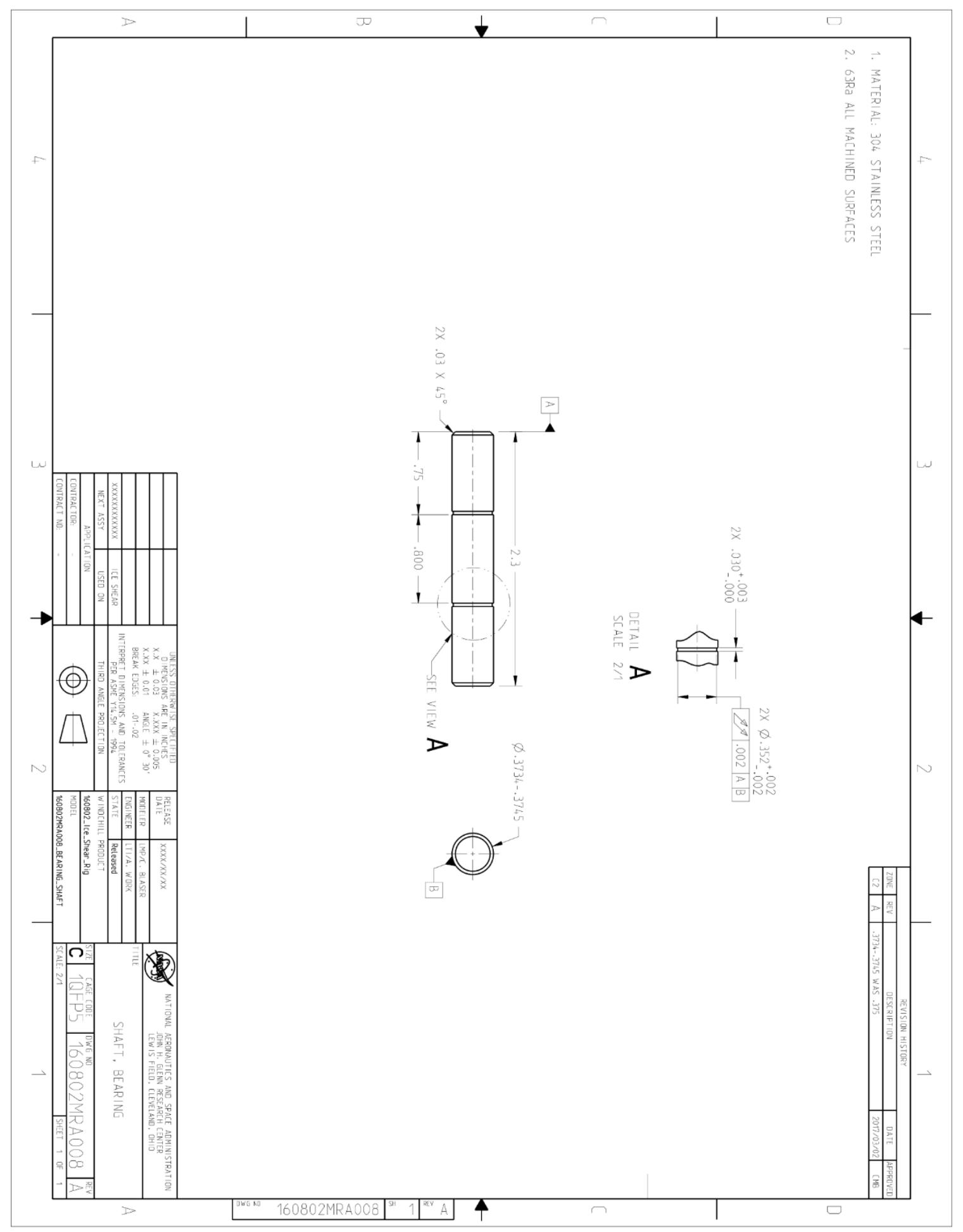




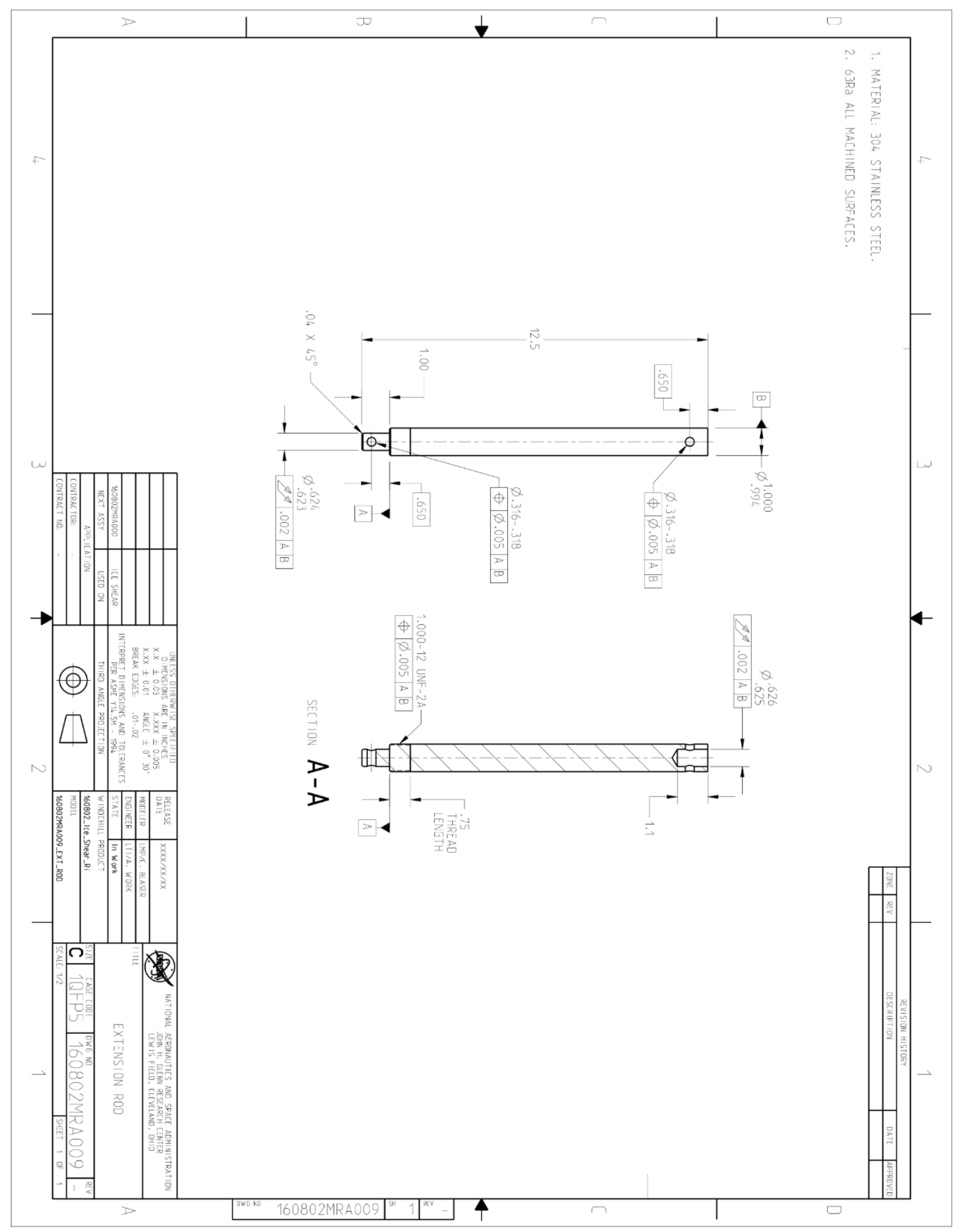




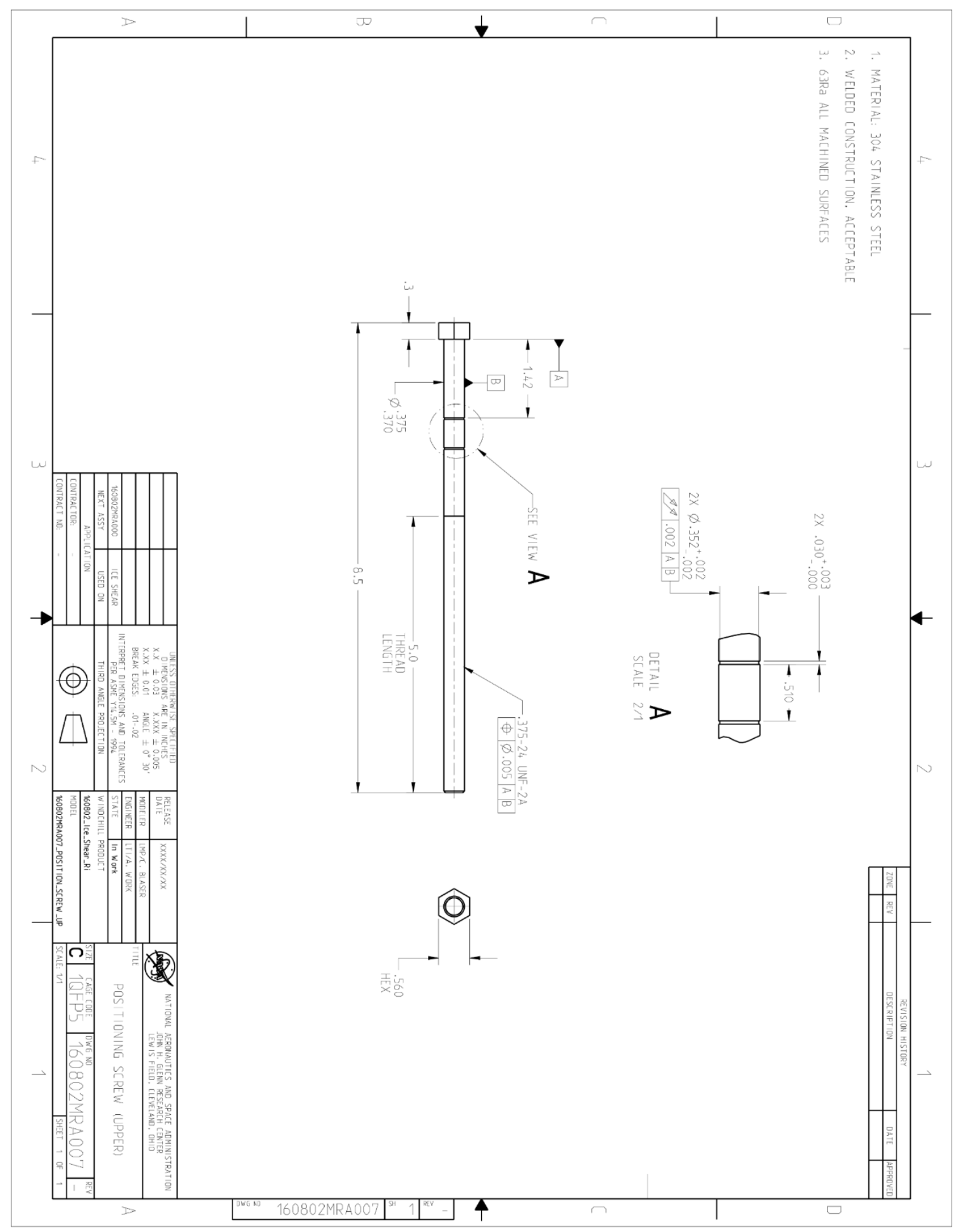




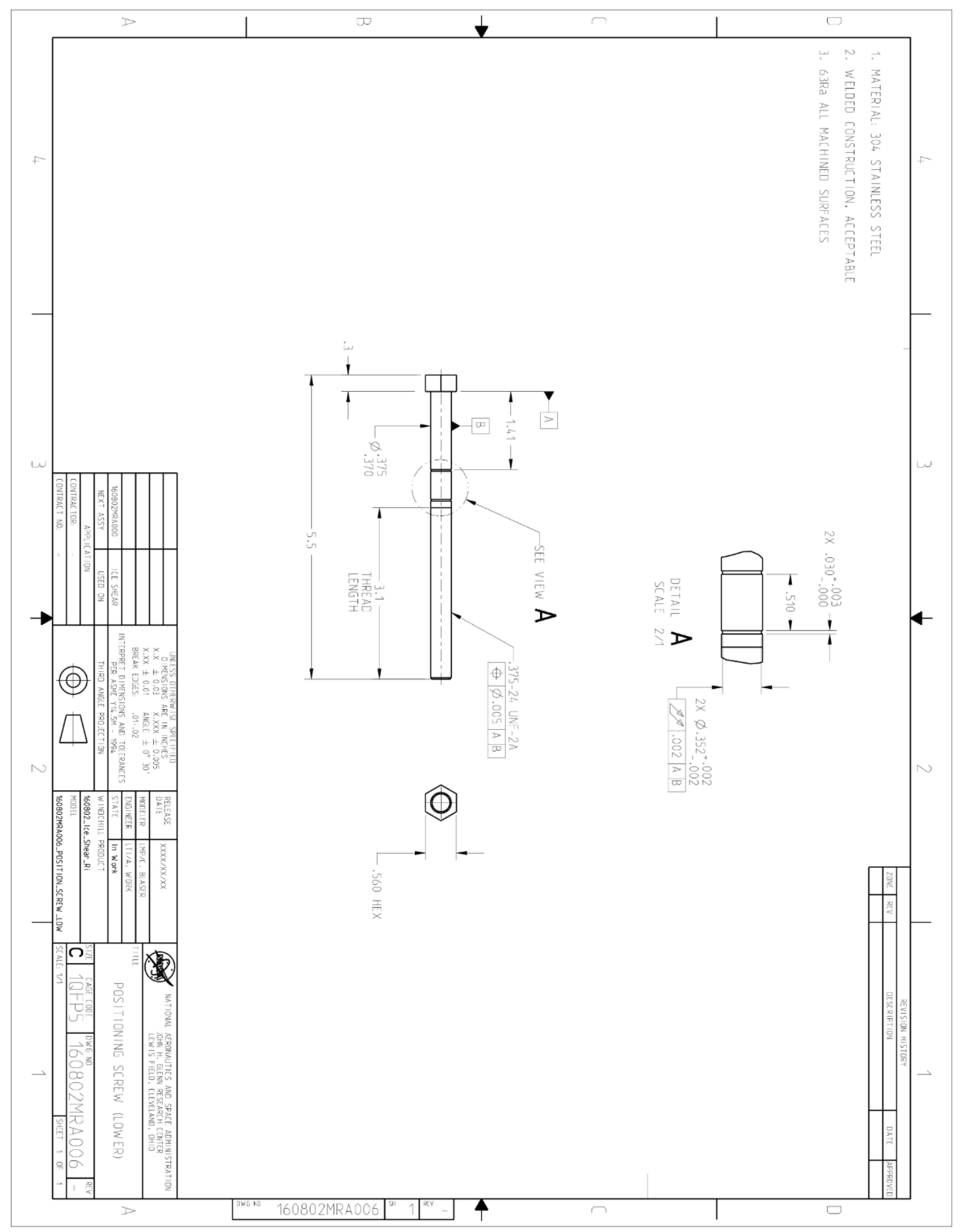




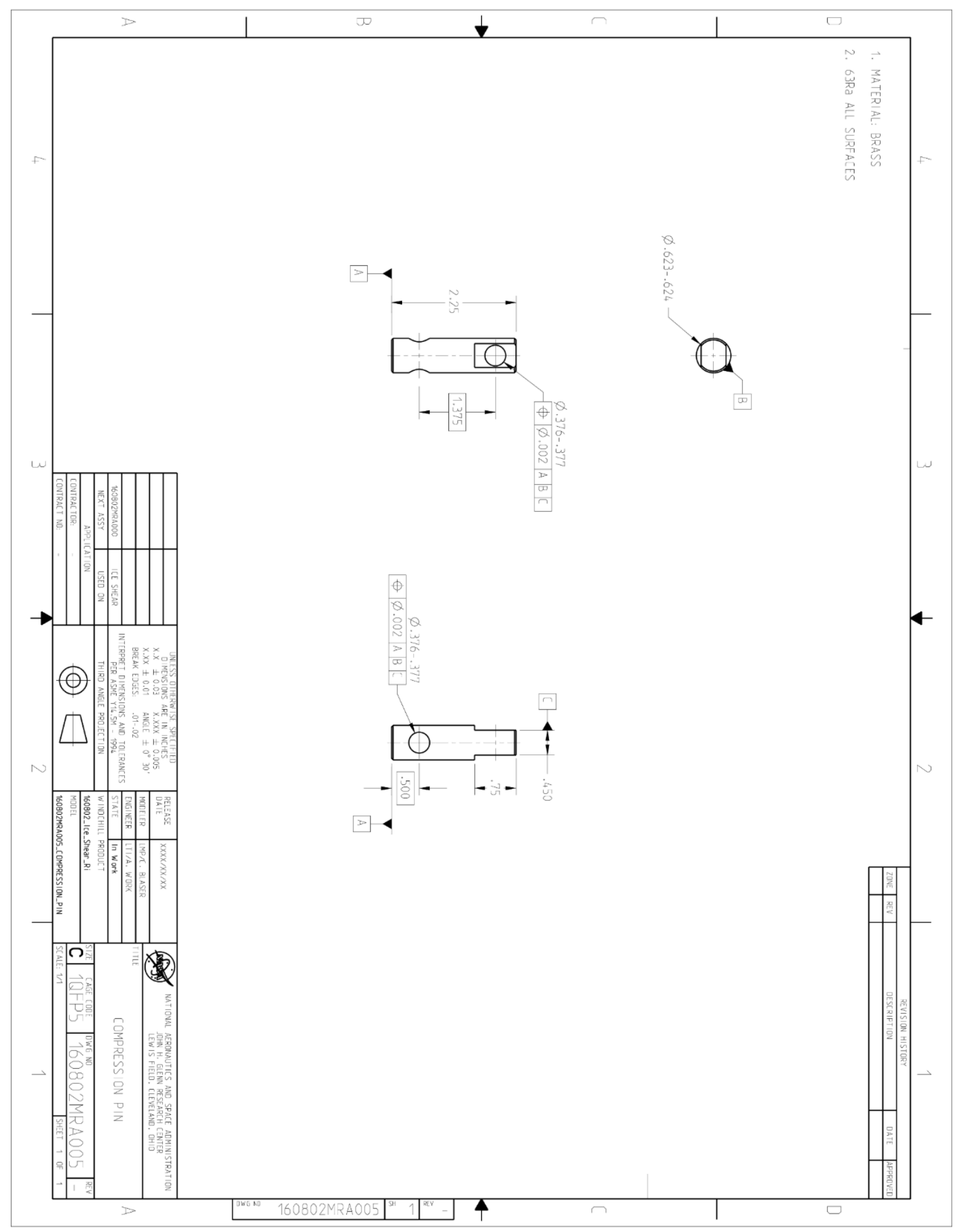




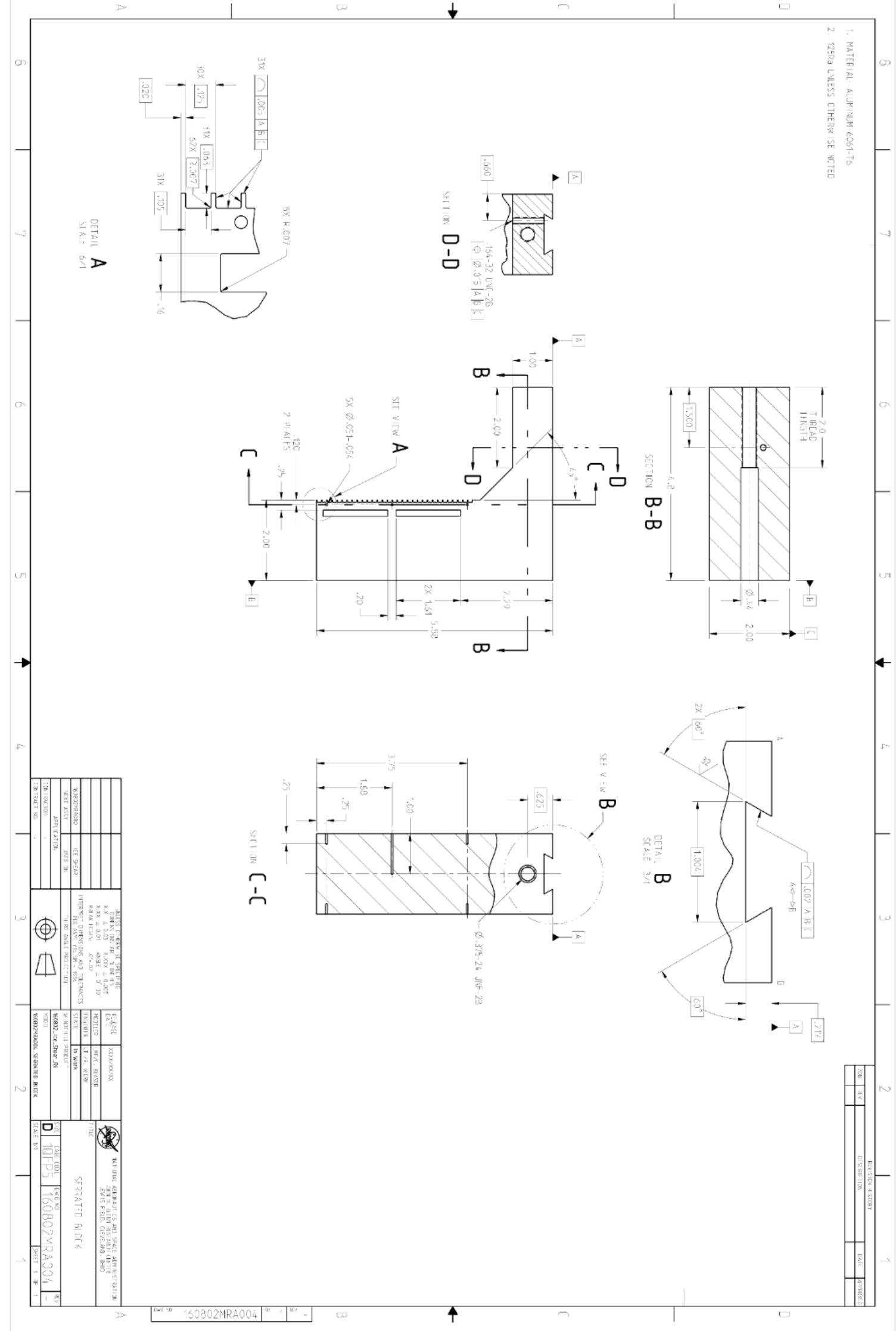




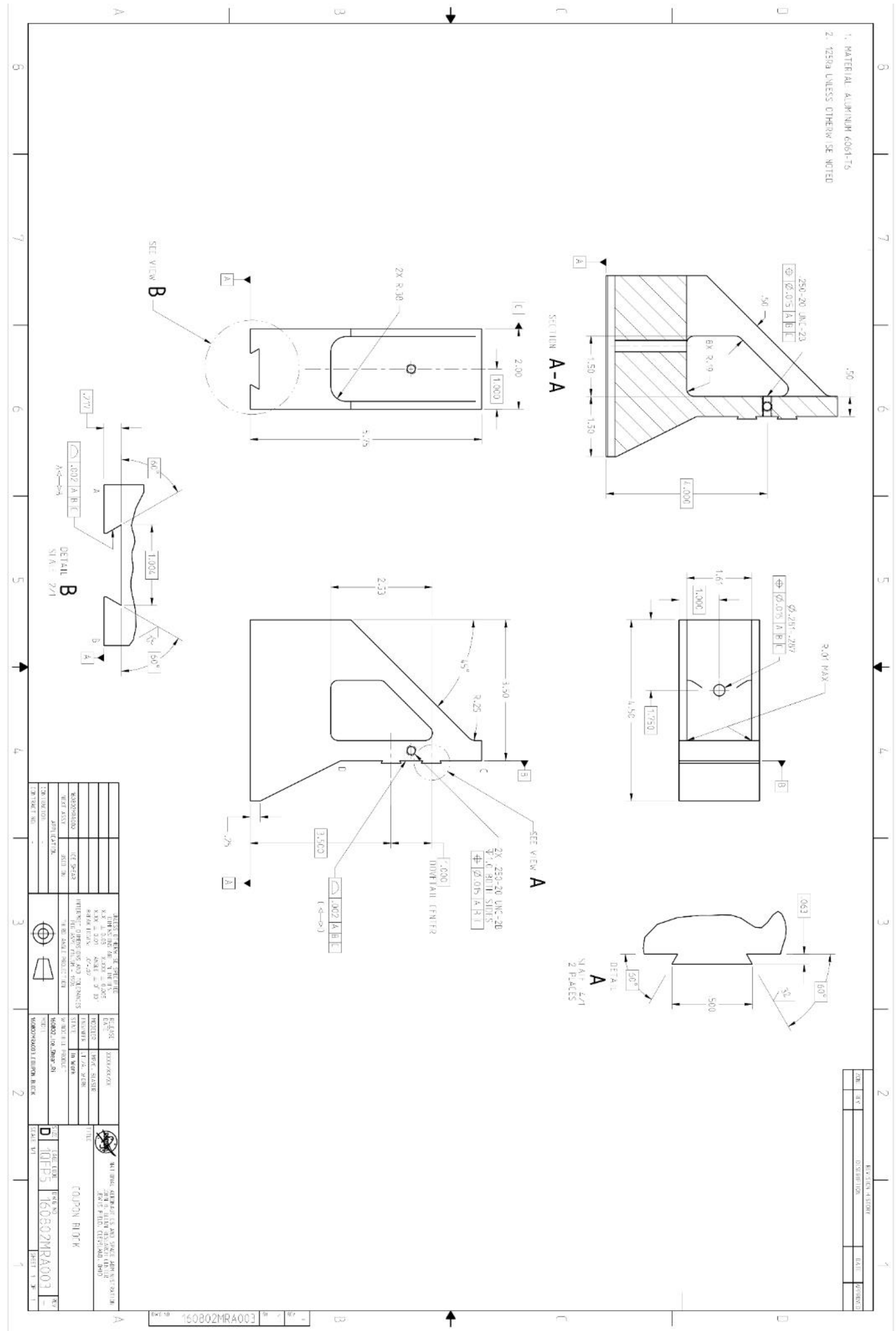




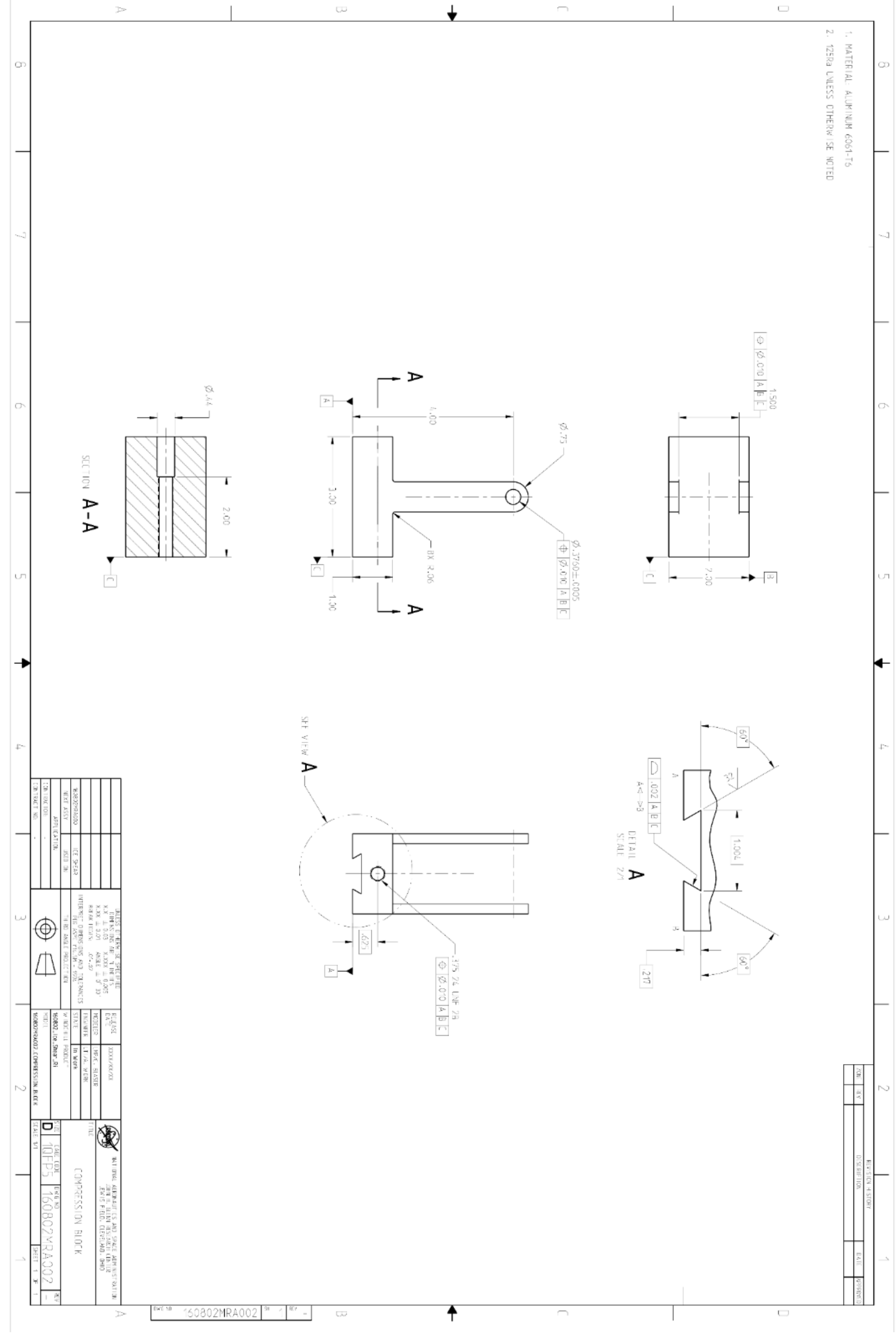




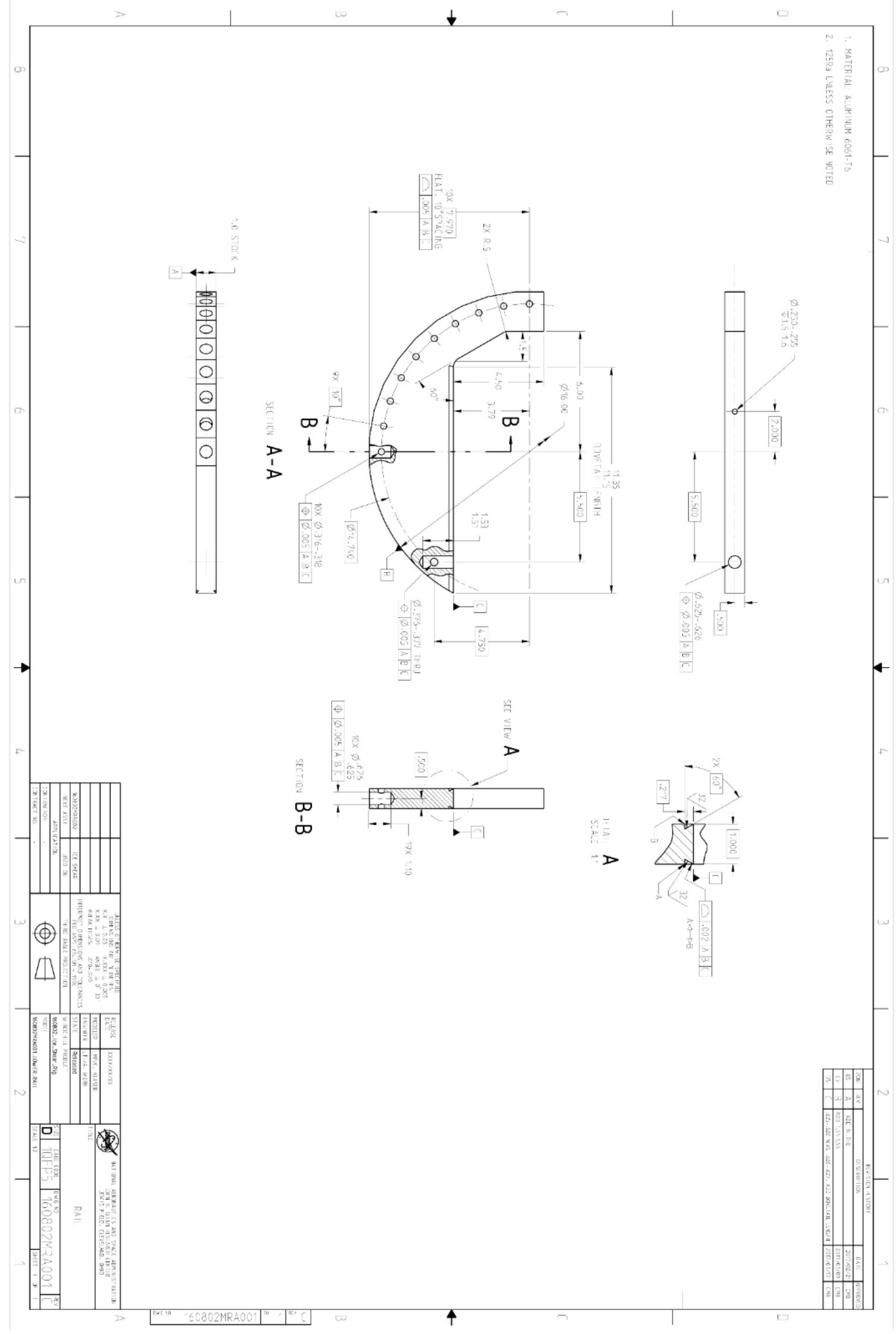




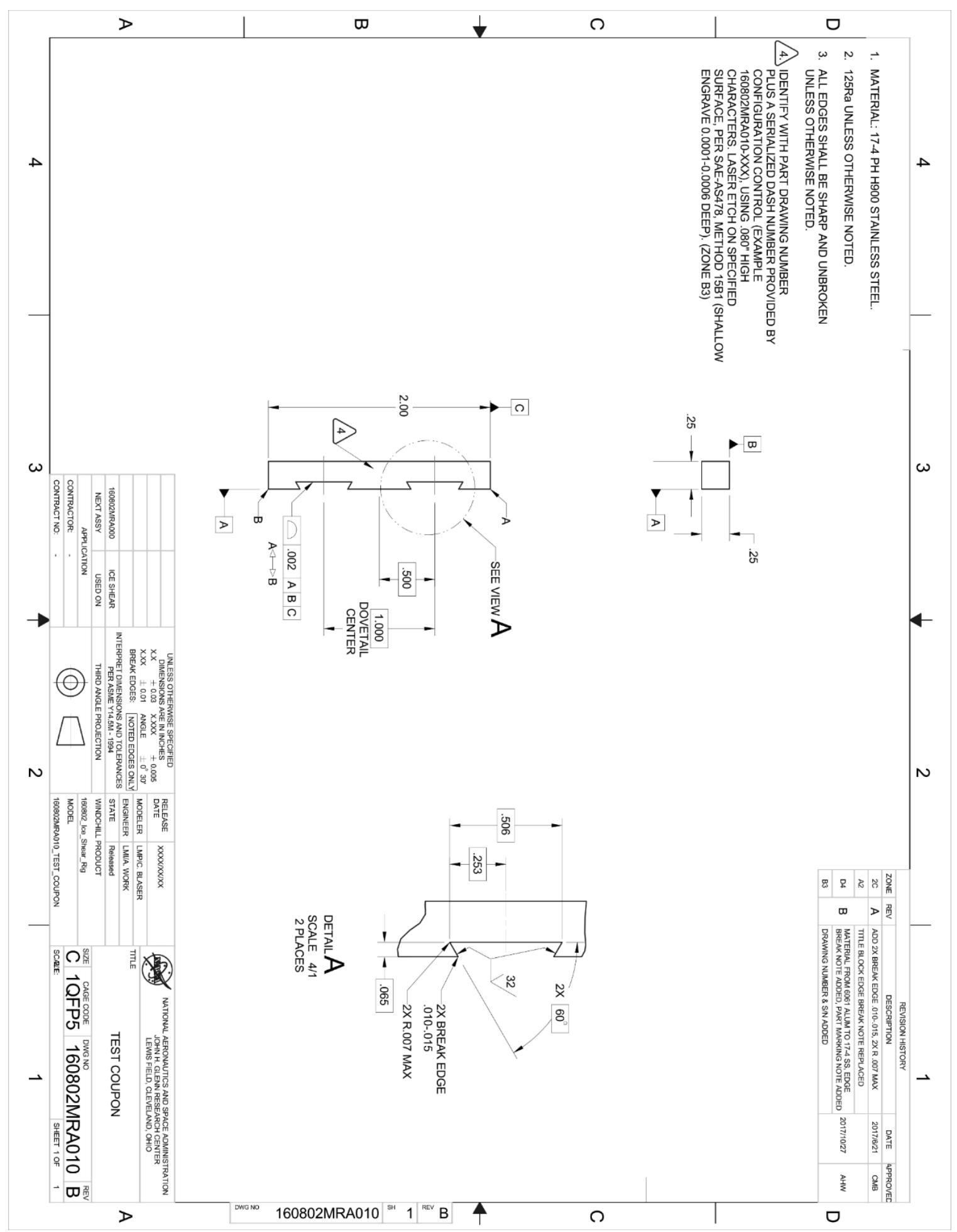




\section{CURRICULUM VITA}

Andrew H. Work Jr.

Phone: (502)-648-8186 or Email: andrew@wrks.us

Professional Experience

Ohio Aerospace Institute

Senior Researcher working in NASA GRC Icing Branch

Cleveland, $\mathrm{OH}$

- Developed test methods to measure the adhesion strength of ice and evaluate icephobic surfaces for aircraft safety, including the design of a new shear test fixture, leading to improved understanding of the roles of thermal contraction in the adhesion process

- $\quad$ Planned and led complex IRT test campaign including 3 tests and numerous piggyback tests, including the design of the XT Materials wind tunnel model used for the tests

- Designed and established new laboratory including acquiring two lab spaces at NASA GRC, procuring and repairing used and new equipment, associated LabVIEW programming, as well as designing, building, and implementing custom laboratory equipment

- Analyzed high speed imaging of ice shedding and modeled shed ice trajectories

- Mentored and co-mentored 7 interns

University of Louisville

Research Assistant, Graduate Teaching Assistant

Louisville, KY

- Ran multiphase fluid dynamics simulations of droplet splashes to investigate dynamics of splashing and interaction with surface roughness while utilizing FORTRAN code

- Ran experiments on Rapid Electrokinetic Patterning (for Master's thesis)

- Designed experiments for undergraduate laboratory, instructed the laboratory sessions, and graded experimental write-ups

- Grader for Fluid Dynamics and Thermodynamics courses

- Three undergraduate co-ops at Hamilton Sundstrand and one graduate internship at NASA GRC from 2009-2010.

Education

University of Louisville

Doctor of Philosophy in Mechanical Engineering

Louisville, KY

- Completed candidacy requirements with 4.000/4.0

- Hsing Chuang Award for Excellence in Graduate Study

- Pi Tau Sigma Award

- University Fellowship

University of Louisville

Master of Engineering in Mechanical Engineering May 2012

Louisville, KY

- Completed requirements for M.Eng. and M.S. degrees with 4.000/4.0

University of Louisville

Bachelor of Science in Mechanical Engineering, May 2011

Louisville, KY

- Graduated 3.465/4.0

- Passed FE Exam 


\section{Publications and Presentations}

- $\quad$ Work, A., Kreeger, R. E., Gyekenyesi, A., Vargas, M., Drabiak, D., An Experimental Study of the Adhesion Strength of Impact Ice, AIAA Aviation 2018 (in progress)

- Work, A., Lian, Y., A Critical Review of the Measurement of Ice Adhesion to Solid Substrates

- Work, A., Vargas, M., Lian, Y., A Simple Thermoelectric Droplet Generator (accepted with minor revisions 2-20-18)

- $\quad$ Kreeger, R. E., Work, A., Douglass, R., Gazella, M., Koster, Z., \& Turk, J. Analysis and Prediction of Ice Shedding for a Full-Scale Heated Tail Rotor. AIAA Aviation (2016)

- Work, A., \& Williams, S. J. Characterization of 2D colloids assembled by optically-induced electrohydrodynamics. Soft Matter (2015)

- Work, A., \& Williams, S. J. Characterization of 2D colloid aggregations created by optically-induced electrohydrodynamics. Electrophoresis (2015)

- Work, A., Lian, Y., \& Sussman, M. The Effect of Disturbances and Surrounding Air on the Droplet Impact Phenomena, Bulletin of the American Physical Society 58 (2013)

- Velasco, V., Work, A., \& Williams, S. J. Electrokinetic Concentration and Patterning of Colloids with a Scanning Laser, Electrophoresis 33.13 (2012): 1931-1937

- Work, A., Velasco, V., \& Williams, S. J. Electrokinetic Manipulation of Colloids with a Scanning Laser. IMECE (2012), Nov. 9-15, Houston, Texas

- $\quad$ AH Work, Jr., V Velasco, and SJ Williams. Rapid electrokinetic patterning (REP) of hydrosol colloids at a planar electrode, AIChE Annual Meeting, Annual Meeting of the American Electrophoresis Society (2012), Oct. 28 - Nov. 2, Pittsburgh, PA

- Work, A., Velasco, V., \& Williams, S. J. Patterning Simple Geometries with Colloids Using a Scanning Laser. AIChE (420g) (2012). Oct. 28-Nov. 2. Pittsburgh. PA

- V Velasco. AH Work. Jr.. and SJ Williams. Rapid electrokinetic self-assemblv and patterning of colloids, $1^{\text {st }}$ Annual Kentucky Nanotechnology Symposium (2012), Bowling Green, KY, March 30-31 